\title{
Asymmetric Hydrogenation of Racemic Allylic Alcohols via an Isomerization-Dynamic Kinetic Resolution Cascade
}

Kun Wang, ${ }^{\dagger}$ Saisai Niu, ${ }^{\dagger}$ Xin Guo,${ }^{\dagger}$ Weijun Tang, ${ }^{*}, \dagger$ Dong Xue,${ }^{\dagger}$ Jianliang Xiao, ${ }^{\star}$ Huaming Sun ${ }^{\dagger}$, Chao Wang ${ }^{*}, \dagger$

† Key Laboratory of Applied Surface and Colloid Chemistry, Ministry of Education, School of Chemistry and Chemical Engineering, Shaanxi Normal University, Xi'an, 710062, China

${ }^{\star}$ Department of Chemistry, University of Liverpool, Liverpool, L69 7ZD, UK

\section{Supporting Information}

\section{Table of Contents}

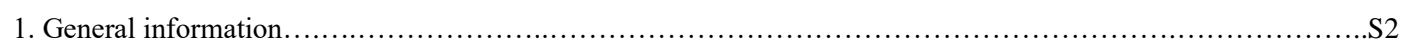

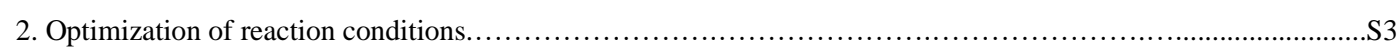

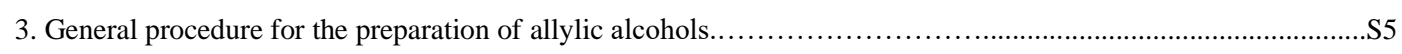

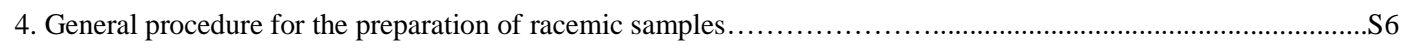

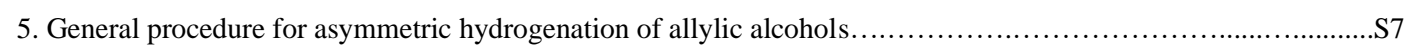

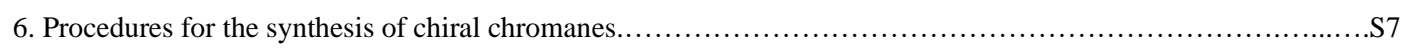

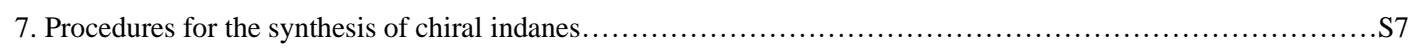

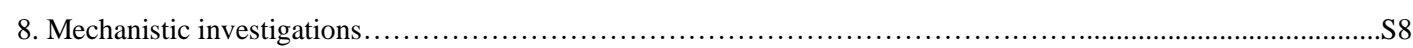

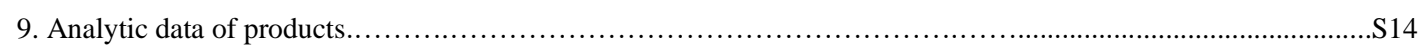

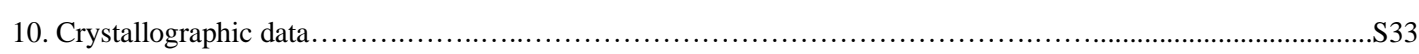

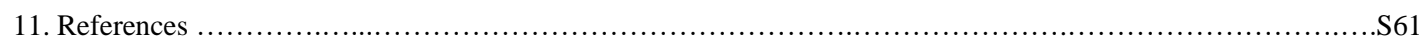

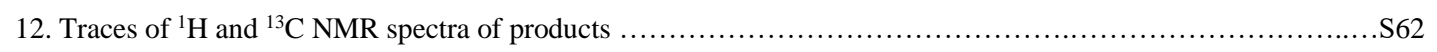

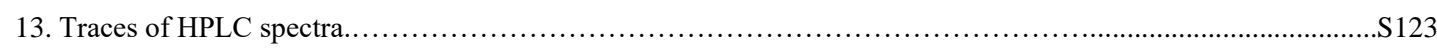




\section{General information}

Unless otherwise specified, the chemicals were obtained commercially and used without further purification. Toluene was refluxed over $\mathrm{Na} /$ benzophenone and distilled under an argon atmosphere. Solvents (ethyl acetate, petroleum ether) used for column chromatography were of technical grade and used after distillation. Analytical thin-layer chromatography (TLC) was conducted with TLC plates (Silica gel 60 F254, Qingdao Haiyang). Flash column chromatography was performed on silica gel 200-300 mesh with freshly distilled solvents. NMR spectra were recorded on a Bruker $400 \mathrm{~Hz}$ NMR spectrometer. HRMS data were recorded on a maXis UHR-TOF mass spectrometer. For reaction condition optimization, the diastereoselectivities were determined by ${ }^{1} \mathrm{H}$ NMR and the enantioselectivities were determined by chiral phase HPLC analysis on a SHIMAZU LC-2010A HT HPLC machine. For substrate scope studies, the diastereo- and enantioselectivities of products were determined by chiral phase HPLC analysis on a SHIMAZU LC-2010A HT HPLC machine (3a-3m, 3o-3af, 5a-5x and 7-11) and Thermo Scientific DIONEX UltiMate U300 HPLC machine (3n). Single-crystal X-Ray diffraction data was collected on a Bruker D8 venture diffractometer $(\mathrm{Cu} / \mathrm{K} \alpha, \lambda=1.5418 \AA)$ at $153 \mathrm{~K}$. The catalysts were purchased from Tokyo Chemical Industry (TCI) (2a, 2b and 2d-2f) and Strem Chemials (2c). 


\section{Optimization of reaction conditions}

Table S1. The effect of different chiral catalysts and the amount of catalyst<smiles>Cc1ccc(C=C2CCc3ccccc3C2O)cc1</smiles>

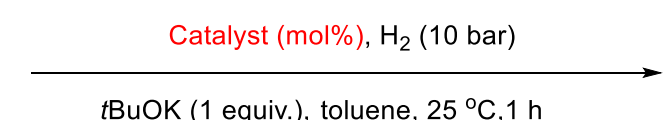<smiles>Cc1ccc(C[C@@H]2CCc3ccccc3[C@@H]2O)cc1</smiles>

$1 \mathrm{a}$

tBuOK (1 equiv.), toluene, $25^{\circ} \mathrm{C}, 1 \mathrm{~h}$

3a

Catalyst loading (mol\%)

${ }^{a}$ Reaction conditions: 1a $(0.25 \mathrm{mmol}), t$ BuOK $\left(1\right.$ equiv.), toluene $(0.8 \mathrm{~mL}), \mathrm{H}_{2}(10 \mathrm{bar}), 25{ }^{\circ} \mathrm{C}, 1 \mathrm{~h} ;{ }^{b}$ Determined by ${ }^{1} \mathrm{H}$ NMR analysis with 1,3,5-trimethoxybenzene as the internal standard; ${ }^{c}$ The $d r$ value was determined by ${ }^{1} \mathrm{H}$ NMR spectroscopy of the crude reaction mixture; ${ }^{d}$ The $e e$ value was determined by HPLC analysis. 
Table S2. The effect of different bases and the amount of base<smiles>Cc1ccc(C=C2CCc3ccccc3C2O)cc1</smiles>
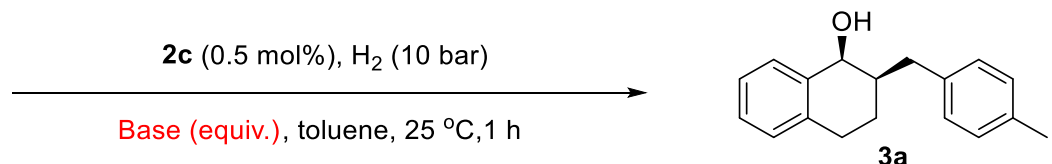

\begin{tabular}{cccccc}
\hline Entry $^{a}$ & Base & Base equivalent & ${\text { Yield }(\%)^{b}}^{b}$ & $\operatorname{dr}(\%)^{c}$ & ${\text { ee }(\%)^{d}}^{c}$ \\
\hline 1 & $t \mathrm{BuOK}$ & 1.0 & 92 & $99: 1$ & - \\
2 & $\mathrm{KOH}$ & 1.0 & trace & - & - \\
3 & $\mathrm{~K}_{2} \mathrm{CO}_{3}$ & 1.0 & 0 & - & - \\
4 & $\mathrm{NaOH}$ & 1.0 & 0 & $95: 5$ & 82 \\
5 & $t \mathrm{BuONa}$ & 1.0 & 12 & $98: 2$ & 93 \\
6 & $\mathrm{CsOH}$ & 1.0 & 10 & - & 82 \\
7 & $\mathrm{DBU}$ & 1.0 & 0 & $98: 2$ & 93 \\
9 & $t \mathrm{BuOK}$ & 0.5 & 50 & $99: 1$ & - \\
\hline
\end{tabular}

${ }^{a}$ Reaction conditions: 1a $(0.25 \mathrm{mmol}), 2 \mathrm{c}(0.5 \mathrm{~mol} \%)$, toluene $(0.8 \mathrm{~mL}), \mathrm{H}_{2}(10 \mathrm{bar}), 25{ }^{\circ} \mathrm{C}, 1 \mathrm{~h} ;{ }^{b}$ Determined by ${ }^{1} \mathrm{H}$ NMR analysis with 1,3,5-trimethoxybenzene as the internal standard; ${ }^{c}$ The $d r$ value was determined by ${ }^{1} \mathrm{H}$ NMR spectroscopy of the crude reaction mixture; ${ }^{d}$ The $e e$ value was determined by HPLC analysis.

Table S3. The effect of the hydrogen pressure<smiles>Cc1ccc(C=C2CCc3ccccc3C2O)cc1</smiles>
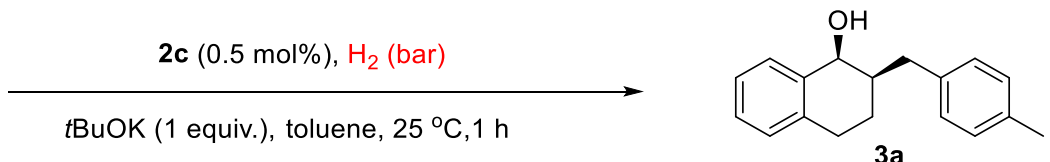

\begin{tabular}{ccccc}
\hline Entry $^{a}$ & $\mathrm{H}_{2}(\mathrm{bar})$ & ${\text { Yield }(\%)^{b}}^{b}$ & $\mathrm{dr}(\%)^{c}$ & ${\operatorname{ee~}(\%)^{d}}^{c}$ \\
\hline 1 & 10 & 92 & $99: 1$ & 93 \\
2 & 20 & 94 & $>99: 1$ & 94 \\
3 & 30 & 96 & $>99: 1$ & 94 \\
4 & 40 & 98 & $>99: 1$ & 95 \\
$5^{e}$ & 40 & 97 & $>99: 1$ & 92 \\
6 & 50 & 96 & $>99: 1$ & 95 \\
\hline
\end{tabular}

${ }^{a}$ Reaction conditions: 1a $(0.25 \mathrm{mmol}), 2 \mathrm{c}(0.5 \mathrm{~mol} \%), t \mathrm{BuOK}\left(1\right.$ equiv.), toluene $(0.8 \mathrm{~mL}), 25{ }^{\circ} \mathrm{C}, 1 \mathrm{~h} ;{ }^{b}$ Determined by ${ }^{1} \mathrm{H}$ NMR analysis with 1,3,5-trimethoxybenzene as the internal standard; ${ }^{c}$ The $d r$ value was determined by ${ }^{1} \mathrm{H}$ NMR spectroscopy of the crude reaction mixture; ${ }^{d}$ The $e e$ value was determined by HPLC analysis; ${ }^{e}$ with $0.1 \mathrm{~mol} \%$ of $2 \mathbf{c}, 1.0 \mathrm{mmol} \mathbf{1 a}, 24 \mathrm{~h}$. 
Table S4. The effect of the different solvents<smiles>Cc1ccc(C=C2CCc3ccccc3C2O)cc1</smiles>

$1 \mathrm{a}$
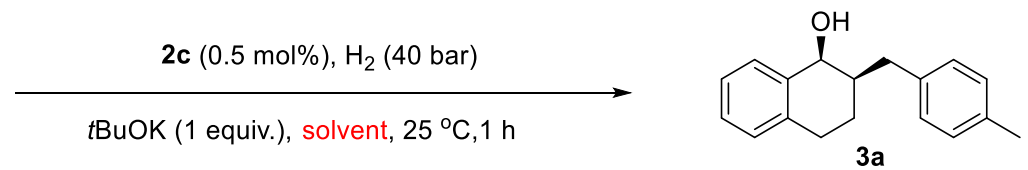

\begin{tabular}{ccccc}
\hline Entry $^{a}$ & Solvent & Yield $(\%)^{b}$ & $\operatorname{dr}(\%)^{c}$ & ${\operatorname{ee~}(\%)^{d}}^{c}$ \\
\hline 1 & toluene & 98 & $>99: 1$ & 95 \\
2 & DCM & 32 & $>99: 1$ & 95 \\
3 & THF & 88 & $>99: 1$ & 89 \\
4 & DMSO & 54 & $>99: 1$ & 54 \\
5 & MeOH & 0 & - & -
\end{tabular}

${ }^{a}$ Reaction conditions: 1a $(0.25 \mathrm{mmol}), 2 \mathrm{c}(0.5 \mathrm{~mol} \%), t \mathrm{BuOK}$ (1 equiv.), $\mathrm{H}_{2}$ (40 bar), solvent $(0.8 \mathrm{~mL}), 25{ }^{\circ} \mathrm{C}, 1 \mathrm{~h} ;{ }^{b}$ Determined by ${ }^{1} \mathrm{H}$ NMR analysis with 1,3,5-trimethoxybenzene as the internal standard; ${ }^{c}$ The $d r$ value was determined by ${ }^{1} \mathrm{H}$ NMR spectroscopy of the crude reaction mixture; ${ }^{d}$ The $e e$ value was determined by HPLC analysis.

Table S5. Optimization of reaction conditions for acyclic allylic alcohols<smiles>OC(/C(=C/c1ccc(Cl)cc1)c1ccccc1)c1ccccc1</smiles>

4 e

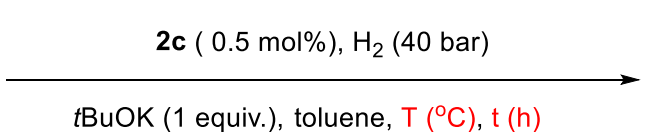

tBuOK (1 equiv.), toluene, $\mathrm{T}\left({ }^{\circ} \mathrm{C}\right), \mathrm{t}(\mathrm{h})$

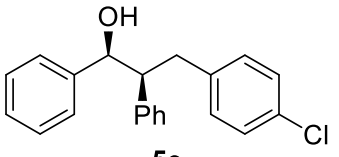

5 e

\begin{tabular}{|c|c|c|c|c|c|}
\hline Entry $^{a}$ & $\mathrm{~T}\left({ }^{\circ} \mathrm{C}\right)$ & $\mathrm{t}(\mathrm{h})$ & Yield $(\%)^{b}$ & $\mathrm{dr}(\%)^{c}$ & ee $(\%)^{d}$ \\
\hline 1 & 25 & 1 & 0 & - & - \\
\hline 2 & 25 & 24 & trace & - & - \\
\hline 3 & 40 & 24 & 70 & $93: 7$ & 89 \\
\hline 4 & 60 & 24 & 84 & $94: 6$ & 90 \\
\hline 5 & 60 & 36 & 96 & $94: 6$ & 90 \\
\hline
\end{tabular}

${ }^{a}$ Reaction conditions: 4 e $(0.25 \mathrm{mmol}), 2 \mathrm{c}$ ( $\left.0.5 \mathrm{~mol} \%\right), t \mathrm{BuOK}$ (1 equiv.), toluene $(0.8 \mathrm{~mL}), \mathrm{H}_{2}(40 \mathrm{bar}) ;{ }^{b}$ Determined by ${ }^{1} \mathrm{H}$ NMR analysis with 1,3,5-trimethoxybenzene as the internal standard; ${ }^{c}$ The $d r$ value was determined by ${ }^{1} \mathrm{H}$ NMR spectroscopy of the crude reaction mixture; ${ }^{d}$ The $e e$ value was determined by HPLC analysis.

\section{General procedure for the preparation of allylic alcohols}

\subsection{General procedure for the preparation of cyclic allylic alcohols}

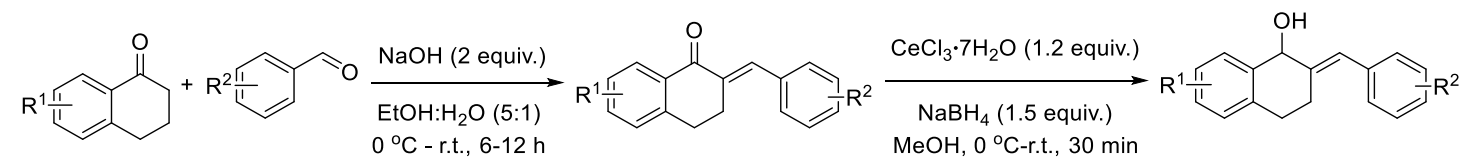

Preparation of cyclic $(\boldsymbol{E})$ - $\boldsymbol{\alpha}, \boldsymbol{\beta}$-unsaturated ketones: ${ }^{[1]}$ A $100 \mathrm{~mL}$ round-bottom flask equipped with a magnetic stir bar was charged with a ketone (10 mmol, 1.0 equiv.), an aldehyde (12 mmol, 1.2 equiv.) and $10 \mathrm{~mL}$ ethanol, then an aqueous solution of $\mathrm{NaOH}$ ( $800 \mathrm{mg}, 20 \mathrm{mmol}, 2$ equiv.) was added dropwisely to the mixture with ice bath cooling. The mixture was stirred at room temperature for 6-12 h. For reactions with precipitates, the reaction mixture was filtered and the solid collected was washed with ethanol, and dried to yield an $(E)-\alpha, \beta$-unsaturated ketone. For reactions without precipitates, 
the solvent was removed under reduced pressure and the crude residue was purified by flash column chromatography (Petroleum ether/Ethyl acetate) on silica gel to give an $(E)-\alpha, \beta$-unsaturated ketone.

Preparation of cyclic $(\boldsymbol{E})$-allylic alcohols: ${ }^{[2]} \mathrm{An}(E)-\alpha, \beta$-unsaturated ketone (1 equiv.) and $\mathrm{CeCl}_{3} \cdot 7 \mathrm{H}_{2} \mathrm{O}(1.2$ equiv.) were stirred in $\mathrm{MeOH}$ at $0{ }^{\circ} \mathrm{C}$, then $\mathrm{NaBH}_{4}$ (1.5 equiv.) was added, and the reaction mixture was further stirred for 30 min at room temperature. After the reaction was finished, the reaction mixture was treated with saturated ammonium chloride solution and extracted with DCM. The combined organic phases were washed with brine, and dried with over anhydrous $\mathrm{Na}_{2} \mathrm{SO}_{4}$. Then the solvent was removed under reduced pressure and the crude residue was purified by flash column chromatography (Petroleum ether/Ethyl acetate) on silica gel to afford a cyclic (E)-allylic alcohol.

\subsection{General procedure for the preparation of acyclic allylic alcohols}

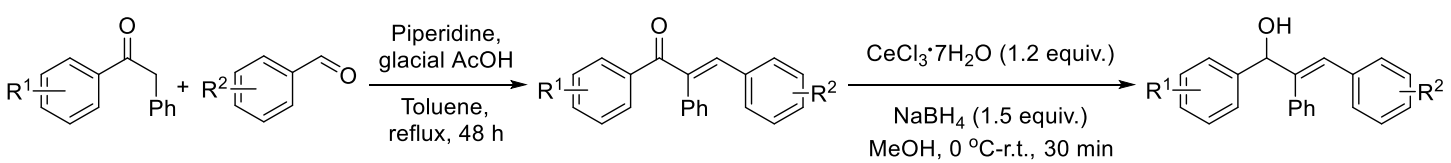

Preparation of acyclic $(\boldsymbol{E})-\boldsymbol{\alpha}, \boldsymbol{\beta}$-unsaturated ketones: ${ }^{[3]}$ To a $200 \mathrm{~mL}$ round-bottom flask equipped with a magnetic stir bar were added a ketone (15 mmol, 1.0 equiv.), an aldehyde (18 mmol, 1.2 equiv.), glacial $\mathrm{AcOH}(0.84 \mathrm{~mL})$, piperidine $(0.3 \mathrm{~mL})$ and anhydrous toluene $(50 \mathrm{ml})$, and the resulting mixture was refluxed in oil bath using a Dean-Stark apparatus for $48 \mathrm{~h}$. After the reaction was finished, the organic solvent was removed under reduced pressure, and the crude residue was purified by flash column chromatography (Petroleum ether/Ethyl acetate) on silica gel to afford a mixture of $Z$ - and $E$ - $\alpha, \beta$-unsaturated ketone. Then the $\alpha, \beta$-unsaturated ketone was reduced following the same procedure in section 3.1 to afford an acyclic $(E)$-allylic alcohol.

\section{General procedure for the preparation of racemic samples}

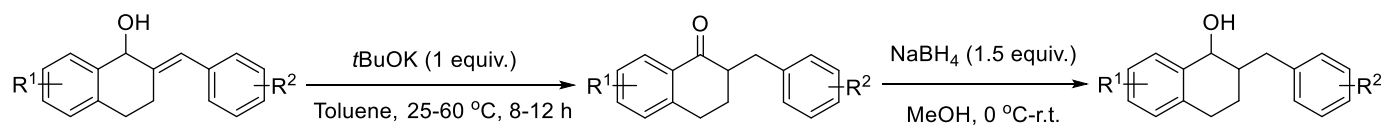

Method 1: The racemic samples for 3a-3d, 3f-3n, 3q-3s, 3aa, and 3ae-3af were prepared by the isomerization of allylic alcohols, followed by the reduction of the corresponding ketones with $\mathrm{NaBH}_{4}$.

General procedure: A pressure tube equipped with a magnetic stir bar was charged with an allylic alcohol $(0.25 \mathrm{mmol})$, $t$ BuOK $(28 \mathrm{mg}, 0.25 \mathrm{mmol})$ and toluene $(2 \mathrm{~mL})$. The mixture was bubbled with argon for $25 \mathrm{~min}$ and the tube was sealed. The mixture was then stirred at $25-60^{\circ} \mathrm{C}$ for $12 \mathrm{~h}$. After the reaction, the solvent was evaporated under reduced pressure and the product was purified by silica gel chromatography to give the saturated ketone. The saturated ketone product was then dissolved in $5 \mathrm{~mL}$ methanol. The resulting solution was cooled with an ice bath, and $\mathrm{NaBH}_{4}$ (1.5 equiv.) was added. The reaction mixture was stirred at room temperature until the reaction is completed. The reaction was then quenched with saturated ammonium chloride solution, extracted with ethyl acetate, and dried over $\mathrm{Na}_{2} \mathrm{SO}_{4}$. The organic phase was concentrated under reduced pressure to afford a crude product, which was purified by silica gel chromatography to obtain the cyclic racemic sample.<smiles>OC1CCC2=CCC1=C[R1]=C2</smiles><smiles>[R]OC(=O)[18OH]</smiles><smiles>[R]c1ccc(CC2CCc3cc[R1]cc3C2O)cc1</smiles>

Method 2: ${ }^{[4]}$ The racemic samples for 3e, 3o-3p, 3t-3z, 3ab-3ad and 5a-5x were prepared according to this method. An allylic alcohol $(0.25 \mathrm{mmol}), \mathrm{Pd} / \mathrm{C}(30 \mathrm{mg})$ and $0.8 \mathrm{~mL}$ ethanol were added to a glass tube. The tube was then placed in an autoclave, and the autoclave was charged with $\mathrm{H}_{2}\left(15\right.$ bar). Then the mixture was stirred in water bath at $30{ }^{\circ} \mathrm{C}$ for 12 hours. After cooling to room temperature, the reaction mixture was diluted with $\mathrm{CH}_{2} \mathrm{Cl}_{2}$ and passed through a short plug of silica gel (eluted with $\mathrm{CH}_{2} \mathrm{Cl}_{2}$ ). Then, the collected solution was evaporated under reduced pressure and the product was purified by silica gel chromatography to give the cyclic racemic sample. 


\section{General procedure for asymmetric hydrogenation of allylic alcohols}

\subsection{General procedure for asymmetric hydrogenation of cyclic allylic alcohols}

A cyclic allylic alcohol ( $0.25 \mathrm{mmol}), 2 \mathrm{c}(1.1 \mathrm{mg}, 0.5 \mathrm{~mol} \%), t \mathrm{BuOK}(28 \mathrm{mg}, 0.25 \mathrm{mmol})$ and $0.8 \mathrm{~mL}$ toluene were added to a glass tube. The tube was then placed in an autoclave, and the autoclave was charged with $\mathrm{H}_{2}(40 \mathrm{bar})$. Then the mixture was stirred in water bath at $25{ }^{\circ} \mathrm{C}$ for 1 hour. After the reaction was finished, the solvent was removed under reduced pressure and the product was purified by silica gel chromatography to give the desired product. The $d r$ and $e e$ values were determined by HPLC analysis.

\subsection{General procedure for asymmetric hydrogenation of acyclic allylic alcohols}

An acyclic allylic alcohol $(0.25 \mathrm{mmol}), 2 \mathrm{c}(1.1 \mathrm{mg}, 0.5 \mathrm{~mol} \%), t \mathrm{BuOK}(28 \mathrm{mg}, 0.25 \mathrm{mmol})$ and $0.8 \mathrm{~mL}$ toluene were added to a glass tube. The tube was then placed in an autoclave, and the autoclave was charged with $\mathrm{H}_{2}$ ( 40 bar). The mixture was then stirred in oil bath at $60{ }^{\circ} \mathrm{C}$ for the time required. After cooling to room temperature, the solvent was removed under reduced pressure and the resulting mixture was purified by silica gel chromatography to give the desired product. The $d r$ and $e e$ were determined by HPLC analysis.

\section{Procedure for the synthesis of chiral chromanes}

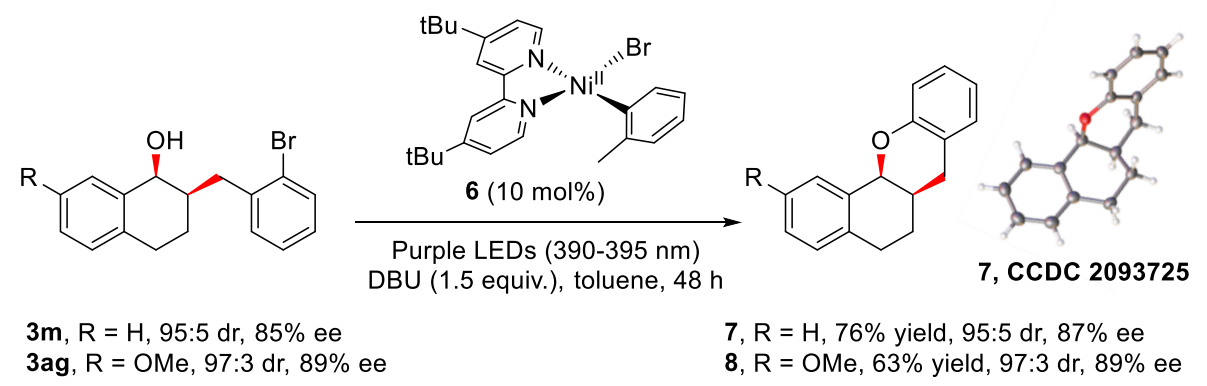

The chiral chromanes were synthesized by Ni-catalyzed intramolecular C-O coupling under visible light. ${ }^{[5]}$ Procedure for the synthesis 7: To an oven-dried $10 \mathrm{~mL}$ Schlenk tube, a magnetic stir bar, Ni catalyst 6 (10 mol \%, $0.025 \mathrm{mmol})$ and the chiral alcohol 3m $(0.25 \mathrm{mmol})$ were added. The tube was degassed with argon for three times. DBU $(0.375 \mathrm{mmol}, 1.5$ equiv.) and toluene $(1.0 \mathrm{~mL})$ were added via syringe under a positive pressure of argon. The tube was then sealed and the reaction mixture was stirred under the irradiation of two purple LED lamps $(1 \mathrm{~cm}$ from the tube, optical power: $260-280 \mathrm{mw} / \mathrm{cm}^{2}$ ) for $48 \mathrm{~h}$ at $80{ }^{\circ} \mathrm{C}$. After cooling to room temperature, the reaction mixture was concentrated under reduced pressure to afford the crude product. The crude product was purified by column chromatography on silica gel to give the desired product 7 (44.8 $\mathrm{mg}, 76 \%$ isolated yield, $95: 5 d r, 87 \% e e$ ).

The chiral chromane $8(41.9 \mathrm{mg}, 63 \%$ isolated yield, $97: 3 d r, 89 \% e e)$ was prepared following the same procedure as 7. The racemic samples for $\mathbf{7}$ and $\mathbf{8}$ were prepared following the same procedure as $\mathbf{7}$ from racemic alcohols.

\section{Procedure for the synthesis of chiral indanes}
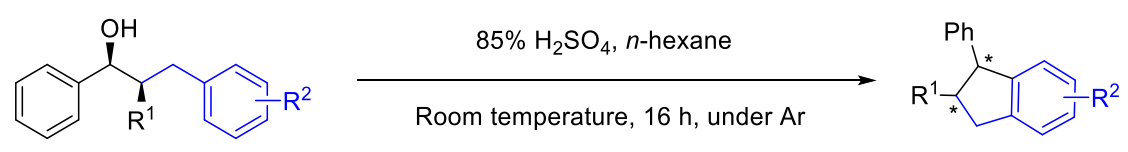

5p, $\mathrm{R}^{1}=\mathrm{Ph}, \mathrm{R}^{2}=2,5-\mathrm{OCH}_{3},>99: 1 \mathrm{dr}, 91 \%$ ee

5q, $R^{1}=P h, R^{2}=3,5-O_{3},>99: 1 d r, 97 \%$ ee

5x, $\mathbf{R}^{1}=p$-Tol, $\mathbf{R}^{2}=3,5-\mathrm{OCH}_{3},>99: 1 \mathrm{dr}, 98 \%$ ee

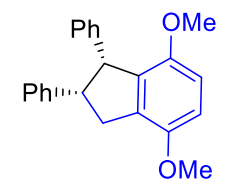

9

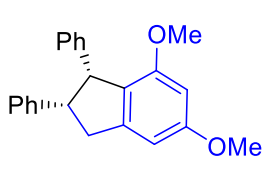

10

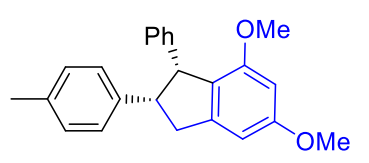

11

$83 \%$ yield, $>99: 1 \mathrm{dr}, 86 \%$ ee $\quad 96 \%$ yield, $>99: 1 \mathrm{dr}, 96 \%$ ee 
The chiral indane products were synthesized by an intramolecular Friedel-Crafts alkylation reaction. ${ }^{[6]}$

Procedure for the synthesis of 9: A pressure tube equipped with a magnetic stir bar was charged with the chiral alcohol $5 \mathbf{p}(0.2 \mathrm{mmol}), 85 \% \mathrm{H}_{2} \mathrm{SO}_{4}(0.2 \mathrm{ml})$ and $n$-hexane $(4 \mathrm{~mL})$. The mixture was bubbled with argon for $25 \mathrm{~min}$ and the tube was sealed. The mixture was then stirred at room temperature for $16 \mathrm{~h}$. After the reaction was finished, the solvent was evaporated under reduced pressure and the product was purified by silica gel chromatography to give the desired product 9 (51.5 mg, 78\% isolated yield, >93:7 dr,91\% ee).

The chiral indanes $\mathbf{1 0}(54.8 \mathrm{mg}, 83 \%$ isolated yield, >99:1 $d r, 86 \% e e)$ and $11(66.0 \mathrm{mg}, 96 \%$ isolated yield, > 99:1 $d r$, $96 \%$ ee) were synthesized following the same procedure as $\mathbf{9}$. The racemic samples for $\mathbf{9}, \mathbf{1 0}$ and 11 were prepared following the same procedure as $\mathbf{9}$ from racemic alcohols.

\section{Mechanistic investigations}

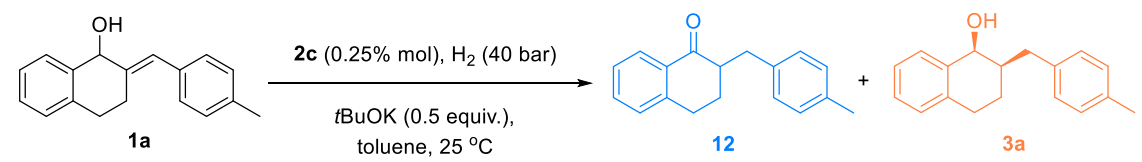
toluene, $25^{\circ} \mathrm{C}$

12

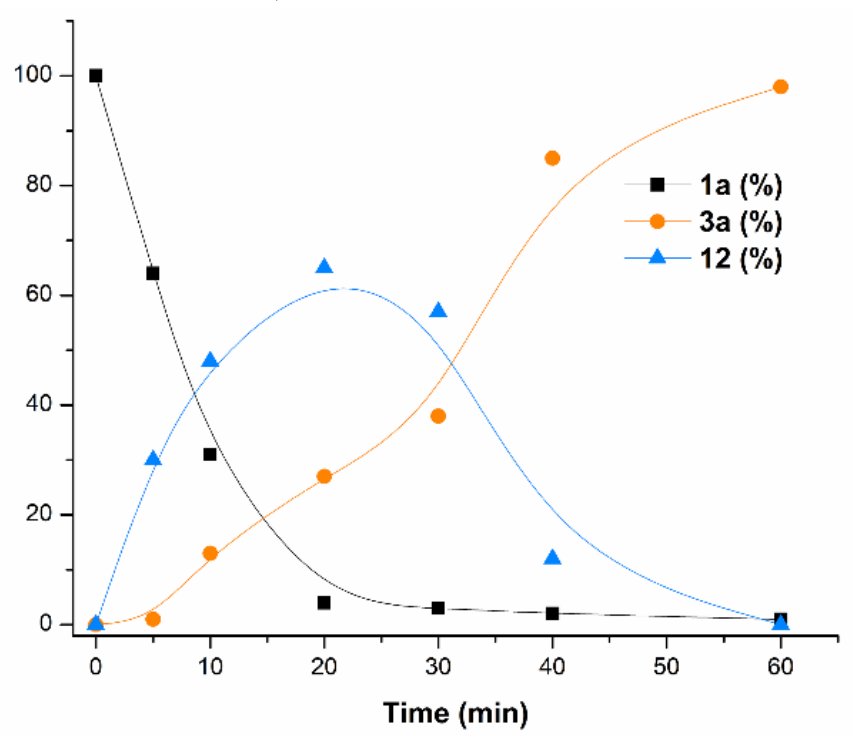

Figure S1. The time course for hydrogenation of 1 a with $\mathbf{2 c}$.

\subsection{Hydrogenation of 12 to 3a catalyzed by $2 \mathrm{c}$}<smiles>Cc1ccc(CC2CCc3ccccc3C2=O)cc1</smiles>

12

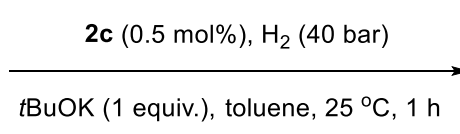

tBuOK (1 equiv.), toluene, $25^{\circ} \mathrm{C}, 1 \mathrm{~h}$

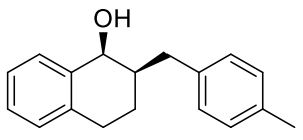

$3 \mathbf{a}$

$96 \%$ yield, $>99: 1 \mathrm{dr}, 94 \%$ ee

12 ( $0.25 \mathrm{mmol}), 2 \mathrm{c}(1.1 \mathrm{mg}, 0.5 \mathrm{~mol} \%), t \mathrm{BuOK}(28 \mathrm{mg}, 0.25 \mathrm{mmol})$ and $0.8 \mathrm{~mL}$ toluene were added to a glass tube. The tube was then placed in an autoclave, and the autoclave was charged with $\mathrm{H}_{2}(40 \mathrm{bar})$. Then the mixture was stirred at 25 ${ }^{\circ} \mathrm{C}$ for 1 hour. After the reaction was finished, the solvent was removed under reduced pressure. The yield (96\%) was determined by ${ }^{1} \mathrm{H}$ NMR analysis of the crude mixture with 1,3,5-trimethoxybenzene as an internal standard. The $d r$ and $e e$ were determined by HPLC analysis of pure 3a (purified by silica gel chromatography).

\subsection{Isomerization of 1a to 12}<smiles>Cc1ccc(C=C2CCc3ccccc3C2O)cc1</smiles>

1a
Toluene, $25^{\circ} \mathrm{C}, 5 \mathrm{~min}$, under $\mathrm{N}_{2}$

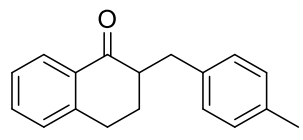

12

with 1 equiv. of $t \mathrm{BuOK}, 36 \%$ yield with 2c only, 0 yield with 1 equiv. of $t$ BuOK, $2 c, 38 \%$ yield 
In a glovebox, a pressure tube equipped with a magnetic stir bar was charged with $\mathbf{1 a}(0.25 \mathrm{mmol})$, with or without $t$ BuOK $(0.25 \mathrm{mmol}, 1$ equiv.), toluene $(1 \mathrm{~mL})$ and with or without $2 \mathbf{c}(0.5 \mathrm{~mol} \%)$. The tube was sealed, and the mixture was stirred at $25{ }^{\circ} \mathrm{C}$ for $5 \mathrm{~min}$. The solvent was then removed under reduced pressure. The yield of $\mathbf{1 2}$ was determined by by ${ }^{1} \mathrm{H}$ NMR analysis with 1,3,5-trimethoxybenzene as an internal standard. These results suggest that the allylic alcohol isomerization was promoted by $t \mathrm{BuOK}$ only.

\subsection{Deuterium labelling studies}

8.3.1 Isomerization of 1a-D under $\mathrm{N}_{2}$ in the absence of $2 \mathrm{c}$<smiles>Cc1ccc(/C=C2\CCc3ccccc3C2(O)O)cc1</smiles>

1a-D

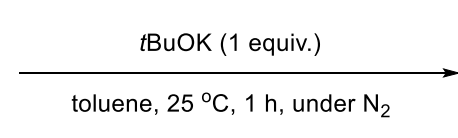

toluene, $25^{\circ} \mathrm{C}, 1 \mathrm{~h}$, under $\mathrm{N}_{2}$

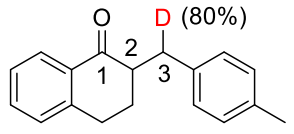

13

In a glovebox, a pressure tube equipped with a magnetic stir bar was charged with 1a-D $(0.25 \mathrm{mmol}, 93 \% \mathrm{D}$ labelled, Figure S2a), $t \mathrm{BuOK}(0.25 \mathrm{mmol}, 1$ equiv.), and toluene $(1 \mathrm{~mL})$. The tube was sealed, and the mixture was stirred at 25 ${ }^{\circ} \mathrm{C}$ for 1 hour. The solvent was then removed under reduced pressure and the resulting mixture was purified by silica gel chromatography. The ${ }^{1} \mathrm{H}$ NMR spectrum of the product $\mathbf{1 3}$ in $\mathrm{CDCl}_{3}$ is shown in Figure $\mathbf{S 2 \mathbf { b }}$. There is $80 \%$ of deuterium labelling at the $\mathrm{C}^{3}$-position. This result support a $t \mathrm{BuOK}$ promoted intramolecular 1,3-hydrogen transfer process for the allylic alcohol isomerization. The signals corresponding to the $\mathrm{C}^{3}$-protons of $\mathbf{1 3}$ were determined by HSQC studies (Figure S2e).

8.3.2 Hydrogenation of 1a-D under standard catalytic conditions<smiles>Cc1ccc(/C=C2\CCc3ccccc32)cc1</smiles>
1a-D

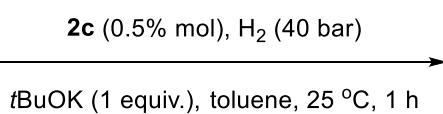

tBuOK (1 equiv.), toluene, $25^{\circ} \mathrm{C}, 1 \mathrm{~h}$

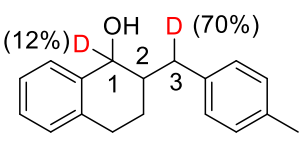

14

The deuterium-labelling product 1a-D $(0.25 \mathrm{mmol}, 93 \% \mathrm{D}$ labelled $), \mathbf{2 c}(0.5 \mathrm{~mol} \%), t \mathrm{BuOK}(0.25 \mathrm{mmol}, 1$ equiv. $)$ and $0.8 \mathrm{~mL}$ toluene were added to a glass tube. The tube was placed in an autoclave, and the autoclave was charged with $\mathrm{H}_{2}$ (40 bar). The mixture was stirred at $25{ }^{\circ} \mathrm{C}$ for 1 hour. The solvent was then removed under reduced pressure and the resulting mixture was purified by silica gel chromatography. The ${ }^{1} \mathrm{H}$ NMR spectrum of the product 14 in $\mathrm{CDCl}_{3}$ is shown in Figure S2c. There are $12 \%$ the deuterium atoms remained at the $\mathrm{C}^{1}$ position and $70 \%$ of the deuterium atoms transferred to the $\mathrm{C}^{3}$-position in $\mathbf{1 4}$. The signals corresponding to the $\mathrm{C}^{3}$-protons were determined by HSQC studies (Figure S2f).

\subsubsection{Reduction of 1a with $\mathrm{D}_{2}$ catalyzed by $2 \mathrm{c}$}<smiles>Cc1ccc(C=C2CCc3ccccc3C2O)cc1</smiles>

1a

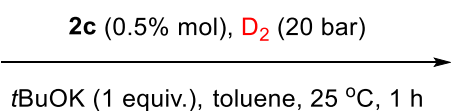

tBuOK ( 1 equiv.), toluene, $25^{\circ} \mathrm{C}, 1 \mathrm{~h}$

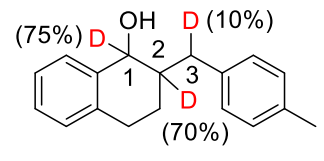

15

Allylic alcohol 1a $(0.25 \mathrm{mmol}), \mathbf{2 c}(0.5 \mathrm{~mol} \%), t \mathrm{BuOK}(0.25 \mathrm{mmol}$, 1equiv.) and $0.8 \mathrm{~mL}$ toluene were added to a glass tube. The tube was placed in an autoclave, and the autoclave was charged with $\mathrm{D}_{2}(20 \mathrm{bar})$. The mixture was stirred at 25 ${ }^{\circ} \mathrm{C}$ for 1 hour. The solvent was then removed under reduced pressure and the resulting mixture was purified by silica gel chromatography. The ${ }^{1} \mathrm{H}$ NMR spectrum of the product $\mathbf{1 5}$ in $\mathrm{CDCl}_{3}$ is shown in Figure $\mathbf{S 2 d}$. There are $75 \%$ deuterium labelling at the $\mathrm{C}^{1}$-position, $70 \%$ at the $\mathrm{C}^{2}$-position and $10 \%$ at the $\mathrm{C}^{3}$-position in $\mathbf{1 5}$. 
${ }^{1} \mathrm{H}$ NMR spectrum of 1a-D $\left(\mathrm{CDCl}_{3}, 400 \mathrm{MHz}\right)$ :
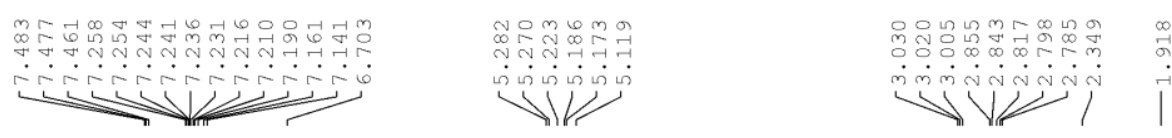

a

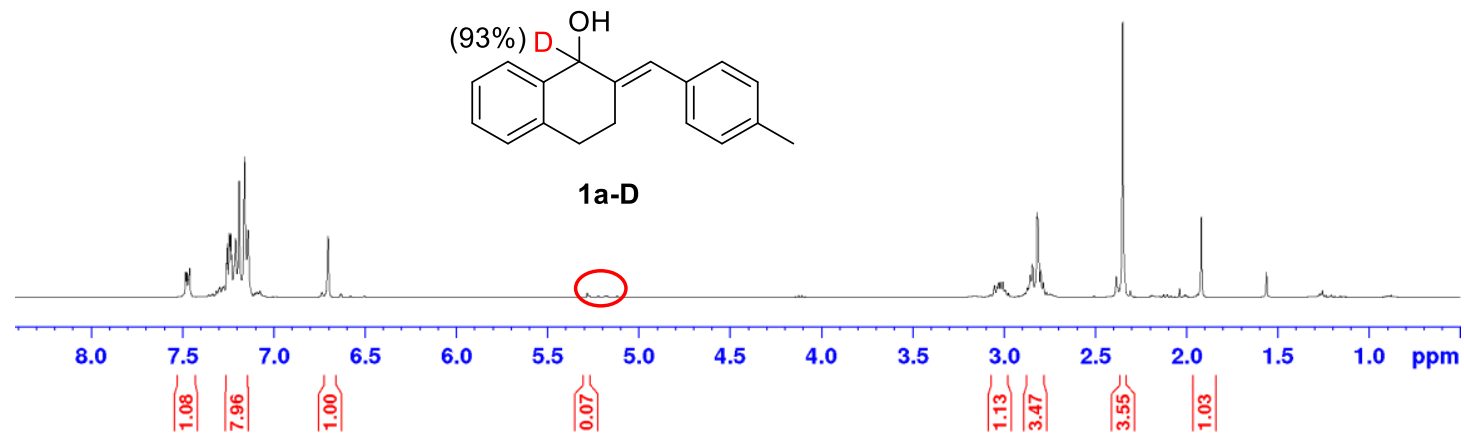

${ }^{1} \mathrm{H}$ NMR spectrum of $\mathbf{1 3}\left(\mathrm{CDCl}_{3}, 400 \mathrm{MHz}\right)$ :

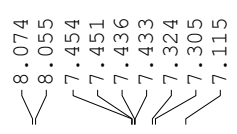

b

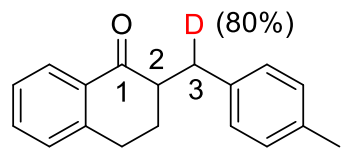

13

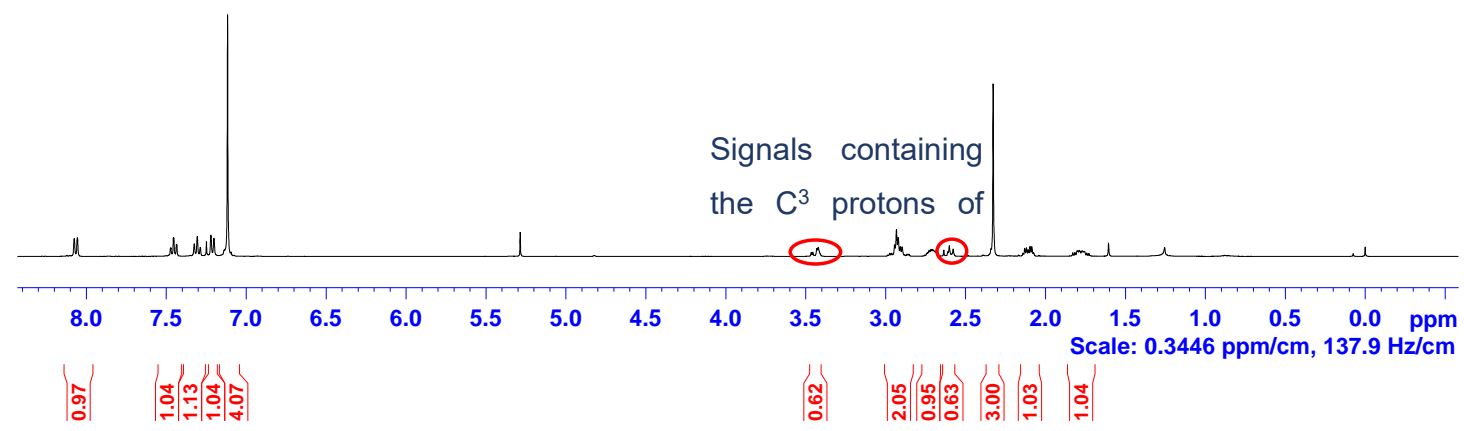


${ }^{1} \mathrm{H}$ NMR spectrum of $\mathbf{1 4}\left(\mathrm{CDCl}_{3}, 400 \mathrm{MHz}\right)$ :
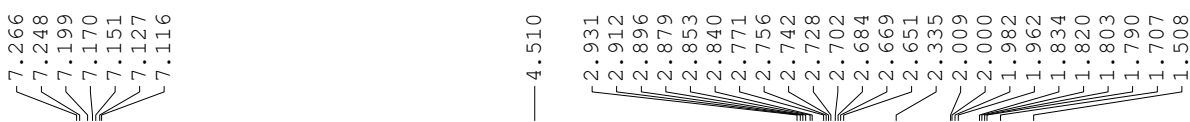

C

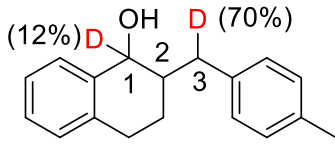

14

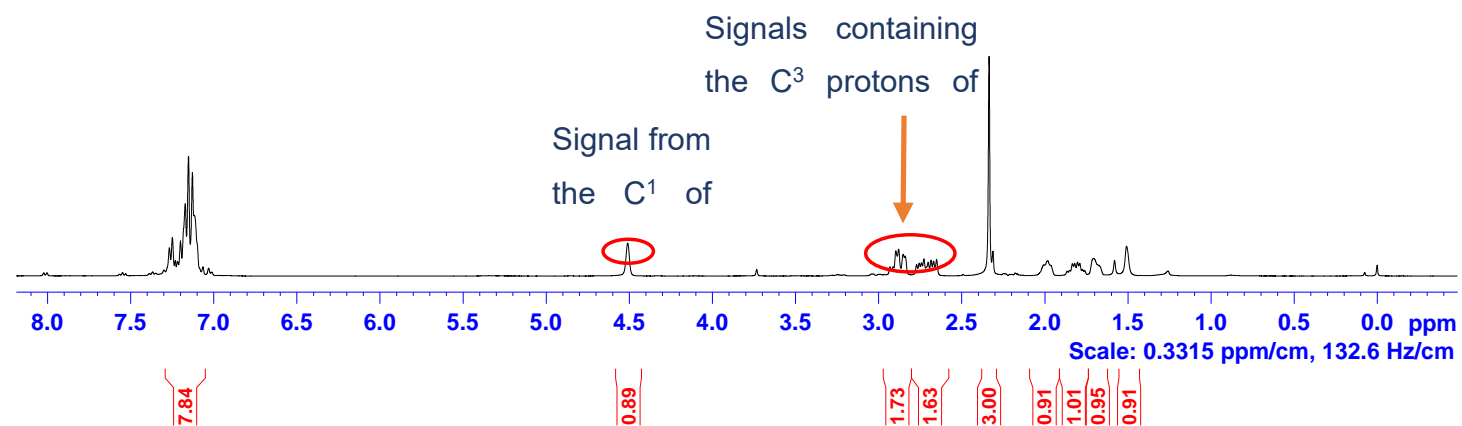

${ }^{1} \mathrm{H}$ NMR spectrum of $\mathbf{1 5}\left(\mathrm{CDCl}_{3}, 400 \mathrm{MHz}\right)$ :
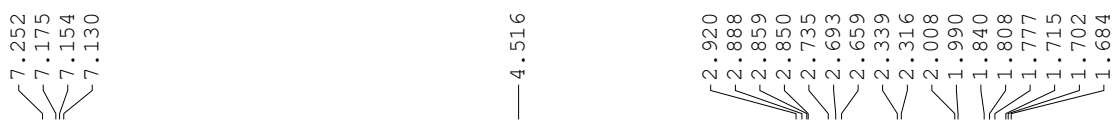

d

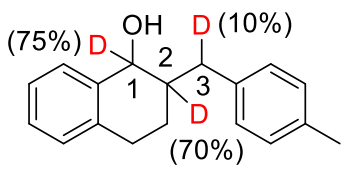

15

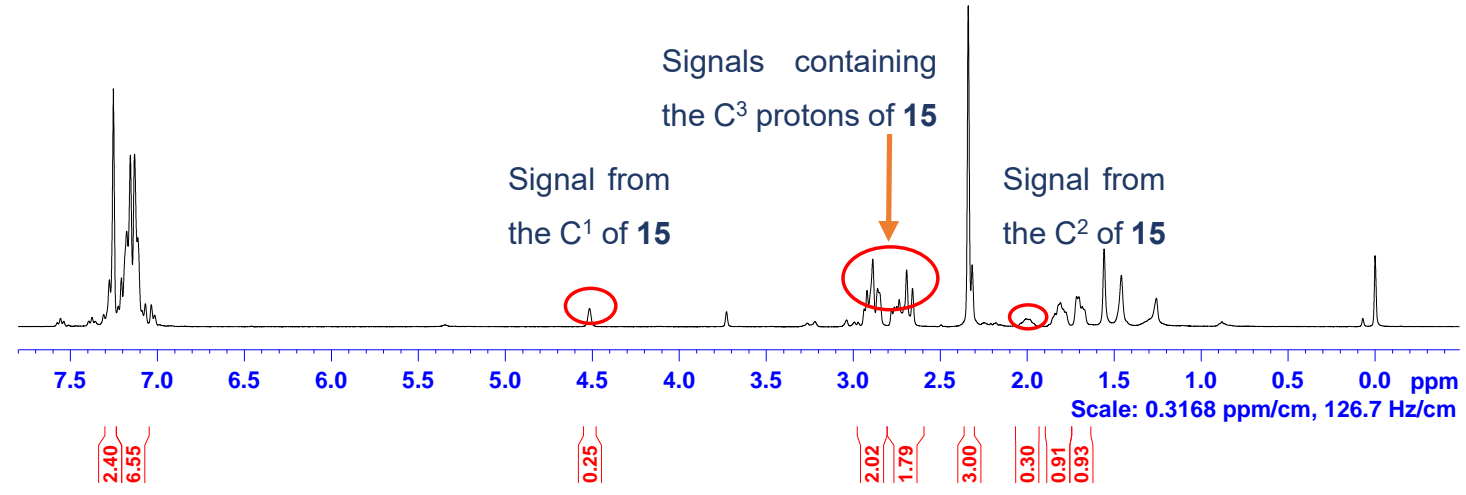



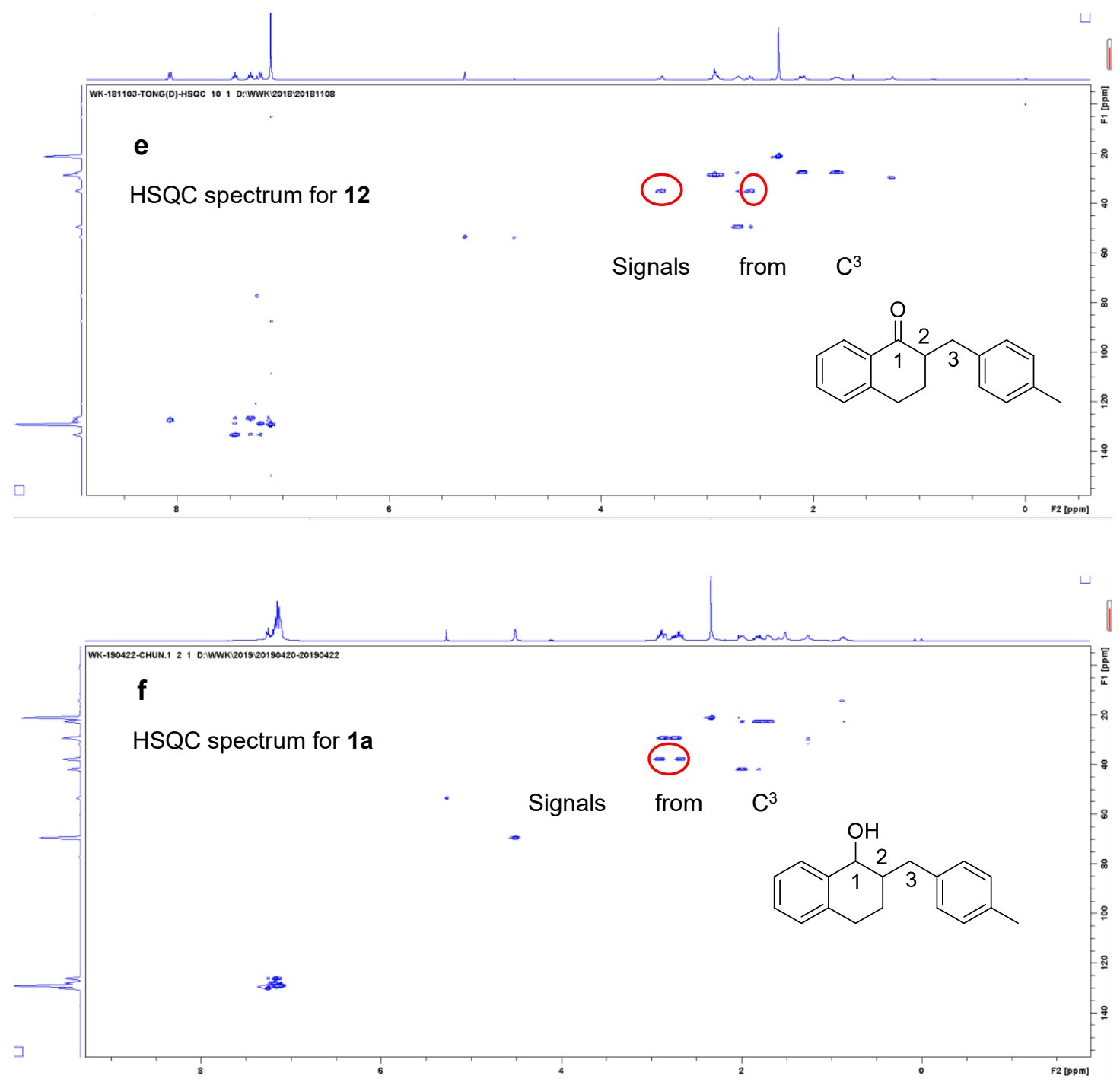

Figure S2. NMR spectra for deuterium labelling studies and HSQC spectra of $\mathbf{1 2}$ and $\mathbf{1 a .}$ 


\subsection{Explanation for the observed enantio- and diastereoselectivity}

Spartan models are used to explain the observed enantio- and diastereoselectivity. The structure of the Ru hydride intermediate from $\mathbf{3 b}$ was modeled using Spartan. And the energy of the intermediate was minimized by Spartan. Then, the models for the reduction of ketones formed from $\boldsymbol{R}-\mathbf{1 a}$ and $\boldsymbol{S}$-1a by the Ru hydride intermediate via a metal-ligand bifunctional mechanism as proposed by Ohkuma and Noyori (Adv. Synth. Catal. 2019, 361, 5540) were constructed respectively. From the models, it can be seemed that the reduction of ketone $\mathbf{K}_{S \text {-1a }}$, leading to the $(S, S)$ alcohol product, suffers steric repulsion between the 4-methylbenezene group from $\mathbf{K}_{\text {s-1a }}$ and a phenyl group on one of the phosphine atoms of the Ru complex (TS-K $\mathrm{K}_{S-1 a}$ ); While the reduction of $\mathbf{K}_{R-1 a}$, leading to the $(S, R)$ alcohol product, via TS-K $\mathrm{K}_{R-1 a}$ is less sterically hindered and energetically favorable. From the analysis of the models, the $(S, R)$ alcohol product from the reduction of $\mathbf{K}_{R-1 a}$ via the energetically favorable TS-K $\mathrm{K}_{R-1 a}$ will be the major product, which fits with the experimental results. However, DFT studies on all the four possible transition states for the reduction of the ketone intermediate would provide more comprehensive and accurate explanation for the observed selectivities.
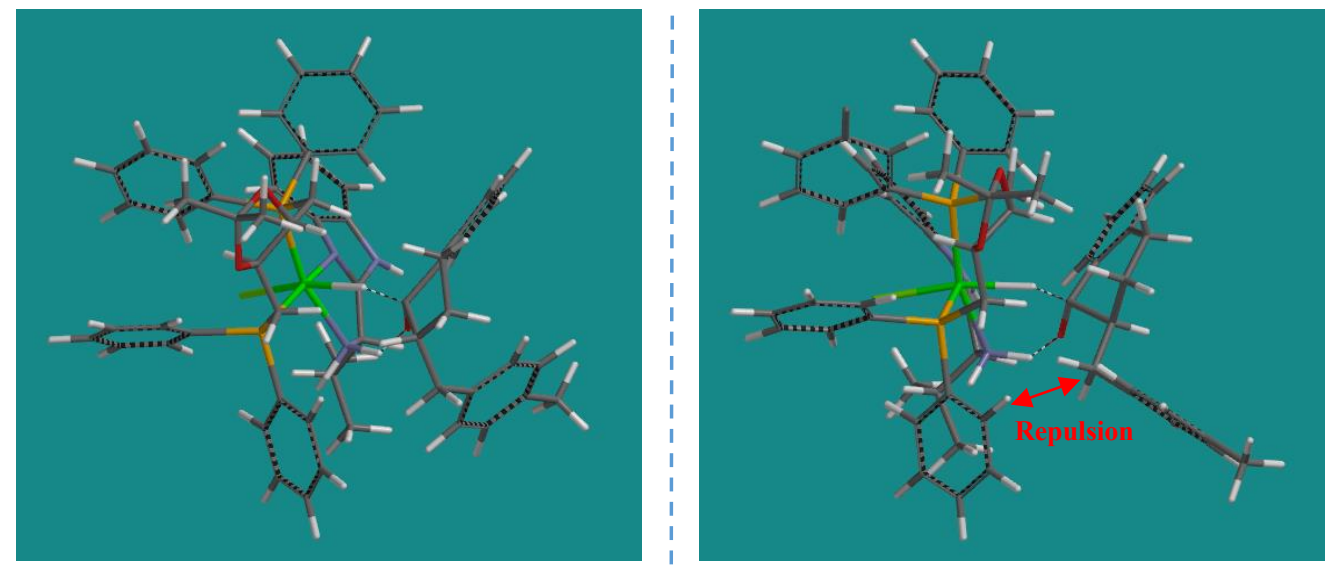

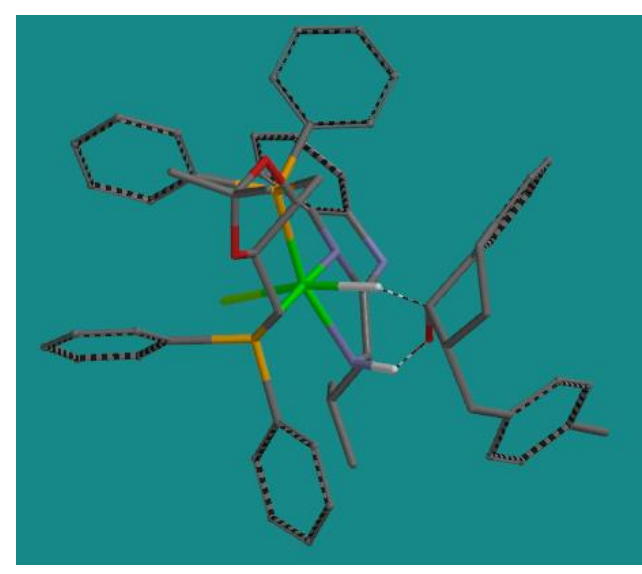

Favored TS-K $\mathrm{K}_{R-1 a} \rightarrow(\mathrm{S}, \mathrm{R})$ product

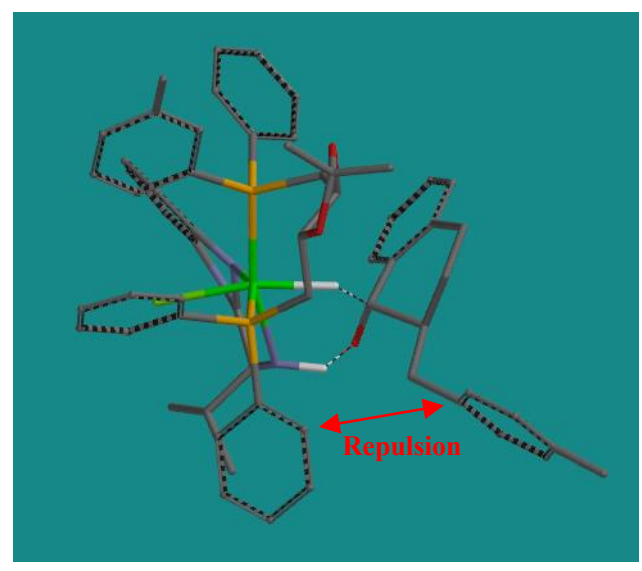

Disfavored TS-K $\mathrm{K}_{\text {-Ia }} \rightarrow(\mathrm{S}, \mathrm{S})$ product

Figure S3. Spartan models for the reduction of ketone intermediates via a metal-ligand bifunctional mechanism. 


\section{Analytic data of products}

(1S,2R)-2-(4-Methylbenzyl)-1,2,3,4-tetrahydronaphthalen-1-ol (3a)<smiles>Cc1ccc(C[C@H]2CCc3ccccc3[C@@H]2O)cc1</smiles>
white solid, $58.6 \mathrm{mg}, 93 \%$ isolated yield. purification on silica gel using a 20:1 petroleum ether/ethyl acetate mixture.

${ }^{1} \mathbf{H}$ NMR $\left(\mathrm{CDCl}_{3}, 400 \mathrm{MHz}\right) \delta(\mathrm{ppm}):$ 7.26-7.10 (m, 8H), $4.51(\mathrm{~s}, 1 \mathrm{H}), 2.93-2.85(\mathrm{~m}, 2 \mathrm{H})$, 2.84-2.65 (m, 2H), $2.33(\mathrm{~s}, 3 \mathrm{H}), 2.03-1.96(\mathrm{~m}, 1 \mathrm{H}), 1.86-1.76(\mathrm{~m}, 1 \mathrm{H}), 1.71-1.67(\mathrm{~m}, 1 \mathrm{H}), 1.52(\mathrm{brs}, 1 \mathrm{H})$.

${ }^{13} \mathbf{C}\left\{{ }^{1} \mathbf{H}\right\} \mathbf{N M R}\left(\mathrm{CDCl}_{3}, 100 \mathrm{MHz}\right) \delta(\mathrm{ppm}): 138.7,137.7,137.1,135.5,130.2,129.24,129.18,128.1,126.2,69.6,41.9$, $37.9,29.3,22.7,21.2$.

HRMS (ESI) m/z: $[\mathrm{M}+\mathrm{Na}]^{+} \mathrm{Calcd}$ for $\mathrm{C}_{18} \mathrm{H}_{20} \mathrm{O} 275.1406$; Found 275.1411.

$>$ 99:1 $d r, 95 \%$ ee HPLC conditions: Chiralpak OJ-H column, $n$-hexane:isopropanol $=95: 5$; flow rate $=0.5 \mathrm{~mL} / \mathrm{min}$; UV detection at $220 \mathrm{~nm} ; \mathrm{t}_{\mathrm{R}}=23.3 \mathrm{~min}$ (major), $\mathrm{t}_{\mathrm{R}}=31.3 \mathrm{~min}$ (minor). The absolute configuration was determined by single-crystal X-ray diffraction analysis. Single crystals suitable for X-ray diffraction measurement were grown from a solution of $\mathbf{3 a}$ in petroleum ether/dichloromethane.

\section{$(1 S, 2 R)$-2-Benzyl-1,2,3,4-tetrahydronaphthalen-1-ol (3b) ${ }^{[7]}$}<smiles>O[C@H]1c2ccccc2CC[C@@H]1Cc1ccccc1</smiles>
white solid, $57.7 \mathrm{mg}, 97 \%$ isolated yield. purification on silica gel using a 20:1 petroleum ether/ethyl acetate mixture.

${ }^{1} \mathbf{H}$ NMR $\left(\mathrm{CDCl}_{3}, 400 \mathrm{MHz}\right) \delta(\mathrm{ppm}):$ 7.33-7.10 (m, 9H), $4.50(\mathrm{~d}, J=2.8 \mathrm{~Hz}, 1 \mathrm{H}), 2.97-2.92$ (m, 1H), 2.89-2.84 (m, 1H), 2.78-2.69 (m, 2H), 2.03-1.99 (m, 1H), 1.85-1.80 (m, 1H), 1.72-1.70 (m, 1H), 1.53 (brs, 1H). ${ }^{13} \mathbf{C}\left\{{ }^{1} \mathbf{H}\right\}$ NMR $\left(\mathrm{CDCl}_{3}, 100 \mathrm{MHz}\right) \delta(\mathrm{ppm}): 140.8,138.7,137.0,130.2,129.4,129.3,128.5,128.1,126.3,126.0,69.5$, $41.9,38.3,29.3,22.7$.

HRMS (ESI) m/z: [M+Na $]^{+}$Calcd for $\mathrm{C}_{17} \mathrm{H}_{18} \mathrm{O} 261.1250$; Found 261.1247.

$>$ 99:1 $d r, 94 \%$ ee HPLC conditions: Chiralpak OJ-H column, $n$-hexane:isopropanol $=95: 5$; flow rate $=0.5 \mathrm{~mL} / \mathrm{min}$; UV detection at $220 \mathrm{~nm} ; \mathrm{t}_{\mathrm{R}}=26.6 \mathrm{~min}$ (major), $\mathrm{t}_{\mathrm{R}}=40.9 \mathrm{~min}$ (minor). The absolute configuration was assigned by analogy to 3a.

\section{(1S,2R)-2-(4-Methoxybenzyl)-1,2,3,4-tetrahydronaphthalen-1-ol (3c) $)^{[7 \mathrm{e}-7 \mathrm{~g}]}$}<smiles>COc1ccc(C[C@H]2CCc3ccccc3[C@@H]2O)cc1</smiles>

white solid, $50.9 \mathrm{mg}, 76 \%$ isolated yield. purification on silica gel using a 10:1 petroleum ether/ethyl acetate mixture.

${ }^{1} \mathbf{H}$ NMR $\left(\mathrm{CDCl}_{3}, 400 \mathrm{MHz}\right) \delta(\mathrm{ppm}): 7.26(\mathrm{~d}, J=7.6 \mathrm{~Hz}, 1 \mathrm{H}), 7.20-7.16(\mathrm{~m}, 4 \mathrm{H})$, $7.11(\mathrm{~d}, J=7.2 \mathrm{~Hz}, 1 \mathrm{H}), 6.86(\mathrm{~d}, J=8.0 \mathrm{~Hz}, 2 \mathrm{H}), 4.50$ (s, 1H), 3.80 (s, 3H), 2.91-2.85 (m, 2H), 2.78-2.64 (m, 2H), 1.99-1.94 (m, 1H), 1.83-1.76 (m, 1H), 1.71-1.67 (m, 1H), 1.49 (brs, 1H).

${ }^{13} \mathbf{C}\left\{{ }^{1} \mathbf{H}\right\}$ NMR $\left(\mathrm{CDCl}_{3}, 100 \mathrm{MHz}\right) \delta(\mathrm{ppm}): 158.0,138.7,137.1,132.8,130.24,130.19,129.3,128.1,126.3,113.9,69.5$, 55.4, 42.0, 37.4, 29.4, 22.7.

HRMS (ESI) m/z: $[\mathrm{M}+\mathrm{Na}]^{+}$Calcd for $\mathrm{C}_{18} \mathrm{H}_{20} \mathrm{O}_{2}$ 291.1356; Found 291.1353 .

$>\mathbf{9 9 : 1} \boldsymbol{d} \boldsymbol{r}, \mathbf{9 8} \%$ ee HPLC conditions: Chiralpak OJ-H column, $n$-hexane:isopropanol $=90: 10$; flow rate $=0.5 \mathrm{~mL} / \mathrm{min}$; $\mathrm{UV}$ detection at $220 \mathrm{~nm} ; \mathrm{t}_{\mathrm{R}}=29.6 \mathrm{~min}$ (major), $\mathrm{t}_{\mathrm{R}}=41.6 \mathrm{~min}$ (minor). The absolute configuration was determined by single-crystal X-ray diffraction analysis. Single crystals suitable for X-ray diffraction measurement were grown from a solution of $\mathbf{3} \mathbf{c}$ in petroleum ether/dichloromethane. 
(1S,2R)-2-([1,1'-Biphenyl]-4-ylmethyl)-1,2,3,4-tetrahydronaphthalen-1-ol (3d)<smiles>O[C@H]1c2ccccc2CC[C@H]1Cc1ccc(-c2ccccc2)cc1</smiles>
pink solid, $59.7 \mathrm{mg}, 76 \%$ isolated yield. purification on silica gel using a 15:1 petroleum ether/ethyl acetate mixture.

${ }^{1} \mathbf{H}$ NMR $\left(\mathrm{CDCl}_{3}, 400 \mathrm{MHz}\right) \delta(\mathrm{ppm}): 7.52-7.45(\mathrm{~m}, 4 \mathrm{H}), 7.33(\mathrm{t}, J=7.6 \mathrm{~Hz}, 2 \mathrm{H})$, 7.26-7.23 (m, 3H), 7.21-7.16 (m, 1H), 7.12-7.02 (m, 3H), 4.45 (s, 1H), 2.93-2.87 (m, $1 \mathrm{H}), 2.78-2.76(\mathrm{~m}, 1 \mathrm{H}), 2.70-2.64(\mathrm{~m}, 2 \mathrm{H}), 1.97-1.94(\mathrm{~m}, 1 \mathrm{H}), 1.78-1.73(\mathrm{~m}, 1 \mathrm{H}), 1.66-1.62(\mathrm{~m}, 1 \mathrm{H}), 1.46(\mathrm{brs}, 1 \mathrm{H})$.

${ }^{13} \mathbf{C}\left\{{ }^{1} \mathbf{H}\right\}$ NMR $\left(\mathrm{CDCl}_{3}, 100 \mathrm{MHz}\right) \delta(\mathrm{ppm}): 141.2,140.0,139.0,138.6,137.0,130.2,129.8,129.2,128.8,128.1,127.19$, $127.15,127.1,126.3,69.5,41.8,37.9,29.3,22.7$.

HRMS (ESI) m/z: $[\mathrm{M}+\mathrm{Na}]^{+}$Calcd for $\mathrm{C}_{23} \mathrm{H}_{22} \mathrm{O} 337.1563$; Found 337.1563 .

98:2 $d r, 92 \%$ ee HPLC conditions: Chiralpak AD-H column, $n$-hexane:isopropanol $=95: 5$; flow rate $=0.5 \mathrm{~mL} / \mathrm{min}$; UV detection at $220 \mathrm{~nm} ; \mathrm{t}_{\mathrm{R}}=30.1 \mathrm{~min}\left(\right.$ minor), $\mathrm{t}_{\mathrm{R}}=38.8 \mathrm{~min}$ (major), $\mathrm{t}_{\mathrm{R}}=60.1 \mathrm{~min}$ (minor). The absolute configuration was assigned by analogy to $\mathbf{3 a}$.

\section{(1S,2R)-2-(4-Fluorobenzyl)-1,2,3,4-tetrahydronaphthalen-1-ol (3e)}<smiles>O[C@H]1c2ccccc2CC[C@@H]1Cc1ccc(F)cc1</smiles>
colorless oil, $54.4 \mathrm{mg}, 85 \%$ isolated yield. purification on silica gel using a 15:1 petroleum ether/ethyl acetate mixture.

${ }^{1} \mathbf{H}$ NMR $\left(\mathrm{CDCl}_{3}, 400 \mathrm{MHz}\right) \delta(\mathrm{ppm}): 7.26-7.11(\mathrm{~m}, 6 \mathrm{H}), 7.04(\mathrm{t}, J=8.8 \mathrm{~Hz}, 2 \mathrm{H}), 4.47(\mathrm{~d}$, $J=2.4 \mathrm{~Hz}, 1 \mathrm{H}), 2.95-2.85(\mathrm{~m}, 2 \mathrm{H}), 2.78-2.66(\mathrm{~m}, 2 \mathrm{H}), 2.00-1.93(\mathrm{~m}, 1 \mathrm{H}), 1.85-1.76(\mathrm{~m}$,

1H), 1.70-1.66 (m, 1H), 1.52 (brs, $1 \mathrm{H})$.

${ }^{13} \mathbf{C}\left\{{ }^{1} \mathbf{H}\right\}$ NMR $\left(\mathrm{CDCl}_{3}, 100 \mathrm{MHz}\right) \delta(\mathrm{ppm}): 161.5\left(\mathrm{~d},{ }^{1} J=241.9 \mathrm{~Hz}\right), 138.6,137.0,136.4\left(\mathrm{~d},{ }^{4} J=3.1 \mathrm{~Hz}\right), 130.7\left(\mathrm{~d},{ }^{3} J=\right.$ $7.7 \mathrm{~Hz}), 130.1,129.3,128.2,126.3,115.2\left(\mathrm{~d},{ }^{2} J=20.8 \mathrm{~Hz}\right), 69.3,42.0,37.5,29.3,22.7$.

HRMS (ESI) m/z: $[\mathrm{M}+\mathrm{Na}]^{+}$Calcd for $\mathrm{C}_{17} \mathrm{H}_{17} \mathrm{OF} 279.1156$; Found 279.1159 .

$>99: 1 d r, 94 \%$ ee HPLC conditions: Chiralpak AD-H column, $n$-hexane:isopropanol = 95:5; flow rate $=0.5 \mathrm{~mL} / \mathrm{min}$; UV detection at $220 \mathrm{~nm} ; \mathrm{t}_{\mathrm{R}}=21.7 \mathrm{~min}\left(\right.$ minor), $\mathrm{t}_{\mathrm{R}}=27.9 \mathrm{~min}$ (major). The absolute configuration was assigned by analogy to 3a.

\section{(1S,2R)-2-(4-Chlorobenzyl)-1,2,3,4-tetrahydronaphthalen-1-ol (3f) ${ }^{[7]]}$}<smiles>O[C@H]1c2ccccc2CC[C@@H]1Cc1ccc(Cl)cc1</smiles>

reddish brown solid, $67.3 \mathrm{mg}, 99 \%$ isolated yield. purification on silica gel using a 15:1 petroleum ether/ethyl acetate mixture.

${ }^{1} \mathbf{H}$ NMR $\left(\mathrm{CDCl}_{3}, 400 \mathrm{MHz}\right) \delta(\mathrm{ppm}):$ 7.27-7.09 (m, 8H), $4.45(\mathrm{~s}, 1 \mathrm{H}), 2.93-2.83(\mathrm{~m}, 2 \mathrm{H})$ 2.75-2.63 (m, 2H), 1.96-1.93 (m, 1H), 1.82-1.77 (m, 1H), 1.68-1.60 (m, 2H).

${ }^{13} \mathbf{C}\left\{{ }^{1} \mathbf{H}\right\} \mathbf{N M R}\left(\mathrm{CDCl}_{3}, 100 \mathrm{MHz}\right) \delta(\mathrm{ppm}): 139.3,138.5,136.9,131.7,130.7,130.1,129.2,128.5,128.2,126.3,69.2$, $41.8,37.6,29.2,22.6$.

HRMS (ESI) m/z: [M+Na] $]^{+}$Calcd for $\mathrm{C}_{17} \mathrm{H}_{17} \mathrm{OCl}$ 295.0860; Found 295.0868.

$>99: 1 d r, 94 \%$ ee HPLC conditions: Chiralpak OJ-H column, $n$-hexane:isopropanol $=95: 5$; flow rate $=0.5 \mathrm{~mL} / \mathrm{min}$; UV detection at $220 \mathrm{~nm} ; \mathrm{t}_{\mathrm{R}}=25.7 \mathrm{~min}$ (major), $\mathrm{t}_{\mathrm{R}}=37.7 \mathrm{~min}$ (minor). The absolute configuration was assigned by analogy to 3a.

\section{(1S,2R)-2-(4-Bromobenzyl)-1,2,3,4-tetrahydronaphthalen-1-ol (3g)}<smiles>O[C@H]1c2ccccc2CC[C@@H]1Cc1ccc(Br)cc1</smiles>
white solid, $70.3 \mathrm{mg}, 89 \%$ isolated yield. purification on silica gel using a 15:1 petroleum ether/ethyl acetate mixture.

${ }^{1} \mathbf{H}$ NMR $\left(\mathrm{CDCl}_{3}, 400 \mathrm{MHz}\right) \delta(\mathrm{ppm}): 7.43(\mathrm{~d}, J=8.4 \mathrm{~Hz}, 2 \mathrm{H}), 7.22-7.12(\mathrm{~m}, 6 \mathrm{H}), 4.48$ $(\mathrm{d}, J=2.4 \mathrm{~Hz}, 1 \mathrm{H}), 2.94-2.86(\mathrm{~m}, 2 \mathrm{H}), 2.79-2.65(\mathrm{~m}, 2 \mathrm{H}), 2.02-1.93(\mathrm{~m}, 1 \mathrm{H}), 1.88-1.77$ (m, 1H), 1.71-1.66 (m, 1H), 1.52 (brs, 1H). 
${ }^{13} \mathbf{C}\left\{{ }^{1} \mathbf{H}\right\}$ NMR $\left(\mathrm{CDCl}_{3}, 100 \mathrm{MHz}\right) \delta(\mathrm{ppm}): 139.8,138.5,136.9,131.5,131.2,130.1,129.3,128.3,126.4,119.8,69.2$, $41.8,37.7,29.3,22.7$.

HRMS (ESI) m/z: $[\mathrm{M}+\mathrm{Na}]^{+}$Calcd for $\mathrm{C}_{17} \mathrm{H}_{17} \mathrm{OBr} 339.0355$; Found 339.0362.

$>$ 99:1 $\boldsymbol{d r}$, 95\% ee HPLC conditions: Chiralpak OJ-H column, $n$-hexane:isopropanol $=95: 5$; flow rate $=0.5 \mathrm{~mL} / \mathrm{min}$; UV detection at $220 \mathrm{~nm} ; \mathrm{t}_{\mathrm{R}}=22.9 \mathrm{~min}$ (minor), $\mathrm{t}_{\mathrm{R}}=30.7 \mathrm{~min}$ (major). The absolute configuration was assigned by analogy to 3a.

(1S,2R)-2-(4-(Trifluoromethyl)benzyl)-1,2,3,4-tetrahydronaphthalen-1-ol (3h)<smiles>O[C@H]1c2ccccc2CC[C@@H]1Cc1ccc(C(F)(F)F)cc1</smiles>

white solid, $64.3 \mathrm{mg}, 84 \%$ isolated yield. purification on silica gel using a 15:1 petroleum ether/ethyl acetate mixture.

${ }^{1} \mathbf{H}$ NMR $\left(\mathrm{CDCl}_{3}, 400 \mathrm{MHz}\right) \delta(\mathrm{ppm}): 7.56(\mathrm{~d}, J=8.0 \mathrm{~Hz}, 2 \mathrm{H}), 7.38(\mathrm{~d}, J=8.0 \mathrm{~Hz}$, 2H), 7.26-7.11 (m, 4H), $4.46(\mathrm{~d}, J=2.8 \mathrm{~Hz}, 1 \mathrm{H}), 3.04-2.99(\mathrm{~m}, 1 \mathrm{H}), 2.91-2.85(\mathrm{~m}, 1 \mathrm{H})$, 2.79-2.73 (m, 2H), 2.03-1.98 (m, 1H), 1.86-1.81 (m, 1H), 1.71-1.66 (m, 1H). 1.55 (brs, $1 \mathrm{H})$.

${ }^{13} \mathbf{C}\left\{{ }^{1} \mathbf{H}\right\}$ NMR $\left(\mathrm{CDCl}_{3}, 100 \mathrm{MHz}\right) \delta(\mathrm{ppm}): 145.1,138.4,136.9,130.1,129.7,129.3,128.5\left(\mathrm{q},{ }^{2} J=32.4 \mathrm{~Hz}\right), 128.3$, 126.4, $125.4\left(\mathrm{q},{ }^{3} \mathrm{~J}=3.7 \mathrm{~Hz}\right), 124.5\left(\mathrm{q},{ }^{1} \mathrm{~J}=270.0 \mathrm{~Hz}\right), 69.3,41.8,38.2,29.3,22.8$.

HRMS (ESI) m/z: $[\mathrm{M}+\mathrm{Na}]^{+}$Calcd for $\mathrm{C}_{18} \mathrm{H}_{17} \mathrm{OF}_{3}$ 329.1124; Found 329.1125.

$>$ 99:1 $d r, 91 \%$ ee HPLC conditions: Chiralpak AD-H column, $n$-hexane:isopropanol $=95: 5$; flow rate $=0.5 \mathrm{~mL} / \mathrm{min}$; $\mathrm{UV}$ detection at $220 \mathrm{~nm} ; \mathrm{t}_{\mathrm{R}}=17.7 \mathrm{~min}$ (minor), $\mathrm{t}_{\mathrm{R}}=24.8 \mathrm{~min}$ (major). The absolute configuration was assigned by analogy to $\mathbf{3 a}$.

\section{(1S,2R)-2-(3-Methylbenzyl)-1,2,3,4-tetrahydronaphthalen-1-ol (3i)}<smiles>Cc1cccc(C[C@@H]2CCc3ccccc3[C@@H]2O)c1</smiles>
white solid, $54.2 \mathrm{mg}, 86 \%$ isolated yield. purification on silica gel using a $18: 1$ petroleum ether/ethyl acetate mixture.

${ }^{1} \mathbf{H}$ NMR $\left(\mathrm{CDCl}_{3}, 400 \mathrm{MHz}\right) \delta(\mathrm{ppm}): 7.17-6.93(\mathrm{~m}, 8 \mathrm{H}), 4.41(\mathrm{~d}, J=2.8 \mathrm{~Hz}, 1 \mathrm{H}), 2.84-2.74$ (m, 2H), 2.68-2.56 (m, 2H), 2.25 (s, $3 \mathrm{H}), 1.95-1.89(\mathrm{~m}, 1 \mathrm{H}), 1.75-1.67(\mathrm{~m}, 1 \mathrm{H}), 1.62-1.57$ (m, $1 \mathrm{H}), 1.51$ (brs, $1 \mathrm{H})$.

${ }^{13} \mathbf{C}\left\{{ }^{1} \mathbf{H}\right\} \mathbf{N M R}\left(\mathrm{CDCl}_{3}, 100 \mathrm{MHz}\right) \delta(\mathrm{ppm}): 140.8,138.7,138.0,137.0,130.17,130.15,129.2,128.3,128.1,126.8,126.4$, $126.2,69.5,41.8,38.2,29.3,22.7,21.6$.

HRMS (ESI) m/z: $[\mathrm{M}+\mathrm{Na}]^{+}$Calcd for $\mathrm{C}_{18} \mathrm{H}_{20} \mathrm{O} 275.1406$; Found 275.1405.

98:2 $d r$, 99\% ee HPLC conditions: Chiralpak OJ-H column, $n$-hexane:isopropanol $=95: 5$; flow rate $=0.5 \mathrm{~mL} / \mathrm{min}$; UV detection at $220 \mathrm{~nm} ; \mathrm{t}_{\mathrm{R}}=21.4 \mathrm{~min}$ (major), $\mathrm{t}_{\mathrm{R}}=25.4 \mathrm{~min}$ (minor). The absolute configuration was determined by single-crystal X-ray diffraction analysis. Single crystals suitable for X-ray diffraction measurement were grown from a solution of $\mathbf{3 i}$ in petroleum ether/dichloromethane.

\section{(1S,2R)-2-(3-Methoxybenzyl)-1,2,3,4-tetrahydronaphthalen-1-ol (3j)}<smiles>COc1cccc(C[C@@H]2CCc3ccccc3[C@H]2O)c1</smiles>
colorless oil, $64.3 \mathrm{mg}, 96 \%$ isolated yield. purification on silica gel using a 18:1 petroleum ether/ethyl acetate mixture.

${ }^{1} \mathbf{H}$ NMR $\left(\mathrm{CDCl}_{3}, 400 \mathrm{MHz}\right) \delta(\mathrm{ppm}):$ 7.18-7.10 (m, 5H), 6.79-6.67 (m, 3H), $4.43(\mathrm{~s}, 1 \mathrm{H})$, OMe $3.71(\mathrm{~s}, 3 \mathrm{H}), 2.86-2.75(\mathrm{~m}, 2 \mathrm{H}), 2.69-2.58(\mathrm{~m}, 2 \mathrm{H}), 1.97-1.91(\mathrm{~m}, 1 \mathrm{H}), 1.90-1.69(\mathrm{~m}, 1 \mathrm{H})$, $1.68-1.60(\mathrm{~m}, 1 \mathrm{H}), 1.59$ (brs, $1 \mathrm{H})$.

${ }^{13} \mathbf{C}\left\{{ }^{1} \mathbf{H}\right\}$ NMR $\left(\mathrm{CDCl}_{3}, 100 \mathrm{MHz}\right) \delta(\mathrm{ppm}): 159.8,142.5,138.6,137.0,130.1,129.4,129.2,128.1,126.2,121.8,115.1$, $111.3,69.5,55.2,41.7,38.3,29.3,22.7$.

HRMS (ESI) m/z: [M+Na $]^{+}$Calcd for $\mathrm{C}_{18} \mathrm{H}_{20} \mathrm{O}_{2}$ 291.1356; Found 291.1354.

$>99: 1 d r, 93 \%$ ee HPLC conditions: Chiralpak AD-H column, $n$-hexane:isopropanol = 95:5; flow rate $=0.5 \mathrm{~mL} / \mathrm{min}$; UV 
detection at $220 \mathrm{~nm} ; \mathrm{t}_{\mathrm{R}}=31.8 \mathrm{~min}$ (minor), $\mathrm{t}_{\mathrm{R}}=40.6 \mathrm{~min}$ (major). The absolute configuration was assigned by analogy to 3a.

(1S,2R)-2-(3-Chlorobenzyl)-1,2,3,4-tetrahydronaphthalen-1-ol (3k)<smiles>O[C@H]1c2ccccc2CC[C@H]1Cc1ccccc1</smiles>
colorless oil, $66.6 \mathrm{mg}, 98 \%$ isolated yield. purification on silica gel using a 15:1 petroleum ether/ethyl acetate mixture.

${ }^{1} \mathbf{H}$ NMR $\left(\mathrm{CDCl}_{3}, 400 \mathrm{MHz}\right) \delta(\mathrm{ppm}):$ 7.20-7.04 (m, 8H), 4.41 (s, 1H), 2.89-2.78 (m, 2H), Cl $\quad 2.72-2.59(\mathrm{~m}, 2 \mathrm{H}), 1.95-1.89(\mathrm{~m}, 1 \mathrm{H}), 1.78-1.73(\mathrm{~m}, 1 \mathrm{H}), 1.64-1.59(\mathrm{~m}, 1 \mathrm{H}), 1.39$ (brd, $J=$ $5.6 \mathrm{~Hz}, 1 \mathrm{H})$.

${ }^{13} \mathbf{C}\left\{{ }^{1} \mathbf{H}\right\}$ NMR $\left(\mathrm{CDCl}_{3}, 100 \mathrm{MHz}\right) \delta(\mathrm{ppm}): 143.0,138.5,136.9,134.3,130.1,129.7,129.4,129.3,128.3,127.6,126.4$, $126.3,69.3,41.8,38.0,29.3,22.7$.

HRMS (ESI) m/z: [M+Na] ${ }^{+}$Calcd for $\mathrm{C}_{17} \mathrm{H}_{17} \mathrm{OCl} 295.0860$; Found 295.0862 .

$>99: 1 d r, 93 \%$ ee HPLC conditions: Chiralpak AD-H column, $n$-hexane:isopropanol $=95: 5$; flow rate $=0.5 \mathrm{~mL} / \mathrm{min}$; UV detection at $220 \mathrm{~nm} ; \mathrm{t}_{\mathrm{R}}=20.6 \mathrm{~min}(\operatorname{minor}), \mathrm{t}_{\mathrm{R}}=25.0 \mathrm{~min}$ (major), $\mathrm{t}_{\mathrm{R}}=28.1 \mathrm{~min}$ (minor). The absolute configuration was assigned by analogy to $\mathbf{3 a}$.

\section{(1S,2R)-2-(2-Methylbenzyl)-1,2,3,4-tetrahydronaphthalen-1-ol (3I)}<smiles>Cc1ccccc1C[C@@H]1CCc2ccccc2[C@H]1O</smiles>

colorless oil, $60.5 \mathrm{mg}, 96 \%$ isolated yield. purification on silica gel using a 20:1 petroleum ether/ethyl acetate mixture.

${ }^{1} \mathbf{H}$ NMR $\left(\mathrm{CDCl}_{3}, 400 \mathrm{MHz}\right) \delta(\mathrm{ppm}):$ 7.18-7.04 (m, 8H), 4.45 (s, 1H), 2.91-2.86 (m, 1H), 2.78-2.76 (m, 1H), 2.67-2.62 (m, 2H), $2.27(\mathrm{~s}, 3 \mathrm{H}), 1.89-1.80(\mathrm{~m}, 1 \mathrm{H}), 1.78-1.75(\mathrm{~m}, 1 \mathrm{H}), 1.65-1.63(\mathrm{~m}, 1 \mathrm{H}), 1.45$ (brs, $1 \mathrm{H})$.

${ }^{13} \mathbf{C}\left\{{ }^{1} \mathbf{H}\right\}$ NMR $\left(\mathrm{CDCl}_{3}, 100 \mathrm{MHz}\right) \delta(\mathrm{ppm}): 139.0,138.7,137.0,136.6,130.5,130.23,130.16,129.2,128.1,126.23$, 126.16, 125.8, 69.9, 40.5, 35.6, 29.4, 22.5, 19.7.

HRMS (ESI) m/z: $[\mathrm{M}+\mathrm{Na}]^{+}$Calcd for $\mathrm{C}_{18} \mathrm{H}_{20} \mathrm{O} 275.1406$; Found 275.1406.

$>$ 99:1 $d r, 95 \%$ ee HPLC conditions: Chiralpak OJ-H column, $n$-hexane:isopropanol $=98: 2$; flow rate $=0.5 \mathrm{~mL} / \mathrm{min}$; UV detection at $220 \mathrm{~nm} ; \mathrm{t}_{\mathrm{R}}=38.1 \mathrm{~min}$ (major), $\mathrm{t}_{\mathrm{R}}=44.5 \mathrm{~min}$ (minor), $\mathrm{t}_{\mathrm{R}}=48.2 \mathrm{~min}$ (minor). The absolute configuration was assigned by analogy to $\mathbf{3 a}$.

\section{(1S,2R)-2-(2-Bromobenzyl)-1,2,3,4-tetrahydronaphthalen-1-ol (3m)}<smiles>O[C@H]1c2ccccc2CC[C@@H]1Cc1ccccc1Br</smiles>

white solid, $73.5 \mathrm{mg}, 93 \%$ isolated yield. purification on silica gel using a 15:1 petroleum ether/ethyl acetate mixture.

${ }^{1} \mathbf{H}$ NMR $\left(\mathrm{CDCl}_{3}, 400 \mathrm{MHz}\right) \delta(\mathrm{ppm}): 7.47(\mathrm{~d}, J=8.0 \mathrm{~Hz}, 1 \mathrm{H}), 7.28-7.26(\mathrm{~m}, 1 \mathrm{H}), 7.20-6.98$ $(\mathrm{m}, 6 \mathrm{H}), 4.44(\mathrm{~s}, 1 \mathrm{H}), 3.03-2.98(\mathrm{~m}, 1 \mathrm{H}), 2.83-2.75(\mathrm{~m}, 2 \mathrm{H}), 2.71-2.62(\mathrm{~m}, 1 \mathrm{H}), 2.09-2.03(\mathrm{~m}$, 1H), 1.81-1.76 (m, 1H), 1.65-1.60 (m, 1H), 1.51 (brs, $1 \mathrm{H})$.

${ }^{13} \mathbf{C}\left\{{ }^{1} \mathbf{H}\right\}$ NMR $\left(\mathrm{CDCl}_{3}, 100 \mathrm{MHz}\right) \delta(\mathrm{ppm}): 140.2,138.6,137.0,133.0,131.9,130.2,129.3,128.2,127.9,127.3,126.3$, $125.1,69.6,39.9,38.6,29.4,22.4$.

HRMS (ESI) m/z: $[\mathrm{M}+\mathrm{Na}]^{+} \mathrm{Calcd}$ for $\mathrm{C}_{17} \mathrm{H}_{17} \mathrm{OBr} 339.0355$; Found 339.0355.

96:4 $d r$, 85\% ee HPLC conditions: Chiralpak AD-H column, $n$-hexane:isopropanol $=95: 5$; flow rate $=0.5 \mathrm{~mL} / \mathrm{min}$; UV detection at $220 \mathrm{~nm} ; \mathrm{t}_{\mathrm{R}}=22.5 \min \left(\right.$ minor), $\mathrm{t}_{\mathrm{R}}=24.9 \min \left(\right.$ major), $\mathrm{t}_{\mathrm{R}}=34.7 \mathrm{~min}$ (minor). The absolute configuration was assigned by analogy to $\mathbf{3 a}$. 
(1S,2R)-2-(2,5-Dimethoxybenzyl)-1,2,3,4-tetrahydronaphthalen-1-ol (3n)

petroleum ether/ethyl acetate mixture.
${ }_{\mathrm{H}}^{1} \mathbf{H M R}\left(\mathrm{CDCl}_{3}, 400 \mathrm{MHz}\right) \delta(\mathrm{ppm}): 7.16(\mathrm{~d}, J=5.2 \mathrm{~Hz}, 1 \mathrm{H}), 7.15-7.02(\mathrm{~m}, 3 \mathrm{H})$,
$6.78-6.68(\mathrm{~m}, 2 \mathrm{H}), 6.67-6.65(\mathrm{~m}, 1 \mathrm{H}), 4.29(\mathrm{~s}, 1 \mathrm{H}), 3.75(\mathrm{~s}, 3 \mathrm{H}), 3.69(\mathrm{~s}, 3 \mathrm{H}), 2.95-2.90(\mathrm{~m}$, $1 \mathrm{H}), 2.84-2.80(\mathrm{~m}, 1 \mathrm{H}), 2.74-2.70(\mathrm{~m}, 2 \mathrm{H}), 2.55-2.50(\mathrm{~m}, 1 \mathrm{H}), 1.93-1.86(\mathrm{~m}, 2 \mathrm{H}), 1.66-1.60(\mathrm{~m}, 1 \mathrm{H})$.

${ }^{13} \mathbf{C}\left\{{ }^{1} \mathbf{H}\right\}$ NMR $\left(\mathrm{CDCl}_{3}, 100 \mathrm{MHz}\right) \delta(\mathrm{ppm}): 154.0,151.7,138.2,137.2,130.7,130.2,129.1,127.8,126.0,117.3,111.8$, 111.7, 68.7, 56.4, 55.8, 41.8, 32.6, 29.6, 23.5.

HRMS (ESI) m/z: [M+Na $]^{+}$Calcd for $\mathrm{C}_{19} \mathrm{H}_{22} \mathrm{O}_{3} 321.1461$; Found 321.1459 .

$>99: 1 \boldsymbol{d r}, \mathbf{9 0} \%$ ee HPLC conditions: Chiralpak IA-3 column, $n$-hexane:isopropanol $=95: 5$; flow rate $=0.5 \mathrm{~mL} / \mathrm{min}$; UV detection at $220 \mathrm{~nm} ; t_{R}=31.3 \mathrm{~min}$ (minor), $t_{R}=35.6 \mathrm{~min}$ (major). The absolute configuration was determined by single-crystal X-ray diffraction analysis. Single crystals suitable for X-ray diffraction measurement were grown from a solution of $\mathbf{3 n}$ in petroleum ether/dichloromethane.

(1S,2R)-2-(3-Bromo-4-fluorobenzyl)-1,2,3,4-tetrahydronaphthalen-1-ol (3o)<smiles>O[C@@H]1c2ccccc2CC[C@@H]1Cc1ccc(F)c(Br)c1</smiles>
colorless oil, $79.3 \mathrm{mg}, 95 \%$ isolated yield. purification on silica gel using a 15:1 petroleum ether/ethyl acetate mixture.

${ }^{1} \mathbf{H}$ NMR $\left(\mathrm{CDCl}_{3}, 400 \mathrm{MHz}\right) \delta(\mathrm{ppm}):$ 7.48-7.46 (m, 1H), 7.28-7.12 (m, 5H), 7.08-7.04 $(\mathrm{m}, 1 \mathrm{H}), 4.47(\mathrm{~d}, J=2.4 \mathrm{~Hz}, 1 \mathrm{H}), 2.95-2.87(\mathrm{~m}, 2 \mathrm{H}), 2.80-2.65(\mathrm{~m}, 2 \mathrm{H}), 1.98-1.93(\mathrm{~m}, 1 \mathrm{H}), 1.85-1.81(\mathrm{~m}, 1 \mathrm{H}), 1.71-1.66$ (m, 1H), 1.49 (brs, 1H).

${ }^{13} \mathbf{C}\left\{{ }^{1} \mathbf{H}\right\}$ NMR $\left(\mathrm{CDCl}_{3}, 100 \mathrm{MHz}\right) \delta(\mathrm{ppm}): 157.7\left(\mathrm{~d},{ }^{1} J=243.6 \mathrm{~Hz}\right), 138.4,138.3\left(\mathrm{~d},{ }^{4} \mathrm{~J}=3.8 \mathrm{~Hz}\right), 136.9,134.0,130.1$, $129.8\left(\mathrm{~d},{ }^{3} J=7.0 \mathrm{~Hz}\right), 129.3,128.3,126.4,116.3\left(\mathrm{~d},{ }^{2} J=21.7 \mathrm{~Hz}\right), 108.8\left(\mathrm{~d},{ }^{2} J=20.9 \mathrm{~Hz}\right), 69.1,41.9,37.3,29.2,22.7$.

HRMS (ESI) m/z: $[\mathrm{M}+\mathrm{Na}]^{+}$Calcd for $\mathrm{C}_{17} \mathrm{H}_{16} \mathrm{OBrF}$ 357.0261; Found 357.0266.

$>$ 99:1 $d r, 92 \%$ ee HPLC conditions: Chiralpak AD-H column, $n$-hexane:isopropanol = 95:5; flow rate $=0.5 \mathrm{~mL} / \mathrm{min}$; UV detection at $220 \mathrm{~nm} ; \mathrm{t}_{\mathrm{R}}=21.3 \mathrm{~min}$ (minor), $\mathrm{t}_{\mathrm{R}}=27.6 \mathrm{~min}$ (major). The absolute configuration was assigned by analogy to $3 a$.

(1S,2R)-2-(2-chloro-4-fluorobenzyl)-1,2,3,4-tetrahydronaphthalen-1-ol (3p)

(c)

colorless oil, $66.0 \mathrm{mg}, 91 \%$ isolated yield. purification on silica gel using a 15:1 petroleum ether/ethyl acetate mixture.

${ }^{1} \mathbf{H}$ NMR $\left(\mathrm{CDCl}_{3}, 400 \mathrm{MHz}\right) \delta(\mathrm{ppm}):$ 7.35-7.29 (m, 2H), 7.23-7.18 (m, 2H), 7.14-7.11 $(\mathrm{m}, 2 \mathrm{H}), 6.98-6.93(\mathrm{~m}, 1 \mathrm{H}), 4.48(\mathrm{~d}, J=2.4 \mathrm{~Hz}, 1 \mathrm{H}), 3.06-3.01(\mathrm{~m}, 1 \mathrm{H}), 2.92-2.76(\mathrm{~m}$, $3 \mathrm{H}), 2.11-2.07(\mathrm{~m}, 1 \mathrm{H}), 1.88-1.84(\mathrm{~m}, 1 \mathrm{H}), 1.71-1.66(\mathrm{~m}, 1 \mathrm{H}), 1.54$ (brs, $1 \mathrm{H})$.

${ }^{13} \mathbf{C}\left\{{ }^{1} \mathbf{H}\right\}$ NMR $\left(\mathrm{CDCl}_{3}, 100 \mathrm{MHz}\right) \delta(\mathrm{ppm}): 161.2\left(\mathrm{~d},{ }^{1} J=246.0 \mathrm{~Hz}\right), 138.5,136.9,134.8\left(\mathrm{~d},{ }^{3} J=10.1 \mathrm{~Hz}\right), 134.2\left(\mathrm{~d},{ }^{4} J=\right.$ $3.5 \mathrm{~Hz}), 132.6\left(\mathrm{~d},{ }^{3} J=8.3 \mathrm{~Hz}\right), 130.2,129.3,128.3,126.3,116.9\left(\mathrm{~d},{ }^{2} J=24.2 \mathrm{~Hz}\right), 113.9\left(\mathrm{~d},{ }^{2} J=20.7 \mathrm{~Hz}\right), 69.5,39.8$, $35.5,29.3,22.5$.

HRMS (ESI) m/z: $[\mathrm{M}+\mathrm{Na}]^{+}$Calcd for $\mathrm{C}_{17} \mathrm{H}_{16} \mathrm{OClF}$ 313.0766; Found 313.0768.

97:3 $d r, \mathbf{8 6} \%$ ee HPLC conditions: Chiralpak AD-H column, n-hexane:isopropanol $=98: 2$; flow rate $=0.5 \mathrm{~mL} / \mathrm{min}$; UV detection at $220 \mathrm{~nm} ; \mathrm{t}_{\mathrm{R}}=35.9 \min \left(\right.$ minor), $\mathrm{t}_{\mathrm{R}}=42.5 \min$ (major), $\mathrm{t}_{\mathrm{R}}=67.1 \mathrm{~min}$ (minor). The absolute configuration was assigned by analogy to $\mathbf{3 a}$. 
(1S,2R)-2-(Naphthalen-2-ylmethyl)-1,2,3,4-tetrahydronaphthalen-1-ol (3q)

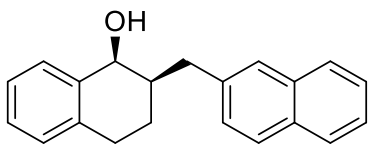

white solid, $67.0 \mathrm{mg}, 93 \%$ isolated yield. purification on silica gel using a 15:1 petroleum ether/ethyl acetate mixture.

${ }^{1} \mathbf{H}$ NMR $\left(\mathrm{CDCl}_{3}, 400 \mathrm{MHz}\right) \delta(\mathrm{ppm}): 7.82-7.78(\mathrm{~m}, 3 \mathrm{H}), 7.71(\mathrm{~s}, 1 \mathrm{H}), 7.47-7.39(\mathrm{~m}$, 3H), 7.23-7.10 (m, 4H), 4.52 (d, $J=2.4 \mathrm{~Hz}, 1 \mathrm{H}), 3.13-3.08(\mathrm{~m}, 1 \mathrm{H}), 2.90-2.85(\mathrm{~m}, 2 \mathrm{H}), 2.78-2.69(\mathrm{~m}, 1 \mathrm{H}), 2.15-2.08(\mathrm{~m}$, $1 \mathrm{H}), 1.92-1.82(\mathrm{~m}, 1 \mathrm{H}), 1.75-1.70(\mathrm{~m}, 1 \mathrm{H}), 1.57$ (brs, $1 \mathrm{H})$.

${ }^{13} \mathbf{C}\left\{{ }^{1} \mathbf{H}\right\}$ NMR $\left(\mathrm{CDCl}_{3}, 100 \mathrm{MHz}\right) \delta(\mathrm{ppm}): 138.7,138.4,137.0,133.8,132.2,130.2,129.3,128.2,128.0,127.8,127.63$, $127.59,126.3,126.1,125.3,69.5,41.8,38.5,29.4,22.8$.

HRMS (ESI) m/z: $[\mathrm{M}+\mathrm{Na}]^{+}$Calcd for $\mathrm{C}_{21} \mathrm{H}_{20} \mathrm{O} 311.1406$; Found 311.1401.

>99: $1 \mathrm{dr}, \mathbf{9 5 \%}$ ee HPLC conditions: Chiralpak OJ-H column, $n$-hexane:isopropanol $=90: 10$; flow rate $=0.5 \mathrm{~mL} / \mathrm{min}$; $\mathrm{UV}$ detection at $220 \mathrm{~nm} ; \mathrm{t}_{\mathrm{R}}=33.4 \mathrm{~min}$ (major), $\mathrm{t}_{\mathrm{R}}=41.0 \mathrm{~min}$ (minor). The absolute configuration was determined by single-crystal X-ray diffraction analysis. Single crystals suitable for X-ray diffraction measurement were grown from a solution of $\mathbf{3 q}$ in petroleum ether/dichloromethane.

(1S,2R)-2-(Naphthalen-2-ylmethyl)-1,2,3,4-tetrahydronaphthalen-1-ol (3r)<smiles>OC1c2ccccc2CCC1Cc1cccc2ccccc12</smiles>
white solid, $69.8 \mathrm{mg}, 97 \%$ isolated yield. purification on silica gel using a 20:1 petroleum ether/ethyl acetate mixture.

${ }^{1} \mathbf{H}$ NMR $\left(\mathrm{CDCl}_{3}, 400 \mathrm{MHz}\right) \delta(\mathrm{ppm}):$ 8.10-8.08 (m, 1H), 7.87-7.84 (m, 1H), 7.75-7.73 (m, 1H), 7.49-7.41 (m, 4H), 7.26-7.08 (m, 4H), $4.57(\mathrm{dd}, J=4.6 \mathrm{~Hz}, 3.0 \mathrm{~Hz}, 1 \mathrm{H}), 3.46-3.41(\mathrm{~m}$, $1 \mathrm{H}), 3.20-3.14(\mathrm{~m}, 1 \mathrm{H}), 2.86-2.84(\mathrm{~m}, 1 \mathrm{H}), 2.73-2.69(\mathrm{~m}, 1 \mathrm{H}), 2.24-2.18(\mathrm{~m}, 1 \mathrm{H}), 1.98-1.87(\mathrm{~m}, 1 \mathrm{H}), 1.77-1.72(\mathrm{~m}, 1 \mathrm{H})$, 1.59 (brd, $J=5.2 \mathrm{~Hz}, 1 \mathrm{H})$.

${ }^{13} \mathbf{C}\left\{{ }^{1} \mathbf{H}\right\}$ NMR $\left(\mathrm{CDCl}_{3}, 100 \mathrm{MHz}\right) \delta(\mathrm{ppm}): 138.7,137.0,136.9,134.2,132.4,130.1,129.3,129.0,128.2,127.6,126.9$, $126.3,125.9,125.58,125.56,124.2,70.0,41.0,35.4,29.3,22.9$.

HRMS (ESI) m/z: $[\mathrm{M}+\mathrm{Na}]^{+}$Calcd for $\mathrm{C}_{21} \mathrm{H}_{20} \mathrm{O}$ 311.1406; Found 311.1403.

$>99: 1 \mathrm{dr}, \mathbf{9 4 \%}$ ee HPLC conditions: Chiralpak OJ-H column, $n$-hexane:isopropanol = 98:2; flow rate $=0.5 \mathrm{~mL} / \mathrm{min}$; UV detection at $220 \mathrm{~nm} ; \mathrm{t}_{\mathrm{R}}=72.6 \mathrm{~min}$ (major), $\mathrm{t}_{\mathrm{R}}=99.1 \mathrm{~min}$ (minor). The absolute configuration was determined by single-crystal X-ray diffraction analysis. Single crystals suitable for X-ray diffraction measurement were grown from a solution of $3 \mathbf{r}$ in petroleum ether/dichloromethane.

(1S,2R)-2-(Pyridin-2-ylmethyl)-1,2,3,4-tetrahydronaphthalen-1-ol (3s)<smiles>O[C@H]1c2ccccc2CC[C@@H]1Cc1ccccn1</smiles>
colorless oil, $59.2 \mathrm{mg}, 99 \%$ isolated yield. purification on silica gel using a 2:1 petroleum ether/ethyl acetate mixture.

${ }^{1} \mathbf{H}$ NMR $\left(\mathrm{CDCl}_{3}, 400 \mathrm{MHz}\right) \delta(\mathrm{ppm}): 8.42(\mathrm{~d}, J=4.4 \mathrm{~Hz}, 1 \mathrm{H}), 7.57-7.53(\mathrm{~m}, 1 \mathrm{H}), 7.26-7.24$ (m, 1H), 7.17-7.14 (m, 1H), 7.08-7.07 (m, 3H), 7.02-6.99 (m, 1H), $4.33(\mathrm{~d}, J=3.2 \mathrm{~Hz}, 1 \mathrm{H}), 3.05-2.99(\mathrm{~m}, 1 \mathrm{H}), 2.84-2.76$ $(\mathrm{m}, 2 \mathrm{H}), 2.72-2.63(\mathrm{~m}, 1 \mathrm{H}), 2.10-2.05(\mathrm{~m}, 1 \mathrm{H}), 1.87-1.82(\mathrm{~m}, 1 \mathrm{H}), 1.65-1.60(\mathrm{~m}, 1 \mathrm{H})$.

${ }^{13} \mathbf{C}\left\{{ }^{1} \mathbf{H}\right\}$ NMR $\left(\mathrm{CDCl}_{3}, 100 \mathrm{MHz}\right) \delta(\mathrm{ppm}): 160.6,148.8,138.2,137.0,136.9,130.6,128.8,127.5,126.0,123.9,121.4$, $68.2,41.1,40.6,29.3,24.3$.

HRMS (ESI) m/z: [M+Na $]^{+}$Calcd for $\mathrm{C}_{16} \mathrm{H}_{17} \mathrm{NO} 262.1202$; Found 262.1203 .

98:2 $d r$, 95\% ee HPLC conditions: Chiralpak OJ-H column, $n$-hexane:isopropanol $=85: 15$; flow rate $=0.5 \mathrm{~mL} / \mathrm{min}$; UV detection at $220 \mathrm{~nm} ; t_{R}=14.5 \min \left(\right.$ minor), $t_{R}=15.3 \min \left(\right.$ major), $t_{R}=18.4 \min \left(\right.$ minor), $t_{R}=26.6 \min ($ minor). The absolute configuration was assigned by analogy to $\mathbf{3 a}$. 
(1S,2R)-2-Isobutyl-1,2,3,4-tetrahydronaphthalen-1-ol (3t)<smiles>CC(C)C[C@H]1CCc2ccccc2[C@H]1O</smiles>
colorless oil, $41.3 \mathrm{mg}, 81 \%$ isolated yield. purification on silica gel using a 30:1 petroleum ether/ethyl acetate mixture.

${ }^{1} \mathbf{H}$ NMR $\left(\mathrm{CDCl}_{3}, 400 \mathrm{MHz}\right) \delta(\mathrm{ppm}): 7.33(\mathrm{~d}, J=7.2 \mathrm{~Hz}, 1 \mathrm{H}), 7.24-7.17(\mathrm{~m}, 2 \mathrm{H}), 7.12(\mathrm{~d}, J=$ $7.2 \mathrm{~Hz}, 1 \mathrm{H}), 4.60(\mathrm{~s}, 1 \mathrm{H}), 2.89-2.73(\mathrm{~m}, 2 \mathrm{H}), 1.85-1.69(\mathrm{~m}, 3 \mathrm{H}), 1.68-1.63(\mathrm{~m}, 1 \mathrm{H}), 1.52-1.47(\mathrm{~m}, 2 \mathrm{H}), 1.31-1.25(\mathrm{~m}, 1 \mathrm{H})$, $0.94(\mathrm{~d}, J=6.8 \mathrm{~Hz}, 6 \mathrm{H})$.

${ }^{13} \mathbf{C}\left\{{ }^{1} \mathbf{H}\right\}$ NMR $\left(\mathrm{CDCl}_{3}, 100 \mathrm{MHz}\right) \delta(\mathrm{ppm}): 138.9,137.2,130.2,129.2,128.0,126.2,70.4,40.9,37.1,29.3,25.0,23.2$, $23.13,23.09$.

HRMS (ESI) m/z: $[\mathrm{M}+\mathrm{Na}]^{+}$Calcd for $\mathrm{C}_{14} \mathrm{H}_{20} \mathrm{O}$ 227.1406; Found 227.1406.

$>\mathbf{9 9 : 1} \boldsymbol{d r}, \mathbf{8 6} \%$ ee HPLC conditions: Chiralpak OD-H column, $n$-hexane:isopropanol $=98: 2$; flow rate $=0.5 \mathrm{~mL} / \mathrm{min}$; UV detection at $220 \mathrm{~nm} ; \mathrm{t}_{\mathrm{R}}=14.8 \mathrm{~min}$ (minor), $\mathrm{t}_{\mathrm{R}}=17.5 \mathrm{~min}$ (major). The absolute configuration was assigned by analogy to 3a.

(1S,2R)-2-Neopentyl-1,2,3,4-tetrahydronaphthalen-1-ol (3u)<smiles>CC(C)(C)C[C@H]1CCc2ccccc2[C@H]1O</smiles>
colorless oil, $48.5 \mathrm{mg}, 89 \%$ isolated yield. purification on silica gel using a $30: 1$ petroleum ether/ethyl acetate mixture.

${ }^{1} \mathbf{H}$ NMR $\left(\mathrm{CDCl}_{3}, 400 \mathrm{MHz}\right) \delta(\mathrm{ppm}): 7.23(\mathrm{~d}, J=7.2 \mathrm{~Hz}, 1 \mathrm{H}), 7.14-7.11(\mathrm{~m}, 2 \mathrm{H}), 7.05(\mathrm{~d}, J=$ $6.8 \mathrm{~Hz}, 1 \mathrm{H}), 4.84$ (s, 1H), 2.77-2.73 (m, 2H), 1.79-1.71 (m, 2H), 1.63-1.58 (m, 2H), 1.44 (brd, $J=3.6 \mathrm{~Hz}, 1 \mathrm{H}), 1.18-1.12$ $(\mathrm{m}, 1 \mathrm{H}), 0.89(\mathrm{~s}, 9 \mathrm{H})$.

${ }^{13} \mathbf{C}\left\{{ }^{1} \mathbf{H}\right\}$ NMR $\left(\mathrm{CDCl}_{3}, 100 \mathrm{MHz}\right) \delta(\mathrm{ppm}): 139.0,136.9,130.1,129.2,128.1,126.2,72.2,45.7,36.1,31.3,30.0,29.4$, 25.1 .

HRMS (ESI) m/z: $[\mathrm{M}+\mathrm{Na}]^{+}$Calcd for $\mathrm{C}_{15} \mathrm{H}_{22} \mathrm{O}$ 241.1563; Found 241.1565.

94:6 dr, 90\% ee HPLC conditions: Chiralpak OJ-H column, $n$-hexane:isopropanol $=98: 2$; flow rate $=0.5 \mathrm{~mL} / \mathrm{min}$; UV detection at $220 \mathrm{~nm} ; \mathrm{t}_{\mathrm{R}}=10.6 \min (\operatorname{minor}), \mathrm{t}_{\mathrm{R}}=11.3 \min \left(\right.$ minor), $\mathrm{t}_{\mathrm{R}}=12.2 \min ($ major$), \mathrm{t}_{\mathrm{R}}=13.2 \min (\operatorname{minor})$. The absolute configuration was assigned by analogy to $\mathbf{3 a}$.

\section{(1S,2R)-2-(Cyclohexylmethyl)-1,2,3,4-tetrahydronaphthalen-1-ol (3v)}

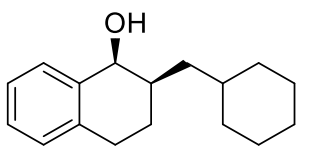

colorless oil, $42.7 \mathrm{mg}, 70 \%$ isolated yield. purification on silica gel using a 30:1 petroleum ether/ethyl acetate mixture.

${ }^{1} \mathbf{H}$ NMR $\left(\mathrm{CDCl}_{3}, 400 \mathrm{MHz}\right) \delta(\mathrm{ppm}):$ 7.35-7.33 (m, 1H), 7.22-7.19 (m, 2H), 7.14-7.12 (m, $1 \mathrm{H}), 4.60(\mathrm{~d}, J=2.8 \mathrm{~Hz}, 1 \mathrm{H}), 2.90-2.77(\mathrm{~m}, 2 \mathrm{H}), 1.88-1.82(\mathrm{~m}, 1 \mathrm{H}), 1.77-1.58(\mathrm{~m}, 7 \mathrm{H}), 1.53-1.48(\mathrm{~m}, 3 \mathrm{H}), 1.32-1.16(\mathrm{~m}$, 4H), 0.97-0.88 (m, 2H),

${ }^{13} \mathbf{C}\left\{{ }^{1} \mathbf{H}\right\}$ NMR $\left(\mathrm{CDCl}_{3}, 100 \mathrm{MHz}\right) \delta(\mathrm{ppm}): 138.9,137.2,130.2,129.2,128.1,126.2,70.4,39.4,36.2,34.6,33.9$, 29.4, $26.9,26.5,23.3$

HRMS (ESI) m/z: [M+Na $]^{+}$Calcd for $\mathrm{C}_{17} \mathrm{H}_{24} \mathrm{O}$ 267.1719; Found 267.1721.

$>\mathbf{9 9 : 1} \boldsymbol{d r}, \mathbf{8 1} \%$ ee HPLC conditions: Chiralpak OJ-H column, $n$-hexane:isopropanol $=98: 2$; flow rate $=0.5 \mathrm{~mL} / \mathrm{min}$; UV detection at $220 \mathrm{~nm} ; \mathrm{t}_{\mathrm{R}}=14.7 \mathrm{~min}$ (major), $\mathrm{t}_{\mathrm{R}}=22.4 \mathrm{~min}$ (minor). The absolute configuration was assigned by analogy to 3a.

\section{(1S,2R)-7-Methoxy-2-(4-methylbenzyl)-1,2,3,4-tetrahydronaphthalen-1-ol (3w)}<smiles>COc1ccc2c(c1)[C@H](O)[C@H](Cc1ccc(C)cc1)CC2</smiles>
orange oil, $63.5 \mathrm{mg}, 90 \%$ isolated yield. purification on silica gel using a 12:1 petroleum ether/ethyl acetate mixture.

${ }^{1} \mathbf{H}$ NMR $\left(\mathrm{CDCl}_{3}, 400 \mathrm{MHz}\right) \delta(\mathrm{ppm}):$ 7.19-7.13 (m, 4H), $7.04(\mathrm{~d}, J=8.0 \mathrm{~Hz}, 1 \mathrm{H})$, 6.81-6.79 (m, 2H), $4.48(\mathrm{t}, J=2.4 \mathrm{~Hz}, 1 \mathrm{H}), 3.77(\mathrm{~s}, 3 \mathrm{H}), 2.94-2.88(\mathrm{~m}, 1 \mathrm{H}), 2.84-2.79(\mathrm{~m}, 1 \mathrm{H}), 2.71-2.66(\mathrm{~m}, 2 \mathrm{H}), 2.35(\mathrm{~s}$, 
$3 \mathrm{H}), 2.02-1.98(\mathrm{~m}, 1 \mathrm{H}), 1.82-1.76(\mathrm{~m}, 1 \mathrm{H}), 1.72-1.68(\mathrm{~m}, 1 \mathrm{H}), 1.53$ (brd, $J=6.0 \mathrm{~Hz}, 1 \mathrm{H})$.

${ }^{13} \mathbf{C}\left\{{ }^{1} \mathbf{H}\right\} \mathbf{N M R}\left(\mathrm{CDCl}_{3}, 100 \mathrm{MHz}\right) \delta(\mathrm{ppm}): 158.0,139.6,137.7,135.5,130.2,129.25,129.19,129.0,115.1,114.1,69.8$, 55.4, 41.9, 37.7, 28.5, 23.0, 21.2 .

HRMS (ESI) m/z: [M+Na] $]^{+}$Calcd for $\mathrm{C}_{19} \mathrm{H}_{22} \mathrm{O}_{2}$ 305.1512; Found 305.1516.

$>$ 99:1 dr, 96\% ee HPLC conditions: Chiralpak OJ-H column, $n$-hexane:isopropanol = 92:8; flow rate $=0.5 \mathrm{~mL} / \mathrm{min}$; UV detection at $220 \mathrm{~nm} ; \mathrm{t}_{\mathrm{R}}=28.0 \mathrm{~min}$ (major), $\mathrm{t}_{\mathrm{R}}=39.0 \mathrm{~min}$ (minor). The absolute configuration was assigned by analogy to $3 a$.

(1S,2R)-7-Fluoro-2-(4-methylbenzyl)-1,2,3,4-tetrahydronaphthalen-1-ol (3x)<smiles>Cc1ccc(C[C@H]2CCc3ccc(F)cc3[C@@H]2O)cc1</smiles>
white solid, $52.7 \mathrm{mg}, 78 \%$ isolated yield. purification on silica gel using a $18: 1$ petroleum ether/ethyl acetate mixture.

${ }^{1} \mathbf{H}$ NMR $\left(\mathrm{CDCl}_{3}, 400 \mathrm{MHz}\right) \delta(\mathrm{ppm}):$ 7.18-7.13 (m, 4H), 7.10-7.06 (m, 1H), 7.00-6.97 (m, 1H), 6.94-6.89 (m, 1H), $4.49(\mathrm{~s}, 1 \mathrm{H}), 2.92-2.83(\mathrm{~m}, 2 \mathrm{H}), 2.70-2.65(\mathrm{~m}, 2 \mathrm{H}), 2.35(\mathrm{~s}, 3 \mathrm{H}), 2.05-1.97(\mathrm{~m}, 1 \mathrm{H})$, $1.82-1.69(\mathrm{~m}, 2 \mathrm{H}), 1.57$ (brd, $J=6.4 \mathrm{~Hz}, 1 \mathrm{H})$.

${ }^{13} \mathbf{C}\left\{{ }^{1} \mathbf{H}\right\}$ NMR $\left(\mathrm{CDCl}_{3}, 100 \mathrm{MHz}\right) \delta(\mathrm{ppm}): 161.2\left(\mathrm{~d},{ }^{1} J=242.7 \mathrm{~Hz}\right), 140.3\left(\mathrm{~d},{ }^{3} \mathrm{~J}=6.1 \mathrm{~Hz}\right), 137.4,135.6,132.5\left(\mathrm{~d},{ }^{4} J=\right.$ $3.1 \mathrm{~Hz}), 130.6\left(\mathrm{~d},{ }^{3} J=7.4 \mathrm{~Hz}\right), 129.3,129.2,116.1\left(\mathrm{~d},{ }^{2} J=20.3 \mathrm{~Hz}\right), 115.3\left(\mathrm{~d},{ }^{2} J=21.2 \mathrm{~Hz}\right), 69.3,41.6,37.5,28.6,22.8$, 21.2 .

HRMS (ESI) m/z: [M+Na $]^{+}$Calcd for $\mathrm{C}_{18} \mathrm{H}_{19} \mathrm{OF}$ 293.1312; Found 293.1318.

$>$ 99: $1 \mathrm{dr}, \mathbf{9 5 \%}$ ee HPLC conditions: Chiralpak AD-H column, $n$-hexane:isopropanol = 95:5; flow rate $=0.5 \mathrm{~mL} / \mathrm{min}$; UV detection at $220 \mathrm{~nm} ; \mathrm{t}_{\mathrm{R}}=21.7 \mathrm{~min}$ (minor), $\mathrm{t}_{\mathrm{R}}=25.1 \mathrm{~min}$ (major). The absolute configuration was assigned by analogy to $3 a$.

(1S,2R)-7-Bromo-2-(4-methylbenzyl)-1,2,3,4-tetrahydronaphthalen-1-ol (3y)<smiles>Cc1ccc(C[C@H]2CCc3ccc(Br)cc3[C@@H]2O)cc1</smiles>
white solid, $75.1 \mathrm{mg}, 91 \%$ isolated yield. purification on silica gel using a 20:1 petroleum ether/ethyl acetate mixture.

${ }^{1} \mathbf{H}$ NMR $\left(\mathrm{CDCl}_{3}, 400 \mathrm{MHz}\right) \delta(\mathrm{ppm}): 7.38(\mathrm{~s}, 1 \mathrm{H}), 7.28(\mathrm{~d}, J=8.0 \mathrm{~Hz}, 1 \mathrm{H})$, 7.14-7.09 (m, 4H), $6.95(\mathrm{~d}, J=8.4 \mathrm{~Hz}, 1 \mathrm{H}), 4.43(\mathrm{~s}, 1 \mathrm{H}), 2.88-2.77(\mathrm{~m}, 2 \mathrm{H}), 2.66-2.58(\mathrm{~m}, 2 \mathrm{H}), 2.33(\mathrm{~s}, 3 \mathrm{H}), 1.96-1.93$ (m, 1H), 1.79-1.74 (m, 1H), 1.69-1.66 (m, 2H).

${ }^{13} \mathbf{C}\left\{{ }^{1} \mathbf{H}\right\} \mathbf{N M R}\left(\mathrm{CDCl}_{3}, 100 \mathrm{MHz}\right) \delta(\mathrm{ppm}): 140.7,137.3,135.9,135.6,132.8,131.0,130.9,129.2,129.1,119.5,69.1$, 41.6, 37.4, 28.7, 22.5, 21.2 .

HRMS (ESI) m/z: [M+Na] ${ }^{+}$Calcd for $\mathrm{C}_{18} \mathrm{H}_{19} \mathrm{OBr} 353.0511$; Found 353.0514.

$>$ 99:1 dr, 99\% ee HPLC conditions: Chiralpak AD-H column, $n$-hexane:isopropanol = 98:2; flow rate $=0.5 \mathrm{~mL} / \mathrm{min}$; UV detection at $220 \mathrm{~nm} ; \mathrm{t}_{\mathrm{R}}=45.8 \mathrm{~min}$ (major), $\mathrm{t}_{\mathrm{R}}=48.4 \mathrm{~min}$ (minor), $\mathrm{t}_{\mathrm{R}}=78.3 \mathrm{~min}$ (minor). The absolute configuration was determined by single-crystal X-ray diffraction analysis. Single crystals suitable for X-ray diffraction measurement were grown from a solution of $\mathbf{3 y}$ in petroleum ether/dichloromethane.

\section{(1S,2R)-5-Methoxy-2-(4-methylbenzyl)-1,2,3,4-tetrahydronaphthalen-1-ol (3z)}<smiles>COc1cccc2c1CC[C@H](Cc1ccc(C)cc1)[C@H]2O</smiles>
white solid, $64.9 \mathrm{mg}, 92 \%$ isolated yield. purification on silica gel using a 15:1 petroleum ether/ethyl acetate mixture.

${ }^{1} \mathbf{H}$ NMR $\left(\mathrm{CDCl}_{3}, 400 \mathrm{MHz}\right) \delta(\mathrm{ppm}): 7.17-7.11(\mathrm{~m}, 5 \mathrm{H}), 6.89(\mathrm{~d}, J=7.6 \mathrm{~Hz}, 1 \mathrm{H}), 6.76(\mathrm{~d}$, $J=8.0 \mathrm{~Hz}, 1 \mathrm{H}), 4.50(\mathrm{~d}, J=2.8 \mathrm{~Hz}, 1 \mathrm{H}), 3.81(\mathrm{~s}, 3 \mathrm{H}), 2.94-2.87(\mathrm{~m}, 2 \mathrm{H}), 2.70-2.65(\mathrm{~m}$, 1H), 2.48-2.39 (m, 1H), $2.34(\mathrm{~s}, 3 \mathrm{H}), 2.01-1.93(\mathrm{~m}, 1 \mathrm{H}), 1.79-1.72(\mathrm{~m}, 2 \mathrm{H}), 1.53(\mathrm{brs}, 1 \mathrm{H})$.

${ }^{13} \mathbf{C}\left\{{ }^{1} \mathbf{H}\right\} \mathbf{N M R}\left(\mathrm{CDCl}_{3}, 100 \mathrm{MHz}\right) \delta(\mathrm{ppm}): 157.3,139.9,137.7,135.4,129.2,129.1,126.7,126.0,122.0,109.1,69.4$, $55.4,41.3,37.7,23.3,22.1,21.2$. 
HRMS (ESI) m/z: $[\mathrm{M}+\mathrm{Na}]^{+}$Calcd for $\mathrm{C}_{19} \mathrm{H}_{22} \mathrm{O}_{2}$ 305.1512; Found 305.1514 .

$>\mathbf{9 9 : 1} \mathbf{d r}, \mathbf{8 8} \%$ ee HPLC conditions: Chiralpak OD-H column, $n$-hexane:isopropanol = 95:5; flow rate $=0.5 \mathrm{~mL} / \mathrm{min}$; UV detection at $220 \mathrm{~nm} ; t_{R}=20.7 \mathrm{~min}$ (minor), $t_{R}=25.4 \mathrm{~min}$ (major). The absolute configuration was determined by single-crystal X-ray diffraction analysis. Single crystals suitable for X-ray diffraction measurement were grown from a solution of $\mathbf{3 z}$ in petroleum ether/dichloromethane.

\section{(4S,5R)-5-(4-Methylbenzyl)-4,5,6,7-tetrahydrobenzo[b]thiophen-4-ol (3aa)}

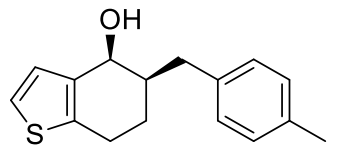

colorless oil, $46.4 \mathrm{mg}, 72 \%$ isolated yield. purification on silica gel using a 15:1 petroleum ether/ethyl acetate mixture.

${ }^{1} \mathbf{H}$ NMR $\left(\mathrm{CDCl}_{3}, 400 \mathrm{MHz}\right) \delta(\mathrm{ppm}):$ 7.17-7.12 (m, 4H), $7.08(\mathrm{~d}, J=5.2 \mathrm{~Hz}, 1 \mathrm{H}), 6.92(\mathrm{~d}$, $J=5.2 \mathrm{~Hz}, 1 \mathrm{H}), 4.52(\mathrm{t}, J=3.0 \mathrm{~Hz}, 1 \mathrm{H}), 2.94-2.87(\mathrm{~m}, 2 \mathrm{H}), 2.74-2.66(\mathrm{~m}, 2 \mathrm{H}), 2.34(\mathrm{~s}, 3 \mathrm{H}), 2.02-1.97(\mathrm{~m}, 1 \mathrm{H})$, $1.87-1.75(\mathrm{~m}, 2 \mathrm{H}), 1.44(\mathrm{brd}, J=6.4 \mathrm{~Hz}, 1 \mathrm{H})$.

${ }^{13} \mathbf{C}\left\{{ }^{1} \mathbf{H}\right\}$ NMR $\left(\mathrm{CDCl}_{3}, 100 \mathrm{MHz}\right) \delta(\mathrm{ppm}): 139.5,138.2,137.5,135.6,129.23,129.22,127.3,123.3,65.6,42.2,37.6$, 25.4, 23.8, 21.2.

HRMS (ESI) m/z: [M+Na $]^{+}$Calcd for $\mathrm{C}_{16} \mathrm{H}_{18} \mathrm{OS} 281.0971$; Found 281.0973.

99:1 dr, 92\% ee HPLC conditions: Chiralpak OJ-H column, $n$-hexane:isopropanol $=95: 5$; flow rate $=0.5 \mathrm{~mL} / \mathrm{min}$; UV detection at $220 \mathrm{~nm} ; \mathrm{t}_{\mathrm{R}}=23.7 \mathrm{~min}$ (major), $\mathrm{t}_{\mathrm{R}}=27.6 \mathrm{~min}$ (minor), $\mathrm{t}_{\mathrm{R}}=30.0 \mathrm{~min}$ (minor). The absolute configuration was assigned by analogy to $\mathbf{3 a}$.

tert-Butyl (3S,4S)-4-hydroxy-3-(4-methylbenzyl)-3,4-dihydroquinoline-1(2H)-carboxylate (3ab)<smiles>Cc1ccc(C[C@@H]2CN(C(=O)OC(C)(C)C)c3ccccc3[C@H]2O)cc1</smiles>
white solid, $81.2 \mathrm{mg}, 92 \%$ isolated yield. purification on silica gel using a 10:1 petroleum ether/ethyl acetate mixture.

${ }^{1} \mathbf{H}$ NMR $\left(\mathrm{CDCl}_{3}, 400 \mathrm{MHz}\right) \delta(\mathrm{ppm}): 7.91$ (d, $\left.J=8.4 \mathrm{~Hz}, 1 \mathrm{H}\right), 7.29-7.24(\mathrm{~m}, 2 \mathrm{H}), 7.17(\mathrm{~s}$, 4H), 7.05-7.04 (m, 1H), $4.54(\mathrm{~s}, 1 \mathrm{H}), 4.03-3.99(\mathrm{~m}, 1 \mathrm{H}), 3.39$ (t, $J=12.2 \mathrm{~Hz}, 1 \mathrm{H})$, 2.90-2.87 (m, 1H), 2.75-2.70 (m, 1H), 2.37 (s, 3H), 2.19-2.15 (m, 1H), 1.75 (brs, 1H), $1.50(\mathrm{~s}, 9 \mathrm{H})$.

${ }^{13} \mathbf{C}\left\{{ }^{1} \mathbf{H}\right\}$ NMR $\left(\mathrm{CDCl}_{3}, 100 \mathrm{MHz}\right) \delta(\mathrm{ppm}): 153.8,137.9,136.5,135.8,130.2,129.3,129.1,128.6,123.4,123.2,81.3$, $67.9,44.3,41.5,34.6,28.4,21.1$.

HRMS (ESI) m/z: $[\mathrm{M}+\mathrm{Na}]^{+}$Calcd for $\mathrm{C}_{22} \mathrm{H}_{27} \mathrm{O}_{3} \mathrm{~N}$ 376.1883; Found 376.1890.

$>$ 99: $1 \boldsymbol{d r}, \mathbf{9 7 \%}$ ee HPLC conditions: Chiralpak OJ-H column, $n$-hexane:isopropanol $=95: 5$; flow rate $=0.3 \mathrm{~mL} / \mathrm{min}$; UV detection at $220 \mathrm{~nm} ; \mathrm{t}_{\mathrm{R}}=39.8 \mathrm{~min}$ (major), $\mathrm{t}_{\mathrm{R}}=45.2 \mathrm{~min}$ (minor), $\mathrm{t}_{\mathrm{R}}=51.3 \mathrm{~min}$ (minor). The absolute configuration was assigned by analogy to $\mathbf{3 a}$.

(3S,4S)-3-(4-Methylbenzyl)chroman-4-ol (3ac)<smiles>Cc1ccc(C[C@@H]2COc3ccccc3[C@@H]2O)cc1</smiles>
white solid, $57.8 \mathrm{mg}, 91 \%$ isolated yield. purification on silica gel using a 20:1 petroleum ether/ethyl acetate mixture.

${ }^{1} \mathbf{H}$ NMR $\left(\mathrm{CDCl}_{3}, 400 \mathrm{MHz}\right) \delta(\mathrm{ppm}):$ 7.21-7.12 (m, 6H), 6.90-6.82 (m, 2H), $4.50(\mathrm{~s}, 1 \mathrm{H})$, $4.07(\mathrm{~d}, J=8.4 \mathrm{~Hz}, 2 \mathrm{H}), 2.86-2.80(\mathrm{~m}, 1 \mathrm{H}), 2.65-2.60(\mathrm{~m}, 1 \mathrm{H}), 2.34-2.30(\mathrm{~m}, 4 \mathrm{H}), 1.72$

(brs, 1H).

${ }^{13} \mathbf{C}\left\{{ }^{1} \mathbf{H}\right\}$ NMR $\left(\mathrm{CDCl}_{3}, 100 \mathrm{MHz}\right) \delta(\mathrm{ppm}): 154.5,136.1,135.9,130.3,130.0,129.4,129.1,124.3,120.6,117.0,65.1$, $65.0,40.1,32.5,21.2$.

HRMS (ESI) m/z: [M+Na $]^{+}$Calcd for $\mathrm{C}_{17} \mathrm{H}_{18} \mathrm{O}_{2}$ 277.1199; Found 277.1202.

$>99: 1 \boldsymbol{d r},>\mathbf{9 9} \% \boldsymbol{e e}$ HPLC conditions: Chiralpak OD-H column, $n$-hexane:isopropanol $=98: 2$; flow rate $=0.5 \mathrm{~mL} / \mathrm{min}$; $\mathrm{UV}$ detection at $220 \mathrm{~nm} ; \mathrm{t}_{\mathrm{R}}=32.5$ (major). The absolute configuration was determined by single-crystal X-ray diffraction analysis. Single crystals suitable for X-ray diffraction measurement were grown from a solution of 3ac in petroleum 
ether/dichloromethane.

(3S,4S)-6-Methyl-3-(4-methylbenzyl)chroman-4-ol (3ad)<smiles>Cc1ccc(C[C@@H]2COc3ccc(C)cc3[C@@H]2O)cc1</smiles>
yellow solid, $50.3 \mathrm{mg}, 75 \%$ isolated yield. purification on silica gel using a 20:1 petroleum ether/ethyl acetate mixture.

${ }^{1} \mathbf{H}$ NMR $\left(\mathrm{CDCl}_{3}, 400 \mathrm{MHz}\right) \delta(\mathrm{ppm}):$ 7.18-7.13 (m, 4H), $7.02(\mathrm{~s}, 2 \mathrm{H}), 6.76-6.74(\mathrm{~m}$, $1 \mathrm{H}), 4.48(\mathrm{~s}, 1 \mathrm{H}), 4.06(\mathrm{~d}, J=7.6 \mathrm{~Hz}, 2 \mathrm{H}), 2.87-2.81(\mathrm{~m}, 1 \mathrm{H}), 2.67-2.62(\mathrm{~m}, 1 \mathrm{H}), 2.33$ (s, 3H), 2.32-2.28 (m, 4H), 1.70 (brs, $1 \mathrm{H})$.

${ }^{13} \mathbf{C}\left\{{ }^{1} \mathbf{H}\right\}$ NMR $\left(\mathrm{CDCl}_{3}, 100 \mathrm{MHz}\right) \delta(\mathrm{ppm}): 152.2,136.2,135.9,130.7,130.5,129.8,129.4,129.1,124.0,116.8,65.13$, $65.10,40.3,32.5,21.2,20.5$.

HRMS (ESI) m/z: [M+Na $]^{+}$Calcd for $\mathrm{C}_{18} \mathrm{H}_{20} \mathrm{O}_{2}$ 291.1356; Found 291.1359.

$>99: 1 d r,>99 \% e e$ HPLC conditions: Chiralpak OJ-H column, $n$-hexane:isopropanol $=95: 5$; flow rate $=0.5 \mathrm{~mL} / \mathrm{min}$; $\mathrm{UV}$ detection at $220 \mathrm{~nm} ; \mathrm{t}_{\mathrm{R}}=38.7$ (major). The absolute configuration was assigned by analogy to $3 \mathbf{a c}$.

\section{(1S,2S)-2-(4-Methylbenzyl)-2,3-dihydro-1H-inden-1-ol (3ae)}<smiles>Cc1ccc(C[C@@H]2Cc3ccccc3[C@@H]2O)cc1</smiles>
colorless oil, $50.0 \mathrm{mg}, 84 \%$ isolated yield. purification on silica gel using a 20:1 petroleum ether/ethyl acetate mixture.

${ }^{1} \mathbf{H}$ NMR $\left(\mathrm{CDCl}_{3}, 400 \mathrm{MHz}\right) \delta(\mathrm{ppm}): 7.30(\mathrm{~d}, J=7.2 \mathrm{~Hz}, 1 \mathrm{H}), 7.17-7.10(\mathrm{~m}, 5 \mathrm{H}), 7.06-7.04(\mathrm{~m}, 2 \mathrm{H}), 4.90(\mathrm{~s}, 1 \mathrm{H}), 2.97$ (dd, $J=13.2 \mathrm{~Hz}, 7.2 \mathrm{~Hz}, 1 \mathrm{H}), 2.75(\mathrm{~d}, J=8.0 \mathrm{~Hz}, 2 \mathrm{H}), 2.67-2.54(\mathrm{~m}, 2 \mathrm{H}), 2.26(\mathrm{~s}, 3 \mathrm{H}), 1.45$ (brd, $J=4.4 \mathrm{~Hz}, 1 \mathrm{H})$.

${ }^{13} \mathbf{C}\left\{{ }^{1} \mathbf{H}\right\}$ NMR $\left(\mathrm{CDCl}_{3}, 100 \mathrm{MHz}\right) \delta(\mathrm{ppm}): 144.9,143.7,138.3,135.5,129.3,128.9,128.8,126.9,125.2,124.9,76.5$, 47.1, 36.0, 34.6, 21.2 .

HRMS (ESI) m/z: [M+Na] $]^{+}$Calcd for $\mathrm{C}_{17} \mathrm{H}_{18} \mathrm{O} 261.1250$; Found 261.1253.

93:7 $\boldsymbol{d r}, \mathbf{7 9} \%$ ee HPLC conditions: Chiralpak AD-H column, $n$-hexane:isopropanol $=92: 8$; flow rate $=0.5 \mathrm{~mL} / \mathrm{min}$; UV detection at $220 \mathrm{~nm} ; \mathrm{t}_{\mathrm{R}}=16.8 \mathrm{~min}$ (minor), $\mathrm{t}_{\mathrm{R}}=23.3 \mathrm{~min}$ (major), $\mathrm{t}_{\mathrm{R}}=27.1 \mathrm{~min}\left(\right.$ minor), $\mathrm{t}_{\mathrm{R}}=42.5 \mathrm{~min}$ (minor). The absolute configuration was assigned by analogy to $\mathbf{3 a}$.

(5S,6R)-6-(4-Methylbenzyl)-6,7,8,9-tetrahydro-5H-benzo[7]annulen-5-ol (3af)

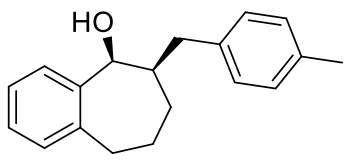

yellow oil, $50.5 \mathrm{mg}, 76 \%$ isolated yield. purification on silica gel using a $20: 1$ petroleum ether/ethyl acetate mixture.

${ }^{1} \mathbf{H}$ NMR $\left(\mathrm{CDCl}_{3}, 400 \mathrm{MHz}\right) \delta(\mathrm{ppm}): 7.22(\mathrm{~s}, 1 \mathrm{H}), 7.16-7.14(\mathrm{~m}, 2 \mathrm{H}), 7.09-7.01(\mathrm{~m}, 5 \mathrm{H})$, $4.88(\mathrm{~s}, 1 \mathrm{H}), 3.07-3.01(\mathrm{~m}, 1 \mathrm{H}), 2.73-2.63(\mathrm{~m}, 2 \mathrm{H}), 2.26(\mathrm{~s}, 3 \mathrm{H}), 2.23-2.11(\mathrm{~m}, 1 \mathrm{H})$, 1.95-1.91 (m, 1H), 1.89-1.88 (m, 1H), 1.80-1.67 (m, 3H), 1.58-1.51 (m, $1 \mathrm{H})$.

${ }^{13} \mathbf{C}\left\{{ }^{1} \mathbf{H}\right\}$ NMR $\left(\mathrm{CDCl}_{3}, 100 \mathrm{MHz}\right) \delta(\mathrm{ppm}): 142.1,141.6,138.2,135.3,129.8,129.13,129.10,127.5,127.3,126.0,77.4$, 45.1, 37.3, 35.7, 31.3, 25.5, 21.1 .

HRMS (ESI) m/z: $[\mathrm{M}+\mathrm{Na}]^{+}$Calcd for $\mathrm{C}_{19} \mathrm{H}_{22} \mathrm{O} 289.1563$; Found 289.1566 .

$>$ 99:1 $d r, 82 \%$ ee HPLC conditions: Chiralpak AD-H column, $n$-hexane:isopropanol $=98: 2$; flow rate $=0.5 \mathrm{~mL} / \mathrm{min}$; UV detection at $220 \mathrm{~nm} ; \mathrm{t}_{\mathrm{R}}=31.8 \mathrm{~min}$ (minor), $\mathrm{t}_{\mathrm{R}}=42.3 \mathrm{~min}$ (major). The absolute configuration was assigned by analogy to 3a. 
$(1 S, 2 S)-1,2,3-T r i p h e n y l p r o p a n-1-o l ~(5 a)^{[7 b]}$<smiles>OC(c1ccccc1)C(c1ccccc1)c1ccccc1</smiles>

white solid, $59.8 \mathrm{mg}, 83 \%$ isolated yield. purification on silica gel using a 20:1 petroleum ether/ethyl acetate mixture.

${ }^{1} \mathbf{H}$ NMR $\left(\mathrm{CDCl}_{3}, 400 \mathrm{MHz}\right) \delta(\mathrm{ppm}):$ 7.40-7.23 (m, 8H), 7.18-7.12 (m, 5H), $6.94(\mathrm{~d}, J=7.2$ $\mathrm{Hz}, 2 \mathrm{H}), 4.90$ (dd, $J=7.2 \mathrm{~Hz}, 2.8 \mathrm{~Hz}, 1 \mathrm{H}), 3.34-3.19$ (m, 1H), 2.99-2.85 (m, 2H), 1.91 (brs, 1H).

${ }^{13} \mathbf{C}\left\{{ }^{1} \mathbf{H}\right\}$ NMR $\left(\mathrm{CDCl}_{3}, 100 \mathrm{MHz}\right) \delta(\mathrm{ppm}): 142.8,140.3,140.1,129.2,129.1,128.4,128.2,127.9,127.04,126.96,125.9$, $77.4,56.3,38.8$

HRMS (ESI) m/z: [M+Na] $]^{+}$Calcd for $\mathrm{C}_{21} \mathrm{H}_{20} \mathrm{O}$ 311.140; Found 311.1409.

96:4 $d r, 90 \%$ ee HPLC conditions: Chiralpak AD-H column, $n$-hexane:isopropanol $=98: 2$; flow rate $=0.5 \mathrm{~mL} / \mathrm{min}$; UV detection at $220 \mathrm{~nm} ; t_{R}=47.5 \min \left(\right.$ minor), $t_{R}=50.2 \min \left(\right.$ minor), $t_{R}=54.8 \min \left(\right.$ minor), $t_{R}=67.4$ min (major). The absolute configuration was determined by single-crystal X-ray diffraction analysis. Single crystals suitable for X-ray diffraction measurement were grown from a solution of $\mathbf{5 a}$ in petroleum ether/dichloromethane.

\section{(1S,2S)-1,2-Diphenyl-3-(p-tolyl)propan-1-ol (5b)}

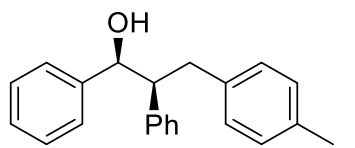

white solid, $45.3 \mathrm{mg}, 60 \%$ isolated yield. purification on silica gel using a 25:1 petroleum ether/ethyl acetate mixture.

${ }^{1} \mathbf{H}$ NMR $\left(\mathrm{CDCl}_{3}, 400 \mathrm{MHz}\right) \delta(\mathrm{ppm}): 7.32-7.19(\mathrm{~m}, 8 \mathrm{H}), 7.12(\mathrm{~d}, J=7.2 \mathrm{~Hz}, 2 \mathrm{H}), 6.92(\mathrm{~d}$, $J=7.6 \mathrm{~Hz}, 2 \mathrm{H}), 6.78(\mathrm{~d}, J=7.6 \mathrm{~Hz}, 2 \mathrm{H}), 4.84(\mathrm{~d}, J=7.2 \mathrm{~Hz}, 1 \mathrm{H}), 3.15-3.11(\mathrm{~m}, 1 \mathrm{H}), 2.91-2.77(\mathrm{~m}, 2 \mathrm{H}), 2.23(\mathrm{~s}, 3 \mathrm{H})$, 1.26 (brs, $1 \mathrm{H})$.

${ }^{13} \mathbf{C}\left\{{ }^{1} \mathbf{H}\right\}$ NMR $\left(\mathrm{CDCl}_{3}, 100 \mathrm{MHz}\right) \delta(\mathrm{ppm}): 142.8,140.3,137.0,135.3,129.2,128.91,128.87,128.41,128.40,127.8$, $127.0,126.9,77.3,56.3,38.2,21.1$.

HRMS (ESI) m/z: $[\mathrm{M}+\mathrm{Na}]^{+}$Calcd for $\mathrm{C}_{22} \mathrm{H}_{22} \mathrm{O} 325.1563$; Found 325.1566 .

95:5 $d r, 89 \%$ ee HPLC conditions: Chiralpak AD-H column, $n$-hexane:isopropanol $=95: 5$; flow rate $=0.5 \mathrm{~mL} / \mathrm{min}$; UV detection at $220 \mathrm{~nm} ; \mathrm{t}_{\mathrm{R}}=25.1 \min \left(\right.$ minor), $\mathrm{t}_{\mathrm{R}}=28.2 \min \left(\right.$ minor), $\mathrm{t}_{\mathrm{R}}=29.9 \min (\operatorname{minor}), \mathrm{t}_{\mathrm{R}}=41.0 \min ($ major). The absolute configuration was assigned by analogy to $\mathbf{5 a}$.

\section{(1S,2S)-3-(4-Methoxyphenyl)-1,2-diphenylpropan-1-ol (5c ${ }^{[8]}$}<smiles>COc1ccc(C[C@H](c2ccccc2)[C@H](O)c2ccccc2)cc1</smiles>

colorless oil, $46.1 \mathrm{mg}, 58 \%$ isolated yield. purification on silica gel using a 20:1 petroleum ether/ethyl acetate mixture.

${ }^{1} \mathbf{H}$ NMR $\left(\mathrm{CDCl}_{3}, 400 \mathrm{MHz}\right) \delta(\mathrm{ppm}): 7.38-7.23(\mathrm{~m}, 8 \mathrm{H}), 7.15(\mathrm{~d}, J=7.6 \mathrm{~Hz}, 2 \mathrm{H})$, $6.83(\mathrm{~d}, J=8.0 \mathrm{~Hz}, 2 \mathrm{H}), 6.70(\mathrm{~d}, J=8.0 \mathrm{~Hz}, 2 \mathrm{H}), 4.88(\mathrm{~d}, J=7.2 \mathrm{~Hz}, 1 \mathrm{H}), 3.74(\mathrm{~s}$,

$3 \mathrm{H}), 3.18-3.12(\mathrm{~m}, 1 \mathrm{H}), 2.92-2.78(\mathrm{~m}, 2 \mathrm{H}), 1.77$ (brs, $1 \mathrm{H})$.

${ }^{13} \mathbf{C}\left\{{ }^{1} \mathbf{H}\right\}$ NMR $\left(\mathrm{CDCl}_{3}, 100 \mathrm{MHz}\right) \delta(\mathrm{ppm}): 157.8,142.9,140.4,132.2,130.0,129.3,128.5,128.4,127.9,127.01,126.96$, 113.6, 77.4, 56.5, 55.2, 37.9.

HRMS (ESI) m/z: [M+Na $]^{+}$Calcd for $\mathrm{C}_{22} \mathrm{H}_{22} \mathrm{O}_{2}$ 341.1512; Found 341.1511.

$>$ 99:1 $d r$, 72\% ee HPLC conditions: Chiralpak AD-H column, $n$-hexane:isopropanol = 95:5; flow rate $=0.5 \mathrm{~mL} / \mathrm{min}$; UV detection at $220 \mathrm{~nm} ; \mathrm{t}_{\mathrm{R}}=46.1 \mathrm{~min}$ (minor), $\mathrm{t}_{\mathrm{R}}=66.2 \mathrm{~min}$ (major). The absolute configuration was assigned by analogy to $5 a$.

\section{(1S,2S)-3-(4-Fluorophenyl)-1,2-diphenylpropan-1-ol (5d)}<smiles>O[C@H](c1ccccc1)[C@H](Cc1ccc(F)cc1)c1ccccc1</smiles>

colorless oil, $64.3 \mathrm{mg}, 84 \%$ isolated yield. purification on silica gel using a 20:1 petroleum ether/ethyl acetate mixture.

${ }^{1} \mathbf{H}$ NMR $\left(\mathrm{CDCl}_{3}, 400 \mathrm{MHz}\right) \delta(\mathrm{ppm}): 7.35-7.20(\mathrm{~m}, 8 \mathrm{H}), 7.10(\mathrm{~d}, J=6.8 \mathrm{~Hz}, 2 \mathrm{H})$, 6.80-6.75 (m, 4H), 4.84 (dd, $J=7.4 \mathrm{~Hz}, 2.6 \mathrm{~Hz}, 1 \mathrm{H}), 3.12-3.07$ (m, 1H), 2.88-2.74 (m, 
2H), 1.84 (brd, $J=2.8 \mathrm{~Hz}, 1 \mathrm{H})$.

${ }^{13} \mathbf{C}\left\{{ }^{1} \mathbf{H}\right\}$ NMR $\left(\mathrm{CDCl}_{3}, 100 \mathrm{MHz}\right) \delta(\mathrm{ppm}): 161.3\left(\mathrm{~d},{ }^{1} J=242.2 \mathrm{~Hz}\right), 142.7,140.1,135.7\left(\mathrm{~d},{ }^{4} J=3.2 \mathrm{~Hz}\right), 130.4\left(\mathrm{~d},{ }^{3} J=\right.$ $7.6 \mathrm{~Hz}), 129.2,128.55,128.52,128.0,127.2,127.0,114.9\left(\mathrm{~d},{ }^{2} J=21.1 \mathrm{~Hz}\right), 77.5,56.6,38.0$.

HRMS (ESI) m/z: [M+Na $]^{+}$Calcd for $\mathrm{C}_{21} \mathrm{H}_{19} \mathrm{OF}$ 329.1312; Found 329.1307.

97:3 dr, 86\% ee HPLC conditions: Chiralpak AD-H column, $n$-hexane:isopropanol $=95: 5$; flow rate $=0.5 \mathrm{~mL} / \mathrm{min}$; UV detection at $220 \mathrm{~nm} ; t_{R}=27.0 \min \left(\right.$ minor), $t_{R}=28.1 \mathrm{~min}\left(\right.$ minor), $t_{R}=34.0 \min \left(\right.$ minor), $t_{R}=47.0 \min$ (major). The absolute configuration was assigned by analogy to $\mathbf{5 a}$.

\section{(1S,2S)-3-(4-Chlorophenyl)-1,2-diphenylpropan-1-ol (5e)}<smiles>O[C@H](c1ccccc1)[C@H](Cc1ccc(Cl)cc1)c1ccccc1</smiles>
white solid, $75.7 \mathrm{mg}, 94 \%$ isolated yield. purification on silica gel using a 20:1 petroleum ether/ethyl acetate mixture.

${ }^{1} \mathbf{H}$ NMR $\left(\mathrm{CDCl}_{3}, 400 \mathrm{MHz}\right) \delta(\mathrm{ppm}):$ 7.38-7.24 (m, 8H), 7.15-7.09 (m, 4H), 6.81 (d, $J=$ $8.4 \mathrm{~Hz}, 2 \mathrm{H}), 4.87$ (dd, $J=7.6 \mathrm{~Hz}, 3.2 \mathrm{~Hz}, 1 \mathrm{H}), 3.16-3.11$ (m, 1H), 2.90-2.78 (m, 2H),

1.89 (brd, $J=3.2 \mathrm{~Hz}, 1 \mathrm{H})$.

${ }^{13} \mathbf{C}\left\{{ }^{1} \mathbf{H}\right\}$ NMR $\left(\mathrm{CDCl}_{3}, 100 \mathrm{MHz}\right) \delta(\mathrm{ppm}): 142.5,139.9,138.6,131.6,130.4,129.1,128.6,128.5,128.3,128.1,127.2$, 127.0, 77.6, 56.4, 38.2.

HRMS (ESI) m/z: $[\mathrm{M}+\mathrm{Na}]^{+}$Calcd for $\mathrm{C}_{21} \mathrm{H}_{19} \mathrm{OCl} 345.1017$; Found 345.1021.

94:6 $d r, 90 \%$ ee HPLC conditions: Chiralpak AD-H column, $n$-hexane:isopropanol $=95: 5$; flow rate $=0.5 \mathrm{~mL} / \mathrm{min}$; UV detection at $220 \mathrm{~nm} ; \mathrm{t}_{\mathrm{R}}=28.0 \min \left(\right.$ minor), $\mathrm{t}_{\mathrm{R}}=31.4 \min \left(\right.$ major), $\mathrm{t}_{\mathrm{R}}=35.6 \min \left(\right.$ minor), $\mathrm{t}_{\mathrm{R}}=64.3 \min ($ major). The absolute configuration was assigned by analogy to $\mathbf{5 a}$.

\section{(1S,2S)-3-(4-Bromophenyl)-1,2-diphenylpropan-1-ol (5f)}<smiles>O[C@H](c1ccccc1)[C@H](Cc1ccc(Br)cc1)c1ccccc1</smiles>

white solid, $82.4 \mathrm{mg}, 90 \%$ isolated yield. purification on silica gel using a 20:1 petroleum ether/ethyl acetate mixture.

${ }^{1} \mathbf{H}$ NMR $\left(\mathrm{CDCl}_{3}, 400 \mathrm{MHz}\right) \delta(\mathrm{ppm}):$ 7.33-7.20 (m, 10H), $7.10(\mathrm{~d}, J=6.8 \mathrm{~Hz}, 2 \mathrm{H}), 6.72$ $(\mathrm{d}, J=8.4 \mathrm{~Hz}, 2 \mathrm{H}), 4.83(\mathrm{dd}, J=7.2 \mathrm{~Hz}, 2.4 \mathrm{~Hz}, 1 \mathrm{H}), 3.11-3.07(\mathrm{~m}, 1 \mathrm{H}), 2.86-2.73(\mathrm{~m}$, 2H), 1.85 (brd, $J=2.8 \mathrm{~Hz}, 1 \mathrm{H})$

${ }^{13} \mathbf{C}\left\{{ }^{1} \mathbf{H}\right\}$ NMR $\left(\mathrm{CDCl}_{3}, 100 \mathrm{MHz}\right) \delta(\mathrm{ppm}): 142.4,139.8,139.0,131.1,130.7,129.0,128.5,128.4,127.9,127.1,126.9$, $119.6,77.5,56.2,38.1$.

HRMS (ESI) m/z: $[\mathrm{M}+\mathrm{Na}]^{+}$Calcd for $\mathrm{C}_{21} \mathrm{H}_{19} \mathrm{OBr} 389.0511$; Found 389.0505.

94:6 dr, 90\% ee HPLC conditions: Chiralpak AD-H column, $n$-hexane:isopropanol $=97: 3$; flow rate $=0.5 \mathrm{~mL} / \mathrm{min}$; UV detection at $220 \mathrm{~nm} ; \mathrm{t}_{\mathrm{R}}=41.2 \min \left(\right.$ minor), $\mathrm{t}_{\mathrm{R}}=48.1 \min \left(\right.$ minor), $\mathrm{t}_{\mathrm{R}}=52.0 \min \left(\right.$ minor), $\mathrm{t}_{\mathrm{R}}=103.4 \min$ (major). The absolute configuration was assigned by analogy to $\mathbf{5 a}$.

\section{(1S,2S)-1,2-Diphenyl-3-(4-(trifluoromethyl)phenyl)propan-1-ol (5g)}<smiles>O[C@H](c1ccccc1)[C@H](Cc1ccc(C(F)(F)F)cc1)c1ccccc1</smiles>
colorless oil, $83.7 \mathrm{mg}, 94 \%$ isolated yield. purification on silica gel using a 15:1 petroleum ether/ethyl acetate mixture.

${ }^{1} \mathbf{H}$ NMR $\left(\mathrm{CDCl}_{3}, 400 \mathrm{MHz}\right) \delta(\mathrm{ppm}): 7.42-7.28(\mathrm{~m}, 10 \mathrm{H}), 7.17(\mathrm{~d}, J=6.8 \mathrm{~Hz}, 2 \mathrm{H})$, $7.02(\mathrm{~d}, J=8.0 \mathrm{~Hz}, 2 \mathrm{H}), 4.89$ (d, $J=6.4 \mathrm{~Hz}, 1 \mathrm{H}), 3.23-3.18(\mathrm{~m}, 1 \mathrm{H}), 3.02-2.90(\mathrm{~m}, 2 \mathrm{H})$

1.98 (brs, $1 \mathrm{H})$

${ }^{13} \mathbf{C}\left\{{ }^{1} \mathbf{H}\right\}$ NMR $\left(\mathrm{CDCl}_{3}, 100 \mathrm{MHz}\right) \delta(\mathrm{ppm}): 144.3,142.4,139.8,129.3,129.1,128.6,128.5,128.1,127.3,127.0,125.1$ (q, $\left.{ }^{3} J=3.7 \mathrm{~Hz}\right), 77.5,56.1,38.6$.

HRMS (ESI) m/z: $[\mathrm{M}+\mathrm{Na}]^{+}$Calcd for $\mathrm{C}_{22} \mathrm{H}_{19} \mathrm{OF}_{3} 379.1280$; Found 379.1285.

97:3 $d r, 99 \%$ ee HPLC conditions: Chiralpak OJ-H column, $n$-hexane:isopropanol $=97: 3$; flow rate $=0.5 \mathrm{~mL} / \mathrm{min}$; UV 
detection at $220 \mathrm{~nm} ; \mathrm{t}_{\mathrm{R}}=36.6 \min (\operatorname{minor}), \mathrm{t}_{\mathrm{R}}=42.8 \min \left(\right.$ major), $\mathrm{t}_{\mathrm{R}}=53.4 \min \left(\right.$ minor), $\mathrm{t}_{\mathrm{R}}=63.0 \min (\operatorname{minor})$. The absolute configuration was assigned by analogy to $\mathbf{5 a}$.

(1S,2S)-1,2-Diphenyl-3-(m-tolyl)propan-1-ol (5h)<smiles>Cc1cccc(C[C@H](c2ccccc2)[C@H](O)c2ccccc2)c1</smiles>
colorless oil, $60.4 \mathrm{mg}, 80 \%$ isolated yield. purification on silica gel using a $30: 1$ petroleum ether/ethyl acetate mixture.

${ }^{1} \mathbf{H}$ NMR $\left(\mathrm{CDCl}_{3}, 400 \mathrm{MHz}\right) \delta(\mathrm{ppm}):$ 7.38-7.24 (m, 8H), $7.17(\mathrm{~d}, J=7.6 \mathrm{~Hz}, 2 \mathrm{H}), 7.07-7.03$ (m, 1H), 6.94-6.92 (m, 1H), 6.75-6.73 (m, 2H), $4.90(\mathrm{~d}, J=7.2 \mathrm{~Hz}, 1 \mathrm{H}), 3.23-3.17(\mathrm{~m}, 1 \mathrm{H})$, 2.97-2.82 (m, 2H), $2.25(\mathrm{~s}, 3 \mathrm{H}), 1.85$ (brs, $1 \mathrm{H})$.

${ }^{13} \mathbf{C}\left\{{ }^{1} \mathbf{H}\right\}$ NMR $\left(\mathrm{CDCl}_{3}, 100 \mathrm{MHz}\right) \delta(\mathrm{ppm}): 142.8,140.3,140.0,137.7,129.9,129.2,128.4,128.0,127.8,127.0,126.9$, 126.7, 126.1, 77.3, 56.2, 38.6, 21.4 .

HRMS (ESI) m/z: $[\mathrm{M}+\mathrm{Na}]^{+}$Calcd for $\mathrm{C}_{22} \mathrm{H}_{22} \mathrm{O} 325.1563$; Found 325.1569 .

97:3 dr, 87\% ee HPLC conditions: Chiralpak AD-H column, $n$-hexane:isopropanol $=98: 2$; flow rate $=0.5 \mathrm{~mL} / \mathrm{min}$; UV detection at $220 \mathrm{~nm} ; \mathrm{t}_{\mathrm{R}}=40.4 \min \left(\right.$ minor), $\mathrm{t}_{\mathrm{R}}=42.0 \mathrm{~min}\left(\right.$ minor), $\mathrm{t}_{\mathrm{R}}=47.8 \min \left(\right.$ minor), $\mathrm{t}_{\mathrm{R}}=52.2 \min$ (major). The absolute configuration was assigned by analogy to $\mathbf{5 a}$.

(1S,2S)-3-(3-Methoxyphenyl)-1,2-diphenylpropan-1-ol (5i)<smiles>COc1cccc(C[C@H](c2ccccc2)[C@H](O)c2ccccc2)c1</smiles>
white solid, $74.7 \mathrm{mg}, 94 \%$ isolated yield. purification on silica gel using a 20:1 petroleum ether/ethyl acetate mixture.

${ }^{1} \mathbf{H}$ NMR $\left(\mathrm{CDCl}_{3}, 400 \mathrm{MHz}\right) \delta(\mathrm{ppm}): 7.37-7.24(\mathrm{~m}, 8 \mathrm{H}), 7.18(\mathrm{~d}, J=7.6 \mathrm{~Hz}, 2 \mathrm{H}), 7.09$ (t, $J$ OMe $=8.0 \mathrm{~Hz}, 1 \mathrm{H}), 6.67(\mathrm{~d}, J=8.0 \mathrm{~Hz}, 1 \mathrm{H}), 6.56(\mathrm{~d}, J=7.2 \mathrm{~Hz}, 1 \mathrm{H}), 6.46(\mathrm{~s}, 1 \mathrm{H}), 4.90(\mathrm{~d}, J=$ $6.0 \mathrm{~Hz}, 1 \mathrm{H}), 3.69$ (s, 3H), 3.23-3.18 (m, 1H), 2.97-2.83 (m, 2H), 1.92 (brs, $1 \mathrm{H})$.

${ }^{13} \mathbf{C}\left\{{ }^{1} \mathbf{H}\right\}$ NMR $\left(\mathrm{CDCl}_{3}, 100 \mathrm{MHz}\right) \delta(\mathrm{ppm}): 159.4,142.8,141.7,140.3,129.2,129.1,128.45,128.42,127.9,127.0,126.9$, 121.5, 114.8, 111.4, 77.4, 56.1, 55.1, 38.8 .

HRMS (ESI) m/z: $[\mathrm{M}+\mathrm{Na}]^{+}$Calcd for $\mathrm{C}_{22} \mathrm{H}_{22} \mathrm{O} 341.1512$; Found 341.1509 .

98:2 $d r$, 93\% ee HPLC conditions: Chiralpak OD-H column, $n$-hexane:isopropanol $=97: 3$; flow rate $=0.5 \mathrm{~mL} / \mathrm{min}$; UV detection at $220 \mathrm{~nm} ; \mathrm{t}_{\mathrm{R}}=59.7 \min$ (minor), $\mathrm{t}_{\mathrm{R}}=82.5 \min$ (major), $\mathrm{t}_{\mathrm{R}}=112.8 \mathrm{~min}$ (minor). The absolute configuration was determined by single-crystal X-ray diffraction analysis. Single crystals suitable for X-ray diffraction measurement were grown from a solution of $\mathbf{5 i}$ in petroleum ether/dichloromethane.

(1S,2S)-3-(3-Fluorophenyl)-1,2-diphenylpropan-1-ol (5j)<smiles>O[C@@H](Cc1cccc(F)c1)c1ccccc1</smiles>
colorless oil, $68.9 \mathrm{mg}, 90 \%$ isolated yield. purification on silica gel using a 30:1 petroleum ether/ethyl acetate mixture.

${ }^{1} \mathbf{H}$ NMR $\left(\mathrm{CDCl}_{3}, 400 \mathrm{MHz}\right) \delta(\mathrm{ppm}):$ 7.27-7.11 (m, 8H), 7.04-6.96 (m, 3H), 6.67 (t, $J=8.4$ $\mathrm{Hz}, 1 \mathrm{H}), 6.57(\mathrm{~d}, J=7.6 \mathrm{~Hz}, 1 \mathrm{H}), 6.50(\mathrm{~d}, J=10.0 \mathrm{~Hz}, 1 \mathrm{H}), 4.75(\mathrm{~d}, J=7.2 \mathrm{~Hz}, 1 \mathrm{H})$, 3.08-3.02 (m, 1H), 2.83-2.70 (m, 2H), 1.80 (brs, 1H).

${ }^{13} \mathbf{C}\left\{{ }^{1} \mathbf{H}\right\}$ NMR $\left(\mathrm{CDCl}_{3}, 100 \mathrm{MHz}\right) \delta(\mathrm{ppm}): 142.6\left(\mathrm{~d},{ }^{3} \mathrm{~J}=7.3 \mathrm{~Hz}\right), 141.1\left(\mathrm{~d},{ }^{1} J=262.8 \mathrm{~Hz}\right), 129.4\left(\mathrm{~d},{ }^{3} \mathrm{~J}=8.2 \mathrm{~Hz}\right), 129.0$, 128.45, 128.40, 127.9, 127.1, 126.8, $124.6\left(\mathrm{~d},{ }^{4} J=2.7 \mathrm{~Hz}\right), 115.7$ (d, $\left.{ }^{2} J=20.7 \mathrm{~Hz}\right), 112.7\left(\mathrm{~d},{ }^{2} J=21.0 \mathrm{~Hz}\right), 77.4,56.0$, 38.4 .

HRMS (ESI) m/z: [M+Na $]^{+}$Calcd for $\mathrm{C}_{21} \mathrm{H}_{19} \mathrm{OF}$ 329.1312; Found 329.1308.

96:4 dr, 91\% ee HPLC conditions: Chiralpak AD-H column, $n$-hexane:isopropanol $=98: 2$; flow rate $=0.5 \mathrm{~mL} / \mathrm{min}$; UV detection at $220 \mathrm{~nm} ; \mathrm{t}_{\mathrm{R}}=49.5 \min (\operatorname{minor}), \mathrm{t}_{\mathrm{R}}=61.3 \mathrm{~min}\left(\right.$ minor), $\mathrm{t}_{\mathrm{R}}=66.4 \mathrm{~min}$ (major). The absolute configuration was assigned by analogy to $\mathbf{5 a}$. 
2.95-2.81 (m, 2H), 1.96 (brs, $1 \mathrm{H})$.

${ }^{13} \mathbf{C}\left\{{ }^{1} \mathbf{H}\right\}$ NMR $\left(\mathrm{CDCl}_{3}, 100 \mathrm{MHz}\right) \delta(\mathrm{ppm}): 142.5,142.2,139.9,133.9,129.4,129.1,128.53,128.49,128.0,127.3,127.2$, $126.9,126.1,77.4,56.1,38.4$.

HRMS (ESI) m/z: $[\mathrm{M}+\mathrm{Na}]^{+}$Calcd for $\mathrm{C}_{21} \mathrm{H}_{19} \mathrm{OCl} 345.1017$; Found 345.1019.

96:4 $d r$, 92\% ee HPLC conditions: Chiralpak AD-H column, $n$-hexane:isopropanol $=98: 2$; flow rate $=0.5 \mathrm{~mL} / \mathrm{min}$; UV detection at $220 \mathrm{~nm} ; \mathrm{t}_{\mathrm{R}}=47.7 \mathrm{~min}$ (minor), $\mathrm{t}_{\mathrm{R}}=58.8 \mathrm{~min}$ (minor), $\mathrm{t}_{\mathrm{R}}=64.5 \mathrm{~min}$ (major). The absolute configuration was assigned by analogy to $\mathbf{5 a}$.

\section{(1S,2S)-3-(3-Bromophenyl)-1,2-diphenylpropan-1-ol (5l)}

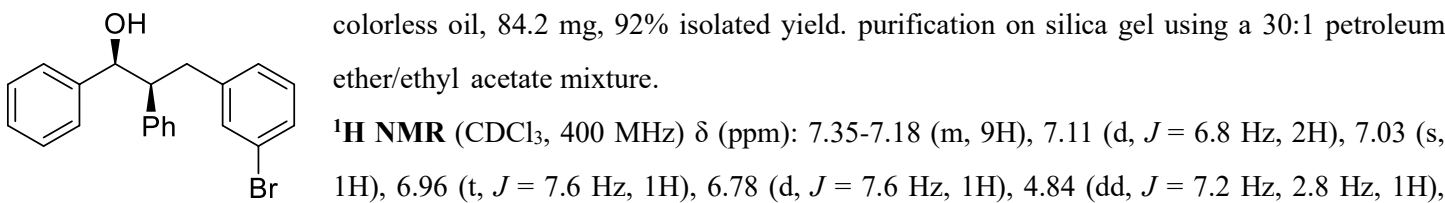
3.15-3.09 (m, 1H), 2.89-2.75 (m, 2H), 1.85 (brd, $J=2.8 \mathrm{~Hz}, 1 \mathrm{H})$.

${ }^{13} \mathbf{C}\left\{{ }^{1} \mathbf{H}\right\}$ NMR $\left(\mathrm{CDCl}_{3}, 100 \mathrm{MHz}\right) \delta(\mathrm{ppm}): 142.44,142.42,139.8,132.0,129.6,129.05,128.98,128.5,128.4,128.0$, $127.7,127.1,126.9,122.1,77.4,56.0,38.4$.

HRMS (ESI) m/z: [M+Na] $]^{+}$Calcd for $\mathrm{C}_{21} \mathrm{H}_{19} \mathrm{OBr} 389.0511$; Found 389.0507

95:5 $d r, 91 \%$ ee HPLC conditions: Chiralpak AD-H column, $n$-hexane:isopropanol $=95: 5$; flow rate $=0.5 \mathrm{~mL} / \mathrm{min}$; UV detection at $220 \mathrm{~nm} ; \mathrm{t}_{\mathrm{R}}=26.0 \mathrm{~min}\left(\right.$ minor), $\mathrm{t}_{\mathrm{R}}=27.0 \min \left(\right.$ minor), $\mathrm{t}_{\mathrm{R}}=33.5 \mathrm{~min}\left(\right.$ minor), $\mathrm{t}_{\mathrm{R}}=36.8 \min$ (major). The absolute configuration was assigned by analogy to $\mathbf{5 a}$.

\section{(1S,2S)-1,2-Diphenyl-3-(3-(trifluoromethyl)phenyl)propan-1-ol (5m)}<smiles>O[C@H](c1ccccc1)[C@@H](c1ccccc1)c1cccc(C(F)(F)F)c1</smiles>
colorless oil, $78.3 \mathrm{mg}, 88 \%$ isolated yield. purification on silica gel using a 20:1 petroleum ether/ethyl acetate mixture.

${ }^{1} \mathbf{H}$ NMR $\left(\mathrm{CDCl}_{3}, 400 \mathrm{MHz}\right) \delta(\mathrm{ppm}):$ 7.25-7.08 (m, 10H), 7.03-7.00 (m, 3H), 6.93 (d, $J=$

$\left.\mathrm{CF}_{3} \quad 7.6 \mathrm{~Hz}, 1 \mathrm{H}\right), 4.76(\mathrm{dd}, J=7.4 \mathrm{~Hz}, 2.2 \mathrm{~Hz}, 1 \mathrm{H}), 3.08-3.03(\mathrm{~m}, 1 \mathrm{H}), 2.87-2.74(\mathrm{~m}, 2 \mathrm{H}), 1.83$ (brd, $J=2.4 \mathrm{~Hz}, 1 \mathrm{H}$ ).

${ }^{13} \mathbf{C}\left\{{ }^{1} \mathbf{H}\right\} \mathbf{N M R}\left(\mathrm{CDCl}_{3}, 100 \mathrm{MHz}\right) \delta(\mathrm{ppm}): 142.4,141.0,139.8,132.4,129.1,128.64,128.59,128.5,128.2,127.3,127.0$, $125.8\left(\mathrm{q},{ }^{3} J=3.9 \mathrm{~Hz}\right), 122.8\left(\mathrm{q},{ }^{3} J=3.9 \mathrm{~Hz}\right), 77.6,56.2,38.7$.

HRMS (ESI) m/z: [M+Na] $]^{+}$Calcd for $\mathrm{C}_{22} \mathrm{H}_{19} \mathrm{OF}_{3} 379.1280$; Found 379.1274.

95:5 dr, 96\% ee HPLC conditions: Chiralpak AD-H column, $n$-hexane:isopropanol $=99: 1$; flow rate $=0.5 \mathrm{~mL} / \mathrm{min}$; UV detection at $220 \mathrm{~nm} ; \mathrm{t}_{\mathrm{R}}=51.0 \mathrm{~min}\left(\right.$ minor), $\mathrm{t}_{\mathrm{R}}=55.3 \min \left(\right.$ minor), $\mathrm{t}_{\mathrm{R}}=64.6 \min ($ major$), \mathrm{t}_{\mathrm{R}}=66.9 \min (\operatorname{minor})$. The absolute configuration was assigned by analogy to $\mathbf{5 a}$.

\section{(1S,2S)-3-(2-Fluorophenyl)-1,2-diphenylpropan-1-ol (5n)}<smiles>O[C@H](c1ccccc1)[C@@H](Cc1ccccc1F)c1ccccc1</smiles>
white solid, $62.7 \mathrm{mg}, 82 \%$ isolated yield. purification on silica gel using a 25:1 petroleum ether/ethyl acetate mixture.

${ }^{1} \mathbf{H}$ NMR $\left(\mathrm{CDCl}_{3}, 400 \mathrm{MHz}\right) \delta(\mathrm{ppm}):$ 7.35-7.28 (m, 5H), 7.24-7.18 (m, 3H), $7.14(\mathrm{~d}, J=6.4$ $\mathrm{Hz}, 2 \mathrm{H}), 7.05-7.02(\mathrm{~m}, 1 \mathrm{H}), 6.87-6.84(\mathrm{~m}, 3 \mathrm{H}), 4.87$ (dd, $J=7.2 \mathrm{~Hz}, 3.2 \mathrm{~Hz}, 1 \mathrm{H}), 3.28-3.22(\mathrm{~m}, 1 \mathrm{H}), 2.97-2.83(\mathrm{~m}, 2 \mathrm{H})$, 
1.88 (brd, $J=3.2 \mathrm{~Hz}, 1 \mathrm{H})$.

${ }^{13} \mathbf{C}\left\{{ }^{1} \mathbf{H}\right\}$ NMR $\left(\mathrm{CDCl}_{3}, 100 \mathrm{MHz}\right) \delta(\mathrm{ppm}): 161.2\left(\mathrm{~d},{ }^{1} \mathrm{~J}=243.2 \mathrm{~Hz}\right), 142.5,140.0,131.3\left(\mathrm{~d},{ }^{4} \mathrm{~J}=4.9 \mathrm{~Hz}\right), 129.1,128.39$, 128.36, 127.9, 127.7 (d, $\left.{ }^{3} J=8.0 \mathrm{~Hz}\right), 127.0,126.9,123.7$ (d, $\left.{ }^{4} J=3.4 \mathrm{~Hz}\right), 115.1\left(\mathrm{~d},{ }^{2} J=22.3 \mathrm{~Hz}\right), 77.5,54.5,32.1$.

HRMS (ESI) m/z: [M+Na $]^{+}$Calcd for $\mathrm{C}_{21} \mathrm{H}_{19} \mathrm{OF}$ 329.1312; Found 329.1309.

93:7 dr, 86\% ee HPLC conditions: Chiralpak AD-H column, $n$-hexane:isopropanol $=95: 5$; flow rate $=0.5 \mathrm{~mL} / \mathrm{min}$; UV detection at $220 \mathrm{~nm} ; \mathrm{t}_{\mathrm{R}}=28.3 \min \left(\right.$ minor), $\mathrm{t}_{\mathrm{R}}=31.2 \min \left(\right.$ minor), $\mathrm{t}_{\mathrm{R}}=32.4 \min \left(\right.$ minor), $\mathrm{t}_{\mathrm{R}}=36.1 \min$ (major). The absolute configuration was assigned by analogy to $\mathbf{5 a}$.

\section{(1S,2S)-3-(2-Bromophenyl)-1,2-diphenylpropan-1-ol (5o)}

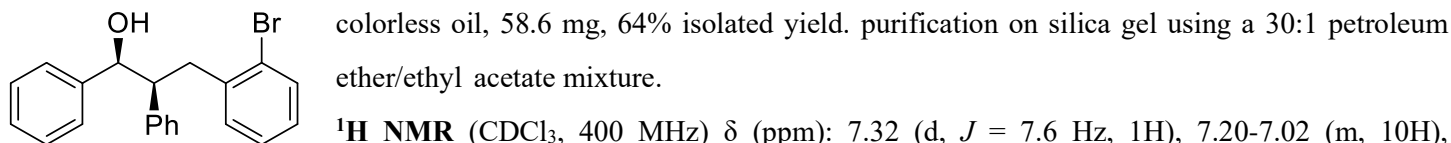
6.86-6.81 (m, 2H), $6.67(\mathrm{~d}, J=7.2 \mathrm{~Hz}, 1 \mathrm{H}), 4.79(\mathrm{~d}, J=7.2 \mathrm{~Hz}, 1 \mathrm{H}), 3.27-3.21(\mathrm{~m}, 1 \mathrm{H}), 3.01-2.97(\mathrm{~m}, 1 \mathrm{H}), 2.85-2.80(\mathrm{~m}$, $1 \mathrm{H}), 1.84$ (brs, $1 \mathrm{H})$.

${ }^{13} \mathbf{C}\left\{{ }^{1} \mathbf{H}\right\}$ NMR $\left(\mathrm{CDCl}_{3}, 100 \mathrm{MHz}\right) \delta(\mathrm{ppm}): 142.5,139.9,139.3,132.8,131.5,129.2,128.4,128.3,127.9,127.7,127.05$, $127.03,126.9,124.6,77.4,53.7,39.2$.

HRMS (ESI) m/z: $[\mathrm{M}+\mathrm{Na}]^{+}$Calcd for $\mathrm{C}_{21} \mathrm{H}_{19} \mathrm{OBr} 389.0511$; Found 389.0512 .

97:3 dr, 85\% ee HPLC conditions: Chiralpak AD-H column, $n$-hexane:isopropanol $=98: 2$; flow rate $=0.5 \mathrm{~mL} / \mathrm{min}$; UV detection at $220 \mathrm{~nm} ; \mathrm{t}_{\mathrm{R}}=46.0 \mathrm{~min}$ (minor), $\mathrm{t}_{\mathrm{R}}=48.2 \min$ (minor), $\mathrm{t}_{\mathrm{R}}=55.9 \min$ (minor), $\mathrm{t}_{\mathrm{R}}=63.4 \mathrm{~min}$ (major). The absolute configuration was assigned by analogy to $\mathbf{5 a}$.

\section{(1S,2S)-3-(2,5-Dimethoxyphenyl)-1,2-diphenylpropan-1-ol (5p)}

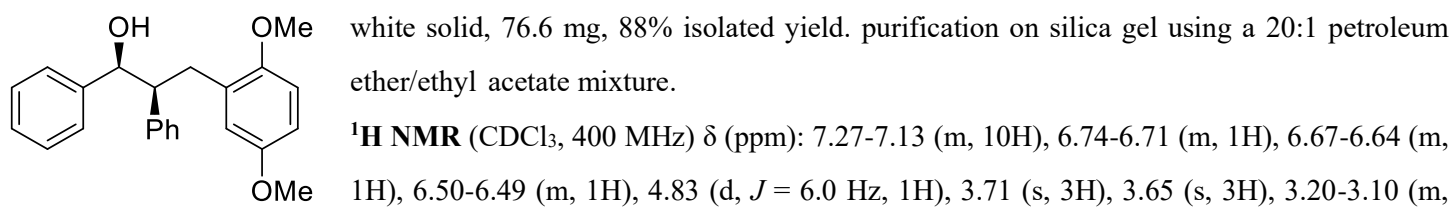
2H), 2.80-2.75 (m, 1H), 2.39 (brs, $1 \mathrm{H})$.

${ }^{13} \mathbf{C}\left\{{ }^{1} \mathbf{H}\right\}$ NMR $\left(\mathrm{CDCl}_{3}, 100 \mathrm{MHz}\right) \delta(\mathrm{ppm}): 153.6,151.9,142.7,140.8,129.8,129.3,128.13,128.08,127.4,126.81$, $126.77,117.1,111.7,111.5,76.3,56.1,55.8,54.7,33.3$.

HRMS (ESI) m/z: [M+Na] $]^{+}$Calcd for $\mathrm{C}_{23} \mathrm{H}_{24} \mathrm{O}_{3} 371.1618$; Found 371.1613.

95:5 dr, 94\% ee HPLC conditions: Chiralpak AD-H column, $n$-hexane:isopropanol $=97: 3$; flow rate $=0.5 \mathrm{~mL} / \mathrm{min}$; UV detection at $220 \mathrm{~nm} ; \mathrm{t}_{\mathrm{R}}=87.8 \mathrm{~min}\left(\right.$ minor), $\mathrm{t}_{\mathrm{R}}=91.6 \mathrm{~min}$ (major), $\mathrm{t}_{\mathrm{R}}=96.4 \mathrm{~min}(\operatorname{minor}), \mathrm{t}_{\mathrm{R}}=109.3 \mathrm{~min}(\operatorname{minor})$. The absolute configuration was determined by single-crystal X-ray diffraction analysis. Single crystals suitable for X-ray diffraction measurement were grown from a solution of $\mathbf{5 p}$ in petroleum ether/dichloromethane.

\section{(1S,2S)-3-(3,5-Dimethoxyphenyl)-1,2-diphenylpropan-1-ol (5q)}<smiles>COc1cc(C[C@H](c2ccccc2)[C@H](O)c2ccccc2)cc(OC)c1</smiles>

white solid, $74.8 \mathrm{mg}, 86 \%$ isolated yield. purification on silica gel using a 15:1 petroleum ether/ethyl acetate mixture.

${ }^{1} \mathbf{H}$ NMR $\left(\mathrm{CDCl}_{3}, 400 \mathrm{MHz}\right) \delta(\mathrm{ppm}): 7.31-7.18(\mathrm{~m}, 8 \mathrm{H}), 7.12(\mathrm{~d}, J=6.8 \mathrm{~Hz}, 2 \mathrm{H})$, $6.19(\mathrm{~s}, 1 \mathrm{H}), 6.04(\mathrm{~s}, 2 \mathrm{H}), 4.84(\mathrm{~d}, J=7.2 \mathrm{~Hz}, 1 \mathrm{H}), 3.61(\mathrm{~s}, 6 \mathrm{H}), 3.16-3.11(\mathrm{~m}, 1 \mathrm{H})$,

2.87-2.74 (m, 2H), 1.91 (brs, $1 \mathrm{H})$.

${ }^{13} \mathbf{C}\left\{{ }^{1} \mathbf{H}\right\} \mathbf{N M R}\left(\mathrm{CDCl}_{3}, 100 \mathrm{MHz}\right) \delta(\mathrm{ppm}): 160.5,142.7,142.5,140.4,129.2,128.42,128.39,127.8,127.0,126.9,107.2$, 98.0, 77.3, 56.0, 55.2, 39.0.

HRMS (ESI) m/z: [M+Na $]^{+}$Calcd for $\mathrm{C}_{23} \mathrm{H}_{24} \mathrm{O}_{3}$ 371.1618; Found 371.1621 . 
$>$ 99: $1 d r, 97 \%$ ee HPLC conditions: Chiralpak OD-H column, $n$-hexane:isopropanol = 97:3; flow rate $=0.5 \mathrm{~mL} / \mathrm{min}$; UV detection at $220 \mathrm{~nm} ; \mathrm{t}_{\mathrm{R}}=73.7 \mathrm{~min}$ (major), $\mathrm{t}_{\mathrm{R}}=78.9 \mathrm{~min}$ (minor). The absolute configuration was determined by single-crystal X-ray diffraction analysis. Single crystals suitable for X-ray diffraction measurement were grown from a solution of $\mathbf{5 q}$ in petroleum ether/dichloromethane.

\section{(1S,2S)-3-(Naphthalen-2-yl)-1,2-diphenylpropan-1-ol (5r)}

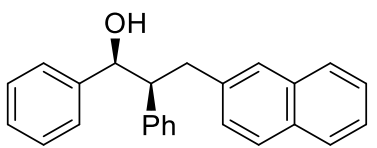

white solid, $76.1 \mathrm{mg}, 90 \%$ isolated yield. purification on silica gel using a 20:1 petroleum ether/ethyl acetate mixture.

${ }^{1} \mathbf{H}$ NMR $\left(\mathrm{CDCl}_{3}, 400 \mathrm{MHz}\right) \delta(\mathrm{ppm}): 7.65-7.33(\mathrm{~m}, 3 \mathrm{H}), 7.28-7.18(\mathrm{~m}, 13 \mathrm{H})$, 7.10-7.07 (m, 1H), 4.94 (d, $J=7.2 \mathrm{~Hz}, 1 \mathrm{H}), 3.32-3.30(\mathrm{~m}, 1 \mathrm{H}), 3.16-3.11(\mathrm{~m}, 1 \mathrm{H}), 3.06-3.01(\mathrm{~m}, 1 \mathrm{H}), 1.93$ (brs, $1 \mathrm{H})$. ${ }^{13} \mathbf{C}\left\{{ }^{1} \mathbf{H}\right\}$ NMR $\left(\mathrm{CDCl}_{3}, 100 \mathrm{MHz}\right) \delta(\mathrm{ppm}): 142.8,140.2,137.7,133.6,132.1,129.3,128.51,128.49,128.0,127.74$, $127.67,127.7,127.6,127.5,127.1,127.0,125.9,125.3,77.2,56.2,39.0$.

HRMS (ESI) m/z: $[\mathrm{M}+\mathrm{Na}]^{+}$Calcd for $\mathrm{C}_{25} \mathrm{H}_{22} \mathrm{O} 361.1563$; Found 361.1570 .

96:4 $d r$, 94\% ee HPLC conditions: Chiralpak AD-H column, $n$-hexane:isopropanol $=95: 5$; flow rate $=0.5 \mathrm{~mL} / \mathrm{min}$; UV detection at $220 \mathrm{~nm} ; \mathrm{t}_{\mathrm{R}}=37.2 \min \left(\right.$ minor), $\mathrm{t}_{\mathrm{R}}=38.5 \min \left(\right.$ minor), $\mathrm{t}_{\mathrm{R}}=41.0 \min \left(\right.$ minor), $\mathrm{t}_{\mathrm{R}}=68.4 \min$ (major). The absolute configuration was assigned by analogy to $\mathbf{5 a}$.

\section{(1S,2S)-3-(Naphthalen-1-yl)-1,2-diphenylpropan-1-ol (5s)}<smiles>OC(c1ccccc1)C(c1ccccc1)c1cccc2ccccc12</smiles>
white solid, $69.3 \mathrm{mg}, 82 \%$ isolated yield. purification on silica gel using a 20:1 petroleum ether/ethyl acetate mixture.

${ }^{1} \mathbf{H}$ NMR $\left(\mathrm{CDCl}_{3}, 400 \mathrm{MHz}\right) \delta(\mathrm{ppm}): 7.85(\mathrm{~d}, J=8.0 \mathrm{~Hz}, 1 \mathrm{H}), 7.67(\mathrm{~d}, J=8.0 \mathrm{~Hz}, 1 \mathrm{H})$, $7.57(\mathrm{~d}, J=8.4 \mathrm{~Hz}, 1 \mathrm{H}), 7.50-7.40(\mathrm{~m}, 7 \mathrm{H}), 7.34-7.15(\mathrm{~m}, 6 \mathrm{H}), 6.92(\mathrm{~d}, J=7.2 \mathrm{~Hz}, 1 \mathrm{H})$, $5.02(\mathrm{dd}, J=7.4 \mathrm{~Hz}, 2.6 \mathrm{~Hz}, 1 \mathrm{H}), 3.58-3.53(\mathrm{~m}, 1 \mathrm{H}), 3.39-3.33(\mathrm{~m}, 1 \mathrm{H}), 3.23-3.18(\mathrm{~m}, 1 \mathrm{H}), 2.00$ (brd, $J=2.4 \mathrm{~Hz}, 1 \mathrm{H})$. ${ }^{13} \mathbf{C}\left\{{ }^{1} \mathbf{H}\right\}$ NMR $\left(\mathrm{CDCl}_{3}, 100 \mathrm{MHz}\right) \delta(\mathrm{ppm}): 142.8,140.6,136.0,133.9,131.9,129.1,128.9,128.48,128.45,128.0,127.4$, $127.2,127.1,126.8,125.8,125.3,125.2,123.8,77.4,55.4,36.1$.

HRMS (ESI) m/z: [M+Na $]^{+}$Calcd for $\mathrm{C}_{25} \mathrm{H}_{22} \mathrm{O} 361.1563$; Found 361.1560 .

96:4 $d r$, 92\% ee HPLC conditions: Chiralpak AD-H column, $n$-hexane:isopropanol $=95: 5$; flow rate $=0.5 \mathrm{~mL} / \mathrm{min}$; UV detection at $220 \mathrm{~nm} ; \mathrm{t}_{\mathrm{R}}=38.1 \mathrm{~min}\left(\right.$ minor), $\mathrm{t}_{\mathrm{R}}=47.8 \mathrm{~min}$ (major), $\mathrm{t}_{\mathrm{R}}=81.8 \min$ (minor). The absolute configuration was assigned by analogy to $\mathbf{5 a}$.

(1S,2S)-1-(4-Chlorophenyl)-2-phenyl-3-(4-(trifluoromethyl)phenyl)propan-1-ol (5t)<smiles>O[C@H](c1ccc(Cl)cc1)[C@H](Cc1ccc(C(F)(F)F)cc1)c1ccccc1</smiles>
colorless oil, $96.5 \mathrm{mg}, 99 \%$ isolated yield. purification on silica gel using a 20:1 petroleum ether/ethyl acetate mixture.

${ }^{1} \mathbf{H}$ NMR $\left(\mathrm{CDCl}_{3}, 400 \mathrm{MHz}\right) \delta(\mathrm{ppm}): 7.37(\mathrm{~d}, J=8.0 \mathrm{~Hz}, 2 \mathrm{H}), 7.30-7.23(\mathrm{~m}, 5 \mathrm{H})$, $7.16(\mathrm{~d}, J=8.4 \mathrm{~Hz}, 2 \mathrm{H}), 7.08$ (d, $J=6.4 \mathrm{~Hz}, 2 \mathrm{H}), 6.99$ (d, $J=8.0 \mathrm{~Hz}, 2 \mathrm{H}), 4.81$ (dd, $J=6.8 \mathrm{~Hz}, 2.4 \mathrm{~Hz}, 1 \mathrm{H}), 3.12-3.06(\mathrm{~m}, 1 \mathrm{H}), 2.98-2.86(\mathrm{~m}, 2 \mathrm{H}), 1.90$ (brd, $J=2.8 \mathrm{~Hz}, 1 \mathrm{H})$.

${ }^{13} \mathbf{C}\left\{{ }^{1} \mathbf{H}\right\}$ NMR $\left(\mathrm{CDCl}_{3}, 100 \mathrm{MHz}\right) \delta(\mathrm{ppm}): 144.1,141.0,139.3,133.7,129.3,129.1,128.69,128.65,128.3,127.5,125.2$ $\left(\mathrm{q},{ }^{3} \mathrm{~J}=3.7 \mathrm{~Hz}\right), 76.8,56.0,38.5$.

HRMS (ESI) m/z: $[\mathrm{M}+\mathrm{Na}]^{+}$Calcd for $\mathrm{C}_{22} \mathrm{H}_{18} \mathrm{OClF}_{3}$ 413.0890; Found 413.0885.

94:6 dr, 99\% ee HPLC conditions: Chiralpak OJ-H column, $n$-hexane:isopropanol $=95: 5$; flow rate $=0.5 \mathrm{~mL} / \mathrm{min}$; UV detection at $220 \mathrm{~nm} ; \mathrm{t}_{\mathrm{R}}=31.5 \min \left(\right.$ minor), $\mathrm{t}_{\mathrm{R}}=39.4 \min \left(\right.$ major), $\mathrm{t}_{\mathrm{R}}=45.7 \min \left(\right.$ minor), $\mathrm{t}_{\mathrm{R}}=54.6 \min ($ minor). The absolute configuration was assigned by analogy to $\mathbf{5 a}$. 
<smiles>O[C@H](c1ccc(Br)cc1)[C@H](c1ccccc1)c1ccc(C(F)(F)F)cc1</smiles>
colorless oil, $94.4 \mathrm{mg}, 87 \%$ isolated yield. purification on silica gel using a 20:1 petroleum ether/ethyl acetate mixture.

${ }^{1} \mathbf{H}$ NMR $\left(\mathrm{CDCl}_{3}, 400 \mathrm{MHz}\right) \delta(\mathrm{ppm}): 7.35(\mathrm{~d}, J=8.4 \mathrm{~Hz}, 2 \mathrm{H}), 7.28(\mathrm{~d}, J=8.0 \mathrm{~Hz}$, $2 \mathrm{H}), 7.18-7.12(\mathrm{~m}, 3 \mathrm{H}), 7.02-6.99(\mathrm{~m}, 4 \mathrm{H}), 6.90(\mathrm{~d}, J=8.0 \mathrm{~Hz}, 2 \mathrm{H}), 4.71(\mathrm{~d}, J=$ $6.8 \mathrm{~Hz}, 1 \mathrm{H}), 3.03-2.97$ (m, 1H), 2.90-2.77 (m, 2H), 1.85 (brs, $1 \mathrm{H})$.

${ }^{13} \mathbf{C}\left\{{ }^{1} \mathbf{H}\right\}$ NMR $\left(\mathrm{CDCl}_{3}, 100 \mathrm{MHz}\right) \delta(\mathrm{ppm}): 144.1,141.5,139.2,131.6,129.3,129.1,128.7,128.6,127.4,125.1\left(\mathrm{q},{ }^{3} J=\right.$ $3.7 \mathrm{~Hz}), 121.8,76.8,55.9,38.4$.

HRMS (ESI) m/z: [M+Na $]^{+}$Calcd for $\mathrm{C}_{22} \mathrm{H}_{18} \mathrm{OBrF}_{3}$ 457.0385; Found 457.0380.

95:5 dr, 96\% ee HPLC conditions: Chiralpak AD-H column, $n$-hexane:isopropanol $=95: 5$; flow rate $=0.5 \mathrm{~mL} / \mathrm{min}$; UV detection at $220 \mathrm{~nm} ; t_{R}=28.6 \min \left(\right.$ minor), $t_{R}=35.6 \min \left(\right.$ minor), $t_{R}=53.2 \min ($ major $), t_{R}=55.1$ min (minor). The absolute configuration was assigned by analogy to $\mathbf{5 a}$.

\section{(1S,2S)-1-(4-Methoxyphenyl)-2-phenyl-3-(4-(trifluoromethyl)phenyl)propan-1-ol (5v)}<smiles>COc1ccc([C@@H](O)[C@H](Cc2ccc(C(F)(F)F)cc2)c2ccccc2)cc1</smiles>

colorless oil, $77.2 \mathrm{mg}, 80 \%$ isolated yield. purification on silica gel using a 15:1 petroleum ether/ethyl acetate mixture.

${ }^{1} \mathbf{H}$ NMR $\left(\mathrm{CDCl}_{3}, 400 \mathrm{MHz}\right) \delta(\mathrm{ppm}): 7.33(\mathrm{~d}, J=8.0 \mathrm{~Hz}, 2 \mathrm{H}), 7.25-7.19(\mathrm{~m}$, $5 \mathrm{H}), 7.11(\mathrm{~d}, J=6.8 \mathrm{~Hz}, 2 \mathrm{H}), 6.93(\mathrm{~d}, J=7.6 \mathrm{~Hz}, 2 \mathrm{H}), 6.87(\mathrm{~d}, J=8.4 \mathrm{~Hz}, 2 \mathrm{H})$, 4.79 (d, $J=7.6 \mathrm{~Hz}, 1 \mathrm{H}), 3.79$ (s, 3H), 3.13-3.08 (m, 1H), 2.93-2.79 (m, 2H), 1.89 (brs, $1 \mathrm{H})$.

${ }^{13} \mathbf{C}\left\{{ }^{1} \mathbf{H}\right\}$ NMR $\left(\mathrm{CDCl}_{3}, 100 \mathrm{MHz}\right) \delta(\mathrm{ppm}): 159.5,144.4,140.0,134.5,129.3,129.1,128.6,128.2,127.3,125.0\left(\mathrm{q},{ }^{3} J=\right.$ $3.7 \mathrm{~Hz}), 113.9,77.4,56.2,55.4,38.7$.

HRMS (ESI) m/z: [M+Na $]^{+}$Calcd for $\mathrm{C}_{23} \mathrm{H}_{21} \mathrm{O}_{2} \mathrm{~F}_{33}$ 409.1386; Found 409.1381 .

91:9 $\boldsymbol{d r}, \mathbf{9 0 \%}$ ee HPLC conditions: Chiralpak AD-H column, $n$-hexane:isopropanol $=92: 8$; flow rate $=0.5 \mathrm{~mL} / \mathrm{min}$; UV detection at $220 \mathrm{~nm} ; \mathrm{t}_{\mathrm{R}}=24.1 \min \left(\right.$ minor), $\mathrm{t}_{\mathrm{R}}=28.5 \min \left(\right.$ minor), $\mathrm{t}_{\mathrm{R}}=45.0 \min (\operatorname{minor}), \mathrm{t}_{\mathrm{R}}=67.9 \min ($ major). The absolute configuration was assigned by analogy to $\mathbf{5 a}$.

\section{(1S,2S)-1-Phenyl-2-(p-tolyl)-3-(4-(trifluoromethyl)phenyl)propan-1-ol (5w)}<smiles>Cc1ccc(C(Cc2ccc(C(F)(F)F)cc2)[C@H](O)c2ccccc2)cc1</smiles>
colorless oil, $85.1 \mathrm{mg}, 92 \%$ isolated yield. purification on silica gel using a 20:1 petroleum ether/ethyl acetate mixture.

${ }^{1} \mathbf{H} \mathbf{N M R}\left(\mathrm{CDCl}_{3}, 400 \mathrm{MHz}\right) \delta(\mathrm{ppm}):$ 7.35-7.29 (m, 7H), 7.07-7.05 (m, 2H), 7.01-6.99 $(\mathrm{m}, 2 \mathrm{H}), 6.95(\mathrm{~d}, J=8.0 \mathrm{~Hz}, 2 \mathrm{H}), 4.79(\mathrm{~d}, J=7.6 \mathrm{~Hz}, 1 \mathrm{H}), 3.14-3.08(\mathrm{~m}, 1 \mathrm{H})$, 2.92-2.81 (m, 2H), 2.29 (s, 3H), 1.90 (brs, 1H).

${ }^{13} \mathbf{C}\left\{{ }^{1} \mathbf{H}\right\}$ NMR $\left(\mathrm{CDCl}_{3}, 100 \mathrm{MHz}\right) \delta(\mathrm{ppm}): 144.4,142.5,136.9,136.6,129.4,129.3,128.9,128.6,128.1,127.0,125.0$ (q, $\left.{ }^{3} J=3.7 \mathrm{~Hz}\right), 77.8,55.7,38.6,21.2$.

HRMS (ESI) m/z: $[\mathrm{M}+\mathrm{Na}]^{+}$Calcd for $\mathrm{C}_{23} \mathrm{H}_{21} \mathrm{OF}_{3}$ 393.1437; Found 393.1430.

95:5 dr, 91\% ee HPLC conditions: Chiralpak AD-H column, $n$-hexane:isopropanol $=95: 5$; flow rate $=0.5 \mathrm{~mL} / \mathrm{min}$; UV detection at $220 \mathrm{~nm} ; \mathrm{t}_{\mathrm{R}}=21.4 \min \left(\right.$ minor), $\mathrm{t}_{\mathrm{R}}=24.4 \min \left(\right.$ minor), $\mathrm{t}_{\mathrm{R}}=27.5 \min \left(\right.$ minor), $\mathrm{t}_{\mathrm{R}}=45.3 \min$ (major). The absolute configuration was assigned by analogy to $\mathbf{5 a}$.

\section{(1S,2S)-1-Phenyl-2-(p-tolyl)-3-(4-(trifluoromethyl)phenyl)propan-1-ol (5x)}<smiles>COc1cc(C[C@H](c2ccc(C)cc2)[C@H](O)c2ccccc2)cc(OC)c1</smiles>

colorless oil, $68.8 \mathrm{mg}, 76 \%$ isolated yield. purification on silica gel using a 20:1 petroleum ether/ethyl acetate mixture.

${ }^{1} \mathbf{H}$ NMR $\left(\mathrm{CDCl}_{3}, 400 \mathrm{MHz}\right) \delta(\mathrm{ppm}): 7.36-7.28(\mathrm{~m}, 5 \mathrm{H}), 7.09-7.03$ (m, 4H), 6.20 (t, $J$ $=2.2 \mathrm{~Hz}, 1 \mathrm{H}), 6.05(\mathrm{~d}, J=2.0 \mathrm{~Hz}, 2 \mathrm{H}), 4.82(\mathrm{~d}, J=7.2 \mathrm{~Hz}, 1 \mathrm{H}), 3.65(\mathrm{~s}, 6 \mathrm{H})$, 
3.16-3.10 (m, 1H), 2.85-2.73 (m, 2H), 2.31 (s, 3H), 1.73 (brs, 1H).

${ }^{13} \mathbf{C}\left\{{ }^{1} \mathbf{H}\right\}$ NMR $\left(\mathrm{CDCl}_{3}, 100 \mathrm{MHz}\right) \delta(\mathrm{ppm}): 160.5,142.8,142.6,137.2,136.6,129.2,129.1,128.4,127.9,127.0,107.2$, 98.0, 77.4, 55.6, 55.3, 39.1, 21.2.

HRMS (ESI) m/z: [M+Na] $]^{+}$Calcd for $\mathrm{C}_{24} \mathrm{H}_{26} \mathrm{O}_{3} 385.1774$; Found 385.1779 .

$>$ 99: 1 dr, 98\% ee HPLC conditions: Chiralpak OJ-H column, $n$-hexane:isopropanol = 90:10; flow rate $=0.5 \mathrm{~mL} / \mathrm{min}$; UV detection at $220 \mathrm{~nm} ; \mathrm{t}_{\mathrm{R}}=68.6 \mathrm{~min}$ (major), $\mathrm{t}_{\mathrm{R}}=77.1 \mathrm{~min}$ (minor). The absolute configuration was assigned by analogy to $5 a$.

(6aR,12aS)-5,6a,7,12a-Tetrahydro-6H-benzo[c]xanthene (7)<smiles>c1ccc2c(c1)CC1CCc3ccccc3[C@H]1O2</smiles>
white solid, $44.8 \mathrm{mg}, 76 \%$ isolated yield. purification on silica gel using a 100:1 petroleum ether/ethyl acetate mixture.

${ }^{1} \mathbf{H}$ NMR $\left(\mathrm{CDCl}_{3}, 400 \mathrm{MHz}\right) \delta(\mathrm{ppm}): 7.43(\mathrm{~d}, J=7.2 \mathrm{~Hz}, 1 \mathrm{H}), 7.28-7.16(\mathrm{~m}, 3 \mathrm{H}), 7.06(\mathrm{~d}, J=7.2$, 2H), 6.86-6.81 (m, 2H), 4.99 (d, $J=2.4 \mathrm{HZ}, 1 \mathrm{H}), 3.23-3.17(\mathrm{~m}, 1 \mathrm{H}), 2.97-2.91(\mathrm{~m}, 2 \mathrm{H}), 2.67-2.62$ (m, 1H), 2.36-2.30 (m, 1H), 2.00-1.95 (m, 1H), 1.75-1.70 (m, 1H).

${ }^{13} \mathbf{C}\left\{{ }^{1} \mathbf{H}\right\}$ NMR $\left(\mathrm{CDCl}_{3}, 100 \mathrm{MHz}\right) \delta(\mathrm{ppm}): 154.0,137.1,135.5,130.4,129.9,129.3,128.7,127.3,126.2,120.5,116.9$, $74.4,31.4,30.5,28.6,23.5$.

HRMS (ESI) m/z: $[\mathrm{M}+\mathrm{Na}]^{+}$Calcd for $\mathrm{C}_{17} \mathrm{H}_{16} \mathrm{O} 259.1093$; Found 259.1091.

95:5 $d r$, 87\% ee HPLC conditions: Chiralpak OD-H column, $n$-hexane:isopropanol $=99: 1$; flow rate $=0.5 \mathrm{~mL} / \mathrm{min}$; UV detection at $220 \mathrm{~nm} ; \mathrm{t}_{\mathrm{R}}=11.6 \min \left(\right.$ minor), $\mathrm{t}_{\mathrm{R}}=13.0 \mathrm{~min}\left(\right.$ minor), $\mathrm{t}_{\mathrm{R}}=22.7 \min ($ major$), \mathrm{t}_{\mathrm{R}}=33.8 \min (\operatorname{minor})$. The absolute configuration was determined by single-crystal X-ray diffraction analysis. Single crystals suitable for X-ray diffraction measurement were grown from a solution of 7 in petroleum ether/dichloromethane.

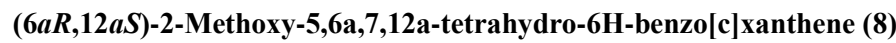<smiles>COc1ccc2c(c1)[C@H]1Oc3ccccc3C[C@H]1CC2</smiles>
white solid, $41.9 \mathrm{mg}, 63 \%$ isolated yield. purification on silica gel using a 100:1 petroleum ether/ethyl acetate mixture.

${ }^{1} \mathbf{H}$ NMR $\left(\mathrm{CDCl}_{3}, 400 \mathrm{MHz}\right) \delta(\mathrm{ppm}): 7.14-7.10(\mathrm{~m}, 3 \mathrm{H}), 7.03(\mathrm{~d}, J=2.4 \mathrm{~Hz}, 1 \mathrm{H})$, 6.92-6.88 (m, 3H), $5.02(\mathrm{~d}, J=2.4 \mathrm{HZ}, 1 \mathrm{H}), 3.86(\mathrm{~s}, 3 \mathrm{H}), 3.25-3.19(\mathrm{~m}, 1 \mathrm{H}), 3.00-2.84(\mathrm{~m}$, $2 \mathrm{H}), 2.72-2.67(\mathrm{~m}, 1 \mathrm{H}), 2.40-2.34(\mathrm{~m}, 1 \mathrm{H}), 2.06-1.95(\mathrm{~m}, 1 \mathrm{H}), 1.80-1.75(\mathrm{~m}, 1 \mathrm{H})$.

${ }^{13} \mathbf{C}\left\{{ }^{1} \mathbf{H}\right\}$ NMR $\left(\mathrm{CDCl}_{3}, 100 \mathrm{MHz}\right) \delta(\mathrm{ppm}): 158.0,153.9,136.4,130.2,129.9,128.9,127.3,120.6,120.5,116.9,115.3$, $114.5,74.6,55.5,31.3,30.2,27.6$.

HRMS (ESI) m/z: $[\mathrm{M}+\mathrm{Na}]^{+}$Calcd for $\mathrm{C}_{18} \mathrm{H}_{18} \mathrm{O}_{2} 289.1199$; Found 289.1197.

97:3 dr, 89\% ee HPLC conditions: Chiralpak OJ-H column, $n$-hexane:isopropanol $=99: 1$; flow rate $=0.5 \mathrm{~mL} / \mathrm{min}$; UV detection at $220 \mathrm{~nm} ; \mathrm{t}_{\mathrm{R}}=24.2 \mathrm{~min}(\operatorname{minor}), \mathrm{t}_{\mathrm{R}}=38.8 \mathrm{~min}$ (major), $\mathrm{t}_{\mathrm{R}}=54.6 \mathrm{~min}$ (minor). The absolute configuration was assigned by analogy to 7 .

(1R,2S)-4,7-Dimethoxy-1,2-diphenyl-2,3-dihydro-1H-indene (9)<smiles>COc1ccc(OC)c2c1CC(c1ccccc1)[C@H]2c1ccccc1</smiles>
colorless oil, $51.5 \mathrm{mg}, 78 \%$ isolated yield. purification on silica gel using a $80: 1$ petroleum ether/ethyl acetate mixture.

${ }^{1} \mathbf{H}$ NMR $\left(\mathrm{CDCl}_{3}, 400 \mathrm{MHz}\right) \delta(\mathrm{ppm}):$ 7.25-7.12 (m, 8H), 7.04-7.02 (m, 2H), 6.75-6.65 (m, 2H), $4.49(\mathrm{~d}, J=3.2 \mathrm{HZ}, 1 \mathrm{H}), 3.82(\mathrm{~s}, 3 \mathrm{H}), 3.53-3.46(\mathrm{~m}, 5 \mathrm{H}), 3.10-3.03(\mathrm{~m}, 1 \mathrm{H})$.

${ }^{13} \mathbf{C}\left\{{ }^{1} \mathbf{H}\right\}$ NMR $\left(\mathrm{CDCl}_{3}, 100 \mathrm{MHz}\right) \delta(\mathrm{ppm}): 150.9,150.2,146.4,145.1,134.5,133.0,128.6$, $128.3,127.4,127.1,126.3,126.1,110.3,109.8,58.6,55.9,55.8,55.2,37.3$.

HRMS (ESI) m/z: [M+Na $]^{+}$Calcd for $\mathrm{C}_{23} \mathrm{H}_{22} \mathrm{O}_{2}$ 353.1512; Found 353.1508.

93:7 dr, 91\% ee HPLC conditions: Chiralpak OD-H column, $n$-hexane:isopropanol $=99: 1$; flow rate $=0.5 \mathrm{~mL} / \mathrm{min}$; UV 
detection at $220 \mathrm{~nm} ; \mathrm{t}_{\mathrm{R}}=12.9 \min \left(\right.$ major), $\mathrm{t}_{\mathrm{R}}=14.6 \min \left(\right.$ minor), $\mathrm{t}_{\mathrm{R}}=17.5 \min \left(\right.$ minor), $\mathrm{t}_{\mathrm{R}}=30.8 \min (\operatorname{minor})$. The absolute configuration was assigned to be $(1 R, 2 S)$. The small $J$ value $(3.2 \mathrm{~Hz})$ for the peak at $4.49 \mathrm{ppm}$ (signal from the $\mathrm{CH}$ of the chiral center at the 2-position on the indane ring) in the ${ }^{1} \mathrm{H}$ NMR suggests a cis relative configuration by comparing with the literature (J. Indian Chem. Soc. 2008, 85, 300-305). And the chiral center at the 2-position on the indane ring of $\mathbf{9}$ is originated from $\mathbf{5 p}$, which should be retained during the ring formation step. However, its configuration should be changed from $R$ in $\mathbf{5 p}$ to $S$ in 10, due to a change of CIP orders for the groups on the chiral center. Thus, the absolute configuration of 9 could be deduced to be $(1 R, 2 S)$.

\section{(1R,2S)-5,7-Dimethoxy-1,2-diphenyl-2,3-dihydro-1H-indene (10)}<smiles>COc1cc2c(c(OC)c1)[C@@H](c1ccccc1)C(c1ccccc1)C2</smiles>
colorless oil, $54.8 \mathrm{mg}, 83 \%$ isolated yield. purification on silica gel using a 200:1 petroleum ether/ethyl acetate mixture.

${ }^{1} \mathbf{H}$ NMR $\left(\mathrm{CDCl}_{3}, 400 \mathrm{MHz}\right) \delta(\mathrm{ppm}):$ 7.18-7.05 (m, 8H), 6.70-6.93 (m, 2H), 6.41 (s, 1H), $6.24(\mathrm{~s}$, $1 \mathrm{H}), 4.33(\mathrm{~d}, J=3.6 \mathrm{HZ}, 1 \mathrm{H}), 3.75(\mathrm{~s}, 3 \mathrm{H}), 3.48(\mathrm{~s}, 3 \mathrm{H}), 3.46-3.36(\mathrm{~m}, 2 \mathrm{H}), 2.98-2.94(\mathrm{~m}, 1 \mathrm{H})$.

${ }^{13} \mathbf{C}\left\{{ }^{1} \mathbf{H}\right\}$ NMR $\left(\mathrm{CDCl}_{3}, 100 \mathrm{MHz}\right) \delta(\mathrm{ppm}): 161.3,157.3,146.5,146.1,145.5,128.6,128.2,127.3$, $127.1,126.3,126.0,124.8,100.6,97.5,57.5,55.61,55.59,55.3,40.8$.

HRMS (ESI) m/z: [M+Na $]^{+}$Calcd for $\mathrm{C}_{23} \mathrm{H}_{22} \mathrm{O}_{2}$ 353.1512; Found 353.1521.

$>\mathbf{9 9 : 1} \boldsymbol{d r}, \mathbf{8 6} \%$ ee HPLC conditions: Chiralpak AD-H column, $n$-hexane:isopropanol $=99.5: 0.5$; flow rate $=0.5 \mathrm{~mL} / \mathrm{min}$; $\mathrm{UV}$ detection at $220 \mathrm{~nm} ; \mathrm{t}_{\mathrm{R}}=24.7 \mathrm{~min}$ (minor), $\mathrm{t}_{\mathrm{R}}=29.9 \mathrm{~min}$ (major). The racemic sample obtained for $\mathbf{1 0}$ contains only a pair of enantiomers. The absolute configuration was assigned to be $(1 R, 2 S)$. The small $J$ value $(3.6 \mathrm{~Hz})$ for the peak at $4.33 \mathrm{ppm}$ (signal from the $\mathrm{CH}$ of the chiral center at the 2-position on the indane ring) in the ${ }^{1} \mathrm{H}$ NMR suggests a cis relative configuration by comparing with the literature (J. Indian Chem. Soc. 2008, 85, 300-305). And the chiral center at the 2-position on the indane ring of $\mathbf{1 0}$ is originated from $\mathbf{5 q}$, which should be retained during the ring formation step. However, its configuration should be changed from $R$ in $\mathbf{5 q}$ to $S$ in $\mathbf{1 0}$, due to a change of CIP orders for the groups on the chiral center. Thus, the absolute configuration of $\mathbf{1 0}$ could be deduced to be $(1 R, 2 S)$.

\section{(1R,2S)-5,7-Dimethoxy-1-phenyl-2-(p-tolyl)-2,3-dihydro-1H-indene (11)}

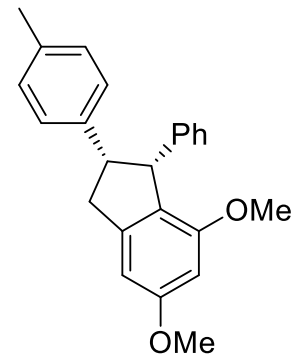

yellow oil, $66.0 \mathrm{mg}, 96 \%$ isolated yield. purification on silica gel using a 100:1 petroleum ether/ethyl acetate mixture.

${ }^{1} \mathbf{H}$ NMR $\left(\mathrm{CDCl}_{3}, 400 \mathrm{MHz}\right) \delta(\mathrm{ppm}):$ 7.15-7.06 (m, 3H), 6.98-6.93 (m, 6H), 6.39 (s, 1H), 6.23 $(\mathrm{s}, 1 \mathrm{H}), 4.31(\mathrm{~d}, J=4.0 \mathrm{HZ}, 1 \mathrm{H}), 3.74(\mathrm{~s}, 3 \mathrm{H}), 3.46(\mathrm{~s}, 3 \mathrm{H}), 3.43-3.34(\mathrm{~m}, 2 \mathrm{H}), 2.95-2.90(\mathrm{~m}$, $1 \mathrm{H}), 2.22(\mathrm{~s}, 3 \mathrm{H})$.

${ }^{13} \mathbf{C}\left\{{ }^{1} \mathbf{H}\right\}$ NMR $\left(\mathrm{CDCl}_{3}, 100 \mathrm{MHz}\right) \delta(\mathrm{ppm}): 161.3,157.3,146.1,145.5,143.4,135.8,129.2$, $128.2,127.2,126.9,125.9,124.8,100.6,97.4,57.5,55.6,55.3,55.2,40.8,21.1$.

HRMS (ESI) m/z: [M+Na $]^{+}$Calcd for $\mathrm{C}_{24} \mathrm{H}_{24} \mathrm{O}_{2}$ 367.1669; Found 367.1671.

$>$ 99: 1 dr, 96\% ee HPLC conditions: Chiralpak OJ-H column, $n$-hexane:isopropanol = 99:1; flow rate $=0.5 \mathrm{~mL} / \mathrm{min}$; UV detection at $220 \mathrm{~nm} ; \mathrm{t}_{\mathrm{R}}=44.1 \mathrm{~min}$ (minor), $\mathrm{t}_{\mathrm{R}}=64.6 \mathrm{~min}$ (major). The racemic sample obtained for 11 contains only a pair of enantiomers. The absolute configuration was assigned to be $(1 R, 2 S)$. The small $J$ value $(4.0 \mathrm{~Hz})$ for the peak at $4.31 \mathrm{ppm}$ (signal from the $\mathrm{CH}$ of the chiral center at the 2-position on the indane ring) in the ${ }^{1} \mathrm{H}$ NMR suggests a cis relative configuration by comparing with the literature (J. Indian Chem. Soc. 2008, 85, 300-305). And the chiral center at the 2-position on the indane ring of $\mathbf{1 1}$ is originated from $\mathbf{5 x}$, which should be retained during the ring formation step. However, its configuration should be changed from $R$ in $\mathbf{5 x}$ to $S$ in $\mathbf{1 1}$, due to a change of CIP orders for the groups on the chiral center. Thus, the absolute configuration of $\mathbf{1 1}$ could be deduced to be $(1 R, 2 S)$. 


\section{Crystallographic data}

CCDC number: CCDC 2093713

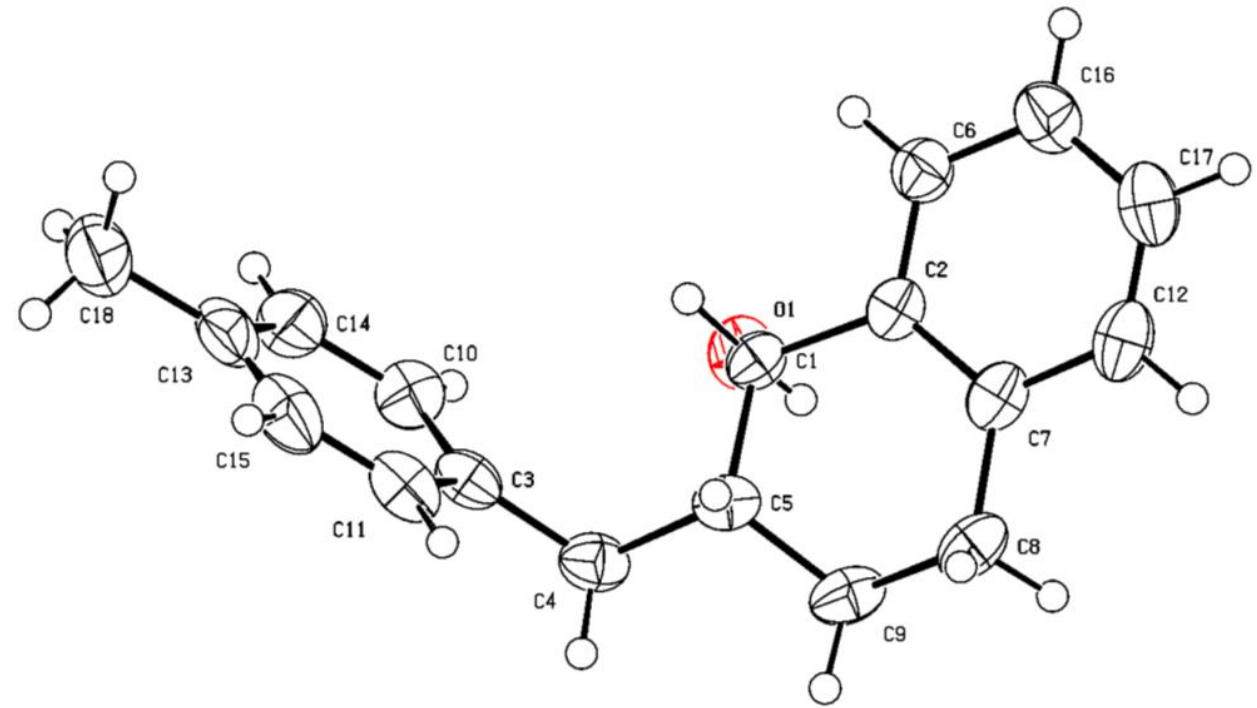

Thermal ellipsoid plot for 3a. Thermal ellipsoids are drawn at the 50\% probability level. 


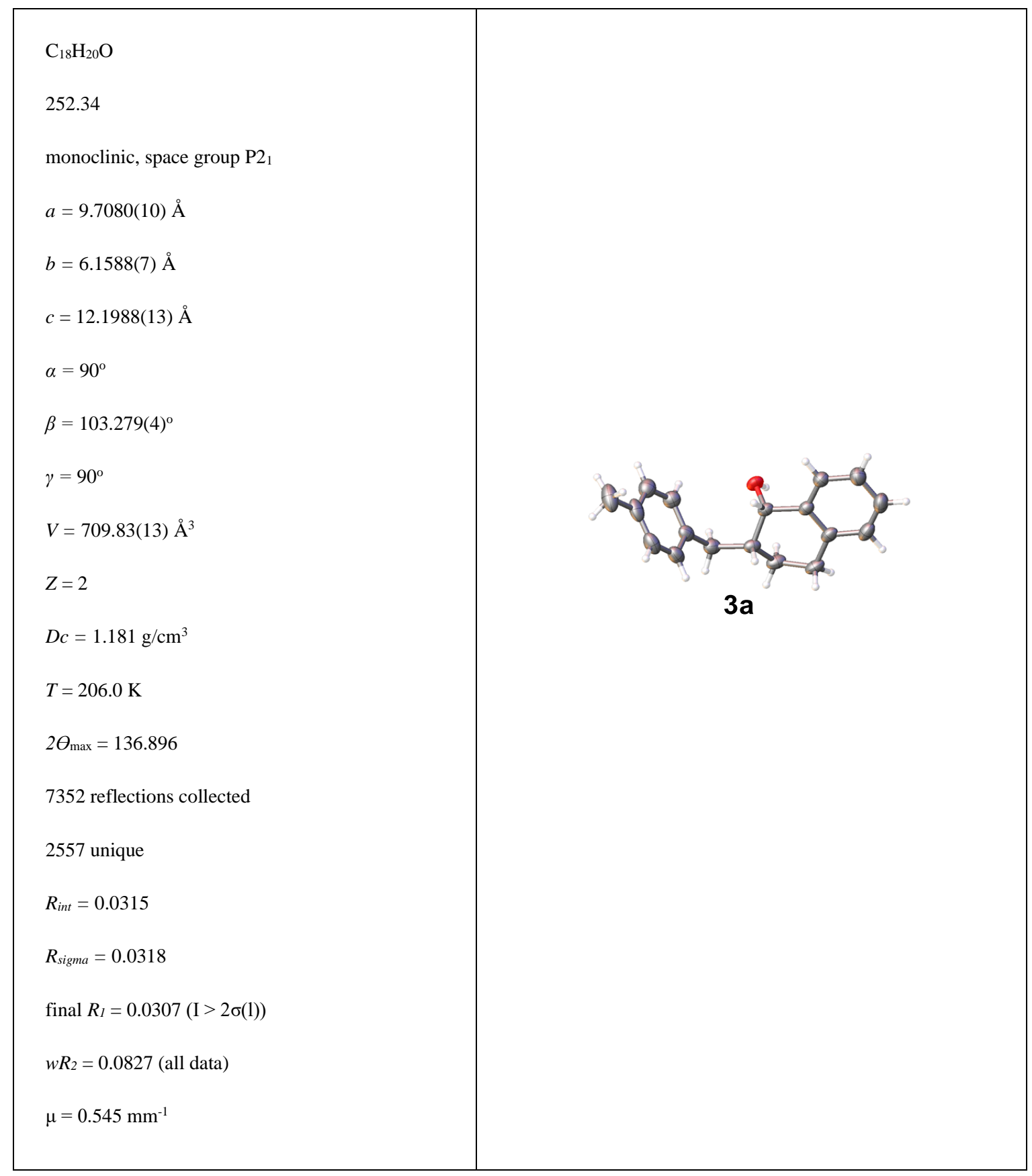


CCDC number: CCDC 2093721

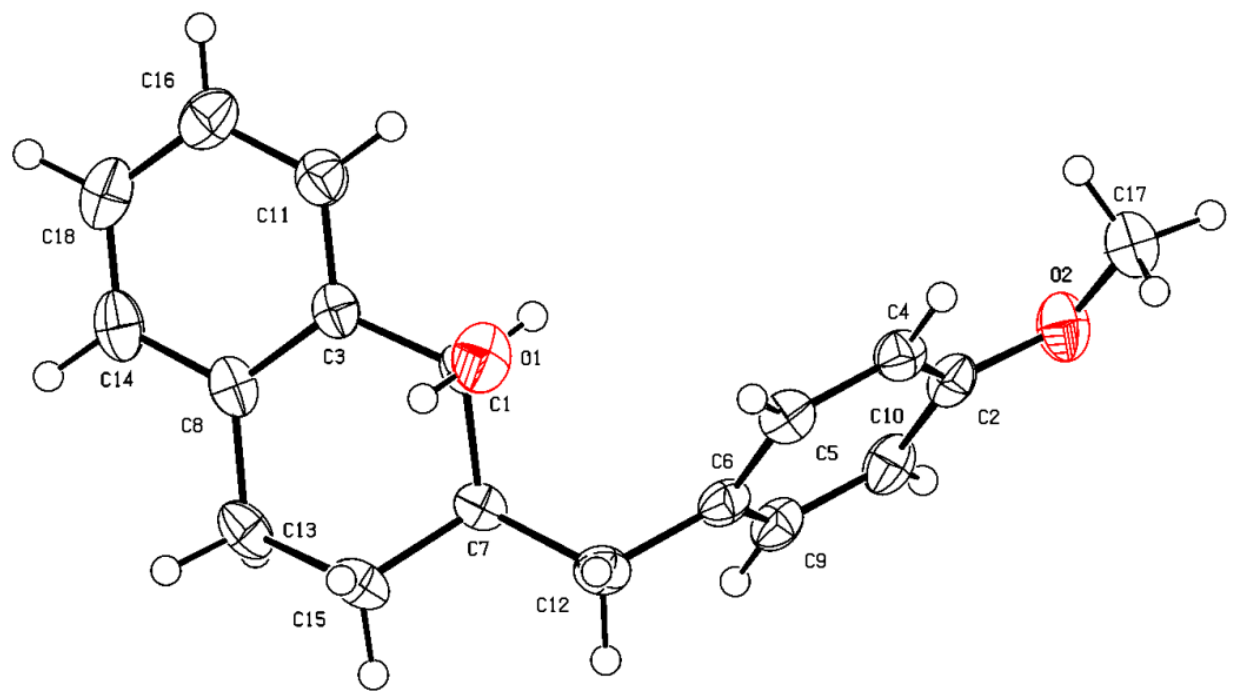

Thermal ellipsoid plot for 3c. Thermal ellipsoids are drawn at the 50\% probability level. 


\begin{tabular}{l}
$\mathrm{C}_{18} \mathrm{H}_{20} \mathrm{O}_{2}$ \\
268.34 \\
monoclinic, space group $\mathrm{P} 21$ \\
$a=9.5057(4) \AA$ \\
$b=6.3276(2) \AA$ \\
$c=12.2052(5) \AA$ \\
$\alpha=90^{\circ}$ \\
$\beta=104.0210(10)^{\circ}$ \\
$\gamma=90^{\circ}$ \\
$V=712.25(5) \AA^{3}$ \\
$Z=2$ \\
$D c=1.251 \mathrm{~g} / \mathrm{cm}^{3}$ \\
$T=153.0 \mathrm{~K}$ \\
$2 \theta_{\text {max }}=136.726^{\circ}$ \\
11727 reflections collected \\
2597 unique \\
$R_{\text {int }}=0.0251$ \\
$R_{\text {sigma }}=0.0191$ \\
final $R_{I}=0.0247$ (I $\left.>2 \sigma(1)\right)$ \\
$w R_{2}=0.0649$ (all data) \\
$\mu=0.629 \mathrm{~mm}-1$ \\
\hline
\end{tabular}




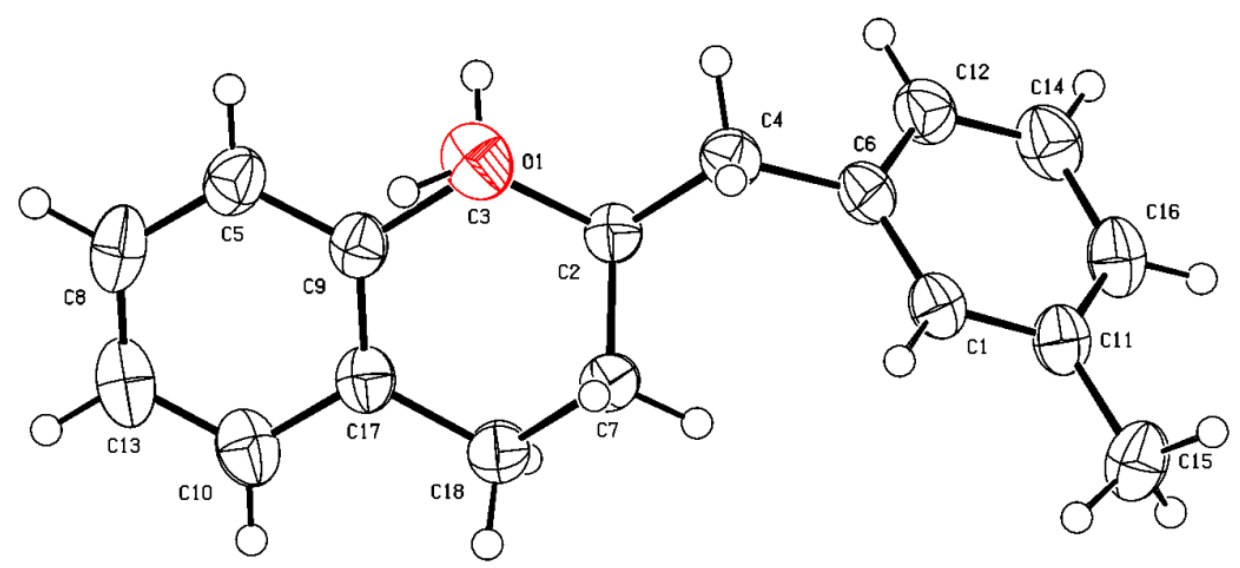

Thermal ellipsoid plot for 3i. Thermal ellipsoids are drawn at the 50\% probability level. 


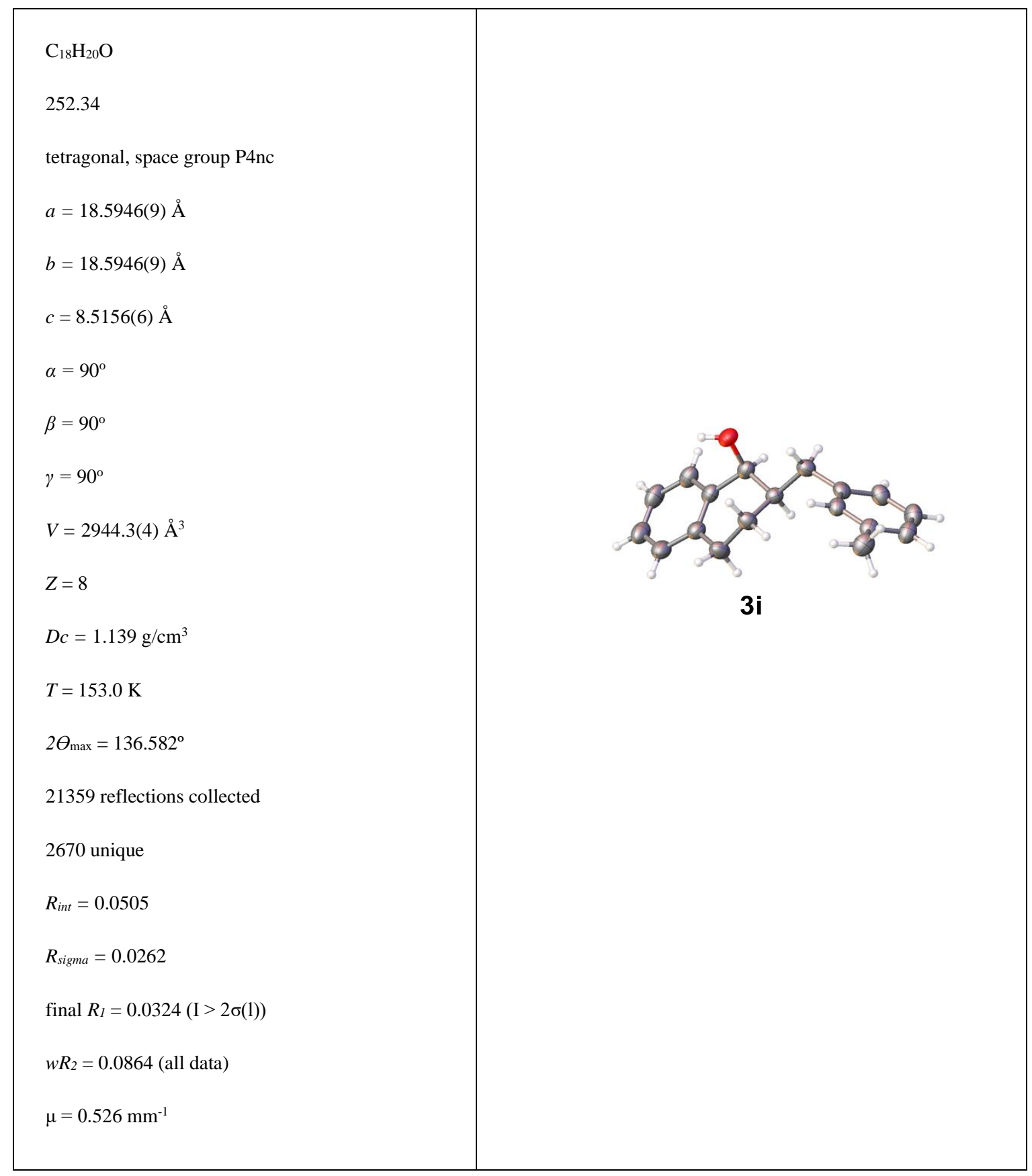




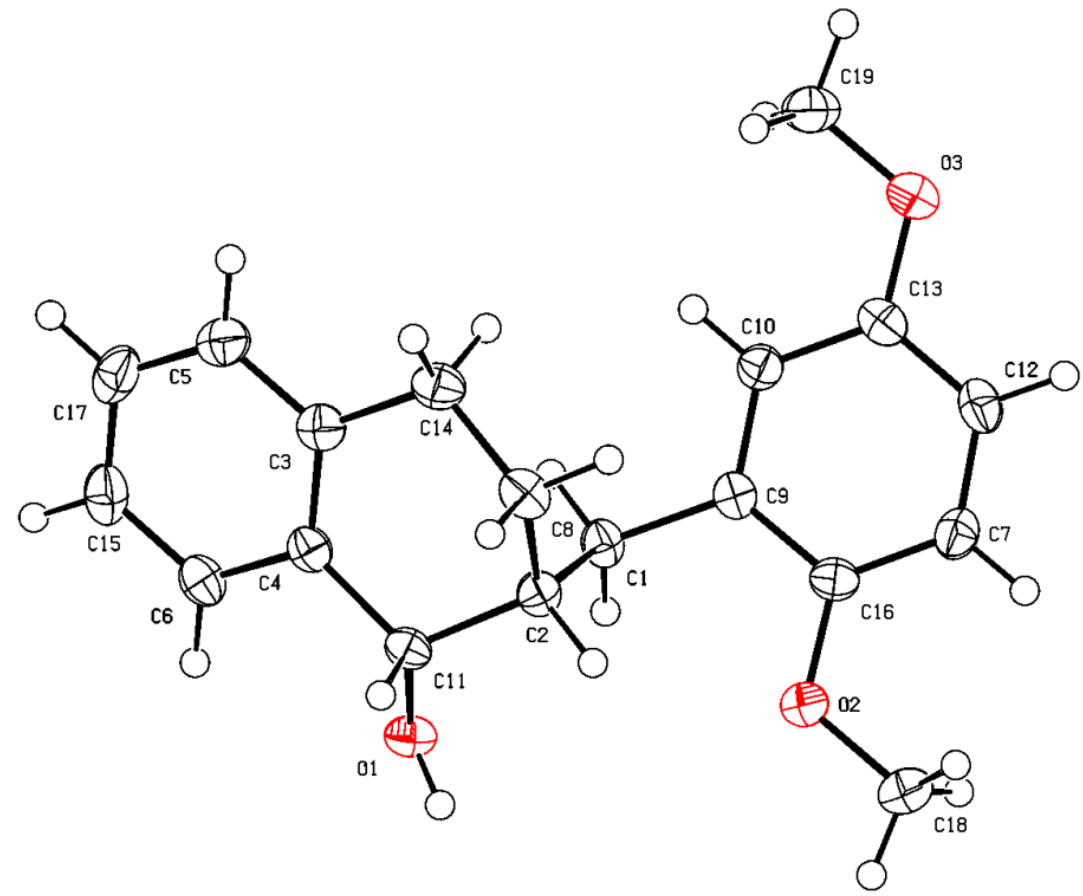

Thermal ellipsoid plot for $3 \mathbf{n}$. Thermal ellipsoids are drawn at the 50\% probability level. 


\begin{tabular}{l}
$\mathrm{C}_{19} \mathrm{H}_{22} \mathrm{O}_{3}$ \\
298.36 \\
orthorhombic, space group $\mathrm{P} 2_{2} 22_{2}$ \\
$a=5.0788(2) \AA$ \\
$b=12.6088$ (7) $\AA$ \\
$c=23.7944(12) \AA$ \\
$\alpha=90^{\circ}$ \\
$\beta=90^{\circ}$ \\
$\gamma=90^{\circ}$ \\
$V=1523.74(13) \AA^{3}$ \\
$Z=4$ \\
$D c=1.301 \mathrm{~g} / \mathrm{cm}^{3}$ \\
$T=153.0 \mathrm{~K}$ \\
$2 \theta_{\text {max }}=136.484^{\circ}$ \\
19722 reflections collected \\
2758 unique \\
$R_{\text {int }}=0.0421$ \\
$R_{\text {sigma }}=0.0221$ \\
final $R_{I}=0.0290$ (I $\left.>2 \sigma(1)\right)$ \\
$w R_{2}=0.0794$ (all data) \\
$\mu=0.692 \mathrm{~mm}-1$ \\
\hline
\end{tabular}




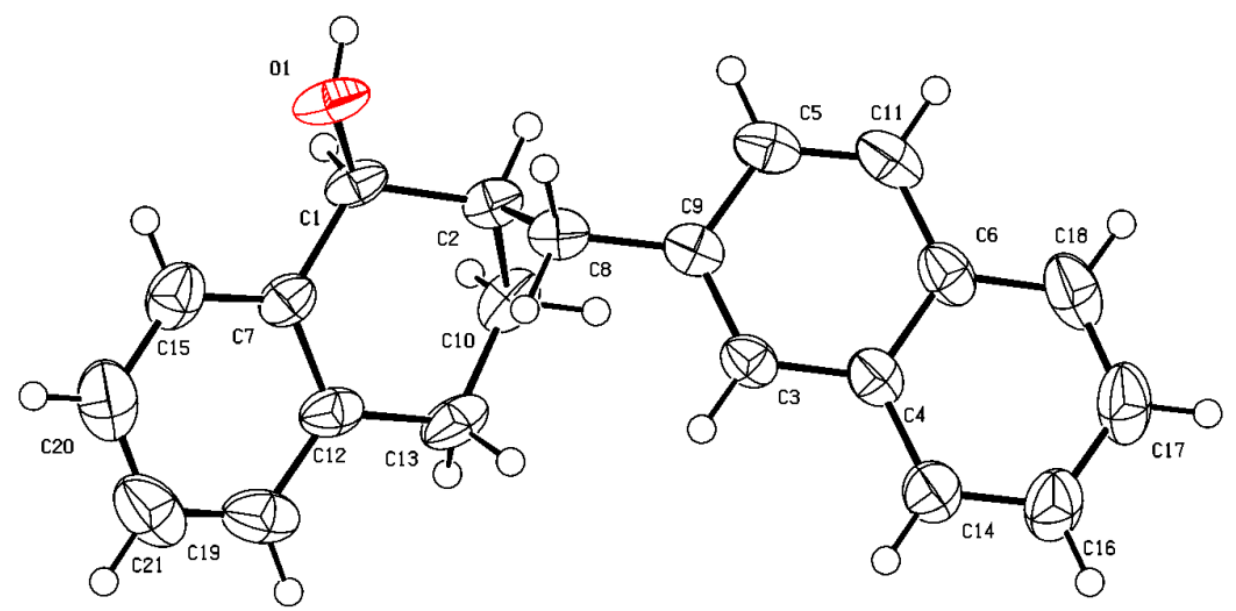

Thermal ellipsoid plot for 3q. Thermal ellipsoids are drawn at the 50\% probability level. 


\begin{tabular}{l}
$\mathrm{C}_{21} \mathrm{H}_{20} \mathrm{O}$ \\
288.37 \\
orthorhombic, space group $\mathrm{P} 212121$ \\
$a=5.2740(4) \AA$ \\
$b=12.1393$ (9) $\AA$ \\
$c=24.1764(17) \AA$ \\
$\alpha=90^{\circ}$ \\
$\beta=90^{\circ}$ \\
$\gamma=90^{\circ}$ \\
$V=1547.8(2) \AA^{3}$ \\
$Z=4$ \\
$D c=1.237 \mathrm{~g} / \mathrm{cm}^{3}$ \\
$T=223.0 \mathrm{~K}$ \\
$2 \theta_{\text {max }}=137.504^{\circ}$ \\
26005 reflections collected \\
2820 unique \\
$R_{\text {int }}=0.0410$ \\
$R_{\text {sigma }}=0.0191$ \\
final $R_{I}=0.0310$ (I $\left.>2 \sigma(1)\right)$ \\
$w R_{2}=0.0835$ (all data) \\
$\mu=0.570 \mathrm{~mm}-1$ \\
\hline
\end{tabular}




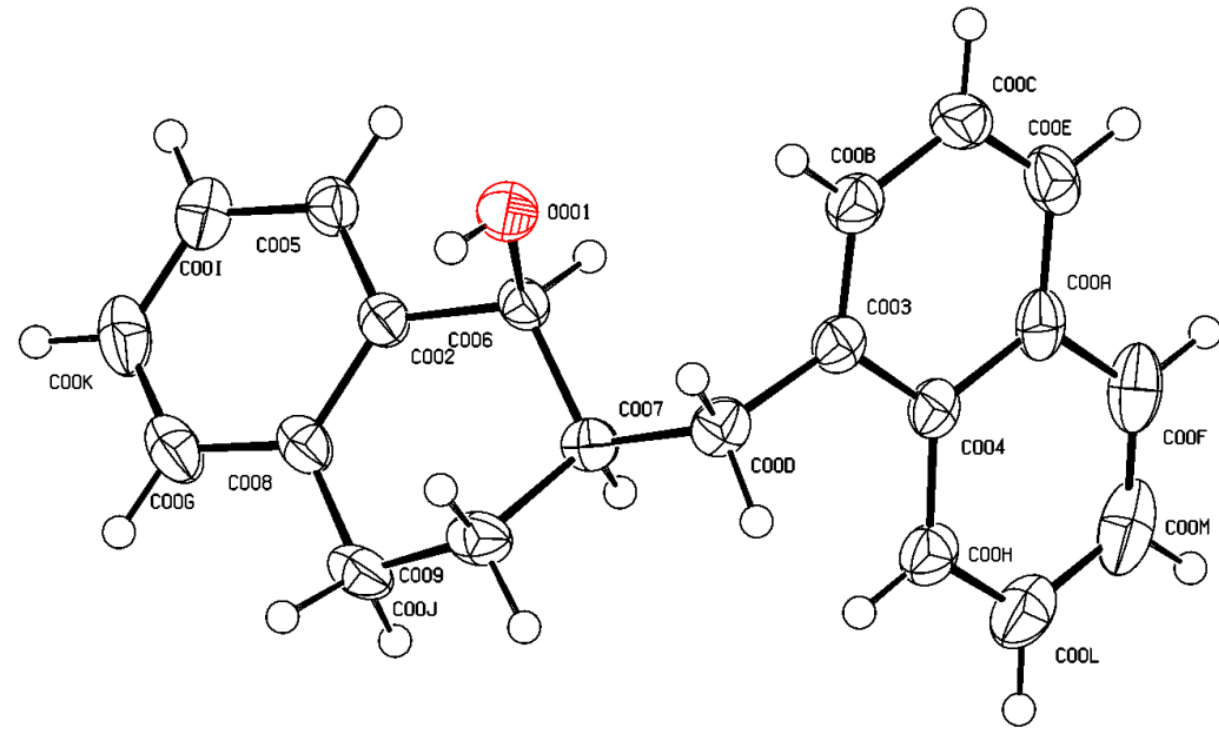

Thermal ellipsoid plot for 3r. Thermal ellipsoids are drawn at the 50\% probability level. 


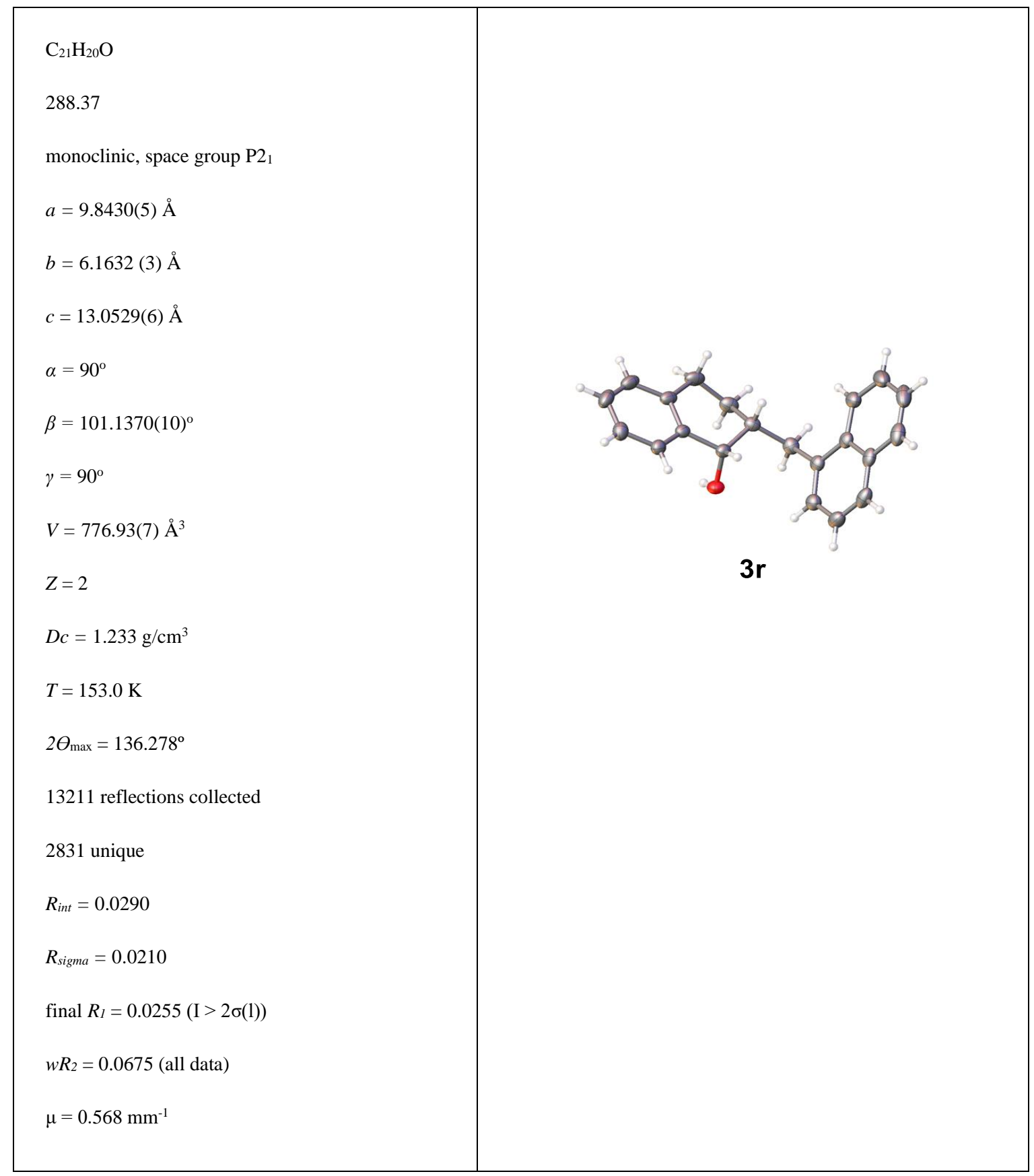




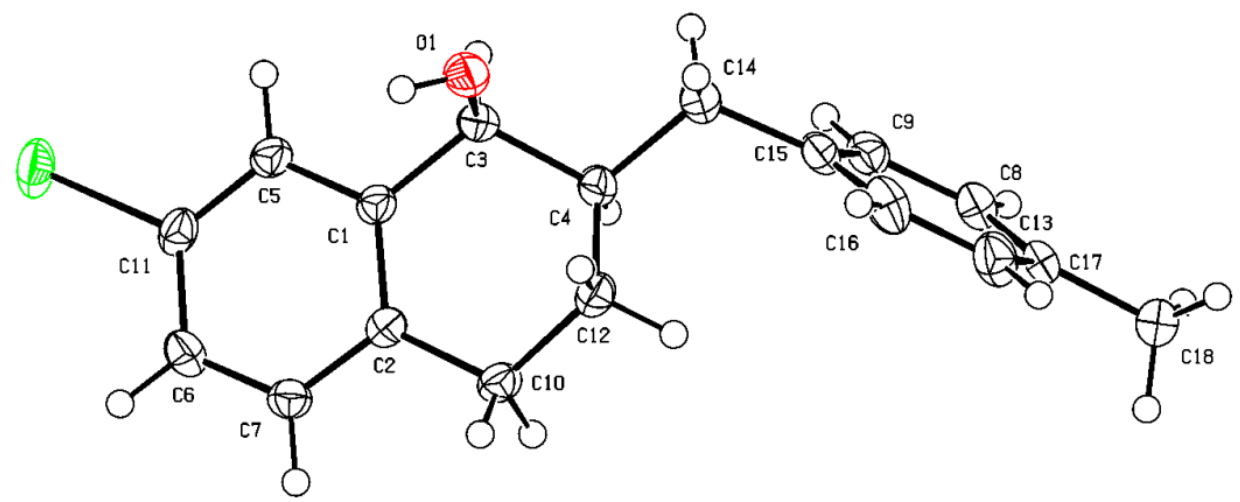

Thermal ellipsoid plot for $\mathbf{3 y}$. Thermal ellipsoids are drawn at the 50\% probability level. 


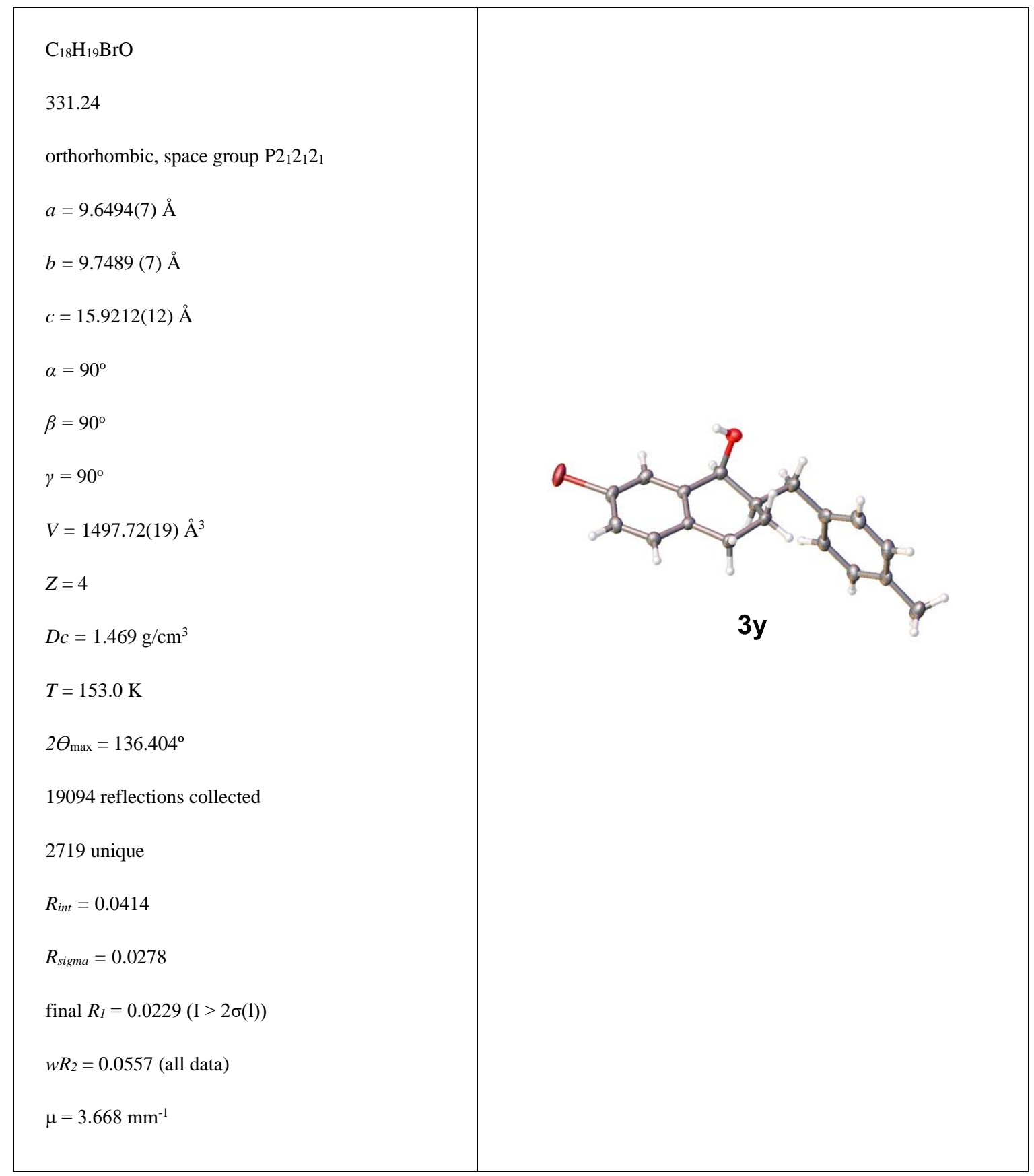




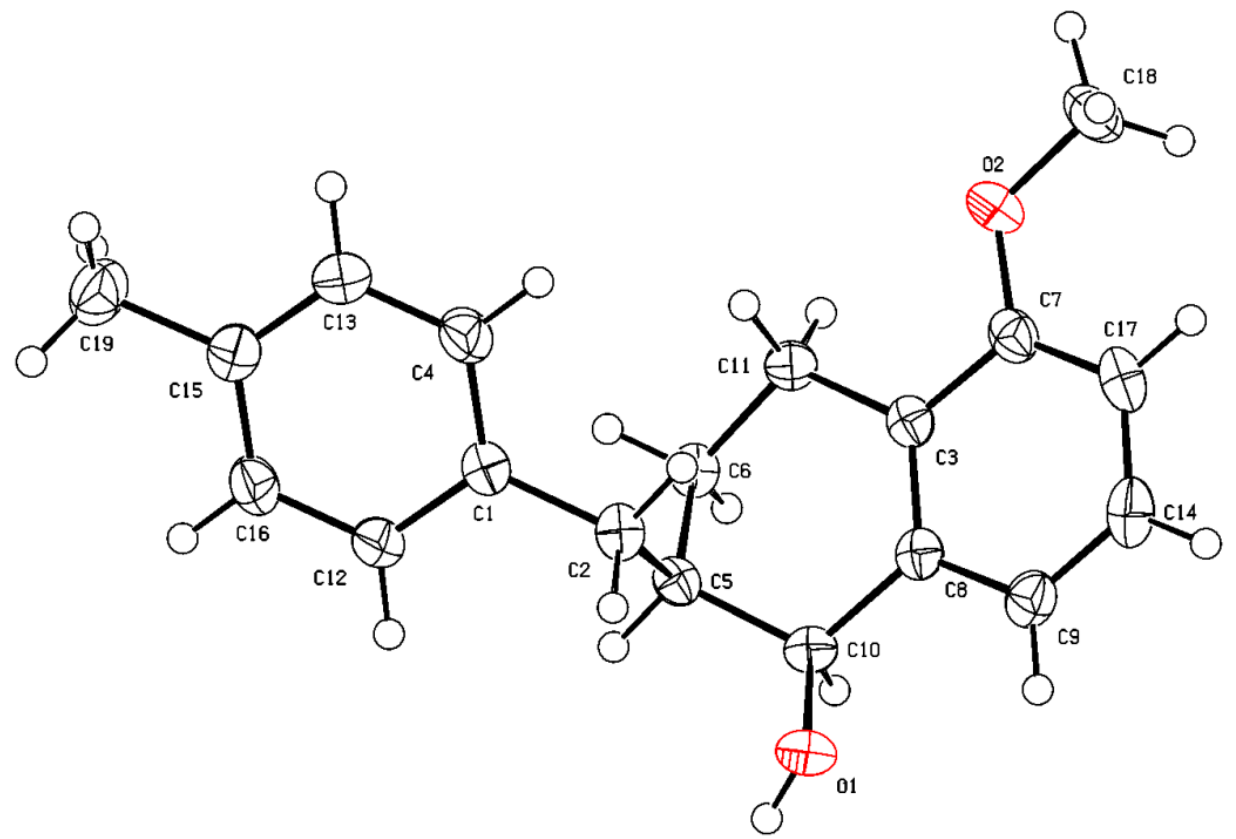

Thermal ellipsoid plot for $\mathbf{3 z}$. Thermal ellipsoids are drawn at the 50\% probability level. 


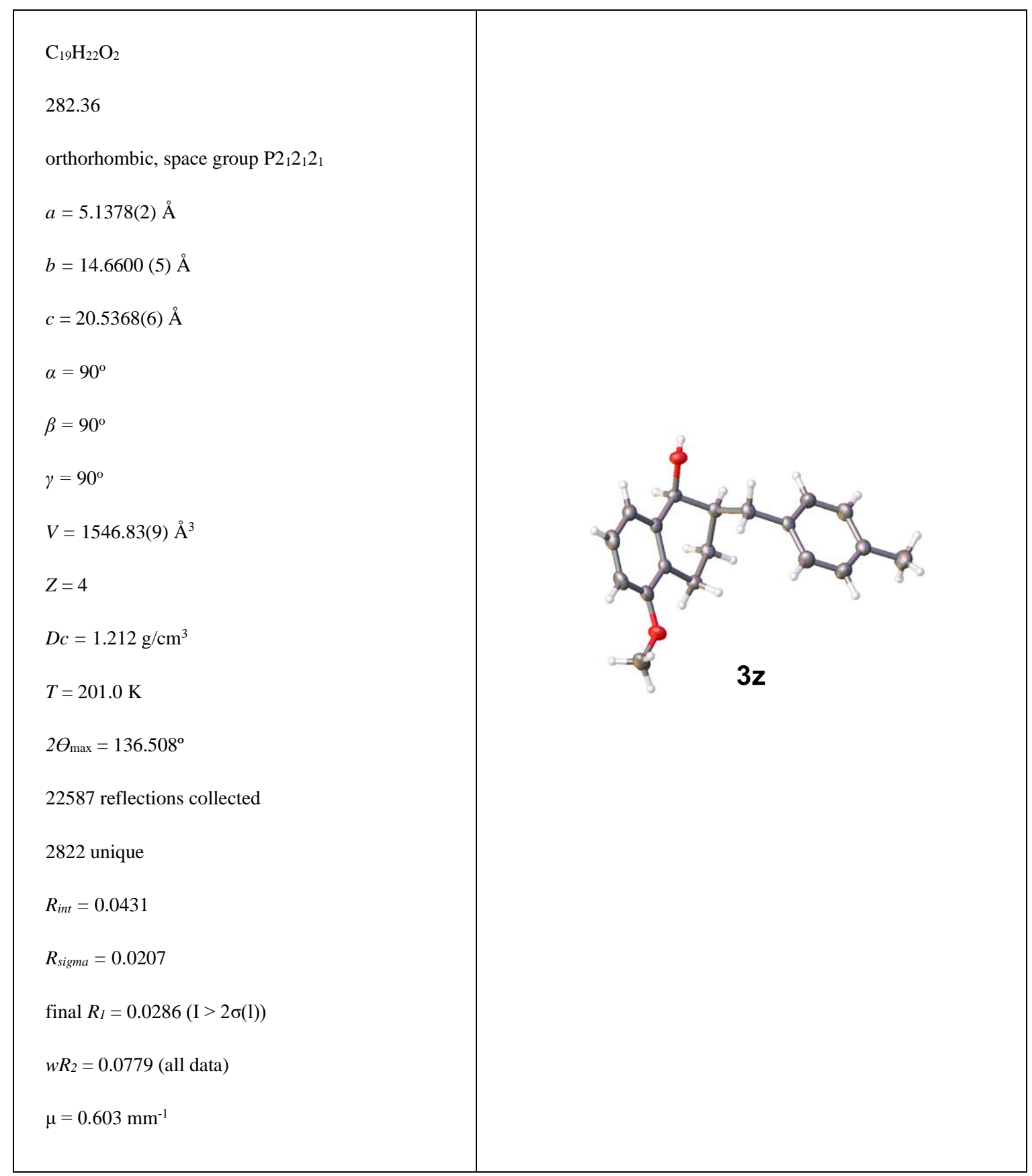




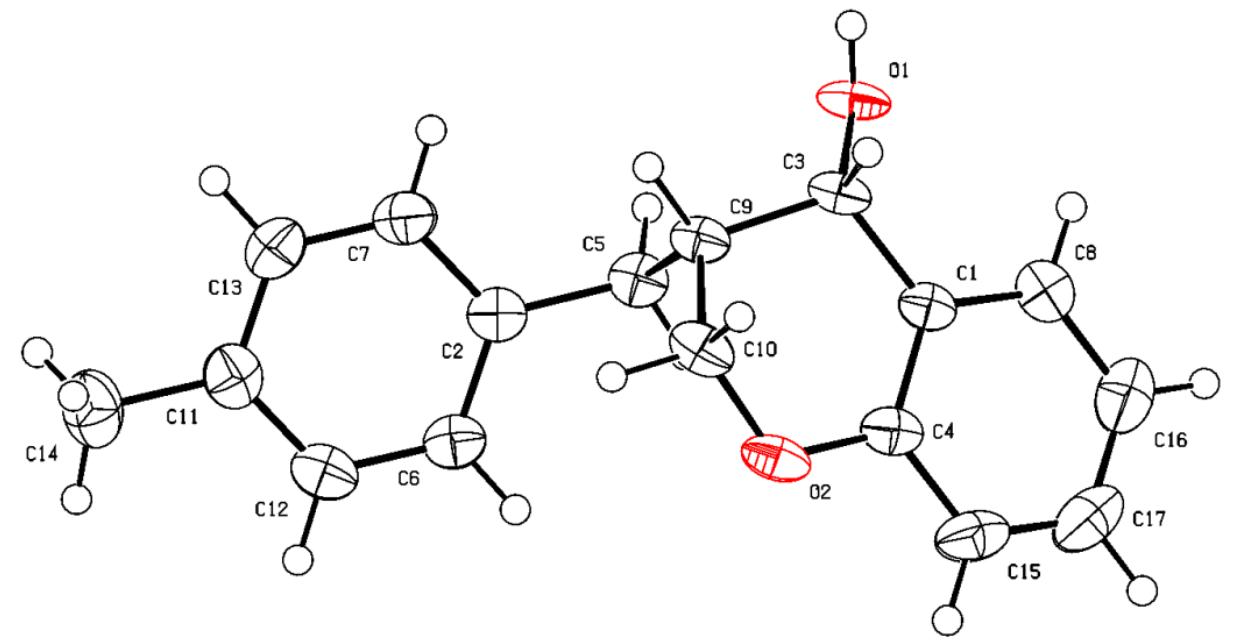

Thermal ellipsoid plot for 3ac. Thermal ellipsoids are drawn at the 50\% probability level. 


\begin{tabular}{l}
$\mathrm{C}_{17} \mathrm{H}_{18} \mathrm{O}_{2}$ \\
254.31 \\
orthorhombic, space group $\mathrm{P} 212121$ \\
$a=5.1016(3) \AA$ \\
$b=11.5169(6) \AA$ \\
$c=23.5278(12) \AA$ \\
$\alpha=90^{\circ}$ \\
$\beta=90^{\circ}$ \\
$\gamma=90^{\circ}$ \\
$V=1382.37(13) \AA^{3}$ \\
$Z=4$ \\
$D c=1.222 \mathrm{~g} / \mathrm{cm}^{3}$ \\
$T=153.0 \mathrm{~K}$ \\
$2 \theta_{\text {max }}=136.176^{\circ}$ \\
20602 reflections collected \\
2482 unique \\
$R_{\text {int }}=0.0392$ \\
$R_{\text {sigma }}=0.0190$ \\
final $R_{I}=0.0279$ (I $\left.>2 \sigma(1)\right)$ \\
$w R_{2}=0.0739$ (all data) \\
$\mu=0.622 \mathrm{~mm}-1$ \\
\hline
\end{tabular}




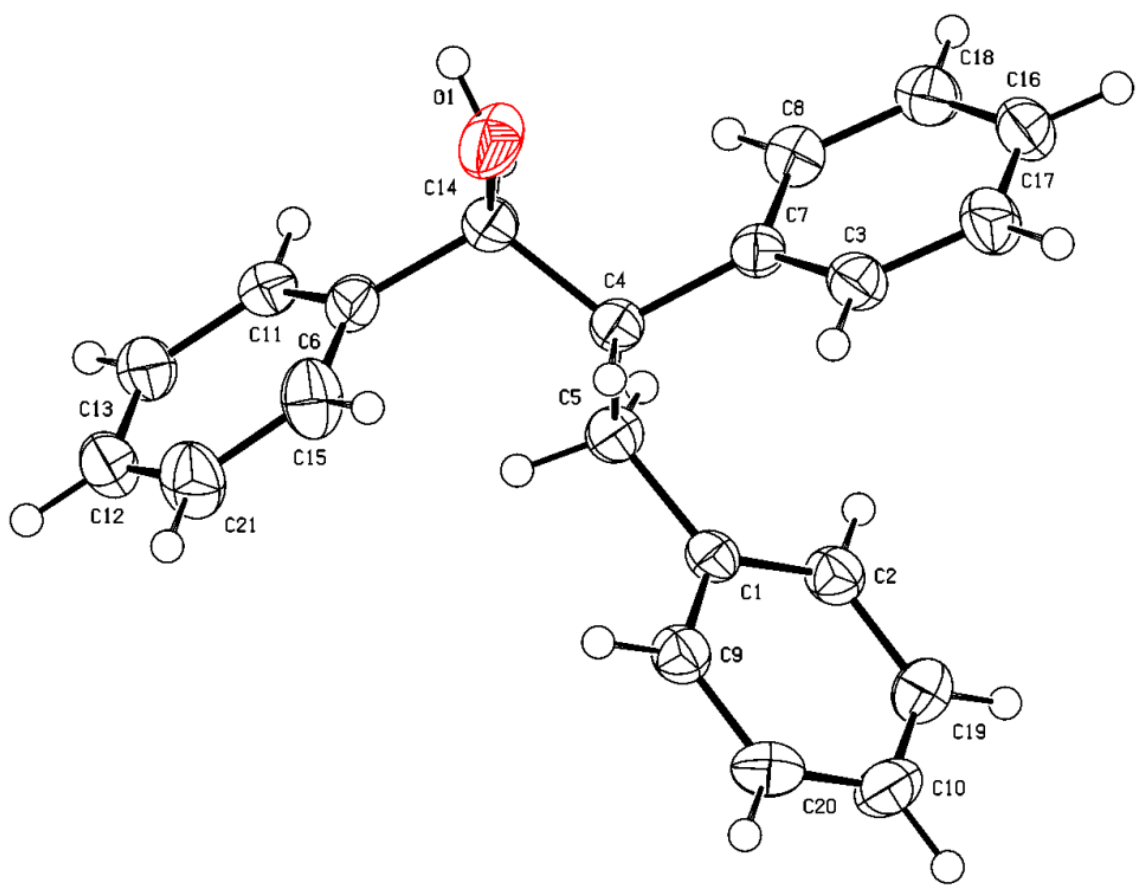

Thermal ellipsoid plot for $\mathbf{5 a}$. Thermal ellipsoids are drawn at the $50 \%$ probability level. 


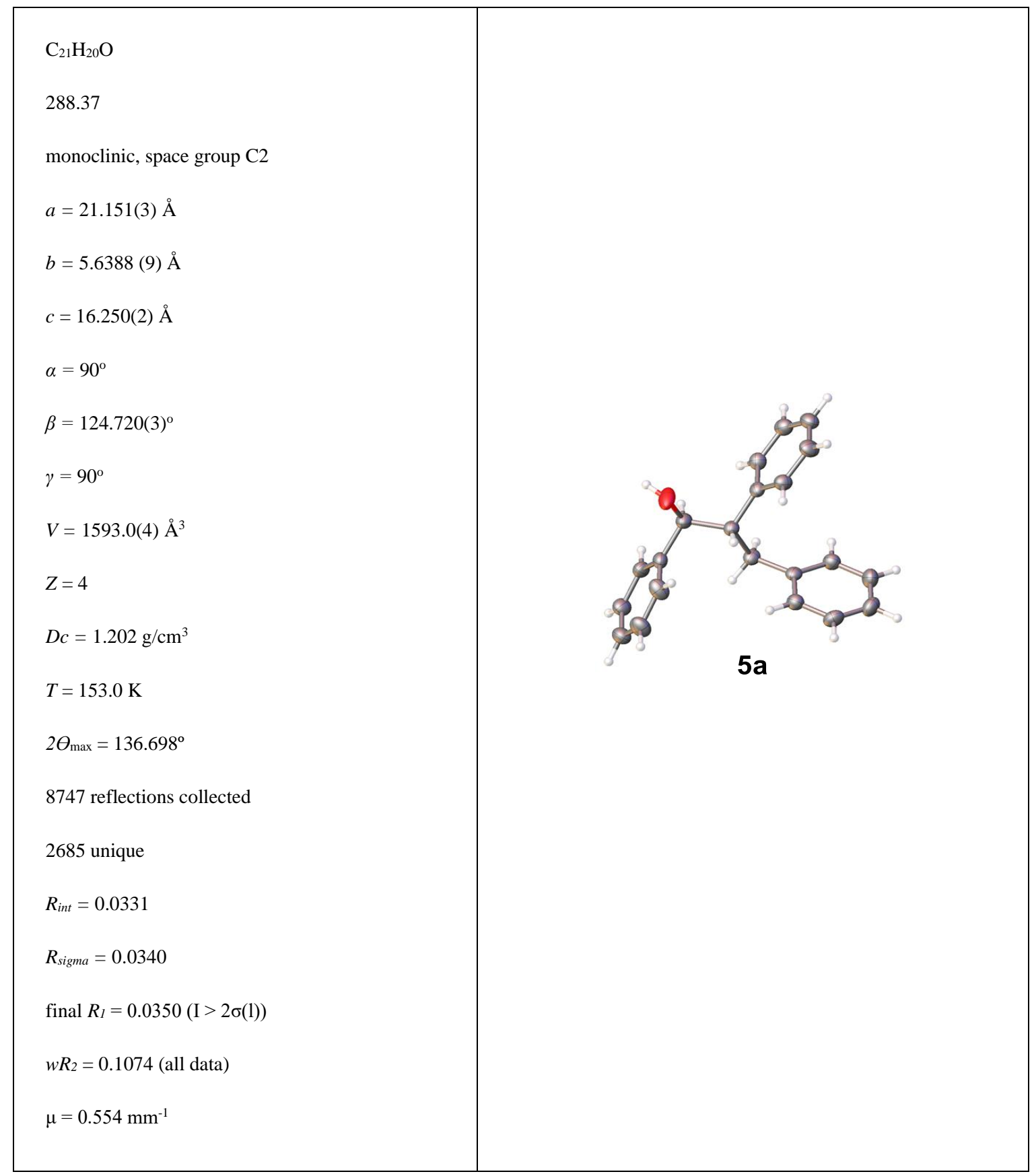




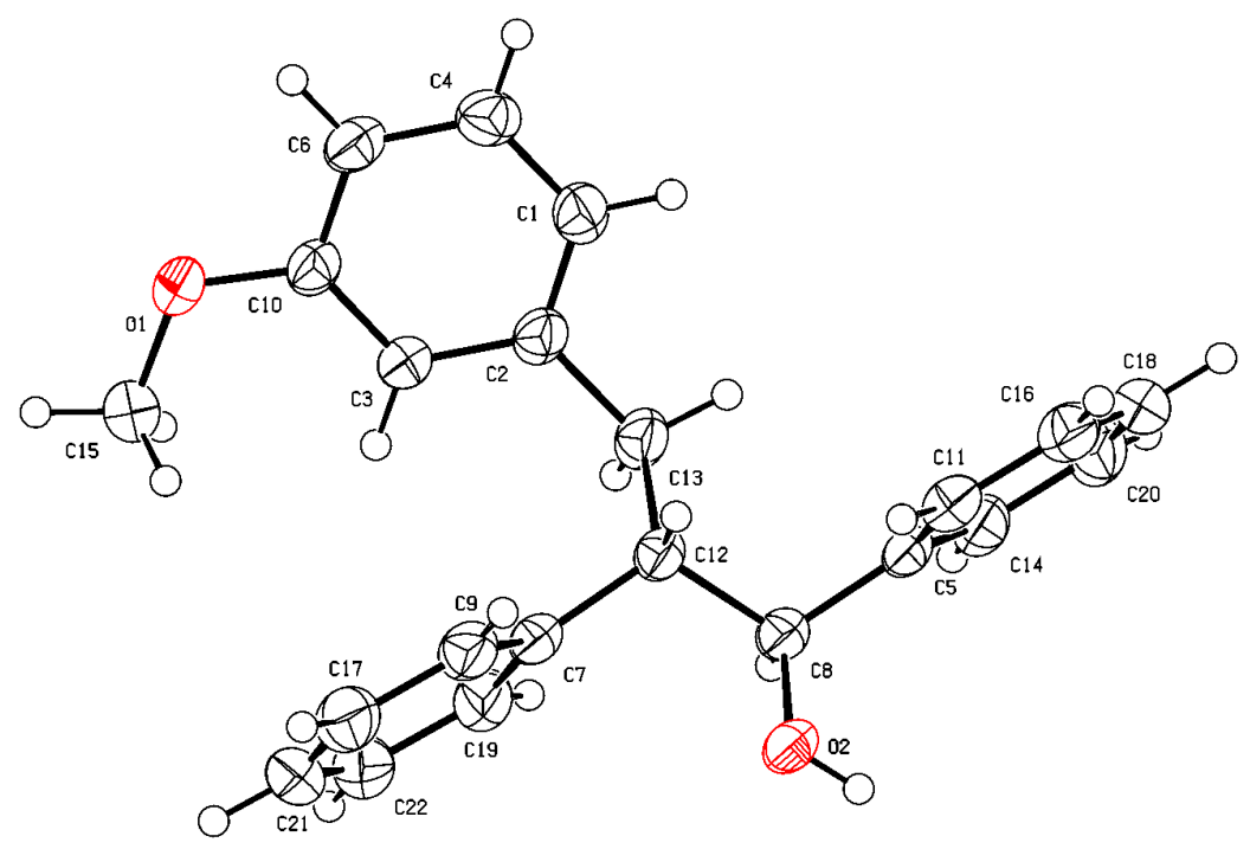

Thermal ellipsoid plot for $\mathbf{5 i}$. Thermal ellipsoids are drawn at the 50\% probability level. 


\begin{tabular}{l}
$\mathrm{C}_{22} \mathrm{H}_{22} \mathrm{O}_{2}$ \\
318.39 \\
monoclinic, space group $\mathrm{P} 21$ \\
$a=5.8390(5) \AA$ \\
$b=8.7267(8) \AA$ \\
$c=17.6306(16) \AA$ \\
$\alpha=90^{\circ}$ \\
$\beta=96.105(2)^{\circ}$ \\
$\gamma=90^{\circ}$ \\
$V=893.28(14) \AA$ \\
$Z=2$ \\
$D c=1.184 \mathrm{~g} / \mathrm{cm}^{3}$ \\
$T=230.0 \mathrm{~K}$ \\
$2 \theta_{\text {max }}=136.82^{\circ}$ \\
12470 reflections collected \\
3220 unique \\
$R_{\text {int }}=0.0407$ \\
$R_{\text {sigma }}=0.0385$ \\
final $R_{I}=0.0329$ (I $\left.>2 \sigma(1)\right)$ \\
$w R_{2}=0.0871$ (all data) \\
$\mu=0.582 \mathrm{~mm}-1$ \\
\hline
\end{tabular}




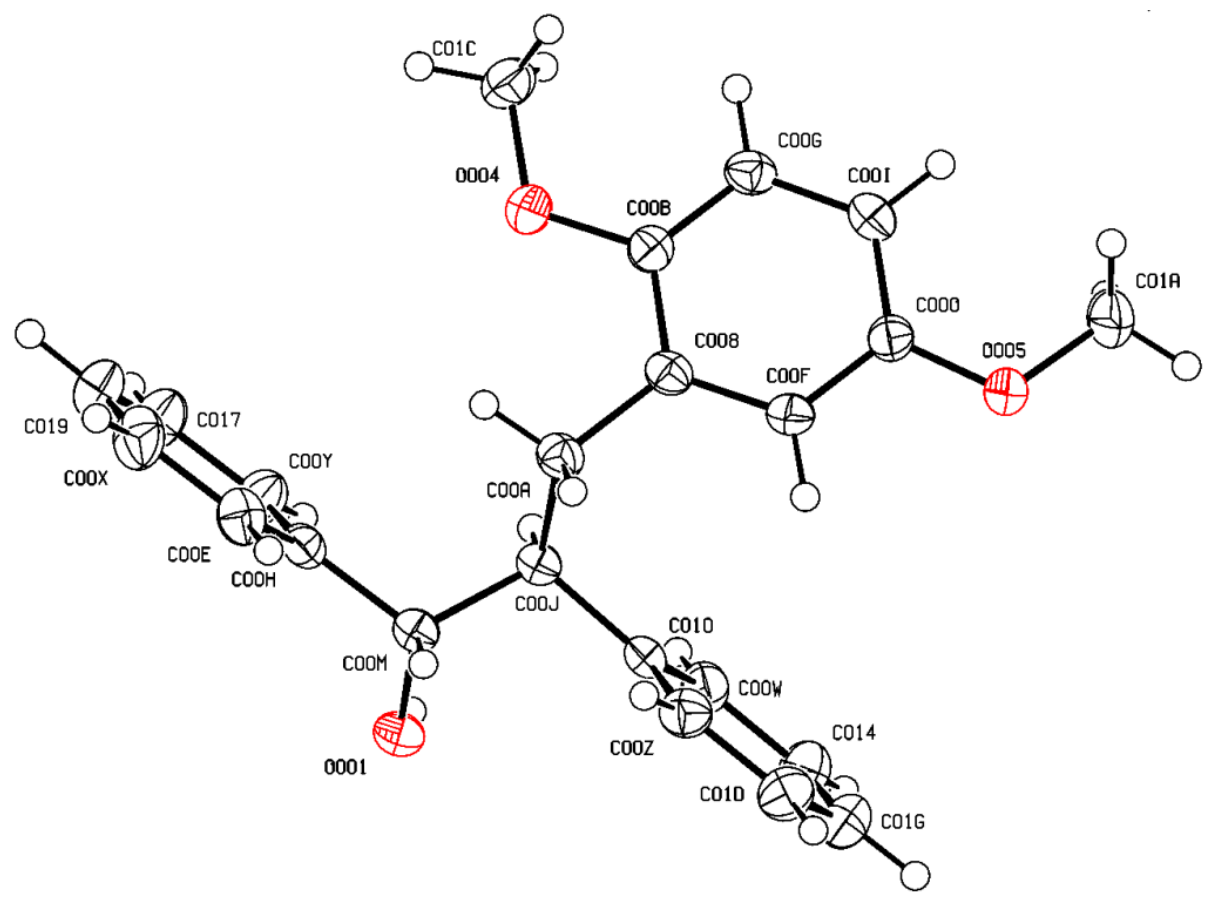

Thermal ellipsoid plot for $\mathbf{5 p}$. Thermal ellipsoids are drawn at the 50\% probability level. 


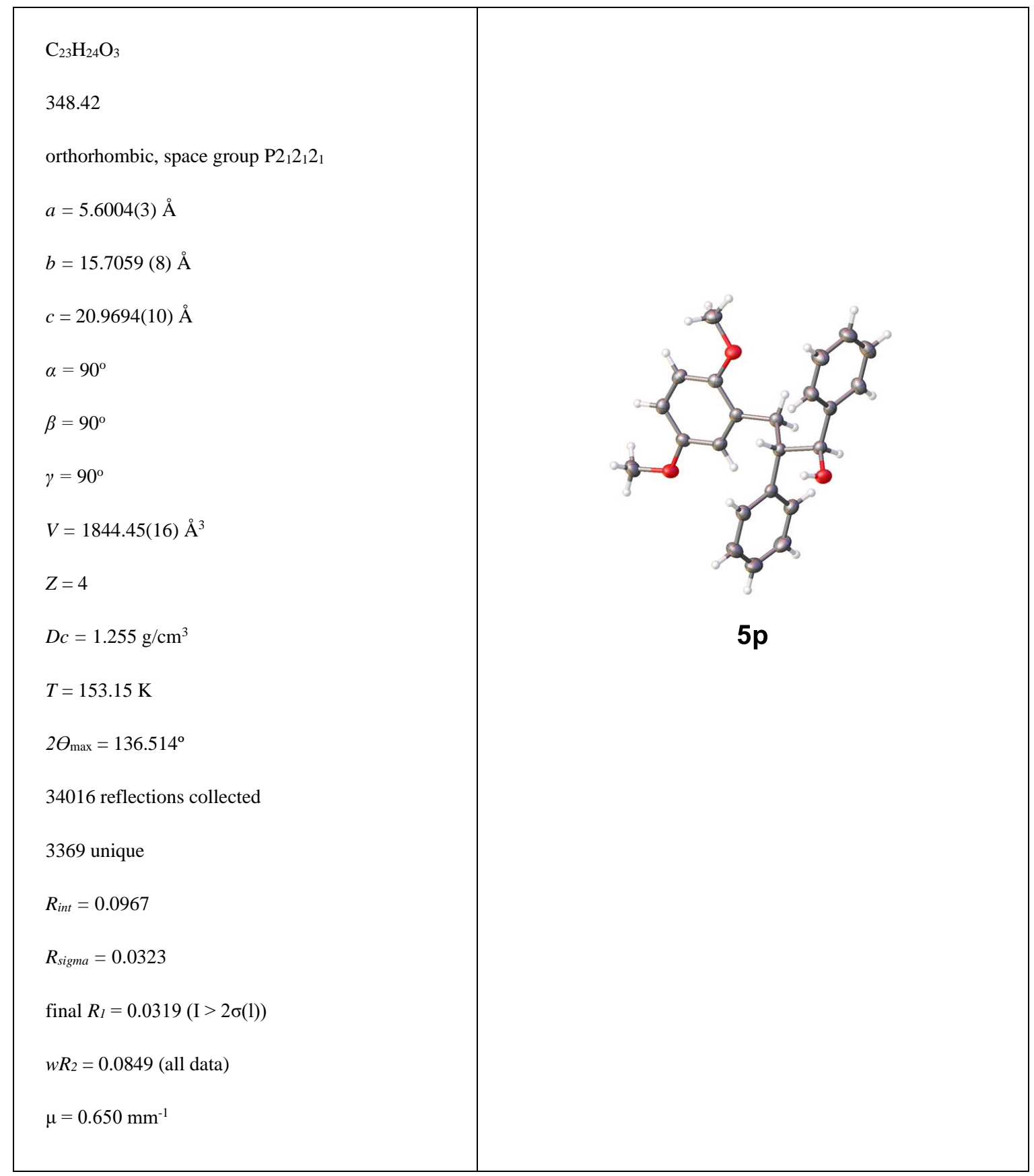




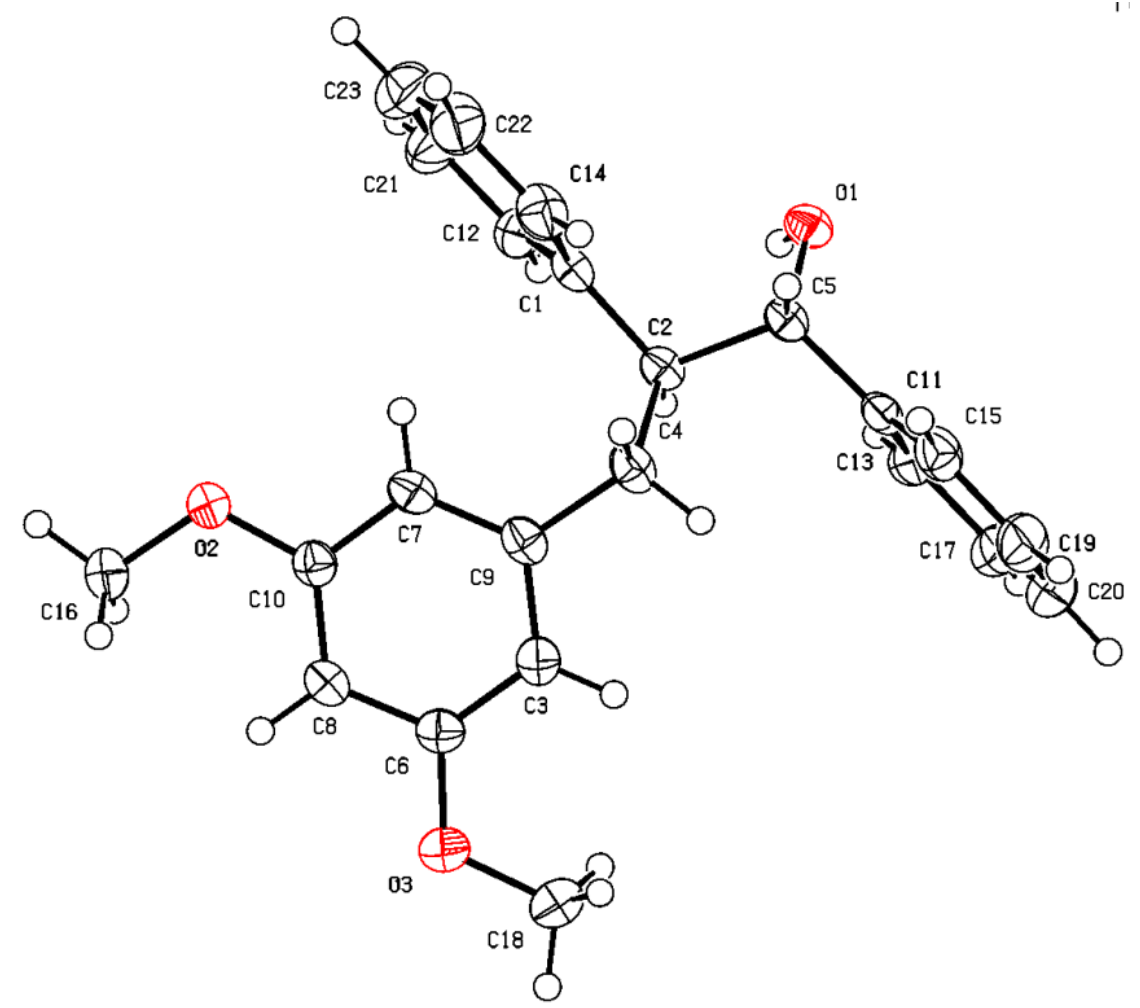

Thermal ellipsoid plot for $\mathbf{5 q}$. Thermal ellipsoids are drawn at the 50\% probability level. 


\begin{tabular}{l}
$\mathrm{C}_{23} \mathrm{H}_{24} \mathrm{O}_{3}$ \\
348.42 \\
orthorhombic, space group $\mathrm{P} 212121$ \\
$a=5.4301(6) \AA$ \\
$b=16.494$ (2) $\AA$ \\
$c=20.957(3) \AA$ \\
$\alpha=90^{\circ}$ \\
$\beta=90^{\circ}$ \\
$\gamma=90^{\circ}$ \\
$V=1876.9(4) \AA^{3}$ \\
$Z=4$ \\
$D c=1.233 \mathrm{~g} / \mathrm{cm}^{3}$ \\
$T=232.0 \mathrm{~K}$ \\
$2 \theta_{\text {max }}=137.864^{\circ}$ \\
29613 reflections collected \\
3427 unique \\
$R_{\text {int }}=0.0612$ \\
$R_{\text {sigma }}=0.0307$ \\
final $R_{I}=0.0312$ (I $\left.>2 \sigma(1)\right)$ \\
$w R_{2}=0.0884$ (all data) \\
$\mu=0.638 \mathrm{~mm}-1$ \\
\hline
\end{tabular}




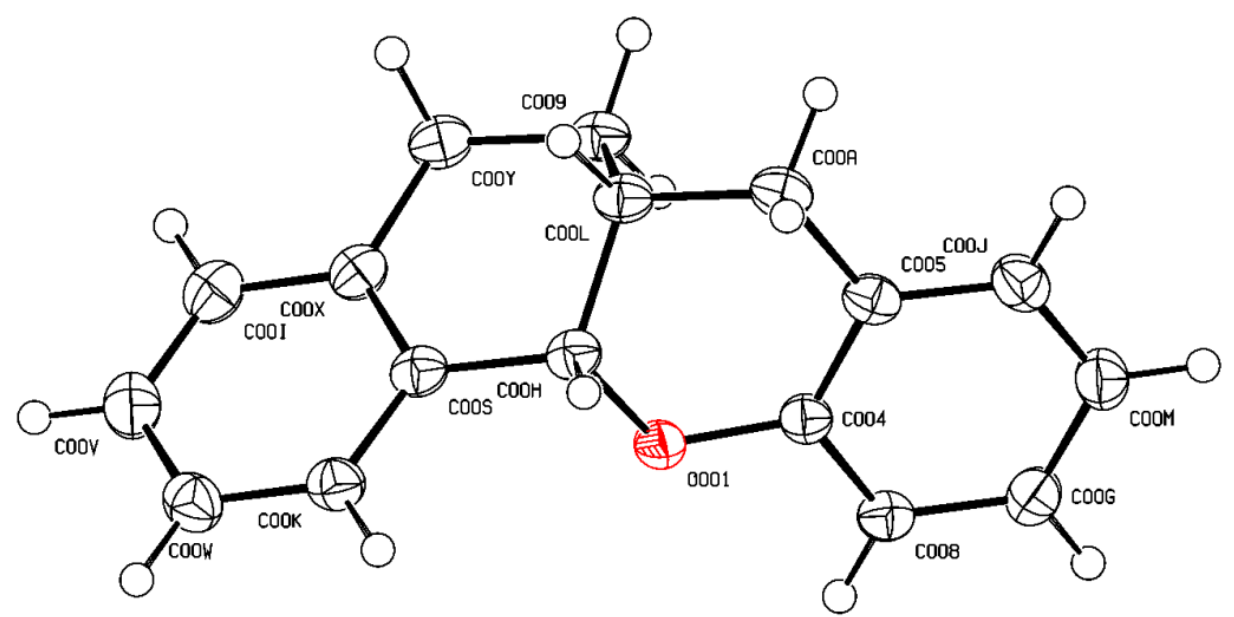

Thermal ellipsoid plot for $\mathbf{7}$. Thermal ellipsoids are drawn at the $50 \%$ probability level. 


\begin{tabular}{l}
$\mathrm{C}_{17} \mathrm{H}_{16} \mathrm{O}$ \\
236.30 \\
monoclinic, space group $\mathrm{P} 21$ \\
$a=5.1405(3) \AA$ \\
$b=19.4930(12) \AA$ \\
$c=6.0747(4) \AA$ \\
$\alpha=90^{\circ}$ \\
$\beta=97.011(2)^{\circ}$ \\
$\gamma=90^{\circ}$ \\
$V=604.16(6) \AA^{3}$ \\
$Z=2$ \\
$D c=1.299 \mathrm{~g} / \mathrm{cm}^{3}$ \\
$T=153.15 \mathrm{~K}$ \\
$2 \theta_{\text {max }}=136.574^{\circ}$ \\
8989 reflections collected \\
2169 unique \\
$R_{\text {int }}=0.0402$ \\
$R_{\text {sigma }}=0.0358$ \\
final $R_{I}=0.0298$ (I $\left.>2 \sigma(1)\right)$ \\
$w R_{2}=0.0751$ (all data) \\
$\mu=0.610 \mathrm{~mm}-1$ \\
\hline
\end{tabular}




\section{Reference}

[1] X. Ren, P. Wang, X. Han, G. Zhang, J. Gu, C. Ding, X. Zheng, F. Cao, ACS Sustain. Chem. Eng. 2017, 5, $6548-6556$.

[2] a) B. V. S. Reddy, G. N. Reddy, M. R. Reddy, J. K. Lakshmi, B. Jagadeesh, B. Sridhar, Asian J. Org. Chem. 2015, 4, 1266-1272; b) C.-K. Chan, Y.-L. Tsai, M.-Y. Chang, Tetrahedron Lett. 2017, 73, 3368-3376.

[3] Z.-L. Shen, X.-P. Xu, S.-J. Ji, J. Org. Chem. 2010, 75, 1162-1167.

[4] N. Morimoto, S.-i. Yamamoto, Y. Takeuchi, Y. Nishina, Rsc Adv. 2013, 3, 15608-15612.

[5] L. Yang, H.-H. Lu, C.-H. Lai, G. Li, W. Zhang, R. Cao, F. Liu, C. Wang, J. Xiao, D. Xue, Angew. Chem. Int. Ed. 2020, $59,12714-12719$.

[6] A. A. Khalaf, I. M. Awad, T. I. El-Emary, H. A. K. A. El-Aal, J. Indian Chem. Soc. 2008, 85, 300-305.

[7] a) H. W. Cheung, T. Y. Lee, H. Y. Lui, C. H. Yeung, C. P. Lau, Adv. Synth. Catal. 2008, 350, 2975-2983; b) T. Miura,

O. Kose, F. Li, S. Kai, S. Saito, Chem. Eur. J. 2011, 17, 11146-11151; c) O. Kose, S. Saito, Org. Biomol. Chem. 2010, 8, 896-900; d) N. Arai, Y. Okabe, T. Ohkuma, Adv. Synth. Catal. 2019, 361, 5540-5547; e) P. Satyanarayana, G. M. Reddy, H. Maheswaran, M. L. Kantam, Adv. Synth. Catal. 2013, 355, 1859-1867; f) Q. Xu, J. Chen, H. Tian, X. Yuan, S. Li, C. Zhou, J. Liu, Angew. Chem. Int. Ed. 2014, 53, 225-229; g) G. S. Caleffi, J. d. O. C. Brum, A. T. Costa, J. L. O. Domingos, P. R. R. Costa, J. Org. Chem. 2021, 86, 4849-4858. 


\section{Traces of ${ }^{1} \mathrm{H}$ NMR and ${ }^{13} \mathrm{C}$ NMR spectra}

${ }^{1} \mathrm{H}$ NMR spectrum of $\mathbf{3 a}\left(\mathrm{CDCl}_{3}, 400 \mathrm{MHz}\right)$ :

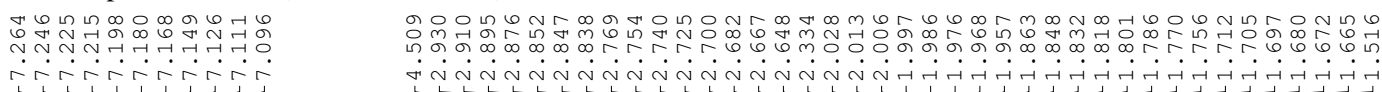<smiles>Cc1ccc(C[C@H]2CCc3ccccc3[C@@H]2O)cc1</smiles>

$3 a$

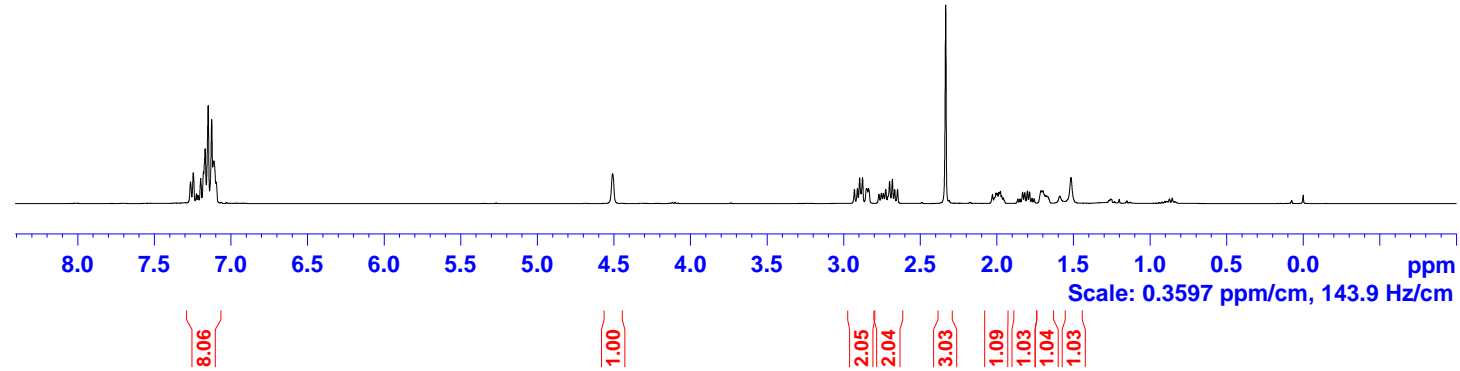

${ }^{13} \mathrm{C}\left\{{ }^{1} \mathrm{H}\right\}$ NMR spectrum of $\mathbf{3 a}\left(\mathrm{CDCl}_{3}, 100 \mathrm{MHz}\right)$ :
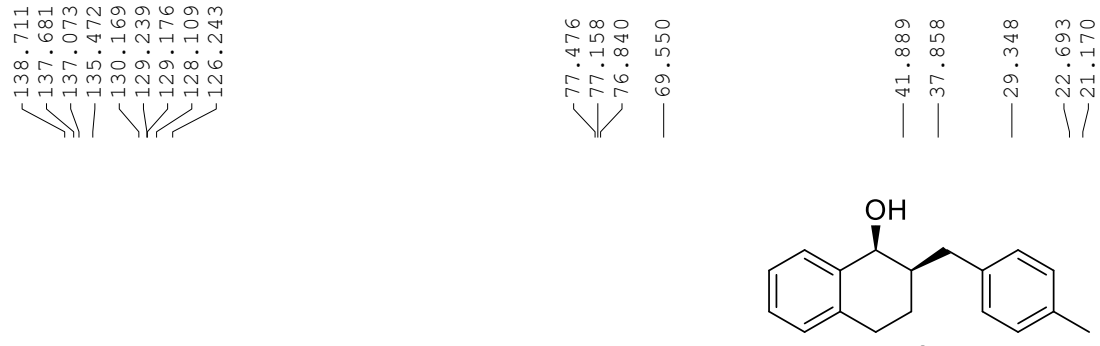

$3 a$

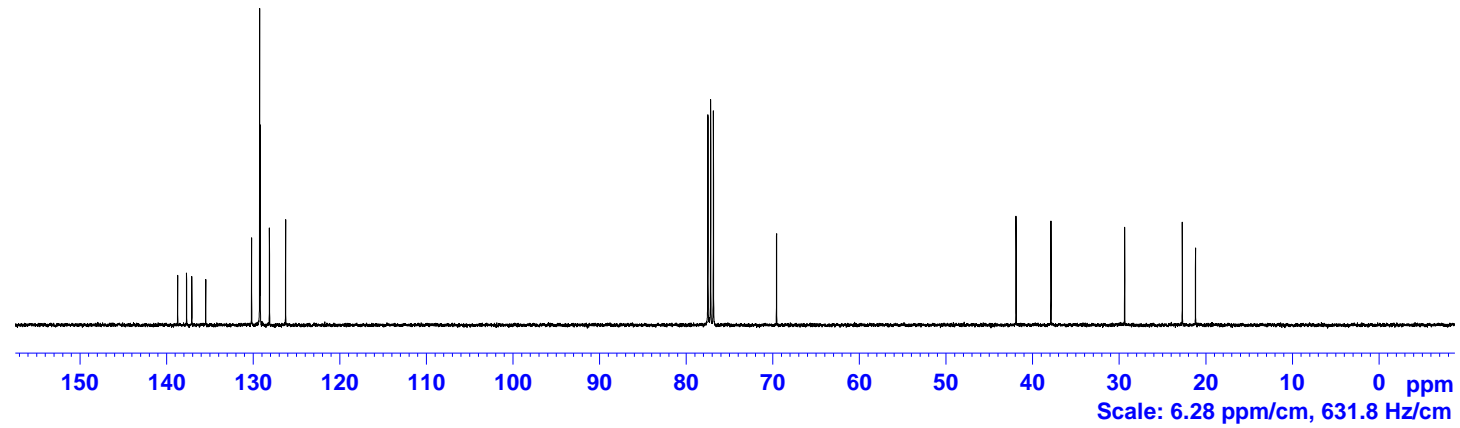


${ }^{1} \mathrm{H}$ NMR spectrum of $\mathbf{3 b}\left(\mathrm{CDCl}_{3}, 400 \mathrm{MHz}\right)$ :

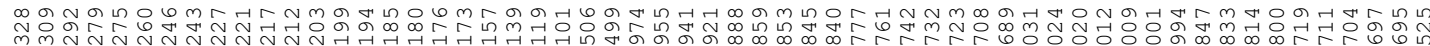
-

$\mathrm{V}$<smiles>O[C@H]1c2ccccc2CC[C@H]1Cc1ccccc1</smiles>

3b

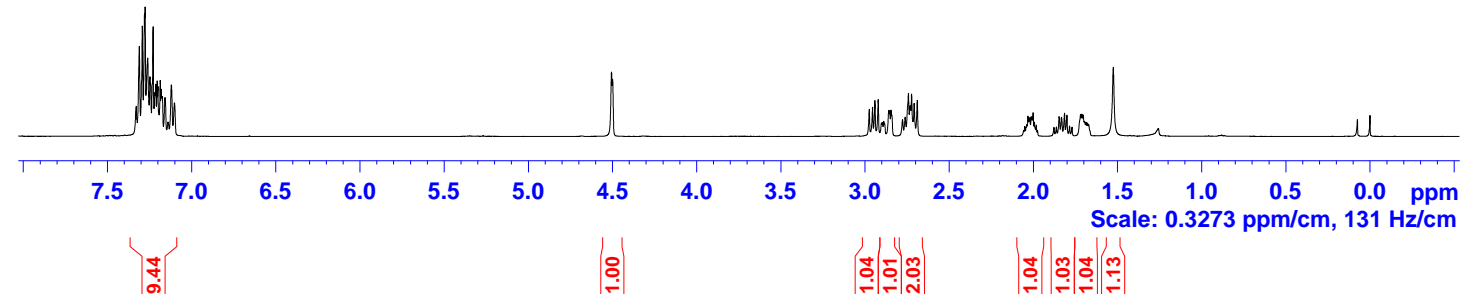

${ }^{13} \mathrm{C}\left\{{ }^{1} \mathrm{H}\right\}$ NMR spectrum of $\mathbf{3 b}\left(\mathrm{CDCl}_{3}, 100 \mathrm{MHz}\right)$ :
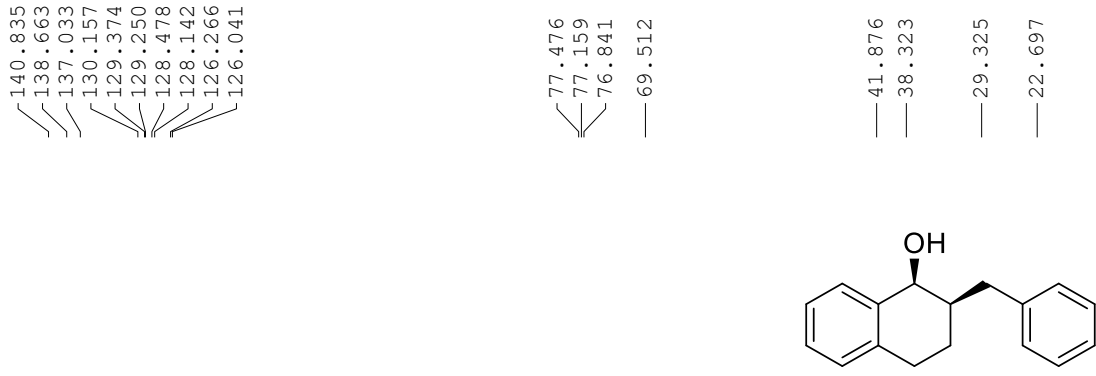

3b

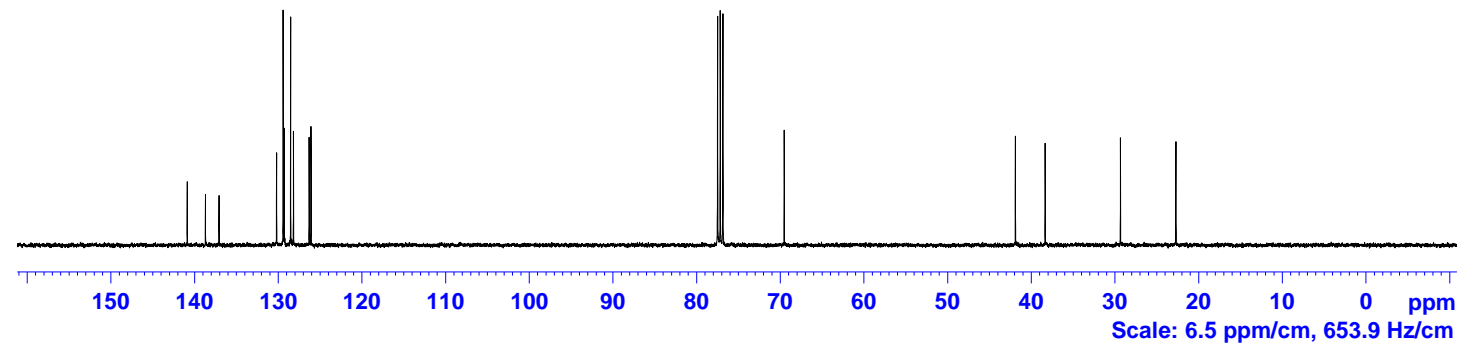


${ }^{1} \mathrm{H}$ NMR spectrum of $\mathbf{3 c}\left(\mathrm{CDCl}_{3}, 400 \mathrm{MHz}\right)$ :
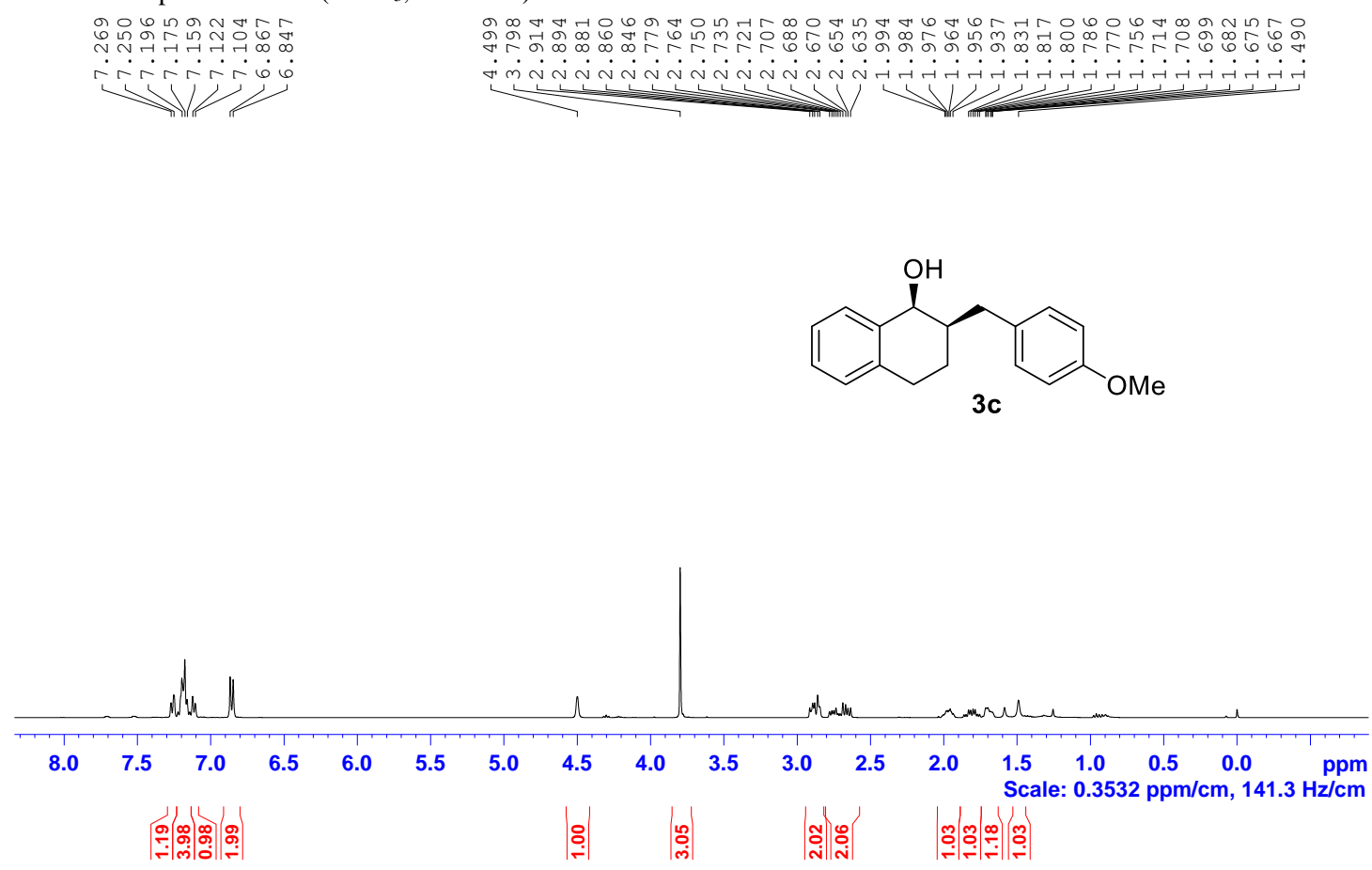

${ }^{13} \mathrm{C}\left\{{ }^{1} \mathrm{H}\right\}$ NMR spectrum of $\mathbf{3 c}\left(\mathrm{CDCl}_{3}, 100 \mathrm{MHz}\right)$ :

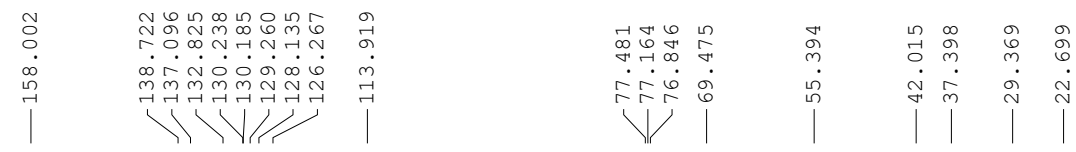

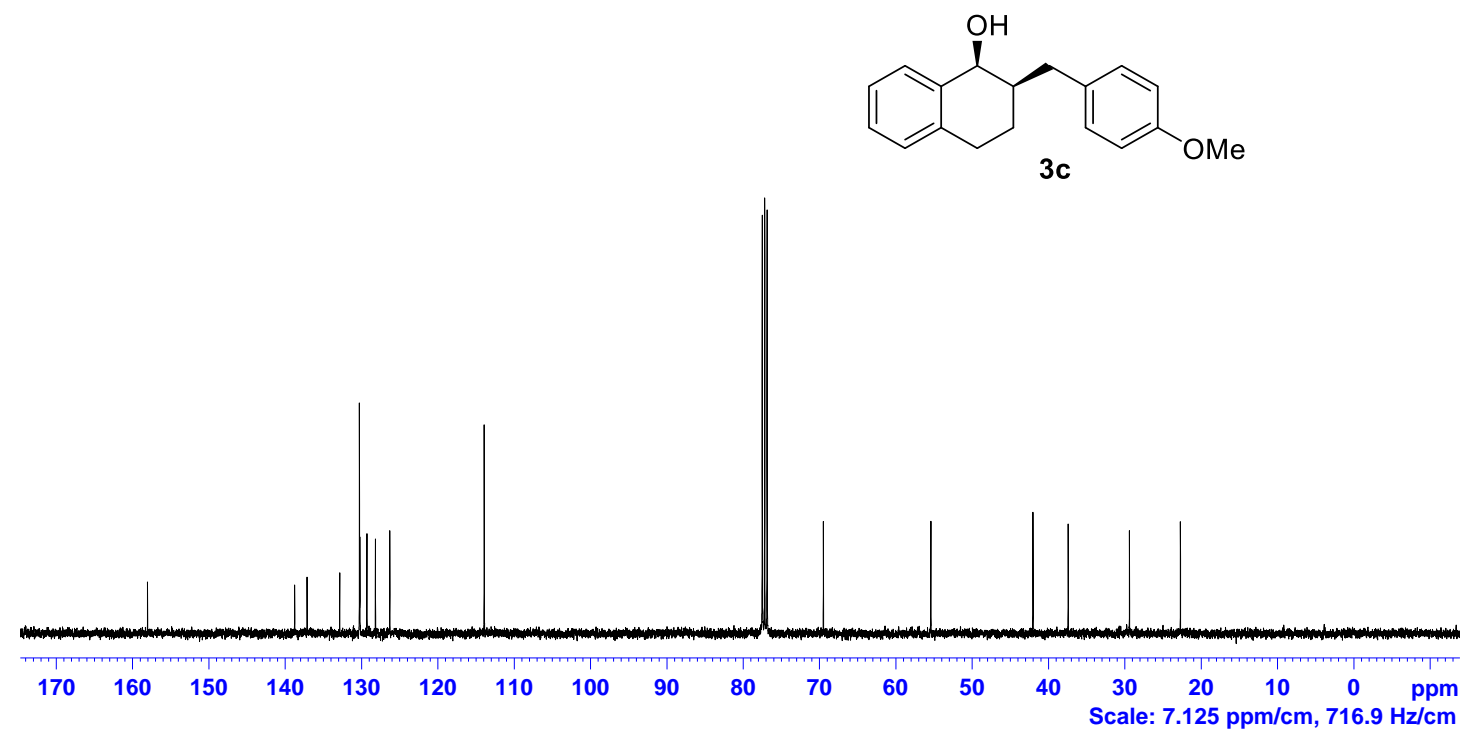


${ }^{1} \mathrm{H}$ NMR spectrum of $\mathbf{3 d}\left(\mathrm{CDCl}_{3}, 400 \mathrm{MHz}\right)$ :

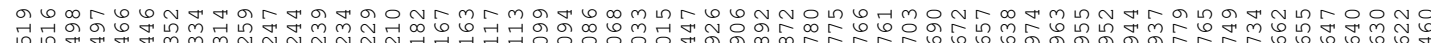
-

(n)<smiles>O[C@H]1c2ccccc2CC[C@H]1Cc1ccc(-c2ccccc2)cc1</smiles>

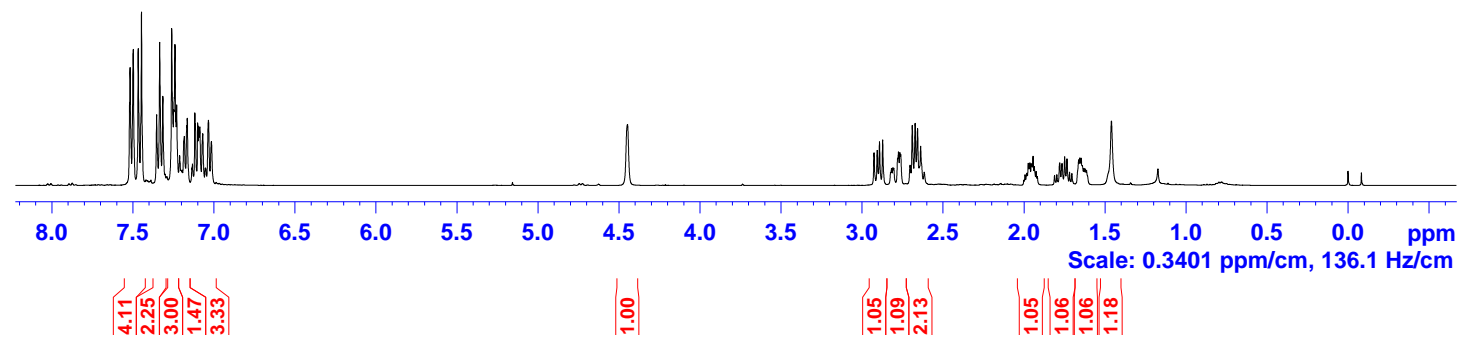

${ }^{13} \mathrm{C}\left\{{ }^{1} \mathrm{H}\right\}$ NMR spectrum of $\mathbf{3 d}\left(\mathrm{CDCl}_{3}, 100 \mathrm{MHz}\right)$ :

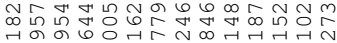

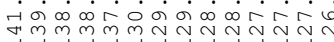

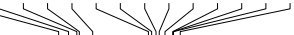

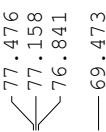

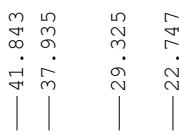<smiles>O[C@H]1c2ccccc2CC[C@H]1Cc1ccc(-c2ccccc2)cc1</smiles>

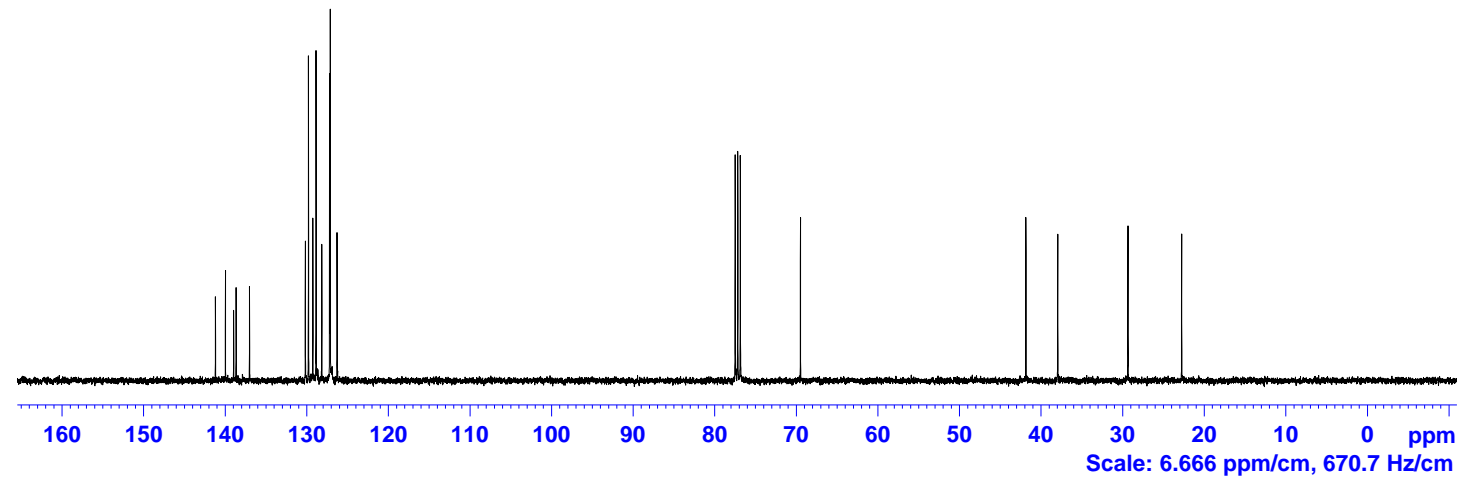


${ }^{1} \mathrm{H}$ NMR spectrum of $\mathbf{3 e}\left(\mathrm{CDCl}_{3}, 400 \mathrm{MHz}\right)$ :
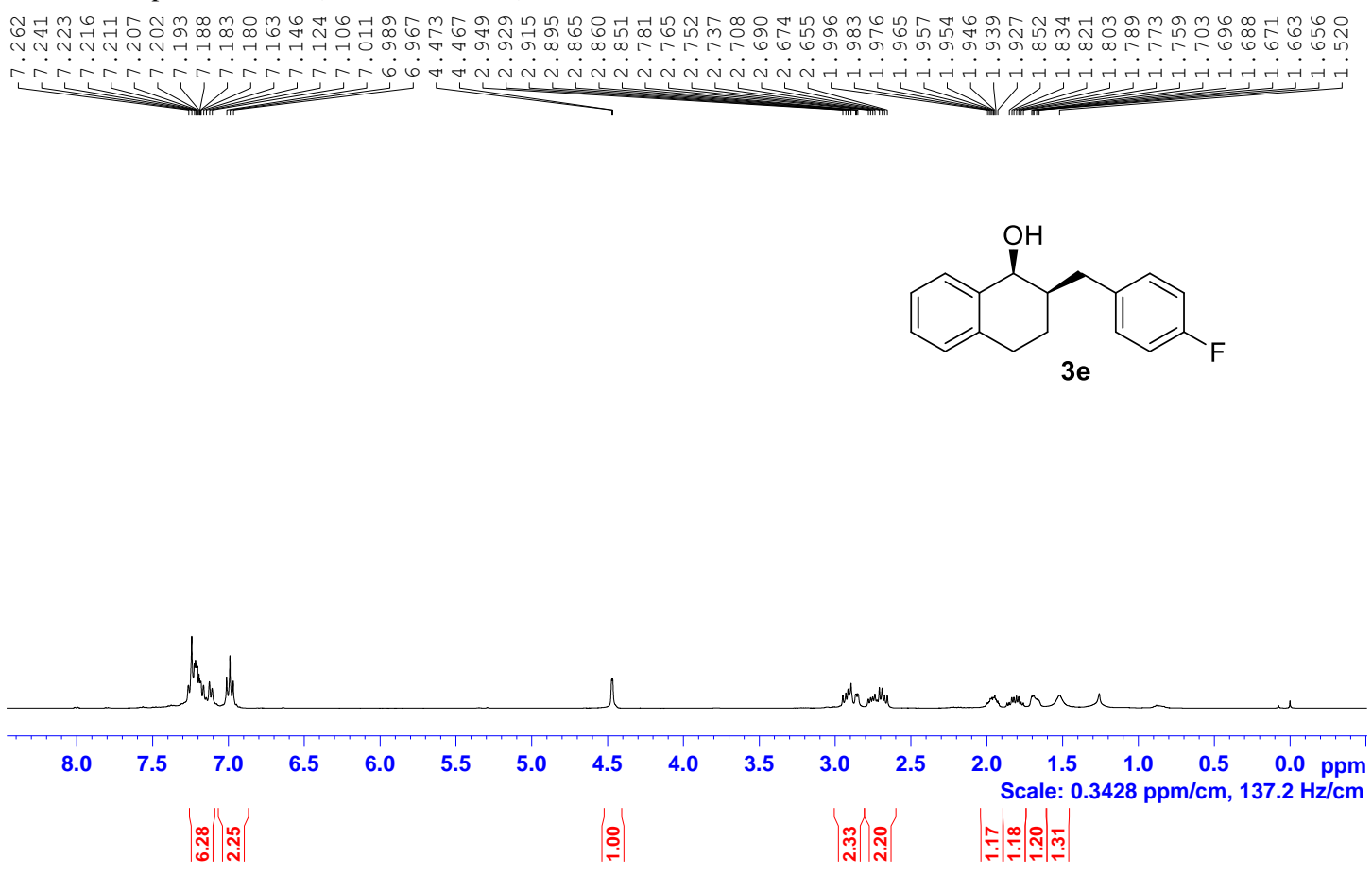

${ }^{13} \mathrm{C}\left\{{ }^{1} \mathrm{H}\right\}$ NMR spectrum of $\mathbf{3 e}\left(\mathrm{CDCl}_{3}, 100 \mathrm{MHz}\right)$ :
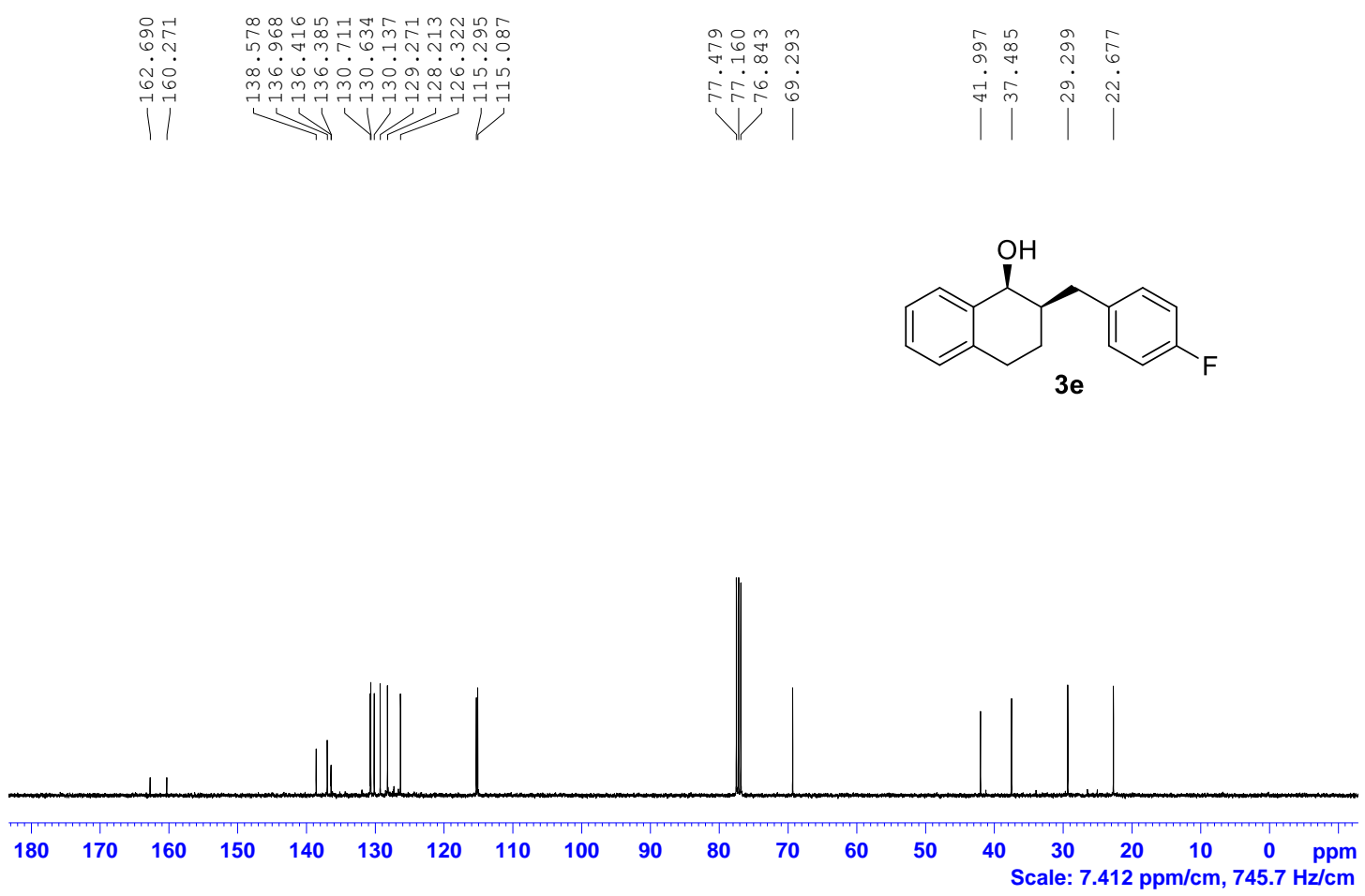

S66 
${ }^{1} \mathrm{H}$ NMR spectrum of $\mathbf{3 f}\left(\mathrm{CDCl}_{3}, 400 \mathrm{MHz}\right)$ :

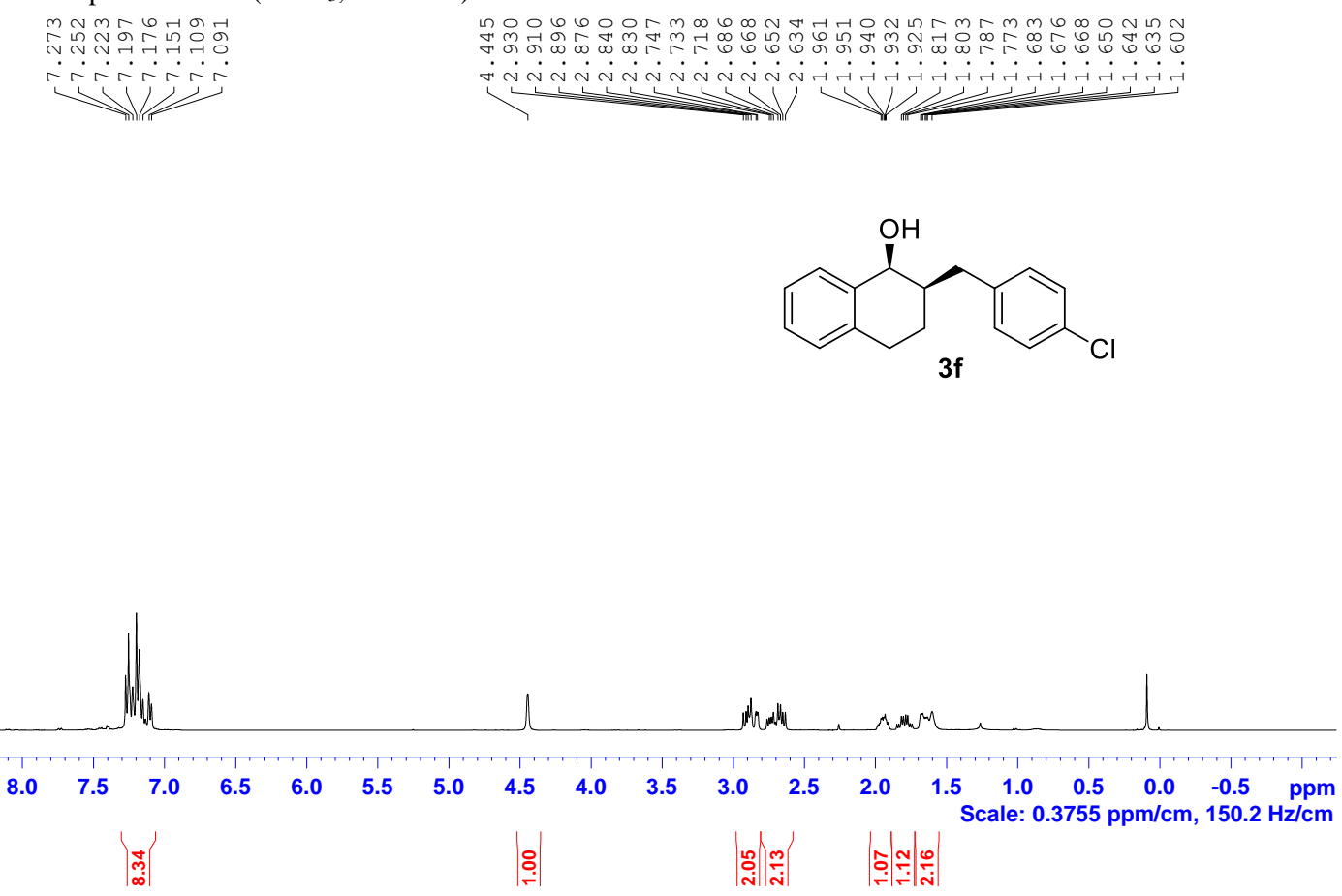

${ }^{13} \mathrm{C}\left\{{ }^{1} \mathrm{H}\right\}$ NMR spectrum of $\mathbf{3 f}\left(\mathrm{CDCl}_{3}, 100 \mathrm{MHz}\right)$ :

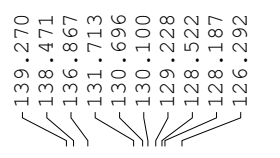

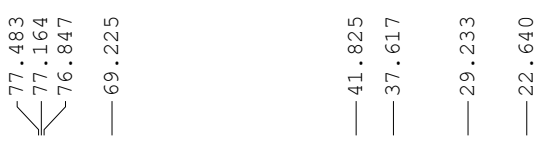
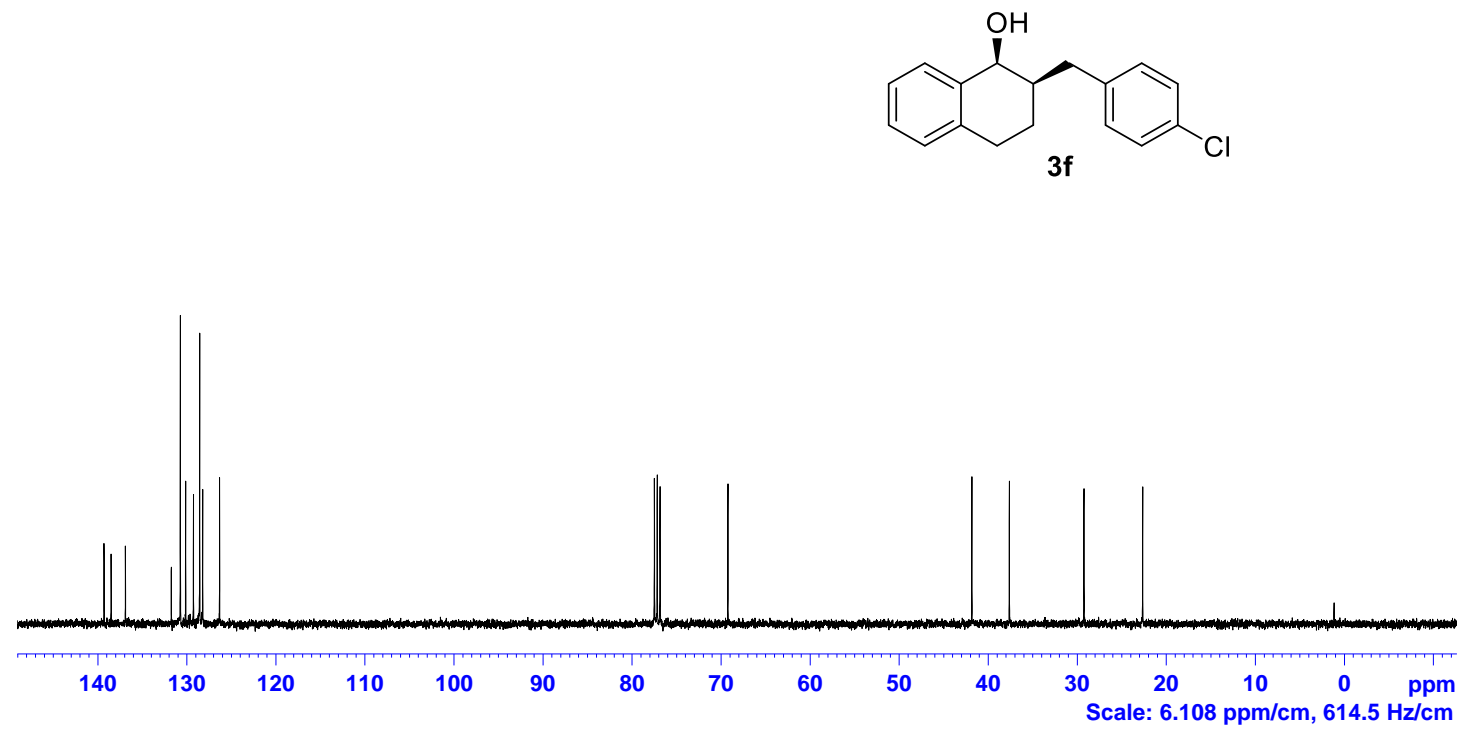
${ }^{1} \mathrm{H}$ NMR spectrum of $\mathbf{3 g}\left(\mathrm{CDCl}_{3}, 400 \mathrm{MHz}\right)$ :

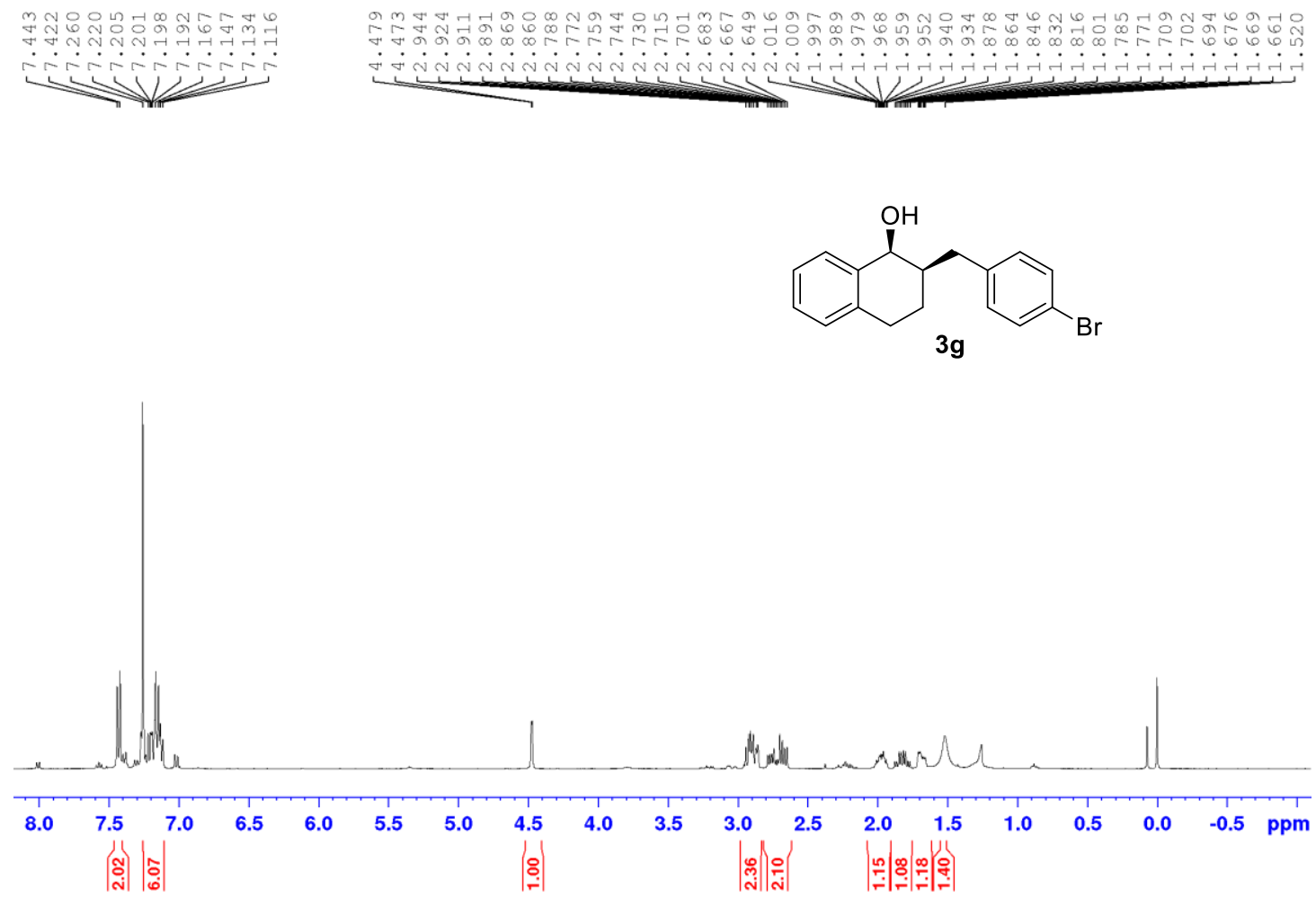

${ }^{13} \mathrm{C}\left\{{ }^{1} \mathrm{H}\right\}$ NMR spectrum of $\mathbf{3 g}\left(\mathrm{CDCl}_{3}, 100 \mathrm{MHz}\right)$ :
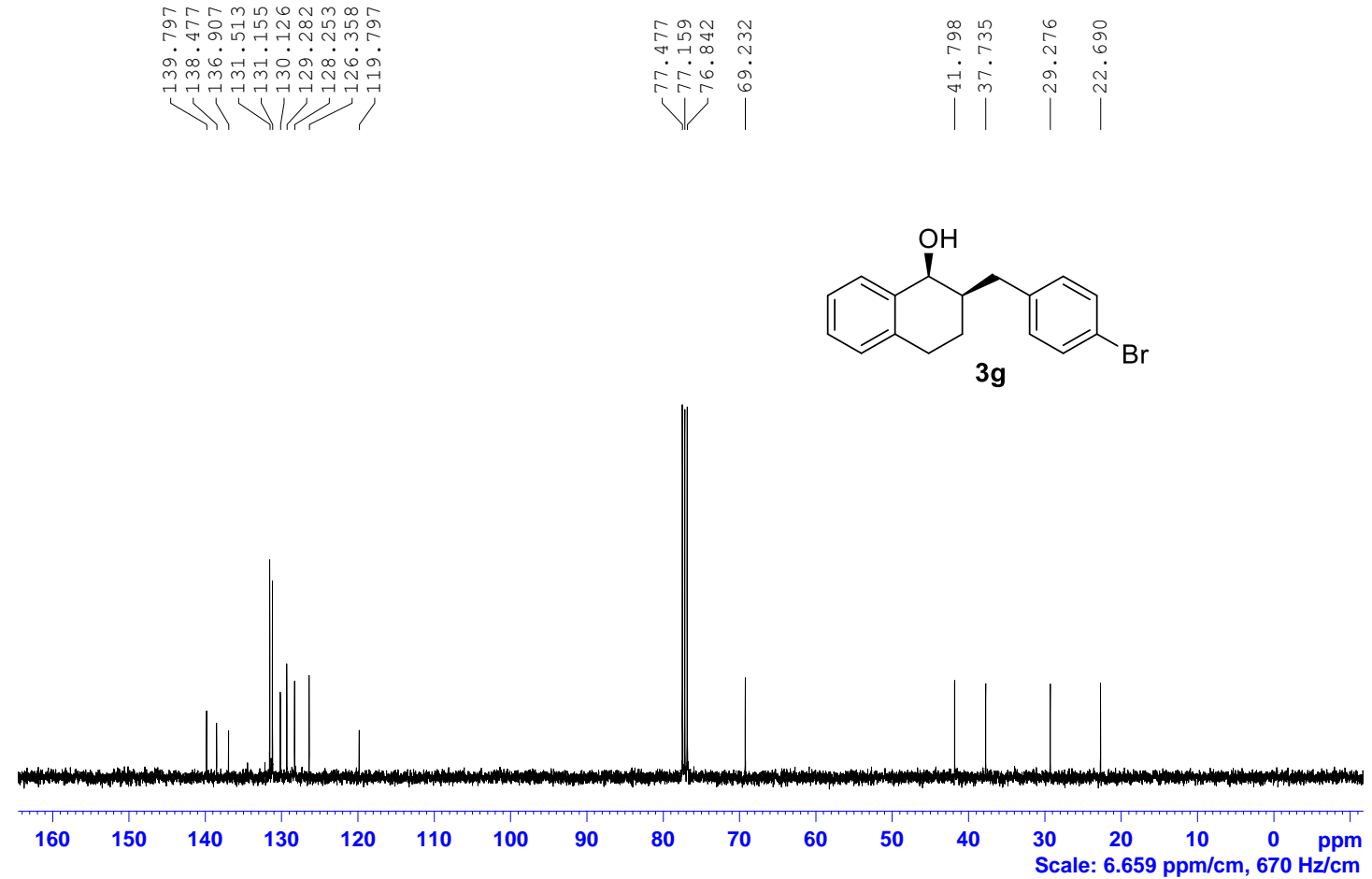
${ }^{1} \mathrm{H}$ NMR spectrum of $\mathbf{3 h}\left(\mathrm{CDCl}_{3}, 400 \mathrm{MHz}\right)$ :
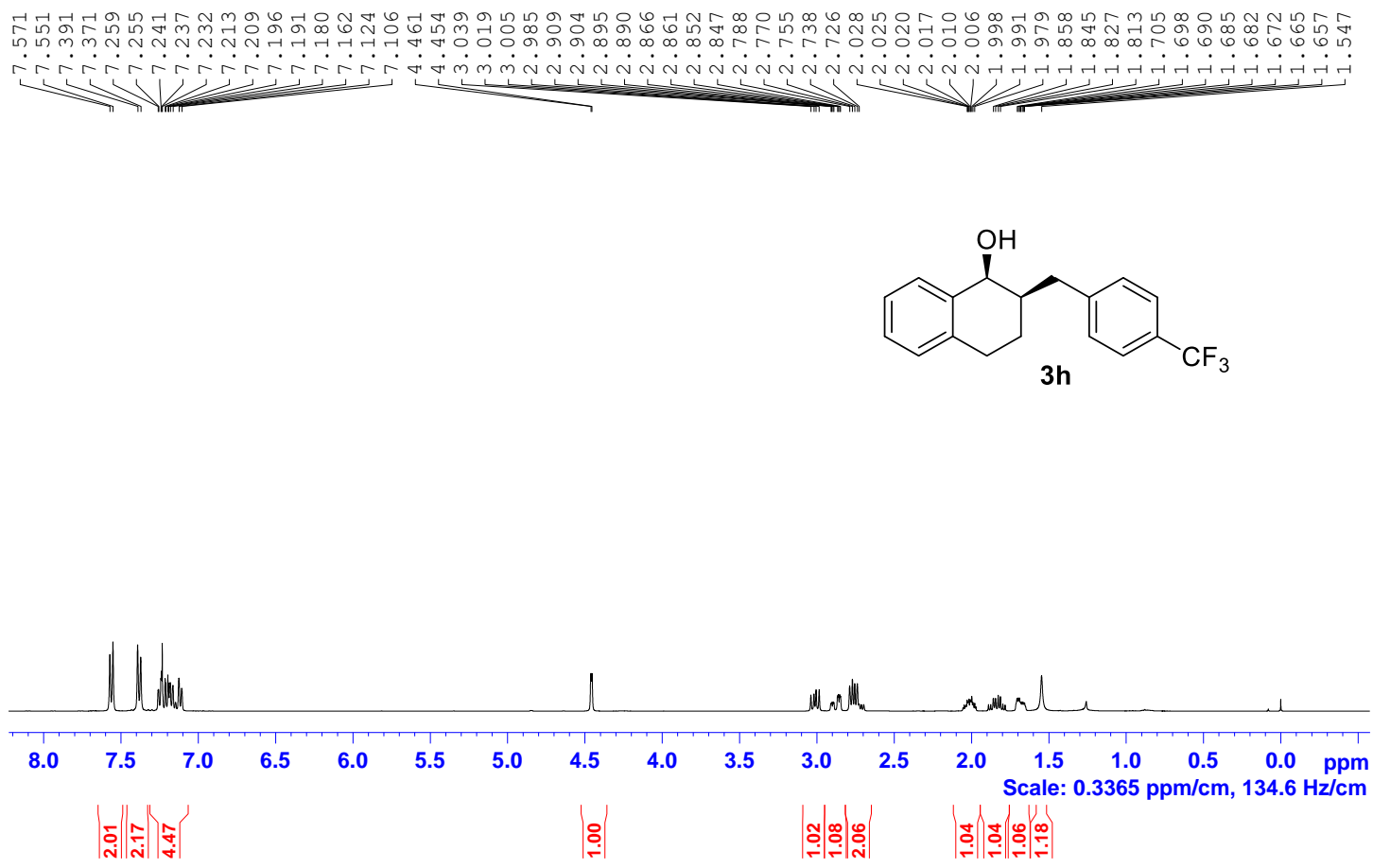

${ }^{13} \mathrm{C}\left\{{ }^{1} \mathrm{H}\right\}$ NMR spectrum of $\mathbf{3 h}\left(\mathrm{CDCl}_{3}, 100 \mathrm{MHz}\right)$ :
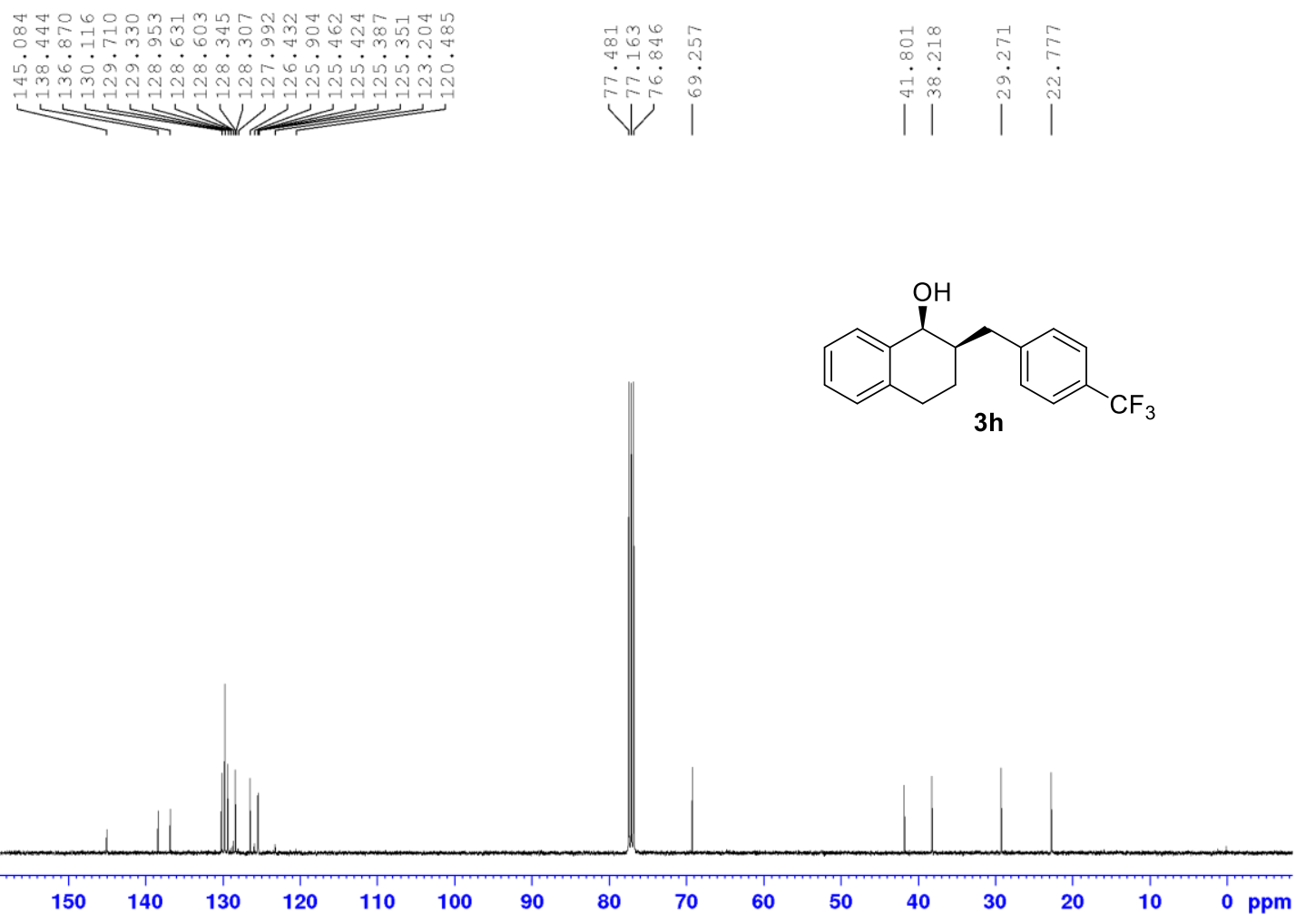

S69 
${ }^{1} \mathrm{H}$ NMR spectrum of $\mathbf{3 i}\left(\mathrm{CDCl}_{3}, 400 \mathrm{MHz}\right)$

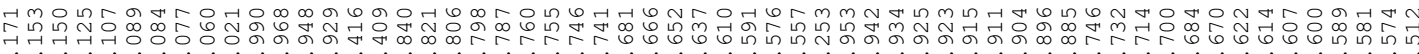

-

W<smiles>Cc1cccc(C[C@@H]2CCc3ccccc3[C@@H]2O)c1</smiles>

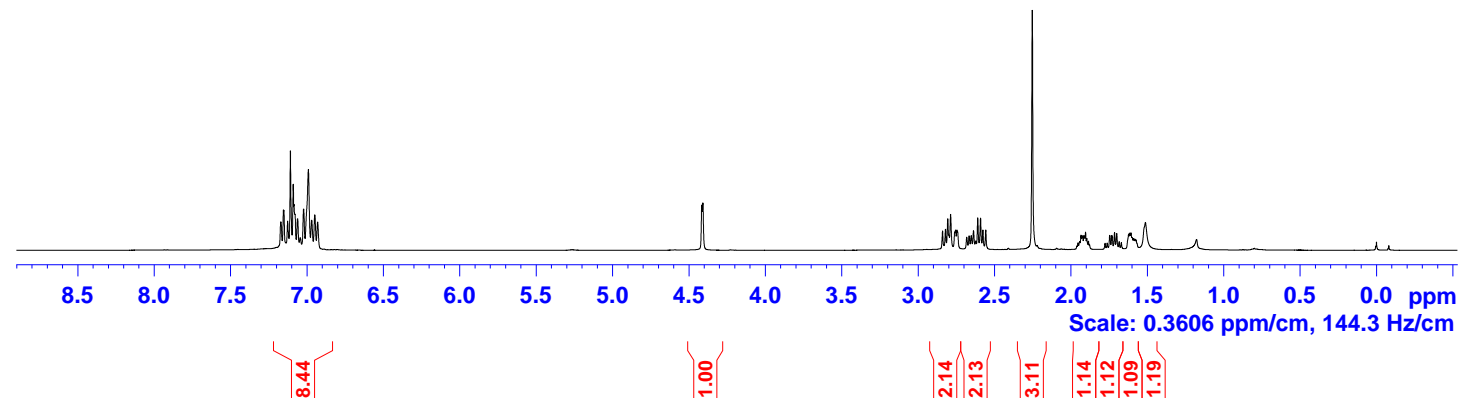

${ }^{13} \mathrm{C}\left\{{ }^{1} \mathrm{H}\right\}$ NMR spectrum of $\mathbf{3 i}\left(\mathrm{CDCl}_{3}, 100 \mathrm{MHz}\right)$ :
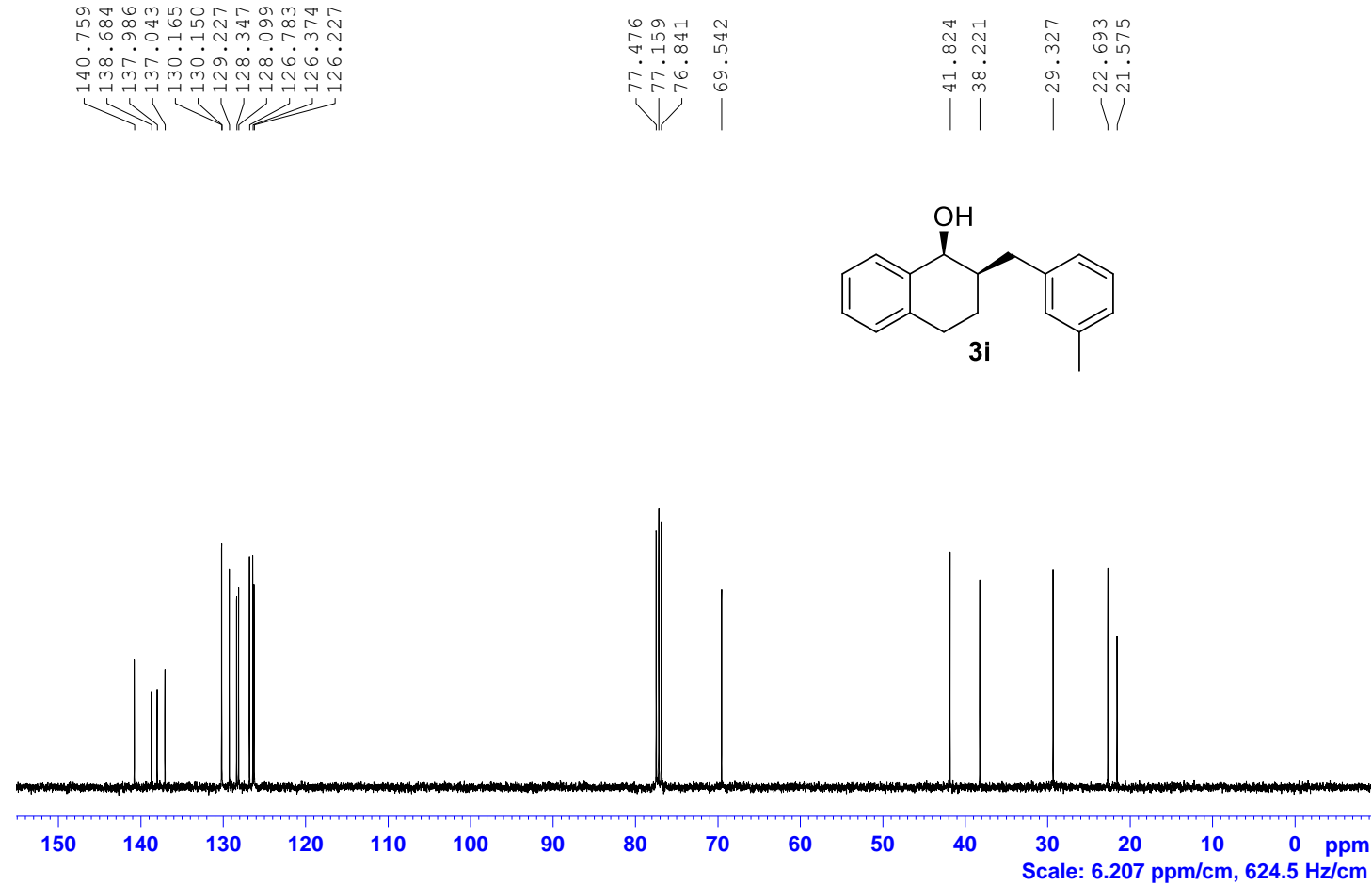
${ }^{1} \mathrm{H}$ NMR spectrum of $\mathbf{3 j}\left(\mathrm{CDCl}_{3}, 400 \mathrm{MHz}\right)$ :
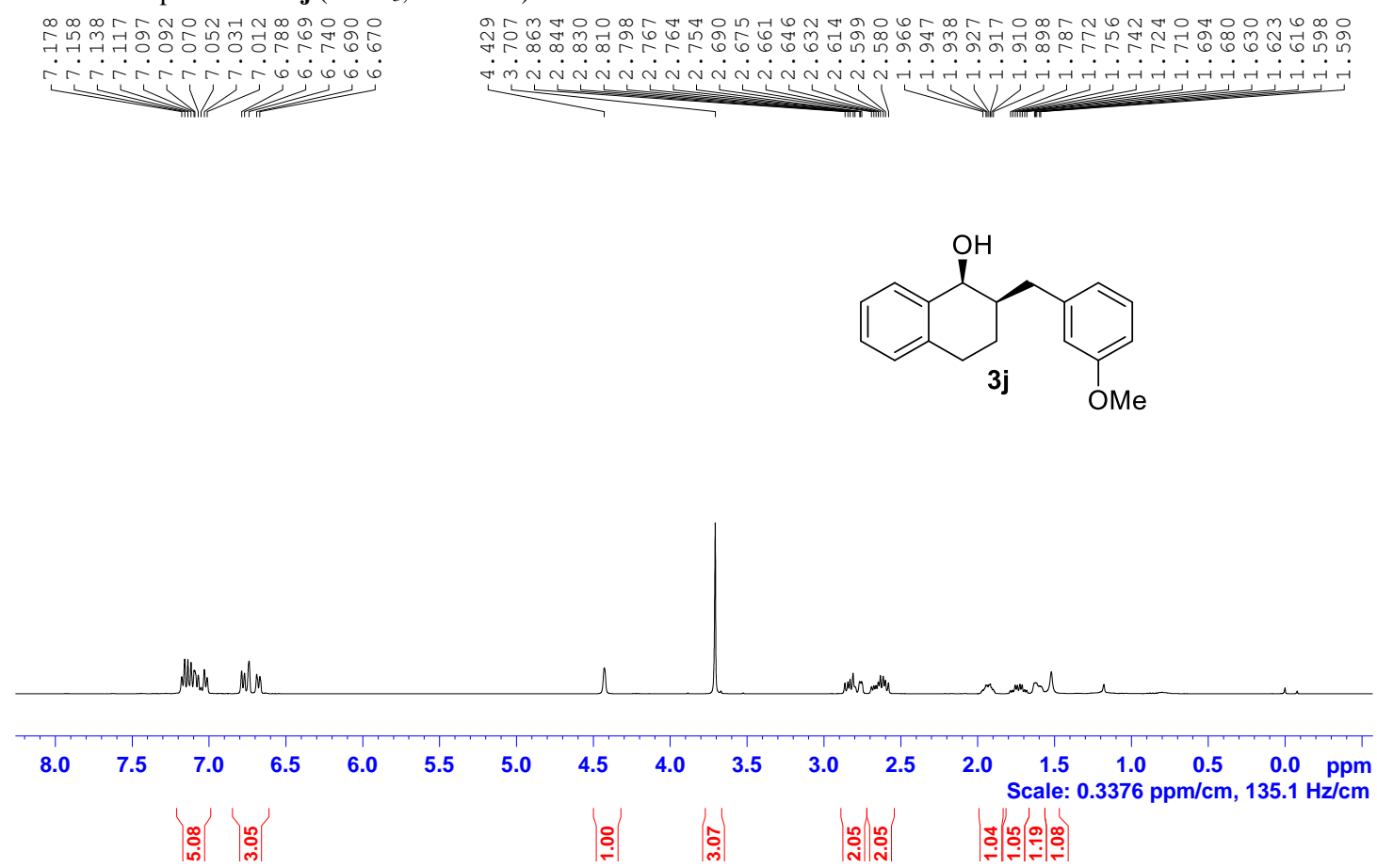

${ }^{13} \mathrm{C}\left\{{ }^{1} \mathrm{H}\right\}$ NMR spectrum of $\mathbf{3 j}\left(\mathrm{CDCl}_{3}, 100 \mathrm{MHz}\right)$ :

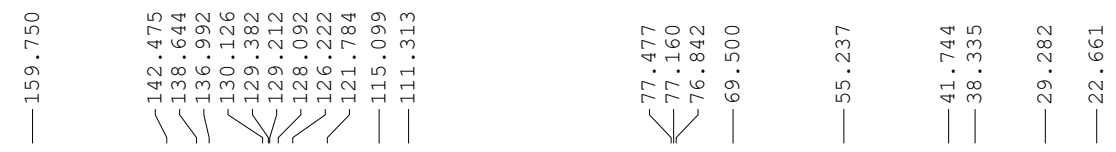<smiles>COc1cccc(C[C@@H]2CCc3ccccc3[C@@H]2O)c1</smiles>

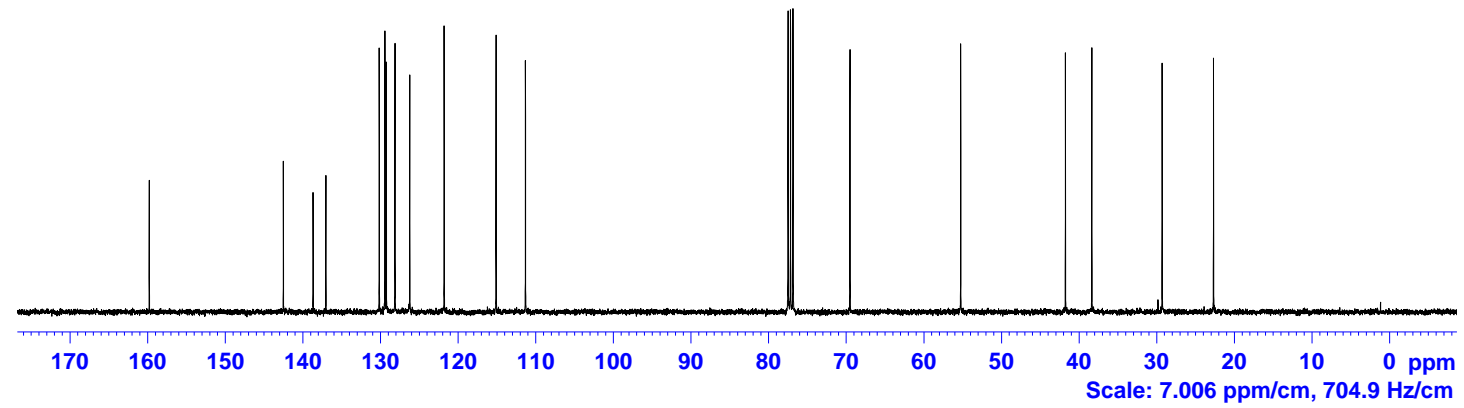


${ }^{1} \mathrm{H}$ NMR spectrum of $\mathbf{3 k}\left(\mathrm{CDCl}_{3}, 400 \mathrm{MHz}\right)$ :

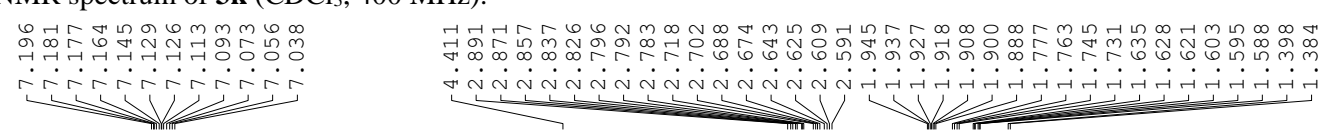<smiles>O[C@H]1c2ccccc2CC[C@@H]1Cc1cccc(Cl)c1</smiles>

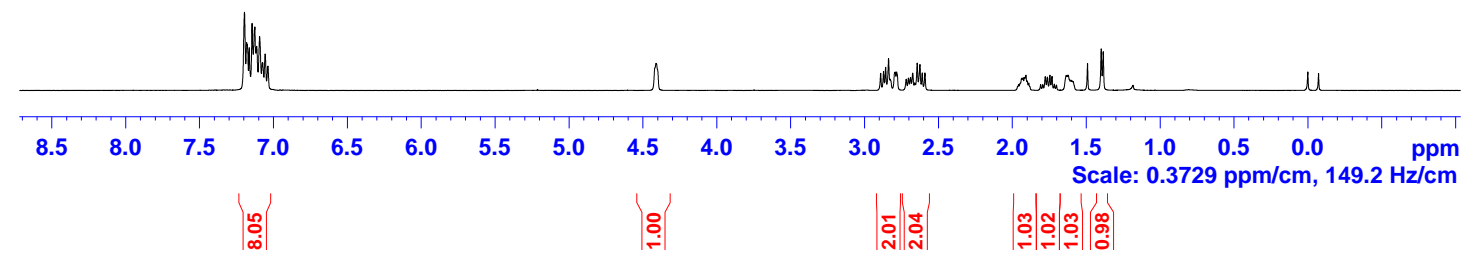

${ }^{13} \mathrm{C}\left\{{ }^{1} \mathrm{H}\right\}$ NMR spectrum of $\mathbf{3 k}\left(\mathrm{CDCl}_{3}, 100 \mathrm{MHz}\right)$ :
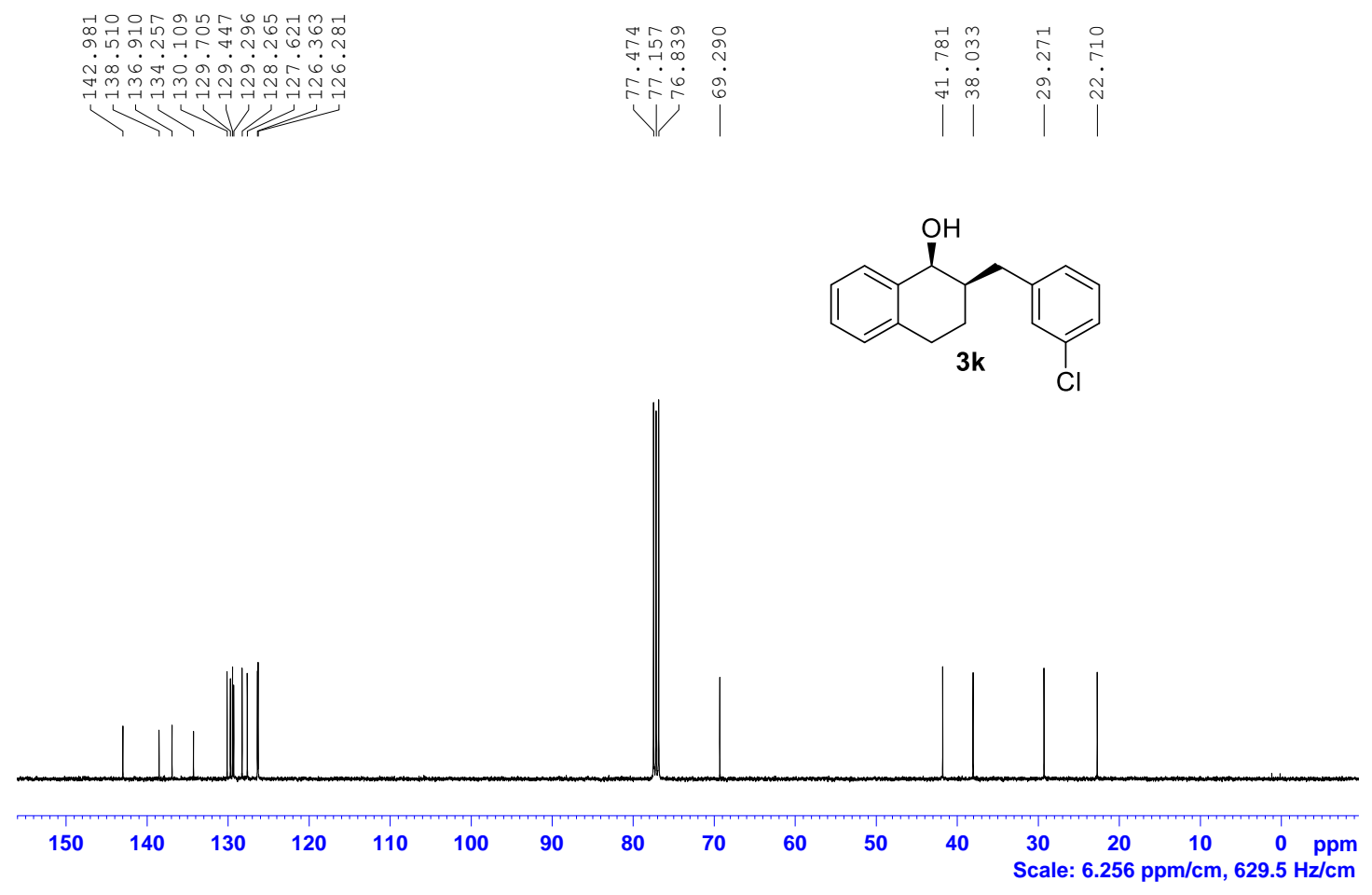
${ }^{1} \mathrm{H}$ NMR spectrum of $\mathbf{3 l}\left(\mathrm{CDCl}_{3}, 400 \mathrm{MHz}\right)$ :

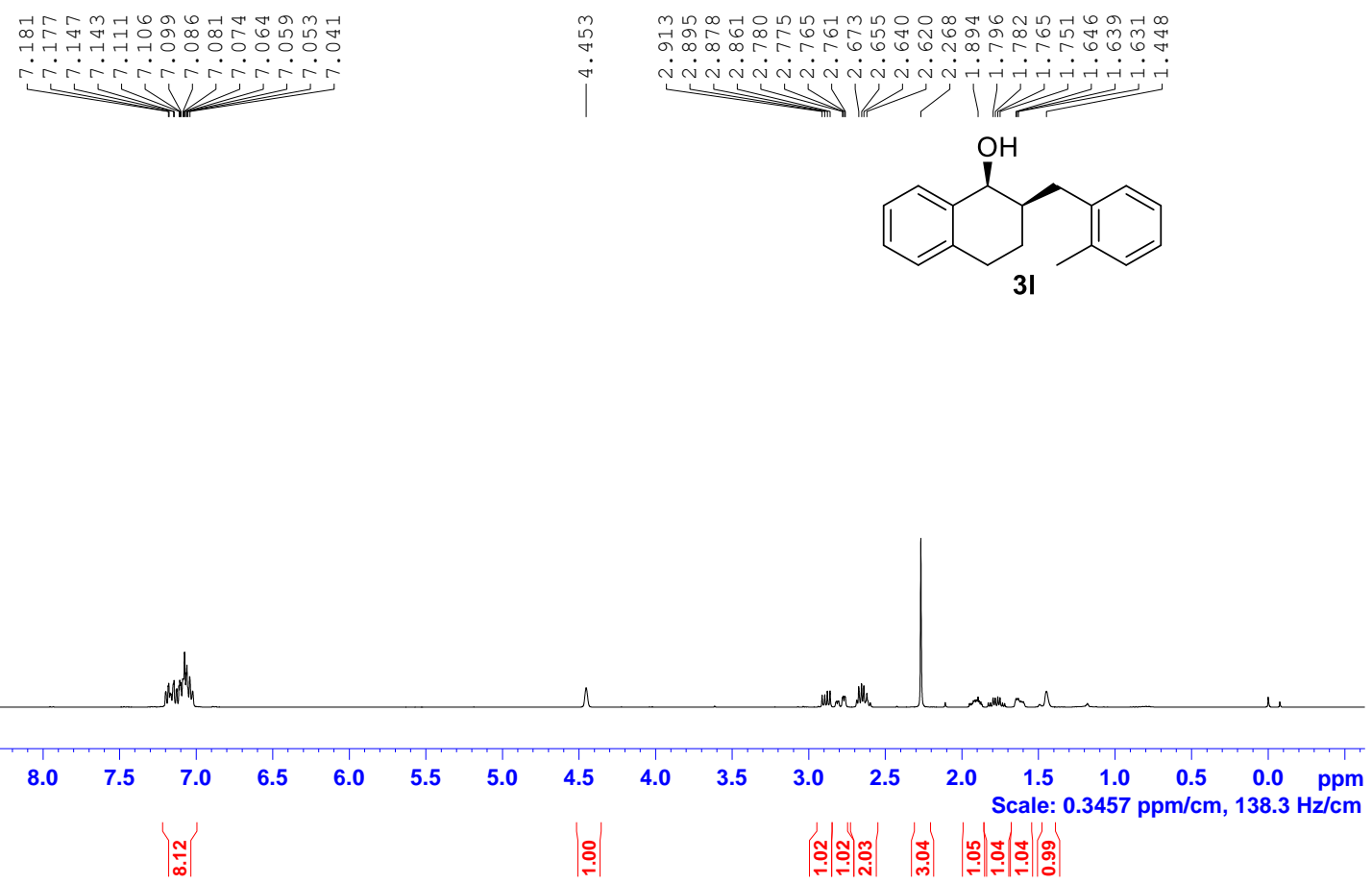

${ }^{13} \mathrm{C}\left\{{ }^{1} \mathrm{H}\right\}$ NMR spectrum of $\mathbf{3 l}\left(\mathrm{CDCl}_{3}, 100 \mathrm{MHz}\right)$ :
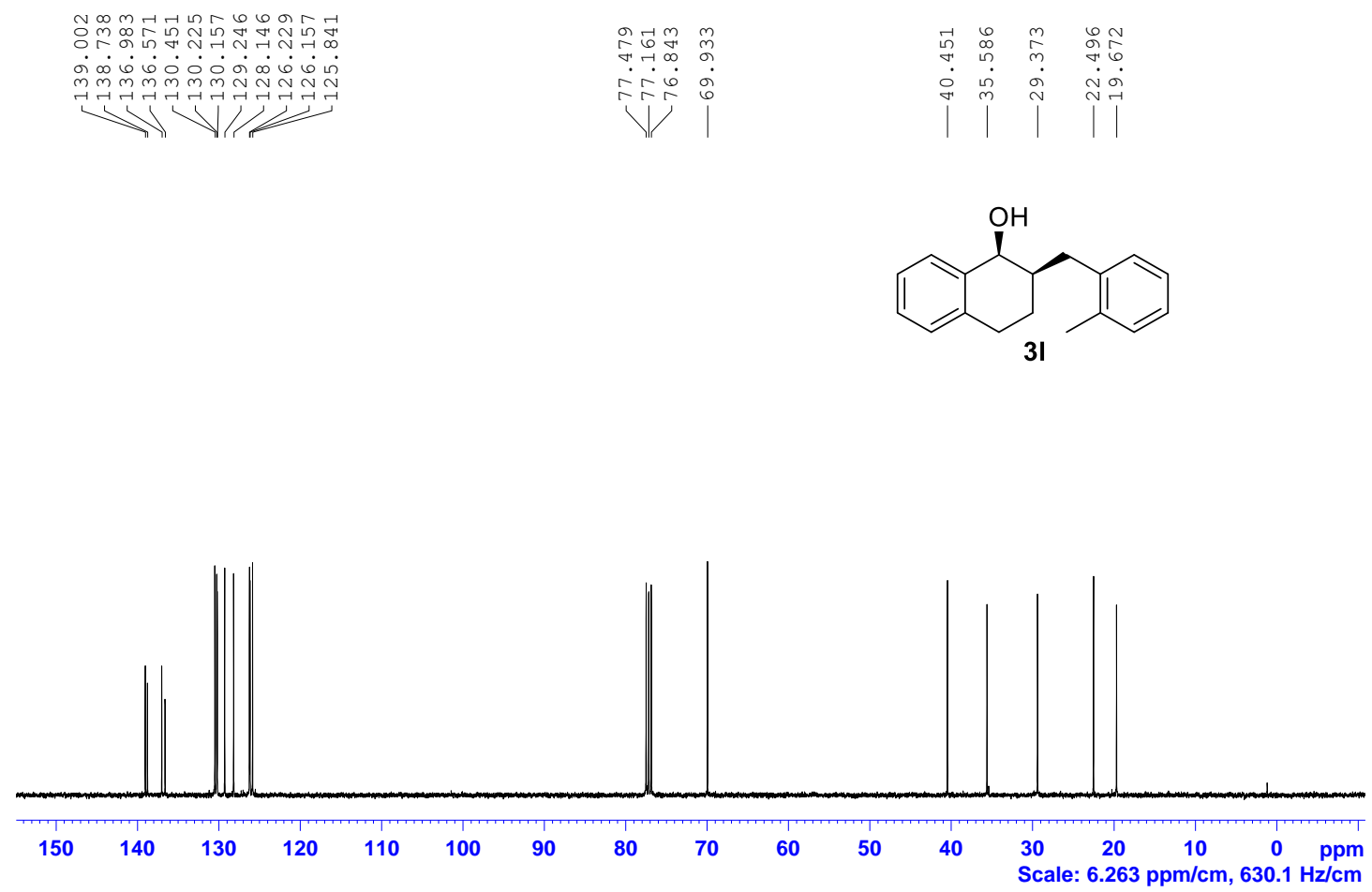
${ }^{1} \mathrm{H}$ NMR spectrum of $\mathbf{3 m}\left(\mathrm{CDCl}_{3}, 400 \mathrm{MHz}\right)$ :

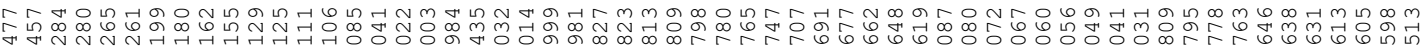

-

W<smiles>O[C@H]1c2ccccc2CC[C@H]1Cc1ccccc1Br</smiles>

$3 \mathrm{~m}$

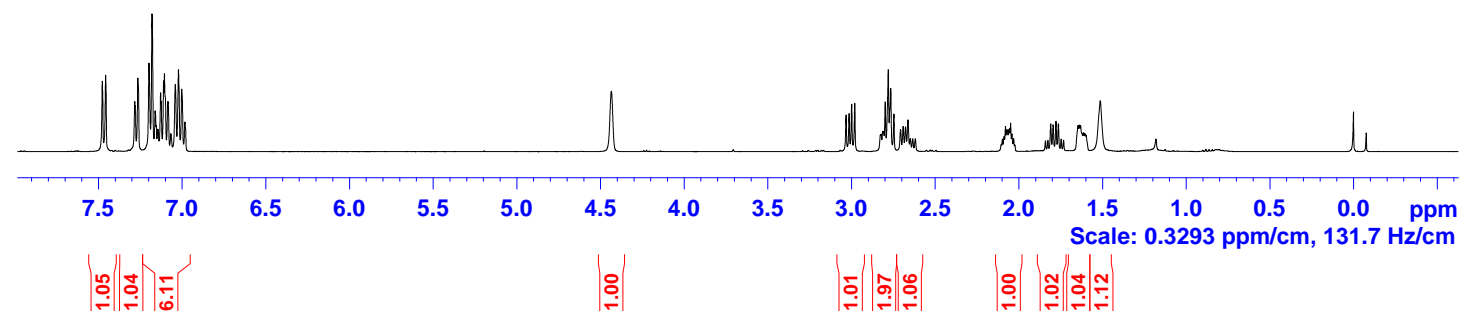

${ }^{13} \mathrm{C}\left\{{ }^{1} \mathrm{H}\right\}$ NMR spectrum of $\mathbf{3 m}\left(\mathrm{CDCl}_{3}, 100 \mathrm{MHz}\right)$ :
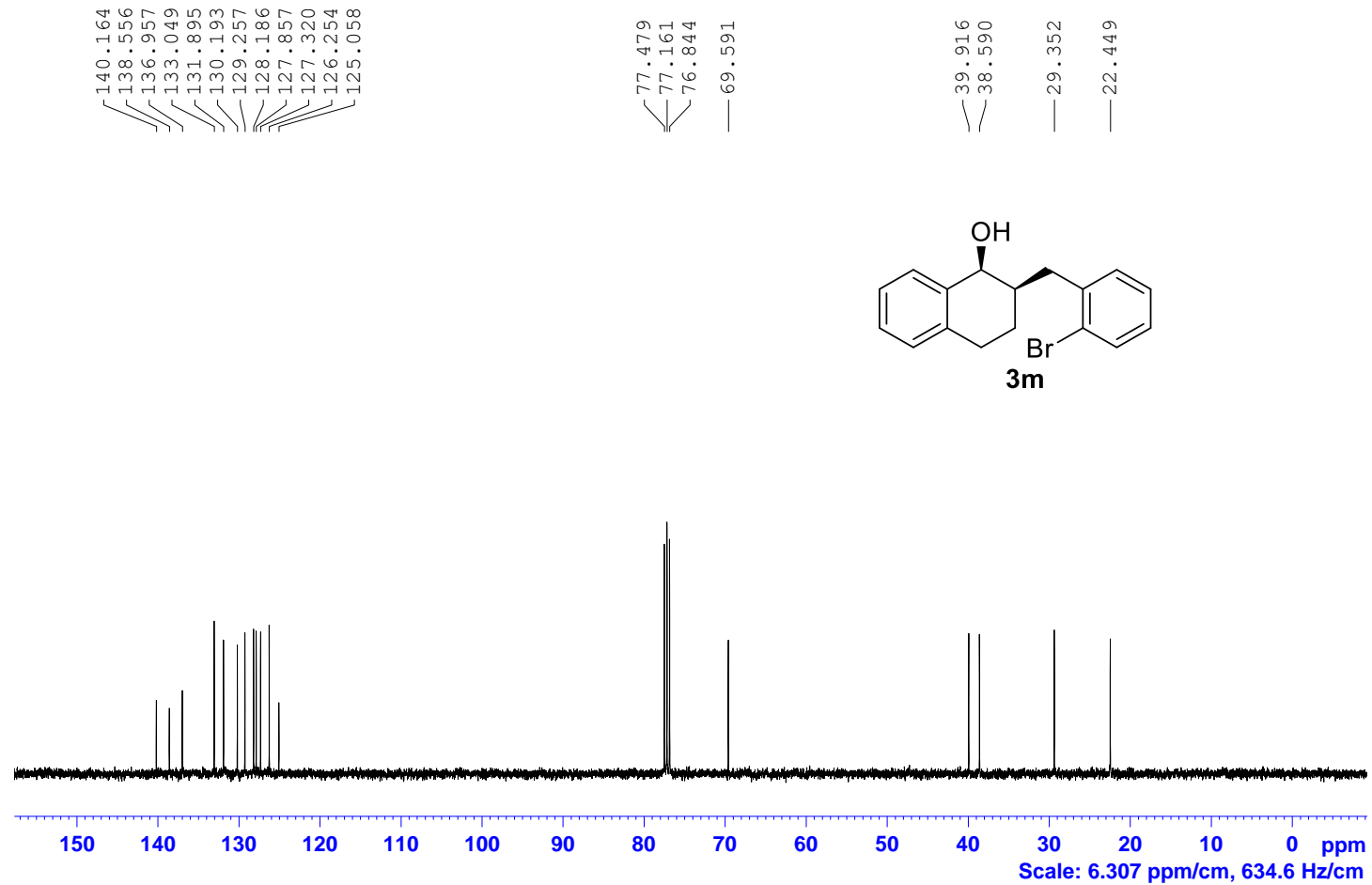
${ }^{1} \mathrm{H}$ NMR spectrum of $\mathbf{3 n}\left(\mathrm{CDCl}_{3}, 400 \mathrm{MHz}\right)$ :

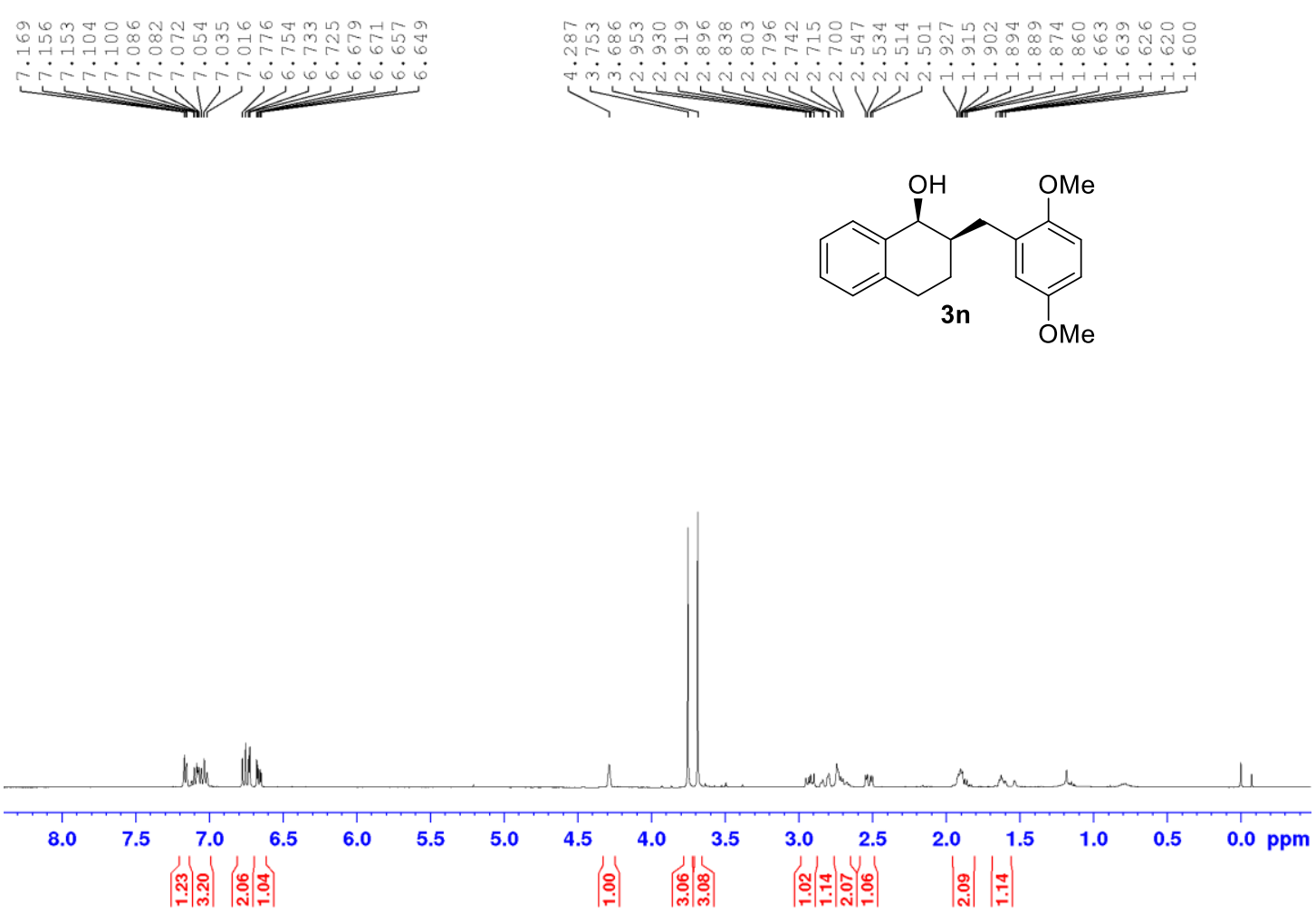

${ }^{13} \mathrm{C}\left\{{ }^{1} \mathrm{H}\right\}$ NMR spectrum of $\mathbf{3 n}\left(\mathrm{CDCl}_{3}, 100 \mathrm{MHz}\right)$ :
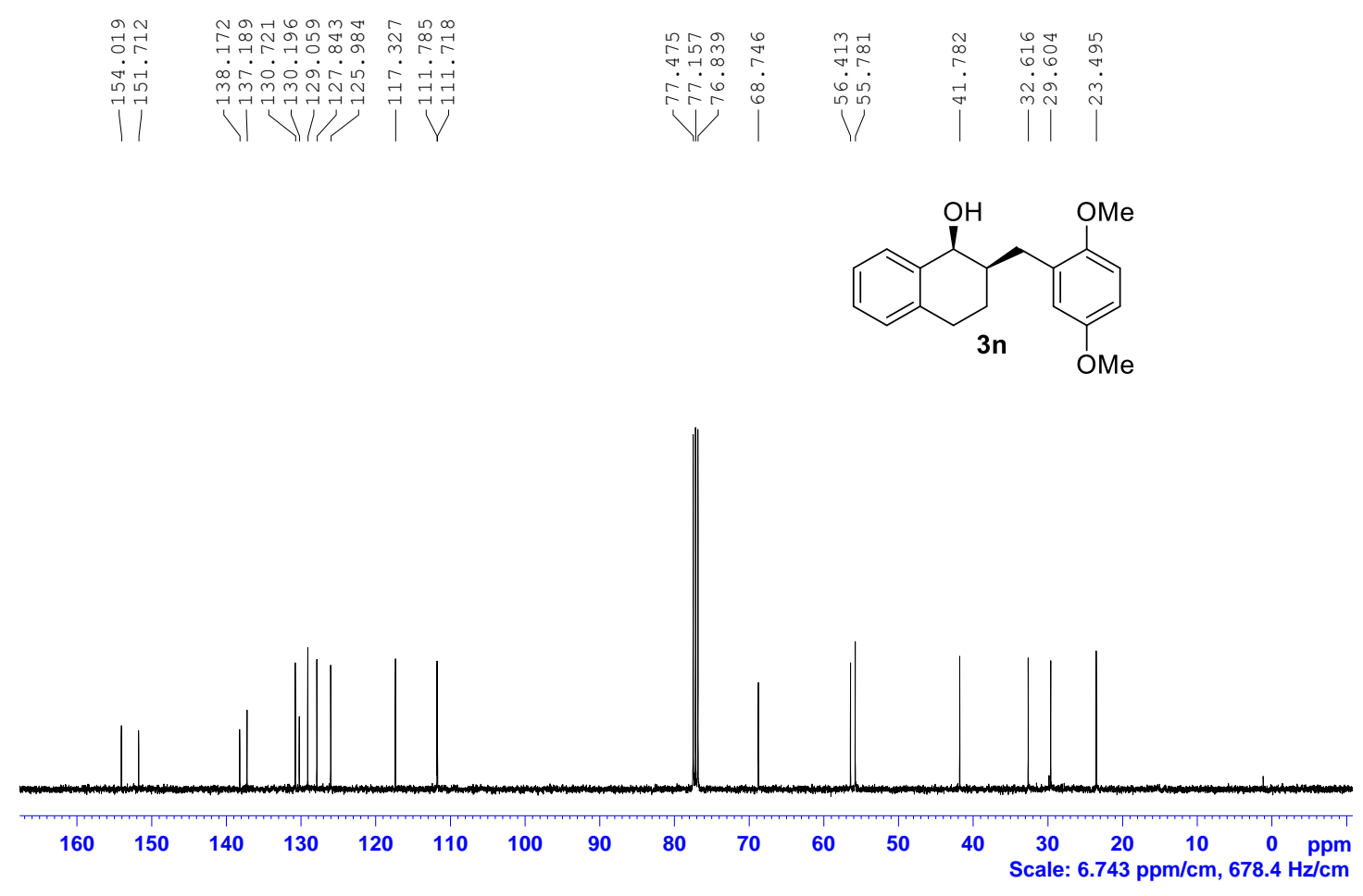
${ }^{1} \mathrm{H}$ NMR spectrum of $\mathbf{3 o}\left(\mathrm{CDCl}_{3}, 400 \mathrm{MHz}\right)$ :

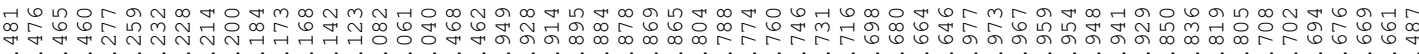

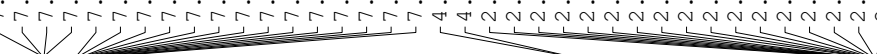<smiles>O[C@@H]1c2ccccc2CC[C@@H]1Cc1ccc(F)c(Br)c1</smiles>

30

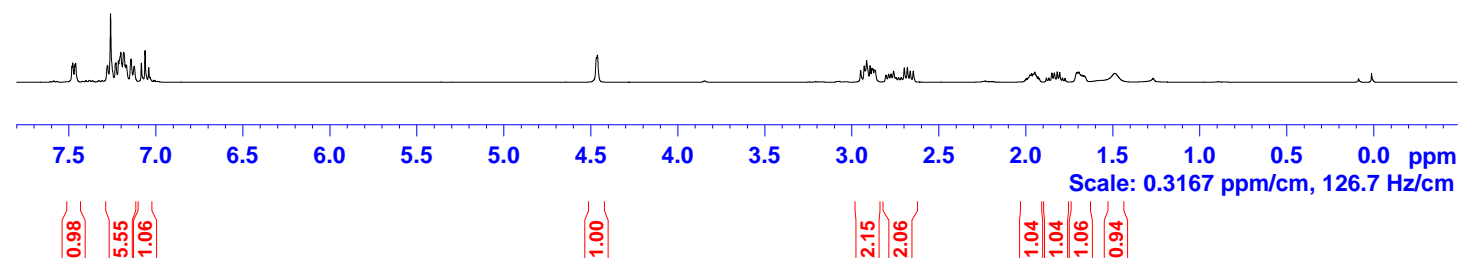

${ }^{13} \mathrm{C}\left\{{ }^{1} \mathrm{H}\right\}$ NMR spectrum of $\mathbf{3 o}\left(\mathrm{CDCl}_{3}, 100 \mathrm{MHz}\right)$ :
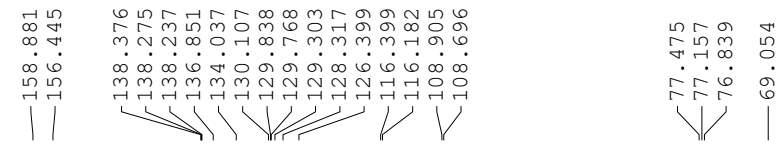

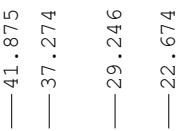<smiles>O[C@@H]1c2ccccc2CC[C@@H]1Cc1ccc(F)c(Br)c1</smiles>

30

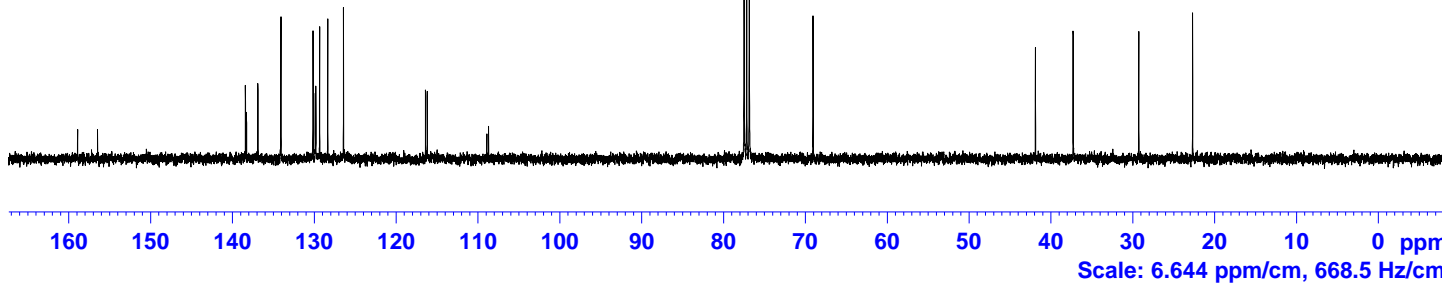


${ }^{1} \mathrm{H}$ NMR spectrum of $\mathbf{3 p}\left(\mathrm{CDCl}_{3}, 400 \mathrm{MHz}\right)$ :

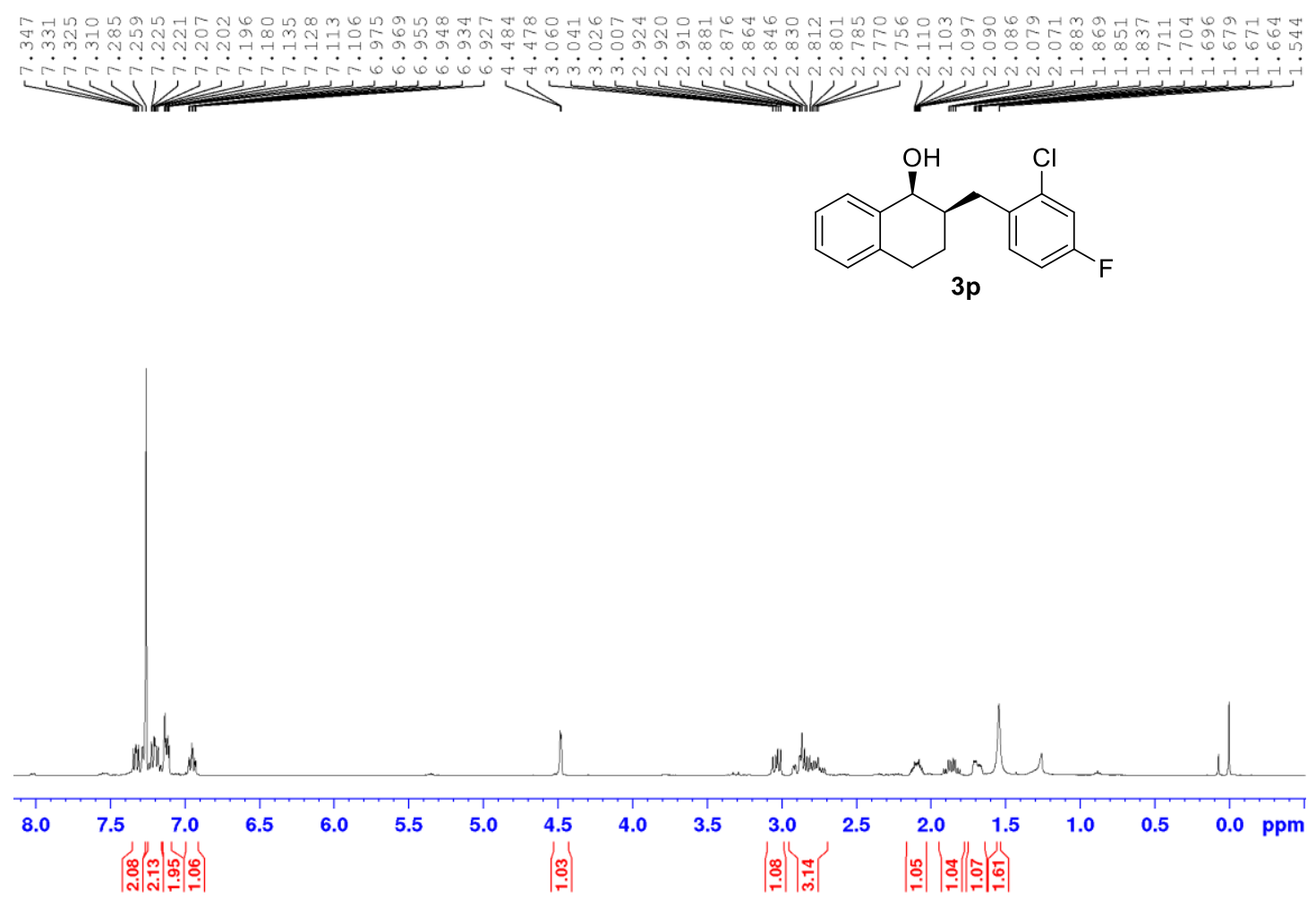

${ }^{13} \mathrm{C}\left\{{ }^{1} \mathrm{H}\right\}$ NMR spectrum of $\mathbf{3 p}\left(\mathrm{CDCl}_{3}, 100 \mathrm{MHz}\right)$ :
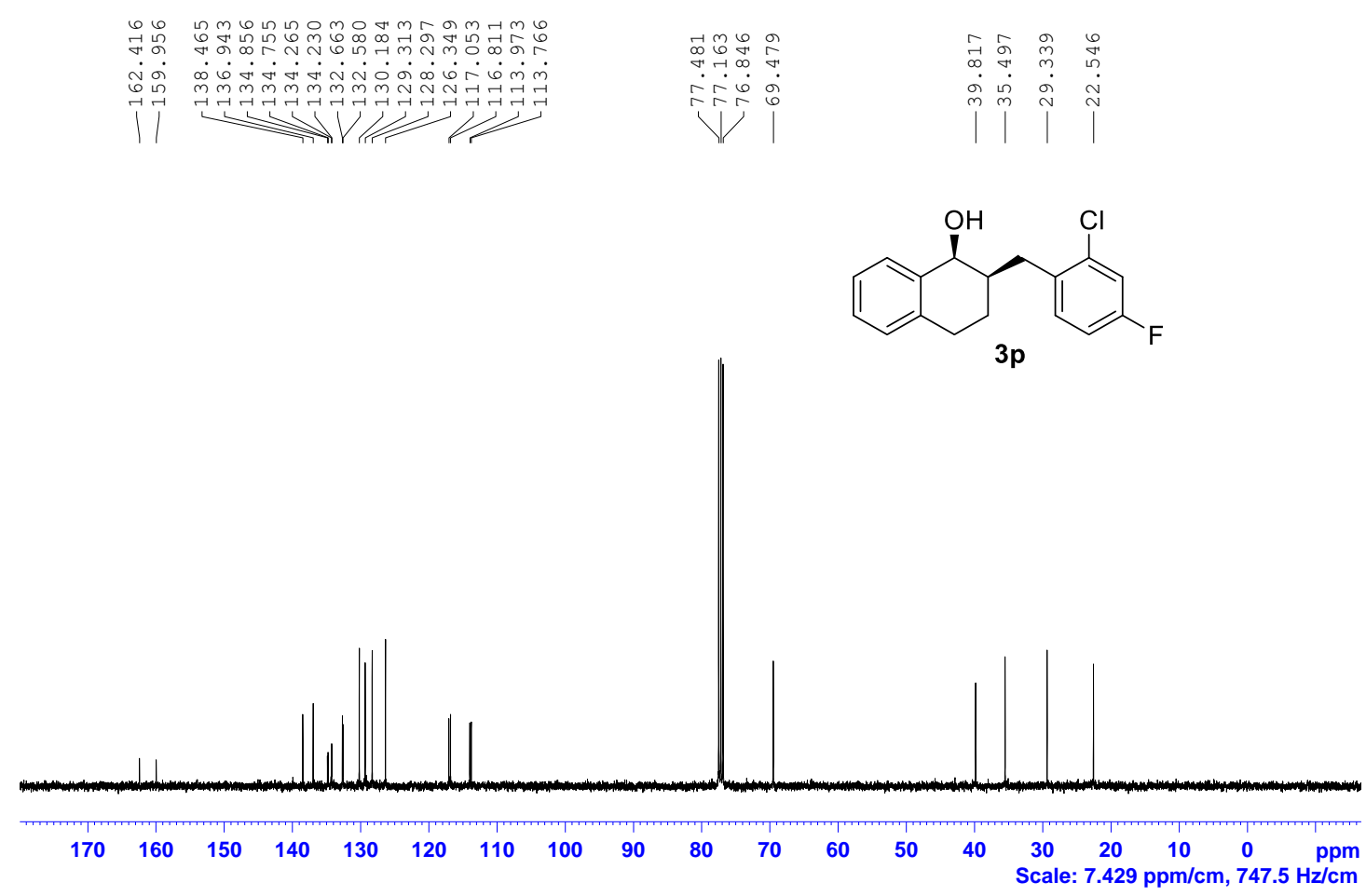
${ }^{1} \mathrm{H}$ NMR spectrum of $\mathbf{3 q}\left(\mathrm{CDCl}_{3}, 400 \mathrm{MHz}\right)$ :

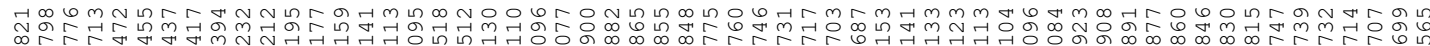
-

$+1 / 2+1$<smiles>O[C@H]1c2ccccc2CC[C@@H]1Cc1ccc2ccccc2c1</smiles>

$3 q$

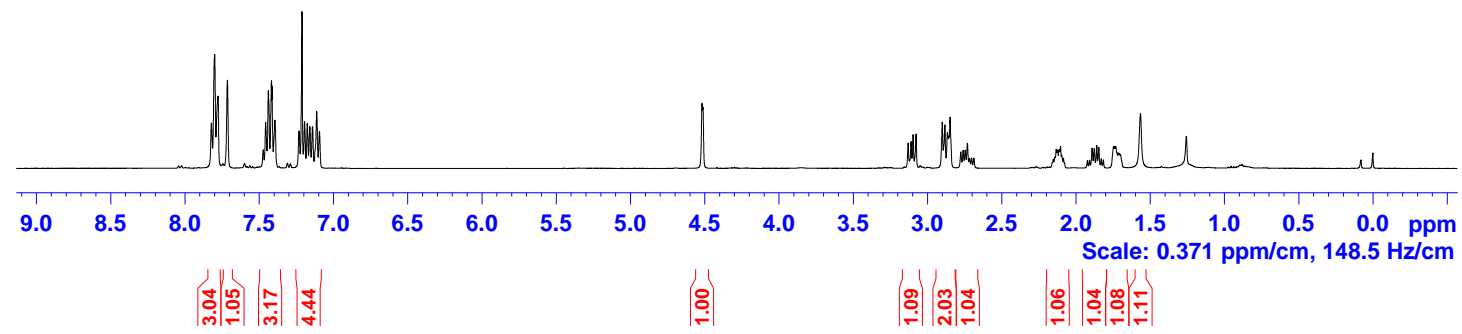

${ }^{13} \mathrm{C}\left\{{ }^{1} \mathrm{H}\right\}$ NMR spectrum of $\mathbf{3 q}\left(\mathrm{CDCl}_{3}, 100 \mathrm{MHz}\right)$ :
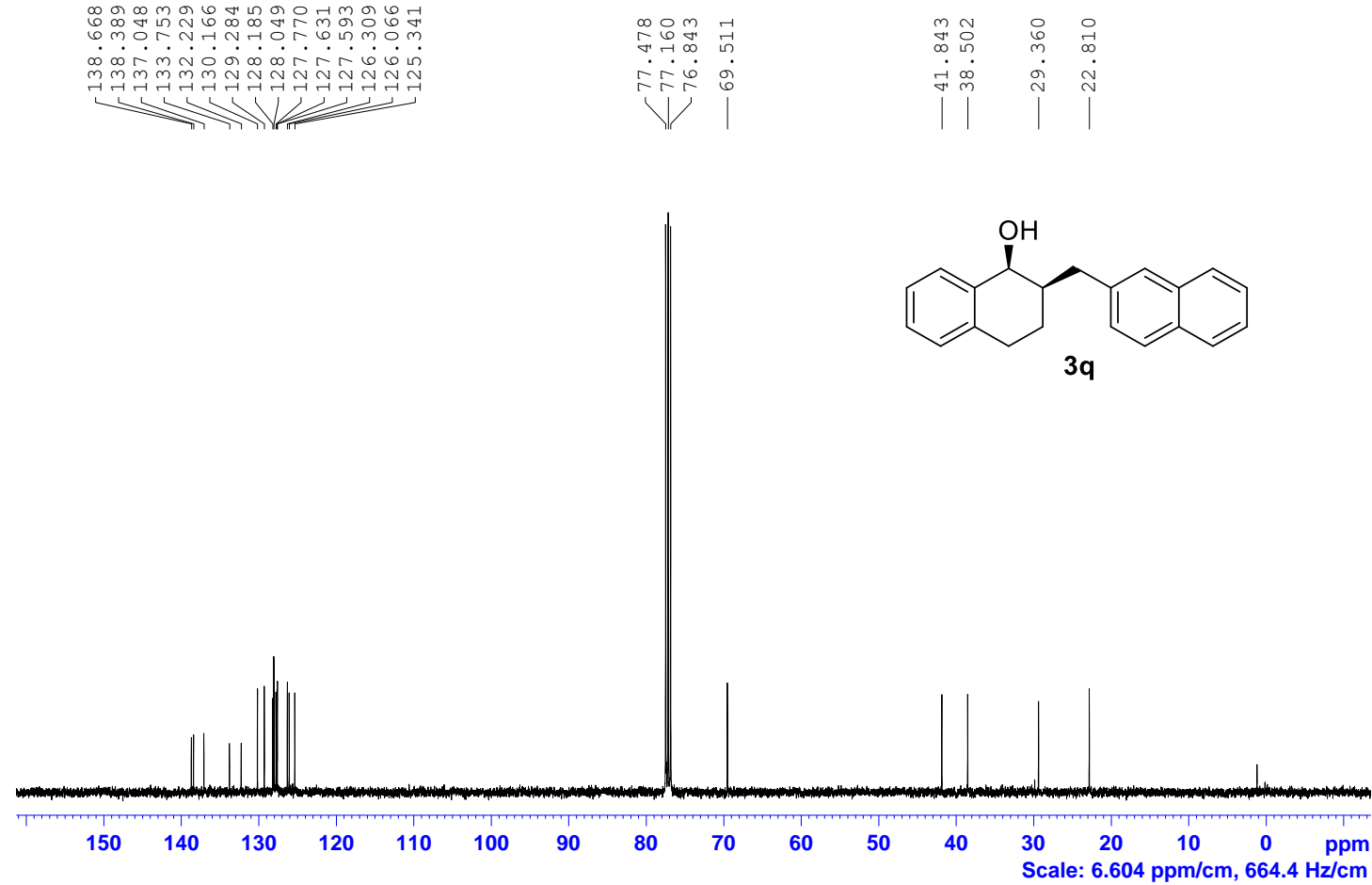
${ }^{1} \mathrm{H}$ NMR spectrum of $\mathbf{3 r}\left(\mathrm{CDCl}_{3}, 400 \mathrm{MHz}\right)$ :

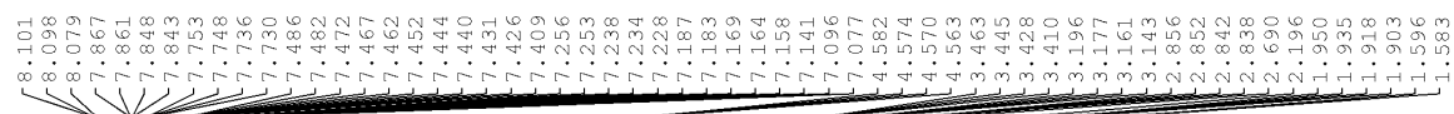<smiles>O[C@H]1c2ccccc2CC[C@@H]1Cc1cccc2ccccc12</smiles>

$3 r$

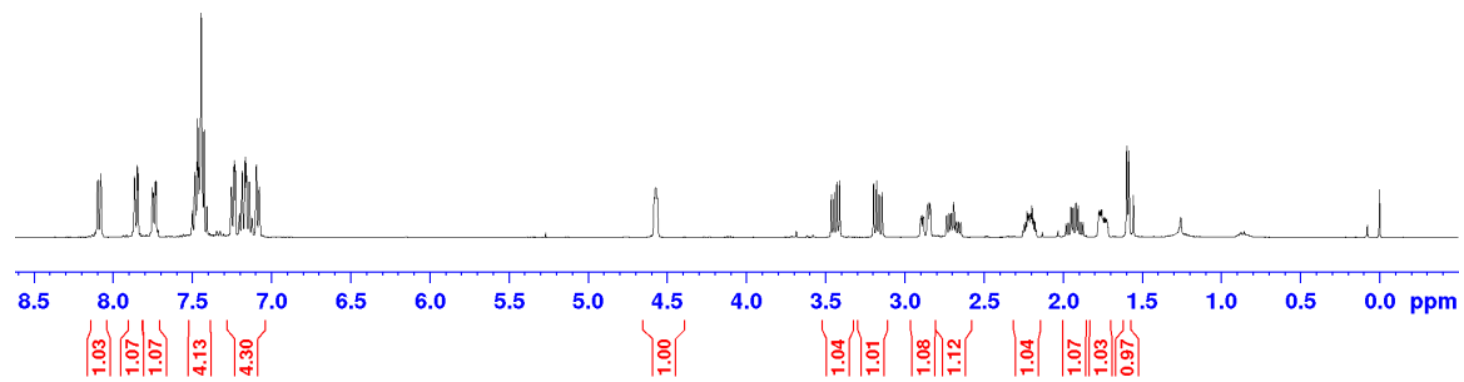

${ }^{13} \mathrm{C}\left\{{ }^{1} \mathrm{H}\right\}$ NMR spectrum of $3 \mathbf{r}\left(\mathrm{CDCl}_{3}, 100 \mathrm{MHz}\right)$ :
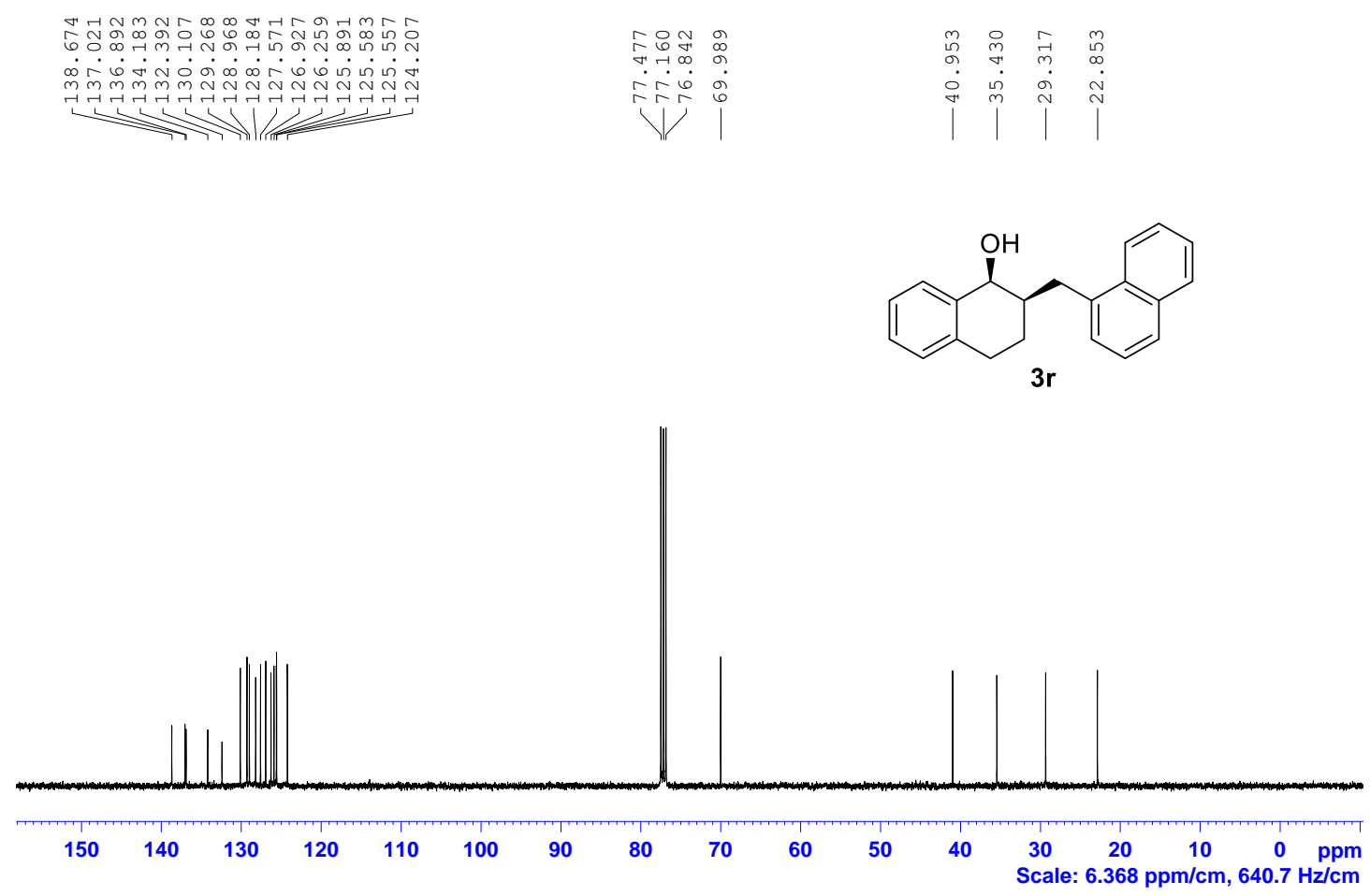
${ }^{1} \mathrm{H}$ NMR spectrum of $3 \mathbf{s}\left(\mathrm{CDCl}_{3}, 400 \mathrm{MHz}\right)$ :

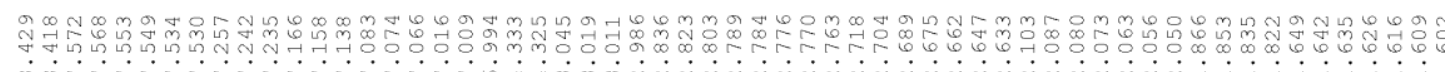

$\underbrace{0}$<smiles>O[C@H]1c2ccccc2CC[C@@H]1Cc1ccccn1</smiles>

$3 \mathrm{~s}$

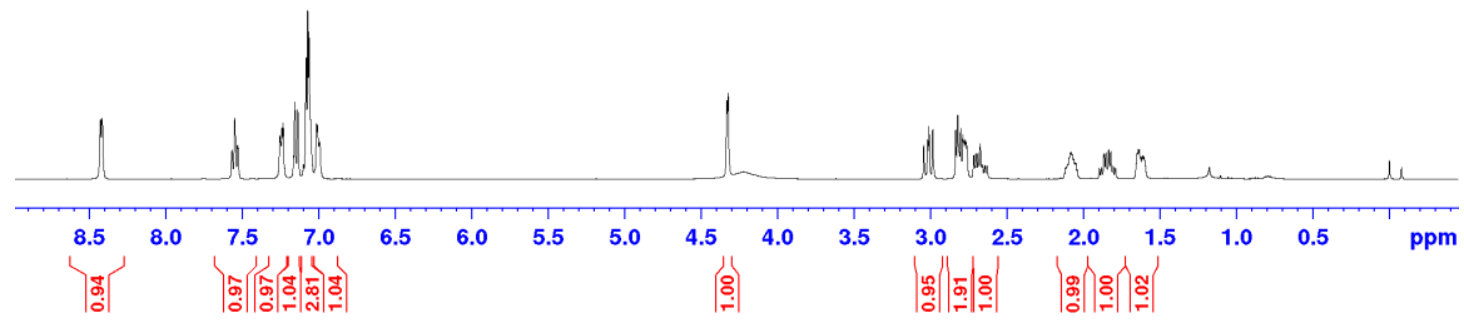

${ }^{13} \mathrm{C}\left\{{ }^{1} \mathrm{H}\right\}$ NMR spectrum of $3 \mathbf{s}\left(\mathrm{CDCl}_{3}, 100 \mathrm{MHz}\right)$ :

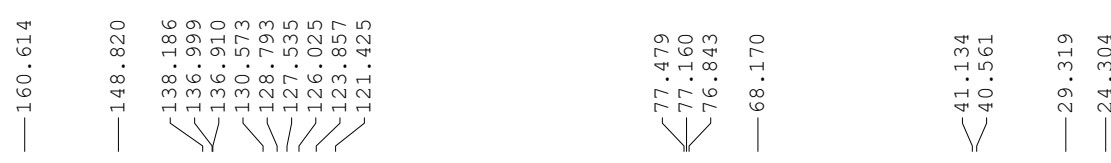<smiles>O[C@H]1c2ccccc2CC[C@H]1Cc1ccccn1</smiles>

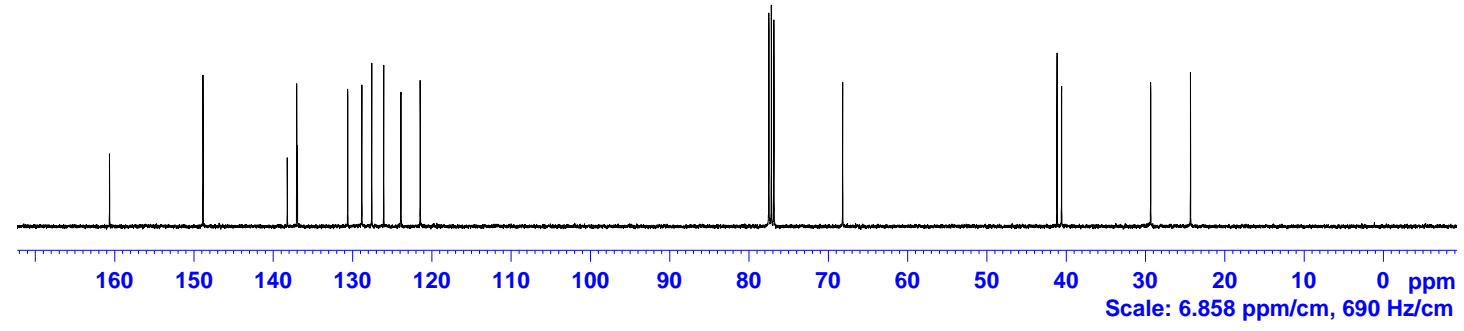


${ }^{1} \mathrm{H}$ NMR spectrum of $\mathbf{3 t}\left(\mathrm{CDCl}_{3}, 400 \mathrm{MHz}\right)$ :

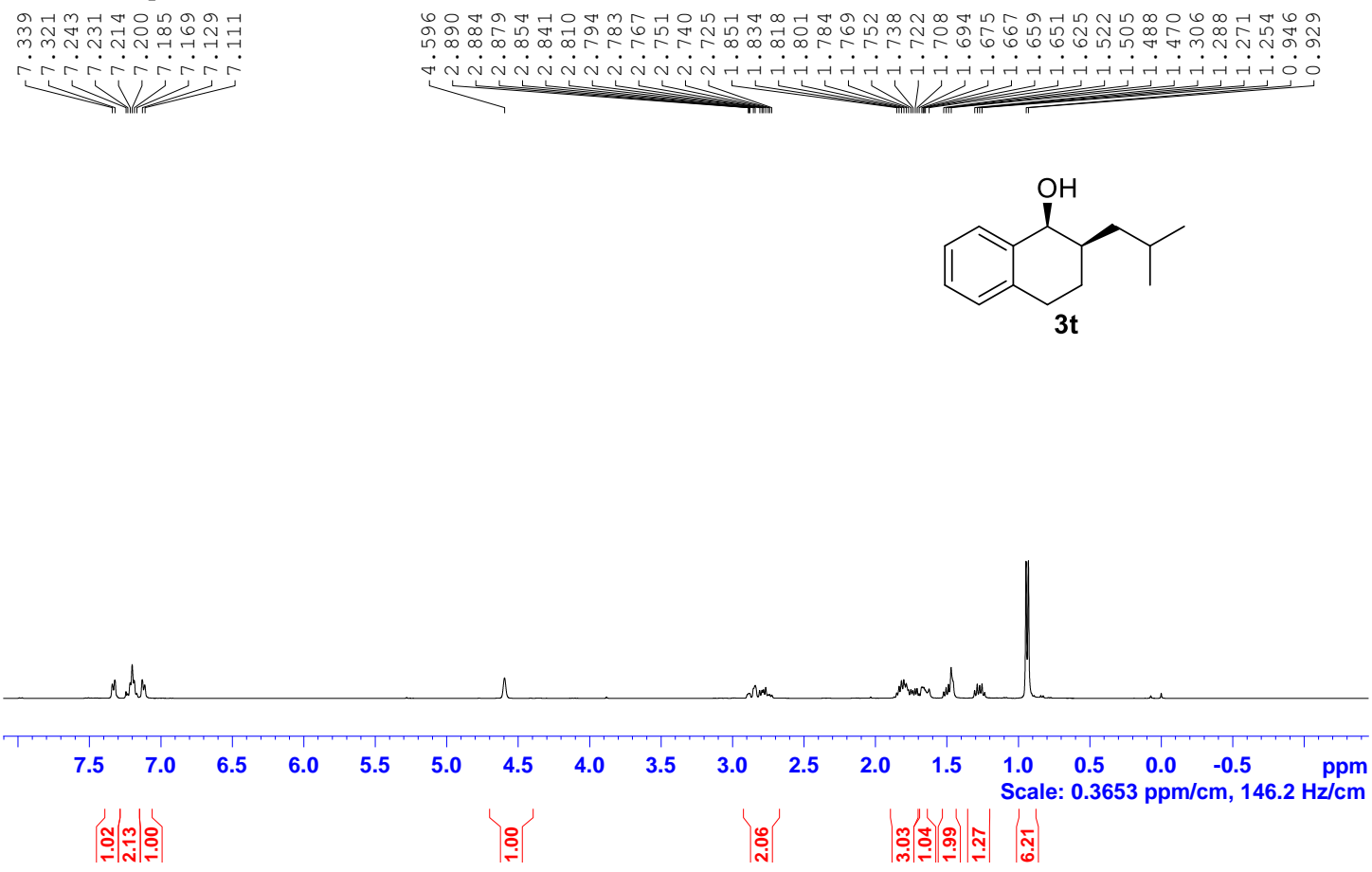

${ }^{13} \mathrm{C}\left\{{ }^{1} \mathrm{H}\right\}$ NMR spectrum of $\mathbf{3 t}\left(\mathrm{CDCl}_{3}, 100 \mathrm{MHz}\right)$ :
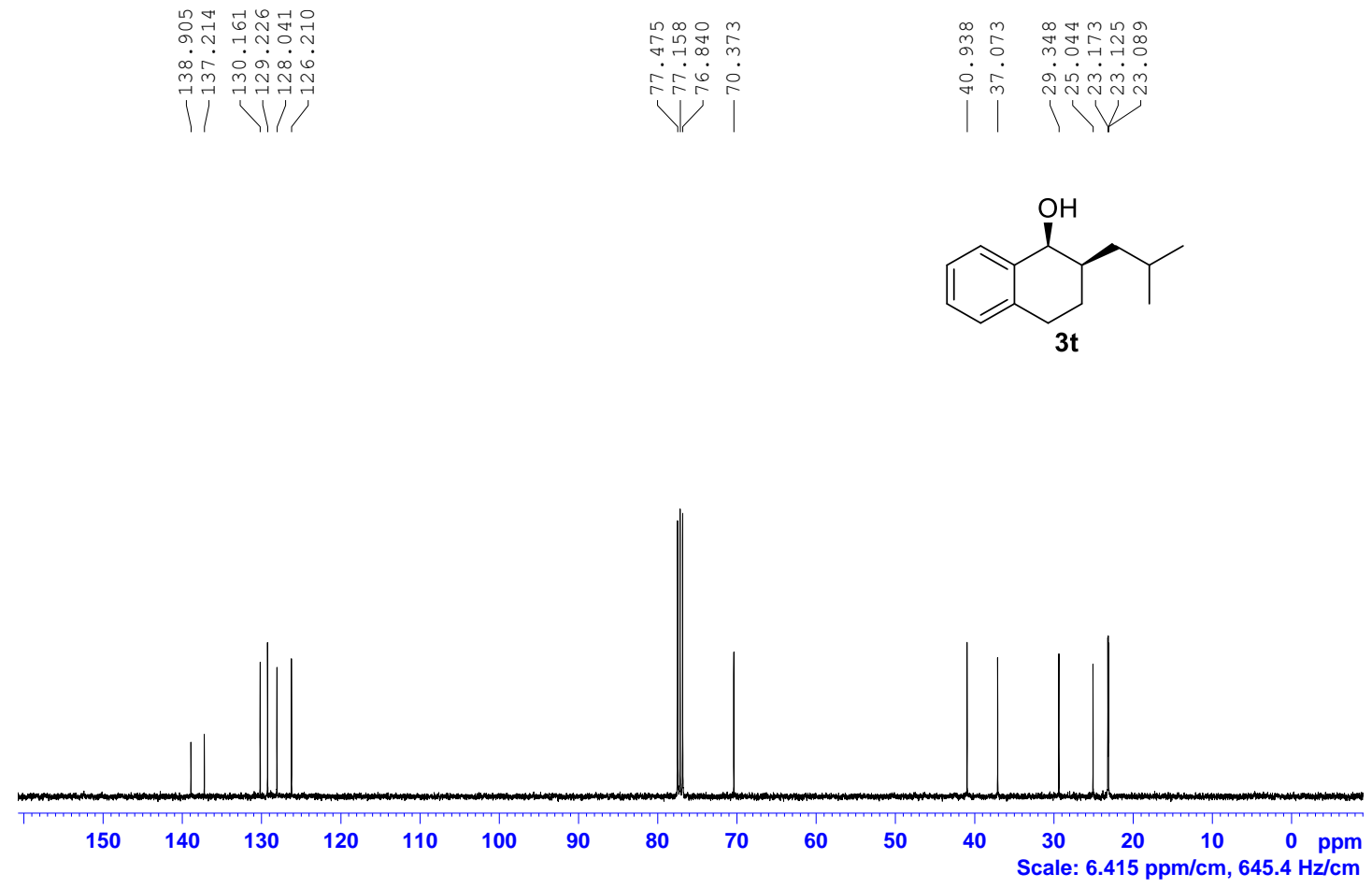
${ }^{1} \mathrm{H}$ NMR spectrum of $\mathbf{3 u}\left(\mathrm{CDCl}_{3}, 400 \mathrm{MHz}\right)$ :

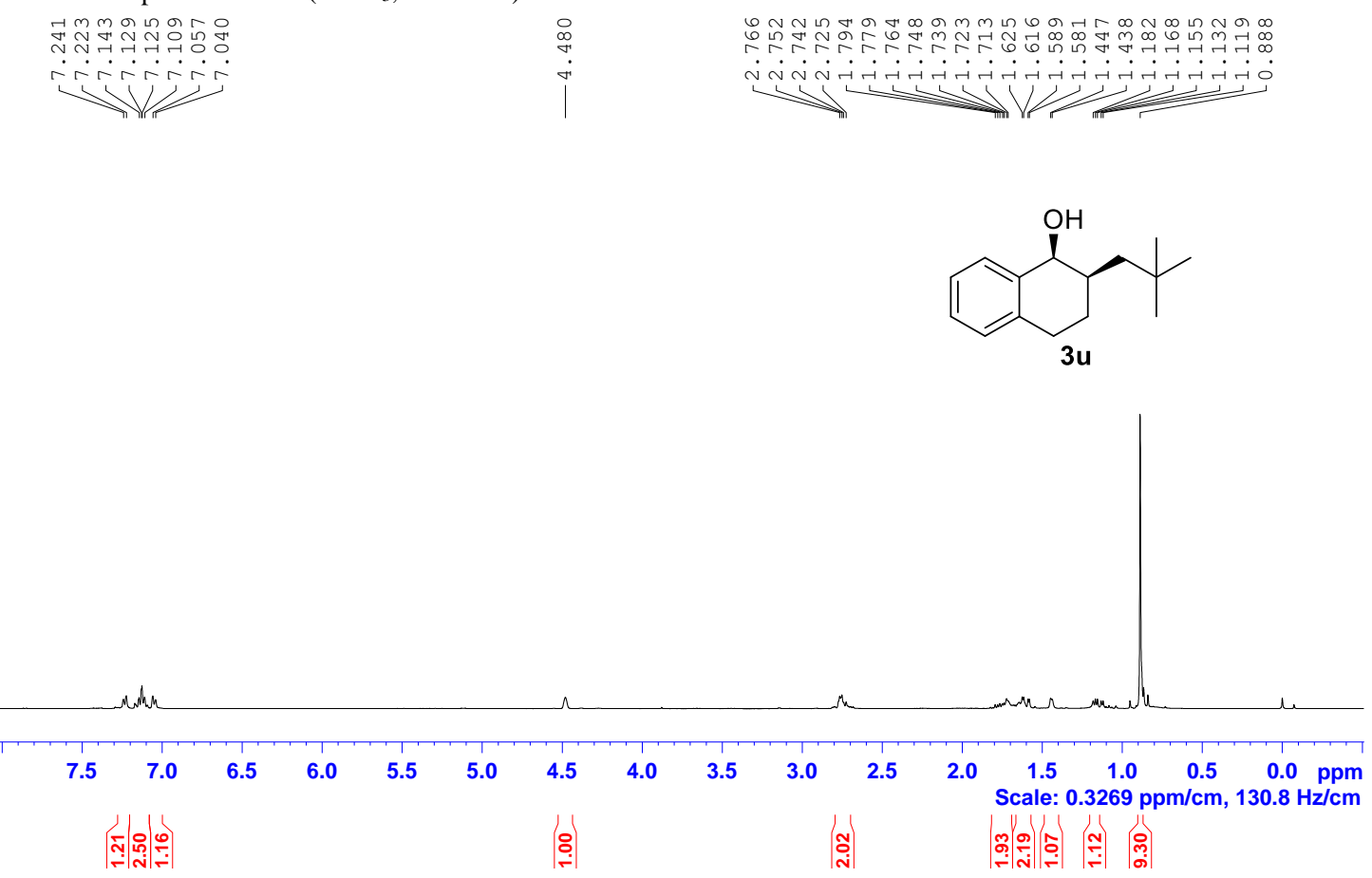

${ }^{13} \mathrm{C}\left\{{ }^{1} \mathrm{H}\right\}$ NMR spectrum of $\mathbf{3 u}\left(\mathrm{CDCl}_{3}, 100 \mathrm{MHz}\right)$ :
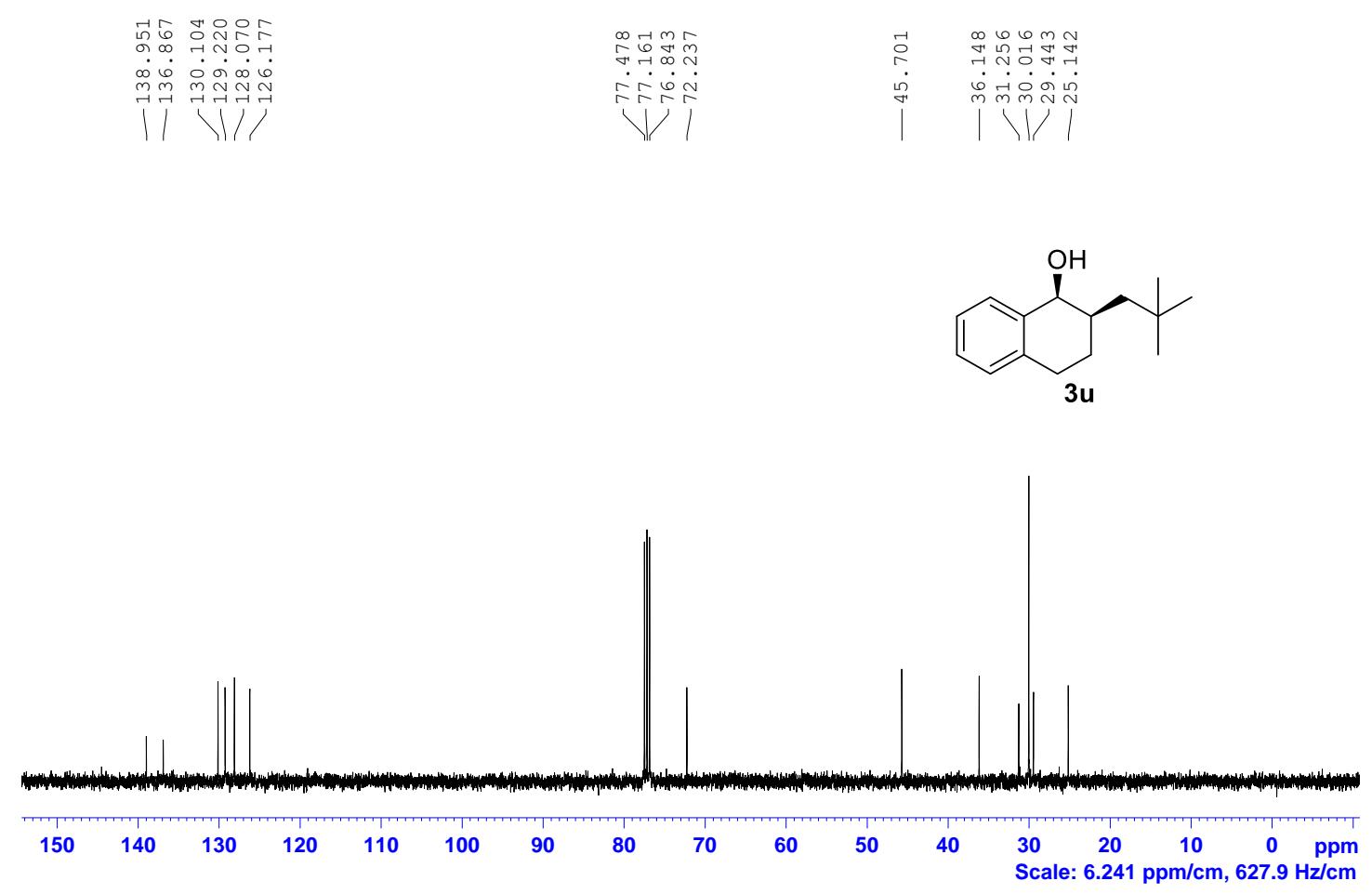
${ }^{1} \mathrm{H}$ NMR spectrum of $\mathbf{3 v}\left(\mathrm{CDCl}_{3}, 400 \mathrm{MHz}\right)$ :

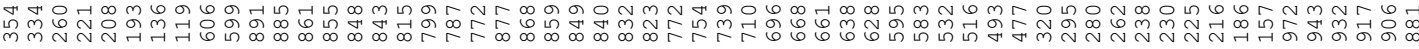

-

Not<smiles>O[C@H]1c2ccccc2CC[C@@H]1CC1CCCCC1</smiles>

$3 v$

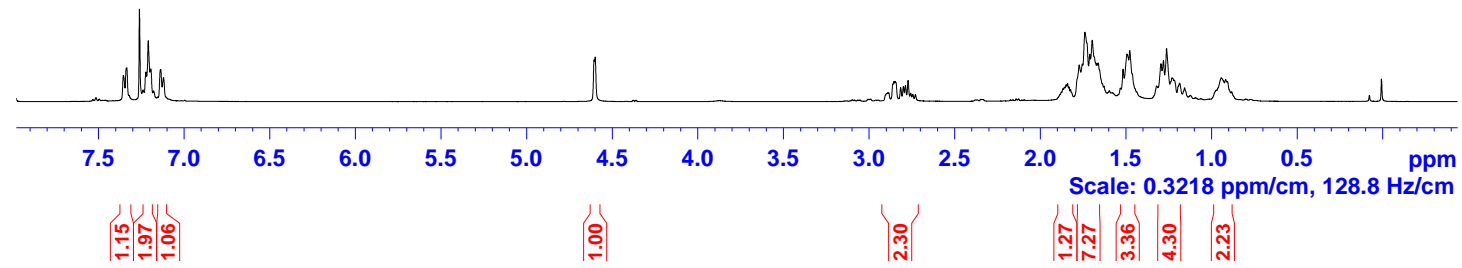

${ }^{13} \mathrm{C}\left\{{ }^{1} \mathrm{H}\right\}$ NMR spectrum of $\mathbf{3 v}\left(\mathrm{CDCl}_{3}, 100 \mathrm{MHz}\right)$ :
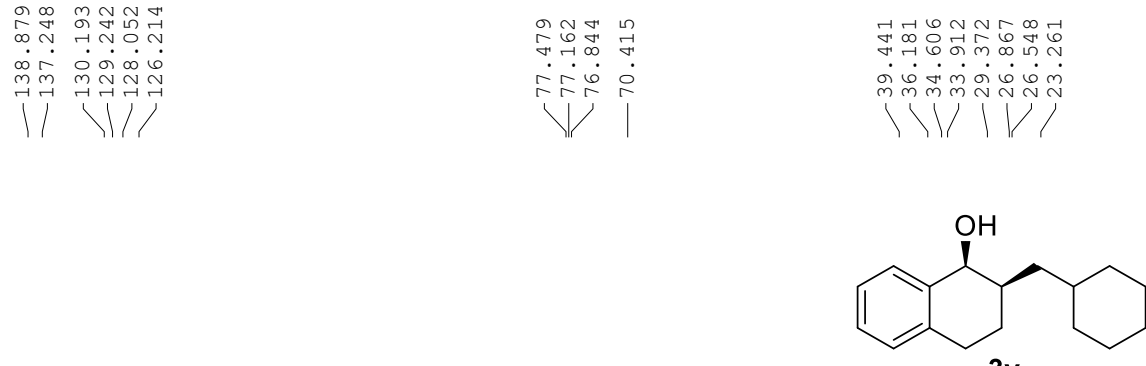

$3 v$

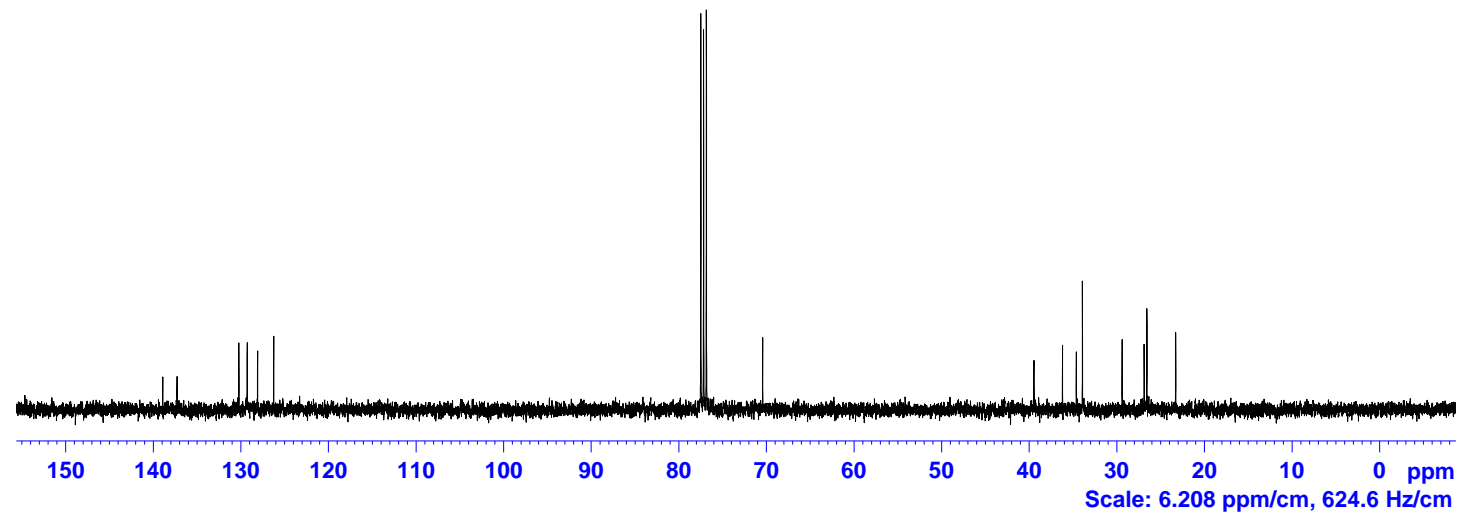


${ }^{1} \mathrm{H}$ NMR spectrum of $\mathbf{3 w}\left(\mathrm{CDCl}_{3}, 400 \mathrm{MHz}\right)$ :
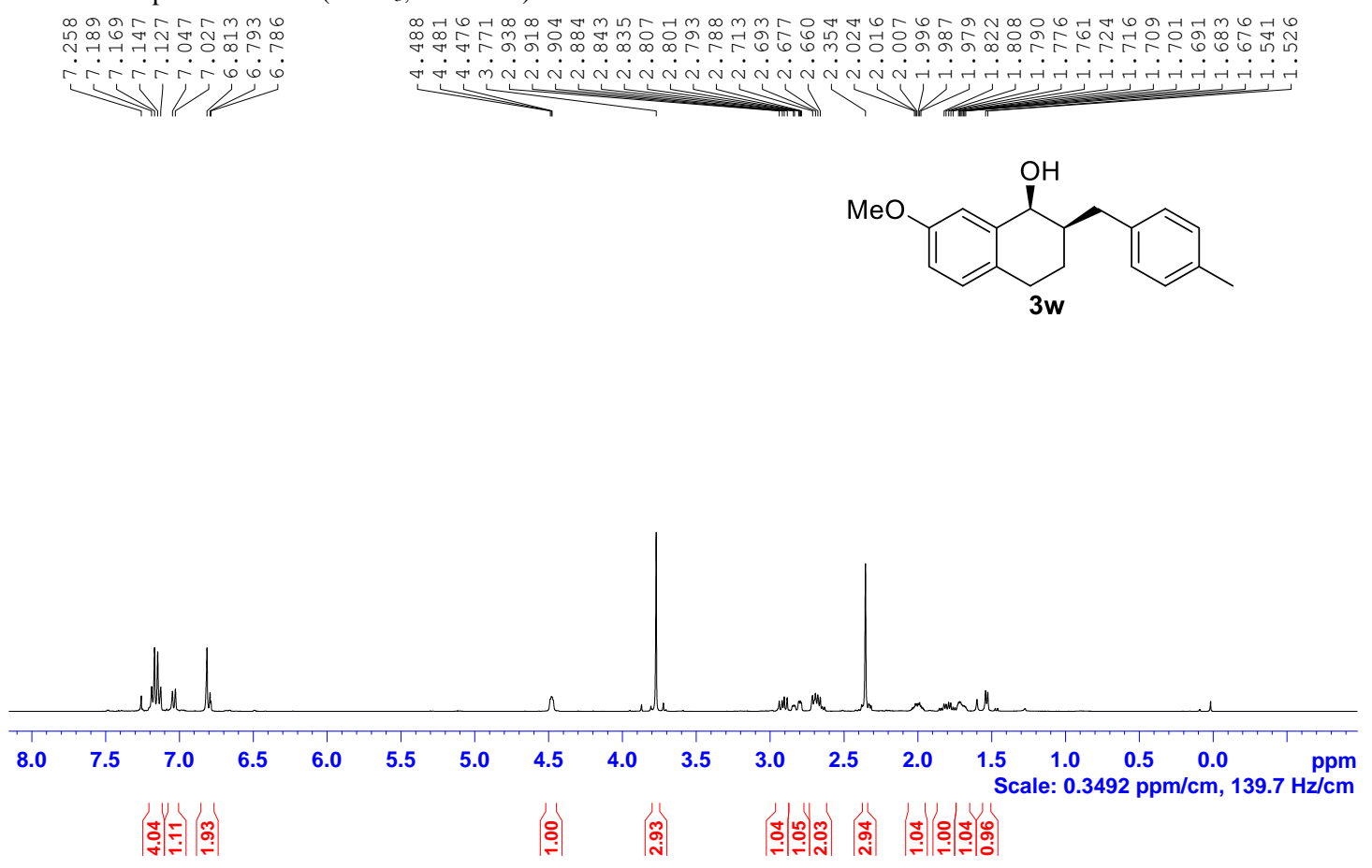

${ }^{13} \mathrm{C}\left\{{ }^{1} \mathrm{H}\right\}$ NMR spectrum of $\mathbf{3 w}\left(\mathrm{CDCl}_{3}, 100 \mathrm{MHz}\right)$ :
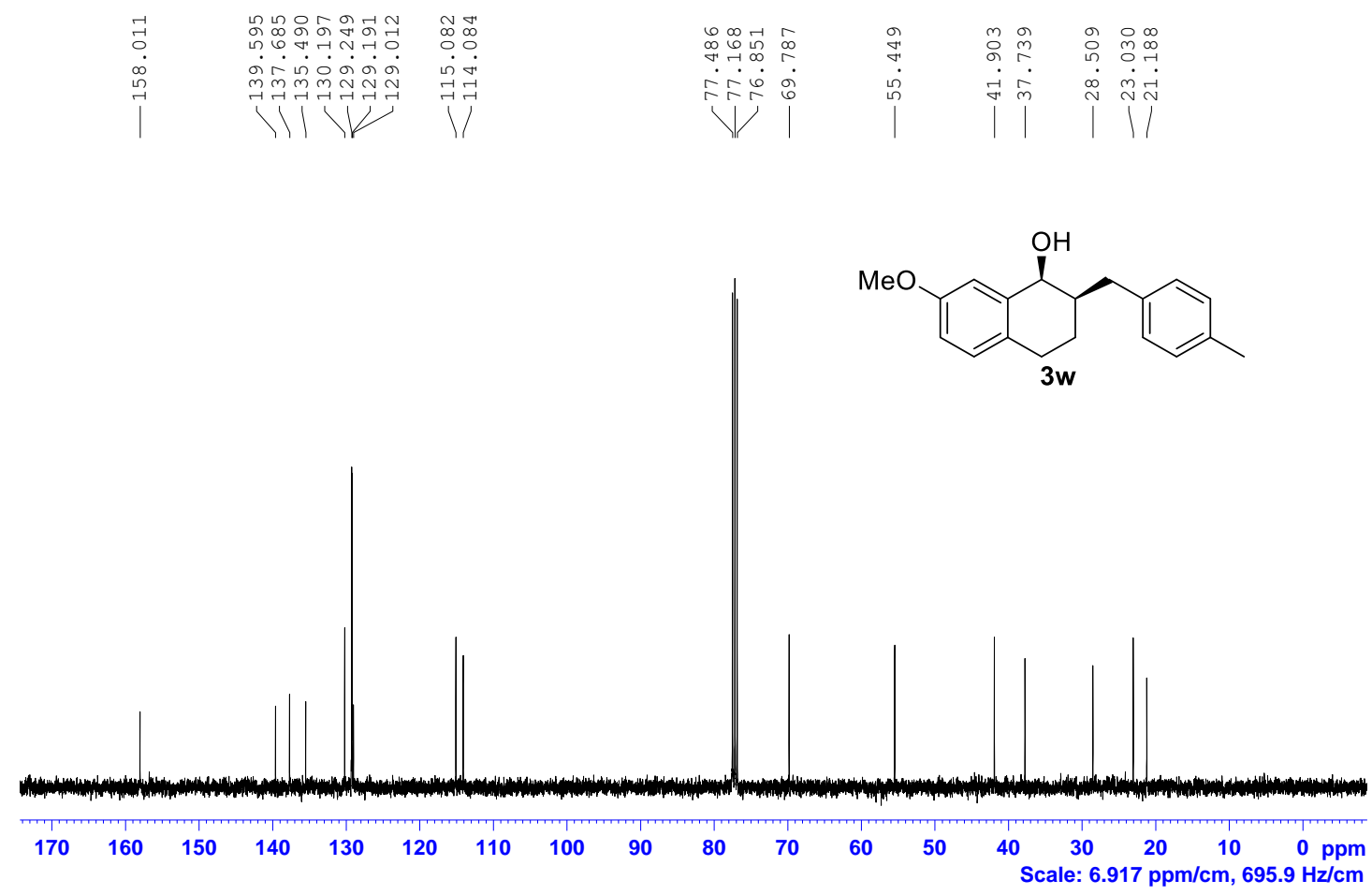

S84 
${ }^{1} \mathrm{H}$ NMR spectrum of $\mathbf{3} \mathbf{x}\left(\mathrm{CDCl}_{3}, 400 \mathrm{MHz}\right)$ :
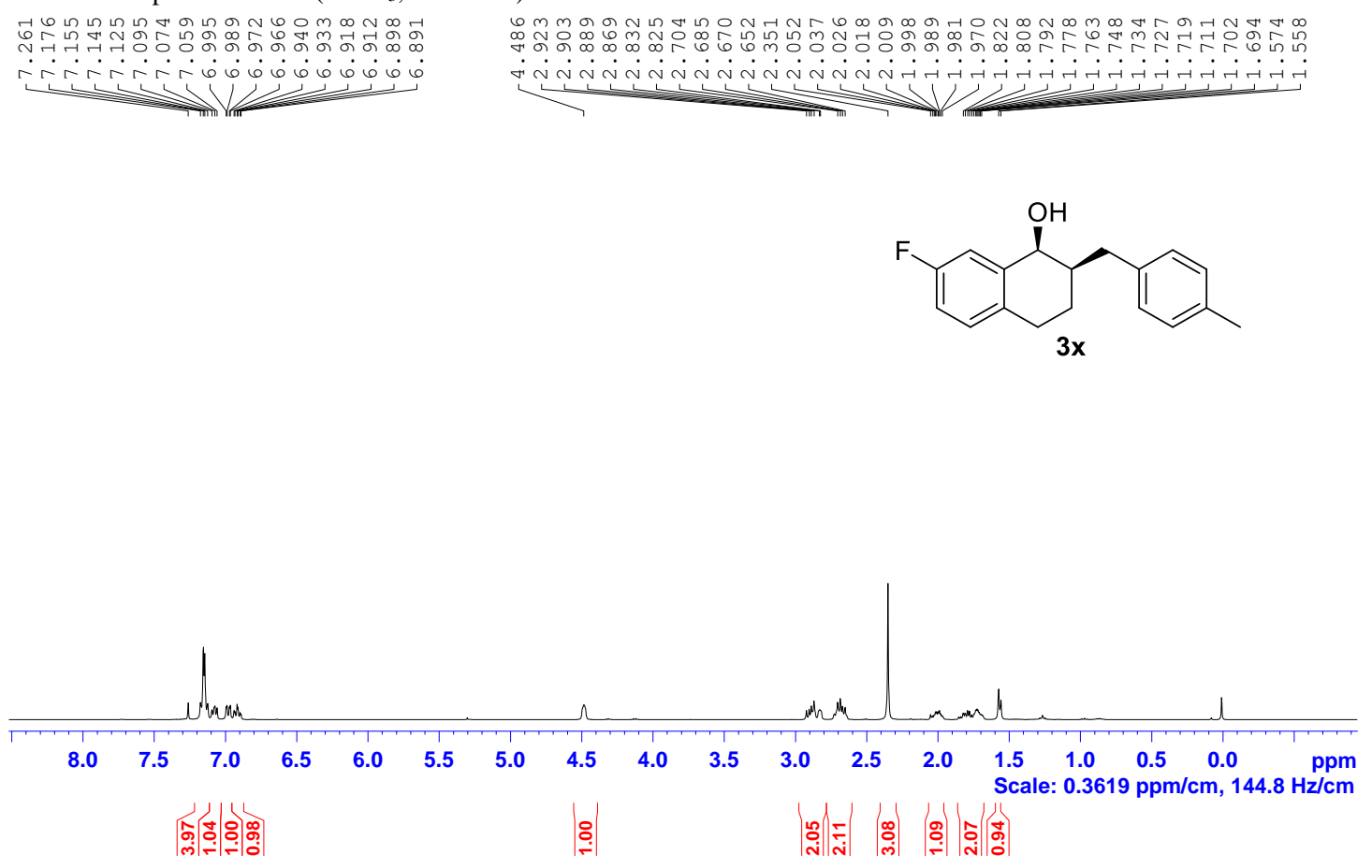

${ }^{13} \mathrm{C}\left\{{ }^{1} \mathrm{H}\right\}$ NMR spectrum of $\mathbf{3 x}\left(\mathrm{CDCl}_{3}, 100 \mathrm{MHz}\right)$ :
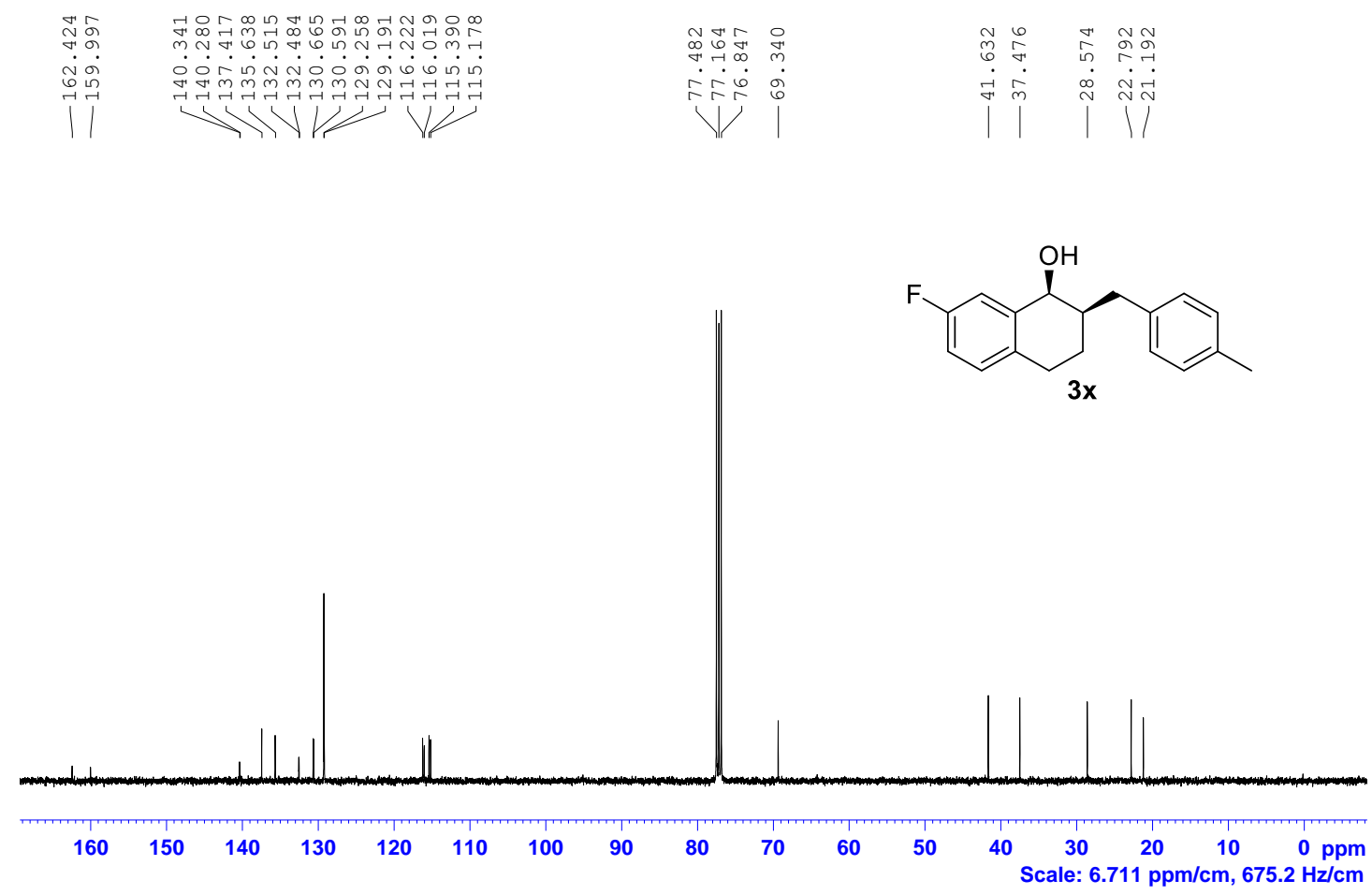

S85 
${ }^{1} \mathrm{H}$ NMR spectrum of $\mathbf{3 y}\left(\mathrm{CDCl}_{3}, 400 \mathrm{MHz}\right)$ :
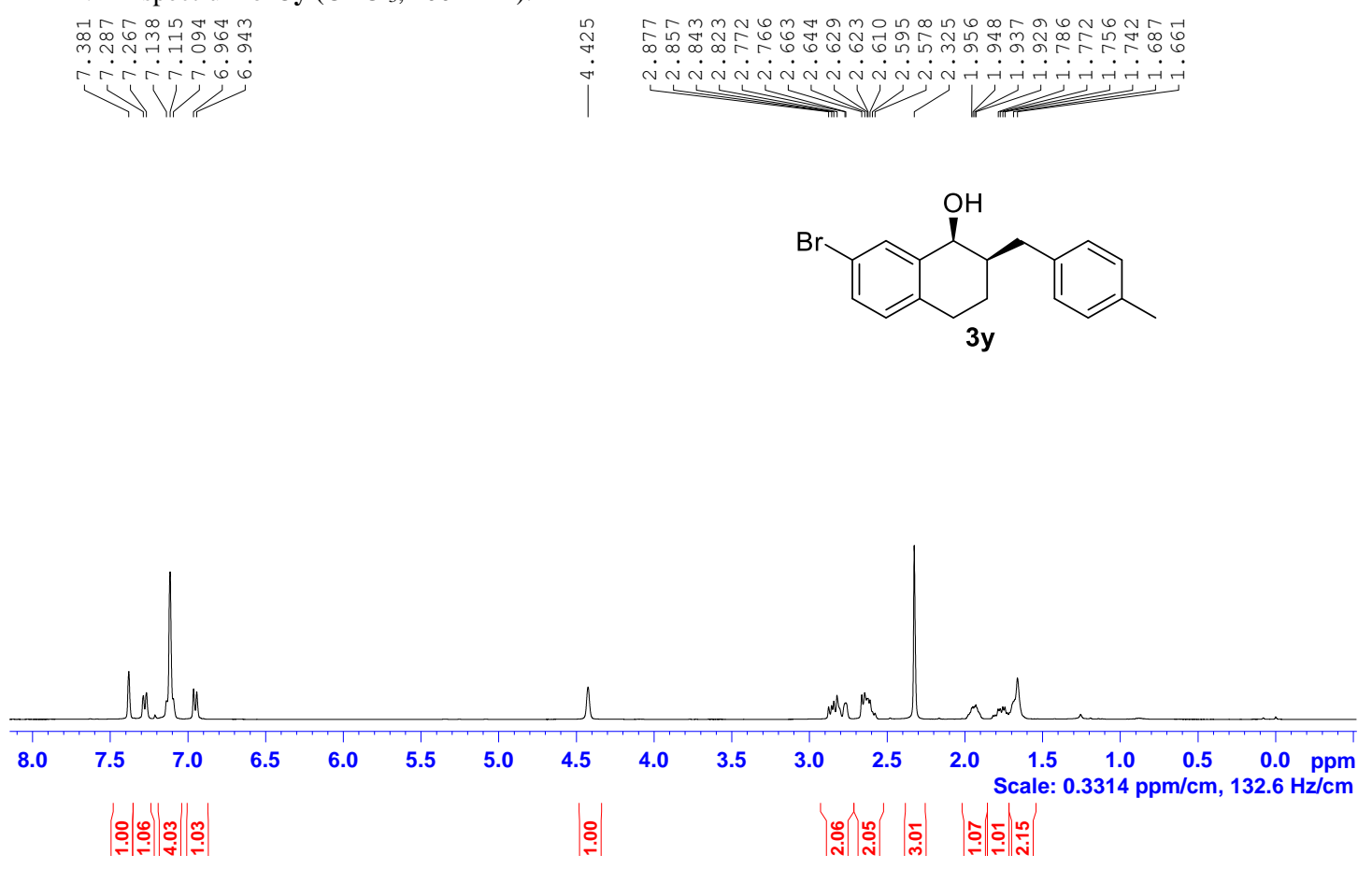

${ }^{13} \mathrm{C}\left\{{ }^{1} \mathrm{H}\right\}$ NMR spectrum of $\mathbf{3 y}\left(\mathrm{CDCl}_{3}, 100 \mathrm{MHz}\right)$ :
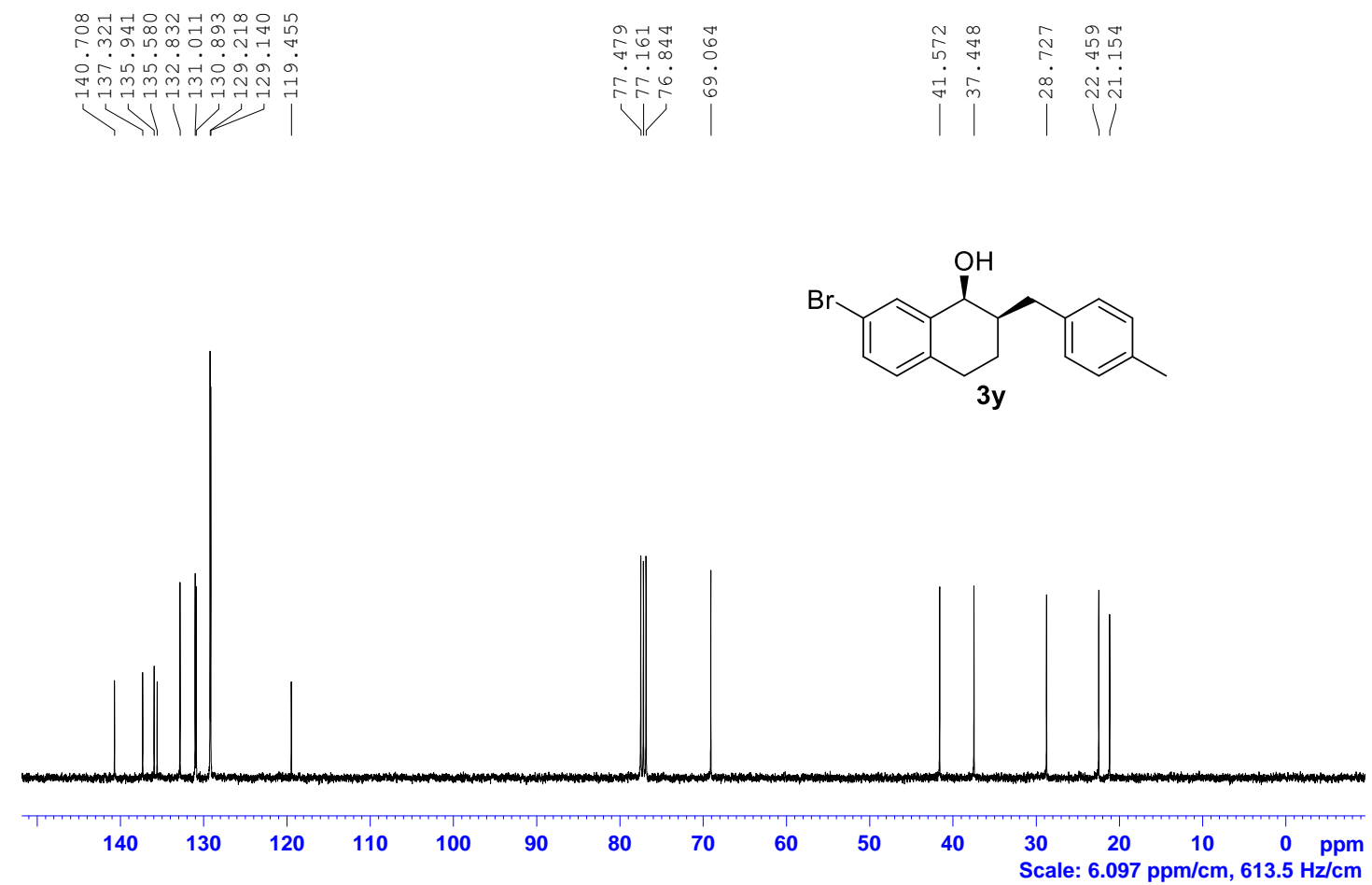

S86 
${ }^{1} \mathrm{H}$ NMR spectrum of $\mathbf{3 z}\left(\mathrm{CDCl}_{3}, 400 \mathrm{MHz}\right)$ :
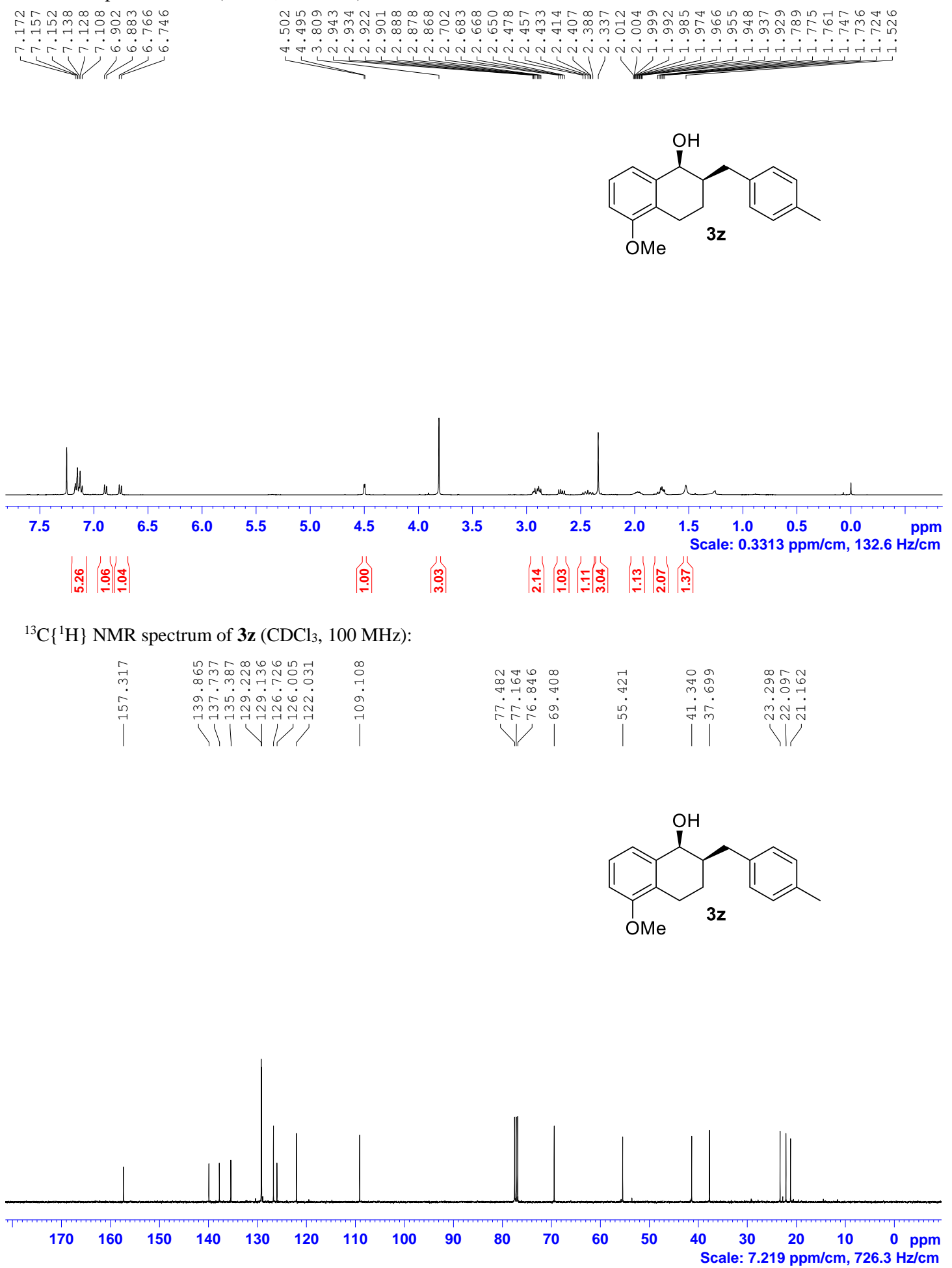

S87 
${ }^{1} \mathrm{H}$ NMR spectrum of $\mathbf{3 a a}\left(\mathrm{CDCl}_{3}, 400 \mathrm{MHz}\right)$ :
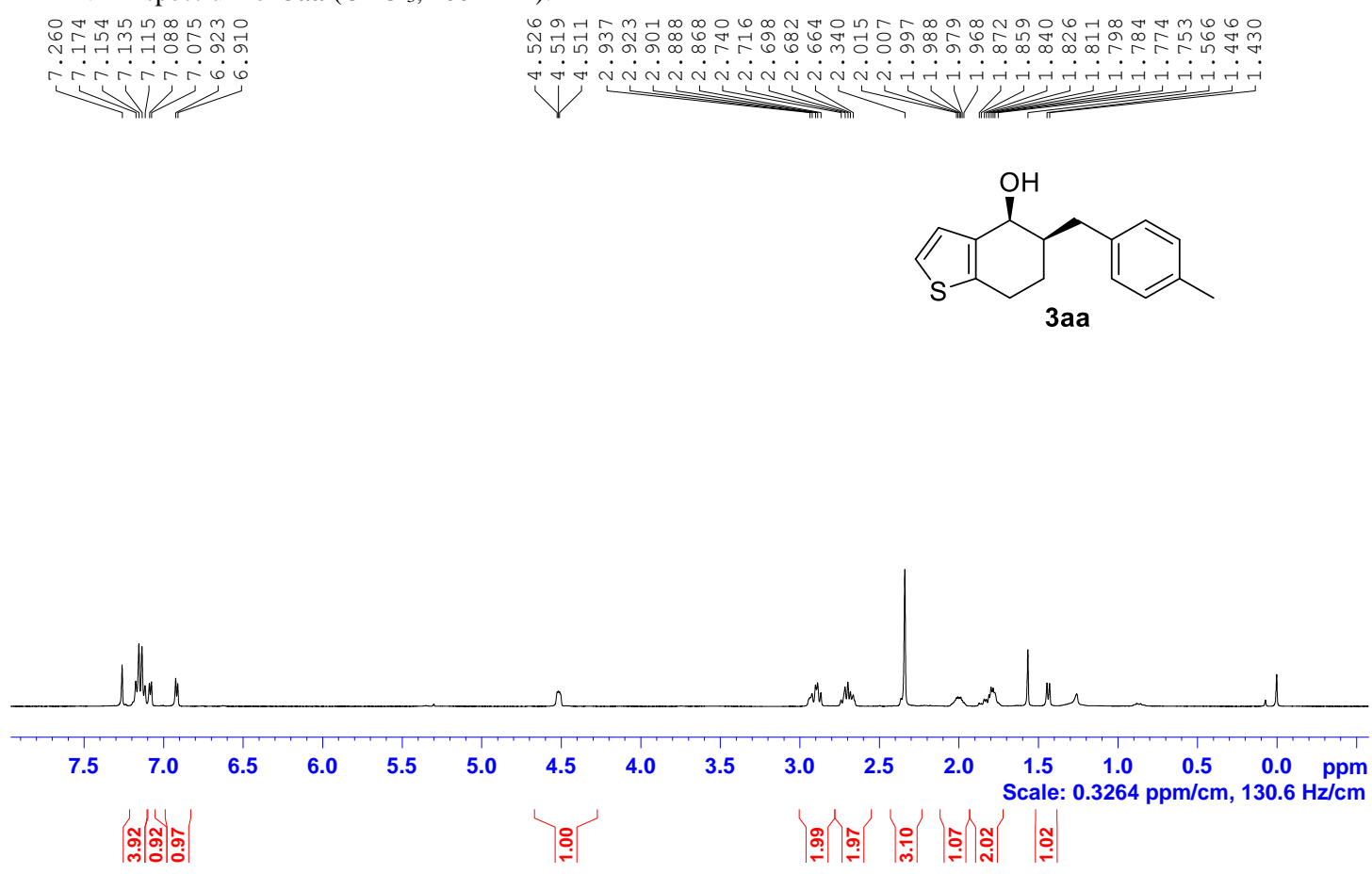

${ }^{13} \mathrm{C}\left\{{ }^{1} \mathrm{H}\right\}$ NMR spectrum of $\mathbf{3 a a}\left(\mathrm{CDCl}_{3}, 100 \mathrm{MHz}\right)$ :
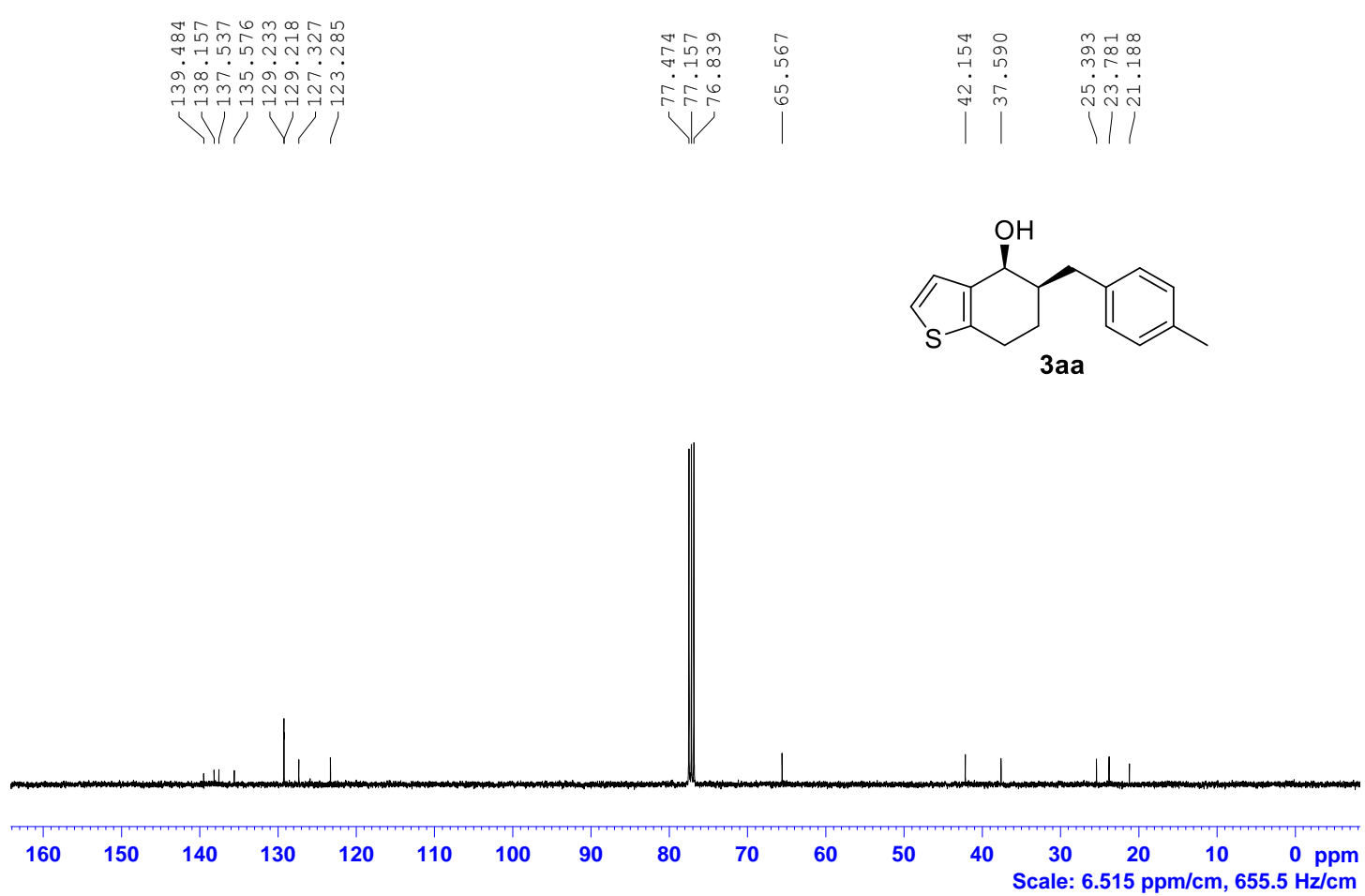

S88 
${ }^{1} \mathrm{H}$ NMR spectrum of $\mathbf{3 a b}\left(\mathrm{CDCl}_{3}, 400 \mathrm{MHz}\right)$ :

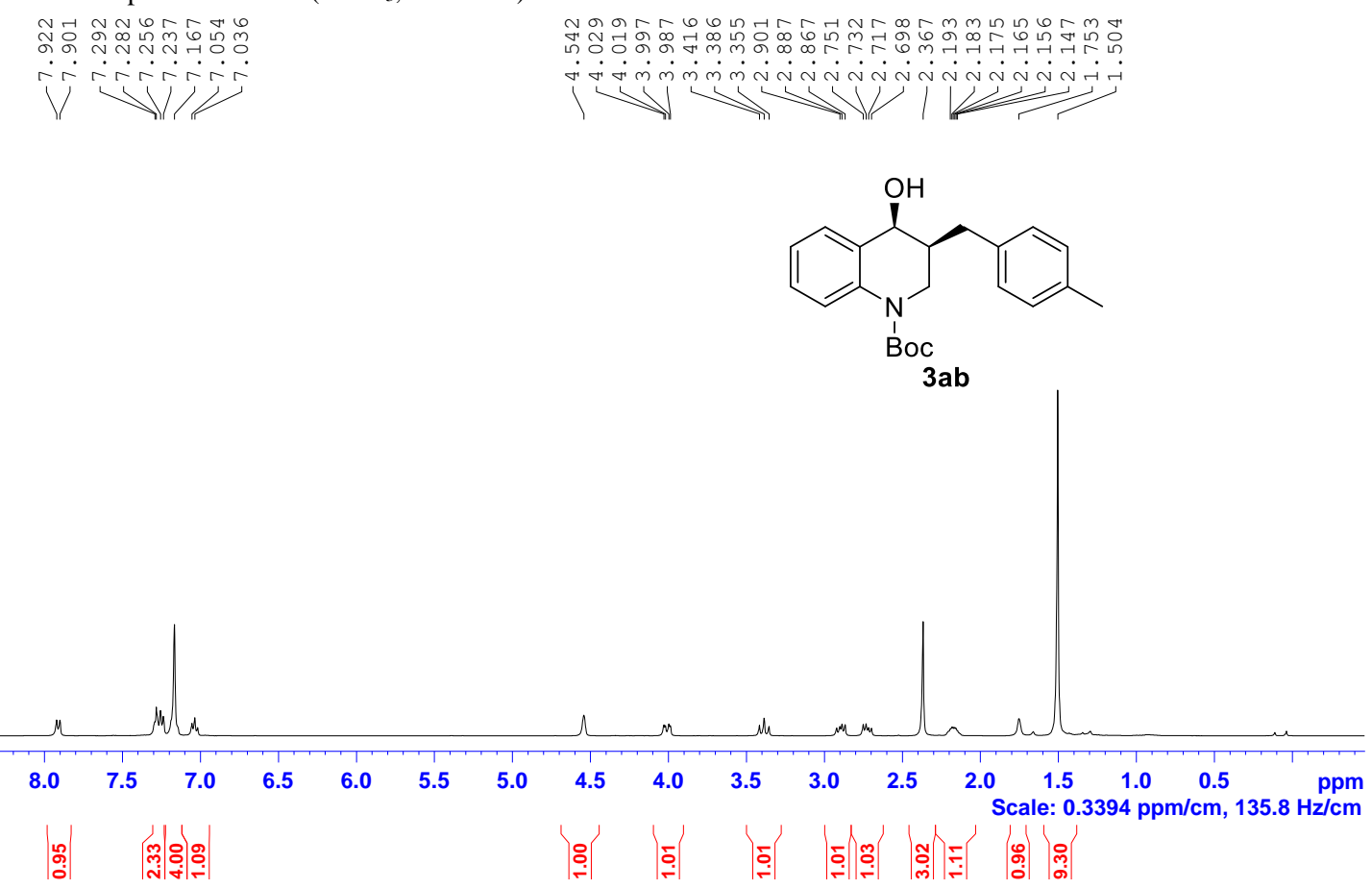

${ }^{13} \mathrm{C}\left\{{ }^{1} \mathrm{H}\right\}$ NMR spectrum of $\mathbf{3 a b}\left(\mathrm{CDCl}_{3}, 100 \mathrm{MHz}\right)$ :
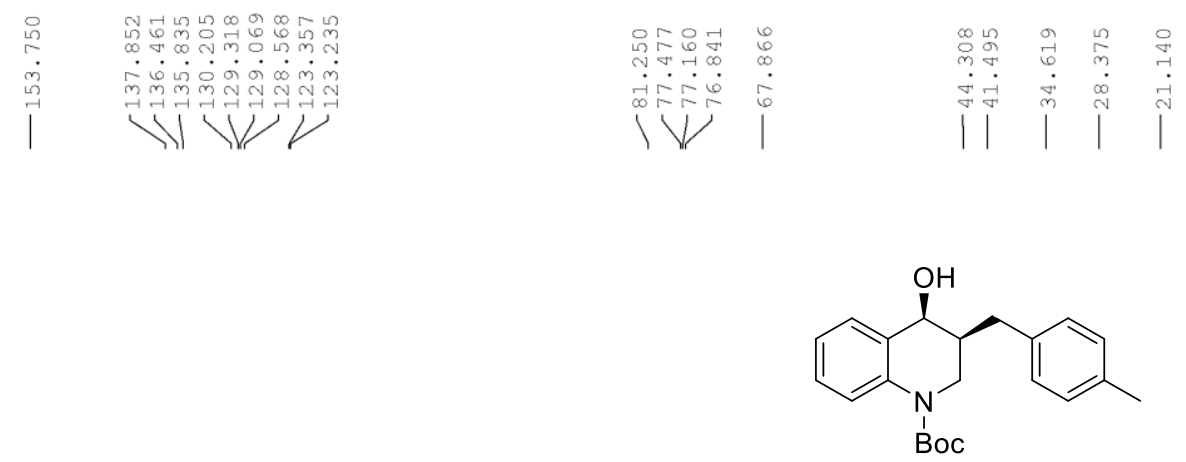

$3 a b$

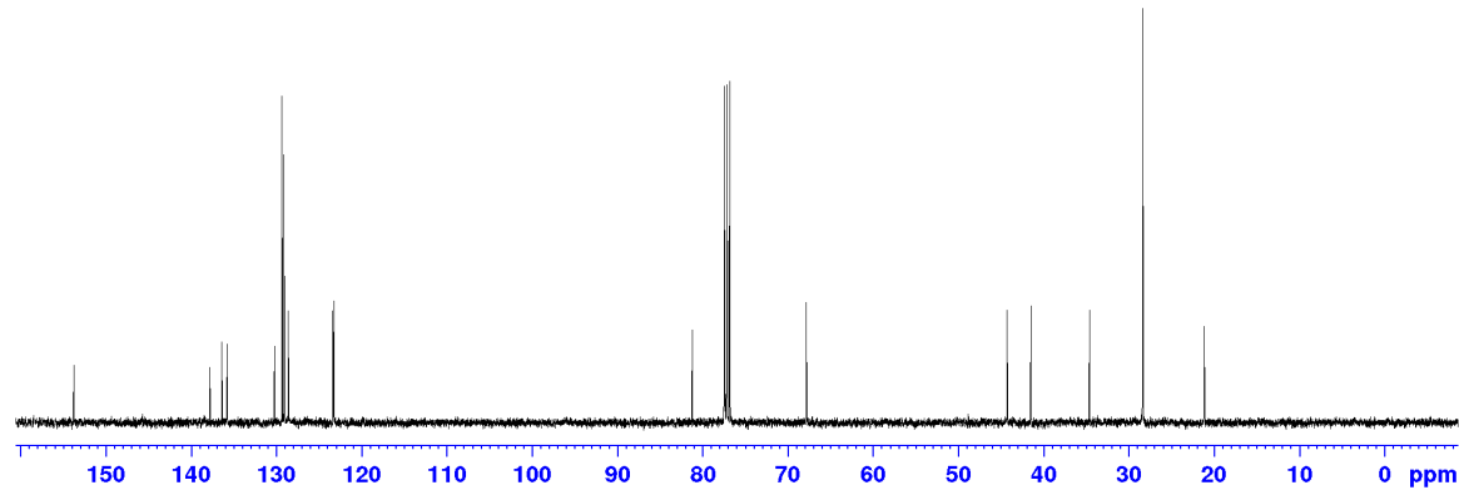


${ }^{1} \mathrm{H}$ NMR spectrum of $\mathbf{3 a c}\left(\mathrm{CDCl}_{3}, 400 \mathrm{MHz}\right)$ :
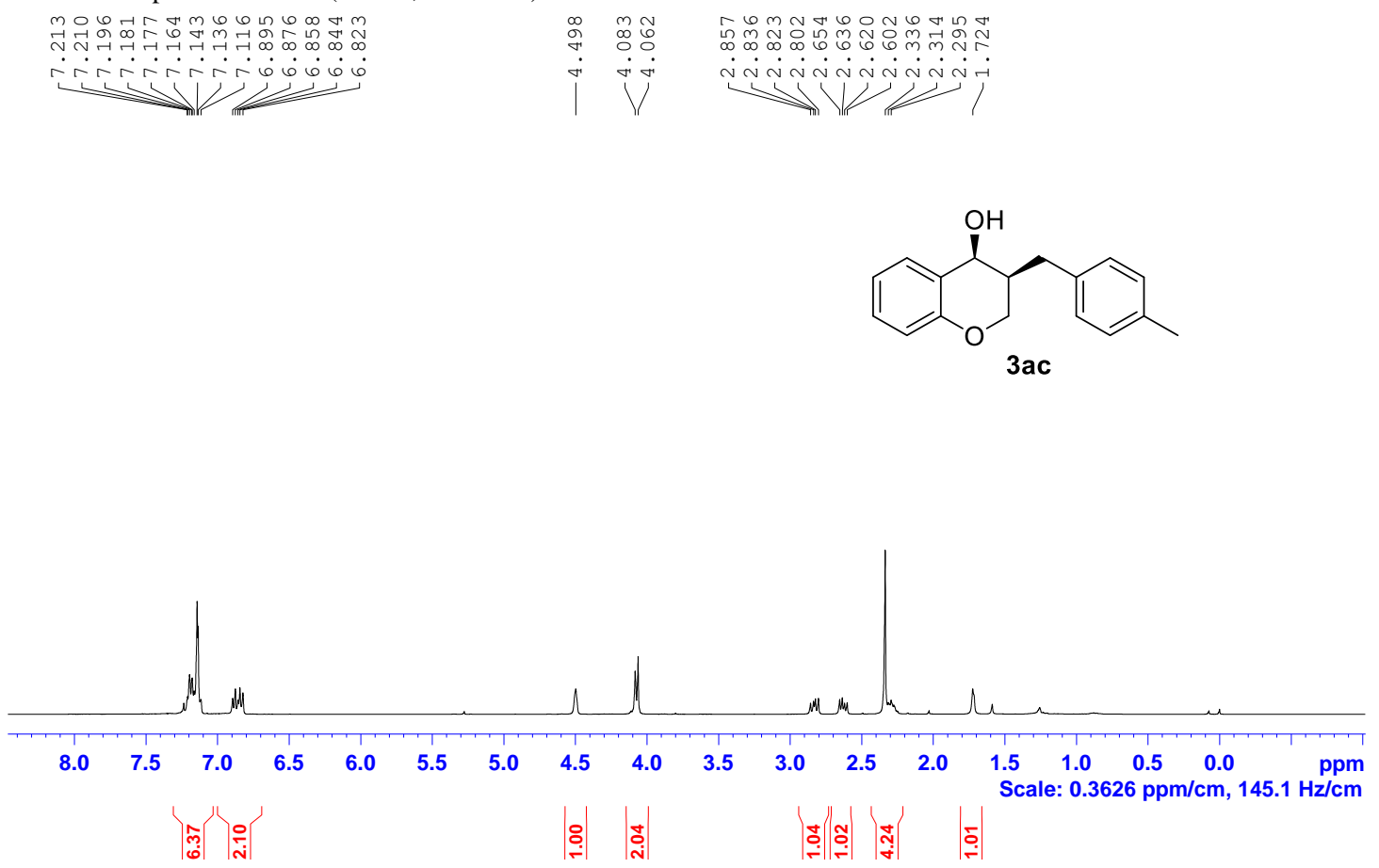

${ }^{13} \mathrm{C}\left\{{ }^{1} \mathrm{H}\right\}$ NMR spectrum of $\mathbf{3 a c}\left(\mathrm{CDCl}_{3}, 100 \mathrm{MHz}\right)$ :
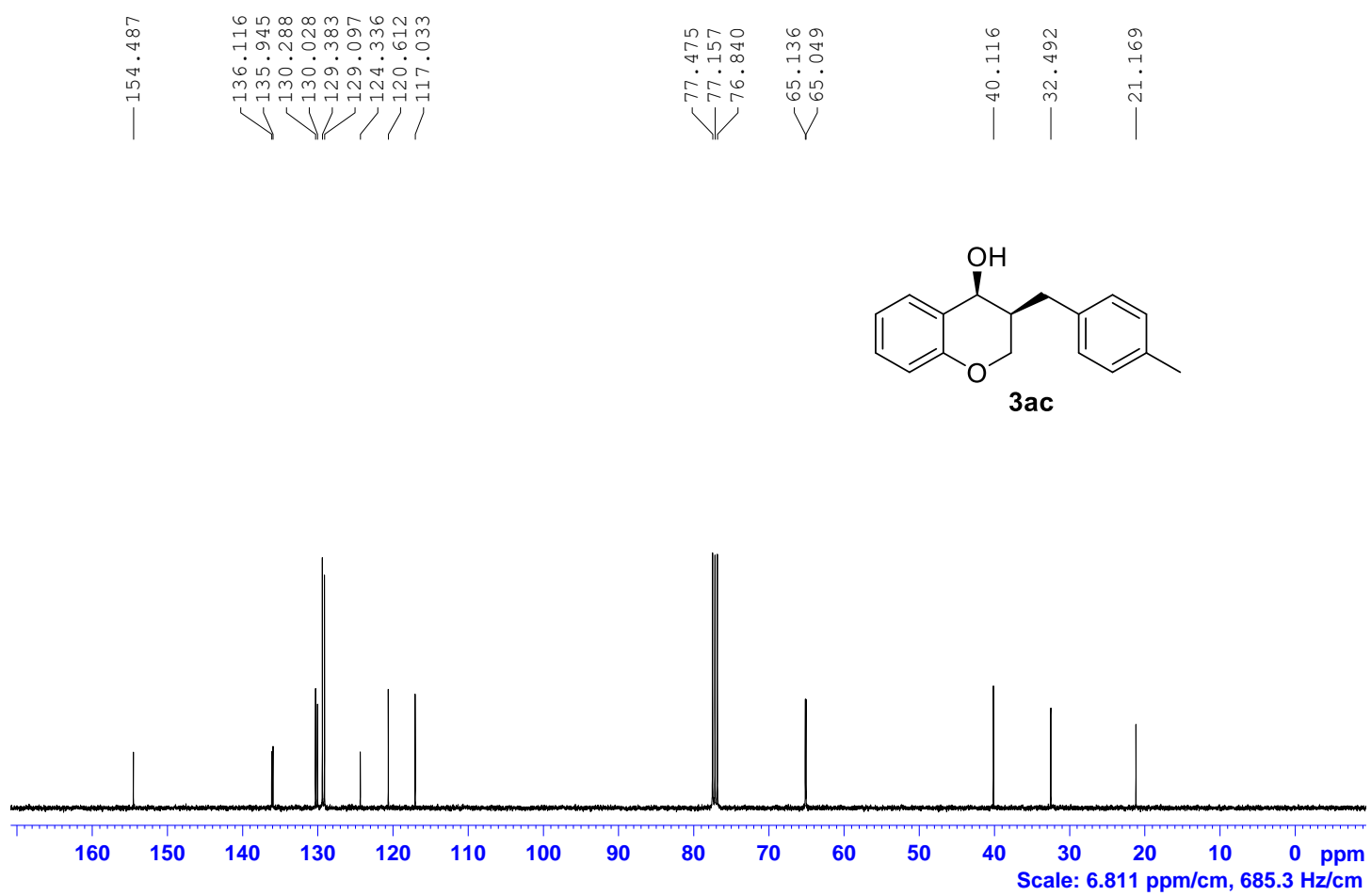

$\mathrm{S} 90$ 
${ }^{1} \mathrm{H}$ NMR spectrum of $\mathbf{3 a d}\left(\mathrm{CDCl}_{3}, 400 \mathrm{MHz}\right)$ :
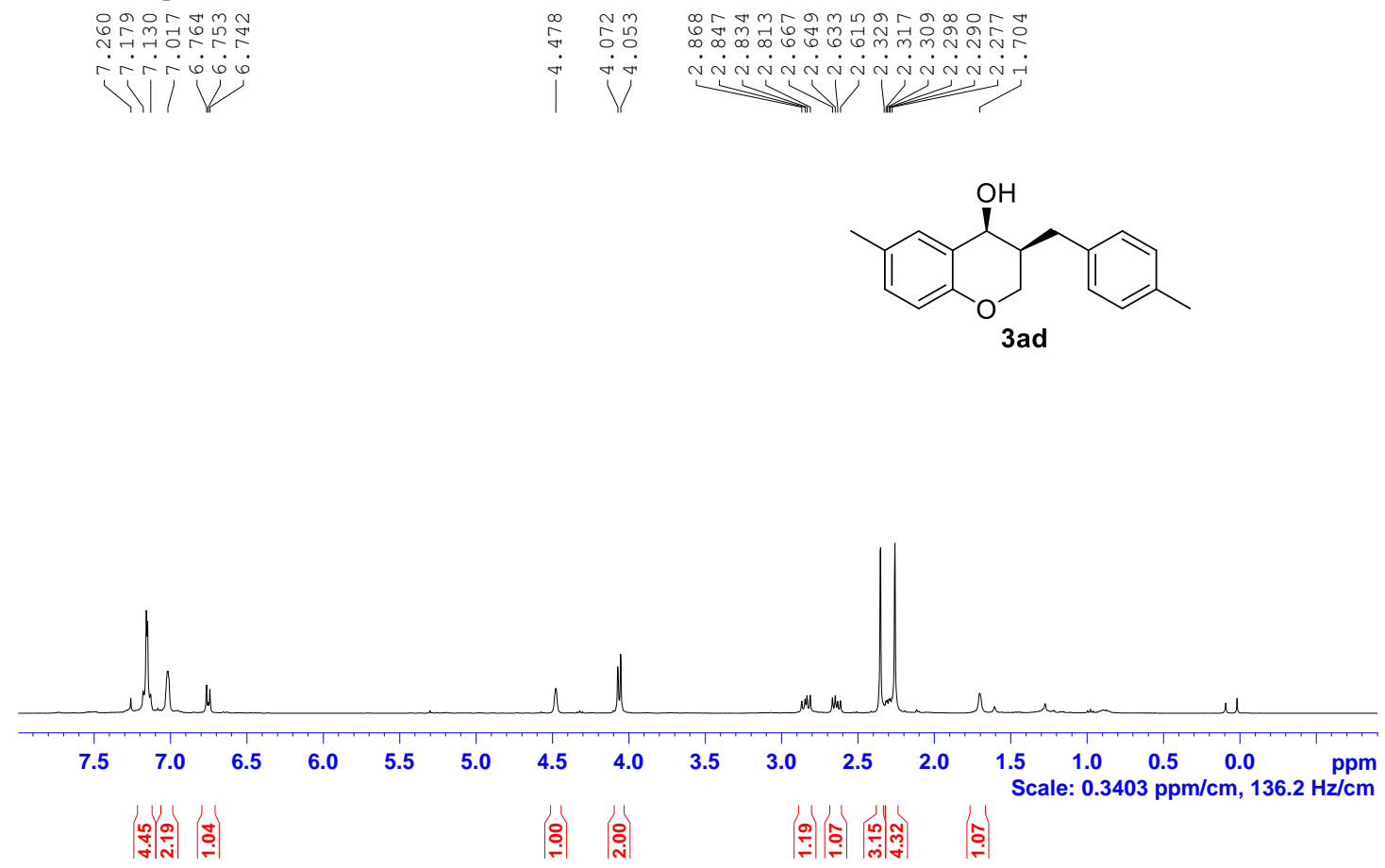

${ }^{13} \mathrm{C}\left\{{ }^{1} \mathrm{H}\right\}$ NMR spectrum of $\mathbf{3 a d}\left(\mathrm{CDCl}_{3}, 100 \mathrm{MHz}\right)$ :
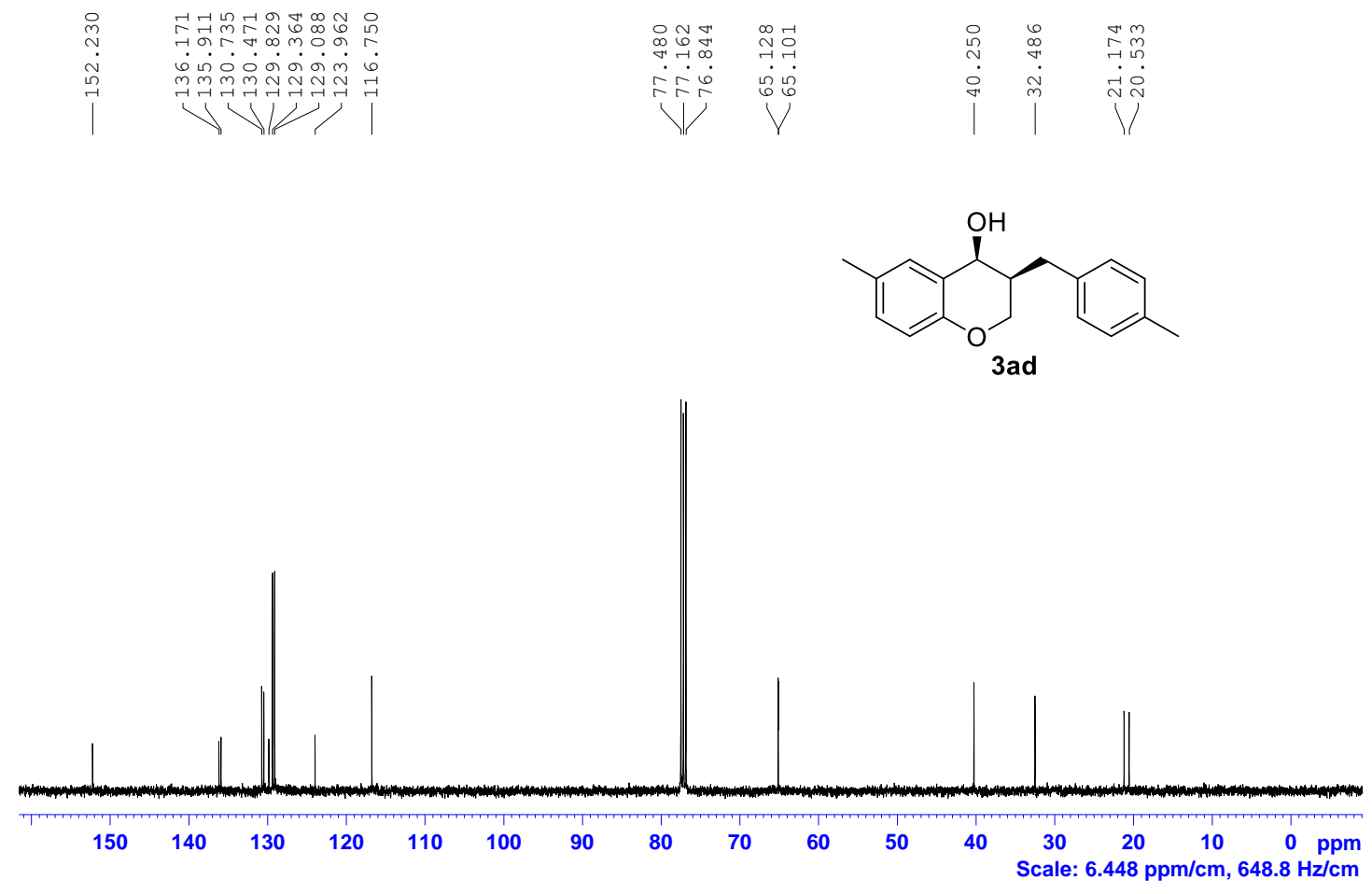
${ }^{1} \mathrm{H}$ NMR spectrum of $\mathbf{3 a e}\left(\mathrm{CDCl}_{3}, 400 \mathrm{MHz}\right)$ :

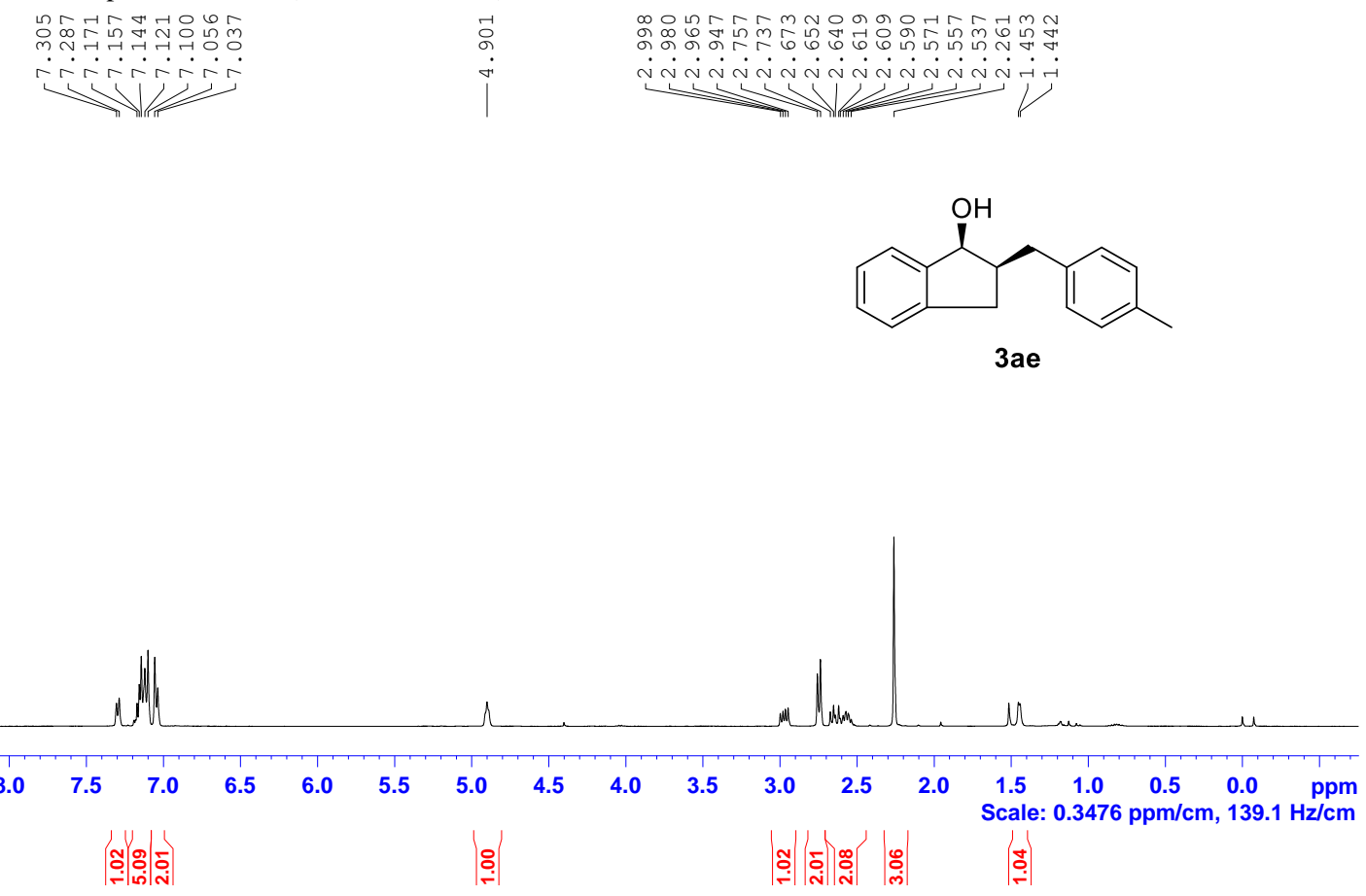

${ }^{13} \mathrm{C}\left\{{ }^{1} \mathrm{H}\right\}$ NMR spectrum of $\mathbf{3 a e}\left(\mathrm{CDCl}_{3}, 100 \mathrm{MHz}\right)$ :
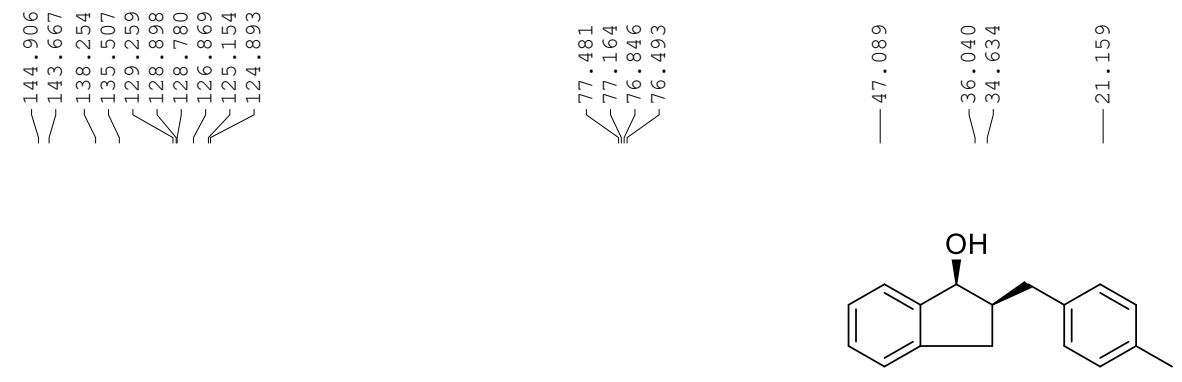

$3 a e$

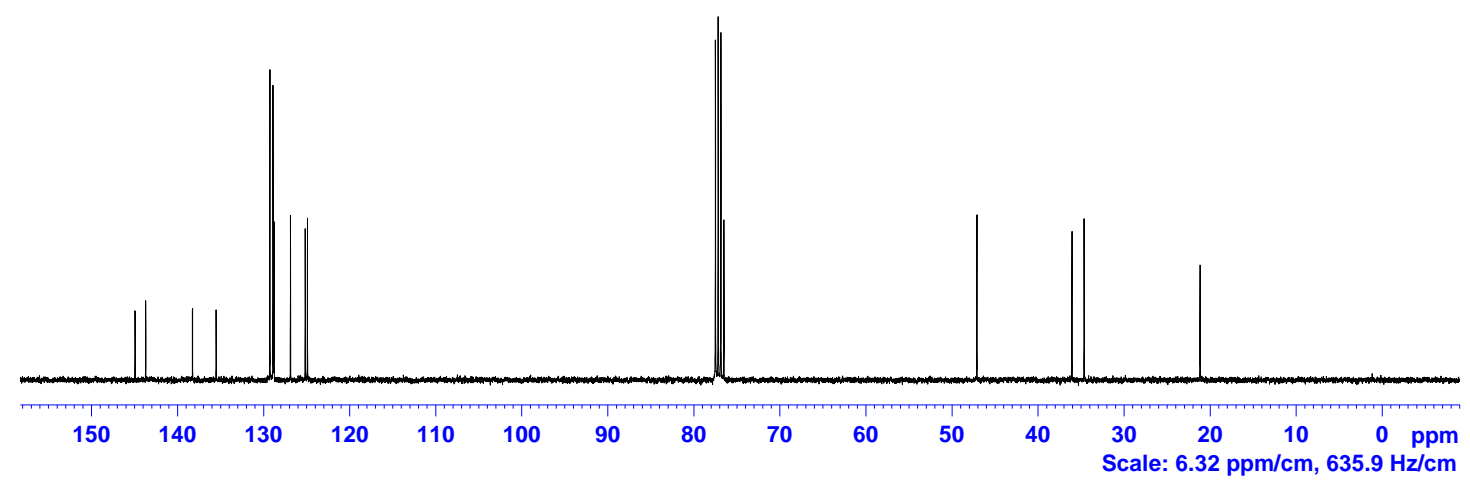


${ }^{1} \mathrm{H} \mathrm{NMR}$ spectrum of $\mathbf{3 a f}\left(\mathrm{CDCl}_{3}, 400 \mathrm{MHz}\right)$ :

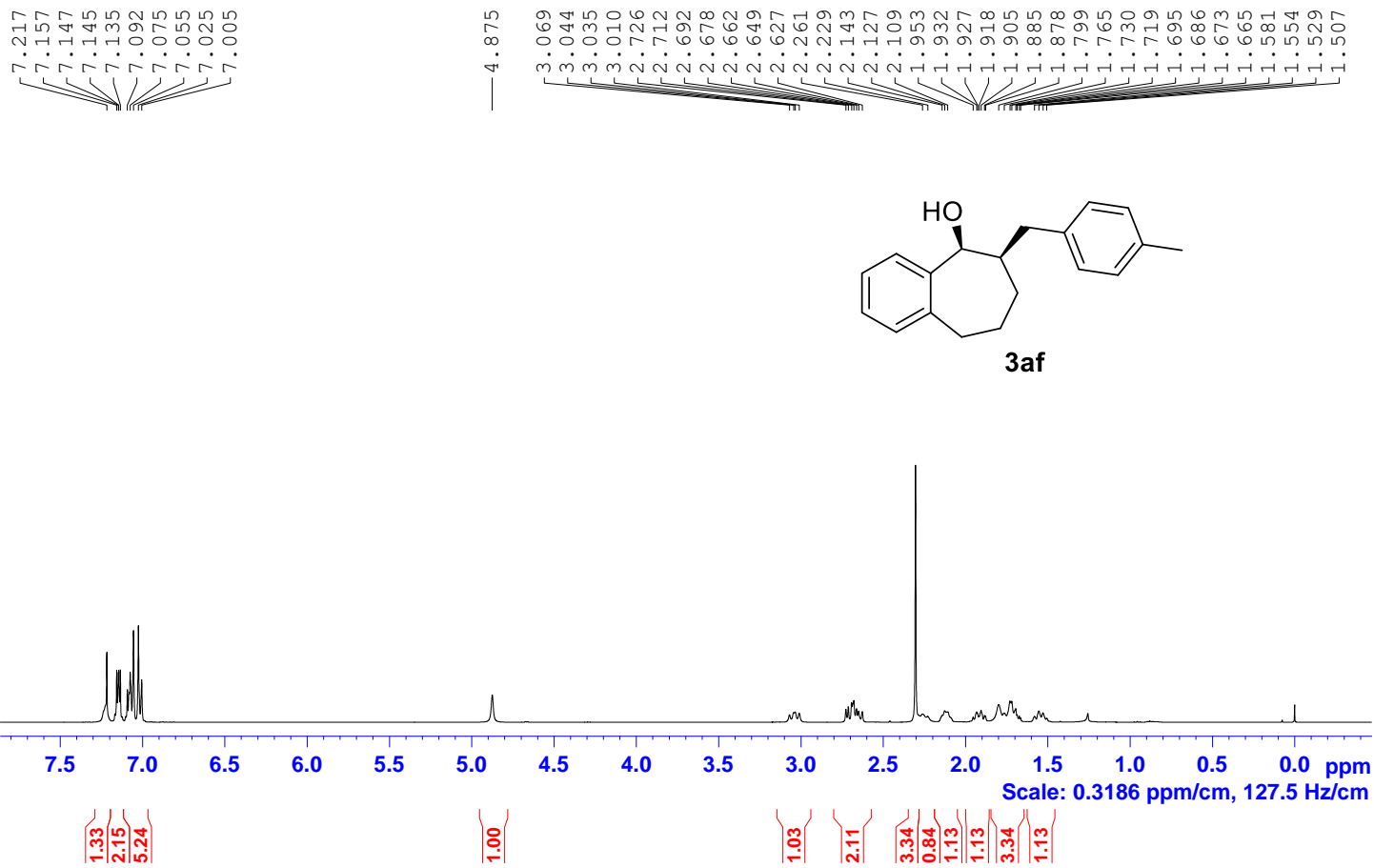

${ }^{13} \mathrm{C}\left\{{ }^{1} \mathrm{H}\right\}$ NMR spectrum of $\mathbf{3 a f}\left(\mathrm{CDCl}_{3}, 100 \mathrm{MHz}\right)$ :
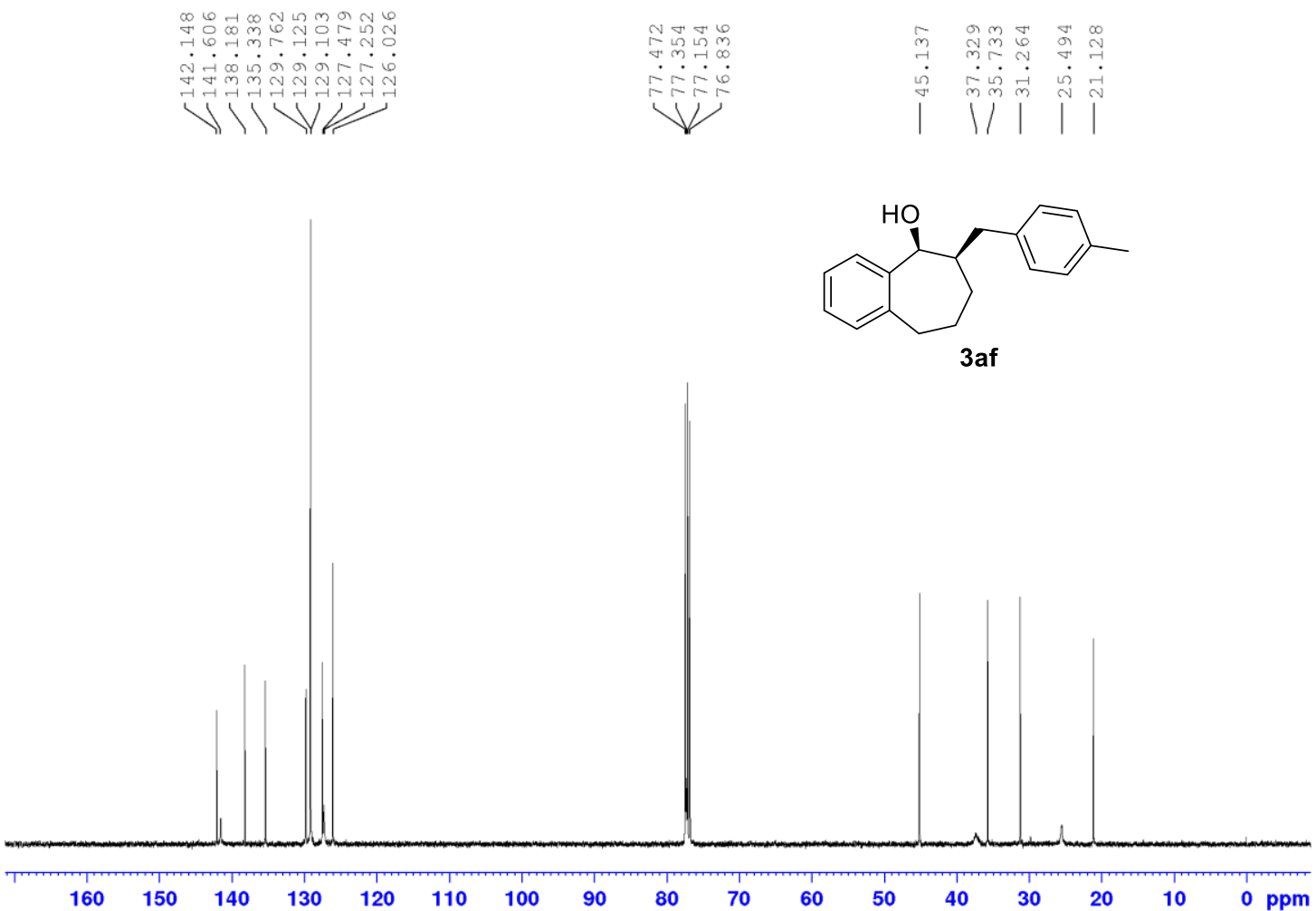
${ }^{1} \mathrm{H}$ NMR spectrum of $\mathbf{5 a}\left(\mathrm{CDCl}_{3}, 400 \mathrm{MHz}\right)$ :

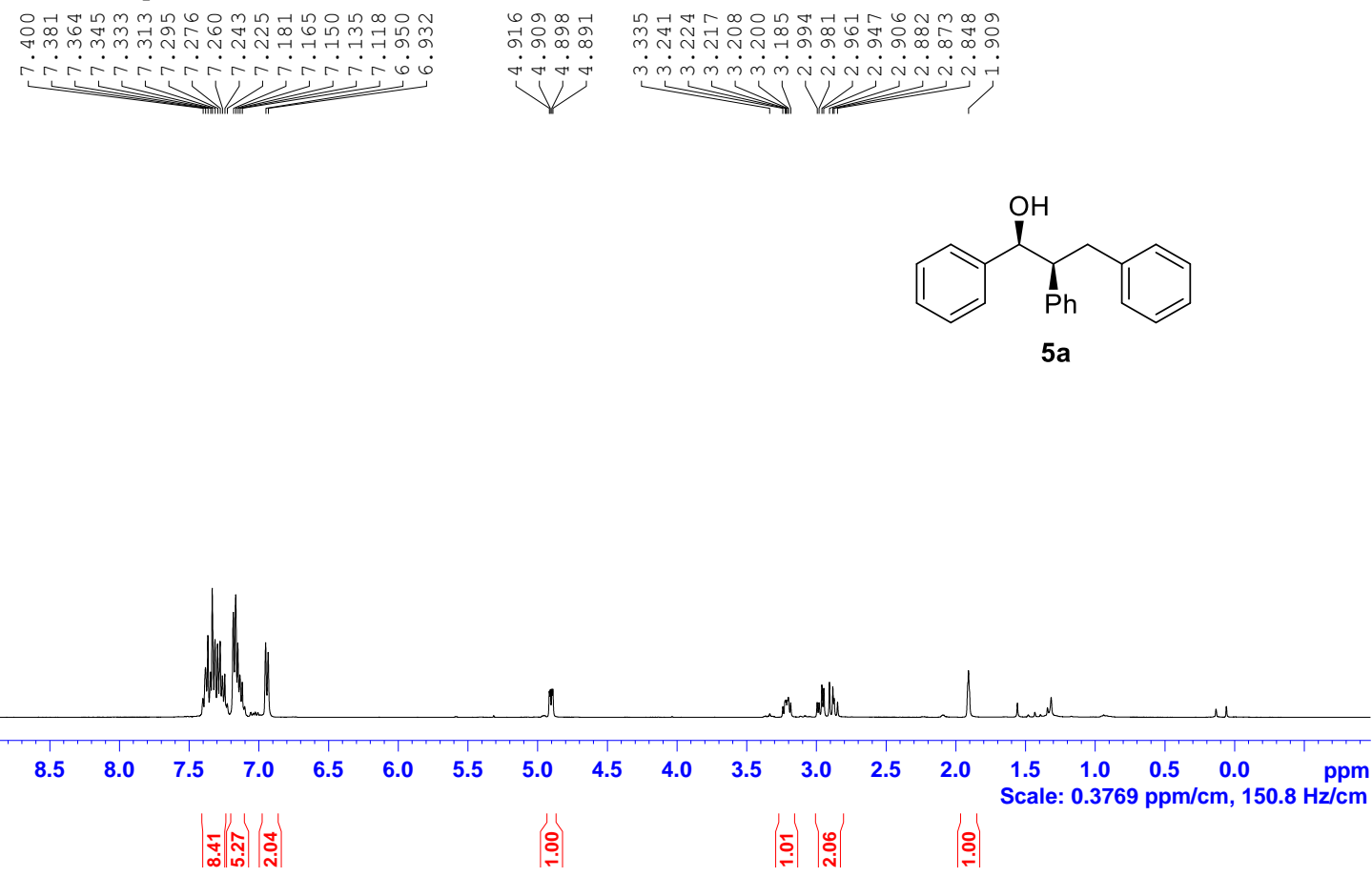

${ }^{13} \mathrm{C}\left\{{ }^{1} \mathrm{H}\right\}$ NMR spectrum of $\mathbf{5 a}\left(\mathrm{CDCl}_{3}, 100 \mathrm{MHz}\right)$ :
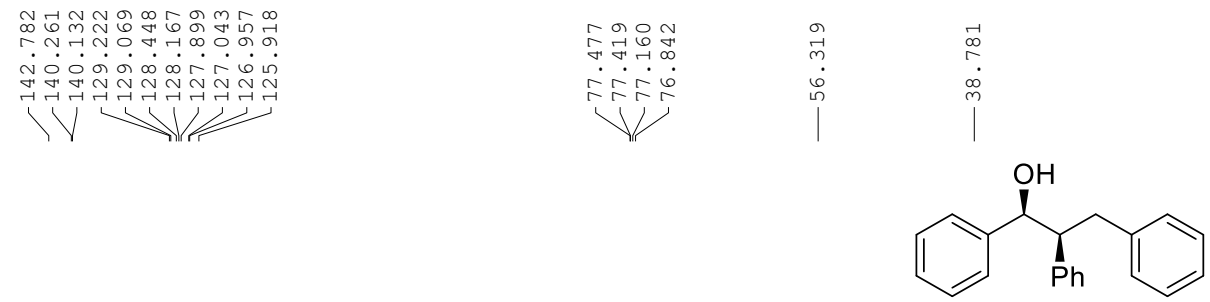

$5 a$

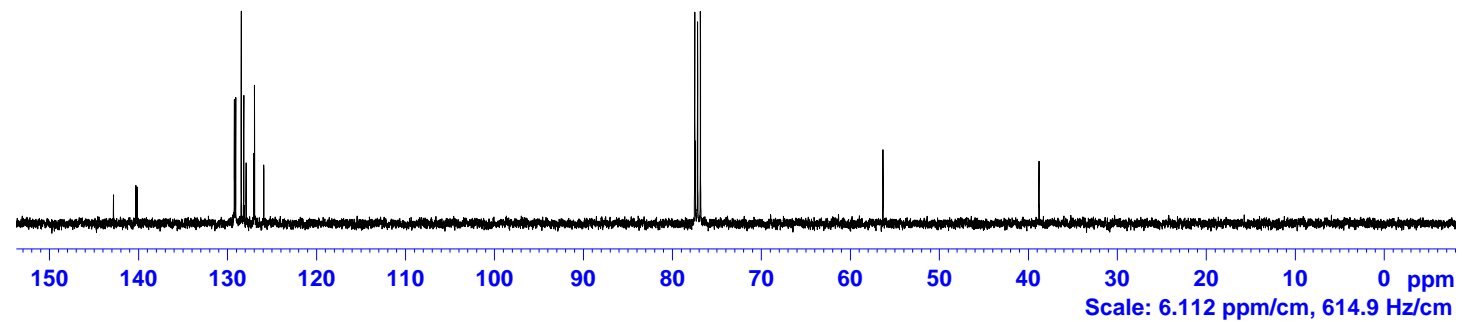


${ }^{1} \mathrm{H}$ NMR spectrum of $\mathbf{5 b}\left(\mathrm{CDCl}_{3}, 400 \mathrm{MHz}\right)$ :
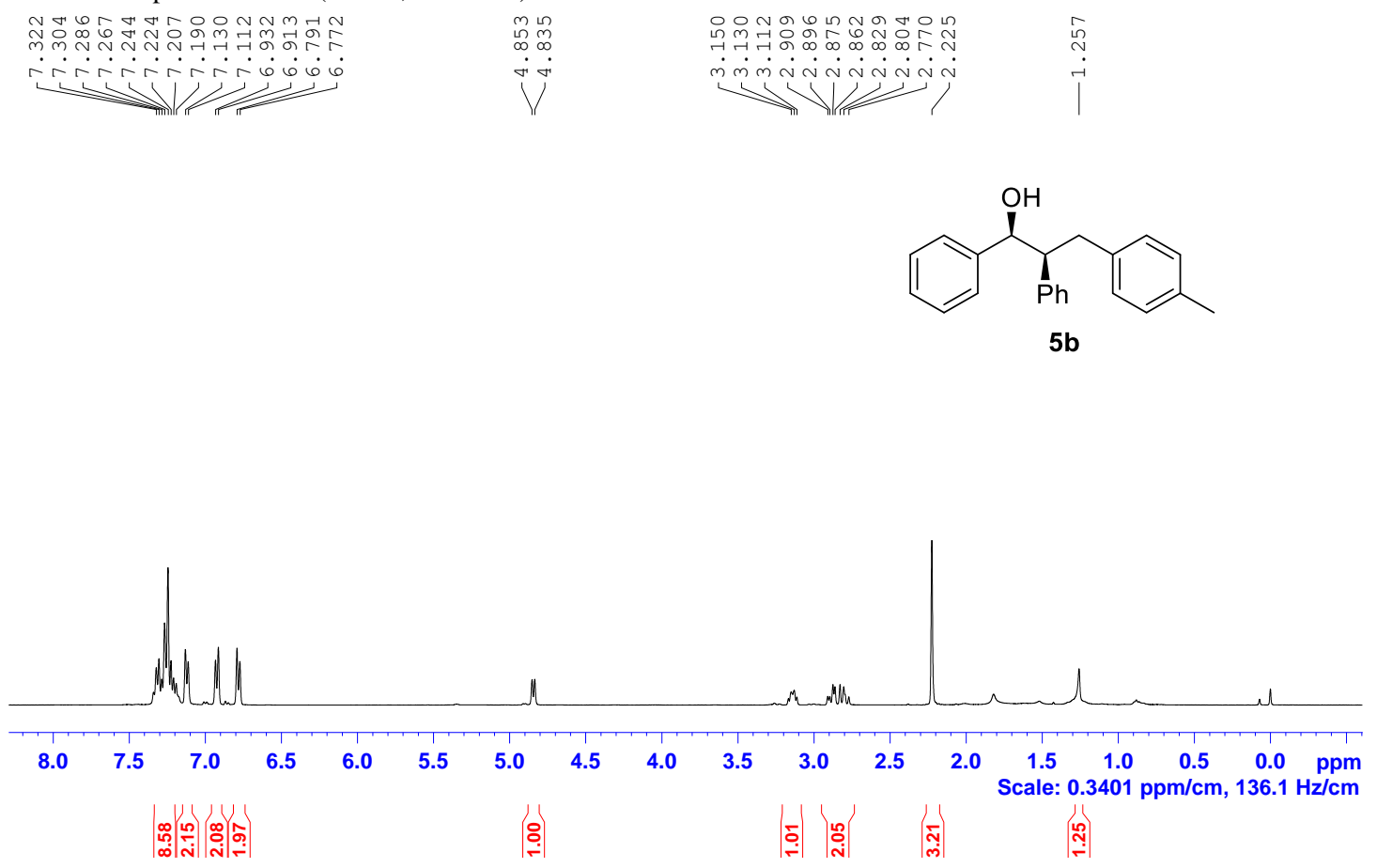

${ }^{13} \mathrm{C}\left\{{ }^{1} \mathrm{H}\right\}$ NMR spectrum of $\mathbf{5 b}\left(\mathrm{CDCl}_{3}, 100 \mathrm{MHz}\right)$ :
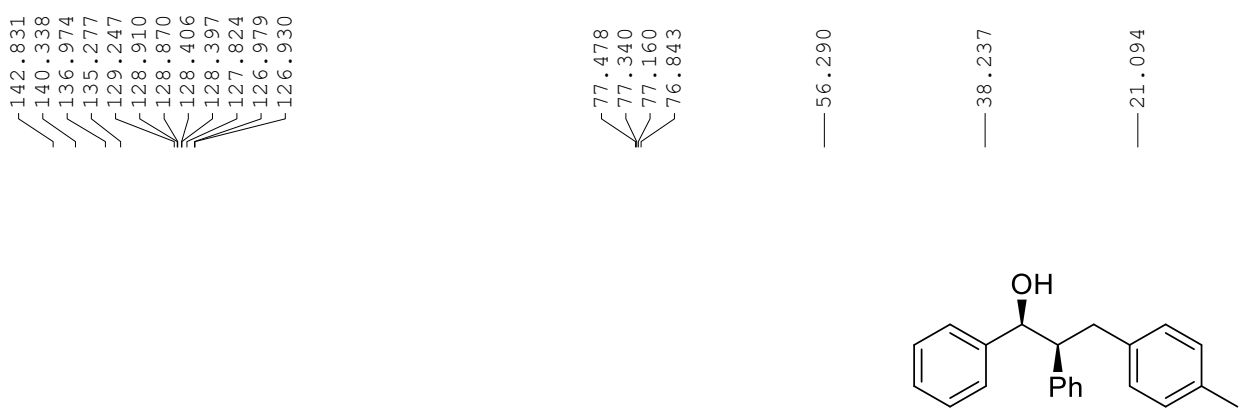

sb

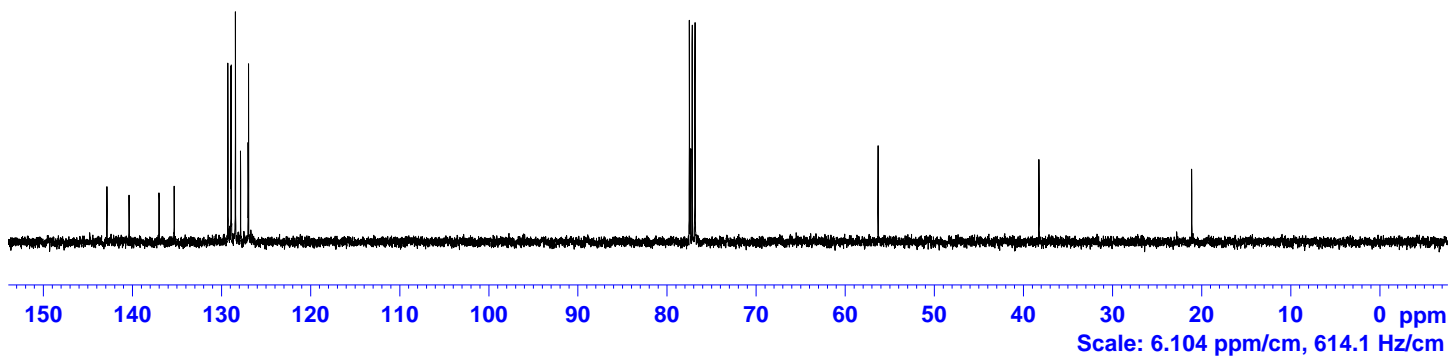

S95 
${ }^{1} \mathrm{H}$ NMR spectrum of $\mathbf{5 c}\left(\mathrm{CDCl}_{3}, 400 \mathrm{MHz}\right)$ :

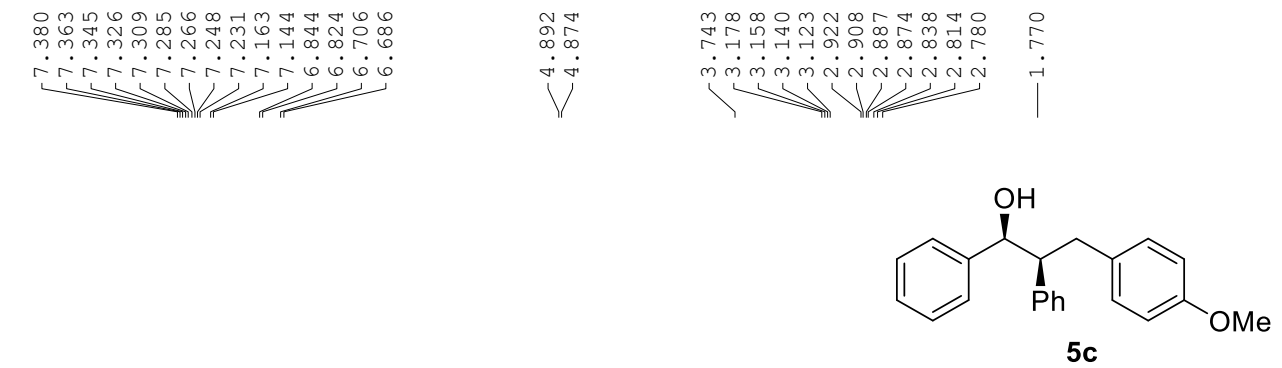

5c

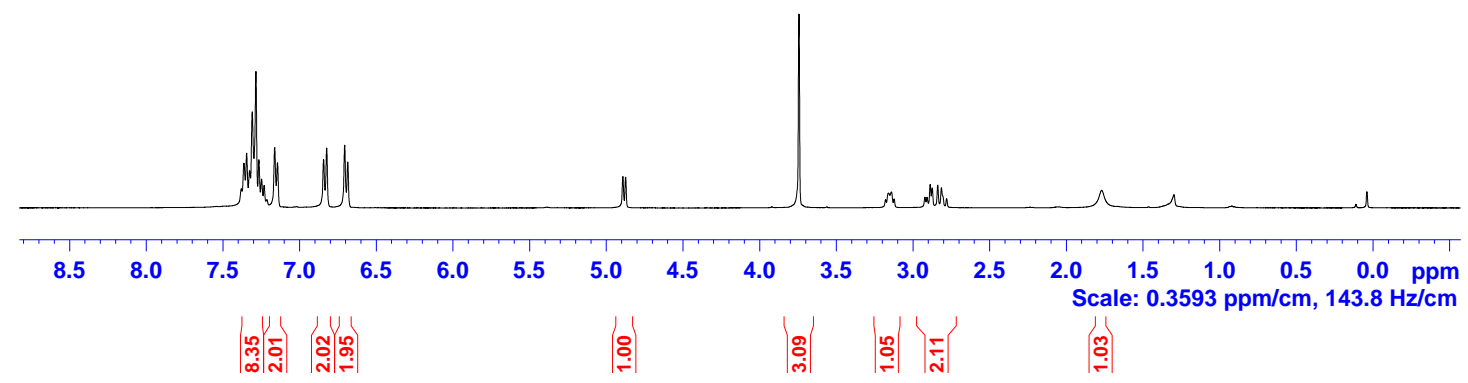

${ }^{13} \mathrm{C}\left\{{ }^{1} \mathrm{H}\right\}$ NMR spectrum of $\mathbf{5 c}\left(\mathrm{CDCl}_{3}, 100 \mathrm{MHz}\right)$ :
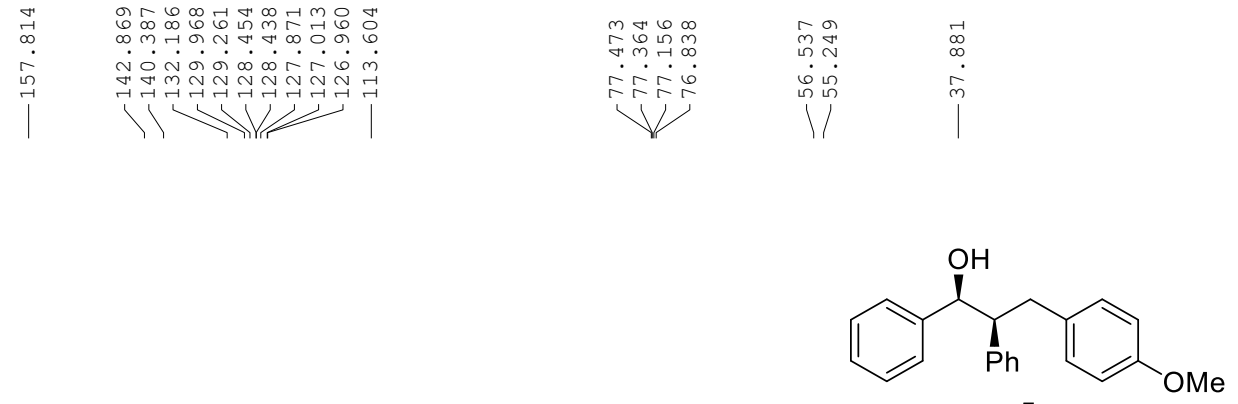

5c

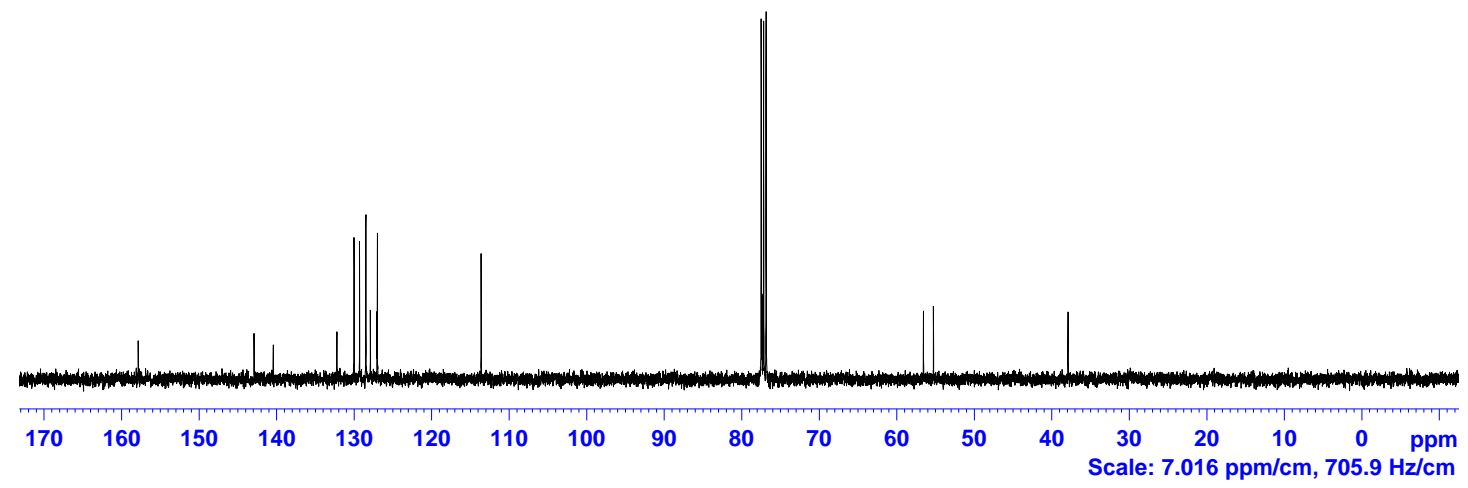


${ }^{1} \mathrm{H}$ NMR spectrum of $\mathbf{5 d}\left(\mathrm{CDCl}_{3}, 400 \mathrm{MHz}\right)$ :
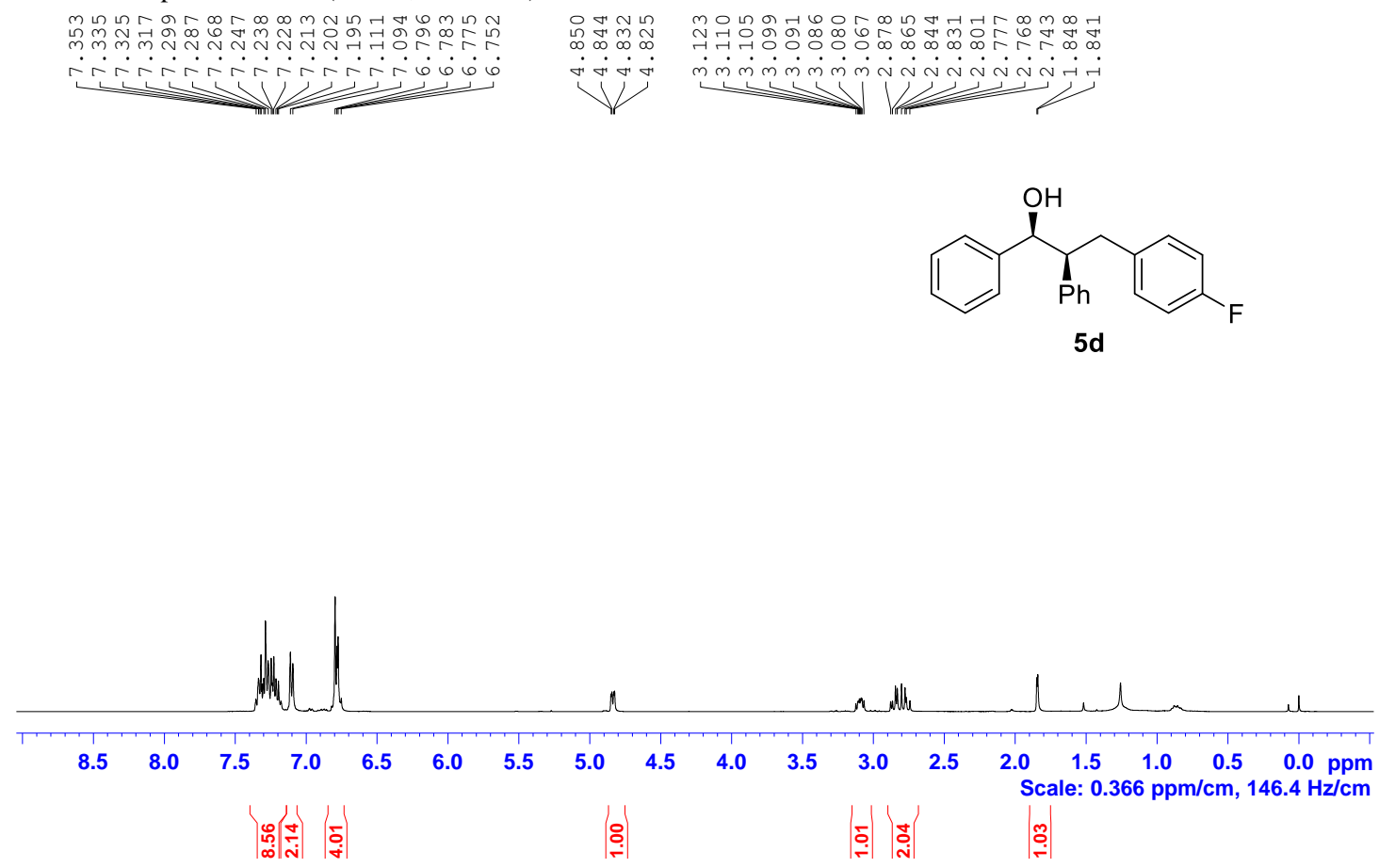

${ }^{13} \mathrm{C}\left\{{ }^{1} \mathrm{H}\right\}$ NMR spectrum of $\mathbf{5 d}\left(\mathrm{CDCl}_{3}, 100 \mathrm{MHz}\right)$ :

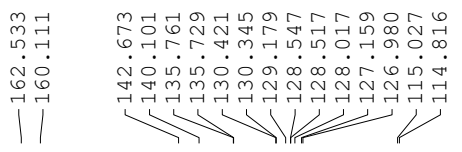

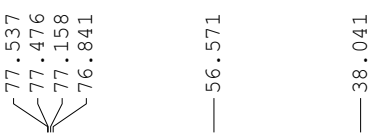<smiles>O[C@H](c1ccccc1)[C@H](Cc1ccc(F)cc1)Pc1ccccc1</smiles>

5d

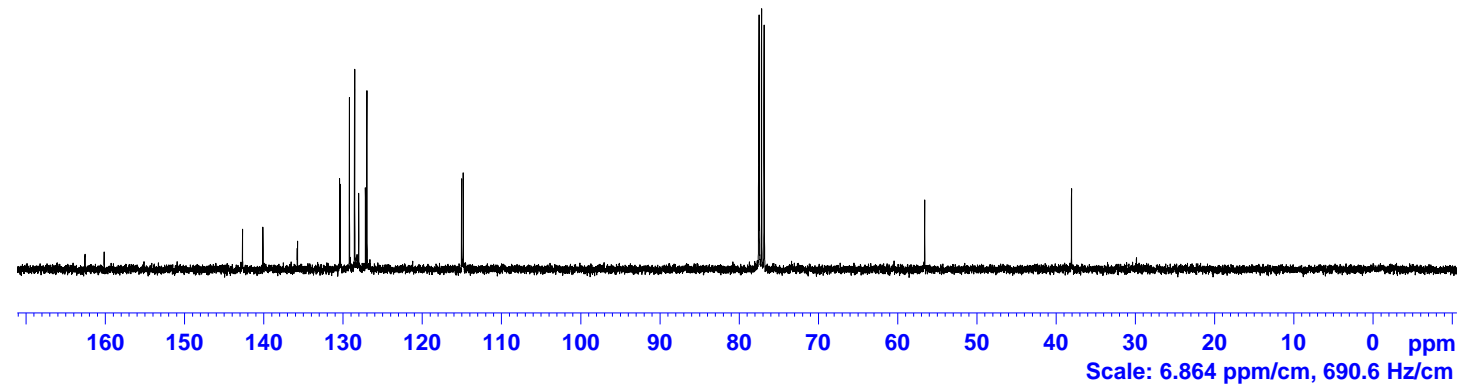


${ }^{1} \mathrm{H}$ NMR spectrum of $\mathbf{5 e}\left(\mathrm{CDCl}_{3}, 400 \mathrm{MHz}\right)$ :

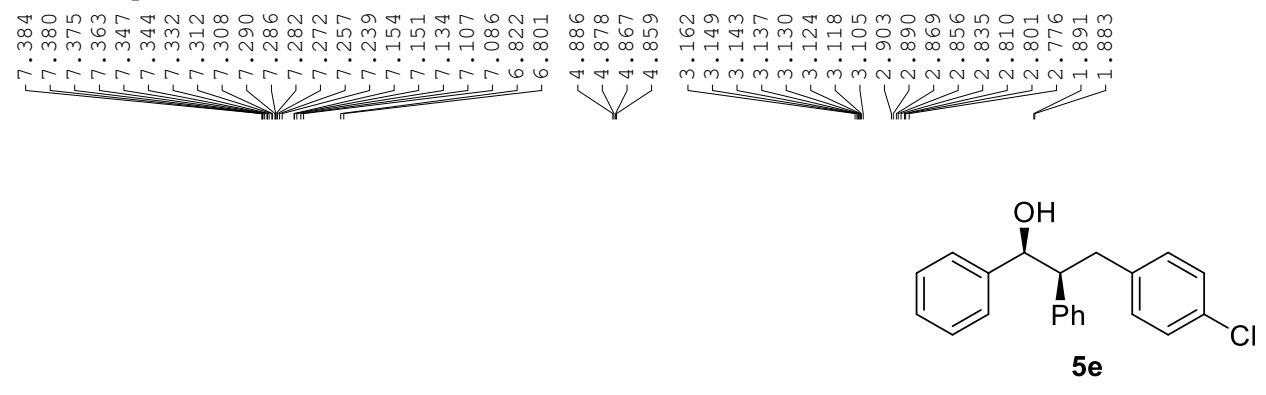

5 e

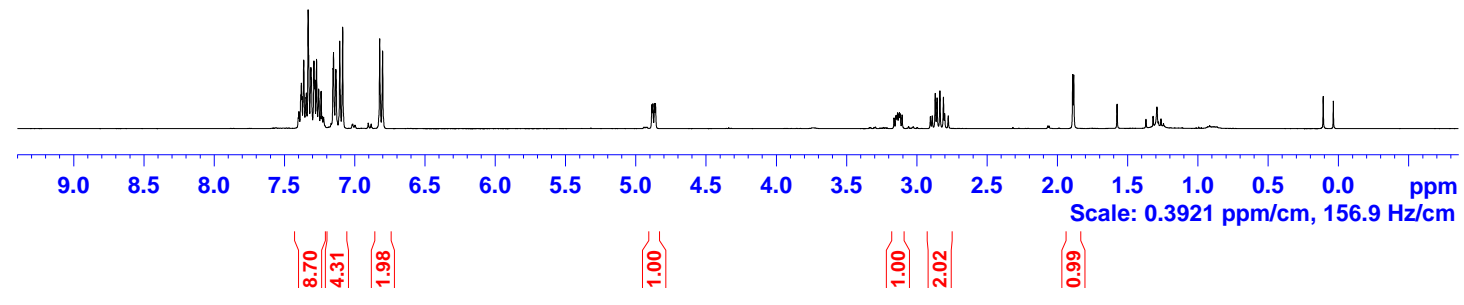

${ }^{13} \mathrm{C}\left\{{ }^{1} \mathrm{H}\right\}$ NMR spectrum of $\mathbf{5 e}\left(\mathrm{CDCl}_{3}, 100 \mathrm{MHz}\right)$ :

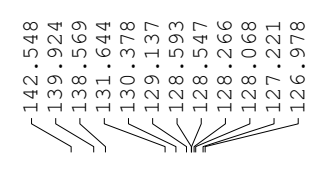

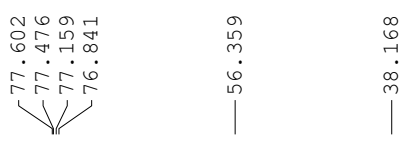

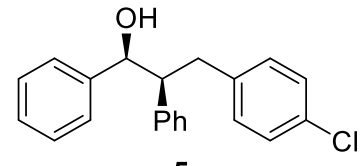

$5 e$

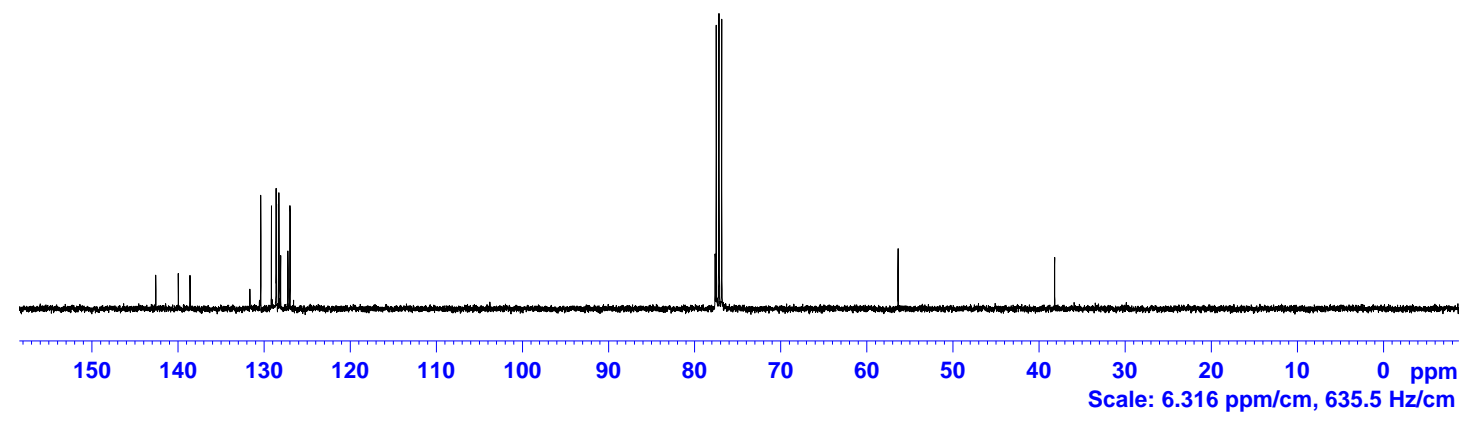


${ }^{1} \mathrm{H}$ NMR spectrum of $\mathbf{5 f}\left(\mathrm{CDCl}_{3}, 400 \mathrm{MHz}\right)$ :
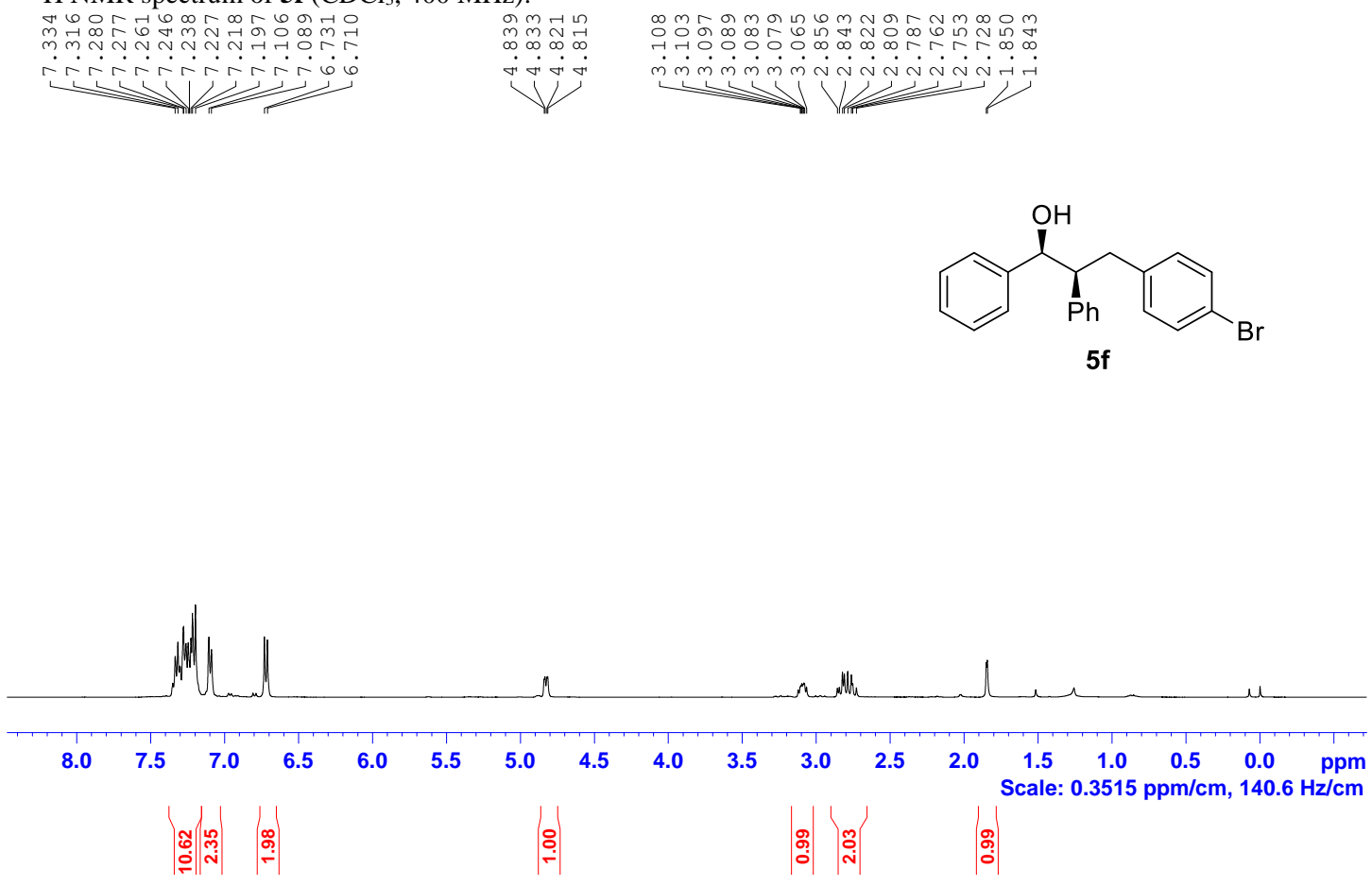

${ }^{13} \mathrm{C}\left\{{ }^{1} \mathrm{H}\right\}$ NMR spectrum of $\mathbf{5 f}\left(\mathrm{CDCl}_{3}, 100 \mathrm{MHz}\right)$ :
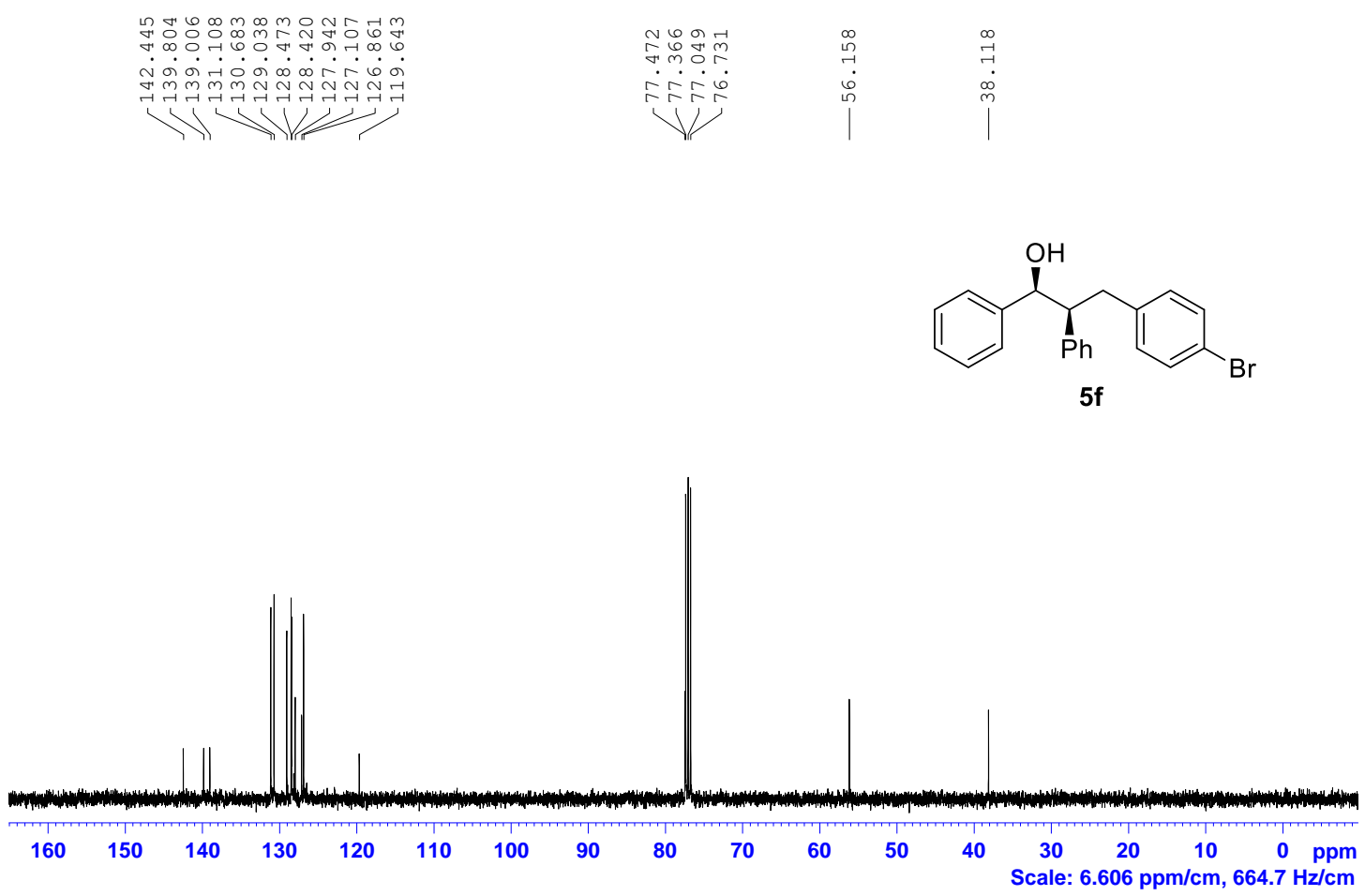

S99 
${ }^{1} \mathrm{H}$ NMR spectrum of $\mathbf{5 g}\left(\mathrm{CDCl}_{3}, 400 \mathrm{MHz}\right)$ :

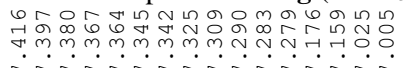

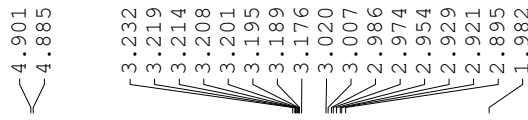<smiles>OC(c1ccccc1)[C@@H](Cc1ccc(C(F)(F)F)cc1)c1ccccc1</smiles>

$5 \mathrm{~g}$

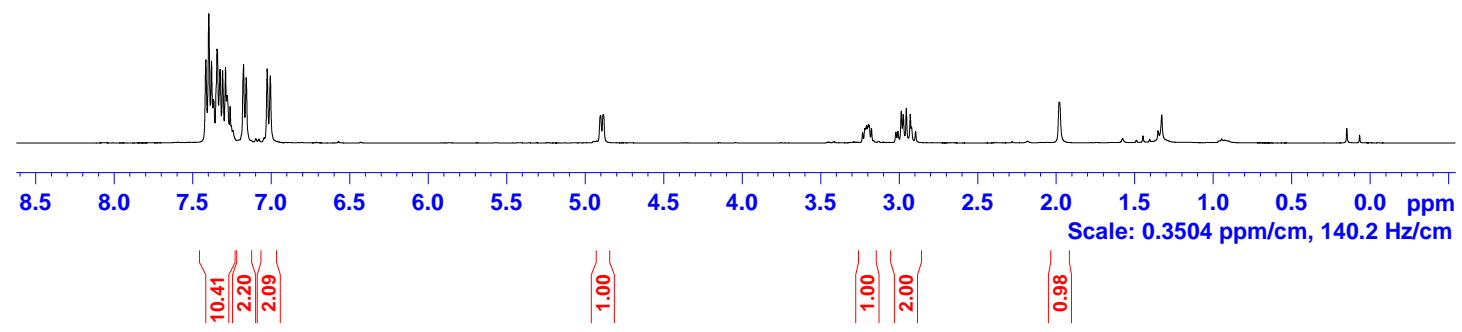

${ }^{13} \mathrm{C}\left\{{ }^{1} \mathrm{H}\right\}$ NMR spectrum of $\mathbf{5 g}\left(\mathrm{CDCl}_{3}, 100 \mathrm{MHz}\right)$ :
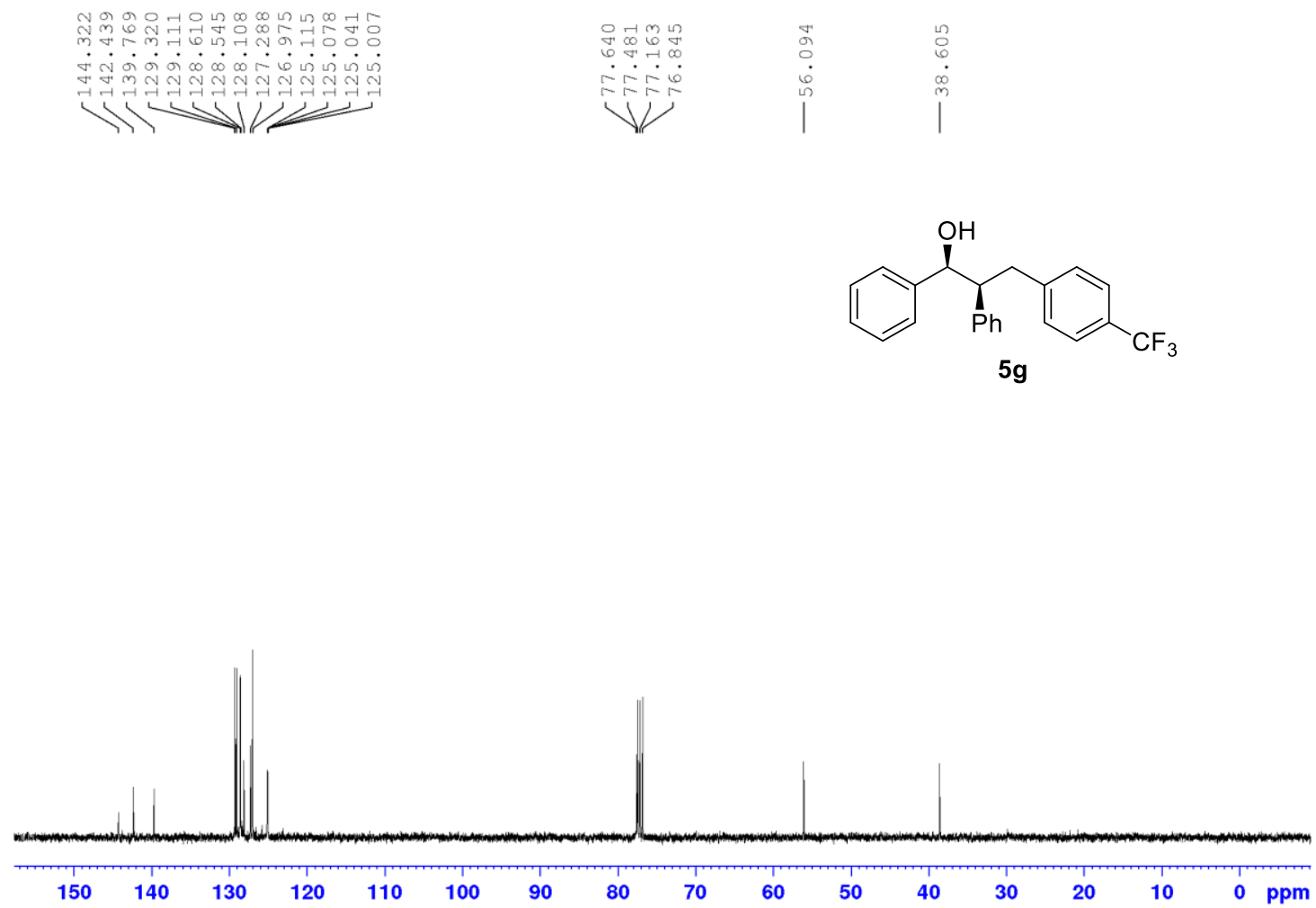
${ }^{1} \mathrm{H}$ NMR spectrum of $\mathbf{5 h}\left(\mathrm{CDCl}_{3}, 400 \mathbf{M H z}\right)$ :

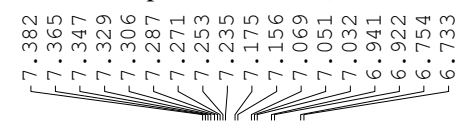

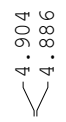
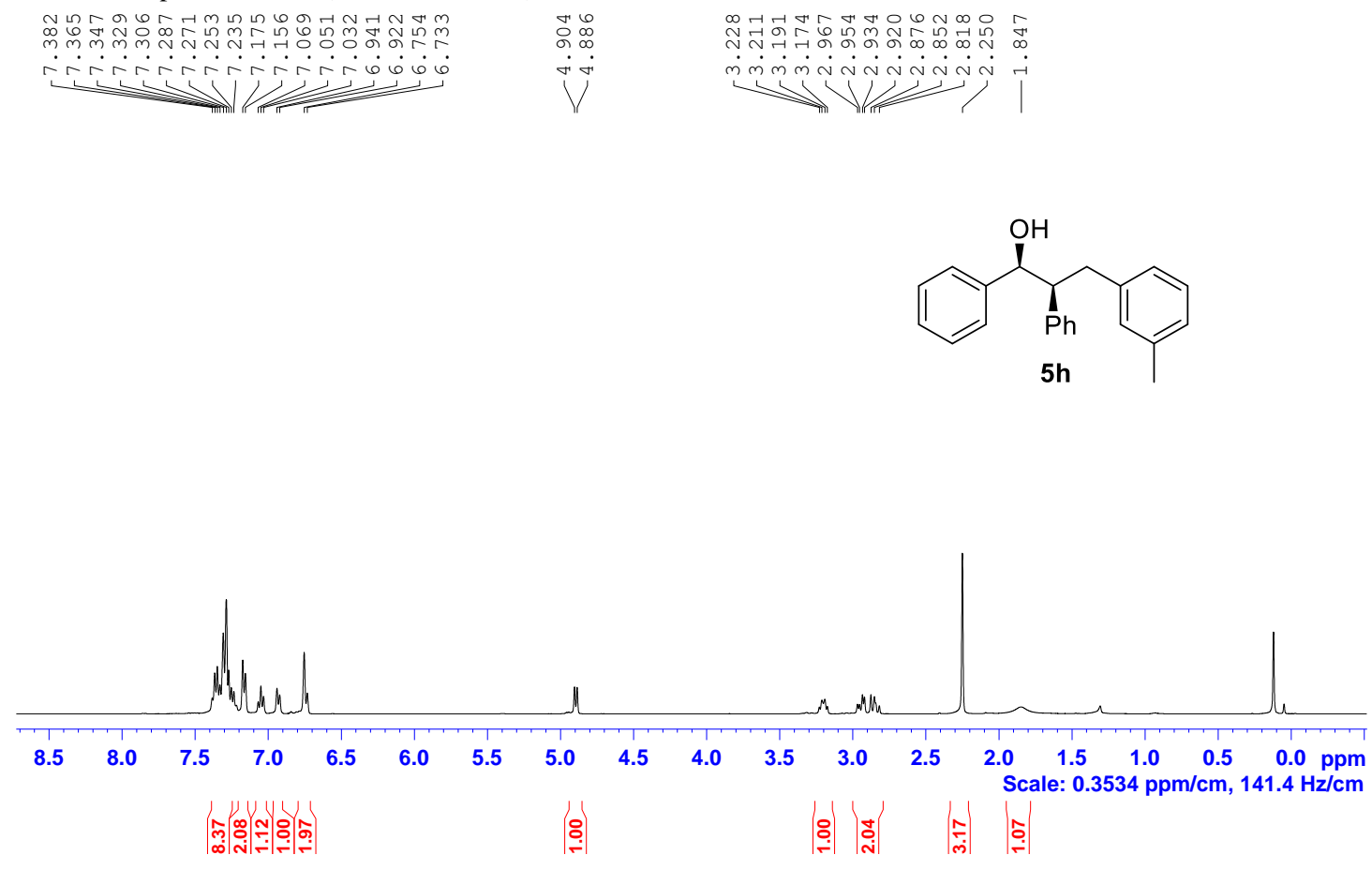

${ }^{13} \mathrm{C}\left\{{ }^{1} \mathrm{H}\right\}$ NMR spectrum of $\mathbf{5 h}\left(\mathrm{CDCl}_{3}, 100 \mathrm{MHz}\right)$ :

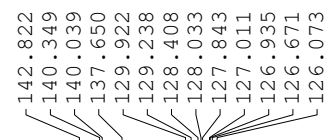

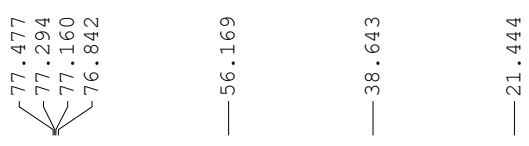<smiles>Cc1cccc(C[C@H](c2ccccc2)[C@@H](O)c2ccccc2)c1</smiles>

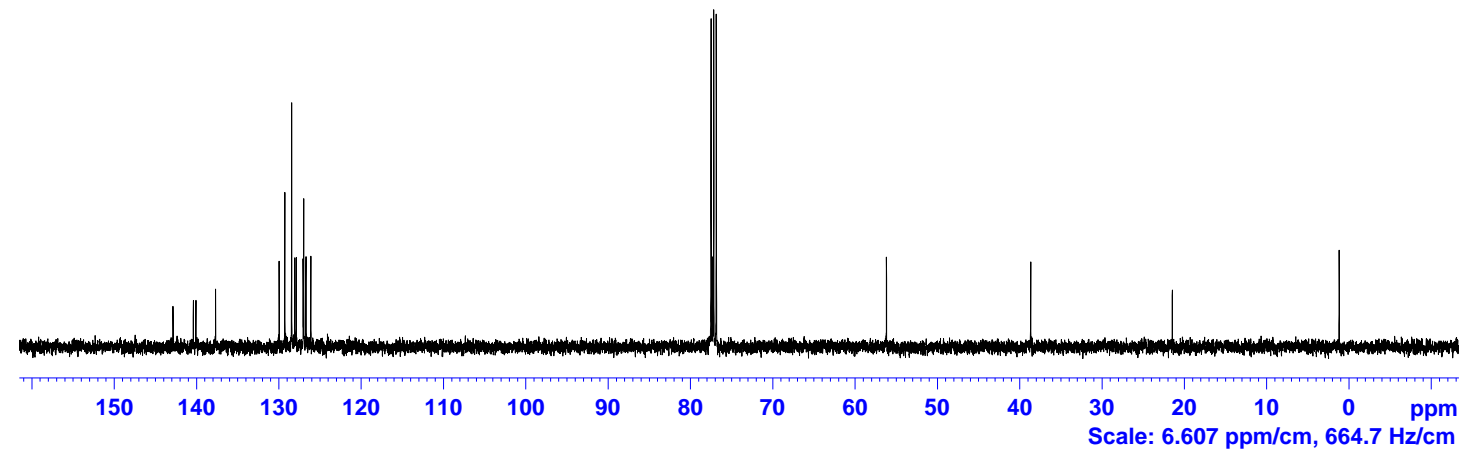


${ }^{1} \mathrm{H}$ NMR spectrum of $\mathbf{5 i}\left(\mathrm{CDCl}_{3}, 400 \mathrm{MHz}\right)$ :
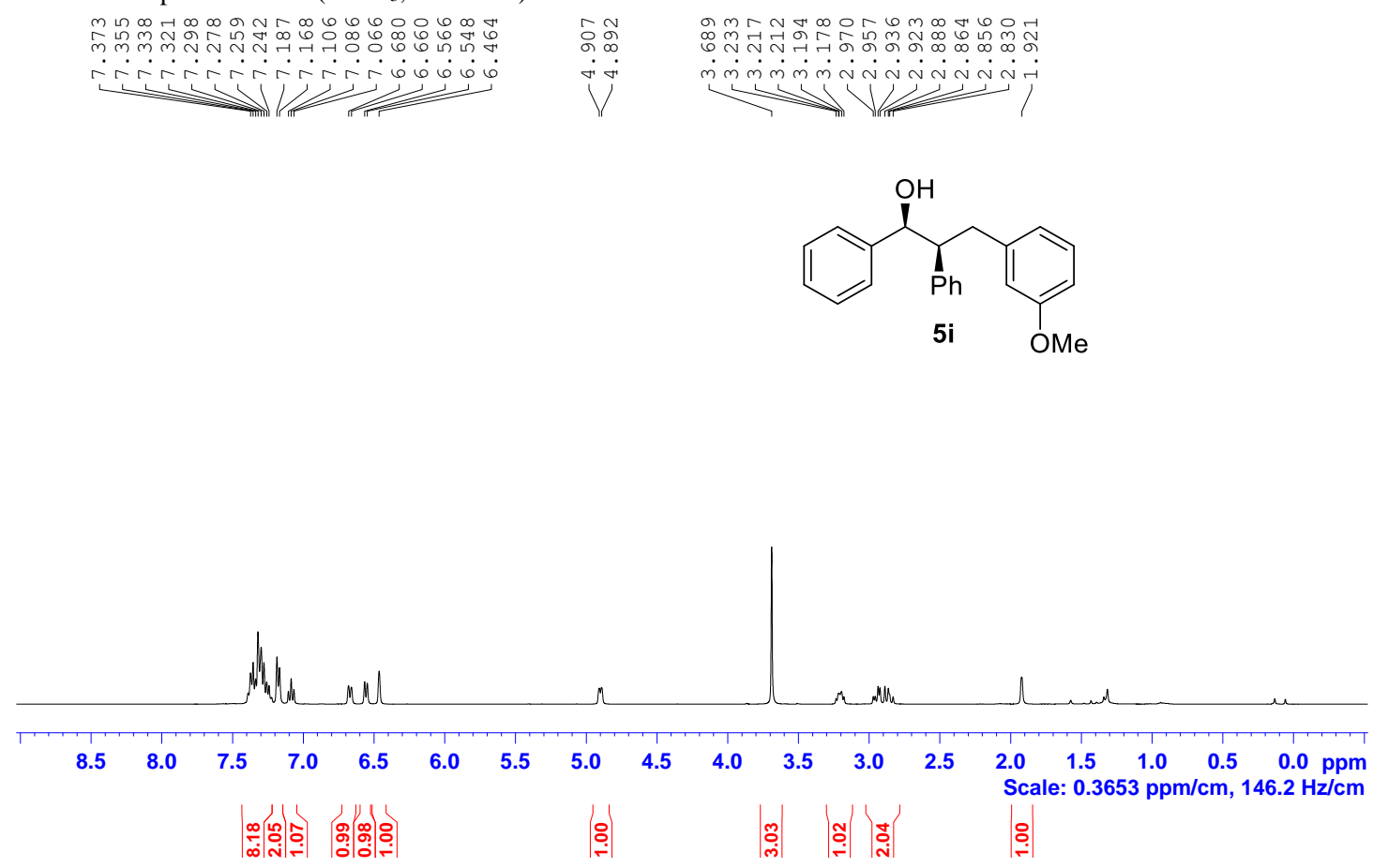

${ }^{13} \mathrm{C}\left\{{ }^{1} \mathrm{H}\right\}$ NMR spectrum of $\mathbf{5 i}\left(\mathrm{CDCl}_{3}, 100 \mathrm{MHz}\right)$ :
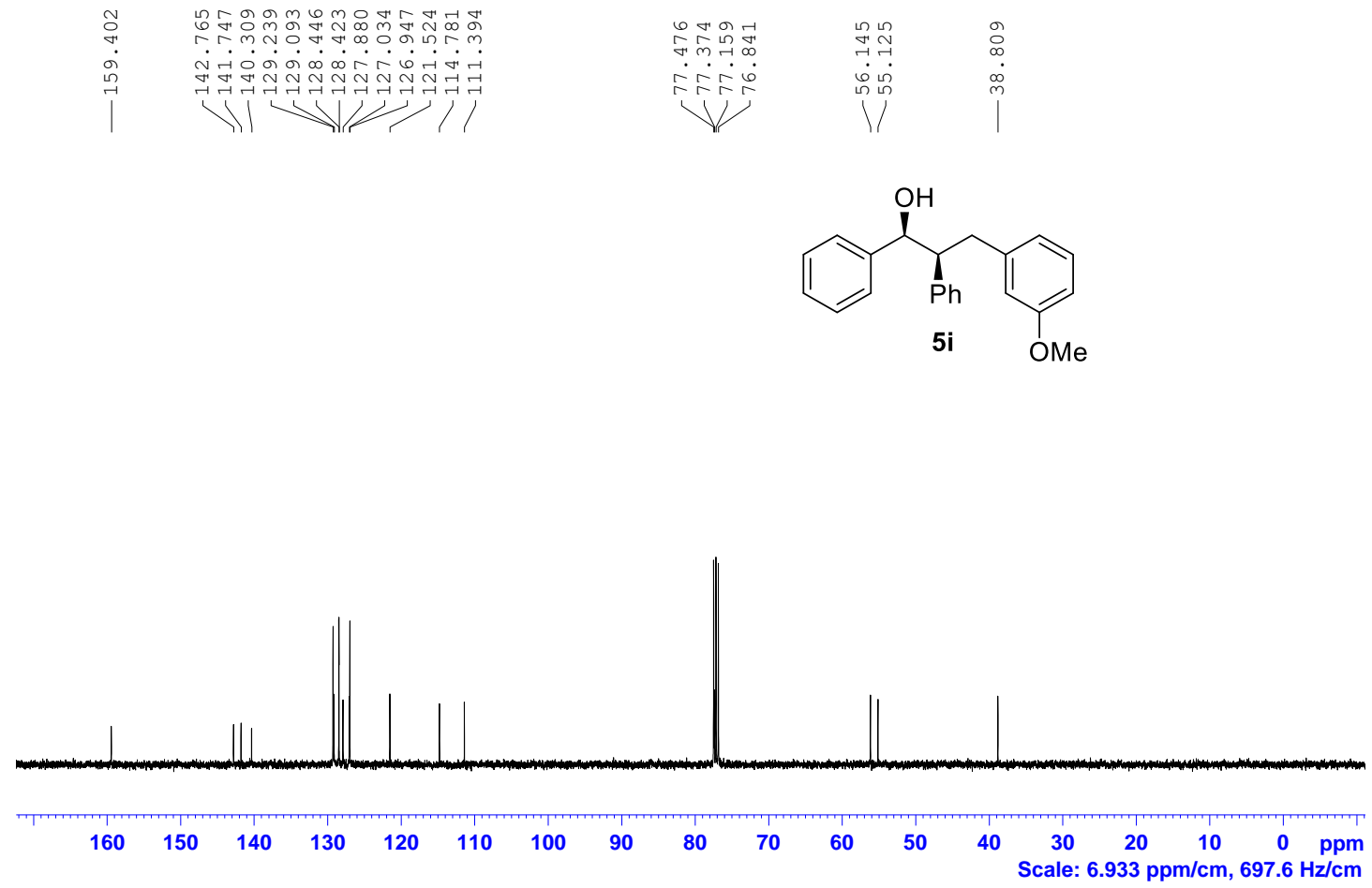
${ }^{1} \mathrm{H}$ NMR spectrum of $\mathbf{5 j}\left(\mathrm{CDCl}_{3}, 400 \mathrm{MHz}\right)$ :
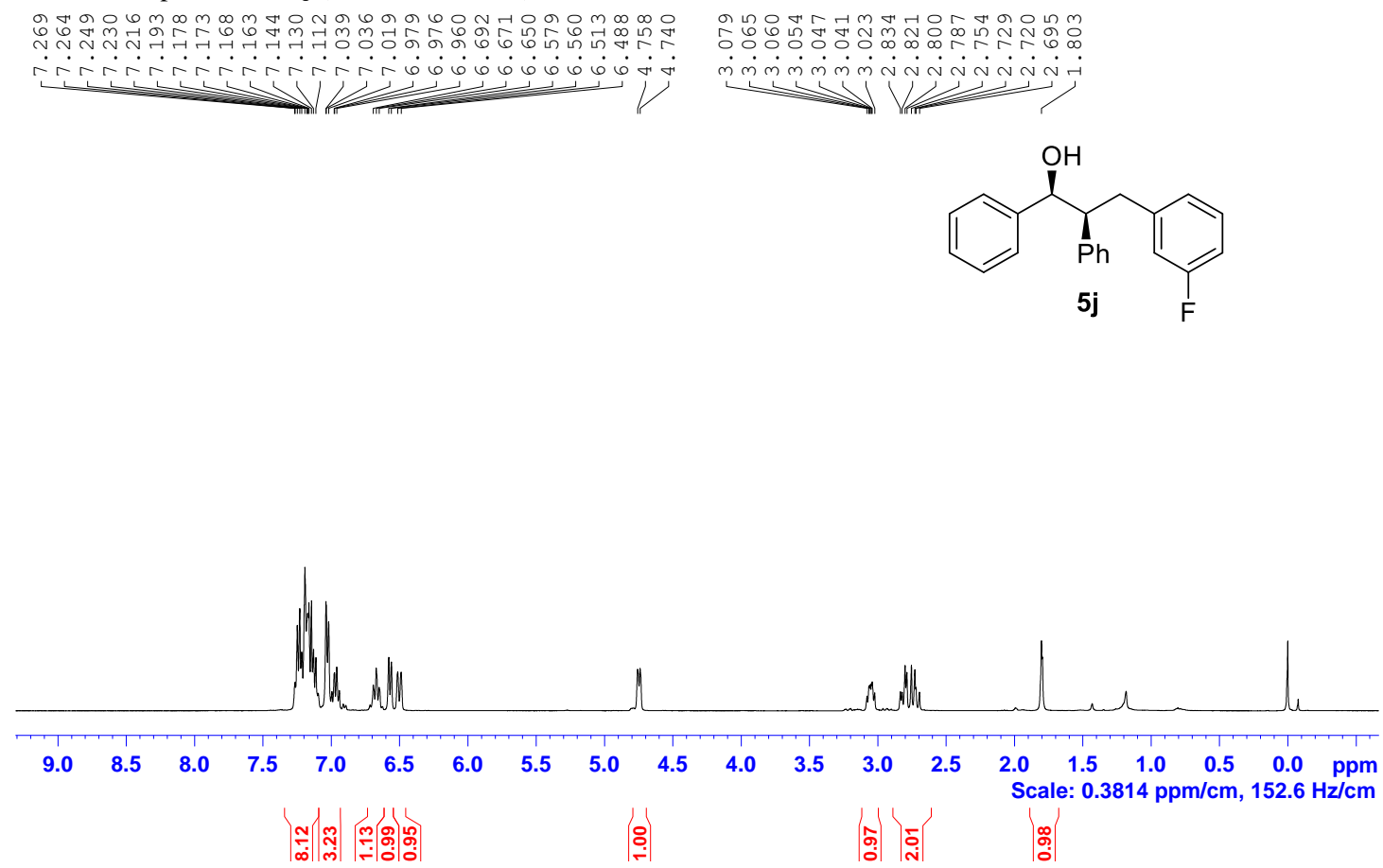

${ }^{13} \mathrm{C}\left\{{ }^{1} \mathrm{H}\right\}$ NMR spectrum of $\mathbf{5 j}\left(\mathrm{CDCl}_{3}, 100 \mathrm{MHz}\right)$ :

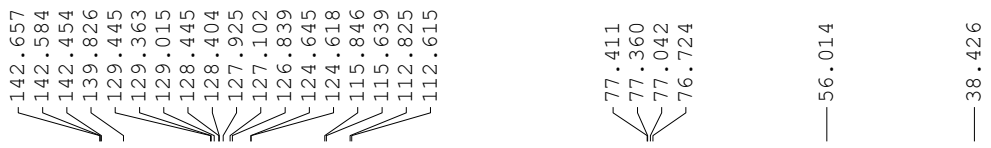<smiles>O[C@H](c1ccccc1)C(c1cccc(F)c1)c1cccc(F)c1</smiles>

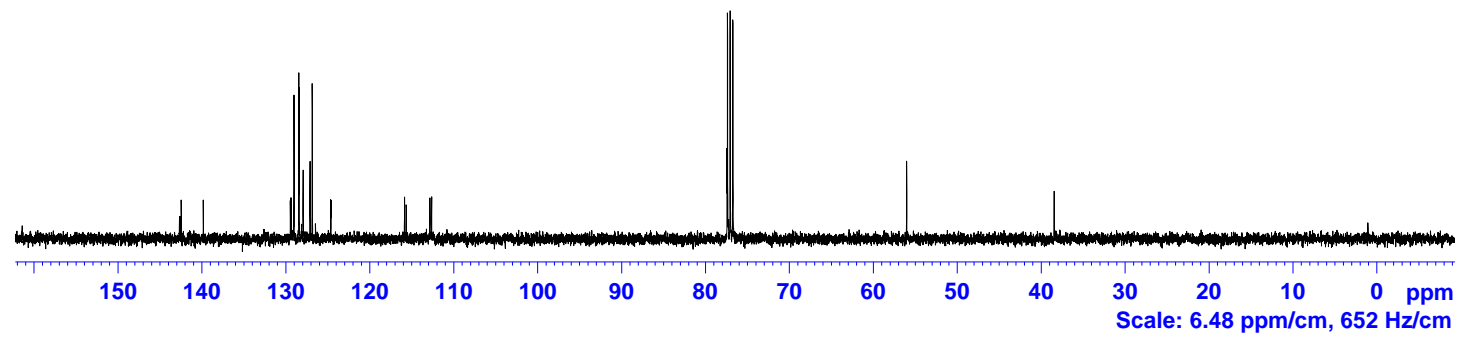


${ }^{1} \mathrm{H}$ NMR spectrum of $\mathbf{5 k}\left(\mathrm{CDCl}_{3}, 400 \mathrm{MHz}\right)$ :
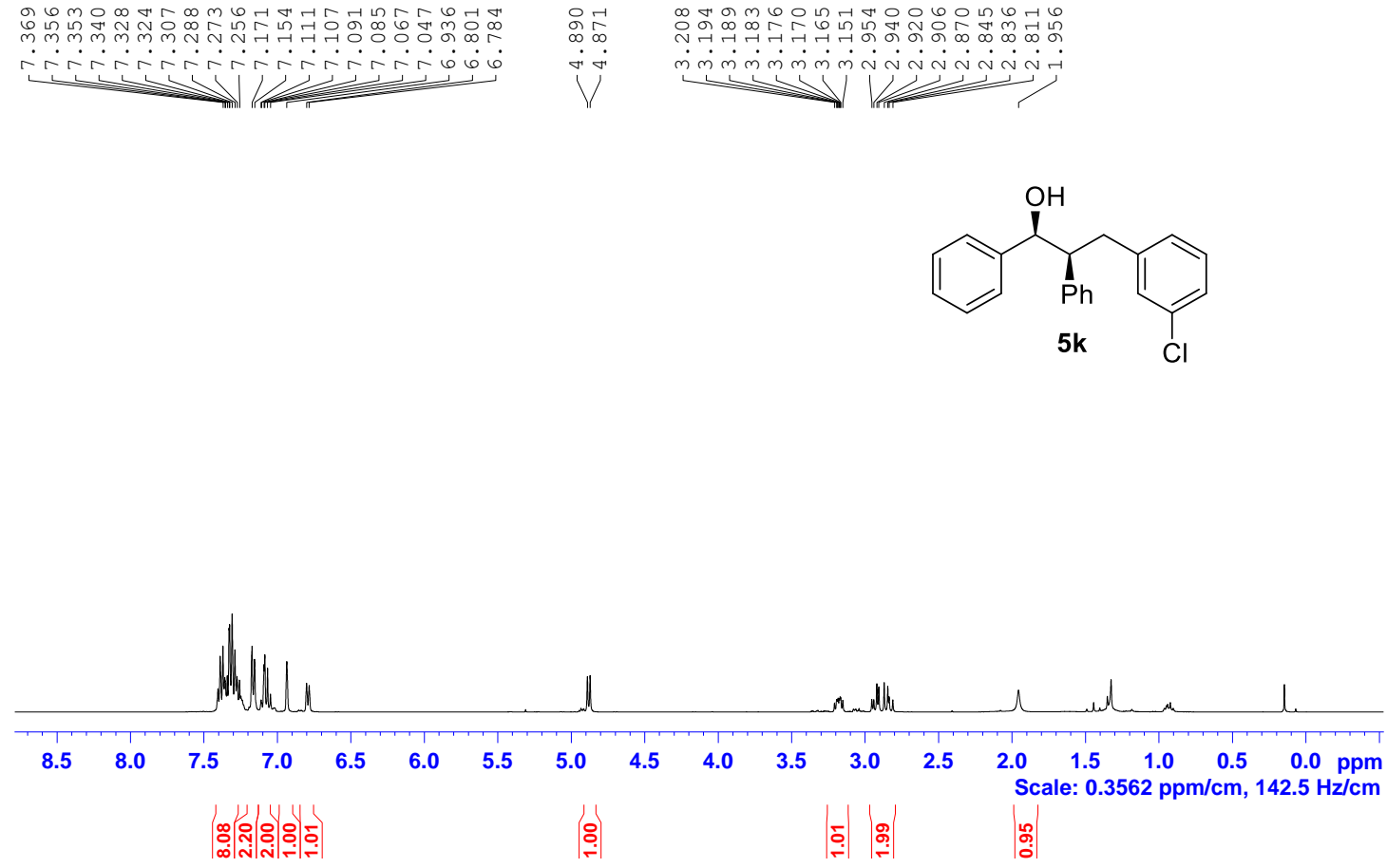

${ }^{13} \mathrm{C}\left\{{ }^{1} \mathrm{H}\right\}$ NMR spectrum of $\mathbf{5 k}\left(\mathrm{CDCl}_{3}, 100 \mathrm{MHz}\right)$ :

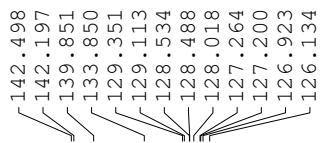

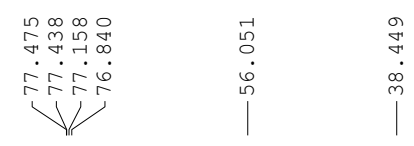<smiles>O[C@H](c1ccccc1)[C@H](P)Cc1cccc(Cl)c1</smiles>

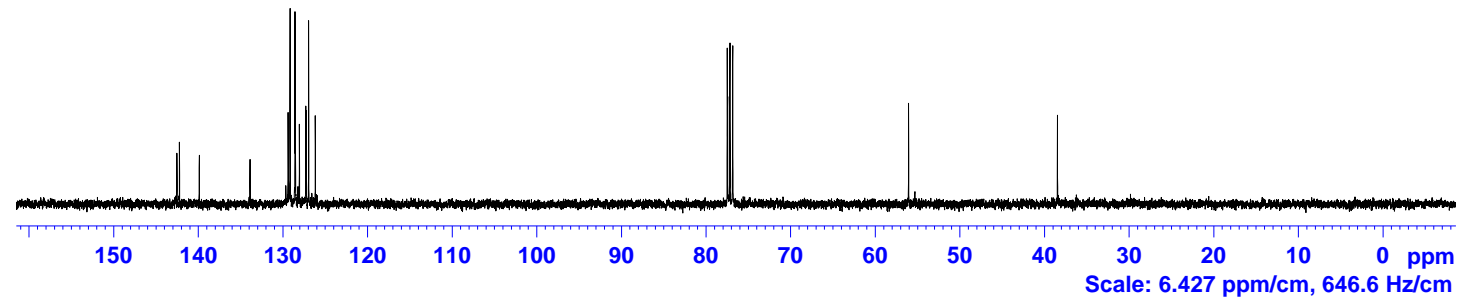


${ }^{1} \mathrm{H}$ NMR spectrum of $\mathbf{5 l}\left(\mathrm{CDCl}_{3}, 400 \mathrm{MHz}\right)$ :
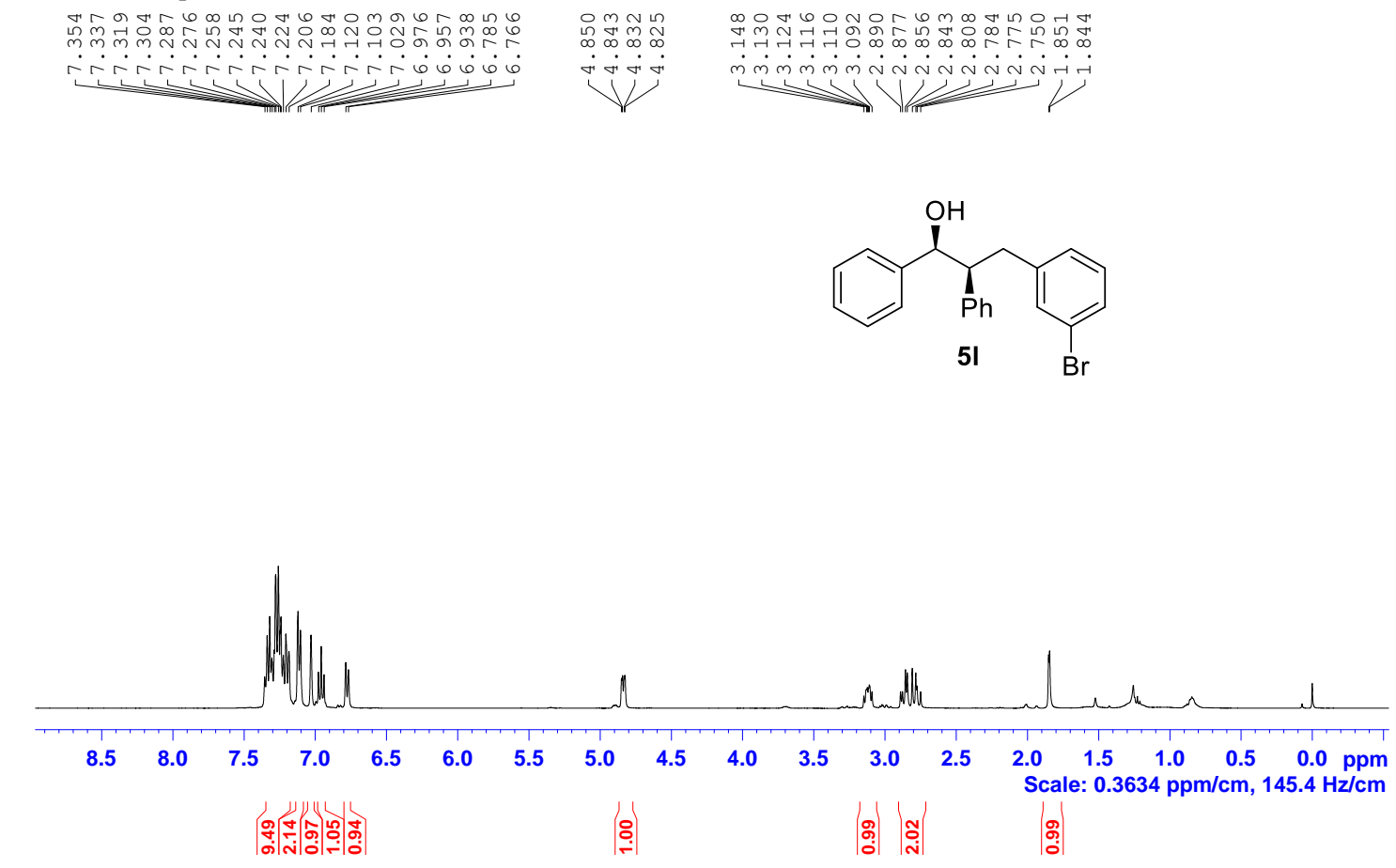

${ }^{13} \mathrm{C}\left\{{ }^{1} \mathrm{H}\right\}$ NMR spectrum of $\mathbf{5 l}\left(\mathrm{CDCl}_{3}, 100 \mathrm{MHz}\right)$ :

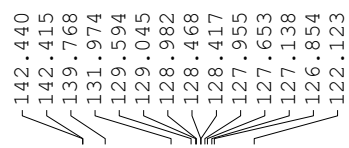

|
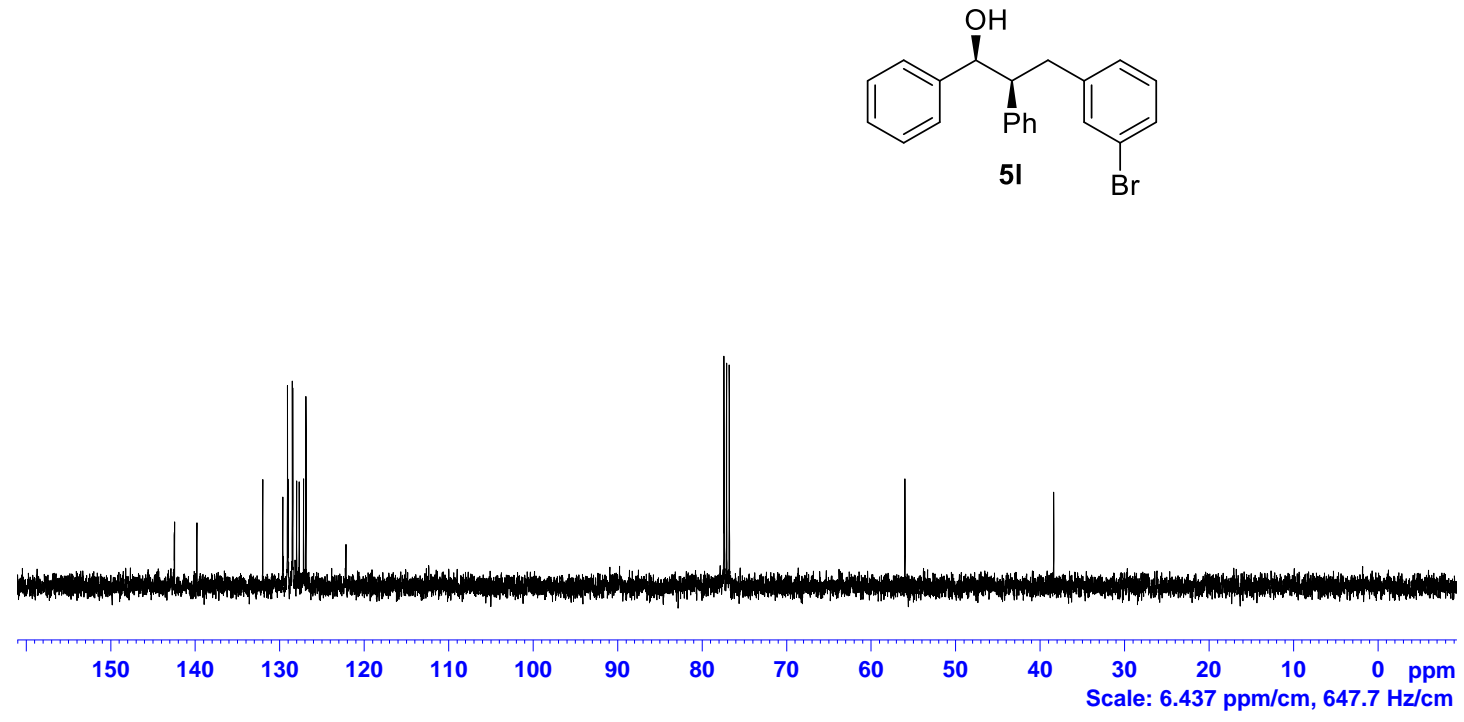
${ }^{1} \mathrm{H}$ NMR spectrum of $\mathbf{5 m}\left(\mathrm{CDCl}_{3}, 400 \mathrm{MHz}\right)$ :
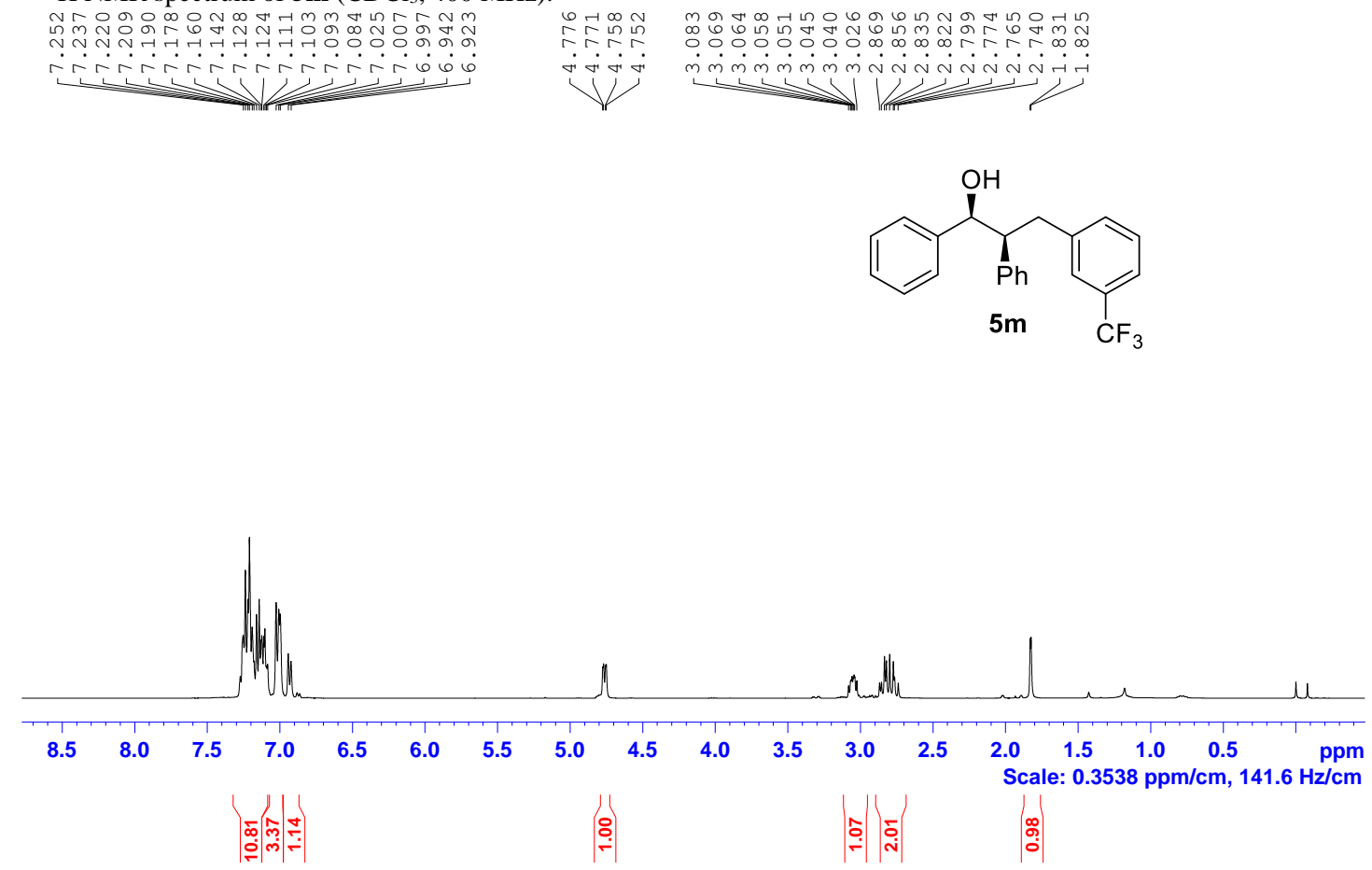

${ }^{13} \mathrm{C}\left\{{ }^{1} \mathrm{H}\right\}$ NMR spectrum of $\mathbf{5 m}\left(\mathrm{CDCl}_{3}, 100 \mathrm{MHz}\right)$ :
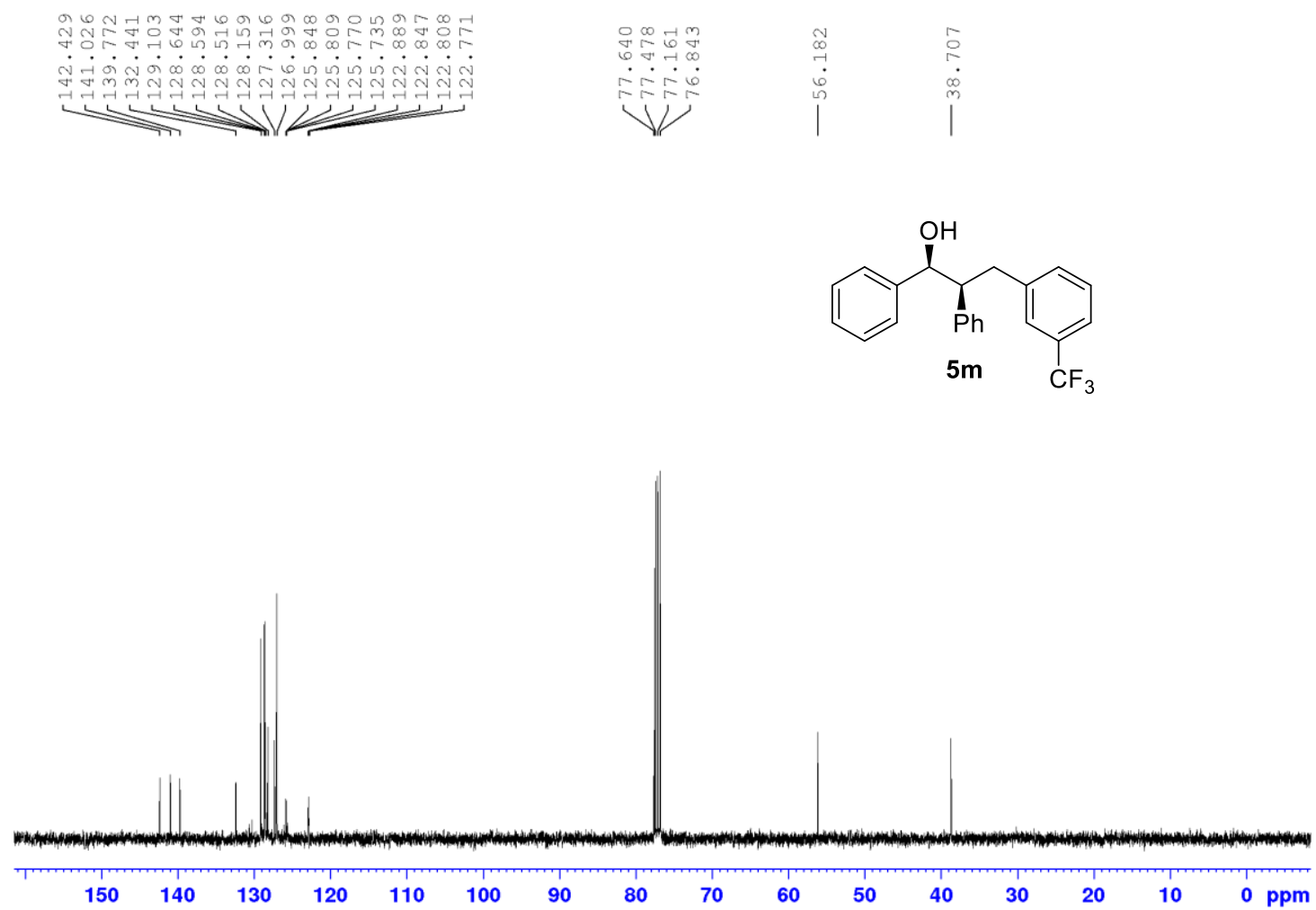
${ }^{1} \mathrm{H}$ NMR spectrum of $\mathbf{5 n}\left(\mathrm{CDCl}_{3}, 400 \mathrm{MHz}\right)$ :
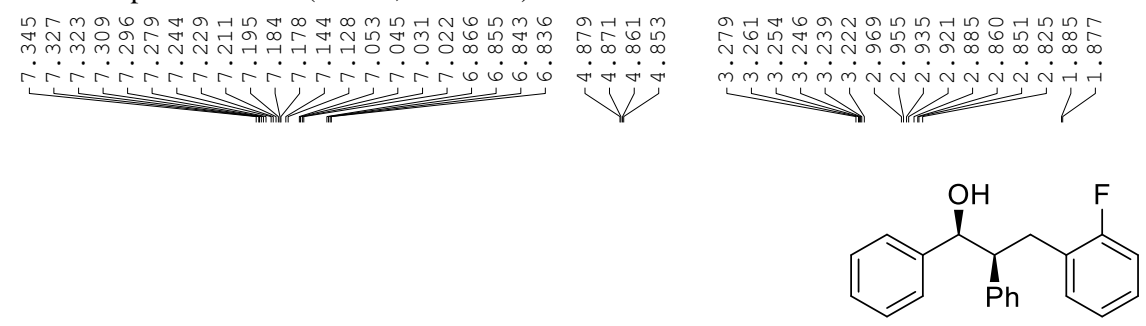

$5 n$

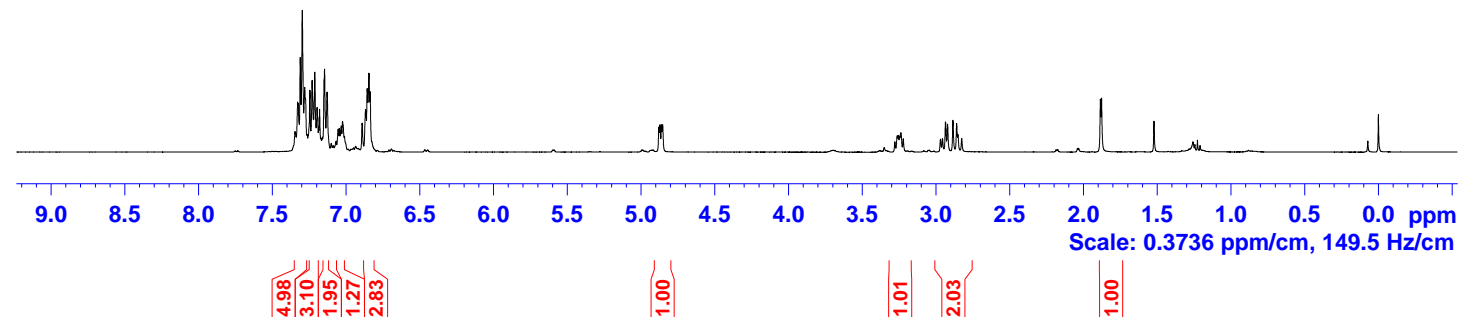

${ }^{13} \mathrm{C}\left\{{ }^{1} \mathrm{H}\right\}$ NMR spectrum of $\mathbf{5 n}\left(\mathrm{CDCl}_{3}, 100 \mathrm{MHz}\right)$ :
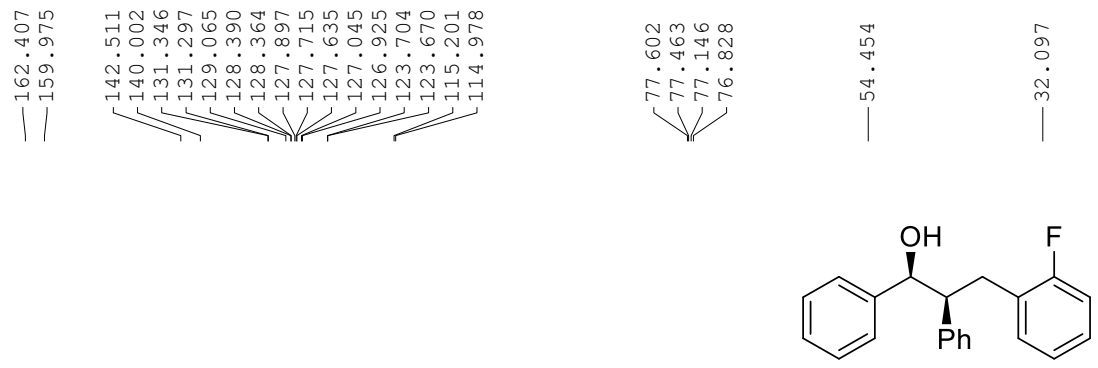

$5 n$

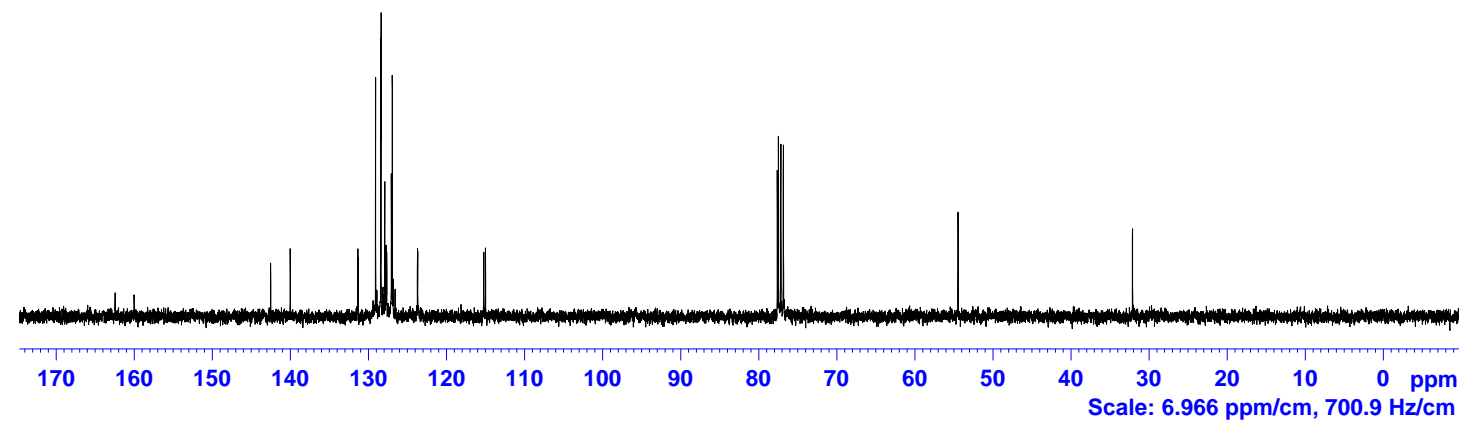


${ }^{1} \mathrm{H}$ NMR spectrum of $\mathbf{5 o}\left(\mathrm{CDCl}_{3}, 400 \mathrm{MHz}\right)$ :

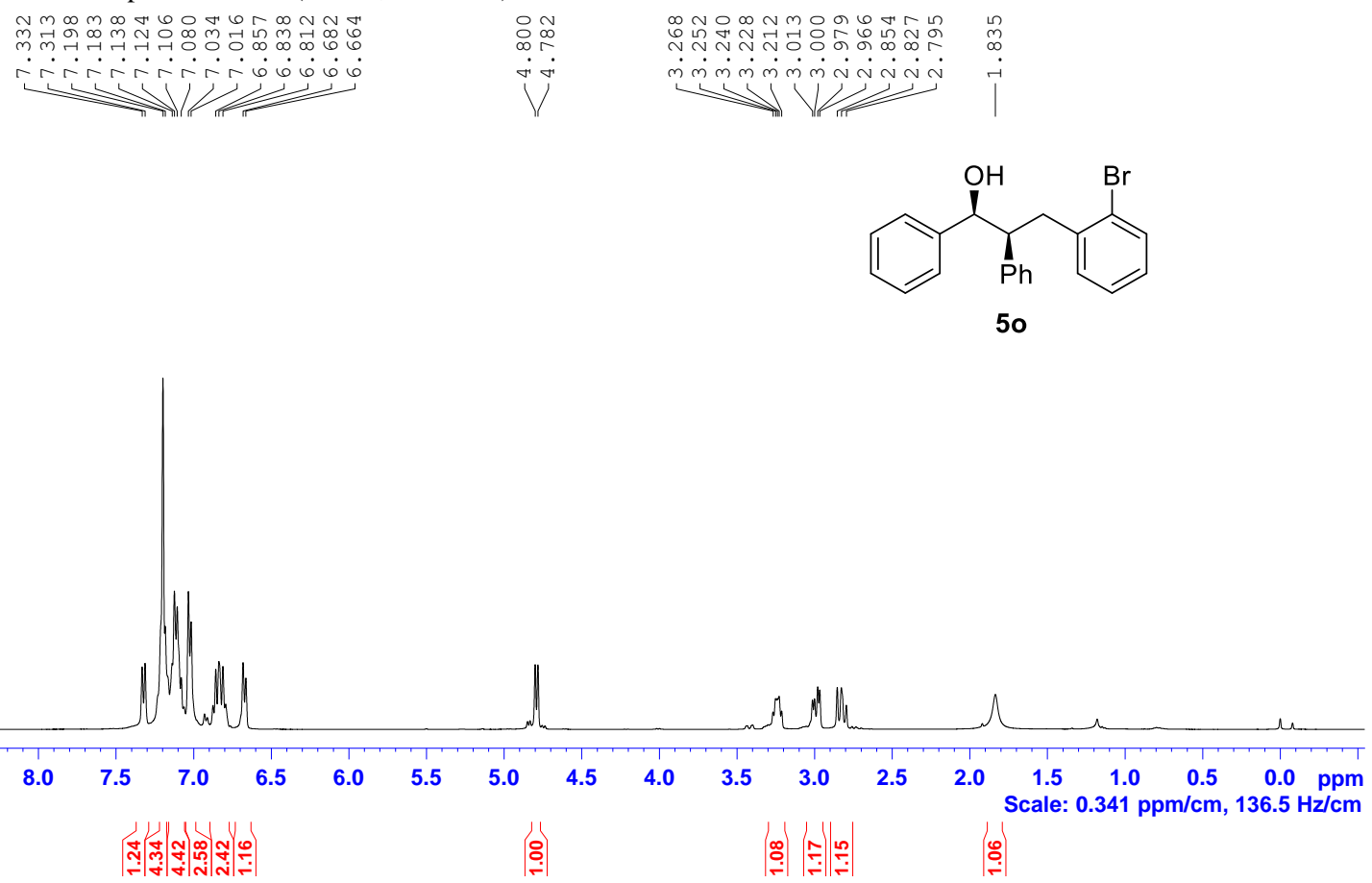

${ }^{13} \mathrm{C}\left\{{ }^{1} \mathrm{H}\right\}$ NMR spectrum of $\mathbf{5 o}\left(\mathrm{CDCl}_{3}, 100 \mathrm{MHz}\right)$ :
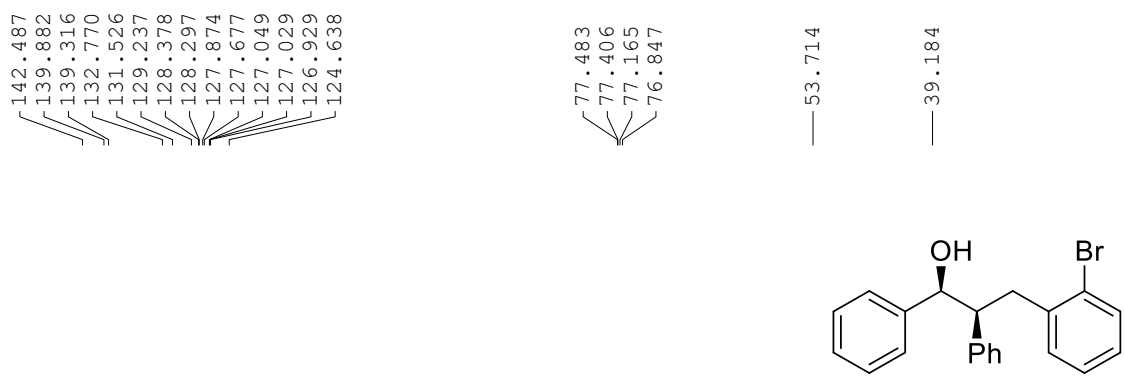

5o

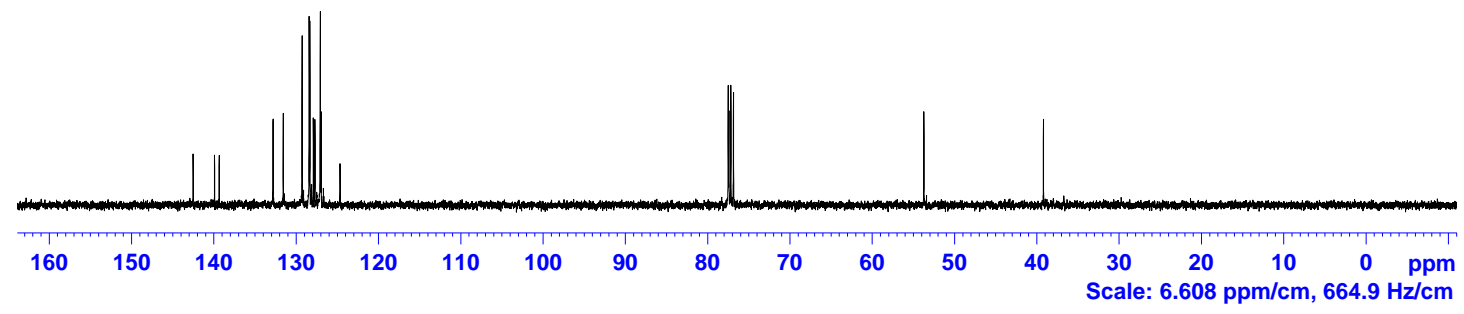


${ }^{1} \mathrm{H}$ NMR spectrum of $\mathbf{5 p}\left(\mathrm{CDCl}_{3}, 400 \mathrm{MHz}\right)$ :

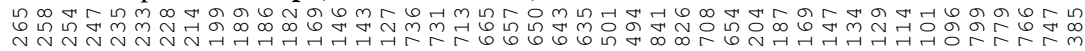

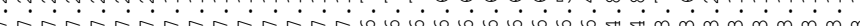

年<smiles>COc1ccc(OC)c(CC(c2ccccc2)[C@H](O)c2ccccc2)c1</smiles>

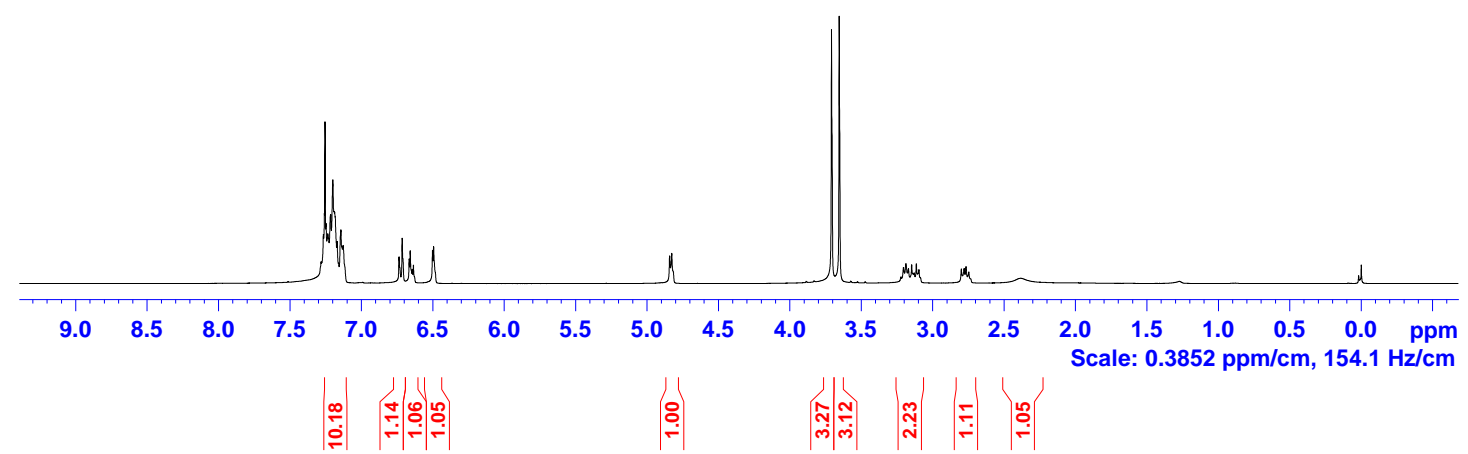

${ }^{13} \mathrm{C}\left\{{ }^{1} \mathrm{H}\right\}$ NMR spectrum of $\mathbf{5 p}\left(\mathrm{CDCl}_{3}, 100 \mathrm{MHz}\right)$ :

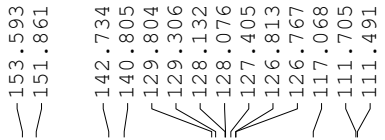

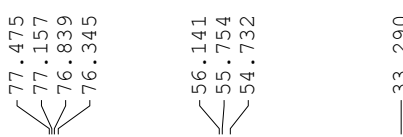
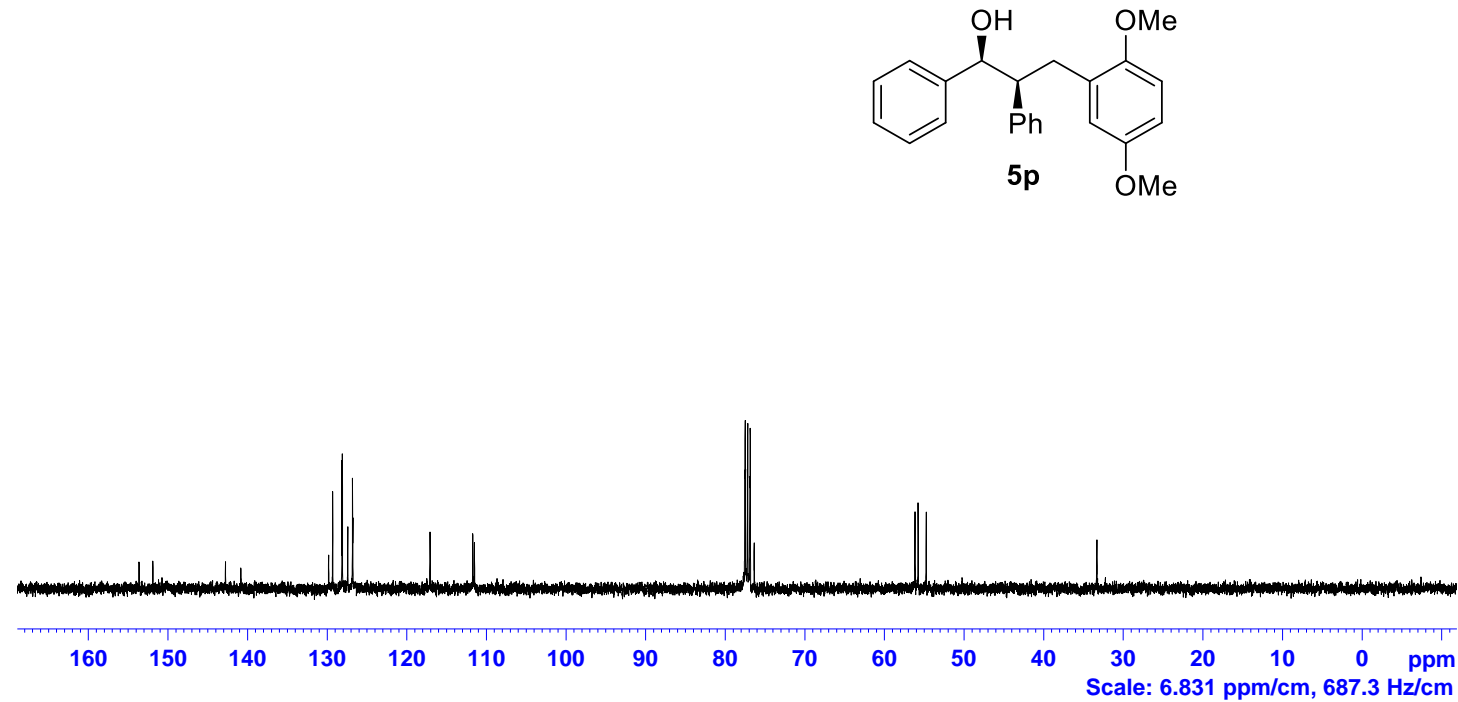
${ }^{1} \mathrm{H}$ NMR spectrum of $\mathbf{5 q}\left(\mathrm{CDCl}_{3}, 400 \mathrm{MHz}\right)$ :

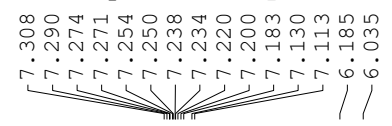

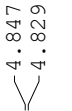
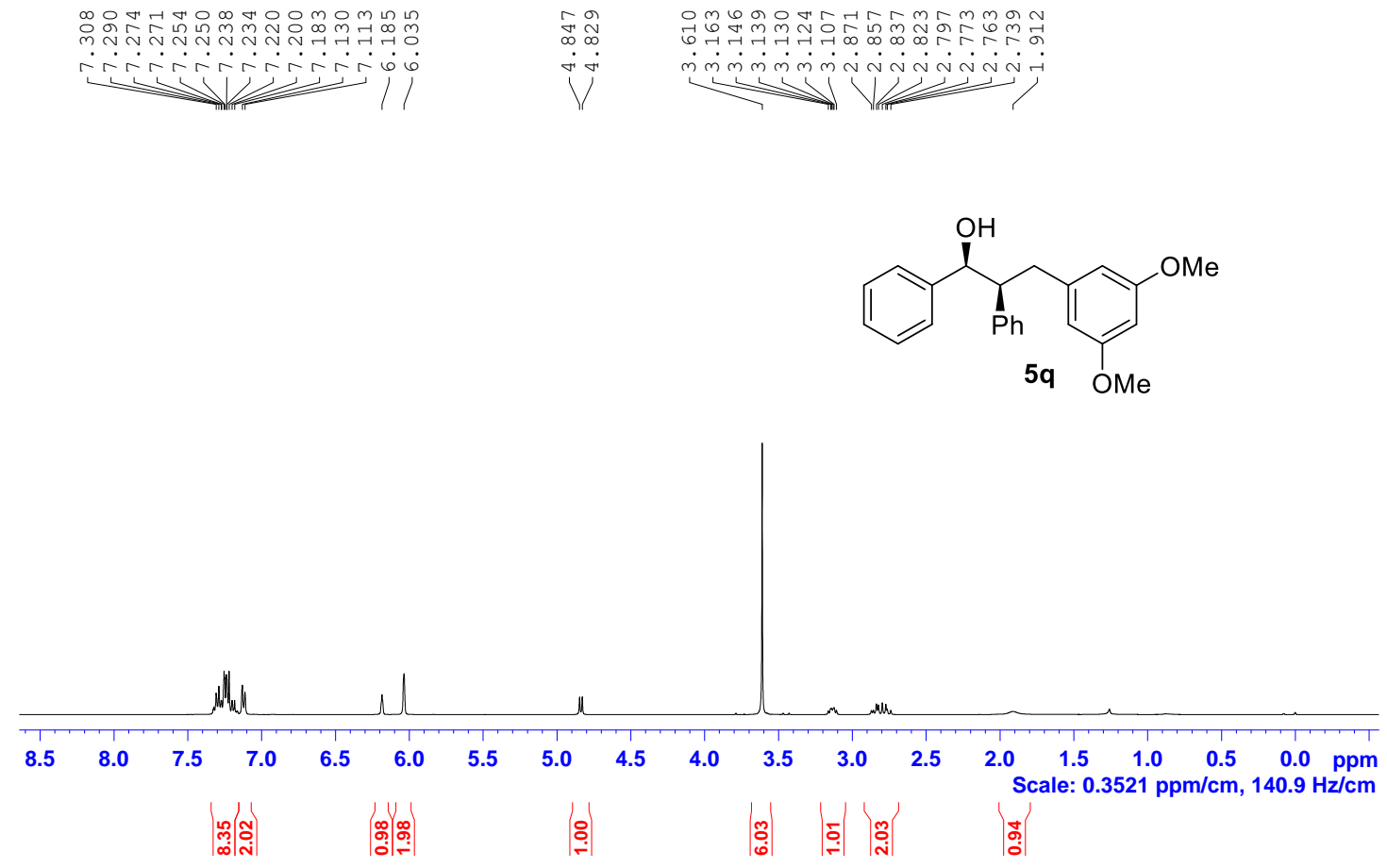

${ }^{13} \mathrm{C}\left\{{ }^{1} \mathrm{H}\right\}$ NMR spectrum of $\mathbf{5 q}\left(\mathrm{CDCl}_{3}, 100 \mathrm{MHz}\right)$ :
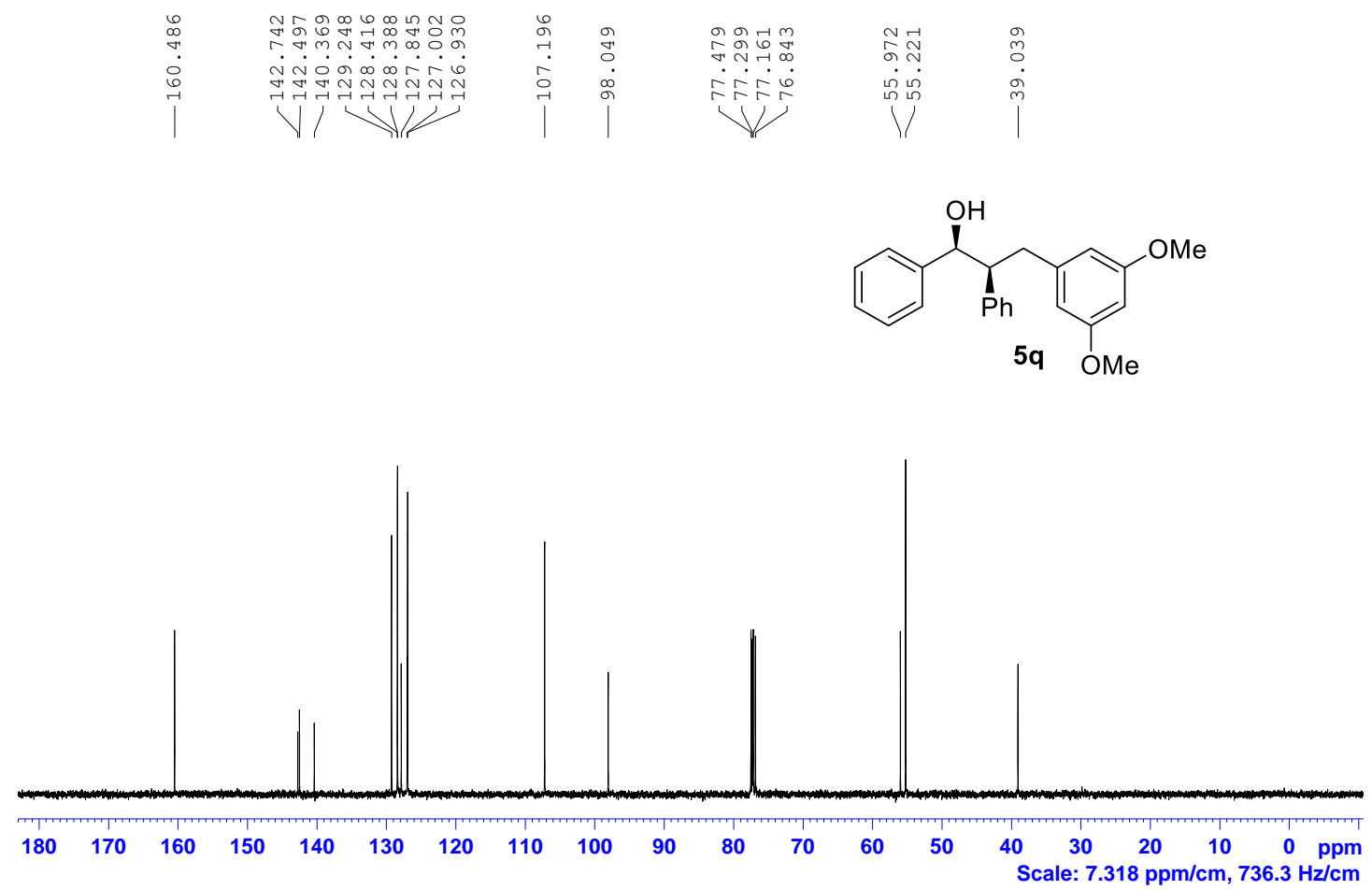
${ }^{1} \mathrm{H}$ NMR spectrum of $\mathbf{5 r}\left(\mathrm{CDCl}_{3}, 400 \mathrm{MHz}\right)$ :
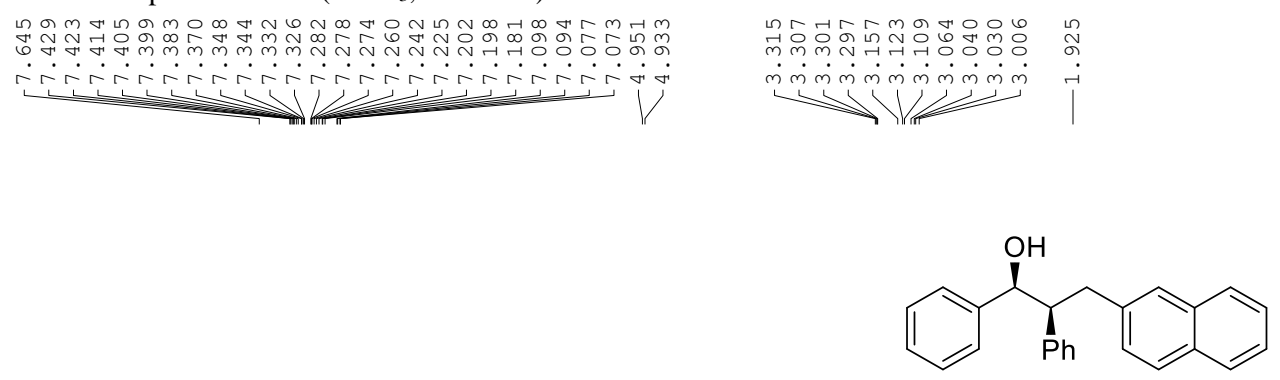

$5 r$

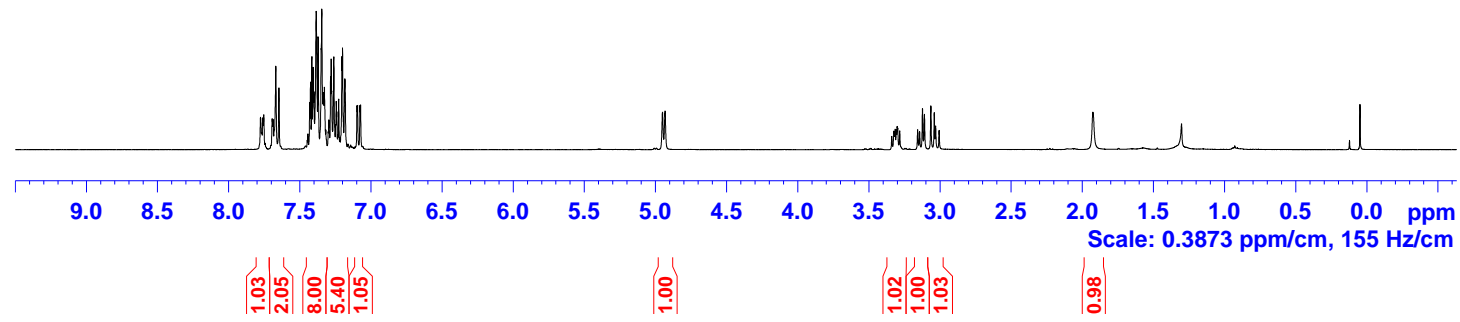

${ }^{13} \mathrm{C}\left\{{ }^{1} \mathrm{H}\right\}$ NMR spectrum of $\mathbf{5 r}\left(\mathrm{CDCl}_{3}, 100 \mathrm{MHz}\right)$ :

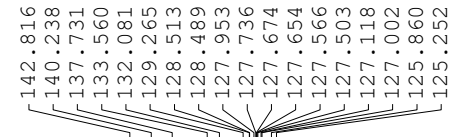

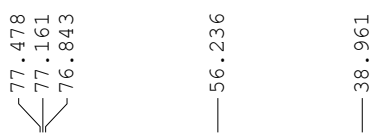<smiles>O[C@H](c1ccccc1)[C@H](Cc1ccc2ccccc2c1)c1ccccc1</smiles>

$5 r$

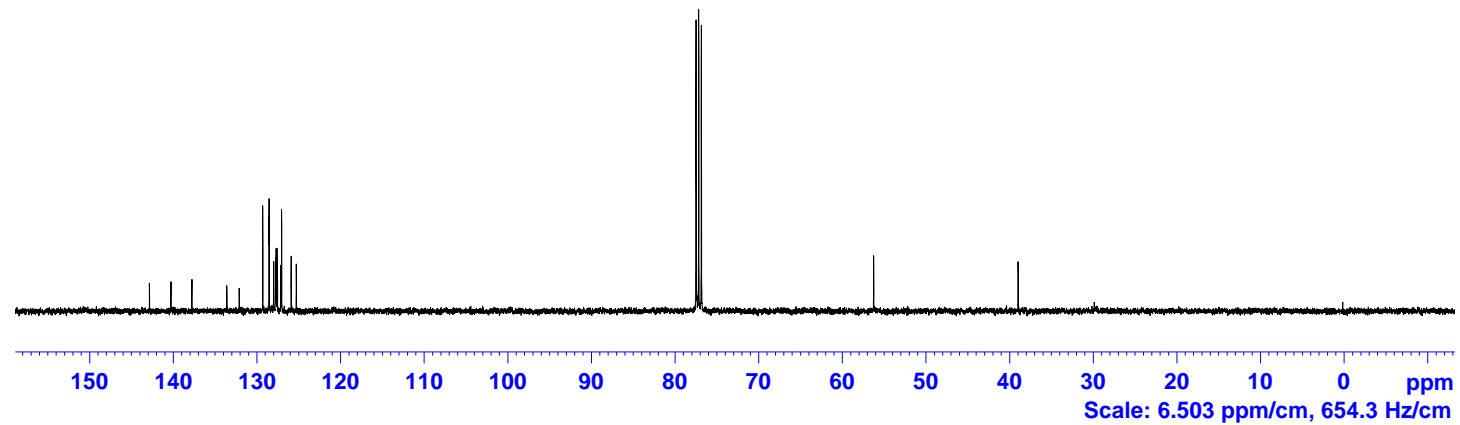




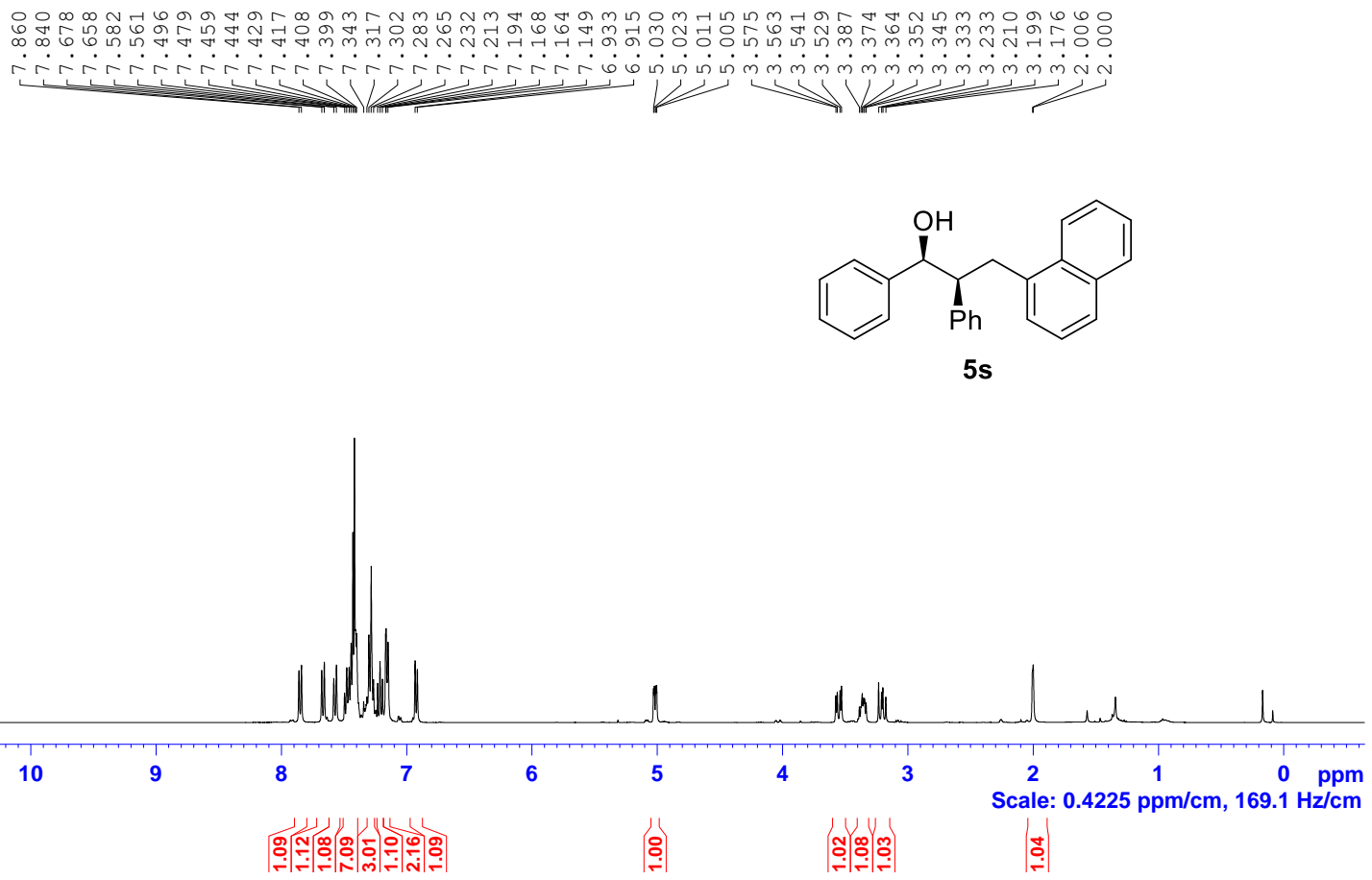

${ }^{13} \mathrm{C}\left\{{ }^{1} \mathrm{H}\right\}$ NMR spectrum of $\mathbf{5 s}\left(\mathrm{CDCl}_{3}, 100 \mathrm{MHz}\right)$ :

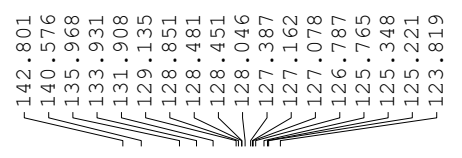

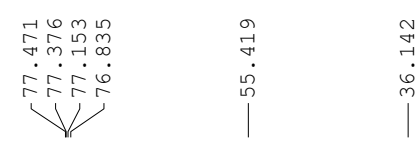

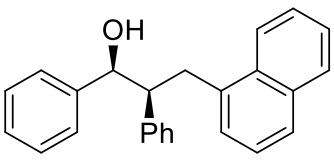

5s

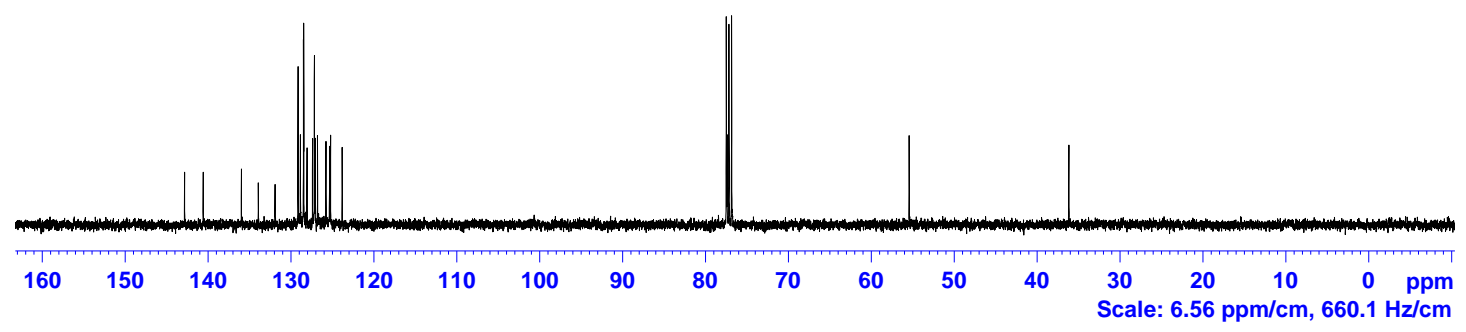


${ }^{1} \mathrm{H}$ NMR spectrum of $\mathbf{5 t}\left(\mathrm{CDCl}_{3}, 400 \mathrm{MHz}\right)$ :
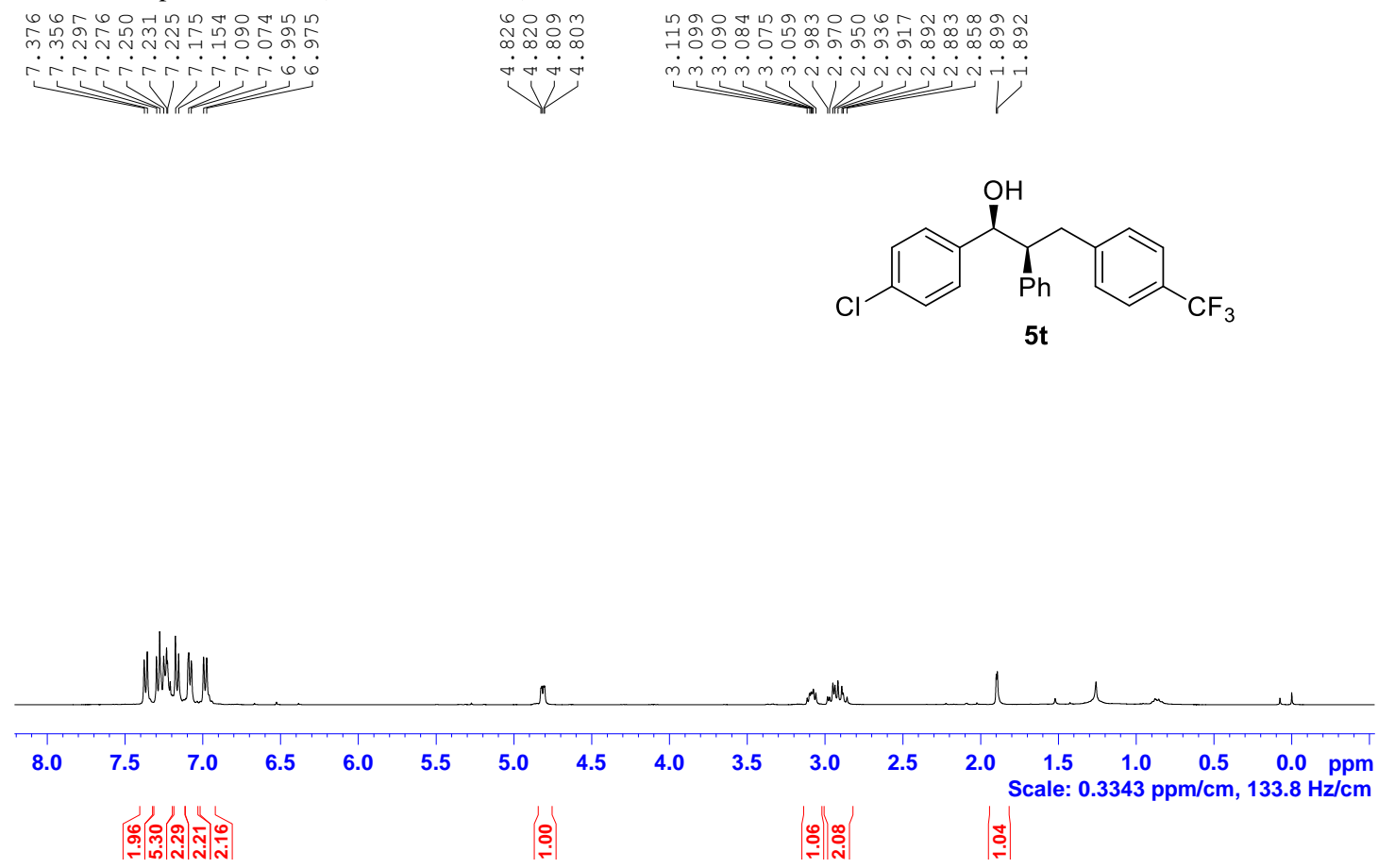

${ }^{13} \mathrm{C}\left\{{ }^{1} \mathrm{H}\right\}$ NMR spectrum of $\mathbf{5 t}\left(\mathrm{CDCl}_{3}, 100 \mathrm{MHz}\right)$ :
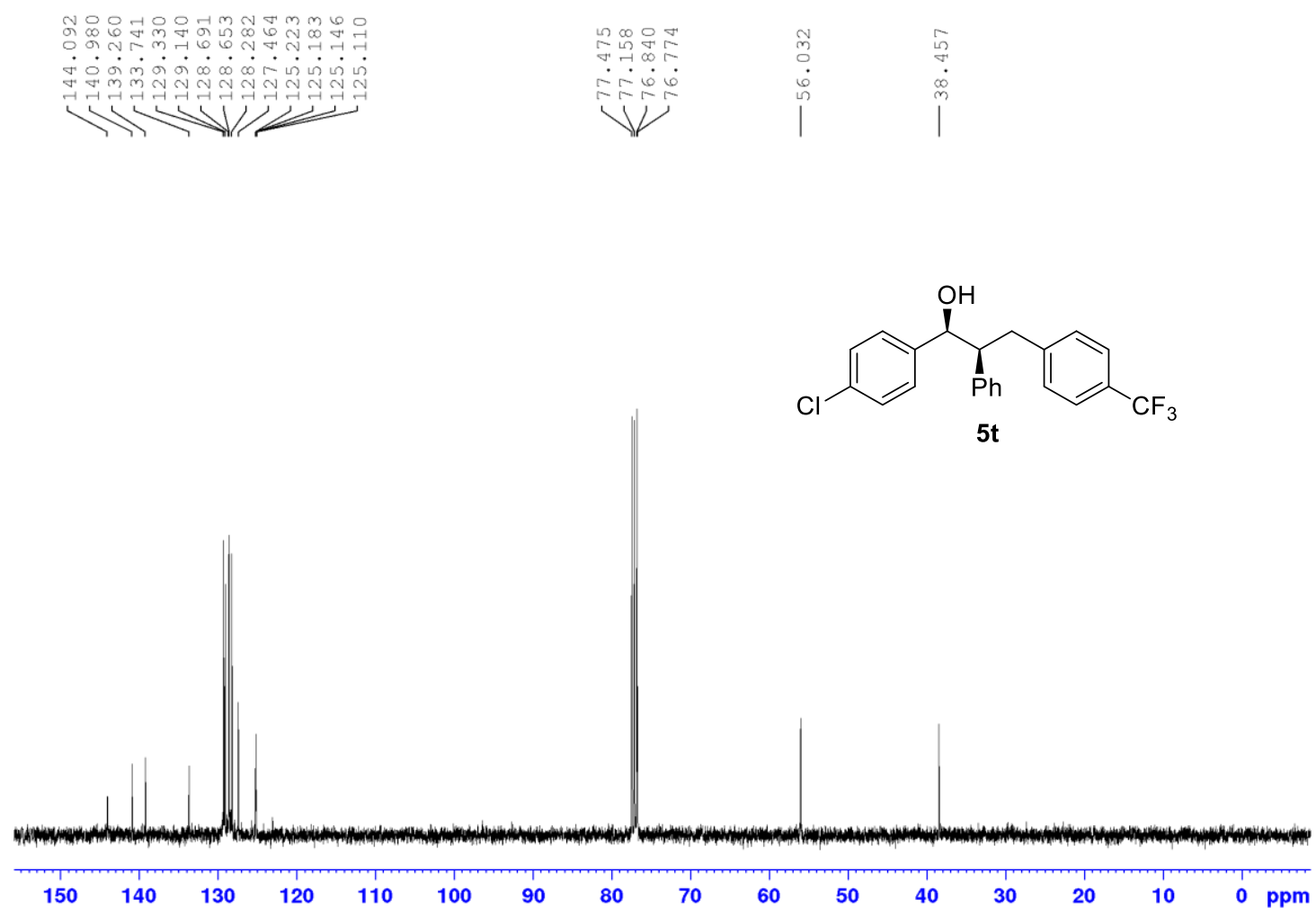
${ }^{1} \mathrm{H}$ NMR spectrum of $\mathbf{5 u}\left(\mathrm{CDCl}_{3}, 400 \mathrm{MHz}\right)$ :

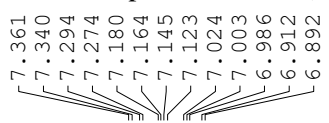

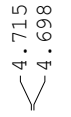

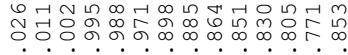

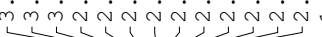<smiles>O[C@H](c1ccc(Br)cc1)[C@@H](P)Cc1ccc(C(F)(F)F)cc1</smiles>

$5 \mathrm{u}$

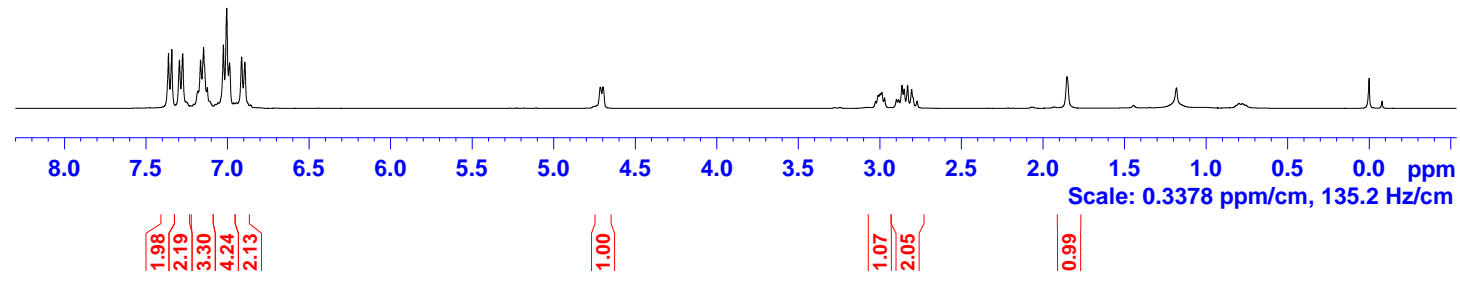

${ }^{13} \mathrm{C}\left\{{ }^{1} \mathrm{H}\right\}$ NMR spectrum of $\mathbf{5 u}\left(\mathrm{CDCl}_{3}, 100 \mathrm{MHz}\right)$ :

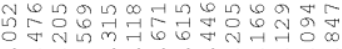

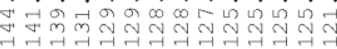

$11+$

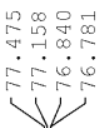

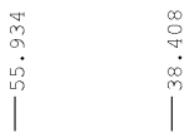<smiles>O[C@H](c1ccc(Br)cc1)[C@@H](c1ccccc1)c1ccc(C(F)(F)F)cc1</smiles>

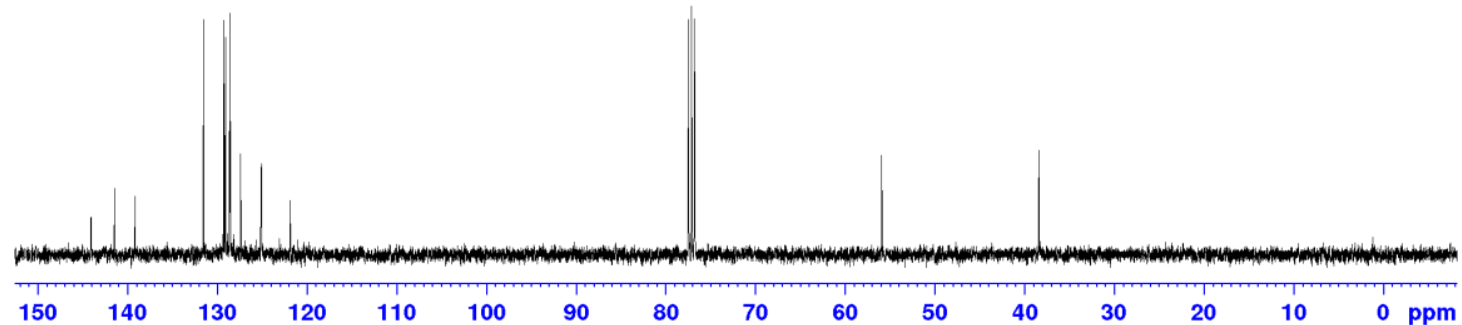


${ }^{1} \mathrm{H}$ NMR spectrum of $\mathbf{5 v}\left(\mathrm{CDCl}_{3}, 400 \mathrm{MHz}\right)$ :

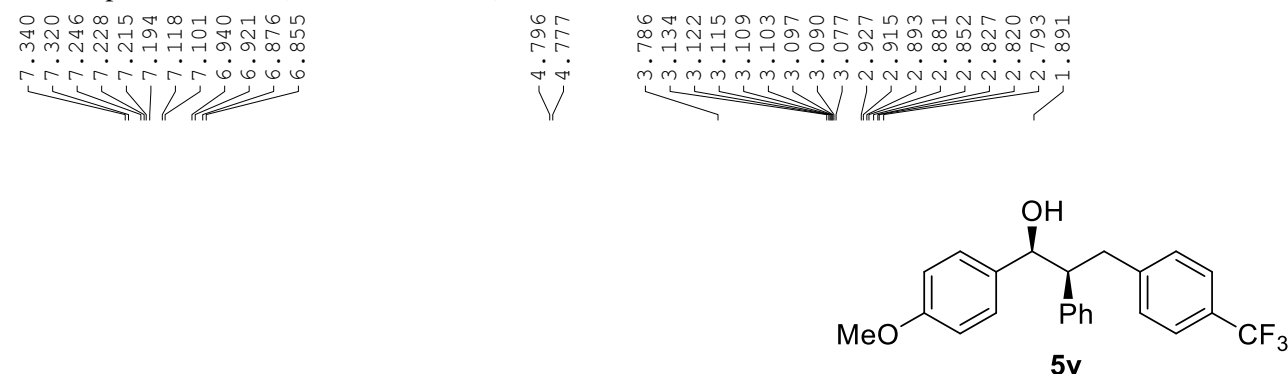

$5 v$

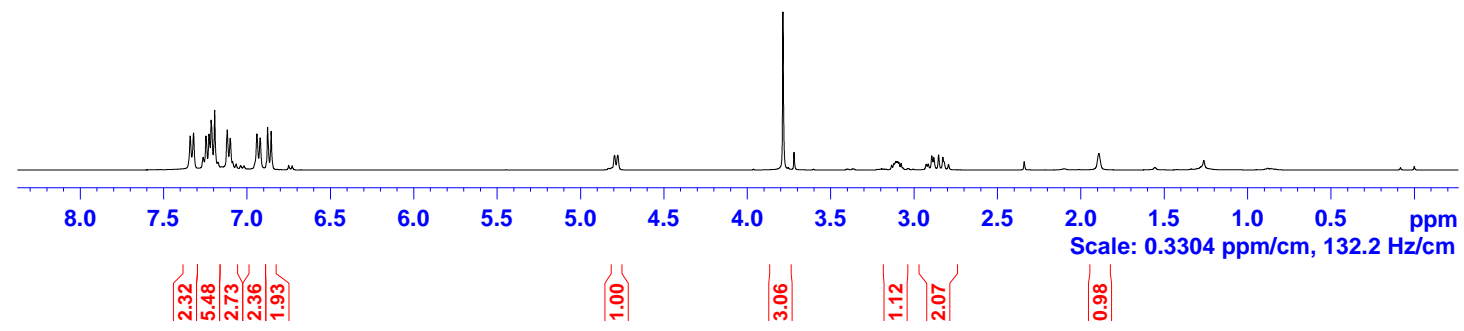

${ }^{13} \mathrm{C}\left\{{ }^{1} \mathrm{H}\right\}$ NMR spectrum of $\mathbf{5 v}\left(\mathrm{CDCl}_{3}, 100 \mathrm{MHz}\right)$ :
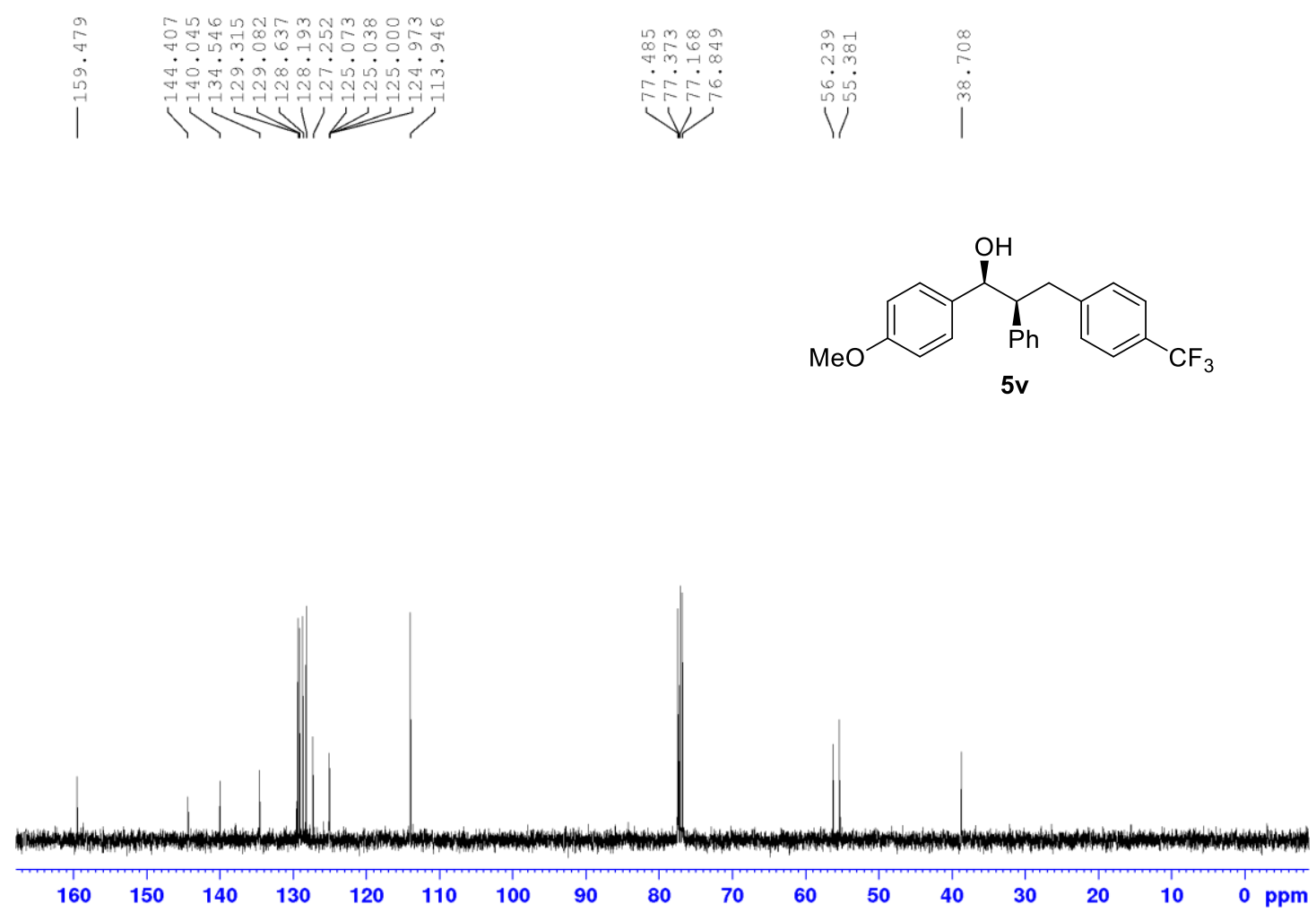
${ }^{1} \mathrm{H}$ NMR spectrum of $\mathbf{5 w}\left(\mathrm{CDCl}_{3}, 400 \mathrm{MHz}\right)$ :

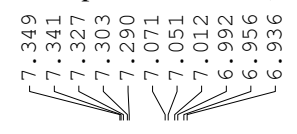

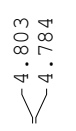
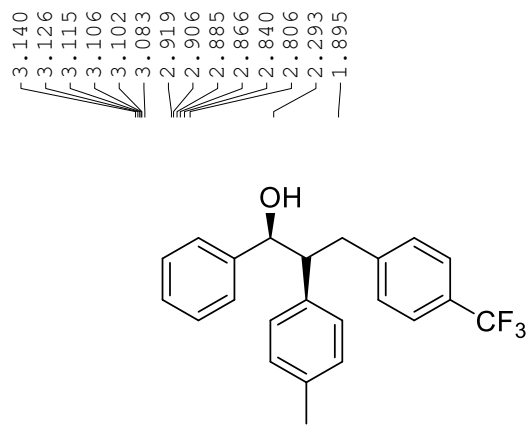

$5 w$

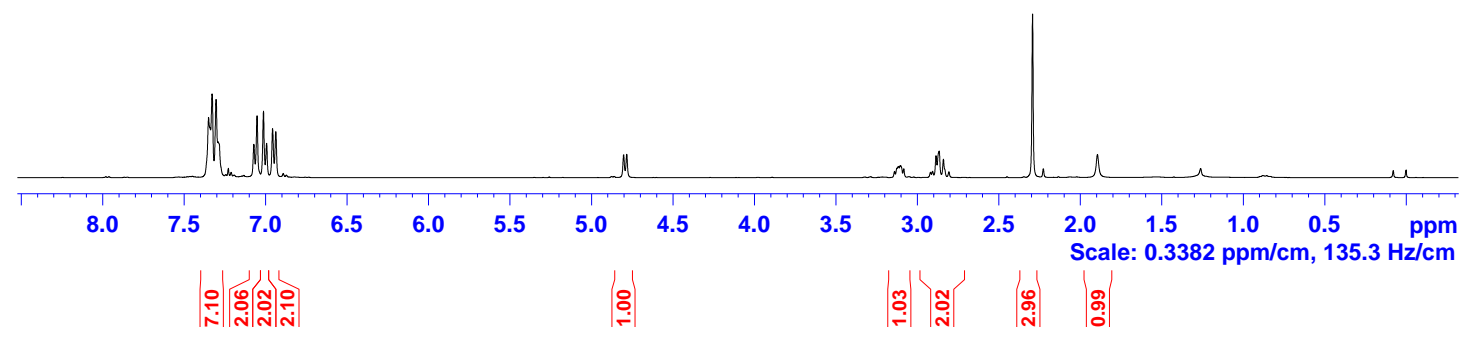

${ }^{13} \mathrm{C}\left\{{ }^{1} \mathrm{H}\right\}$ NMR spectrum of $\mathbf{5 w}\left(\mathrm{CDCl}_{3}, 100 \mathrm{MHz}\right)$ :
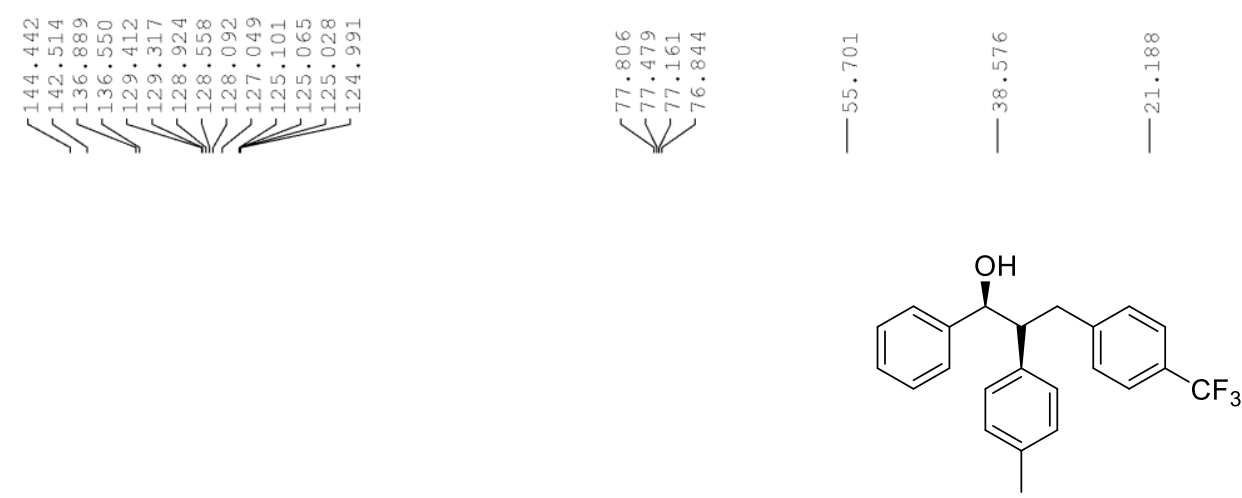

$5 w$

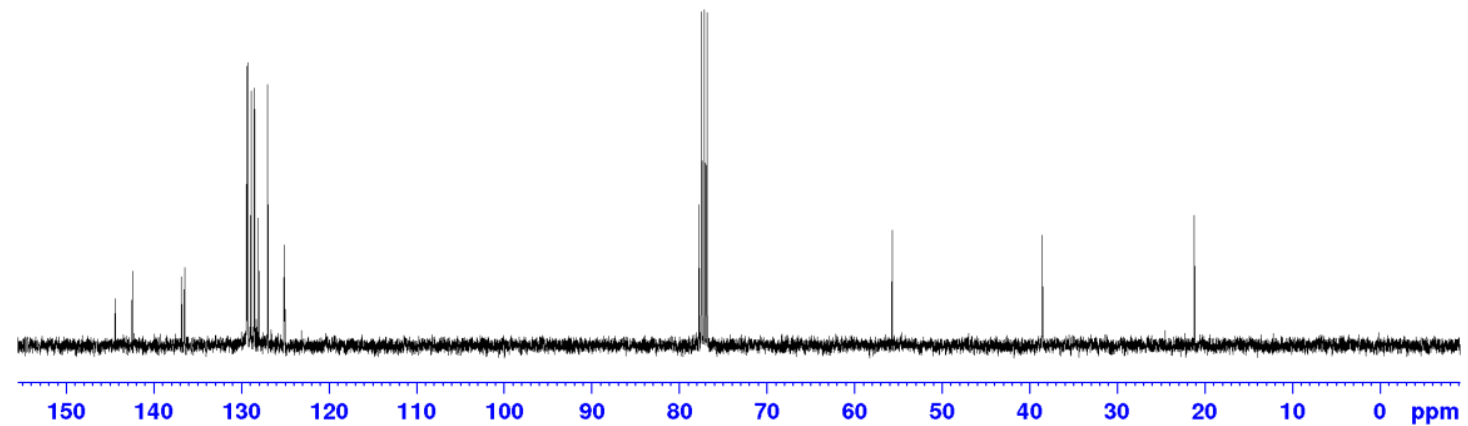


${ }^{1} \mathrm{H}$ NMR spectrum of $\mathbf{5 x}\left(\mathrm{CDCl}_{3}, 400 \mathrm{MHz}\right)$ :

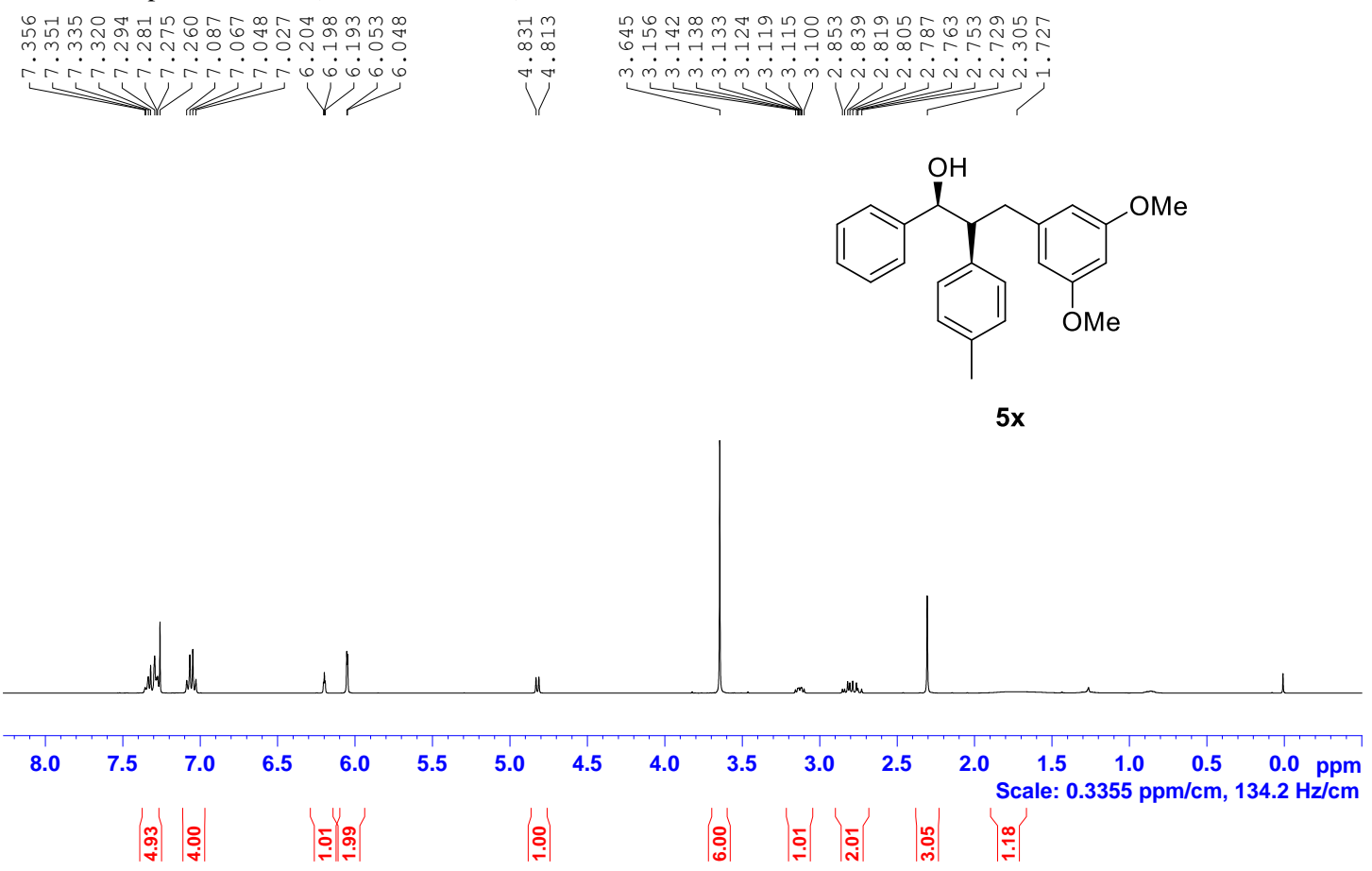

${ }^{13} \mathrm{C}\left\{{ }^{1} \mathrm{H}\right\}$ NMR spectrum of $\mathbf{5 x}\left(\mathrm{CDCl}_{3}, 100 \mathrm{MHz}\right)$ :

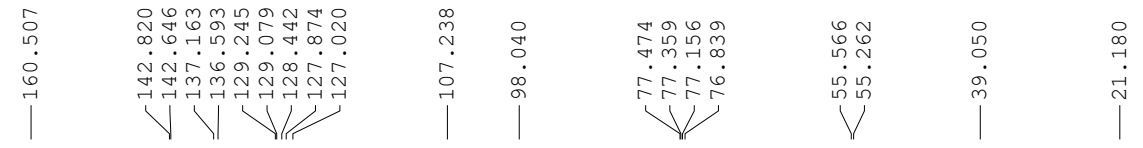

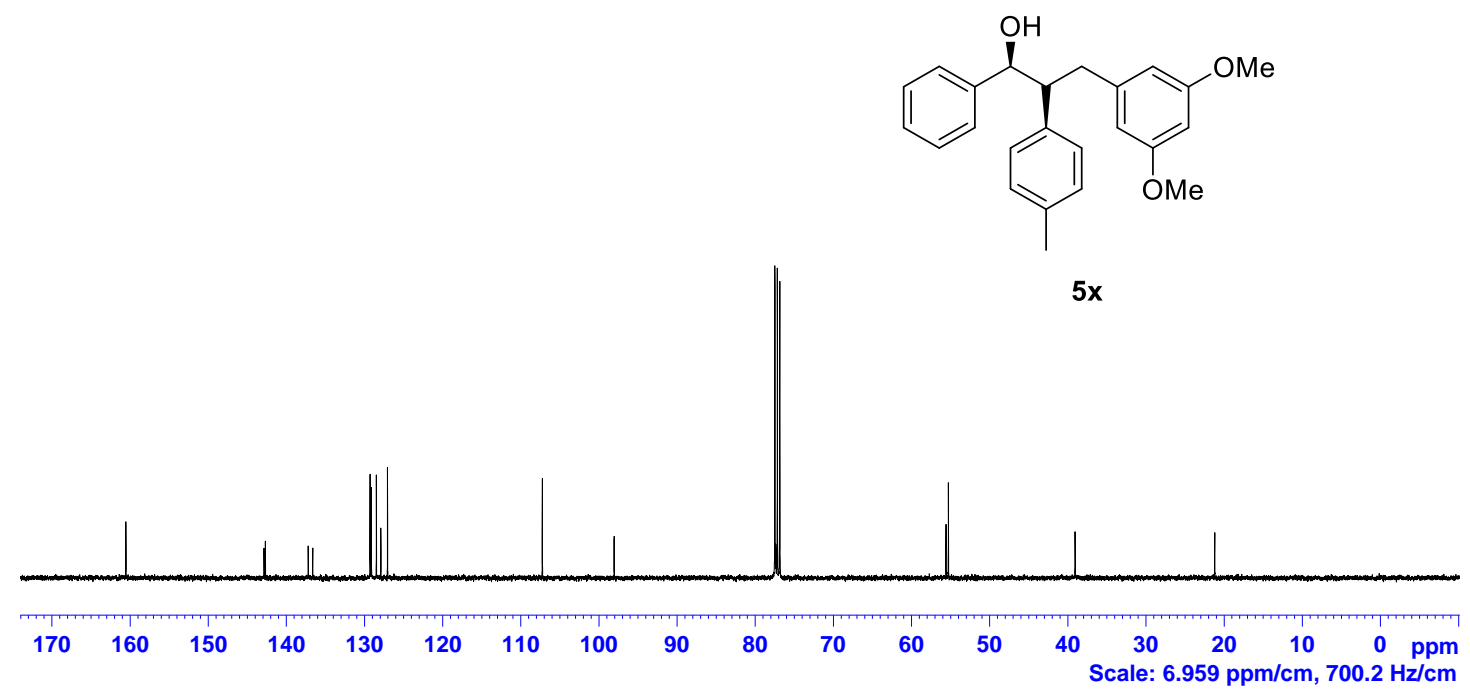


${ }^{1} \mathrm{H}$ NMR spectrum of $7\left(\mathrm{CDCl}_{3}, 400 \mathrm{MHz}\right)$ :

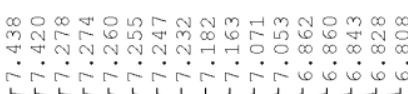

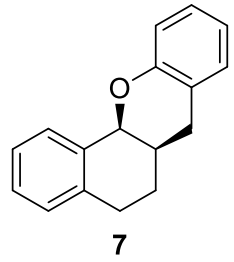

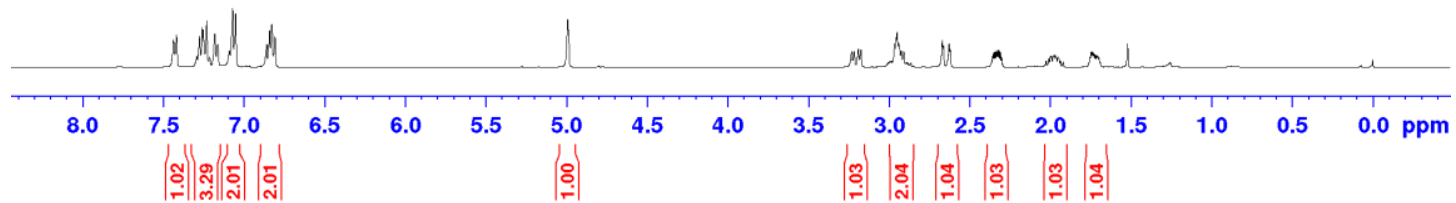

${ }^{13} \mathrm{C}\left\{{ }^{1} \mathrm{H}\right\}$ NMR spectrum of $7\left(\mathrm{CDCl}_{3}, 100 \mathrm{MHz}\right)$ :
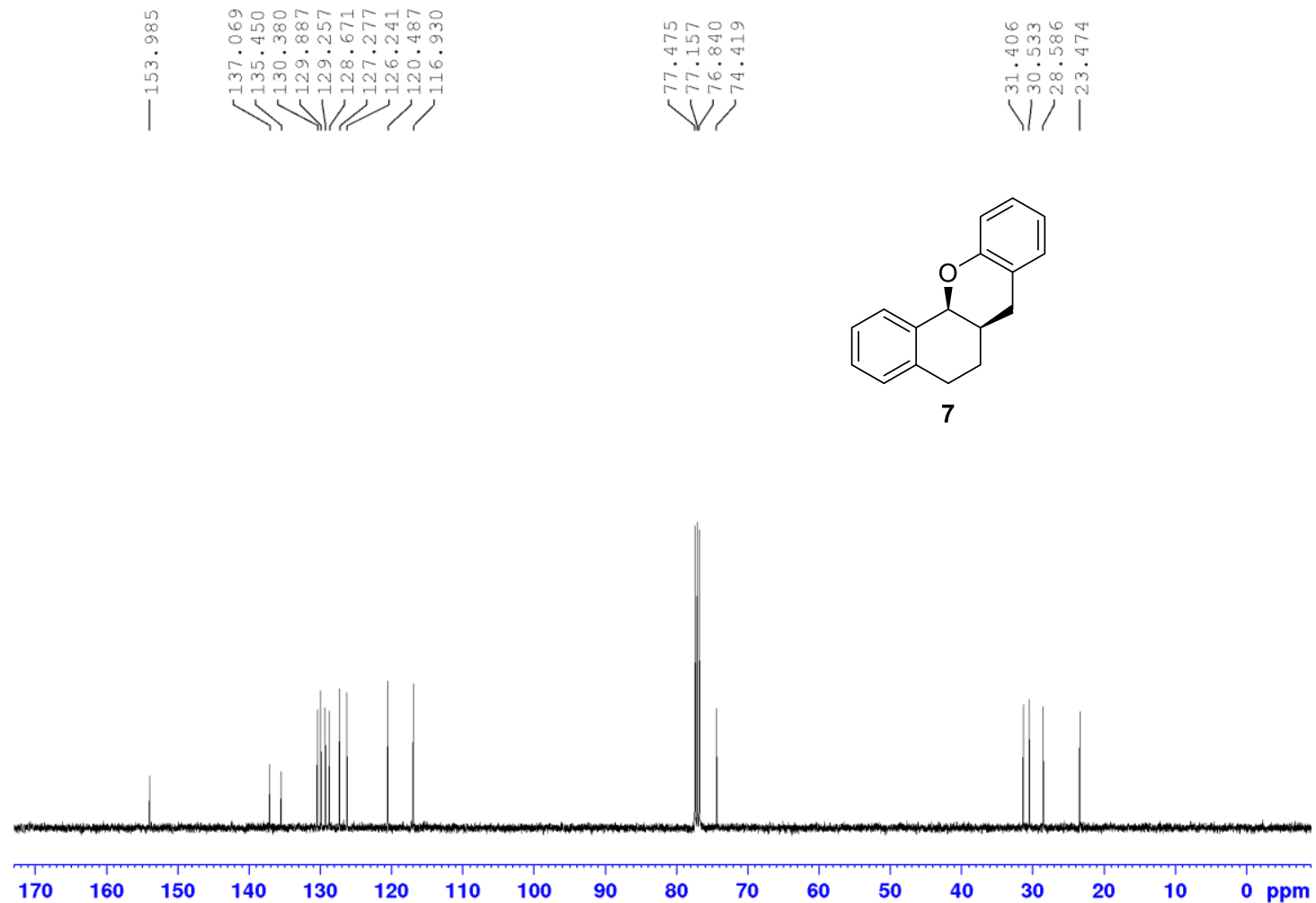
${ }^{1} \mathrm{H}$ NMR spectrum of $\mathbf{8}\left(\mathrm{CDCl}_{3}, 400 \mathrm{MHz}\right)$ :

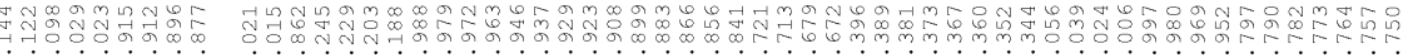<smiles>COc1ccc2c(c1)[C@H]1Oc3ccccc3C[C@H]1CC2</smiles>

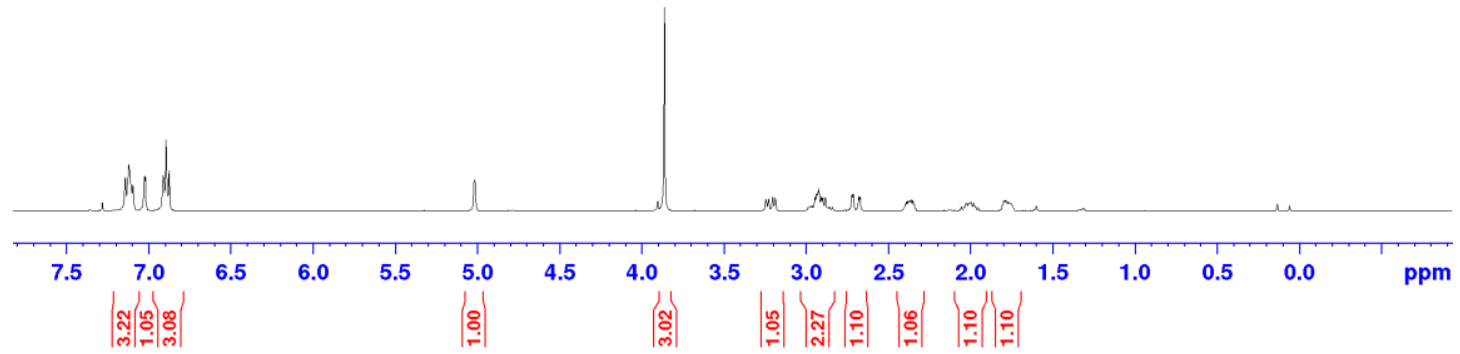

${ }^{13} \mathrm{C}\left\{{ }^{1} \mathrm{H}\right\}$ NMR spectrum of $\mathbf{8}\left(\mathrm{CDCl}_{3}, 100 \mathrm{MHz}\right)$ :
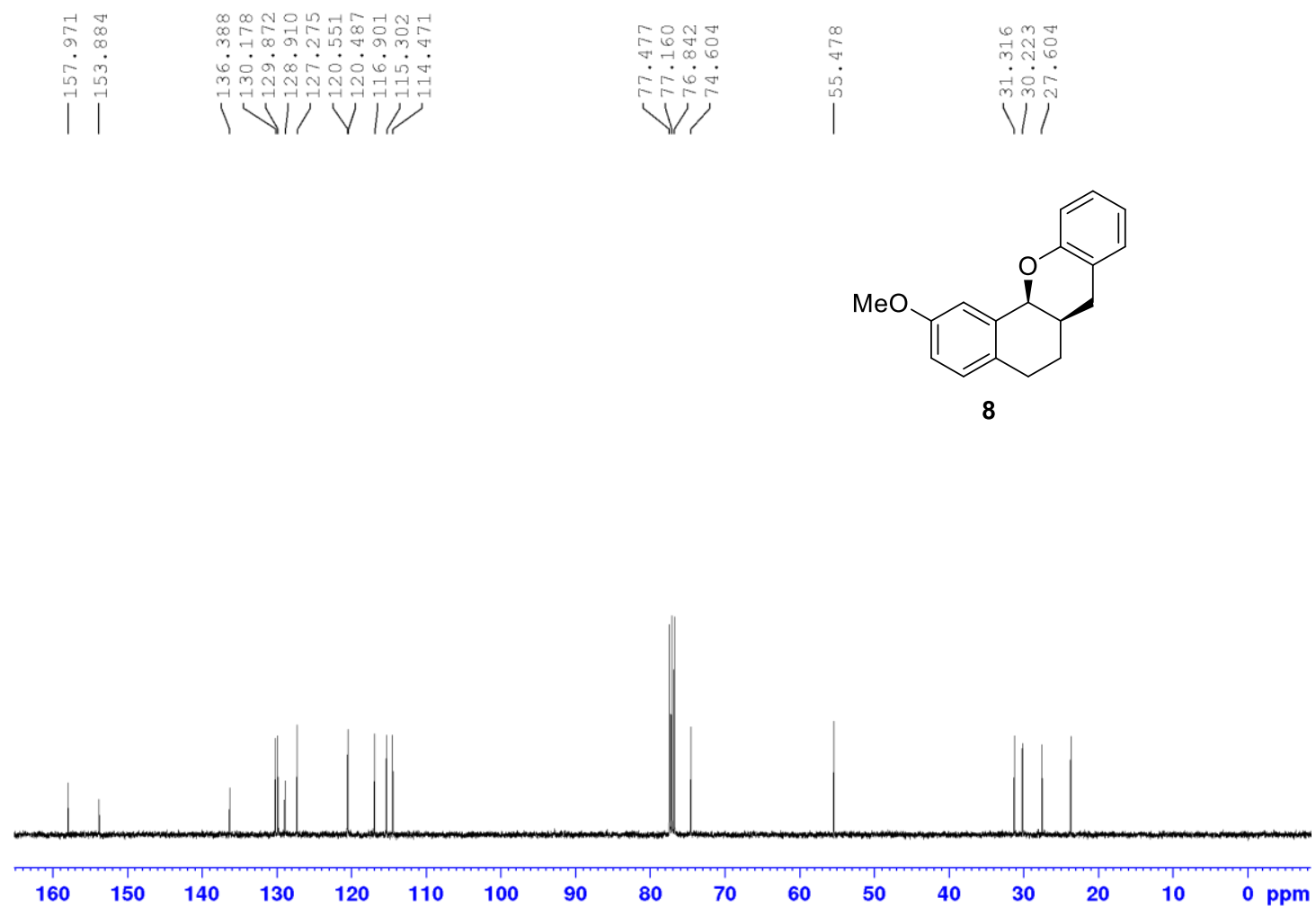
${ }^{1} \mathrm{H}$ NMR spectrum of $9\left(\mathrm{CDCl}_{3}, 400 \mathrm{MHz}\right)$ :

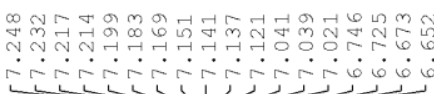

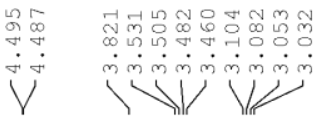

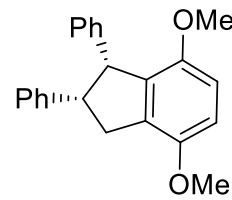

9

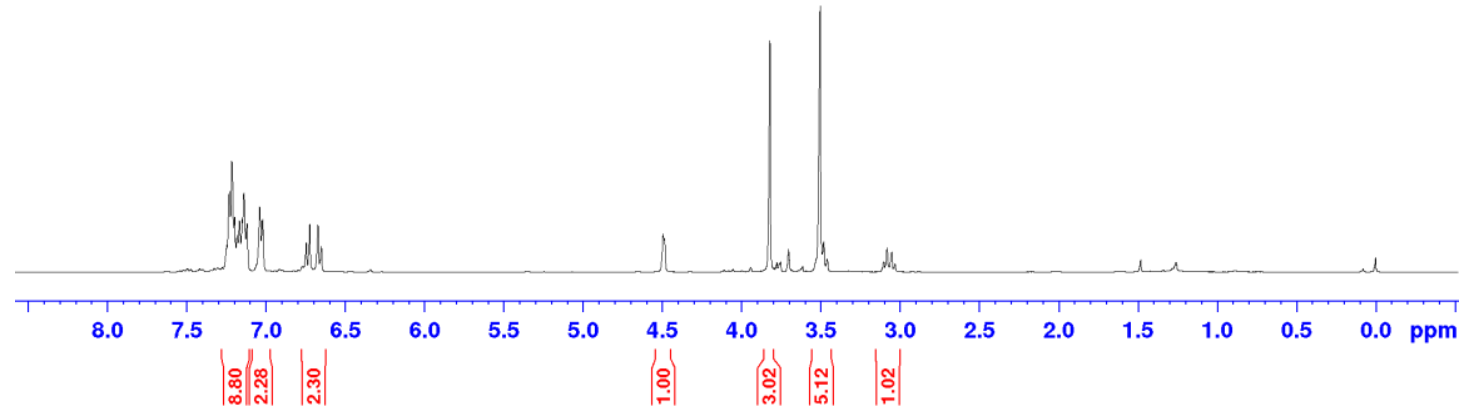

${ }^{13} \mathrm{C}\left\{{ }^{1} \mathrm{H}\right\}$ NMR spectrum of $9\left(\mathrm{CDCl}_{3}, 100 \mathrm{MHz}\right)$ :
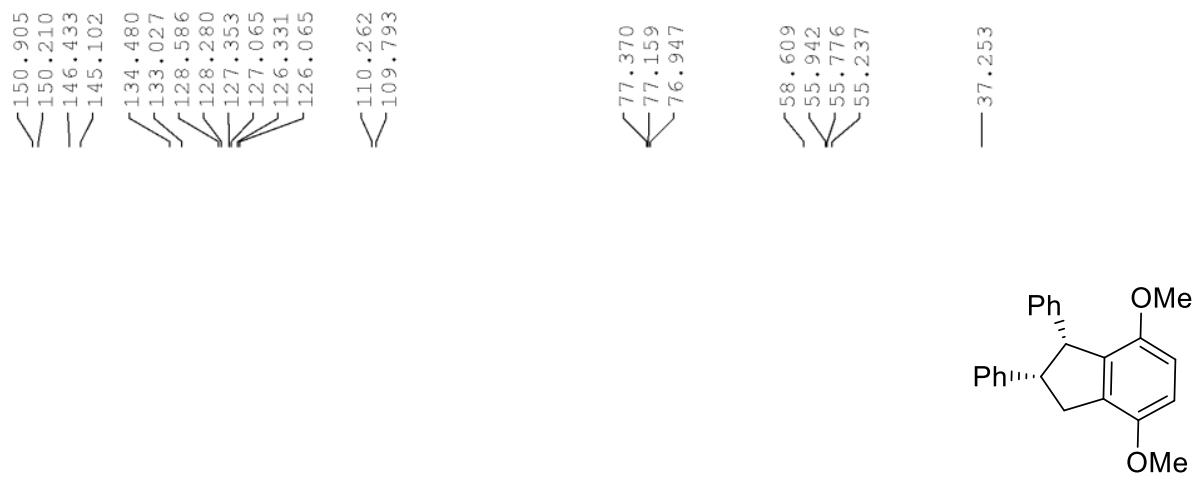

9

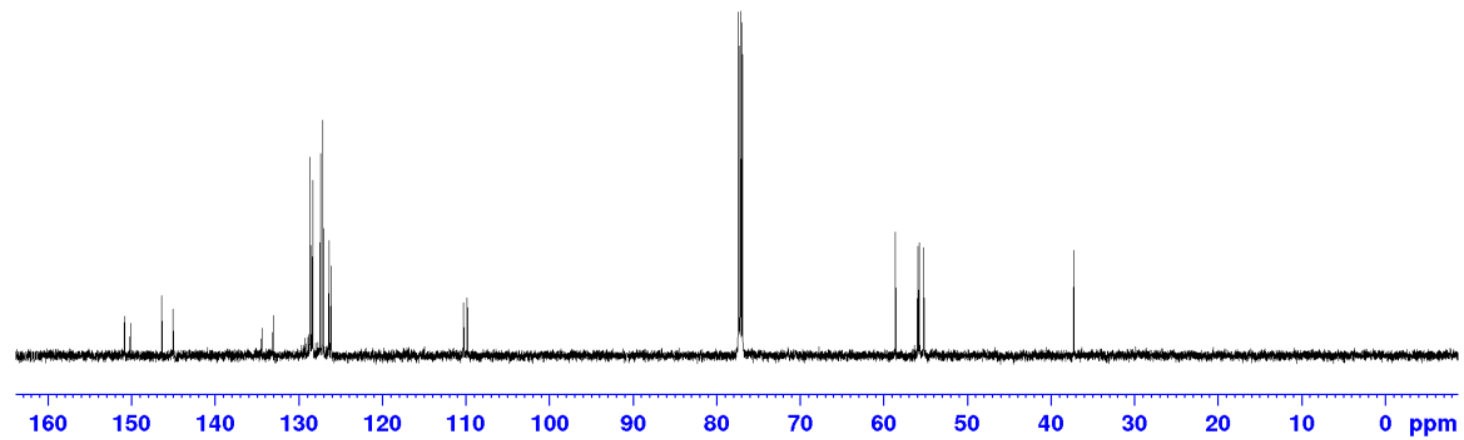


${ }^{1} \mathrm{H}$ NMR spectrum of $\mathbf{1 0}\left(\mathrm{CDCl}_{3}, 400 \mathrm{MHz}\right)$ :

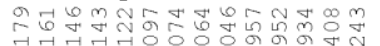

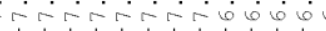
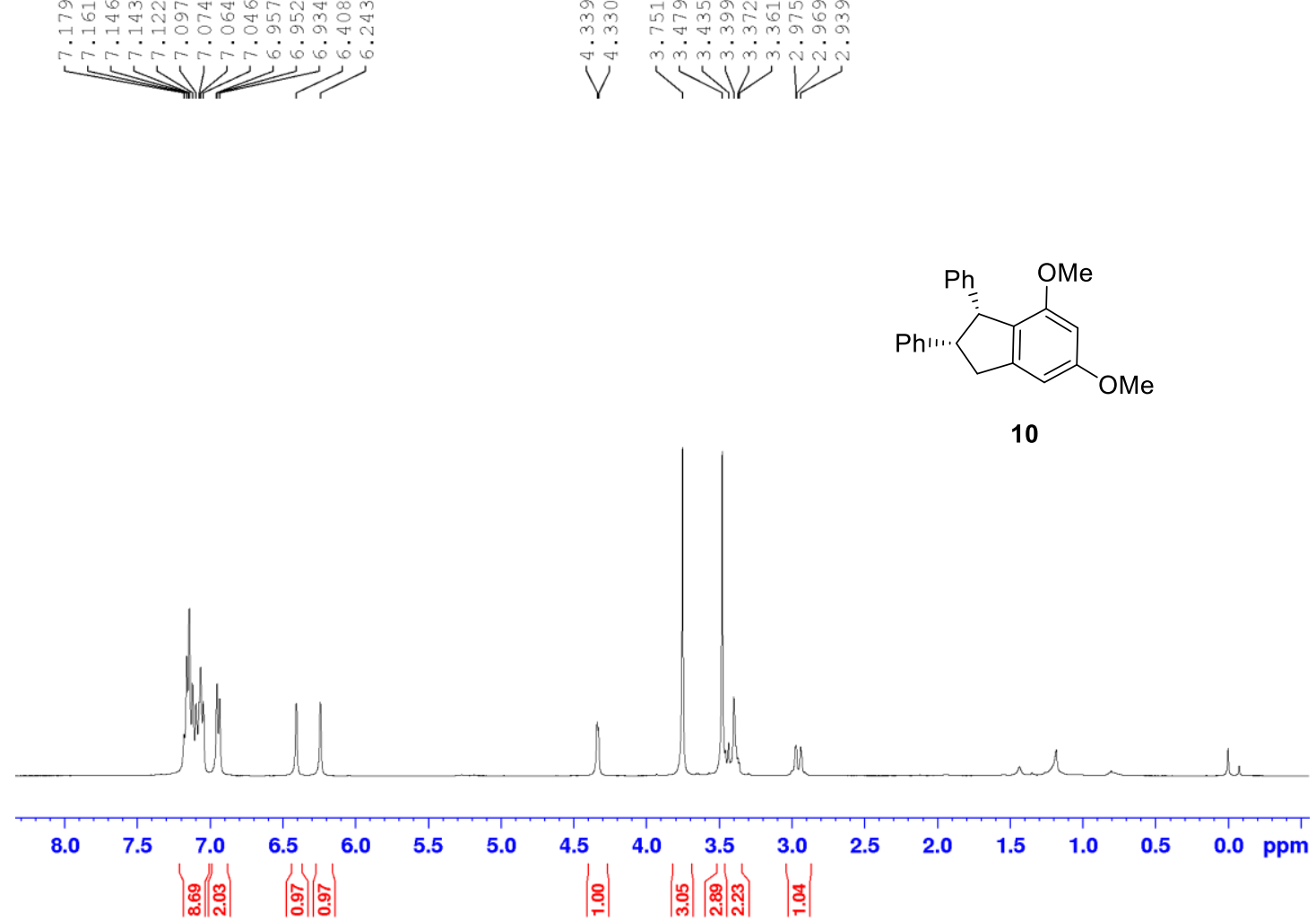

${ }^{13} \mathrm{C}\left\{{ }^{1} \mathrm{H}\right\}$ NMR spectrum of $\mathbf{1 0}\left(\mathrm{CDCl}_{3}, 100 \mathrm{MHz}\right)$ :
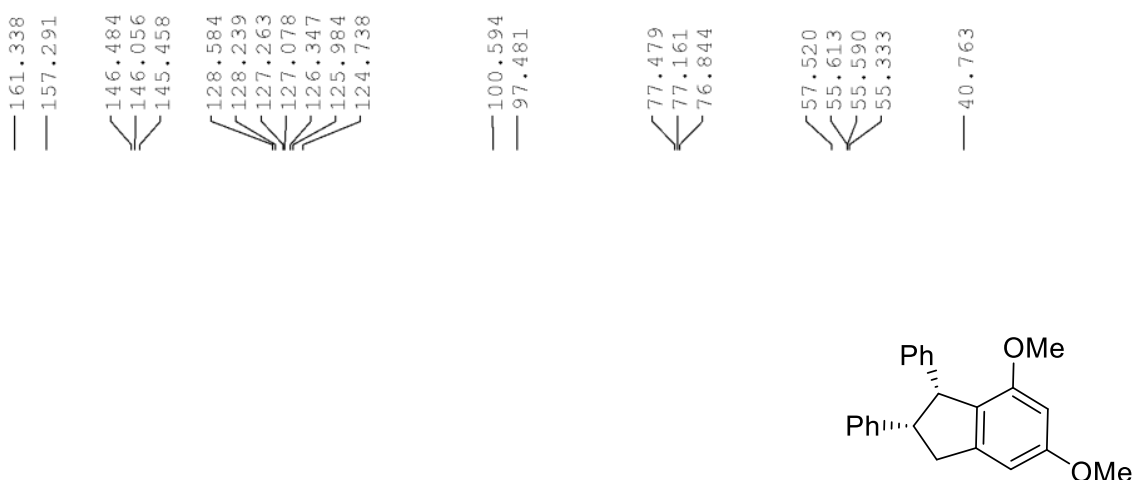

10

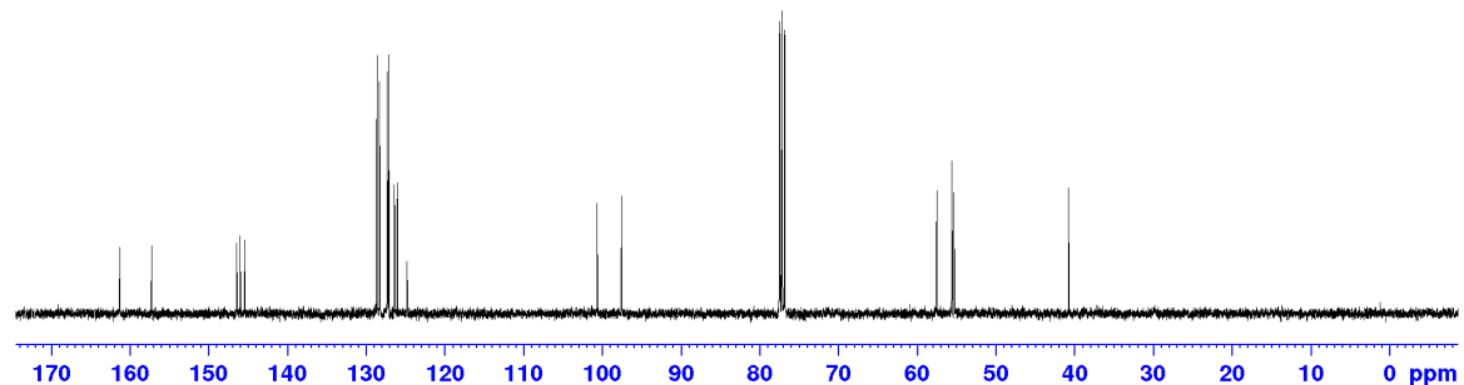


${ }^{1} \mathrm{H}$ NMR spectrum of $\mathbf{1 1}\left(\mathrm{CDCl}_{3}, 400 \mathrm{MHz}\right)$ :
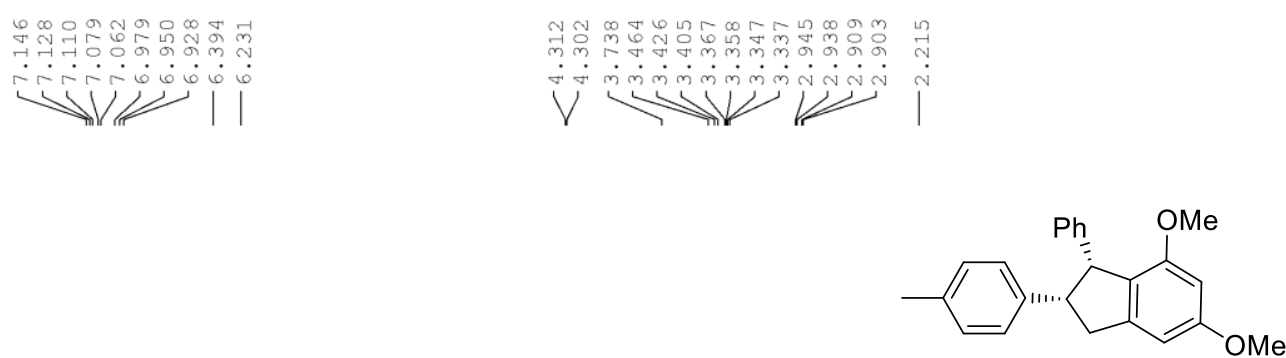

11

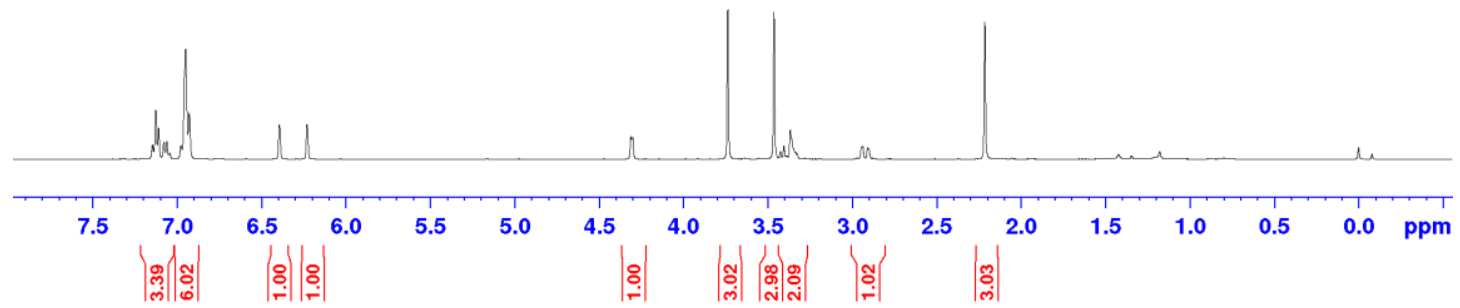

${ }^{13} \mathrm{C}\left\{{ }^{1} \mathrm{H}\right\}$ NMR spectrum of $\mathbf{1 1}\left(\mathrm{CDCl}_{3}, 100 \mathrm{MHz}\right)$ :
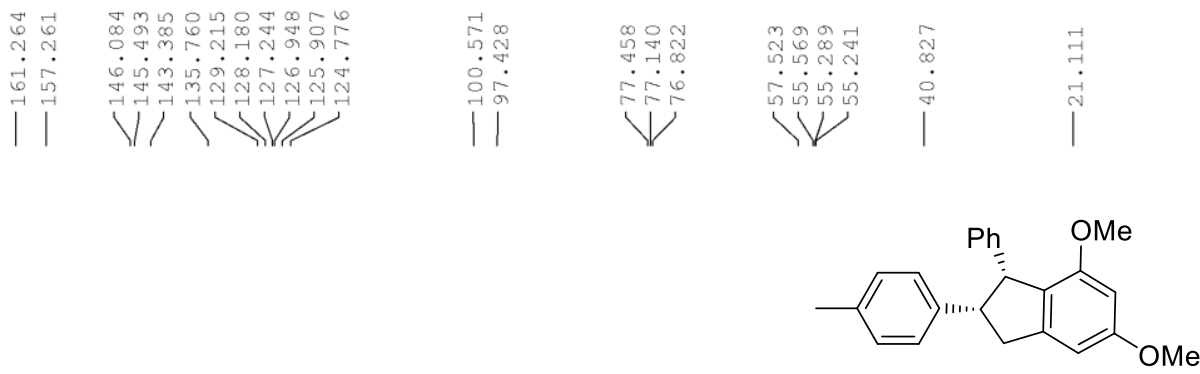

11

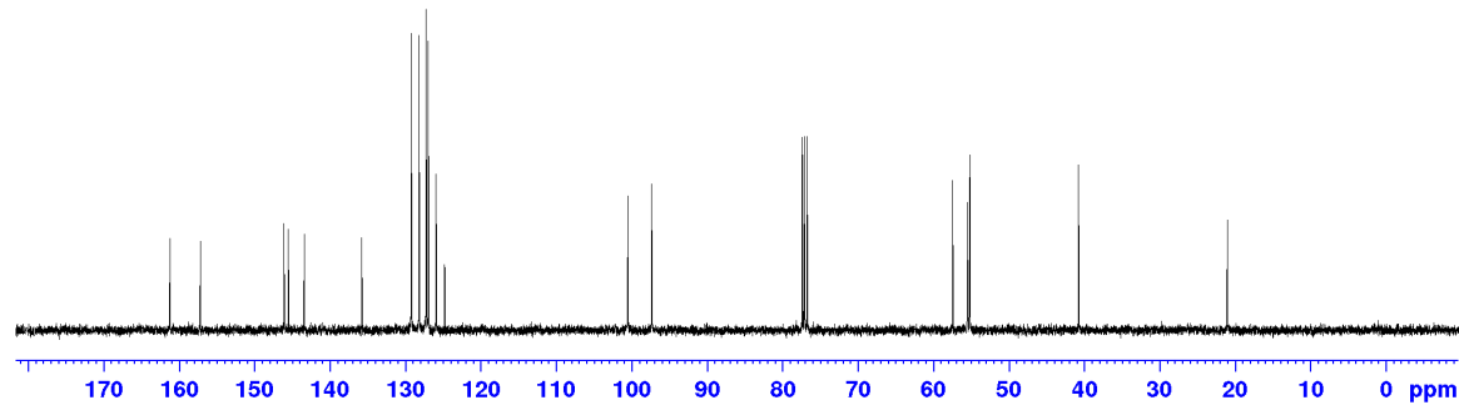




\section{Traces of HPLC spectra}
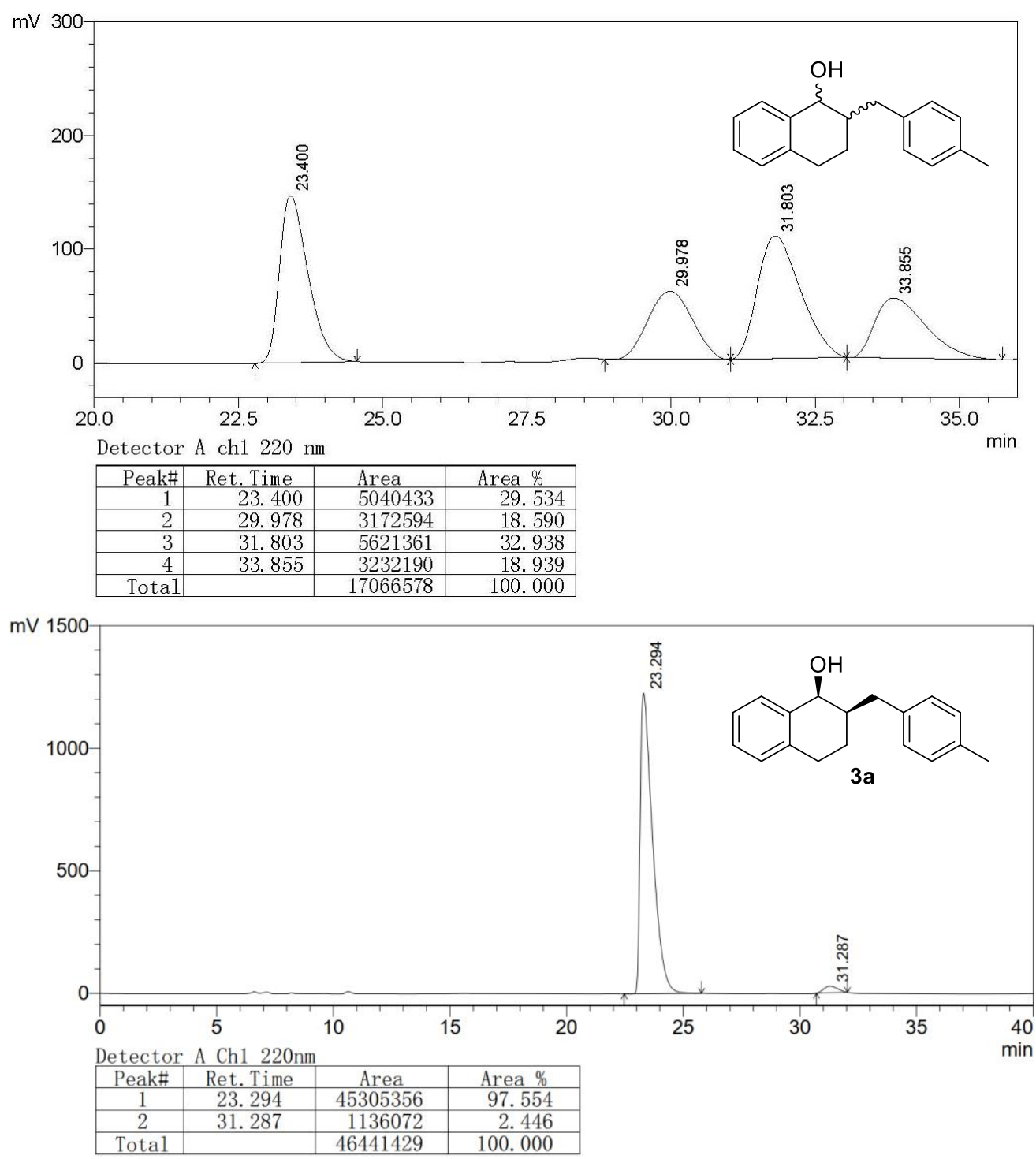

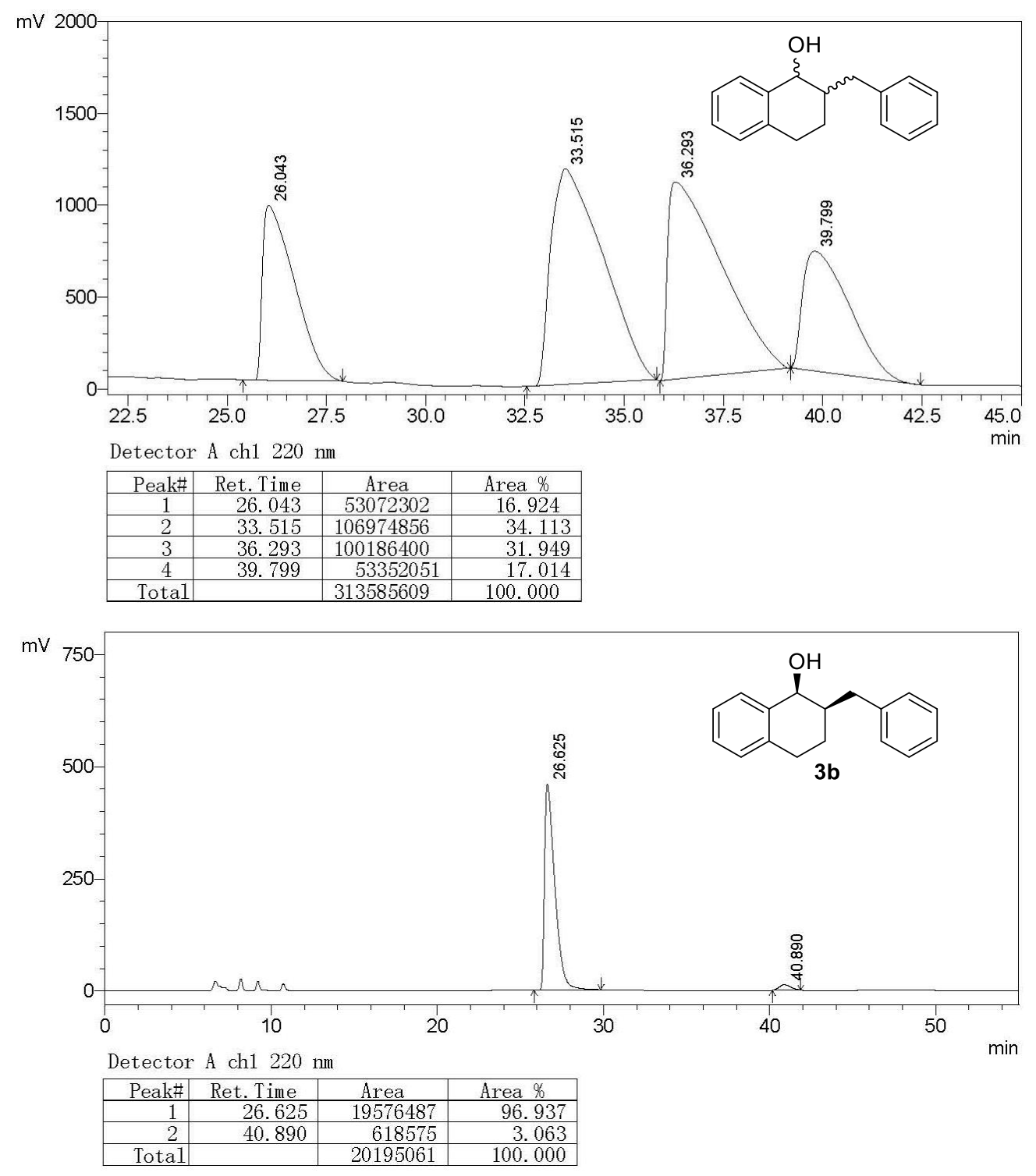


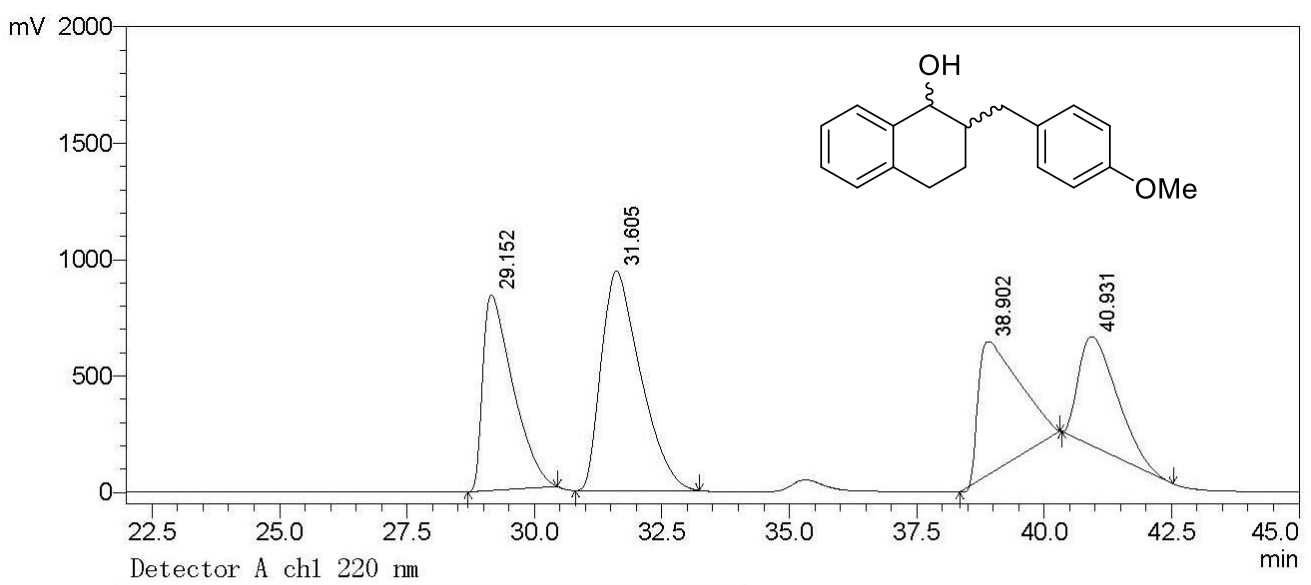

Detector A ch1 $220 \mathrm{~nm}$

\begin{tabular}{|c|c|c|c|}
\hline Peak\# & Ret. Time & Area & Area \% \\
\hline 1 & 29.152 & 34606820 & 24.435 \\
\hline 2 & 31.605 & 50708679 & 35.805 \\
\hline 3 & 38.902 & 31581760 & 22.299 \\
\hline 4 & 40.931 & 24728228 & 17.460 \\
\hline Total & & 141625487 & 100.000 \\
\hline
\end{tabular}

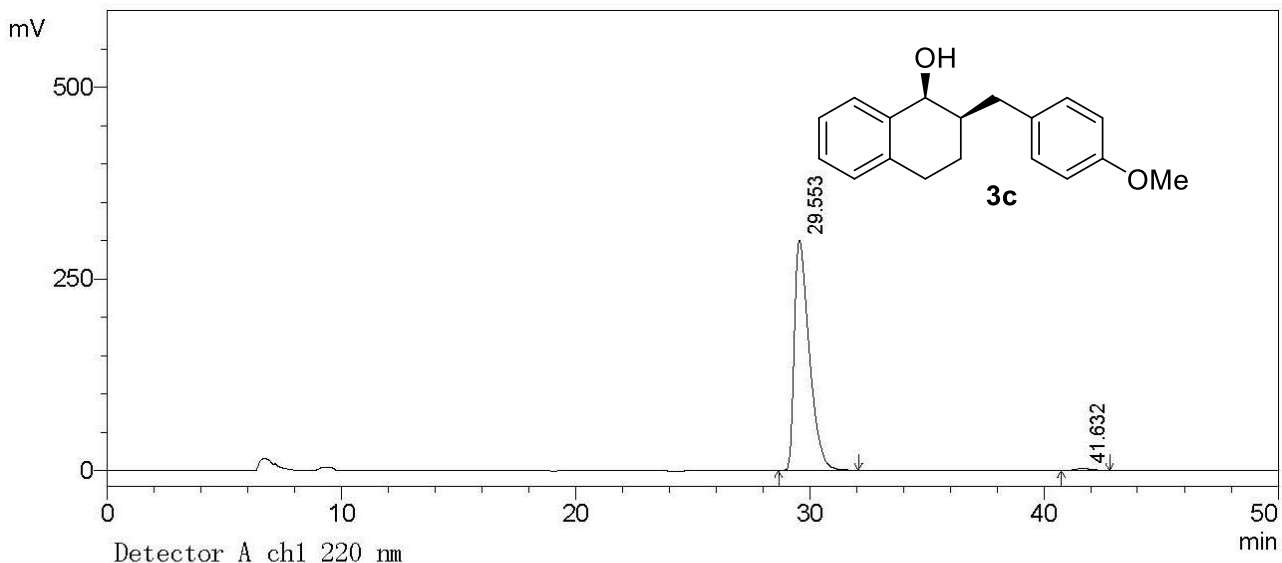

Detector A ch1 $220 \mathrm{~nm}$

\begin{tabular}{|r|r|r|r|}
\hline Peak\# & Ret. Time & \multicolumn{1}{c|}{ Area } & \multicolumn{1}{c|}{ Area \% } \\
\hline 1 & 29.553 & 13277970 & 98.941 \\
\hline 2 & 41.632 & 142127 & 1.059 \\
\hline Total & & 13420097 & 100.000 \\
\hline
\end{tabular}



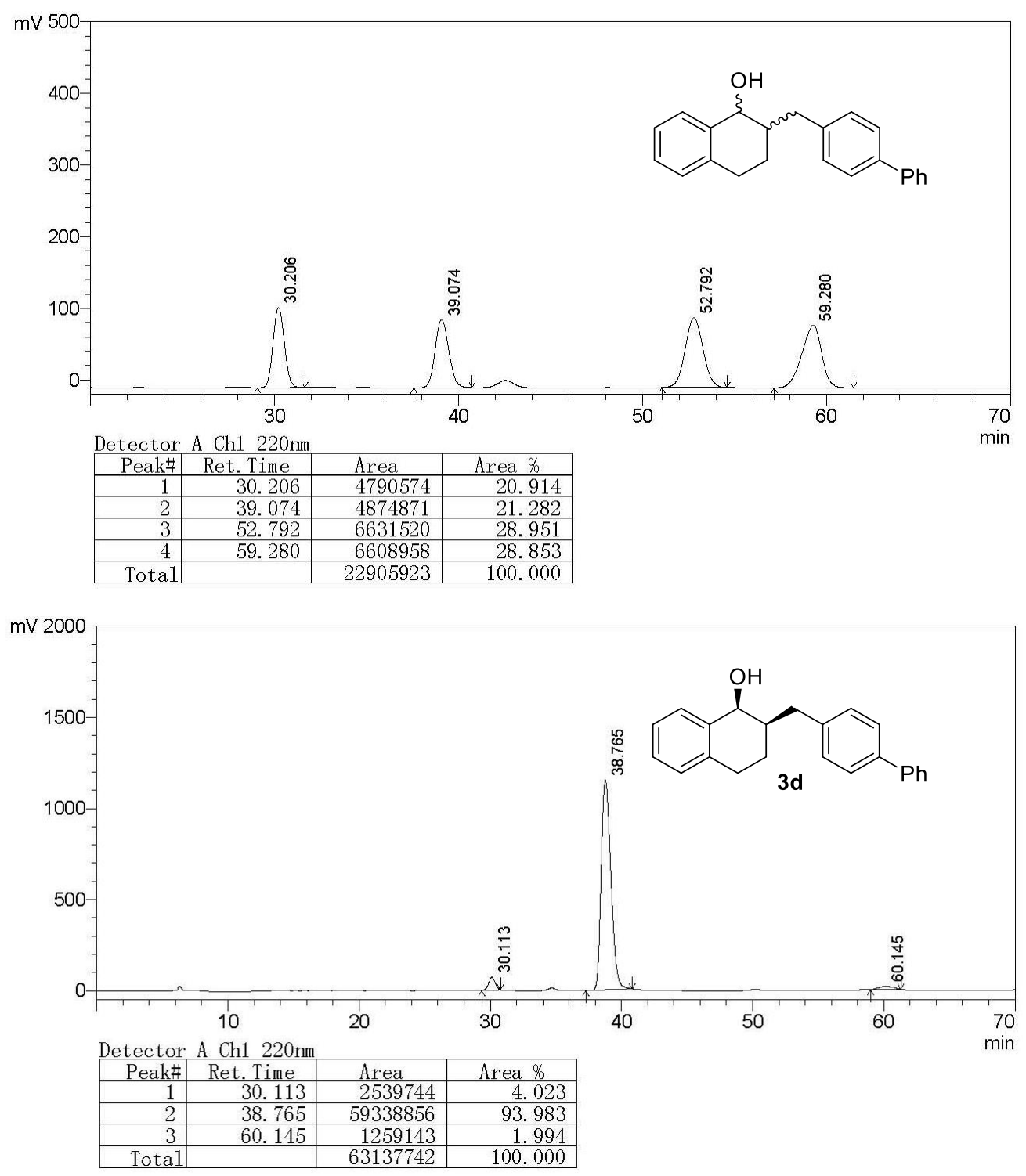

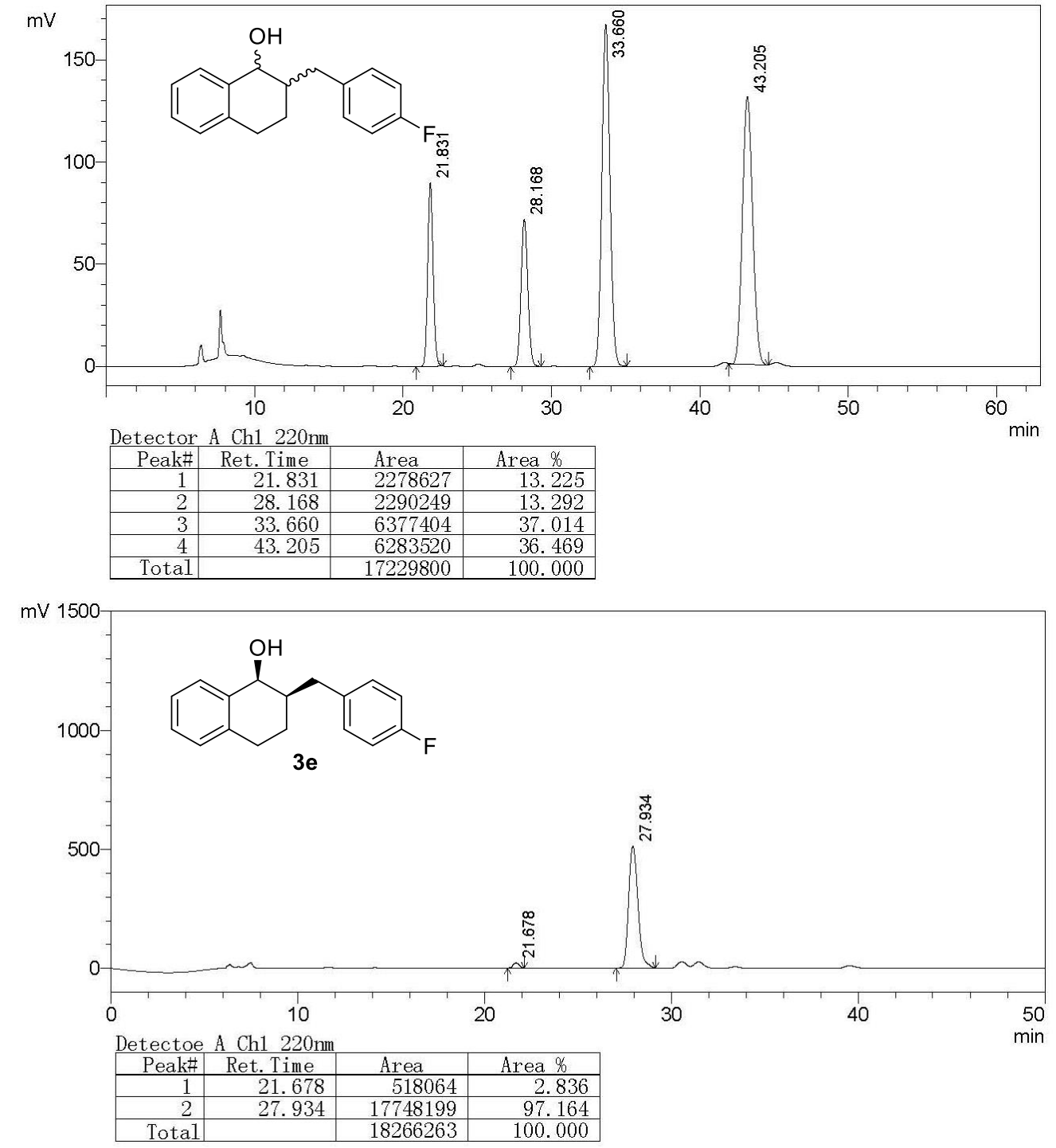


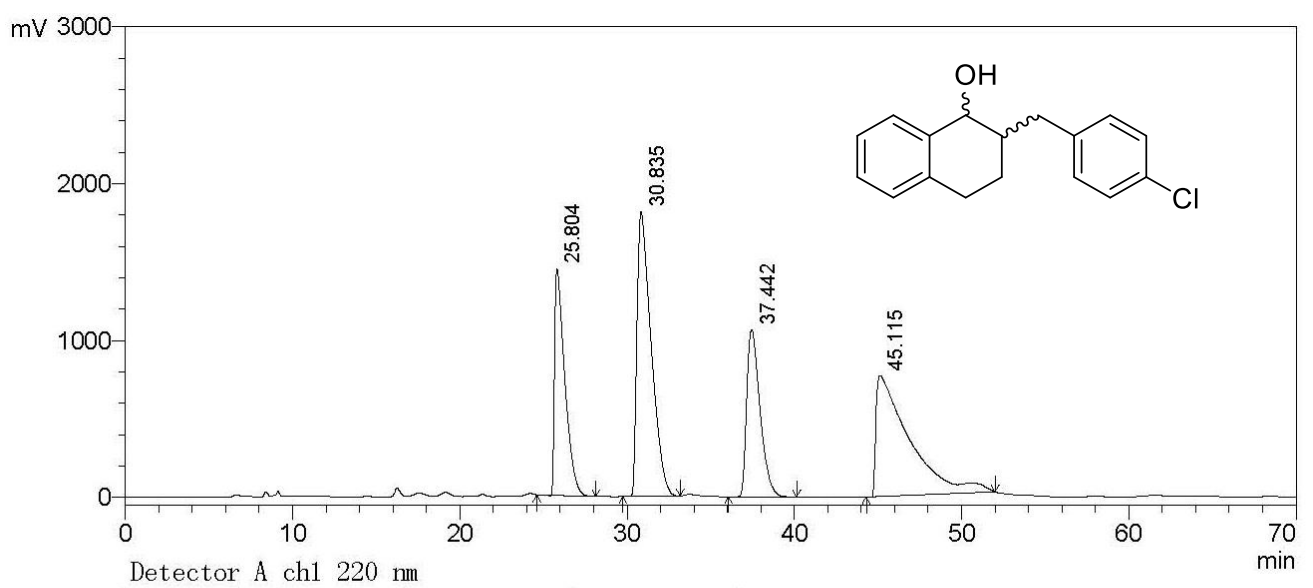

\begin{tabular}{|r|r|r|r|}
\hline Peak\# & Ret. Time & \multicolumn{1}{c|}{ Area } & \multicolumn{1}{c|}{ Area \% } \\
\hline 1 & 25.804 & 61982260 & 18.521 \\
\hline 2 & 30.835 & 106870268 & 31.933 \\
\hline 3 & 37.442 & 58944528 & 17.613 \\
\hline 4 & 45.115 & 106868693 & 31.933 \\
\hline Total & & 334665749 & 100.000 \\
\hline
\end{tabular}

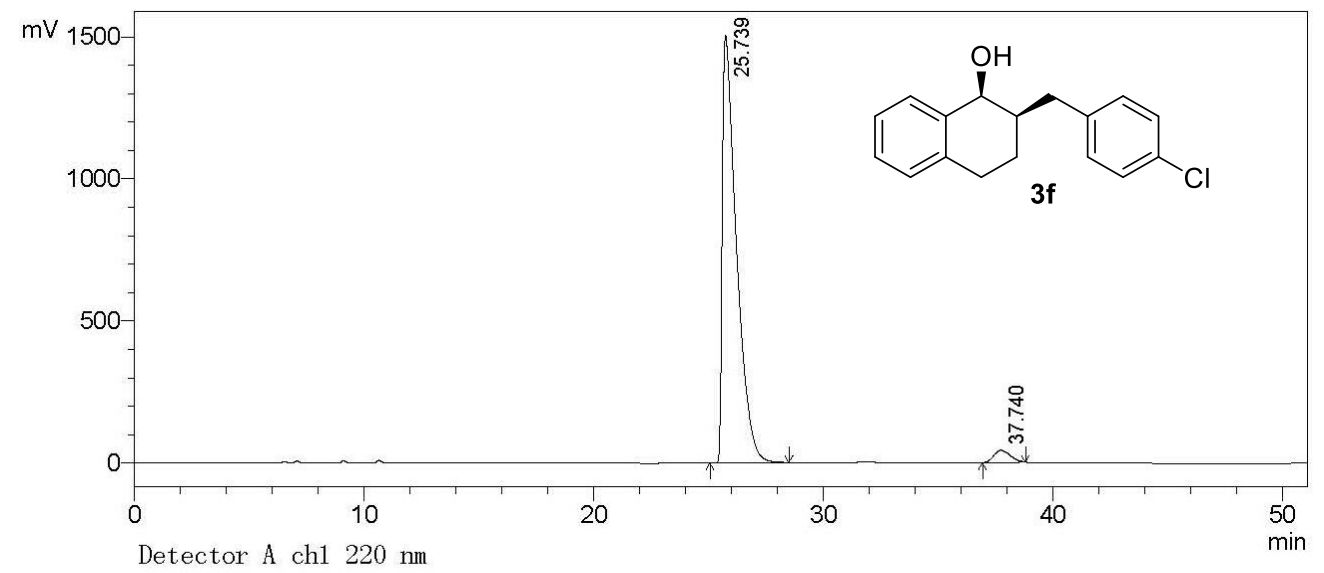

Detector A ch1 $220 \mathrm{~nm}$

\begin{tabular}{|r|r|r|r|}
\hline Peak\# & Ret. Time & \multicolumn{1}{c|}{ Area } & \multicolumn{1}{c|}{ Area \% } \\
\hline 1 & 25.739 & 64238517 & 96.778 \\
\hline 2 & 37.740 & 2138476 & 3.222 \\
\hline Tota1 & & 66376993 & 100.000 \\
\hline
\end{tabular}



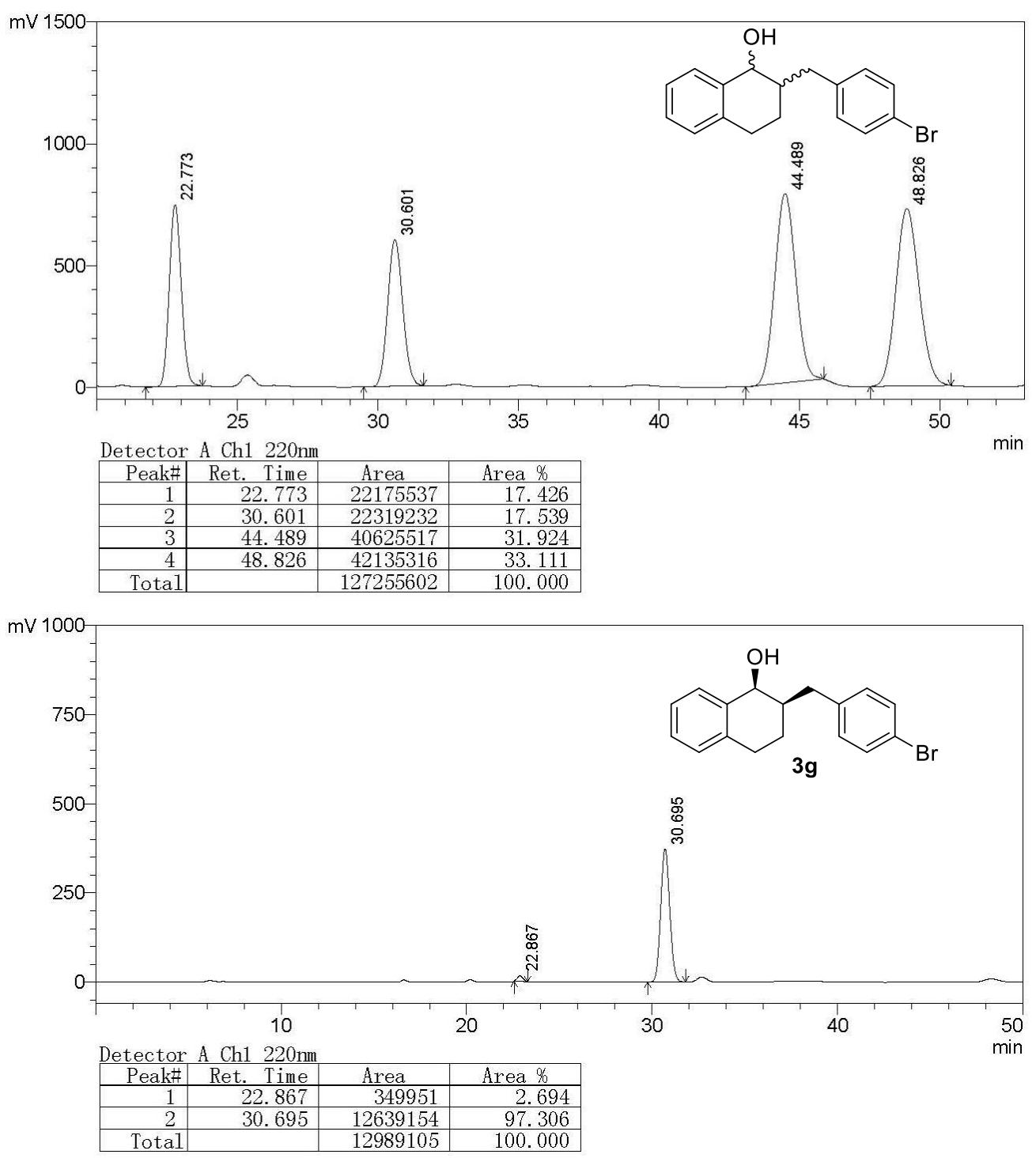

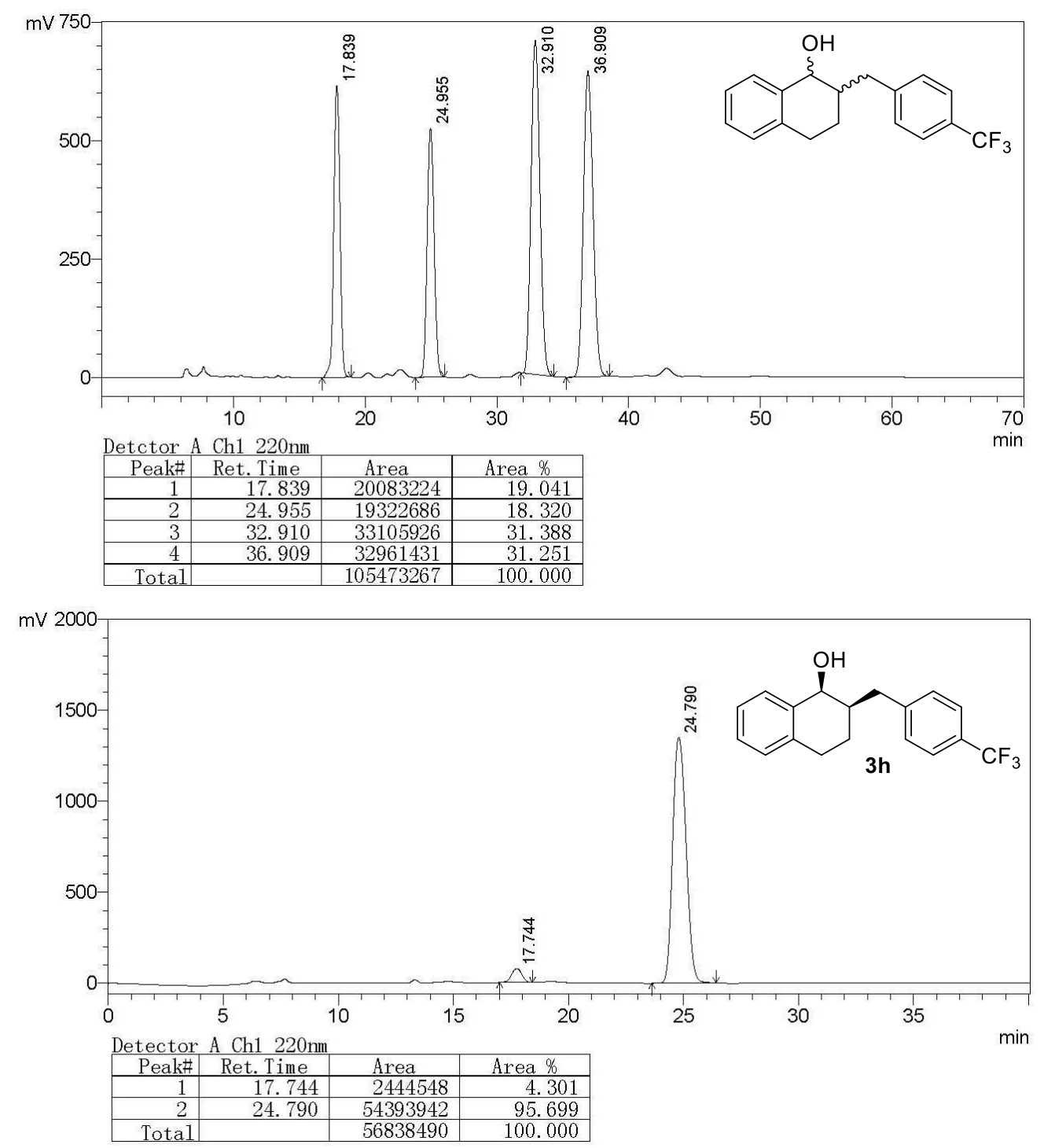

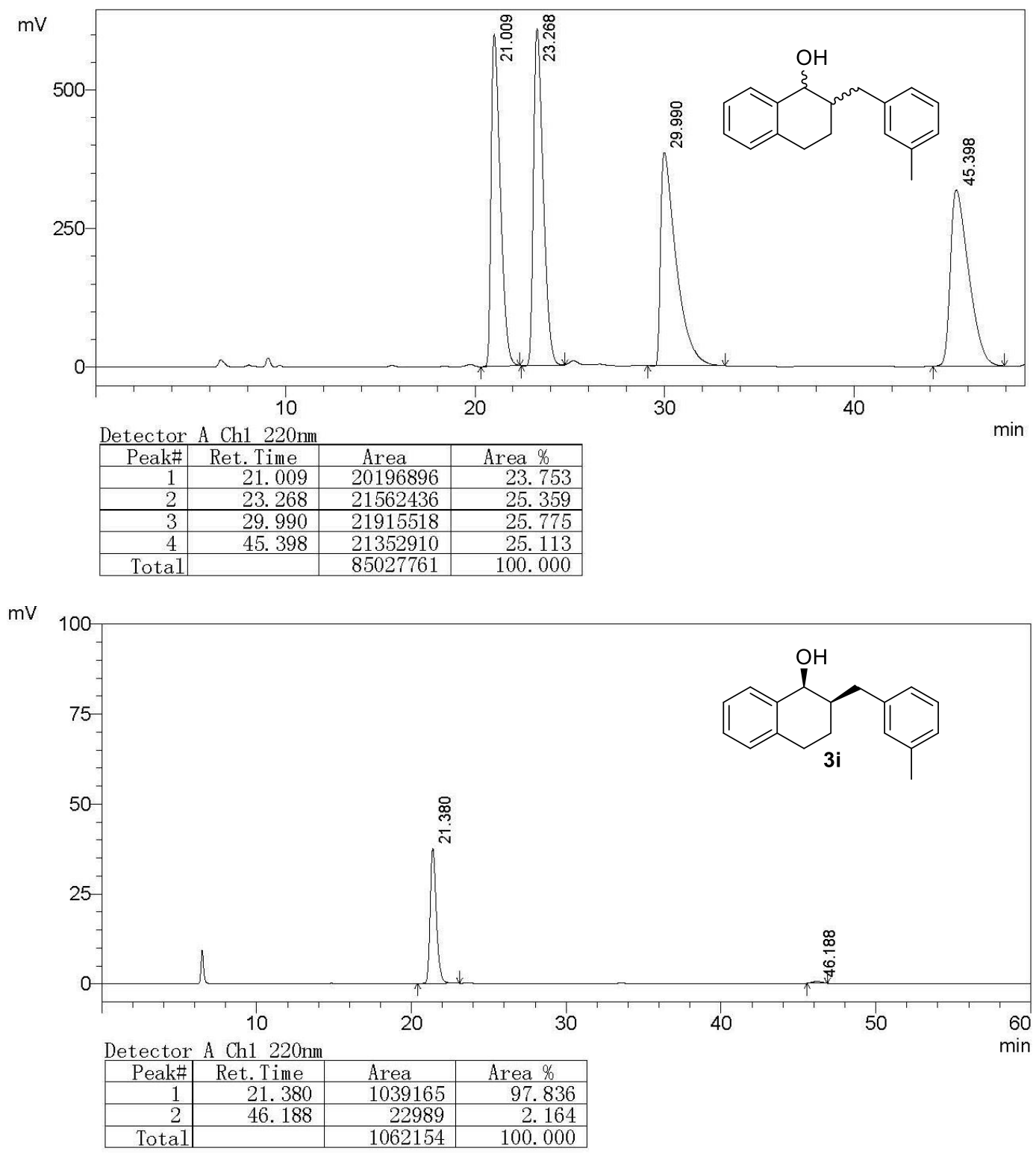

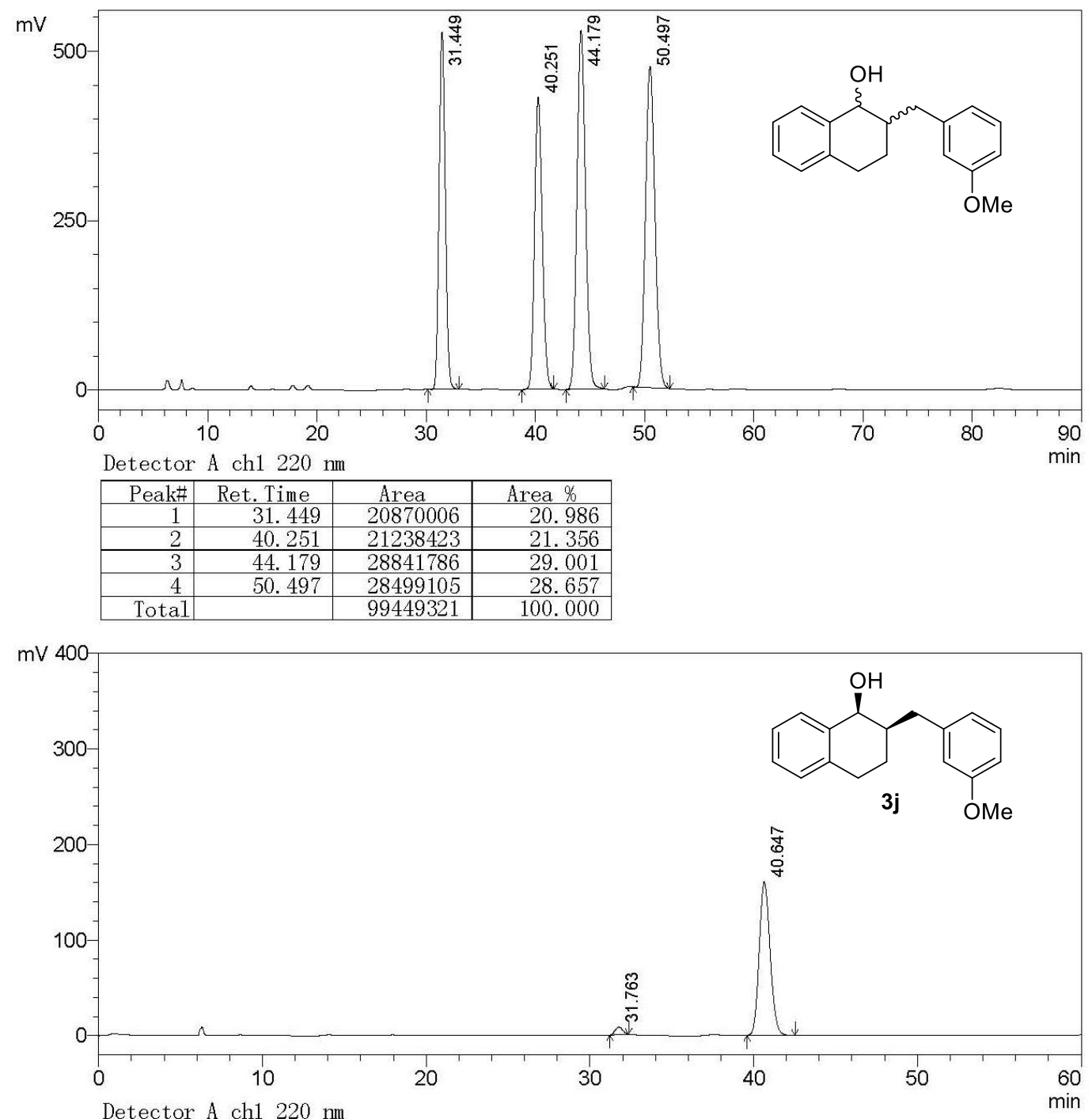

Detector A ch1 $220 \mathrm{~nm}$

\begin{tabular}{|r|r|r|r|}
\hline Peak\# & Ret. Time & \multicolumn{1}{c|}{ Area } & Area \% \\
\hline 1 & 31.763 & 261445 & 3.372 \\
\hline 2 & 40.647 & 7492987 & 96.628 \\
\hline Total & & 7754432 & 100.000 \\
\hline
\end{tabular}




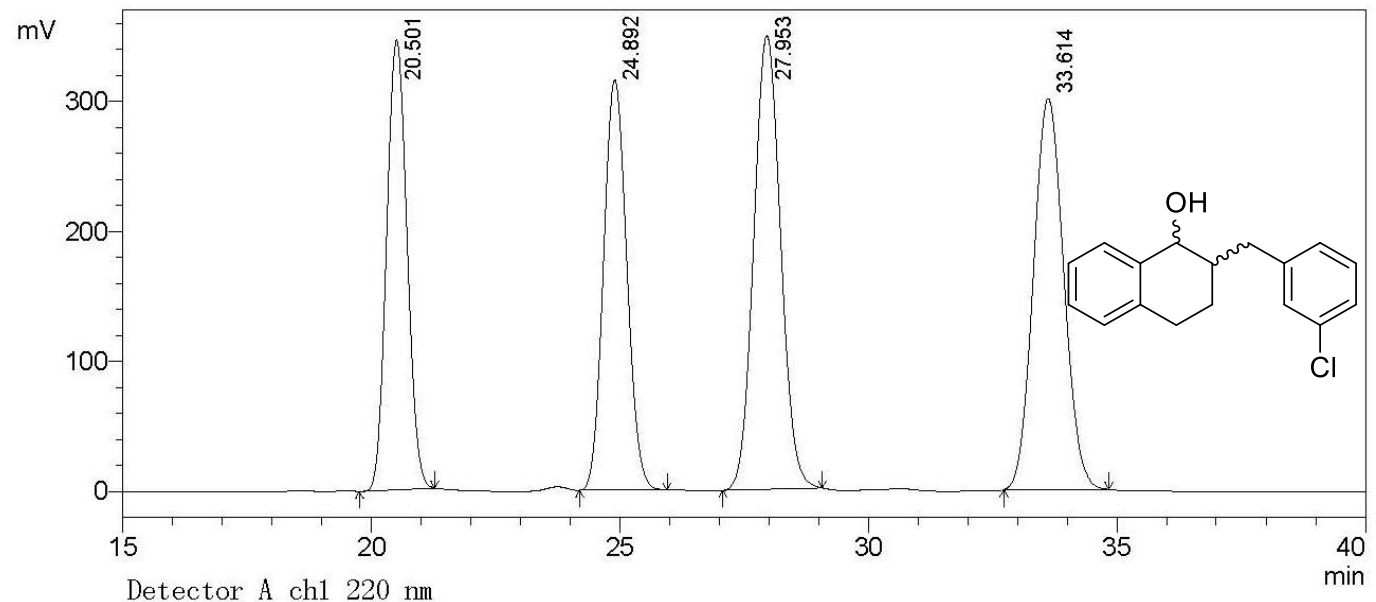

\begin{tabular}{|r|r|r|r|}
\hline Peak\# & Ret. Time & \multicolumn{1}{|c|}{ Area } & Area \% \\
\hline 1 & 20.501 & 9919974 & 21.521 \\
\hline 2 & 24.892 & 10286255 & 22.315 \\
\hline 3 & 27.953 & 13103093 & 28.426 \\
\hline 4 & 33.614 & 12785574 & 27.738 \\
\hline Total & & 46094896 & 100.000 \\
\hline
\end{tabular}

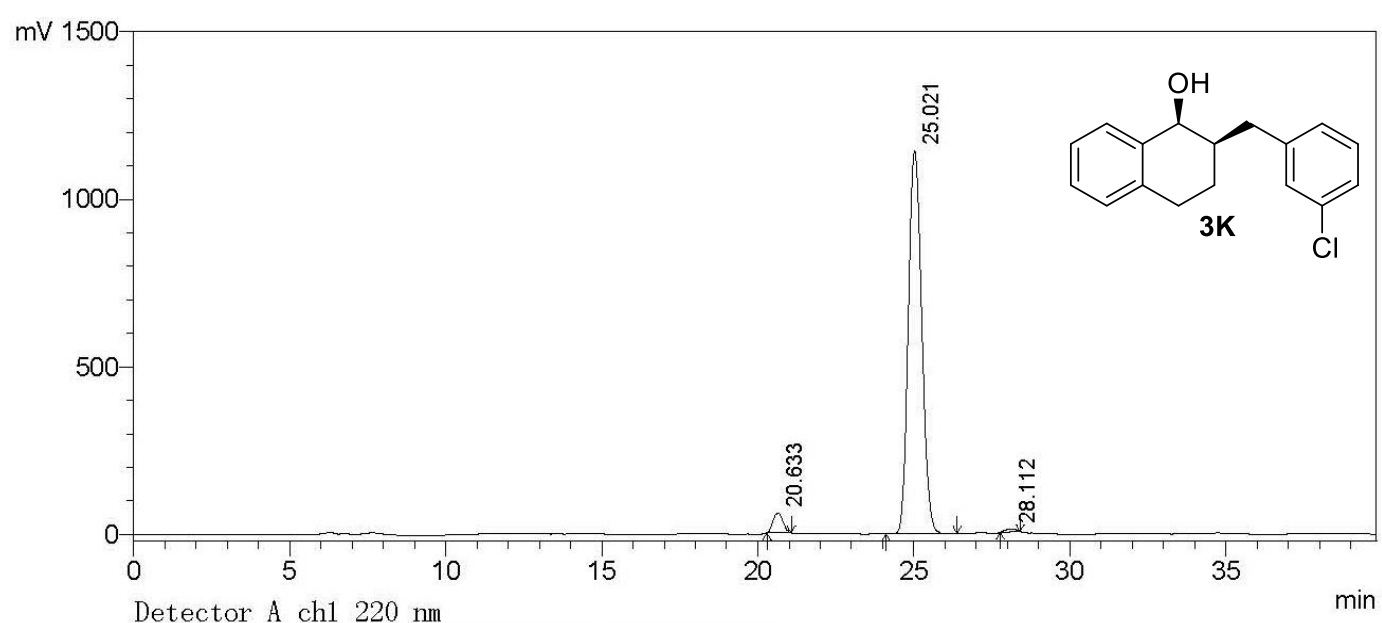

Detector A ch1 $220 \mathrm{~nm}$

\begin{tabular}{|r|r|r|r|}
\hline Peak\# & Ret. Time & \multicolumn{1}{|c|}{ Area } & Area \% \\
\hline 1 & 20.633 & 1260356 & 3.546 \\
\hline 2 & 25.021 & 34073035 & 95.855 \\
\hline 3 & 28.112 & 213080 & 0.599 \\
\hline Tota1 & & 35546471 & 100.000 \\
\hline
\end{tabular}



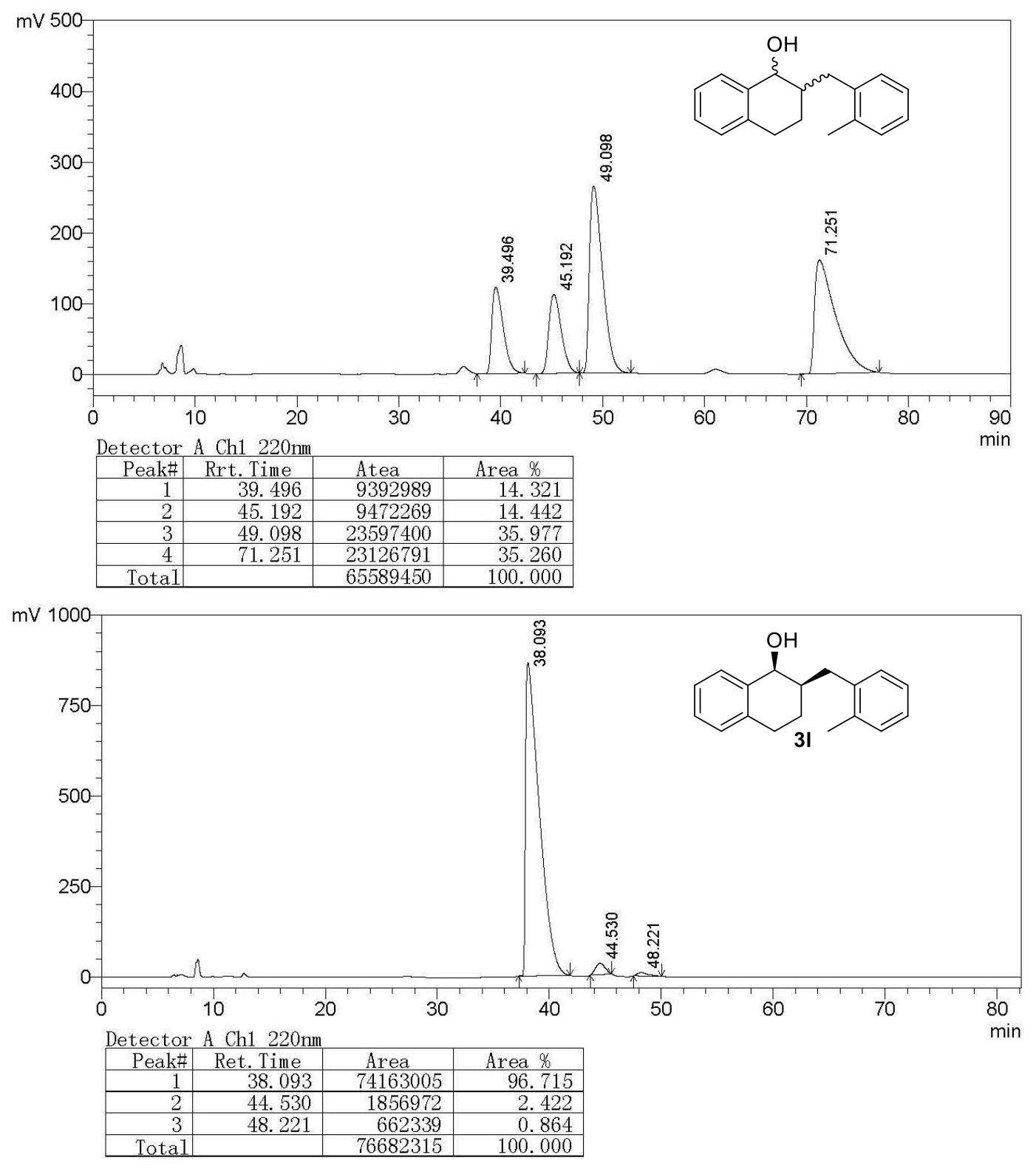

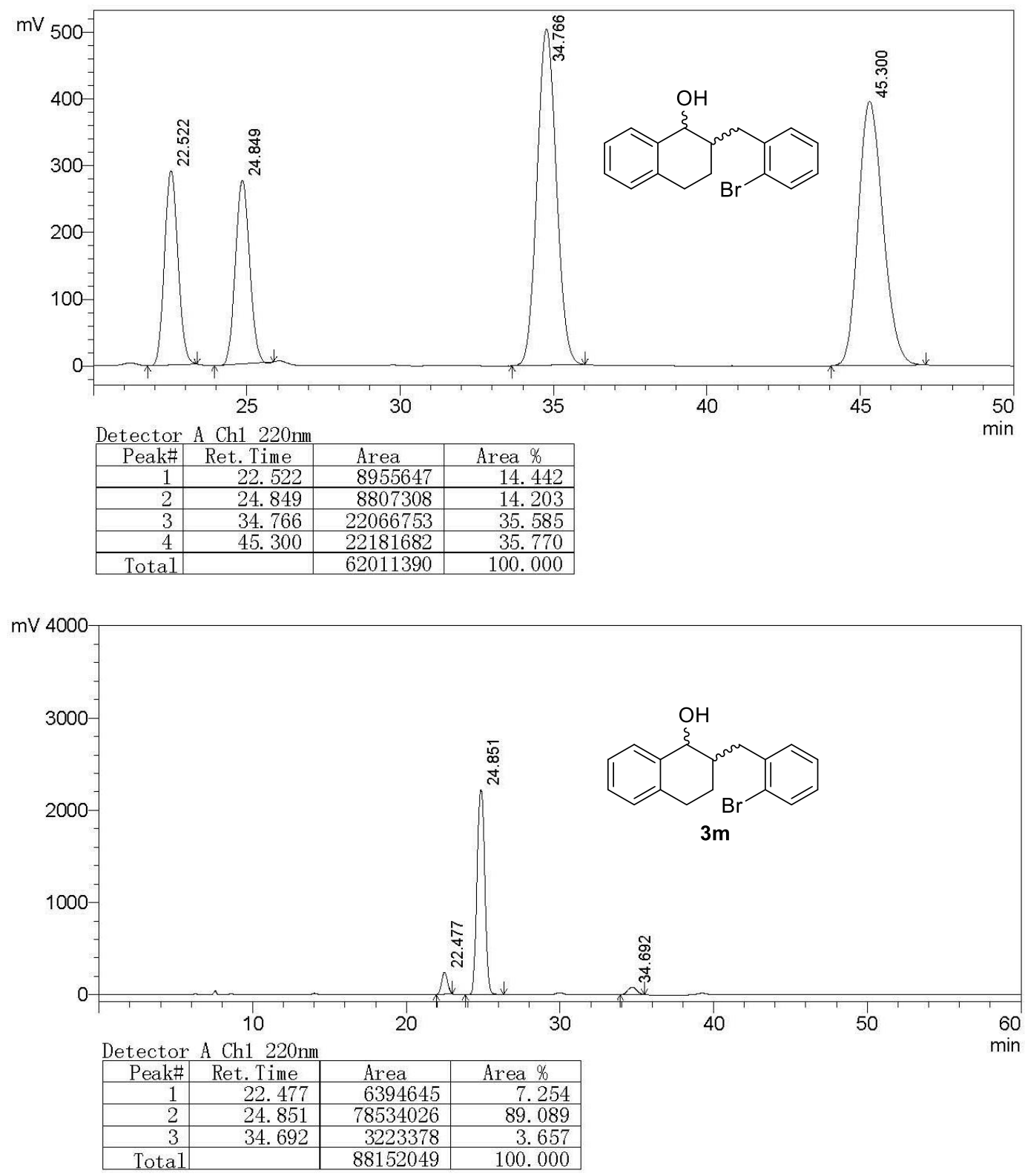

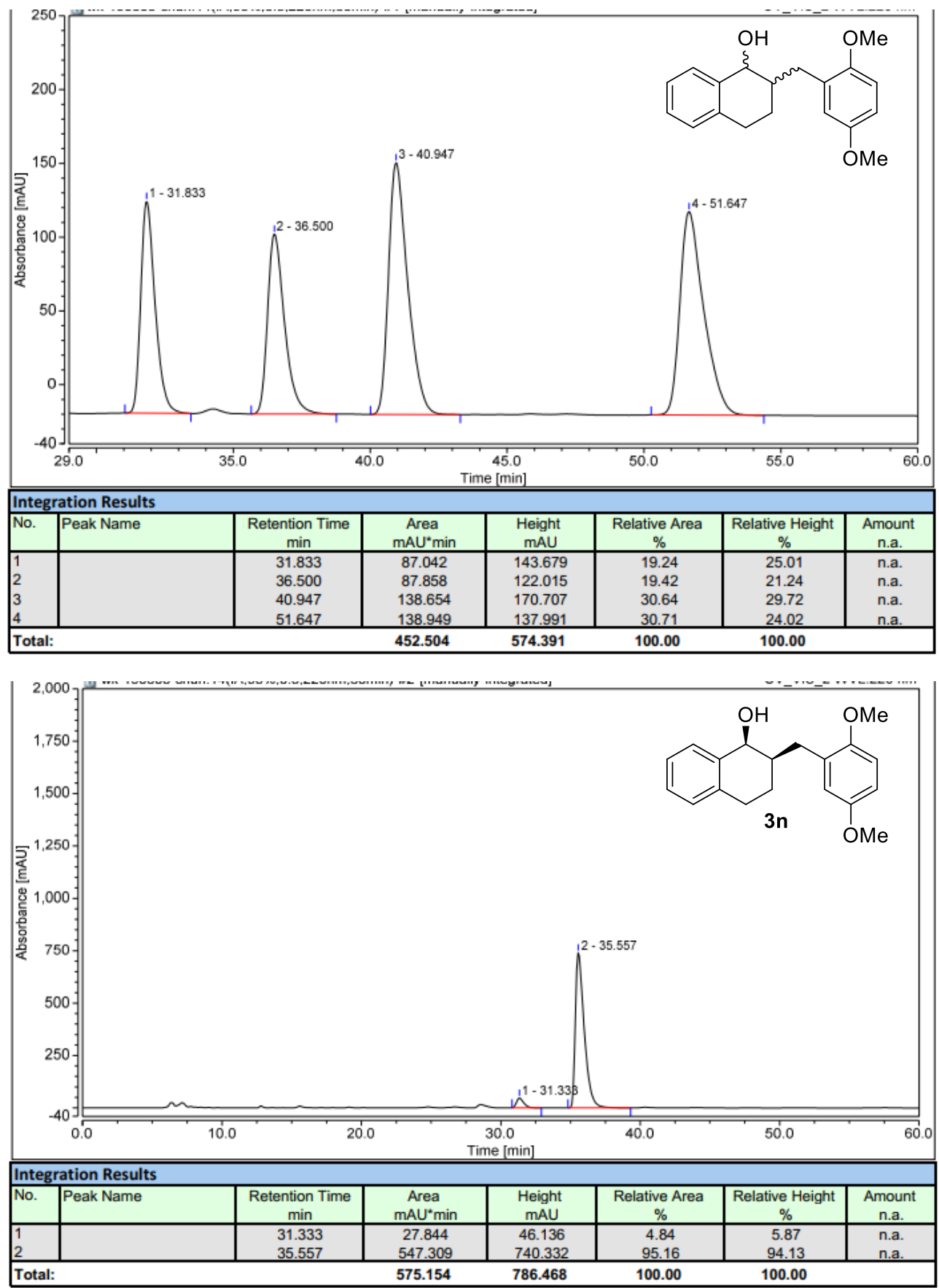

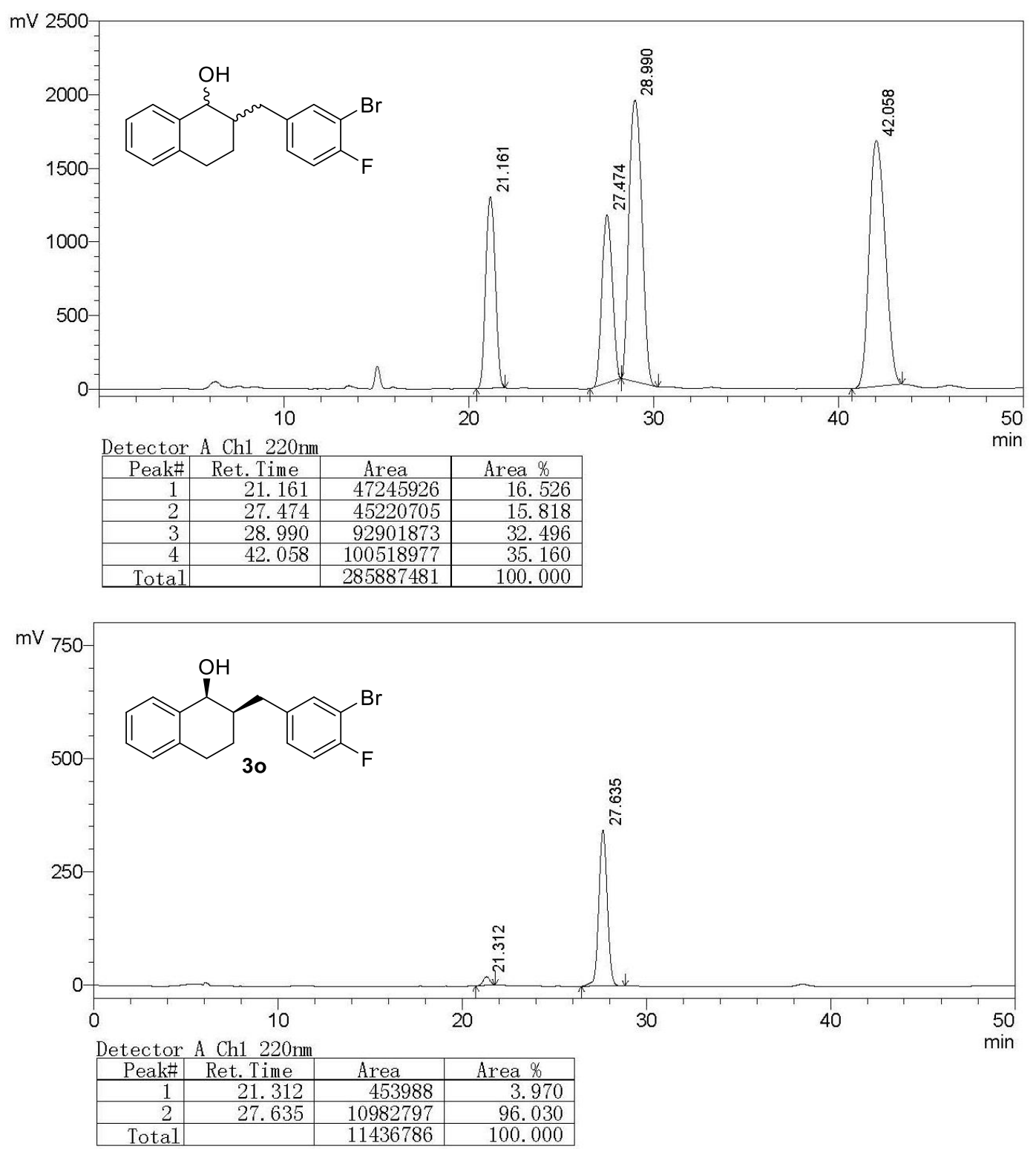

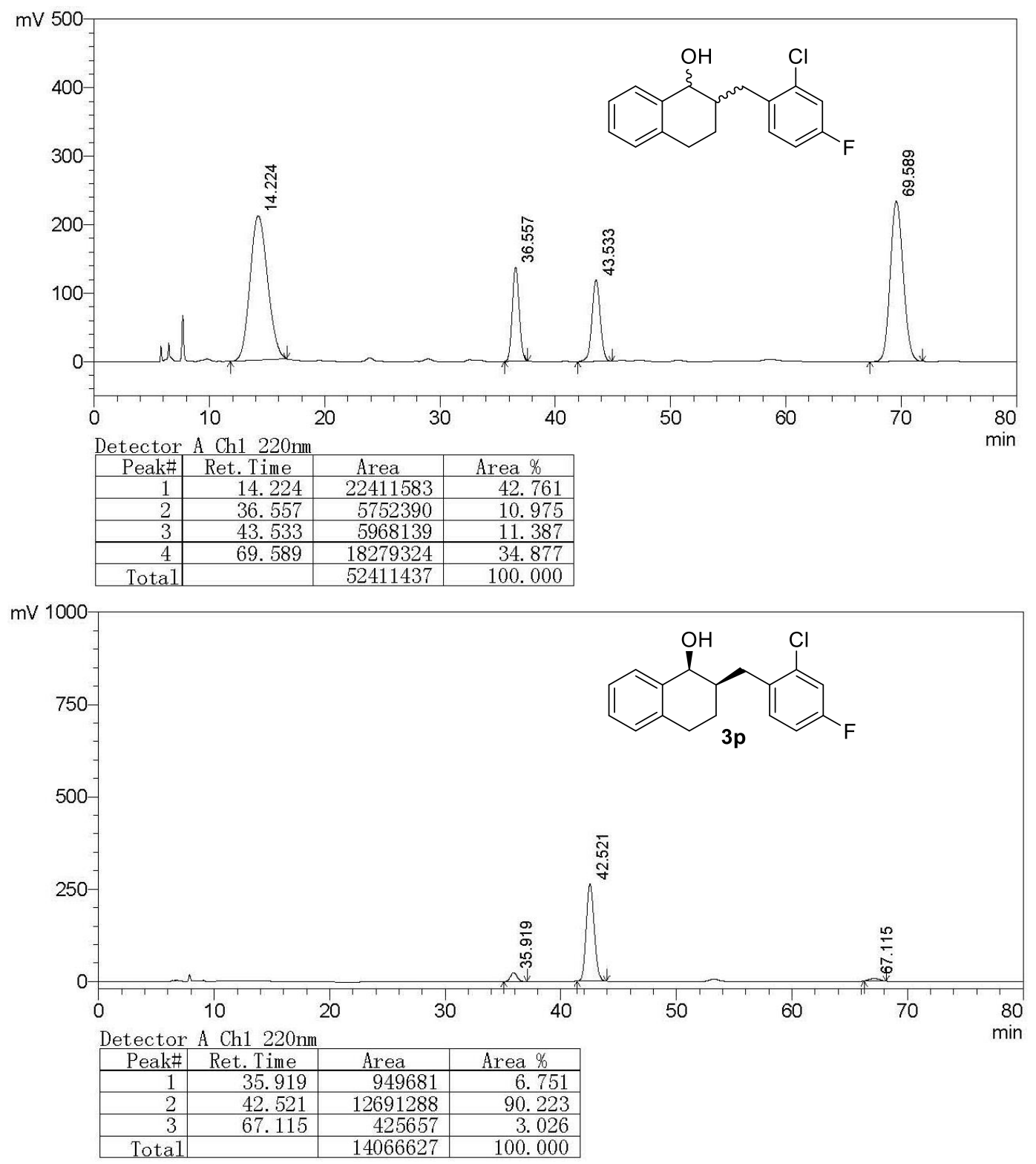


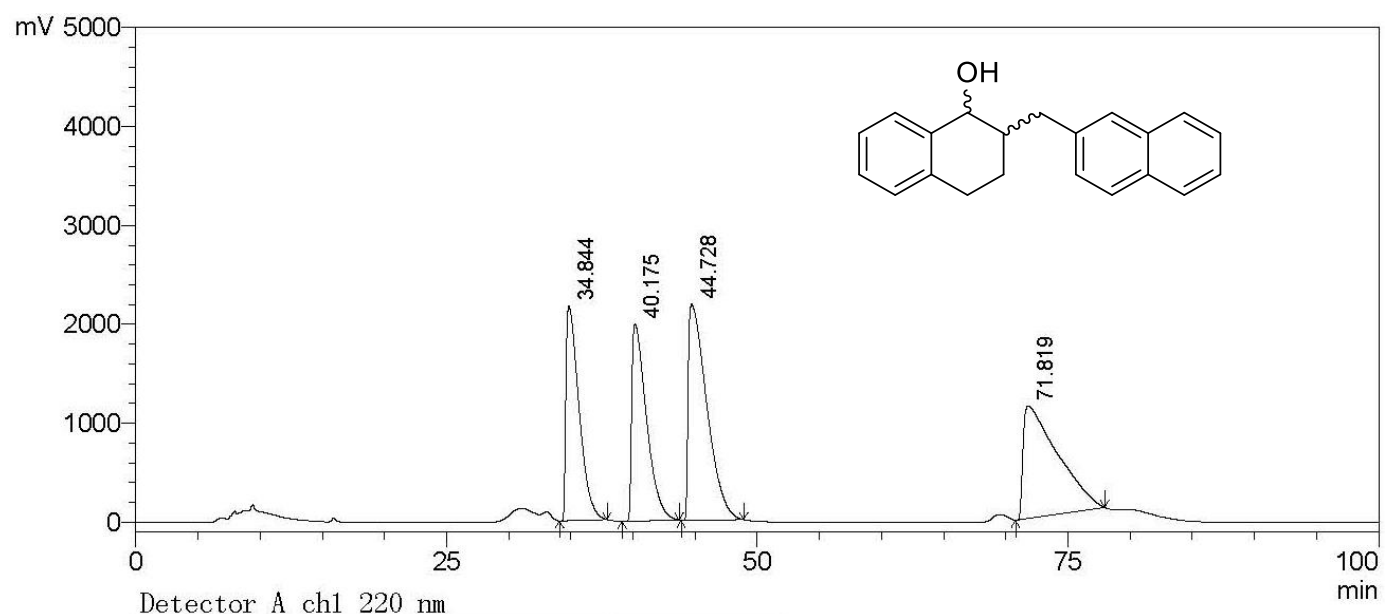

\begin{tabular}{|r|r|r|r|}
\hline Peak\# & Ret. Time & Area & Area \% \\
\hline 1 & 34.844 & 159056817 & 20.704 \\
\hline 2 & 40.175 & 163926989 & 21.338 \\
\hline 3 & 44.728 & 227004569 & 29.549 \\
\hline 4 & 71.819 & 218253607 & 28.409 \\
\hline Total & & 768241982 & 100.000 \\
\hline
\end{tabular}

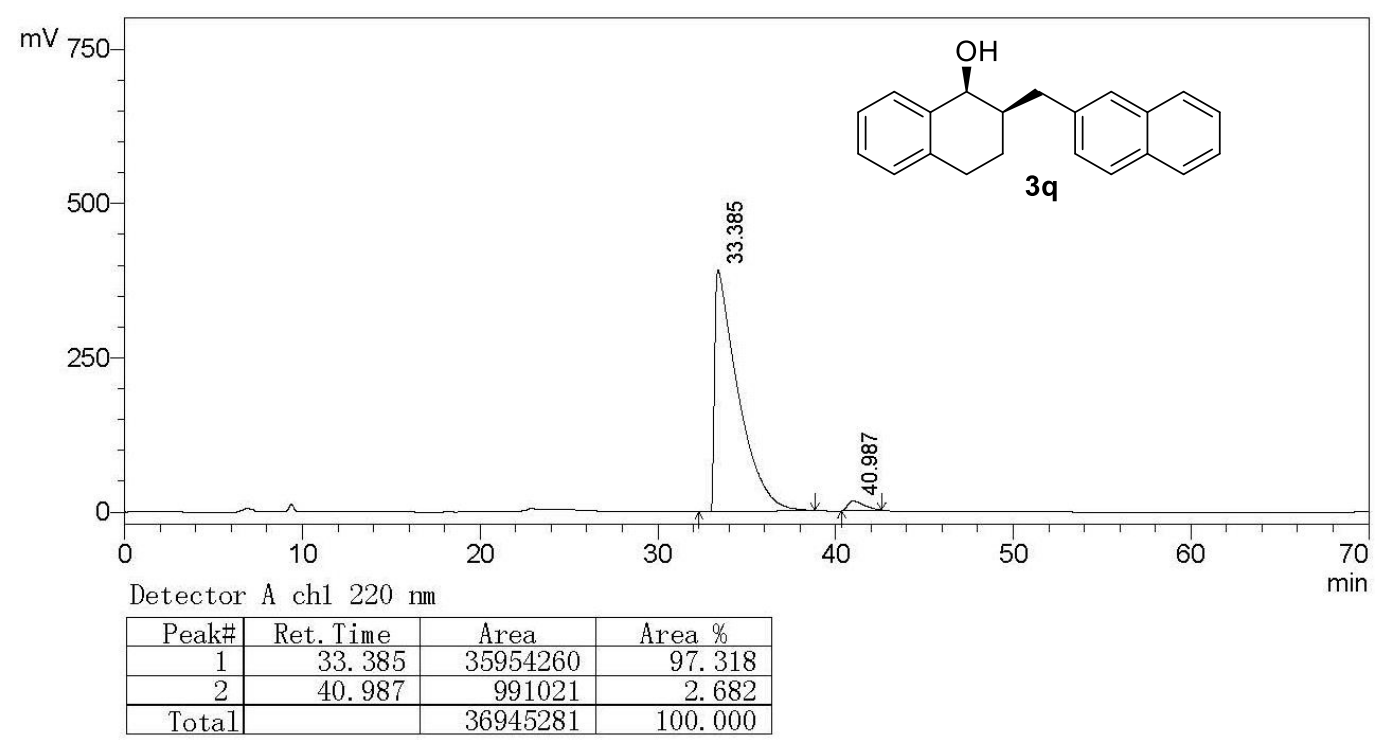



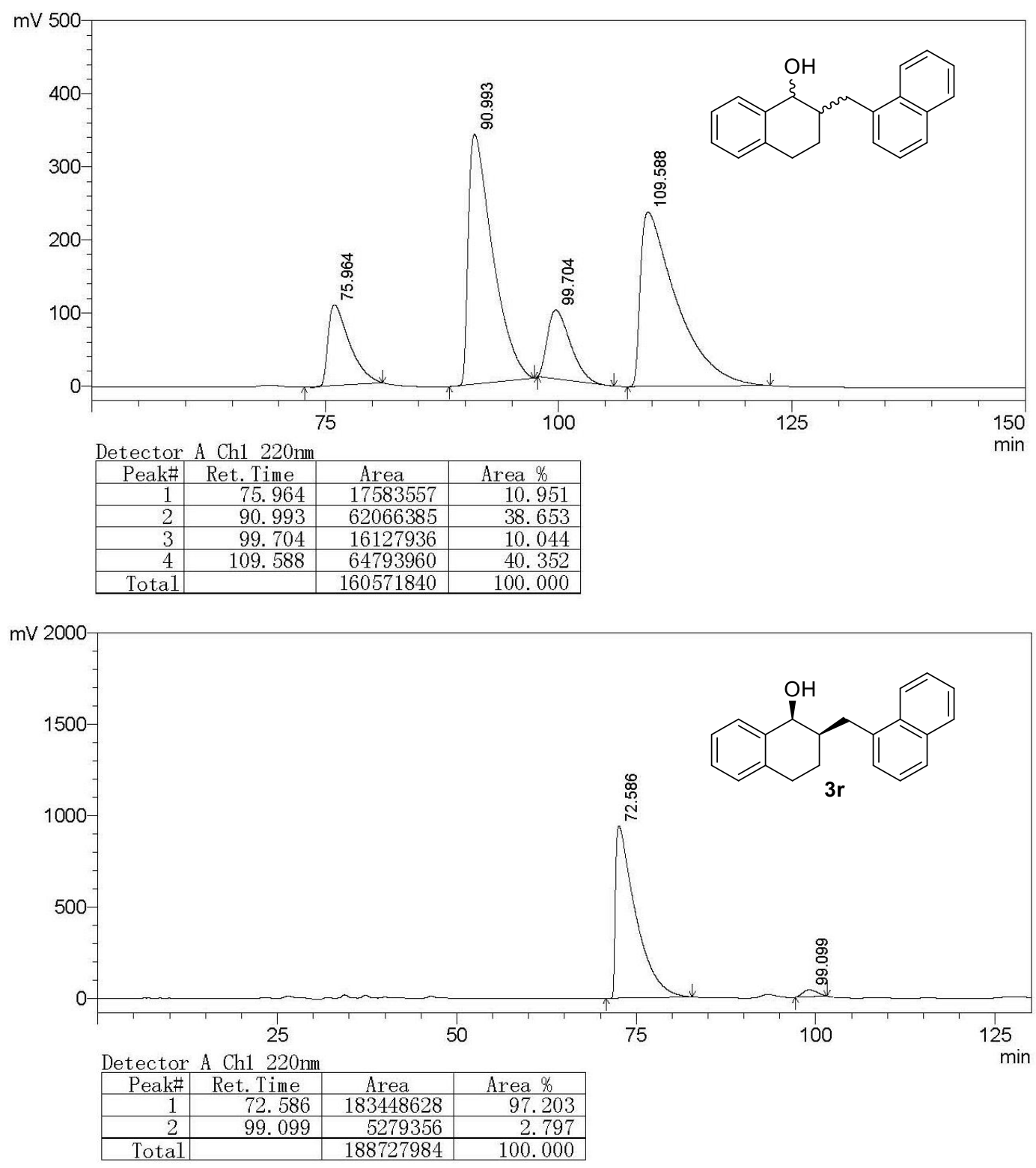

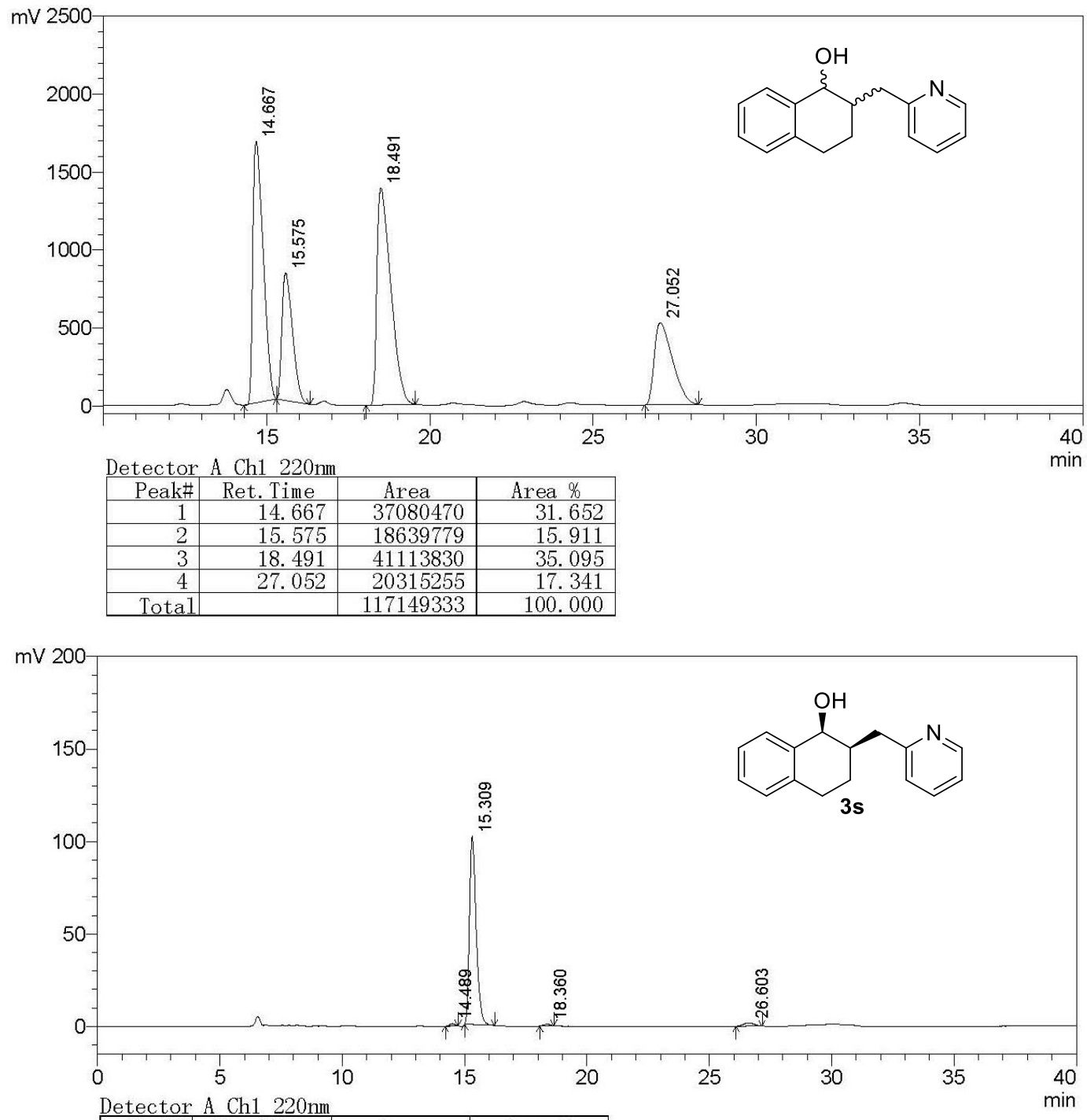

Detector A Ch1 $220 \mathrm{~nm}$
\begin{tabular}{|r|r|r|r|}
\hline Peak\# & Ret. Time & Area & Area \% \\
\hline 1 & 14.489 & 20162 & 0.985 \\
\hline 2 & 15.309 & 1958264 & 95.668 \\
\hline 3 & 18.360 & 17820 & 0.871 \\
\hline 4 & 26.603 & 50699 & 2.477 \\
\hline Total & & 2046946 & 100.000 \\
\hline
\end{tabular}



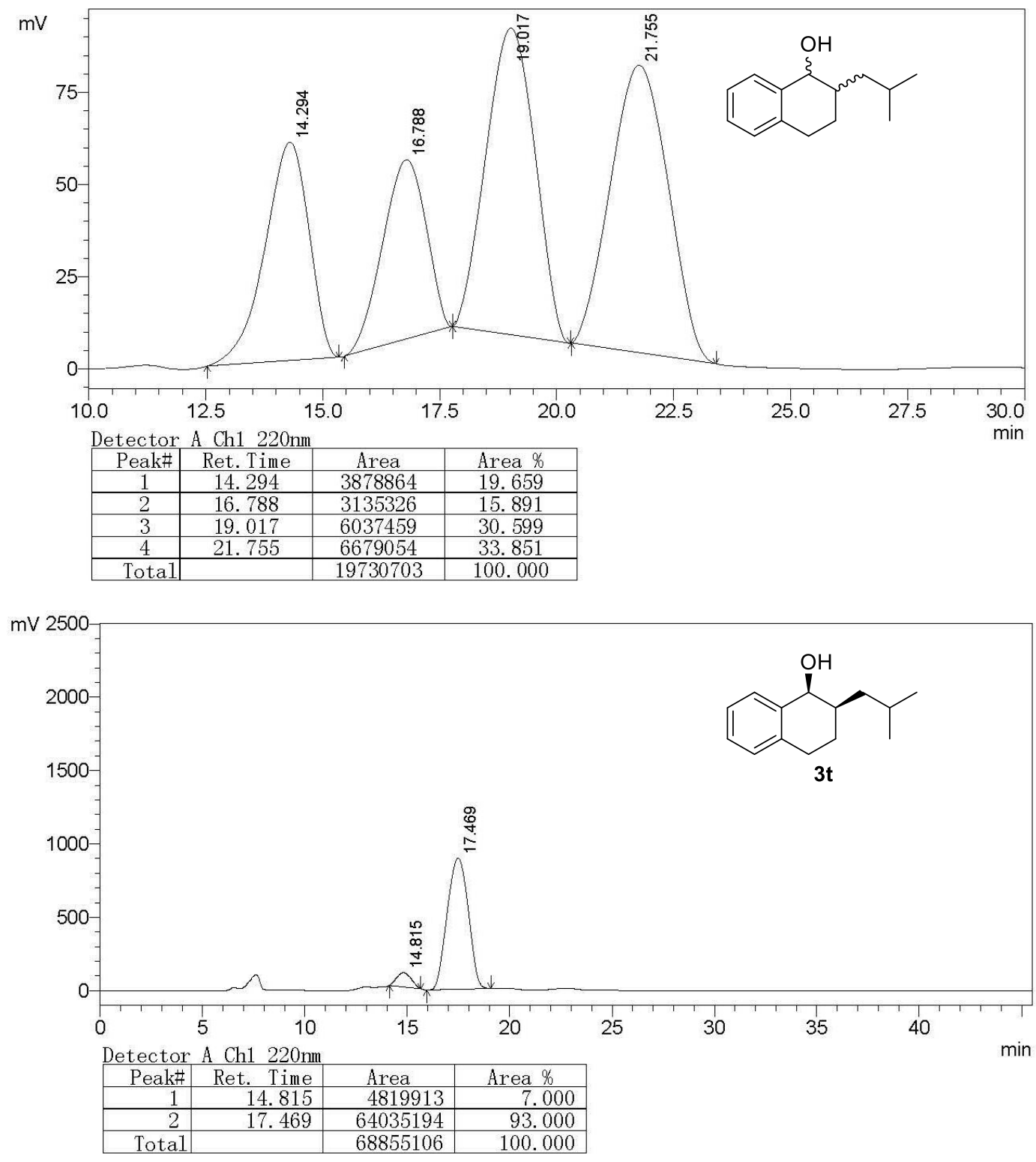

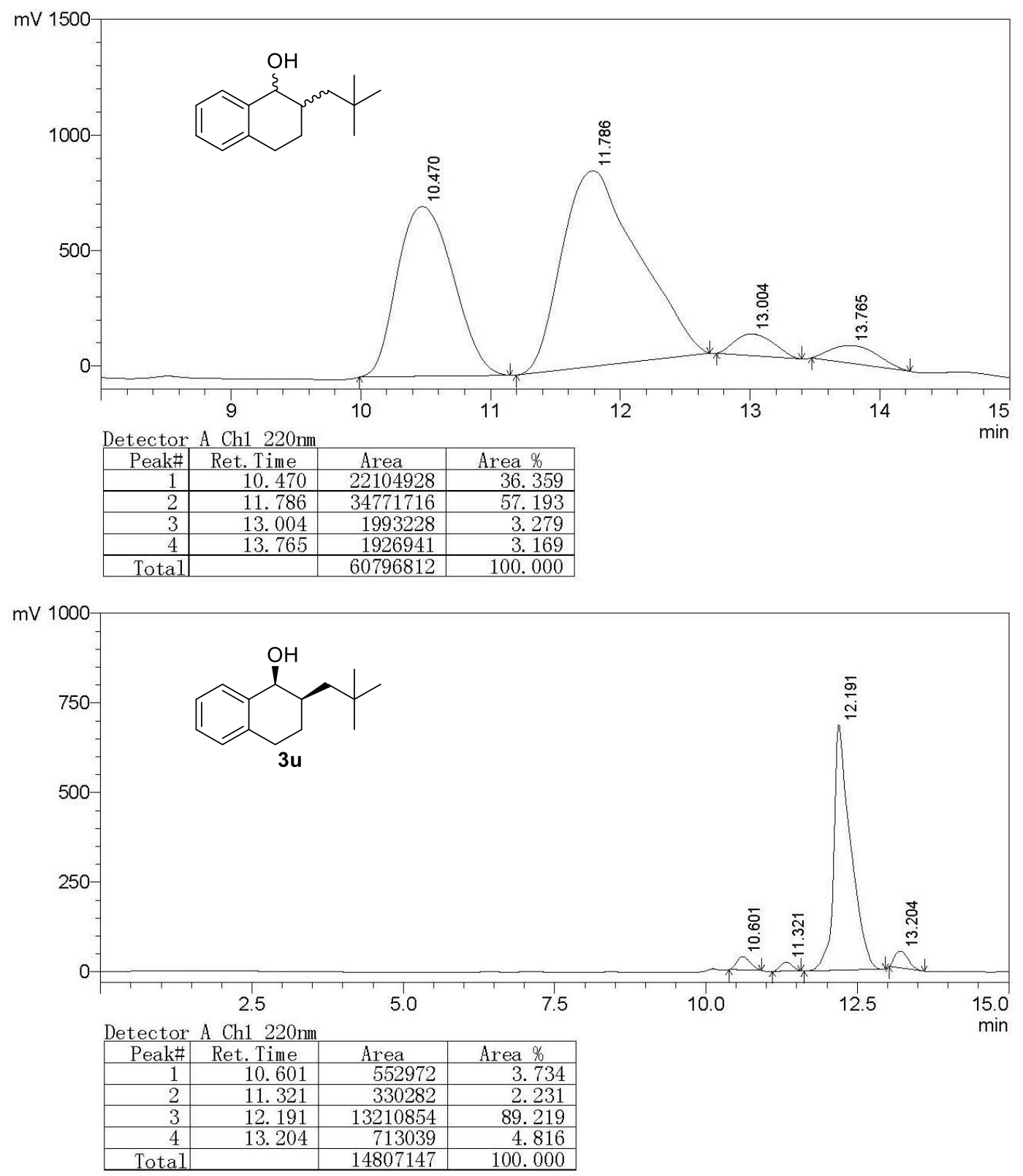

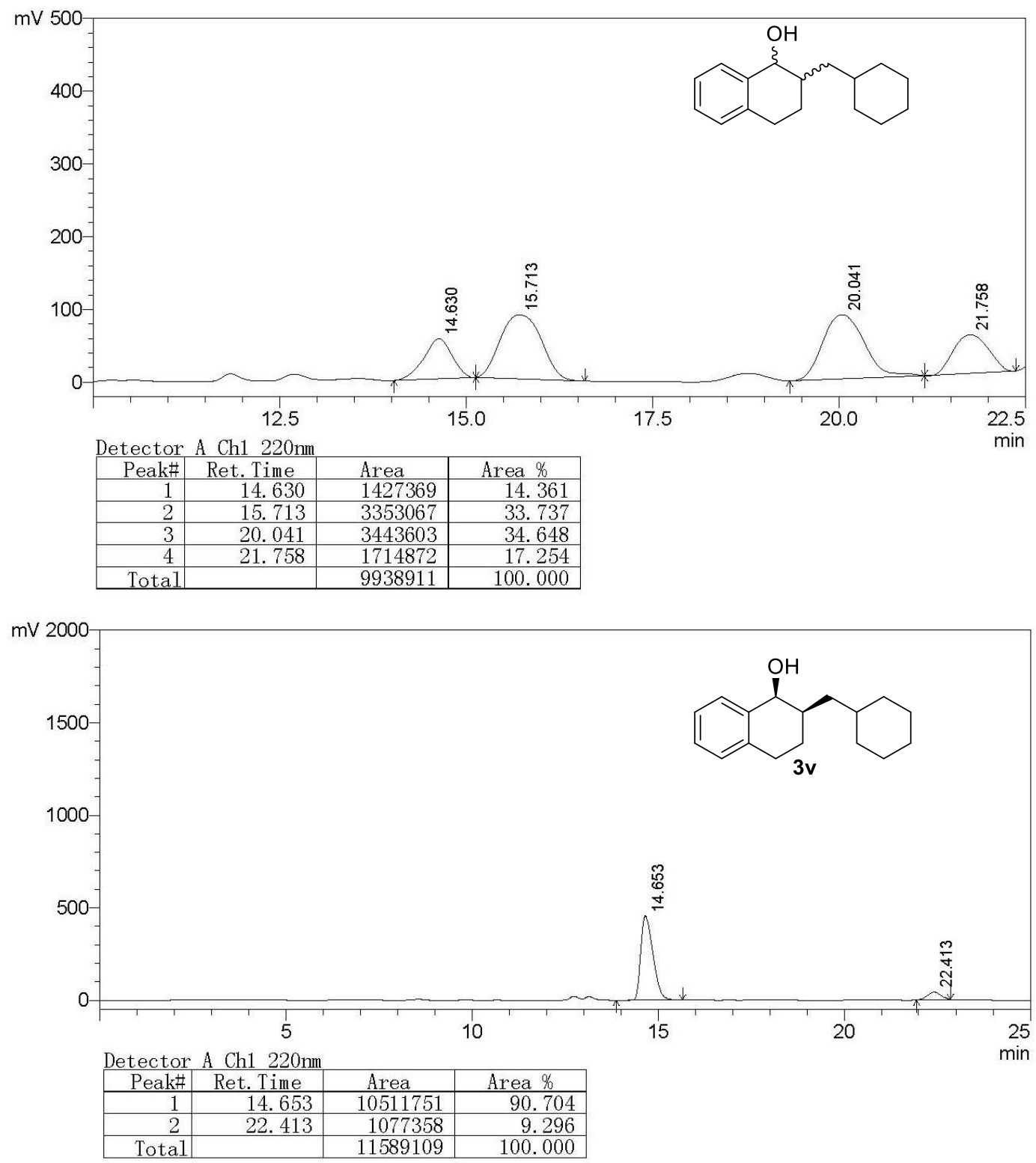

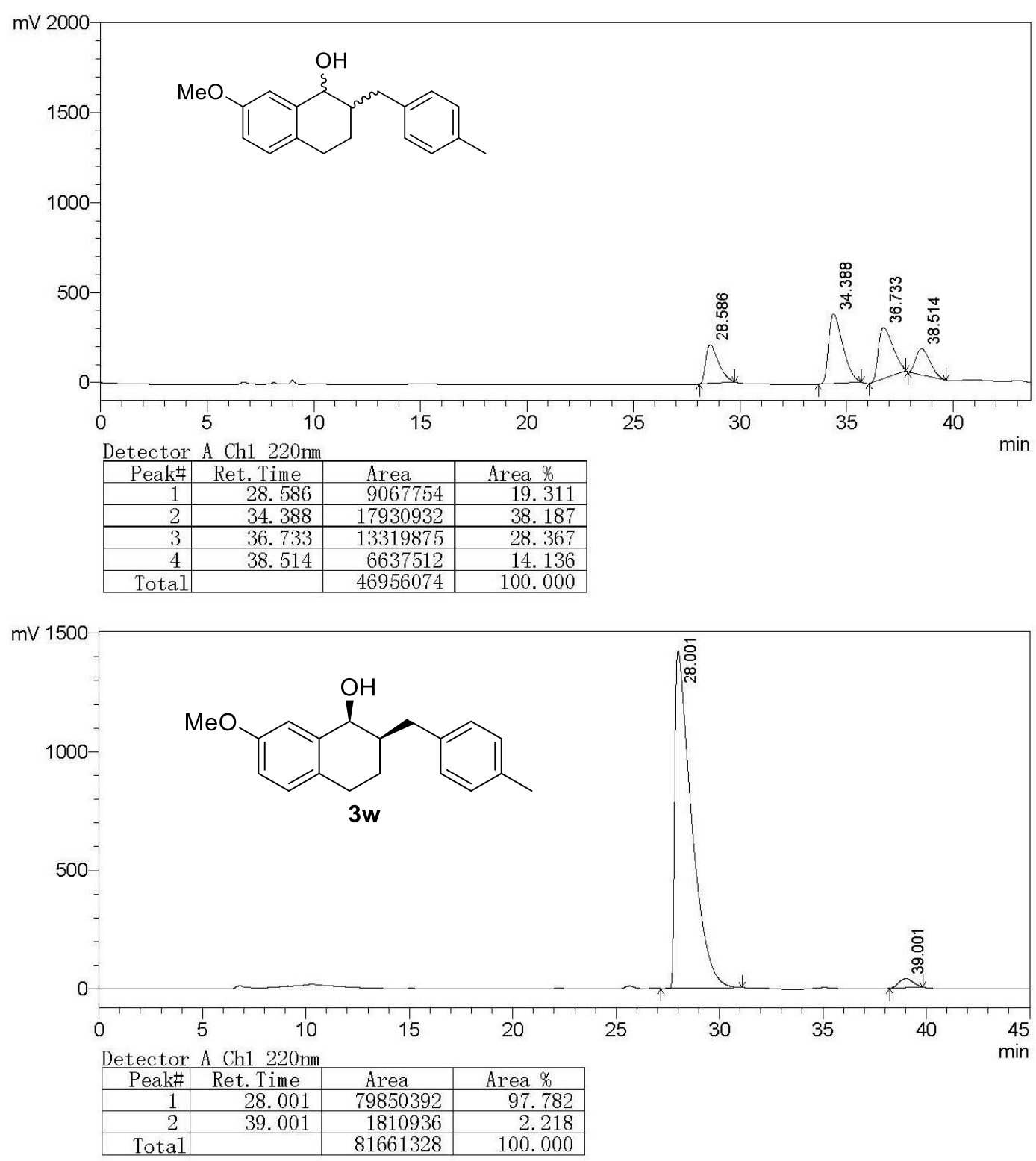

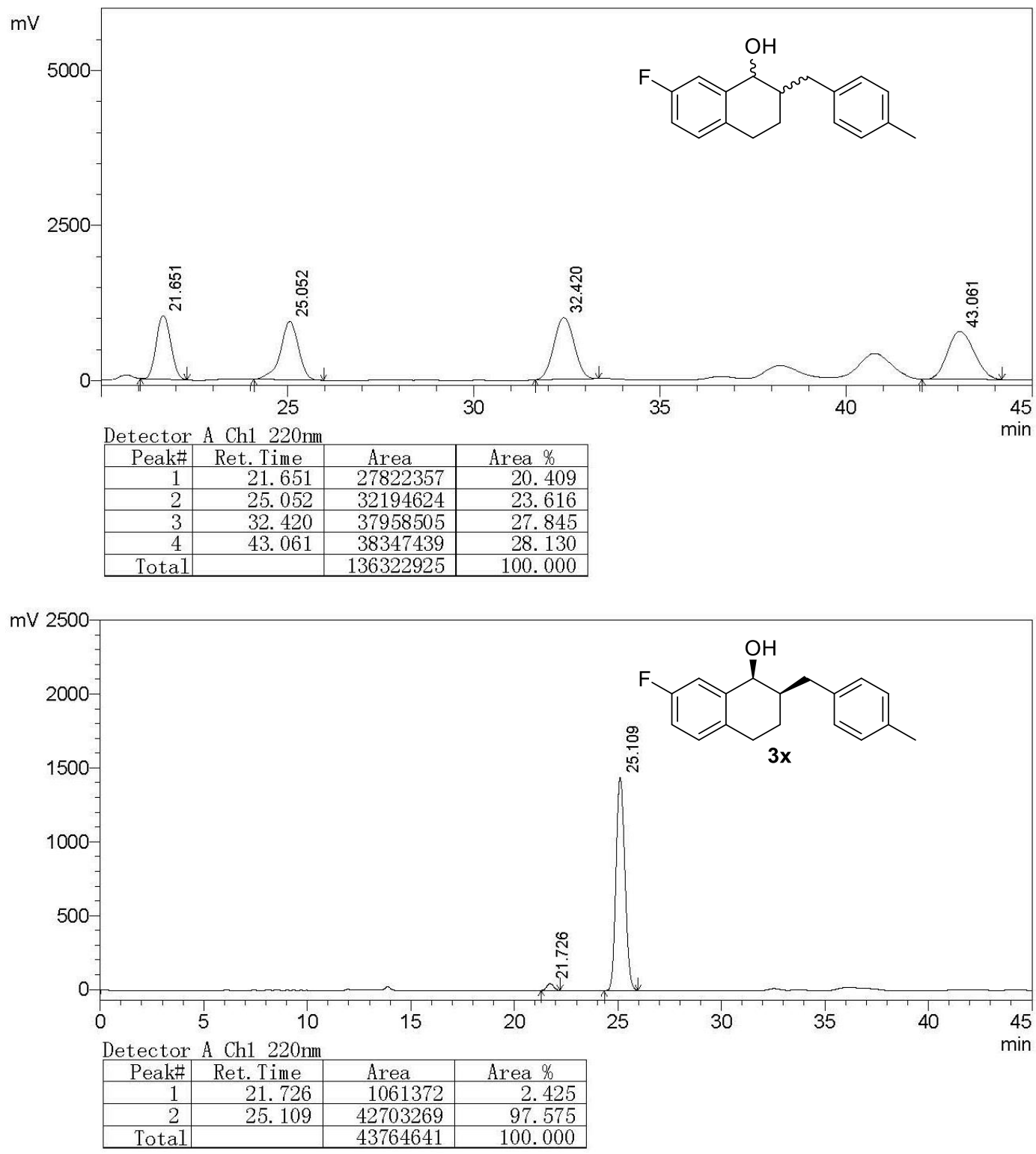

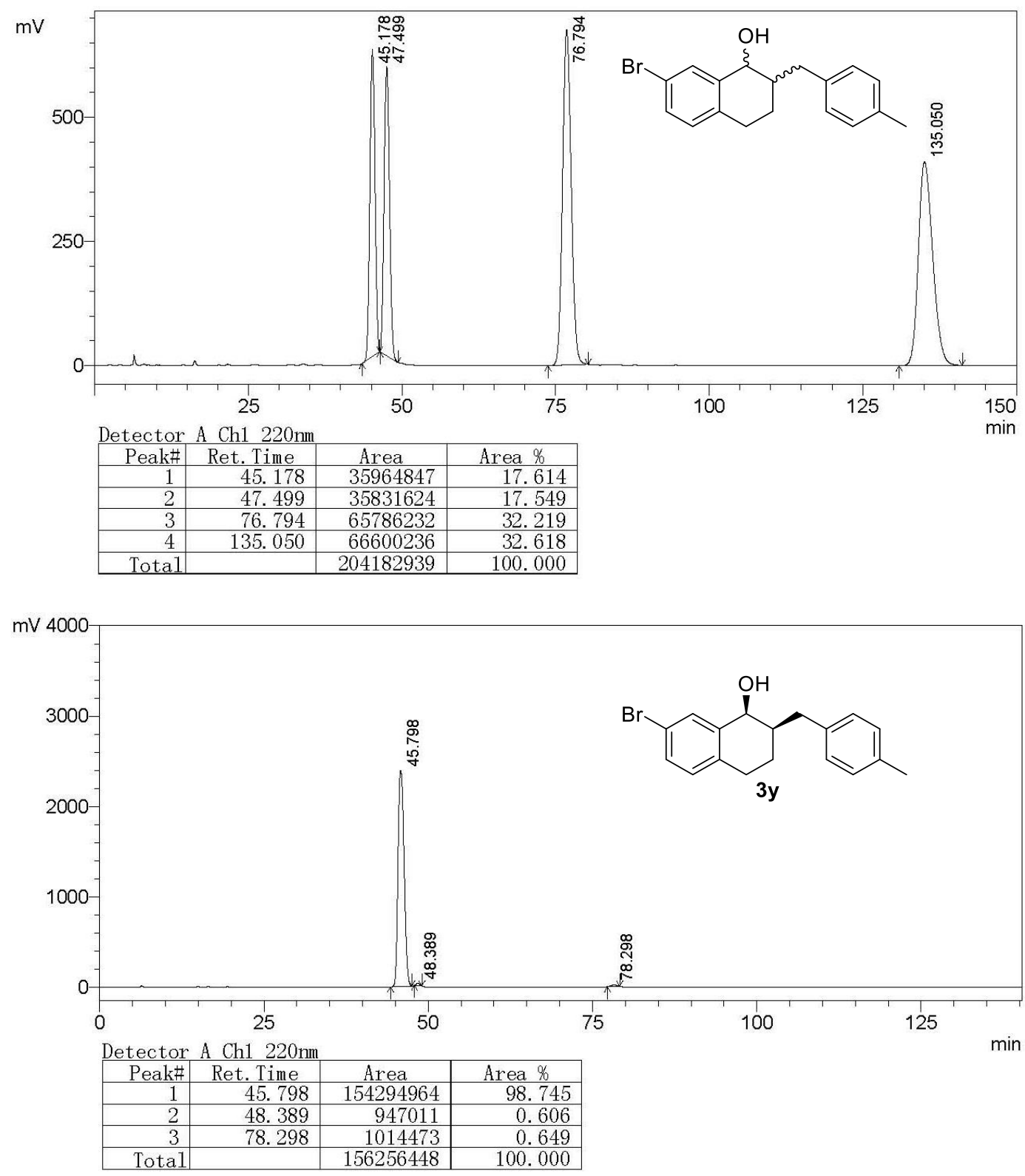

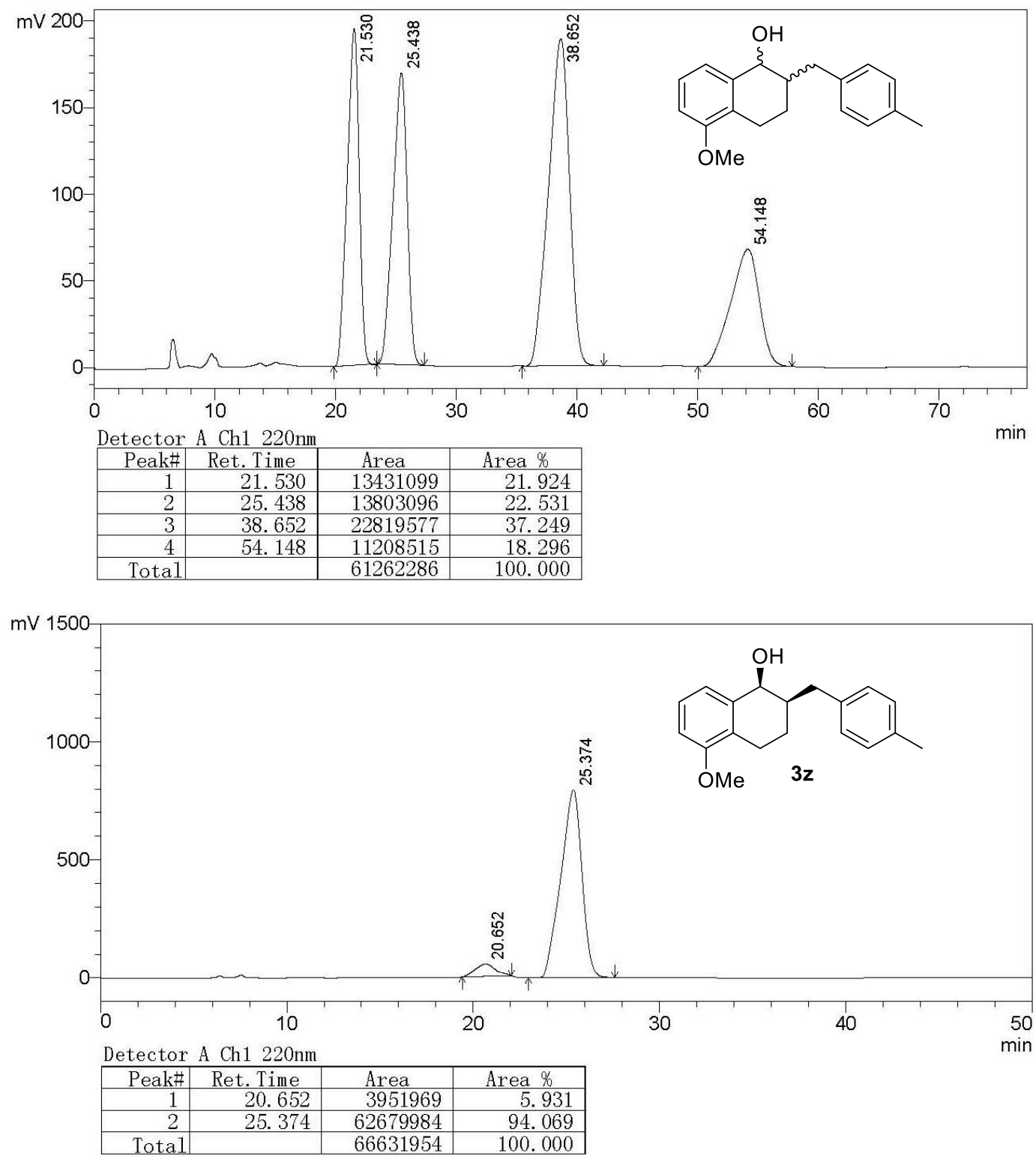

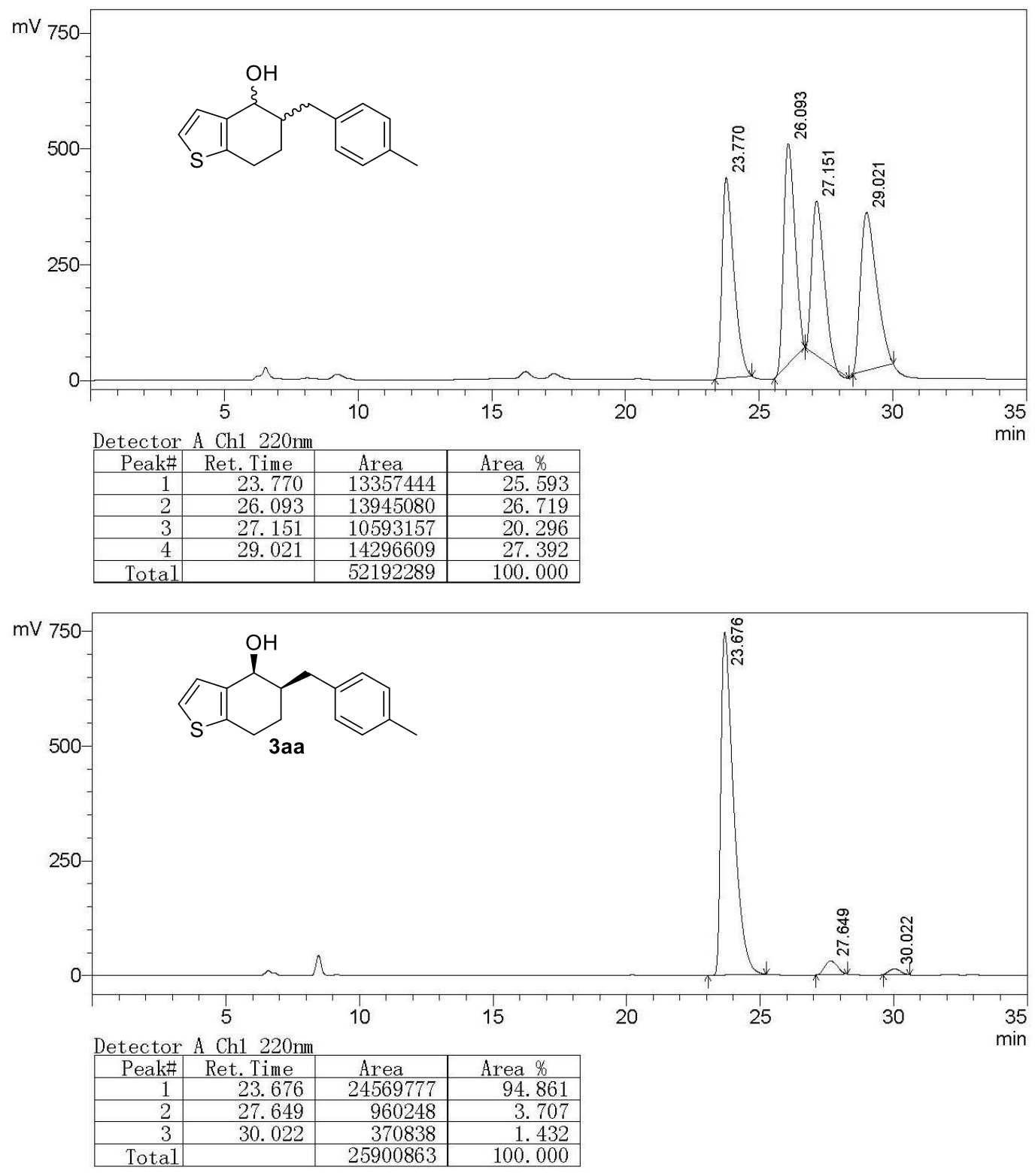

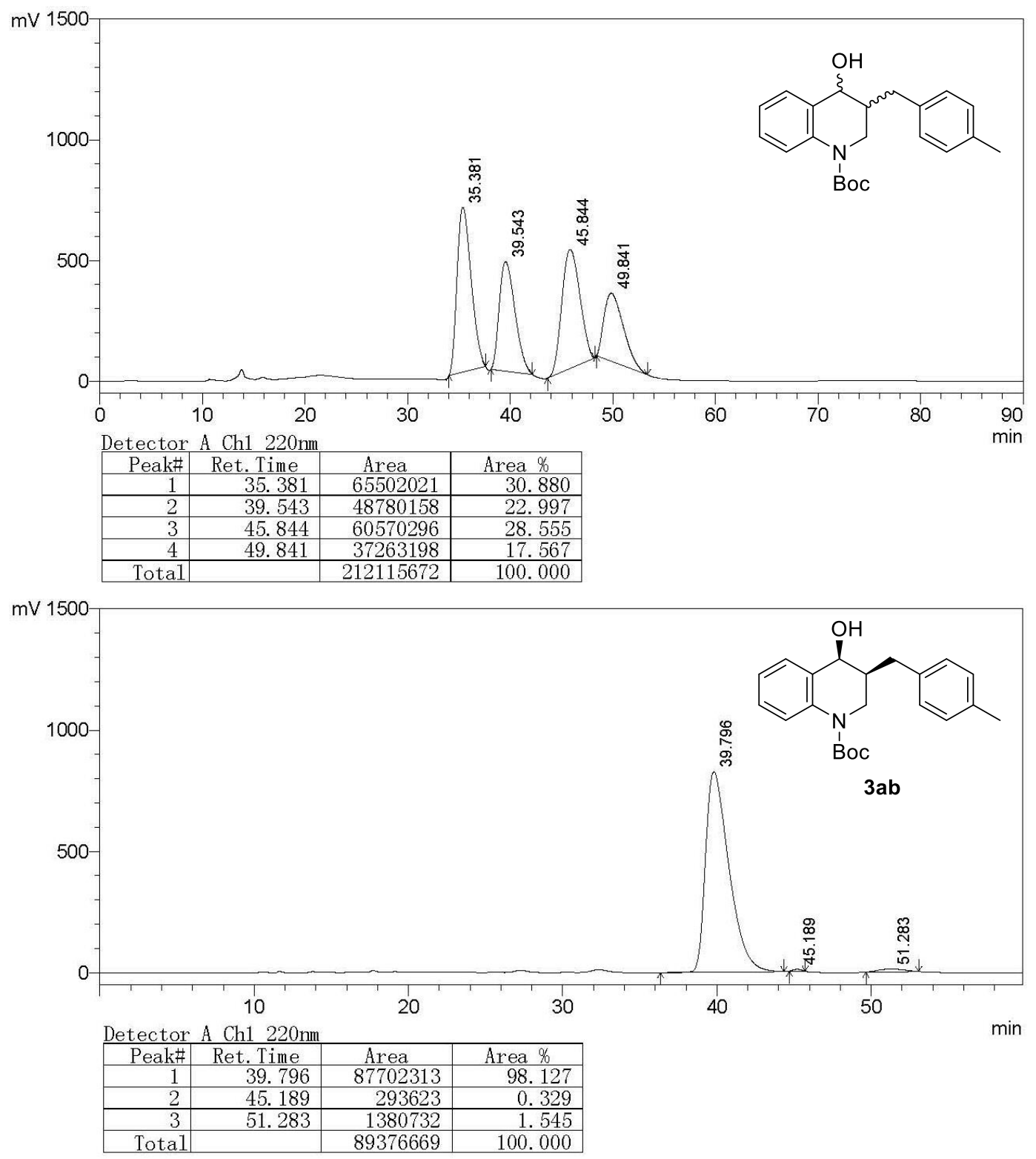

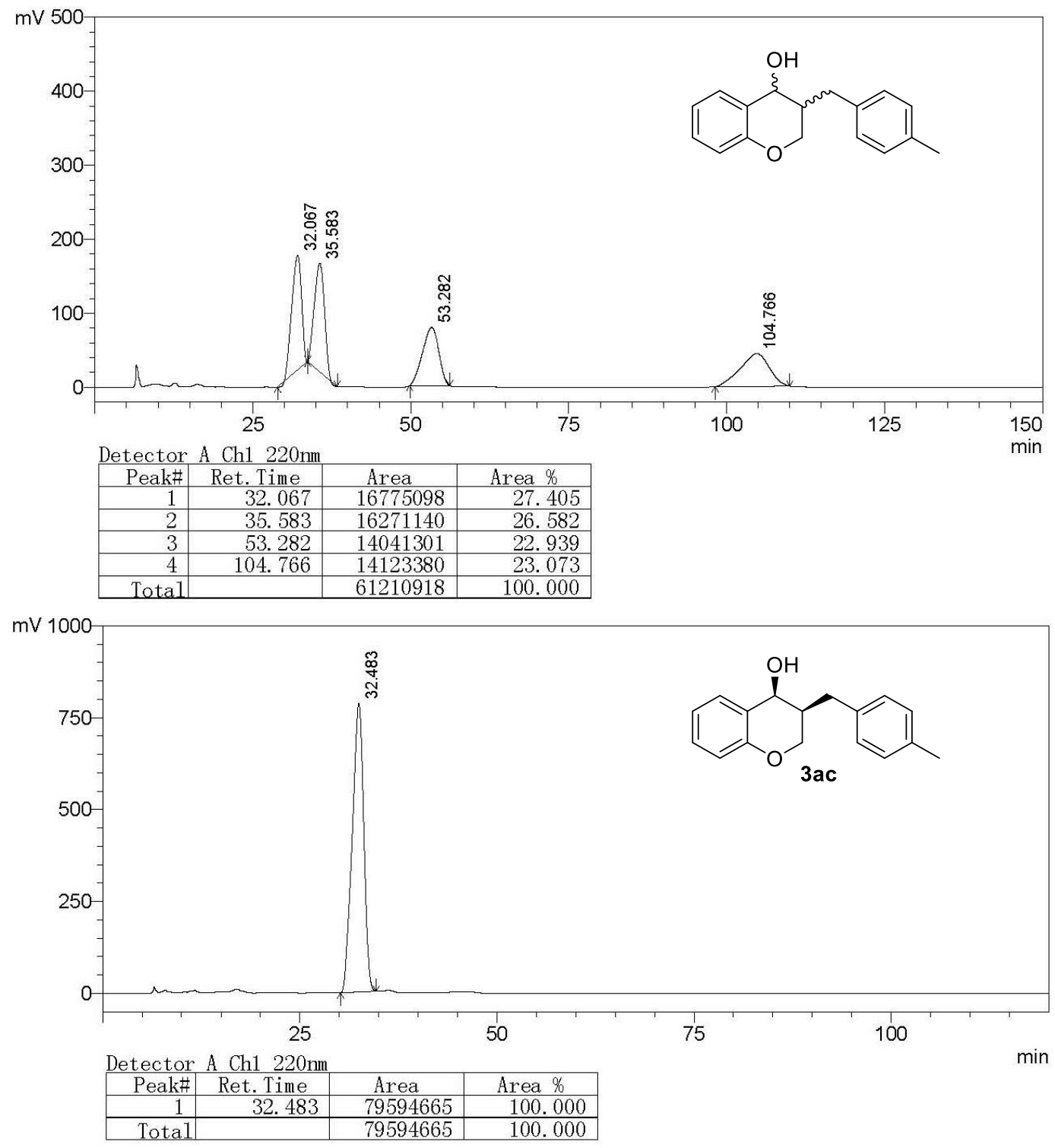


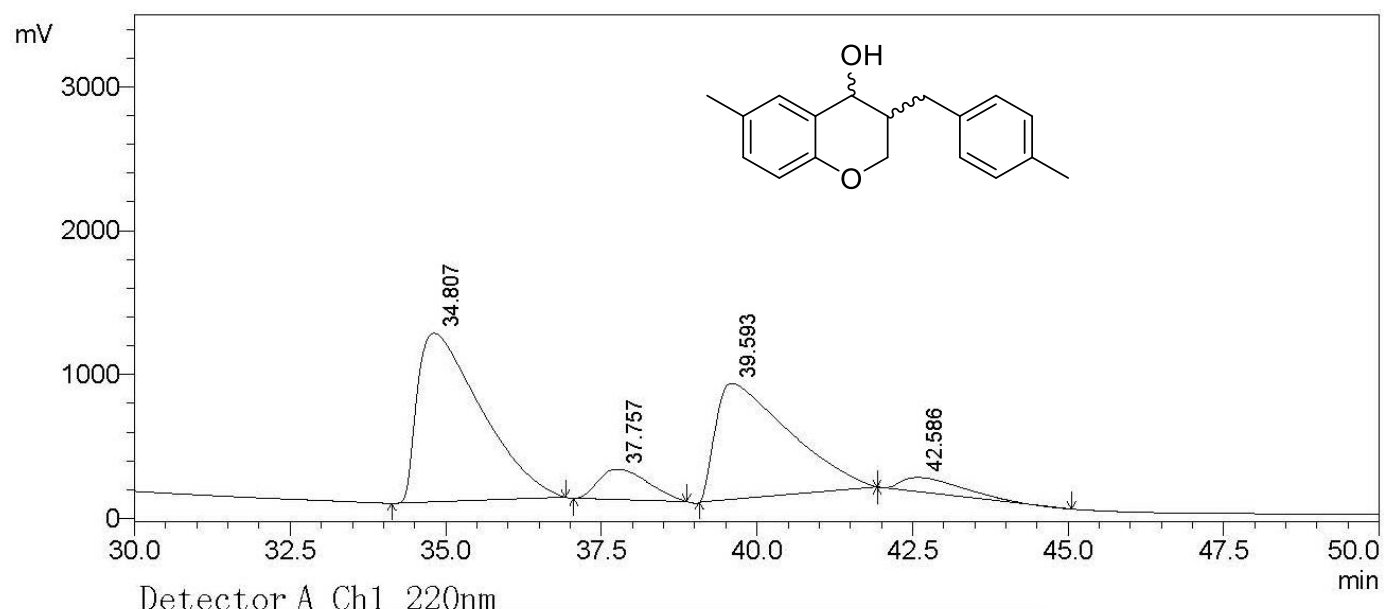

Detector A Ch1 220nm

\begin{tabular}{|r|r|r|r|}
\hline Peak\# & Ret. Time & \multicolumn{1}{|c|}{ Area } & Area \% \\
\hline 1 & 34.807 & 83587991 & 49.795 \\
\hline 2 & 37.757 & 11767732 & 7.010 \\
\hline 3 & 39.593 & 65709620 & 39.144 \\
\hline 4 & 42.586 & 6799104 & 4.050 \\
\hline Total & & 167864447 & 100.000 \\
\hline
\end{tabular}

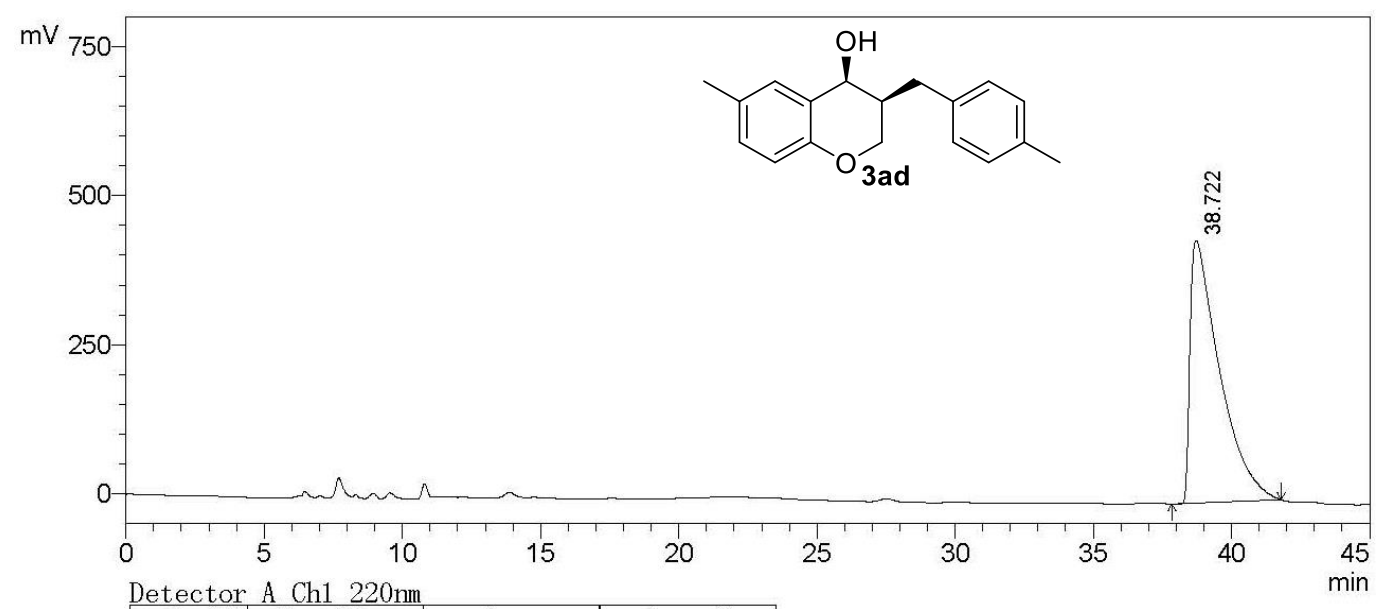

Detector A Ch1 220nm

\begin{tabular}{r|r|r|r} 
Peak\# & Ret. Time & Area & Area \% \\
1 & 38.722 & 32763382 & 100.000 \\
\hline
\end{tabular}

\begin{tabular}{|l|l|l|}
\hline Total & 32763382 & 100.000 \\
\hline
\end{tabular} 

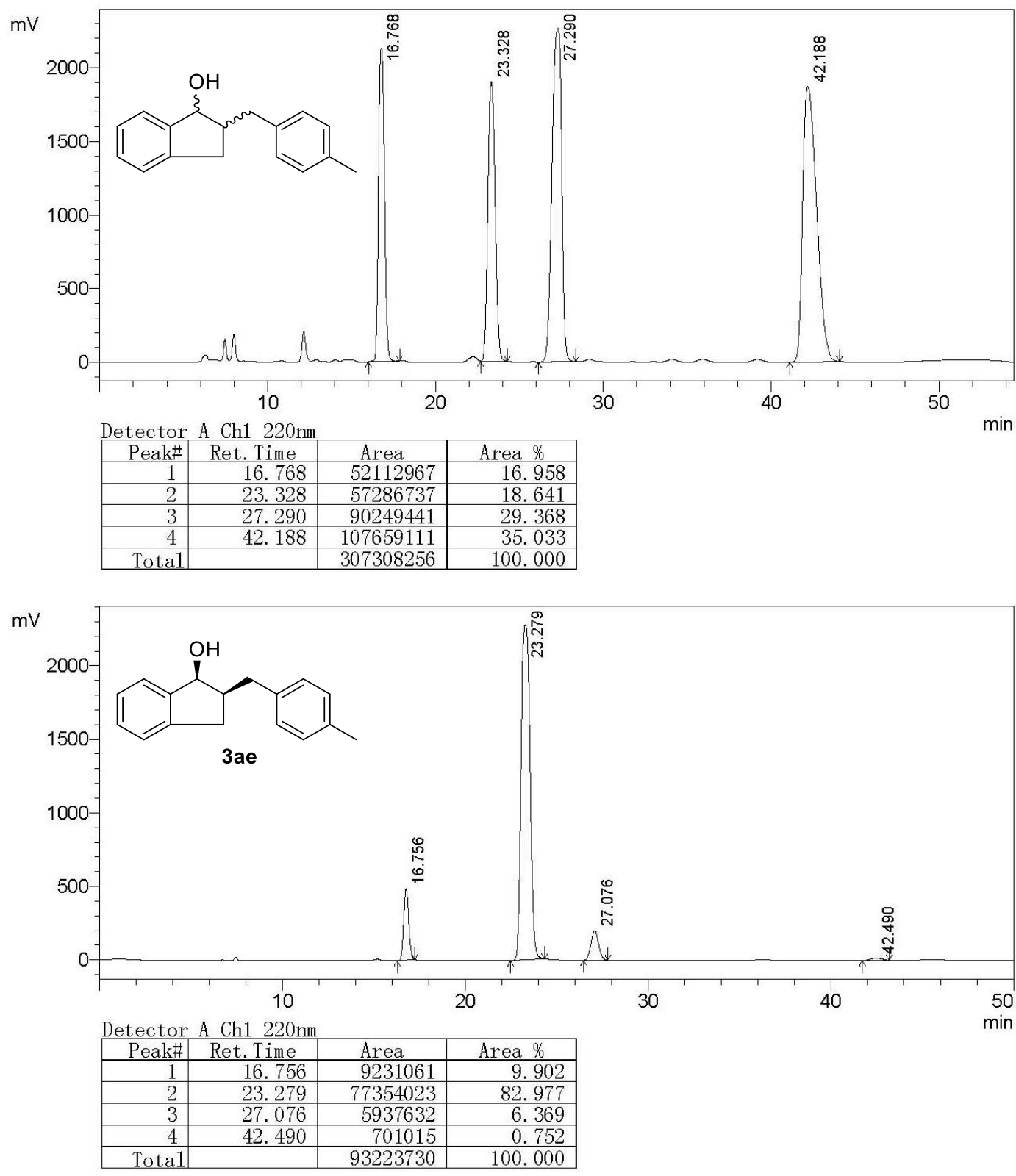

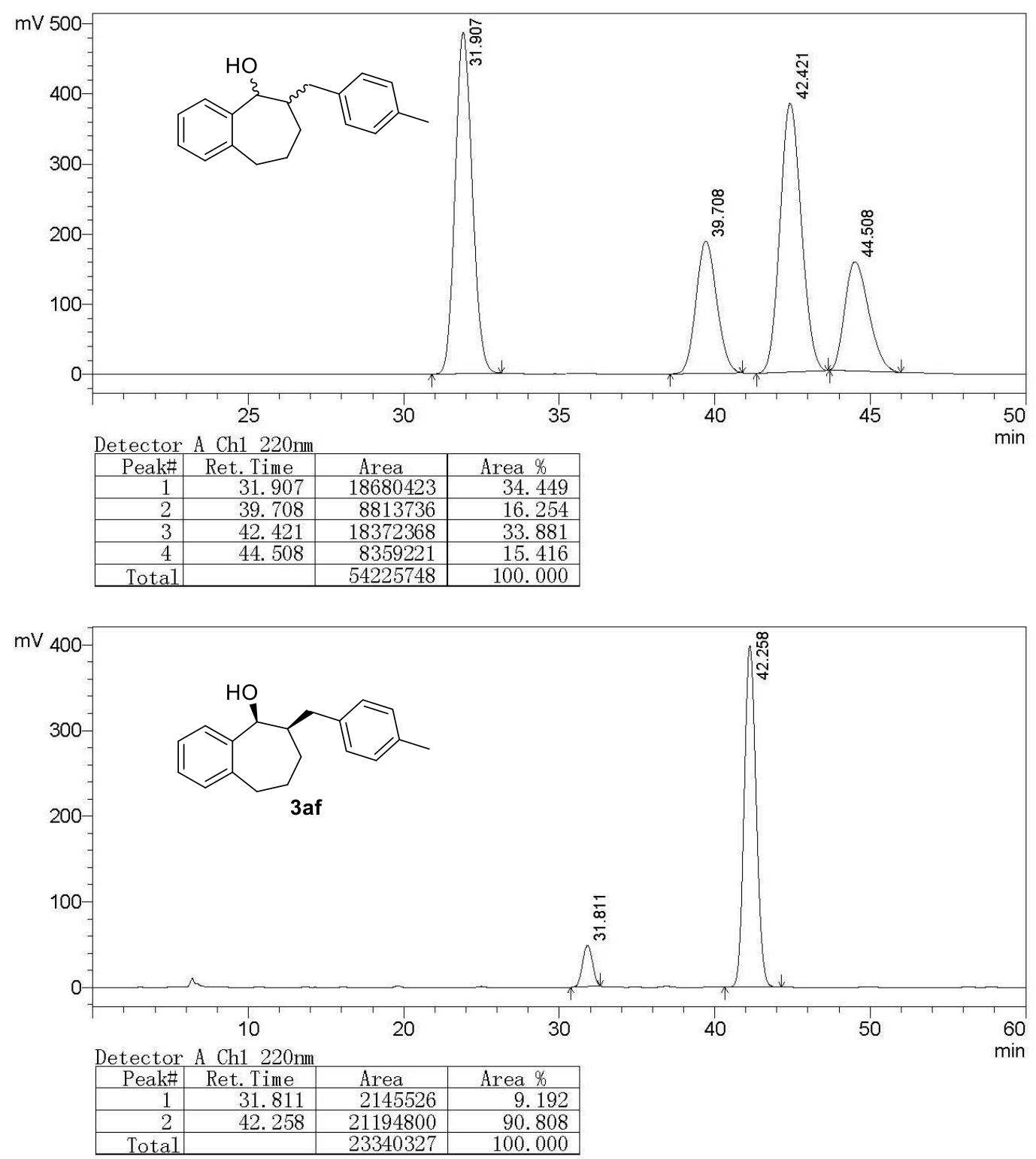

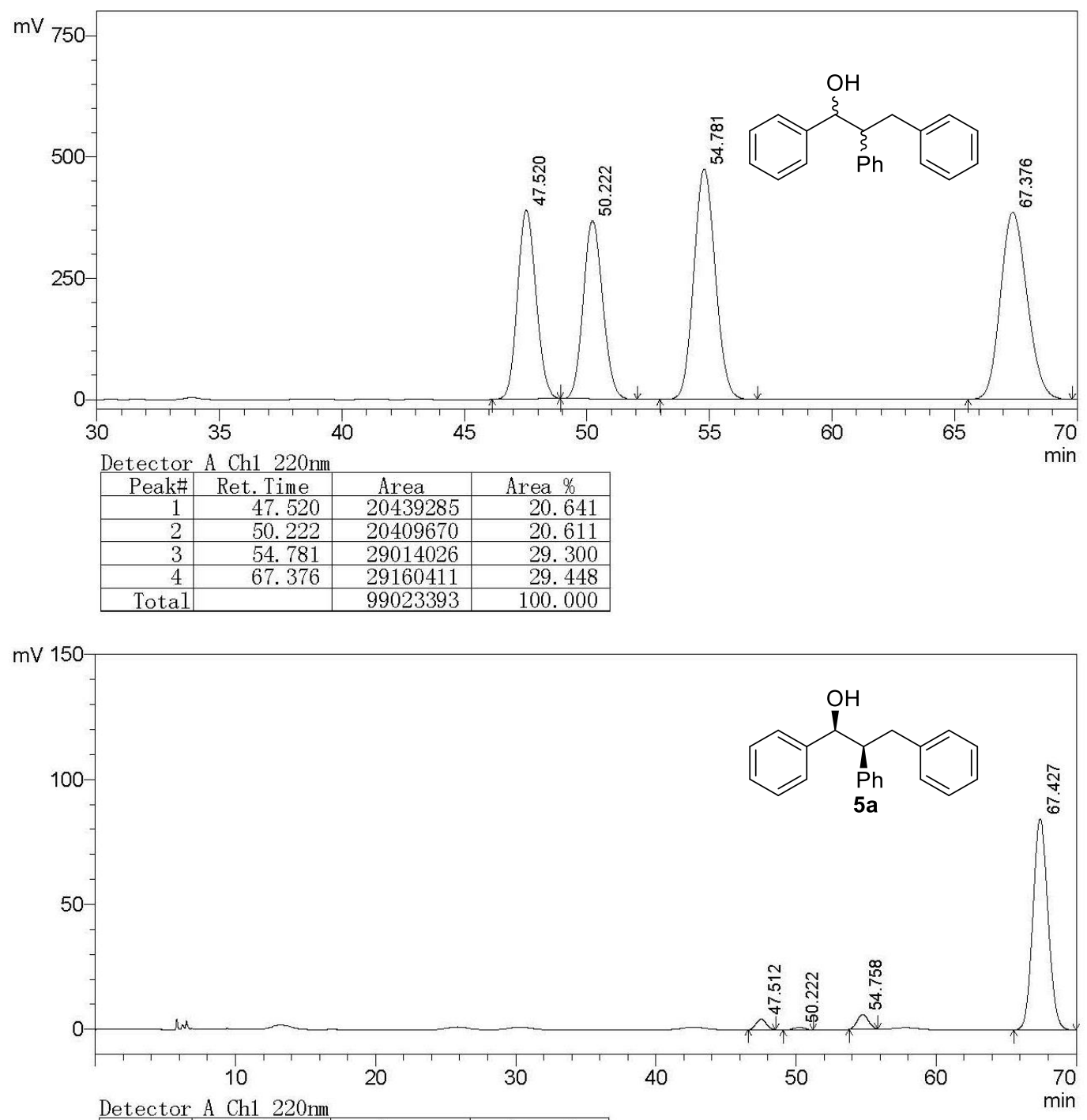

\begin{tabular}{|} 
Detector A Ch1 220nm \\
\hline Peak\# & Ret. Time & \multicolumn{1}{c|}{ Area } & Area \% \\
\hline 1 & 47.512 & 214482 & 3.095 \\
\hline 2 & 50.222 & 57089 & 0.824 \\
\hline 3 & 54.758 & 330093 & 4.764 \\
\hline 4 & 67.427 & 6327864 & 91.317 \\
\hline Total & & 6929528 & 100.000 \\
\hline
\end{tabular}



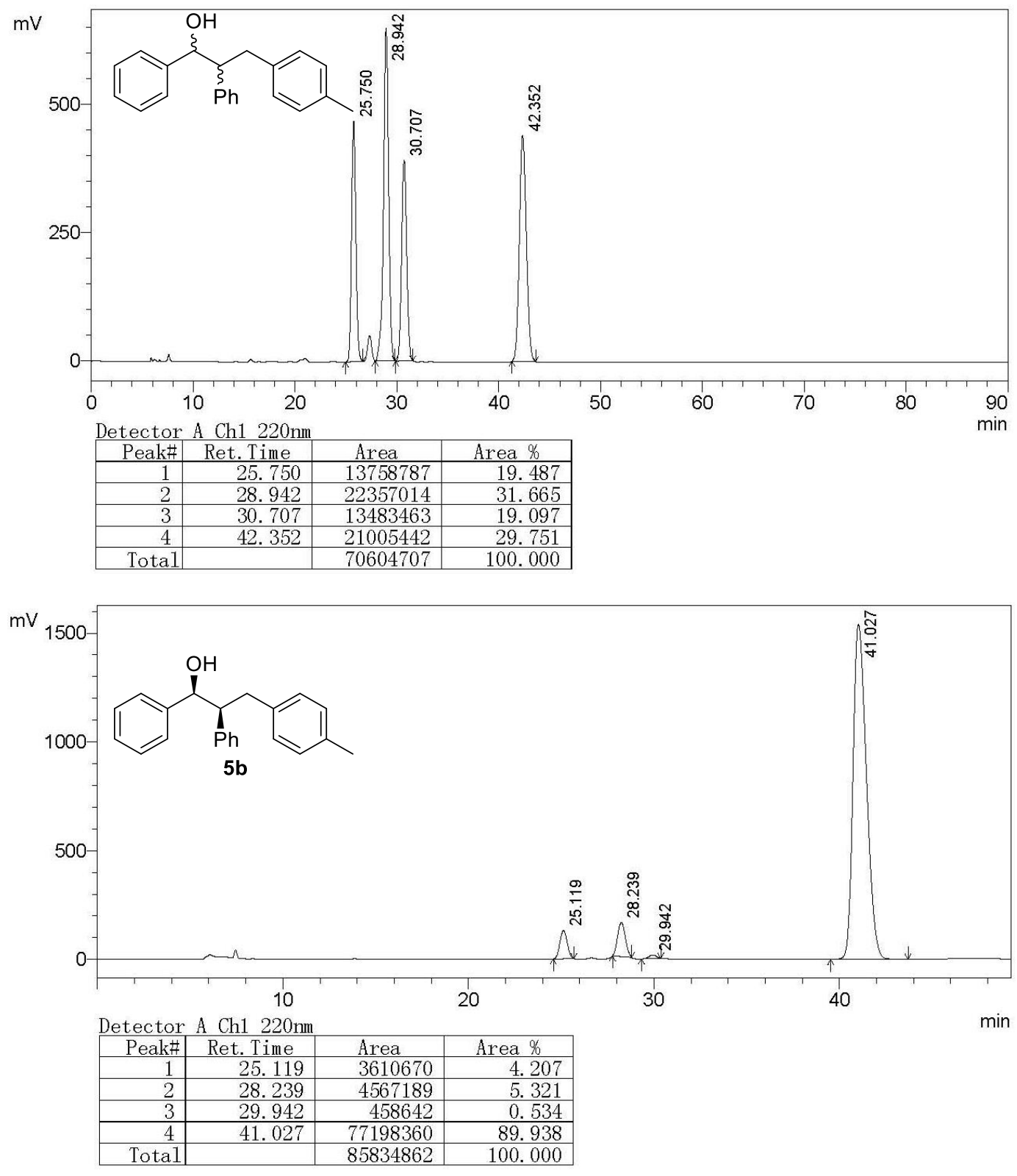

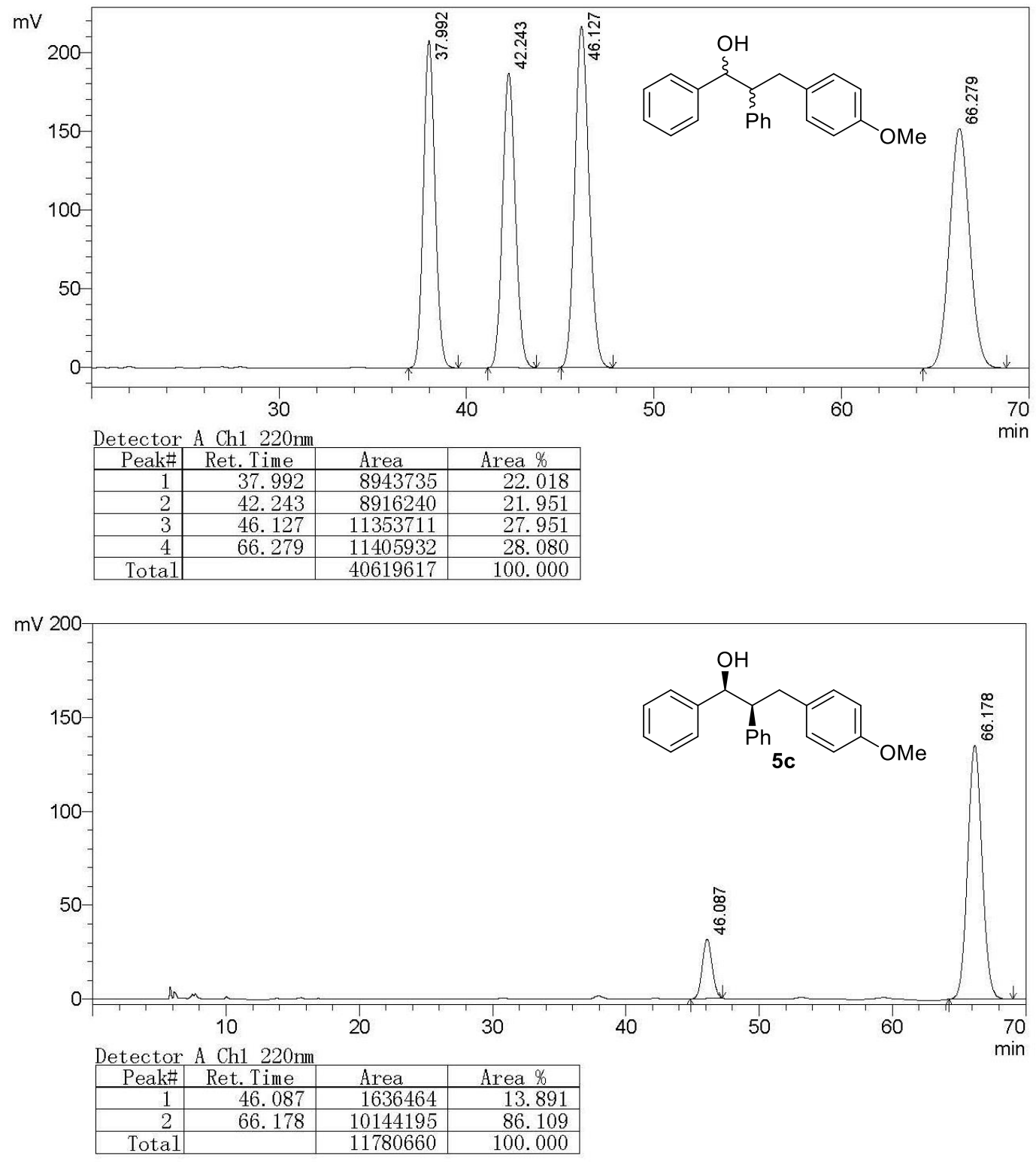

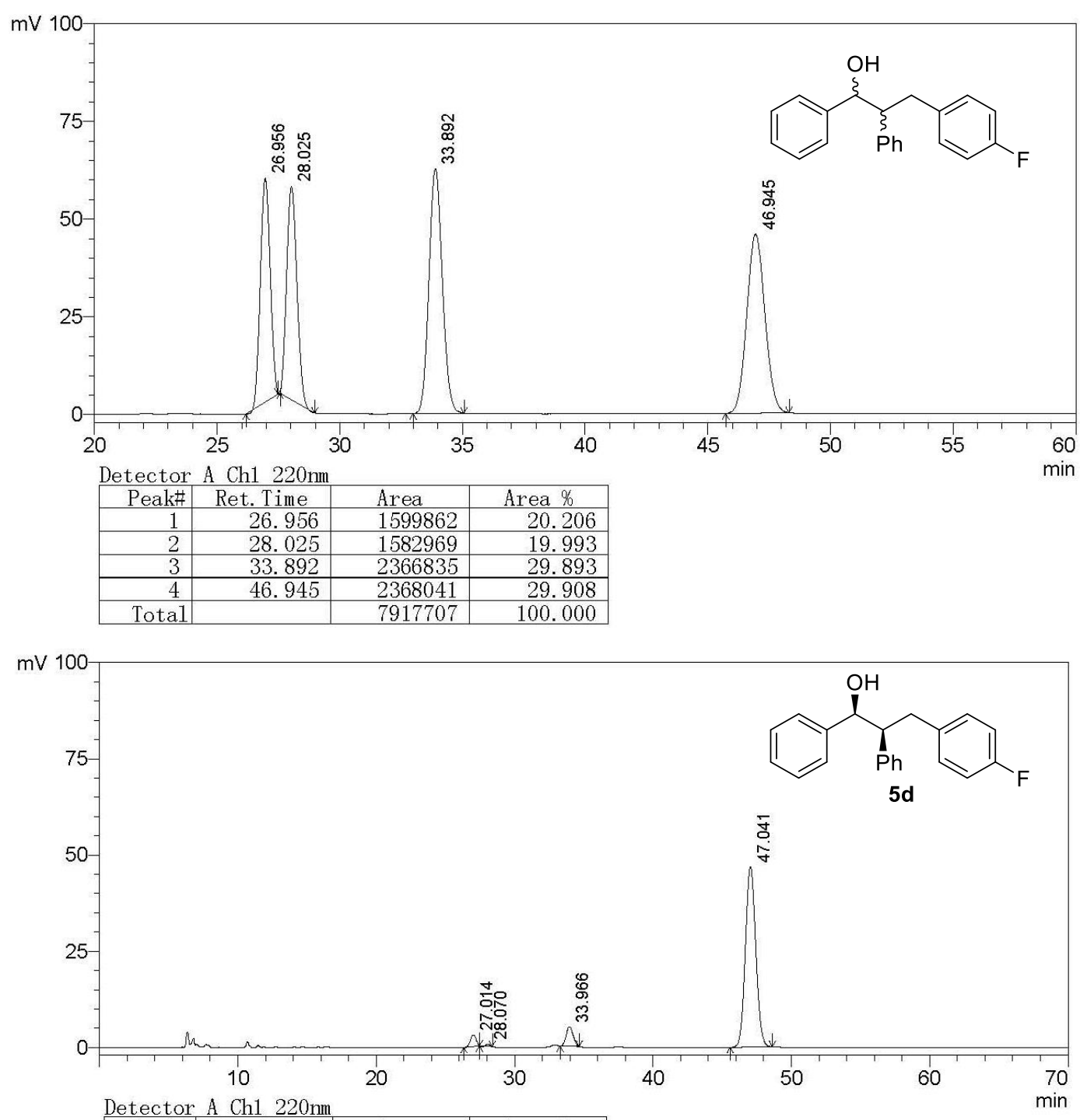

Detector A Ch1 220nm
\begin{tabular}{|r|r|r|r|}
\hline Peak\# & Ret. Time & \multicolumn{1}{|c|}{ Area } & Area \% \\
\hline 1 & 27.014 & 82464 & 3.039 \\
\hline 2 & 28.070 & 7653 & 0.282 \\
\hline 3 & 33.966 & 179532 & 6.616 \\
\hline 4 & 47.041 & 2443769 & 90.062 \\
\hline Total & & 2713418 & 100.000 \\
\hline
\end{tabular}



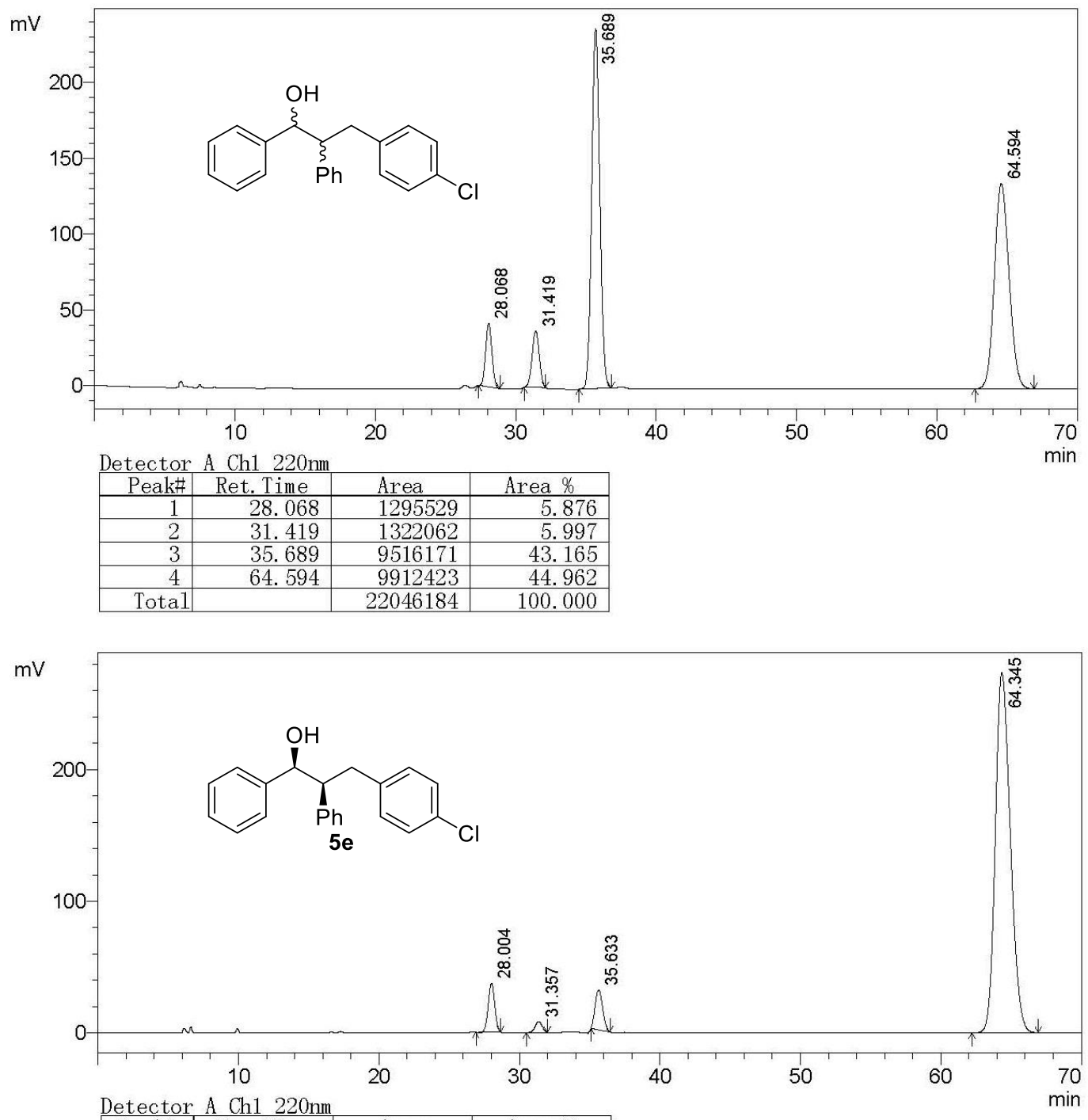

Detector A Ch1 $220 \mathrm{~nm}$
\begin{tabular}{|c|c|c|c|}
\hline Peak\# & Ret. Time & Area & Area \% \\
\hline 1 & 28.004 & 1175643 & 5.145 \\
\hline 2 & 31.357 & 283203 & 1.239 \\
\hline 3 & 35.633 & 1100457 & 4.816 \\
\hline 4 & 64.345 & 20289523 & 88.799 \\
\hline Total & & 22848826 & 100.000 \\
\hline
\end{tabular}



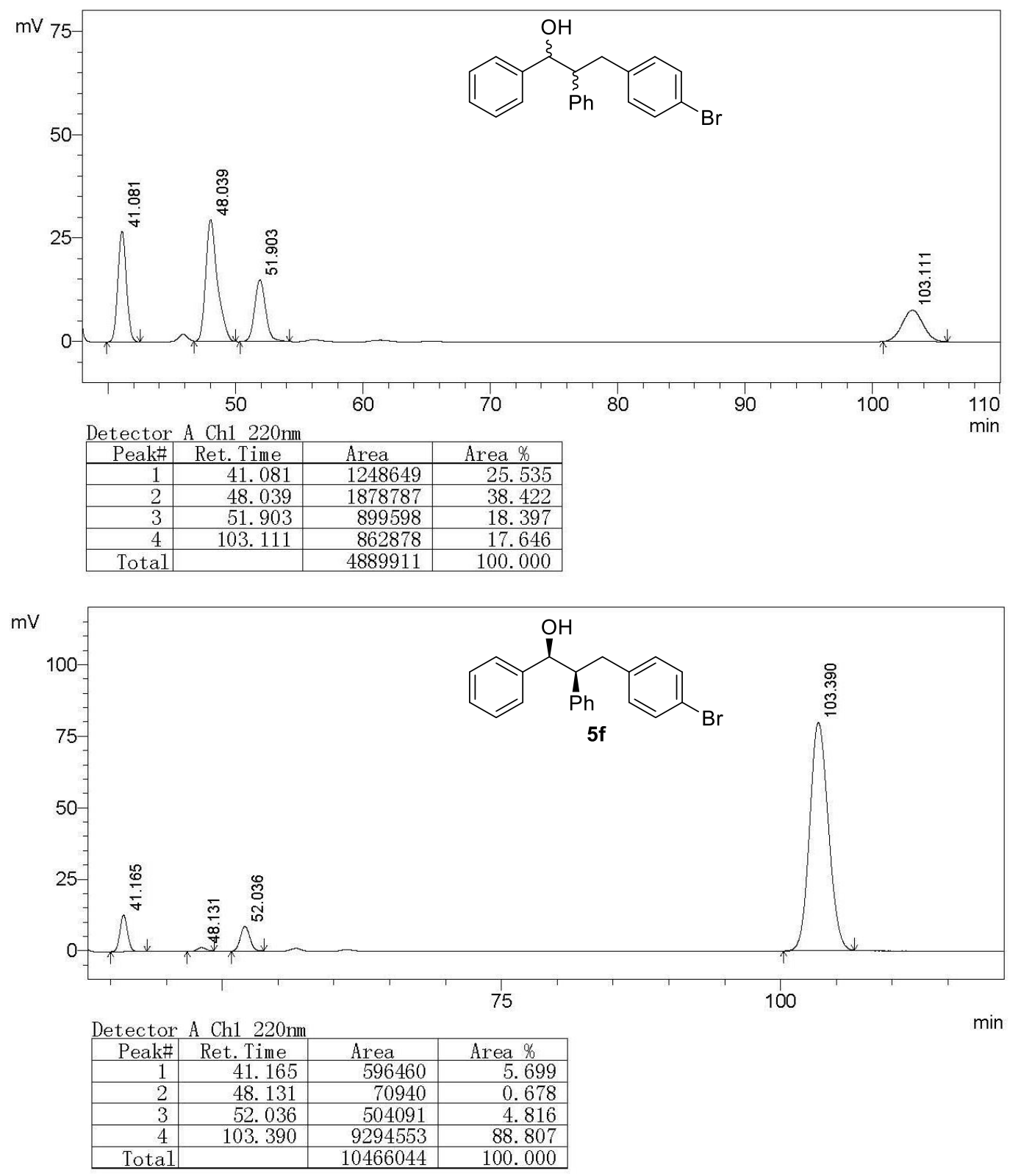

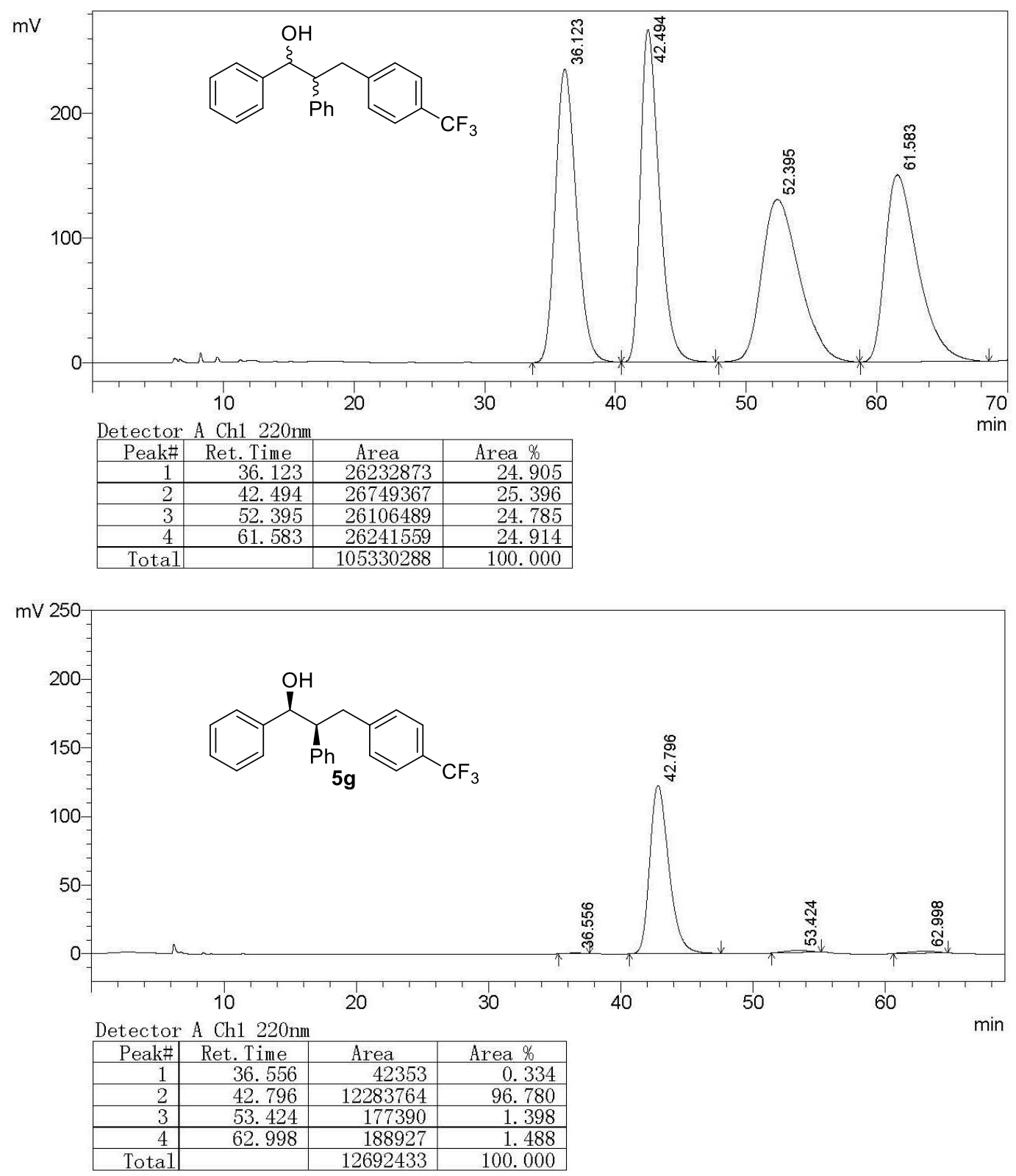

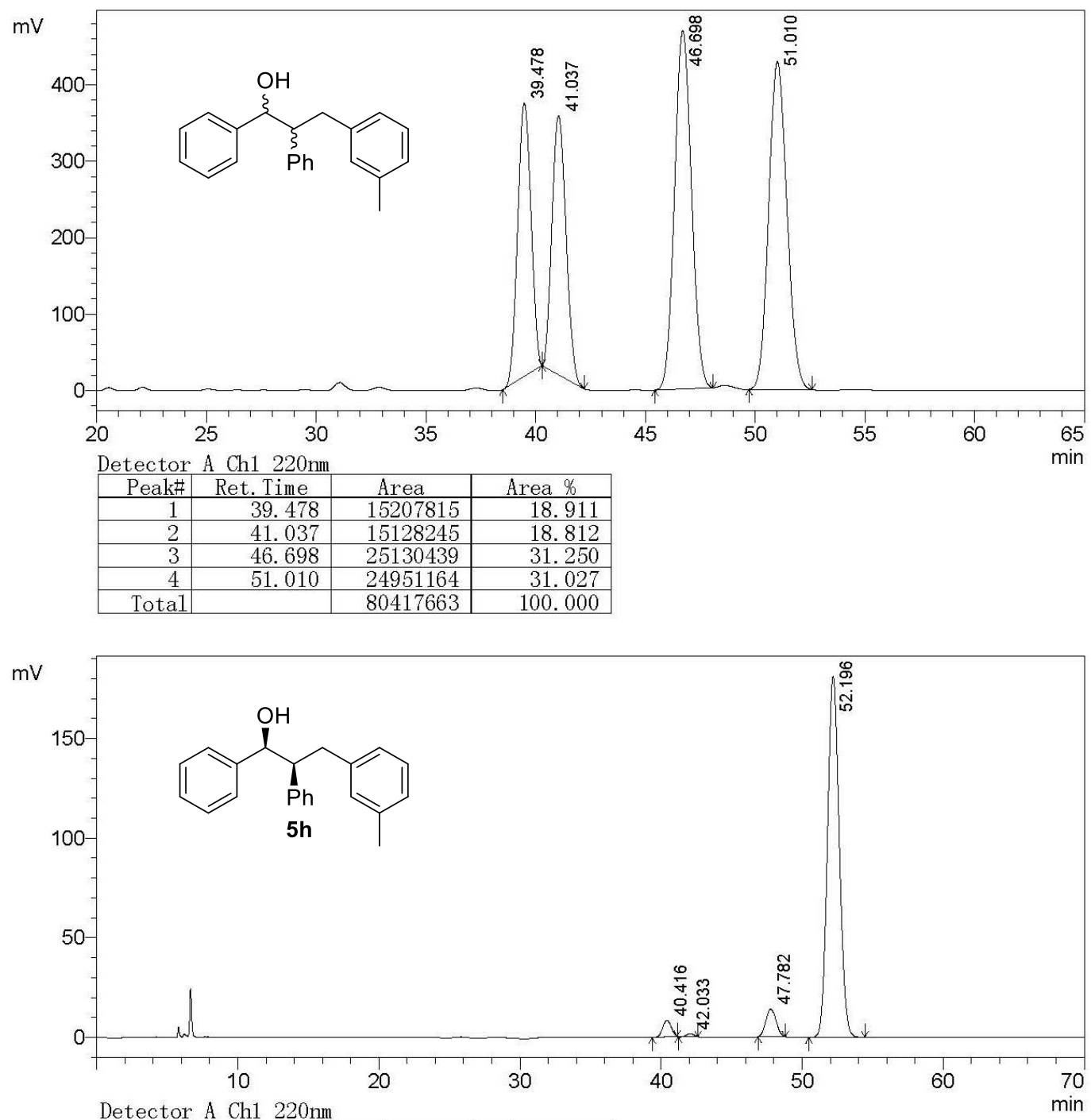

Detector A Ch1 220nm
\begin{tabular}{|r|r|r|r|}
\hline Peak\# & Ret. Time & \multicolumn{1}{c|}{ Area } & Area $\%$ \\
\hline 1 & 40.416 & 342509 & 2.954 \\
\hline 2 & 42.033 & 54838 & 0.473 \\
\hline 3 & 47.782 & 700434 & 6.041 \\
\hline 4 & 52.196 & 10496496 & 90.532 \\
\hline Total & & 11594276 & 100.000 \\
\hline
\end{tabular}



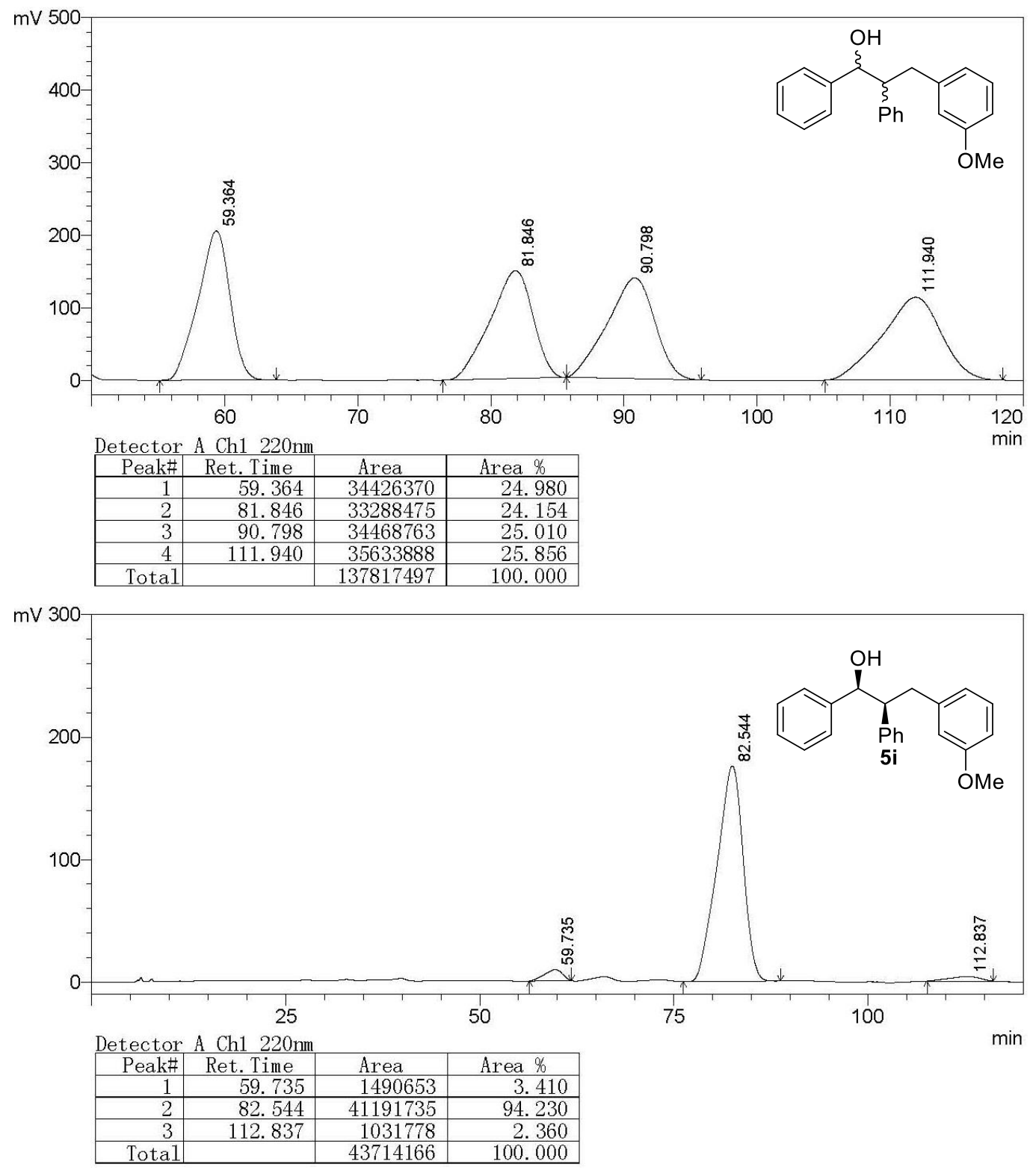


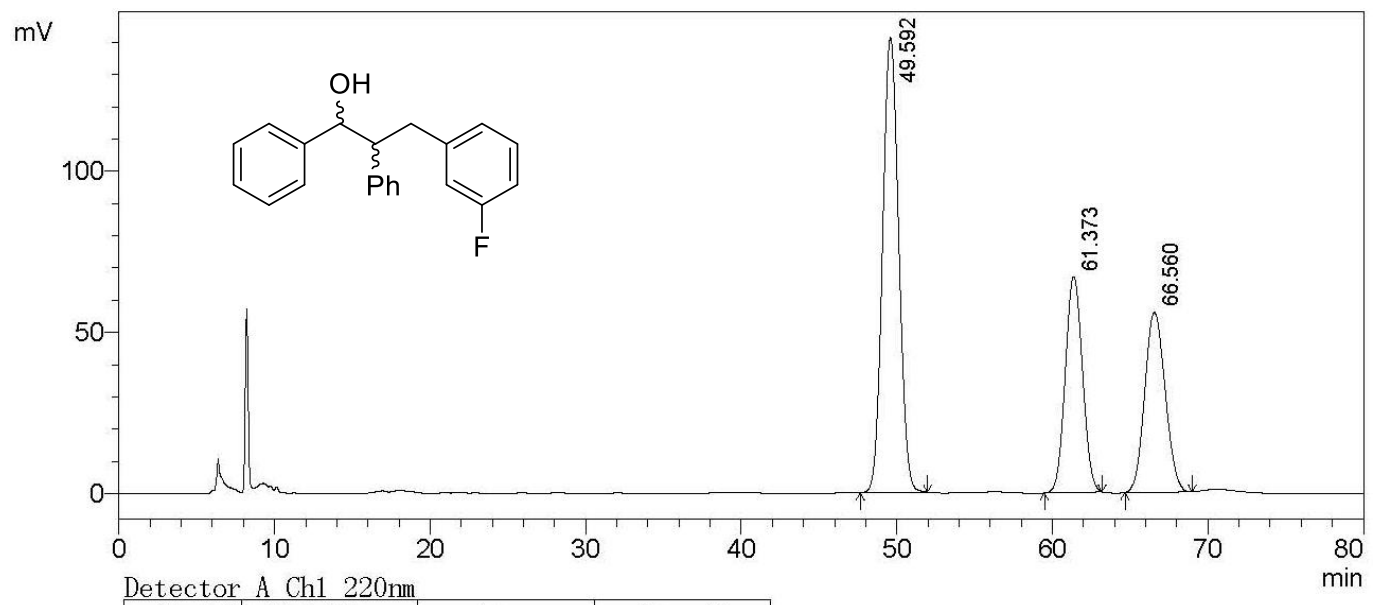

Detector A Ch1 $220 \mathrm{~nm}$
\begin{tabular}{|r|r|r|r|}
\hline Peak\# & Ret. Time & \multicolumn{1}{c|}{ Area } & Area \% \\
\hline 1 & 49.592 & 10166690 & 49.687 \\
\hline 2 & 61.373 & 5167179 & 25.253 \\
\hline 3 & 66.560 & 5127626 & 25.060 \\
\hline Total & & 20461494 & 100.000 \\
\hline
\end{tabular}

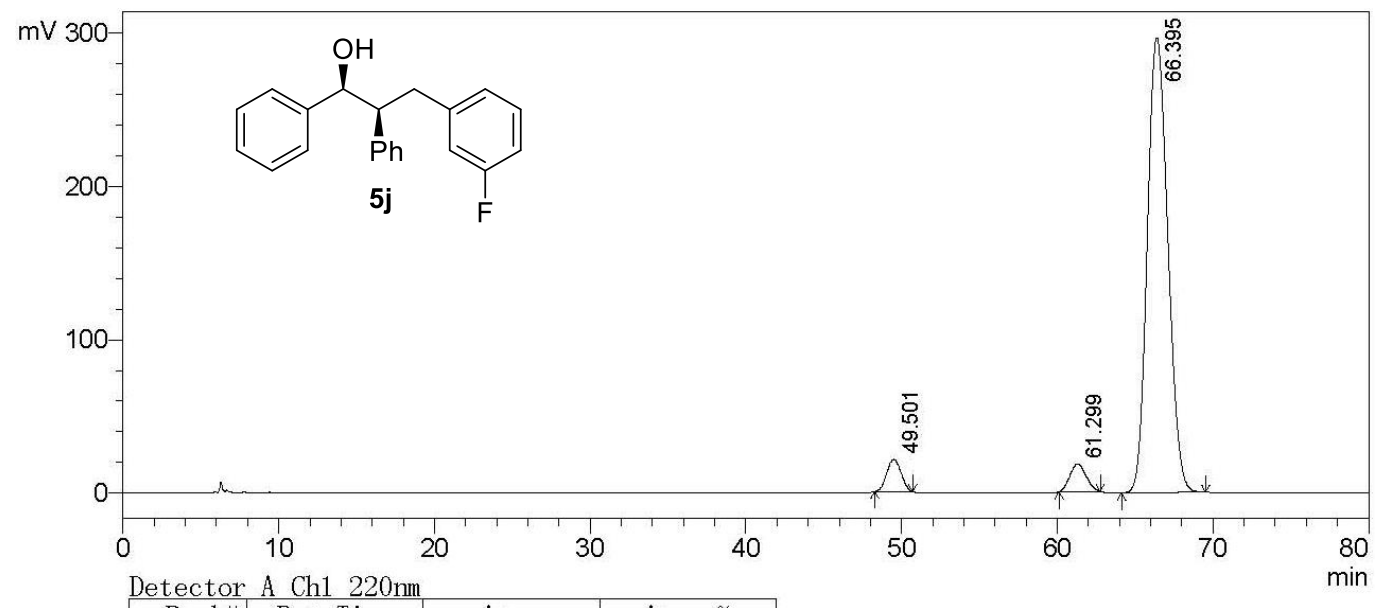

Detector A Ch1 220nm
\begin{tabular}{|r|r|r|r|}
\hline Peak\# & Ret. Time & \multicolumn{1}{|c|}{ Area } & Area \% \\
\hline 1 & 49.501 & 1332309 & 4.465 \\
\hline 2 & 61.299 & 1314431 & 4.405 \\
\hline 3 & 66.395 & 27192122 & 91.130 \\
\hline Tota1 & & 29838862 & 100.000 \\
\hline
\end{tabular}



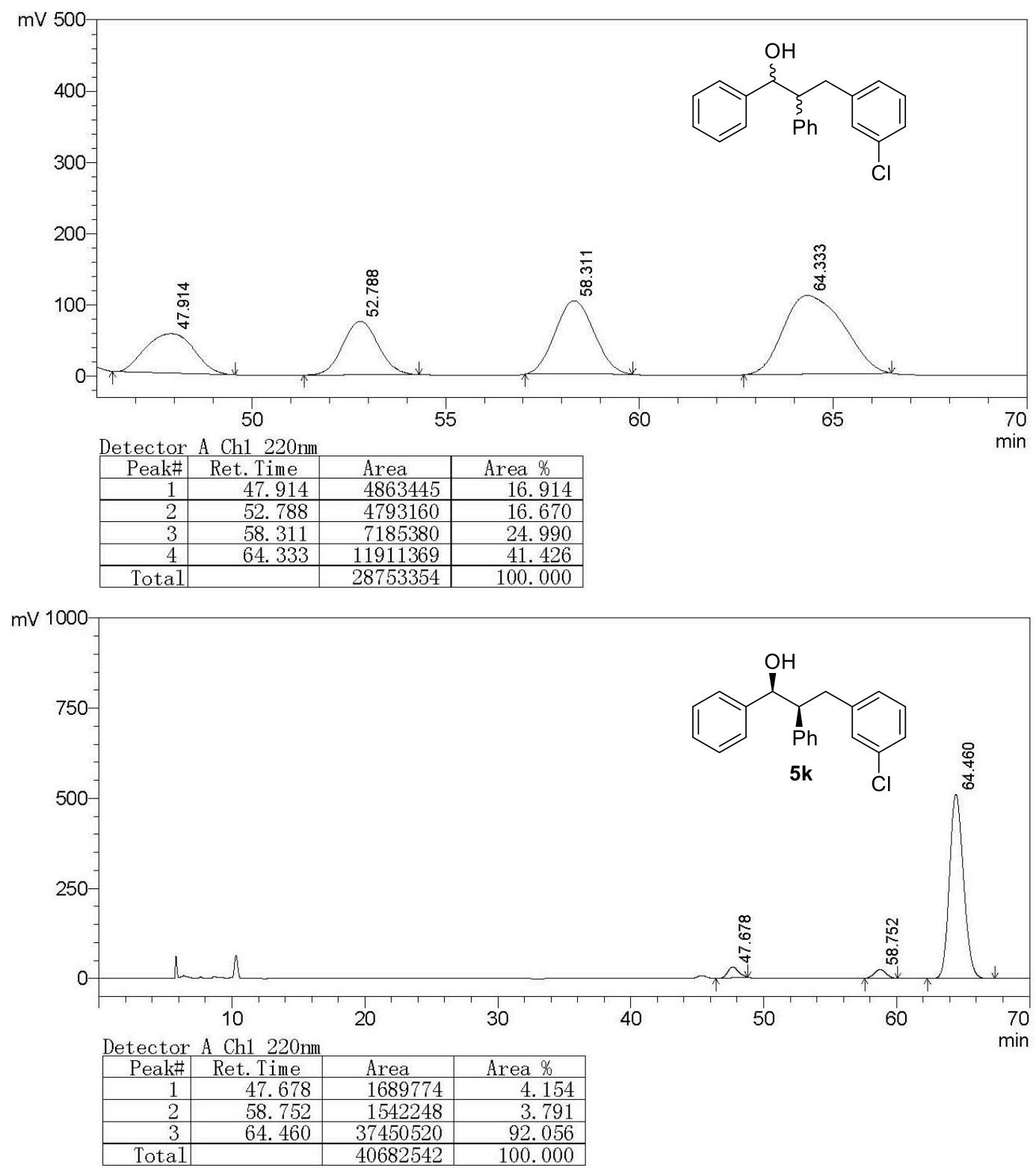

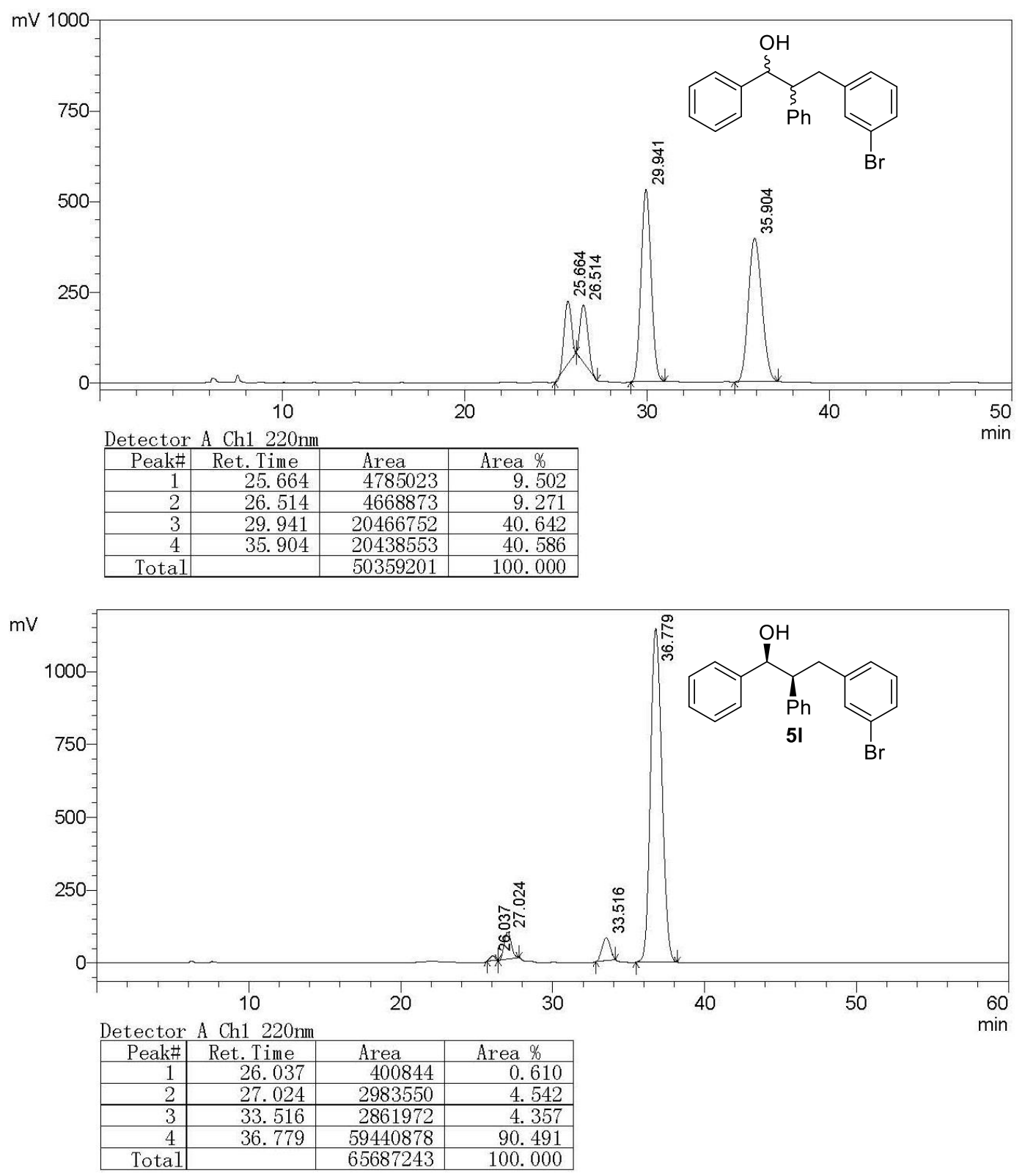

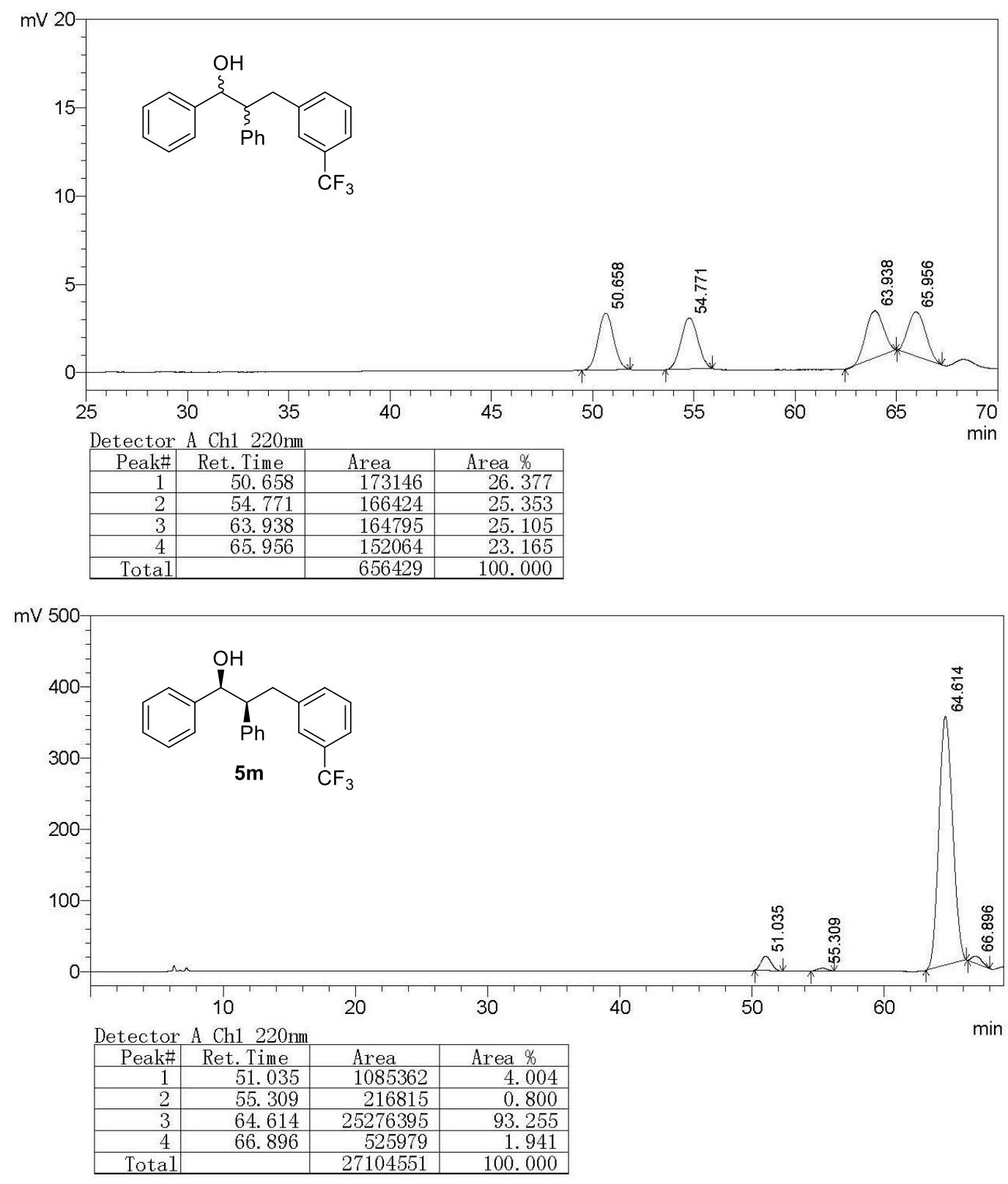

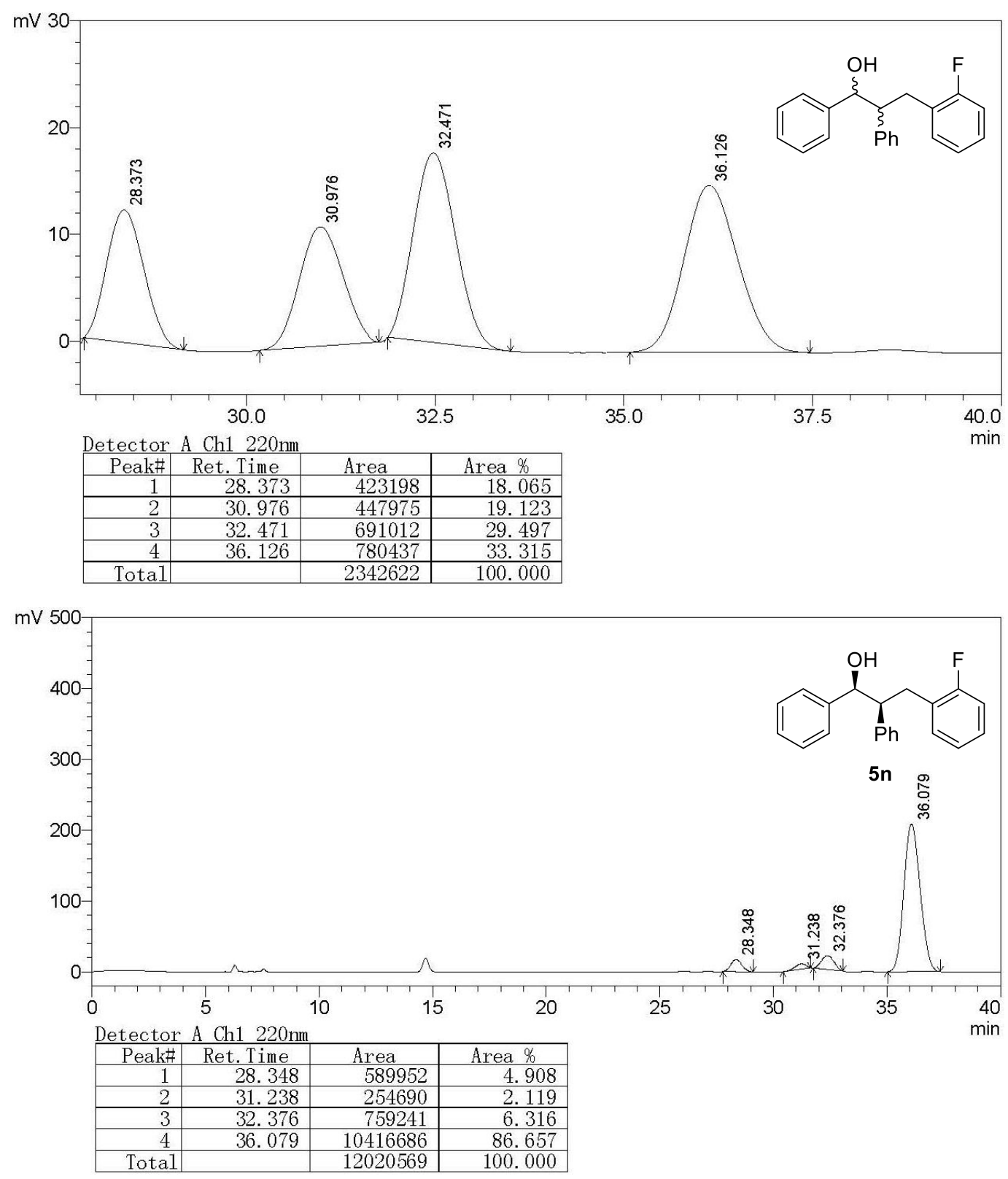


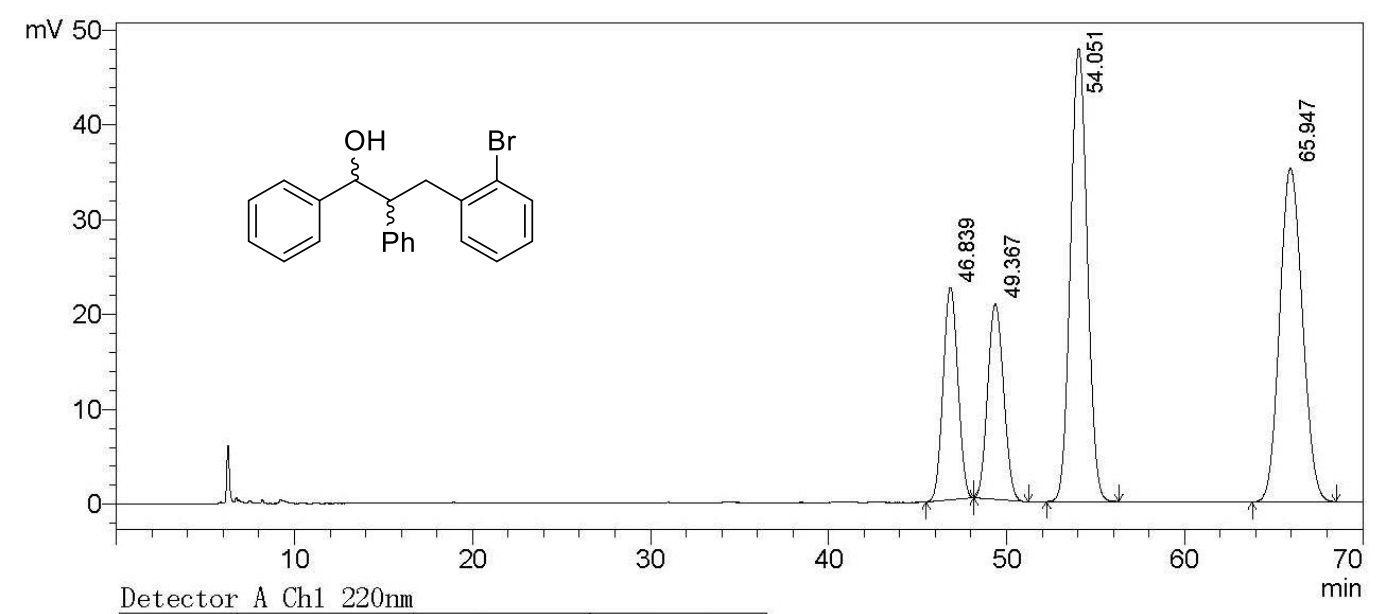

Detector A Ch1 $220 \mathrm{~nm}$
\begin{tabular}{|r|r|r|r|}
\hline Peak\# & Ret. Time & Area & Area \% \\
\hline 1 & 46.839 & 1288504 & 14.594 \\
\hline 2 & 49.367 & 1282059 & 14.521 \\
\hline 3 & 54.051 & 3126233 & 35.408 \\
\hline 4 & 65.947 & 3132279 & 35.477 \\
\hline Total & & 8829075 & 100.000 \\
\hline
\end{tabular}

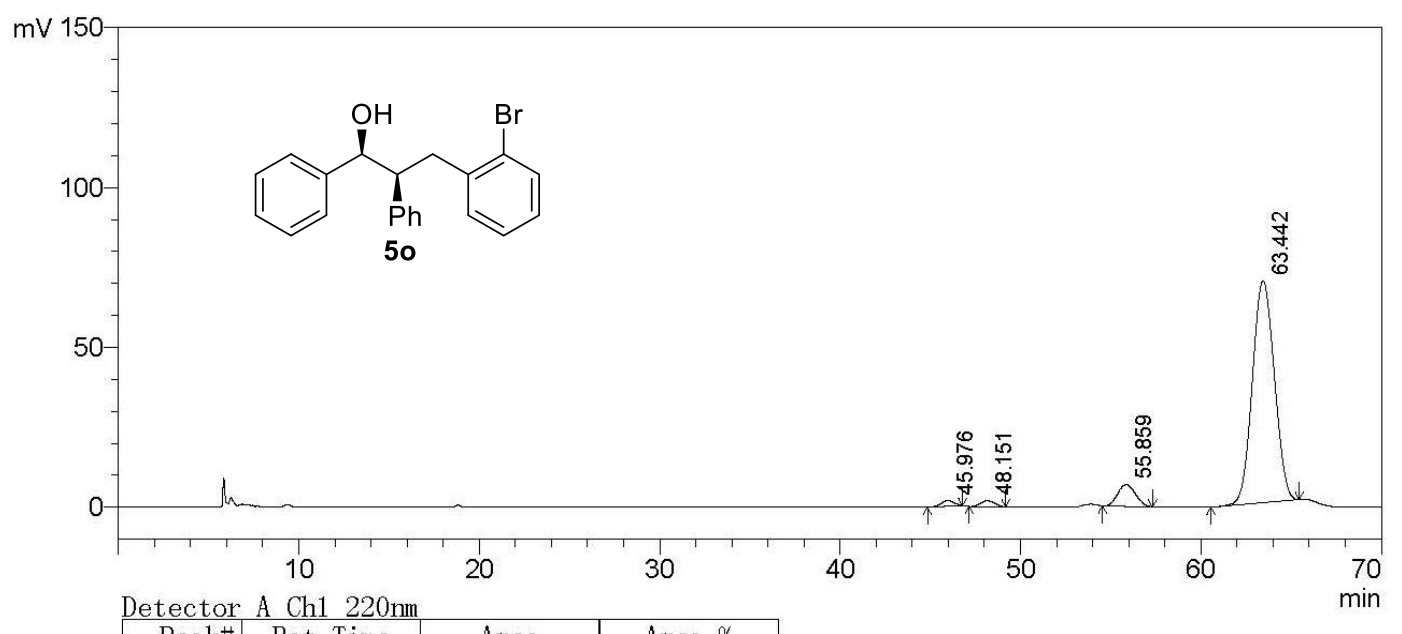

Detector A Ch1 220nm
\begin{tabular}{|r|r|r|r|}
\hline Peak\# & Ret. Time & \multicolumn{1}{c|}{ Area } & Area \% \\
\hline 1 & 45.976 & 88718 & 1.359 \\
\hline 2 & 48.151 & 94880 & 1.453 \\
\hline 3 & 55.859 & 467158 & 7.155 \\
\hline 4 & 63.442 & 5878636 & 90.033 \\
\hline Total & & 6529393 & 100.000 \\
\hline
\end{tabular}



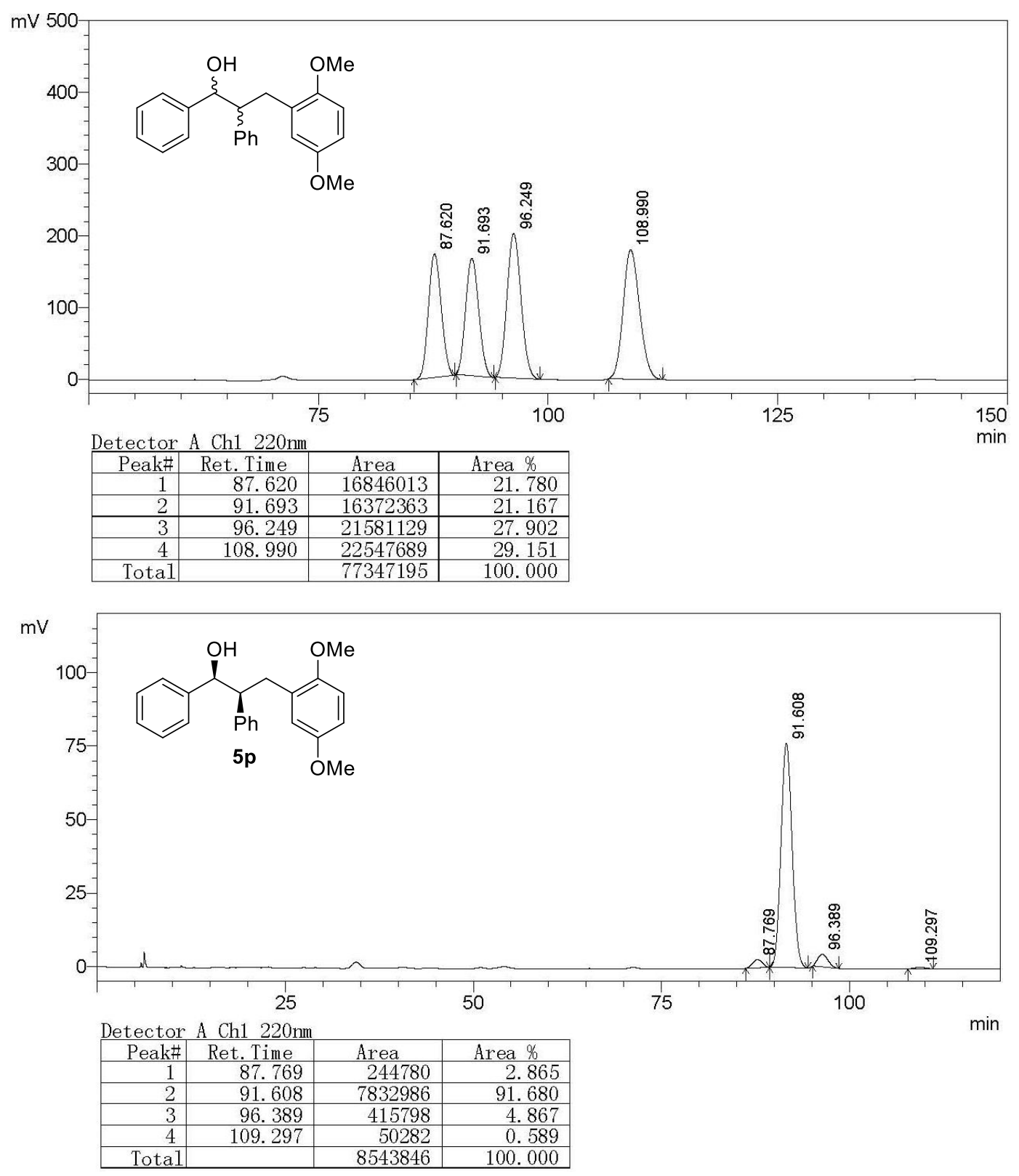

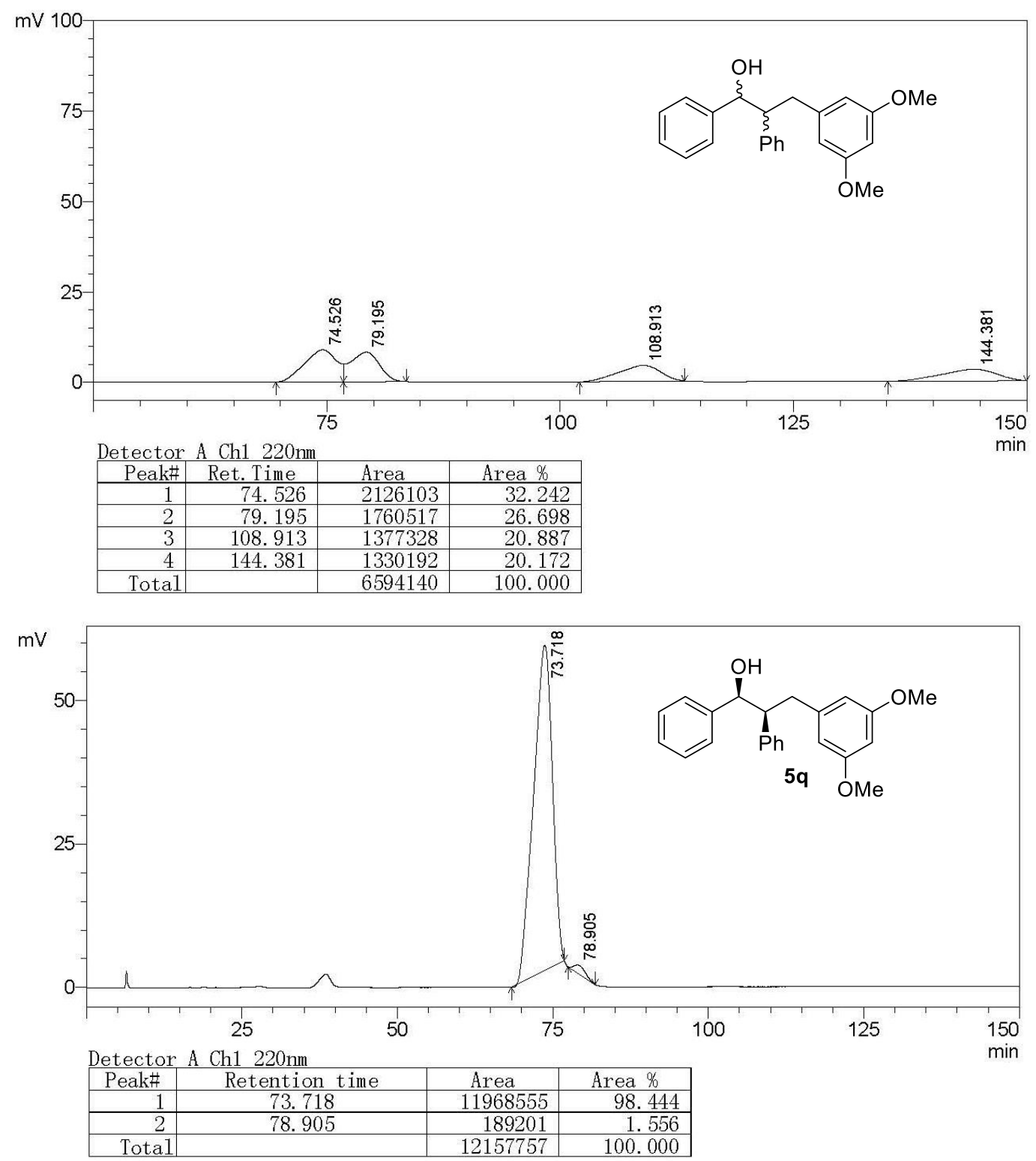

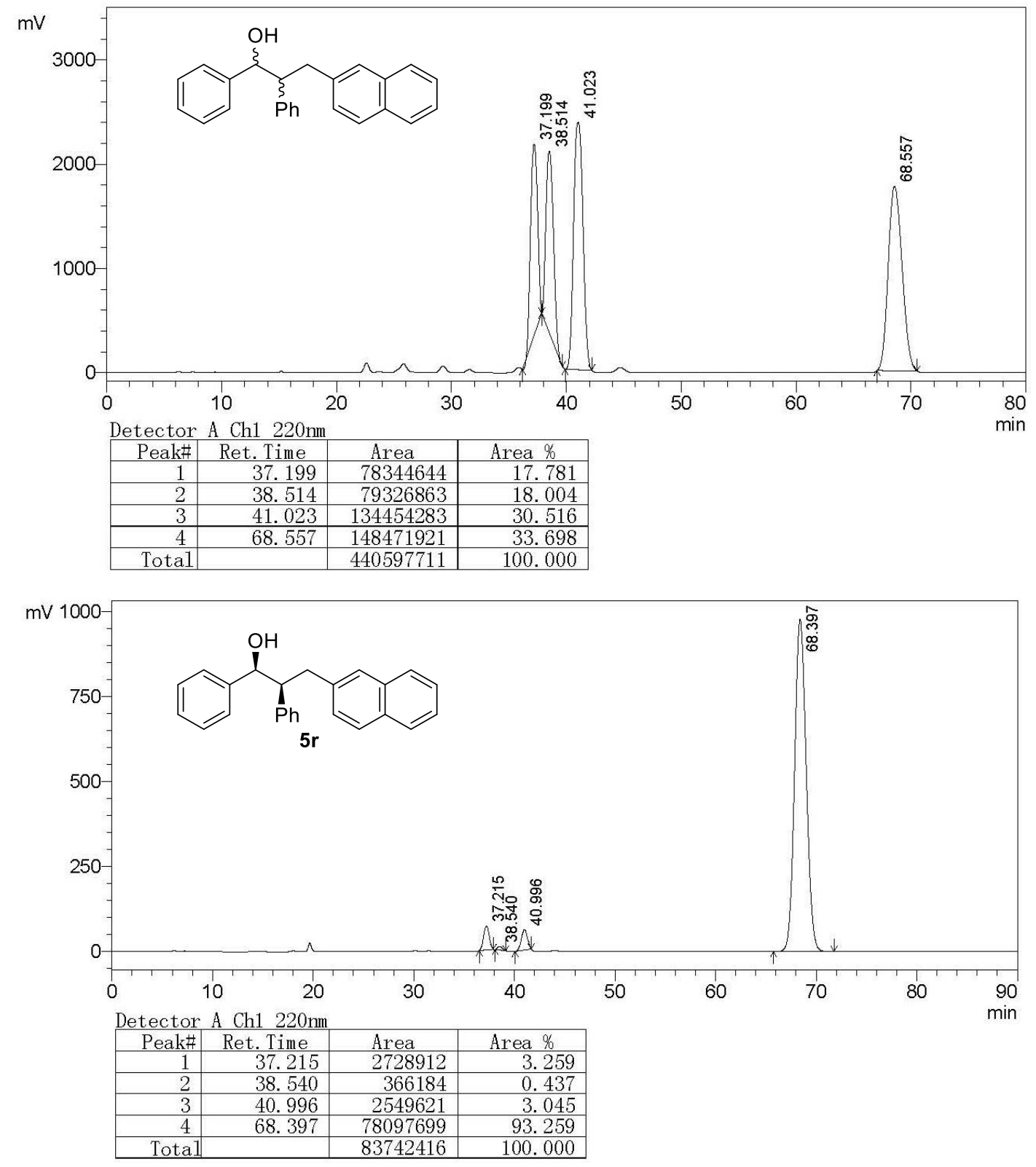

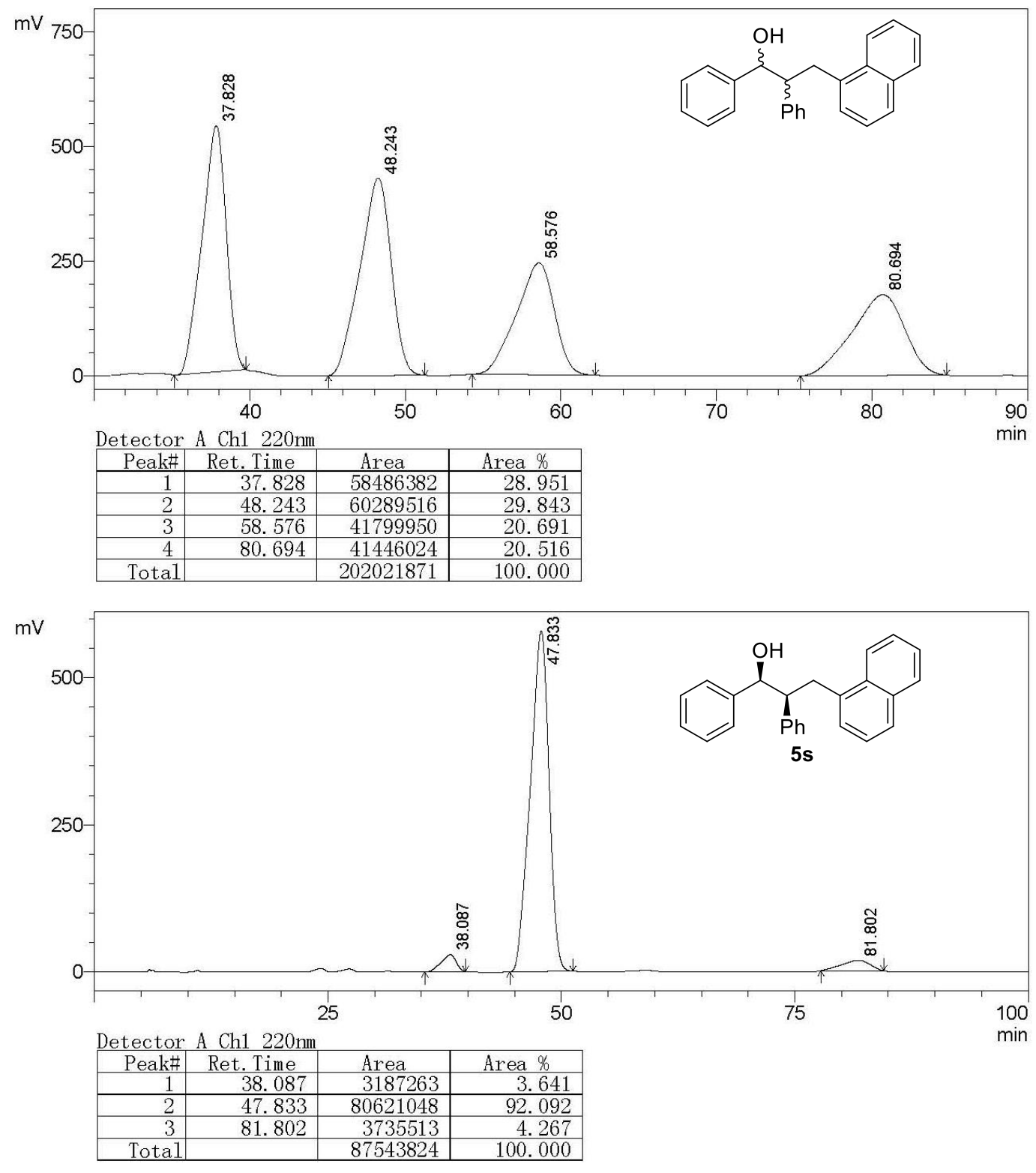

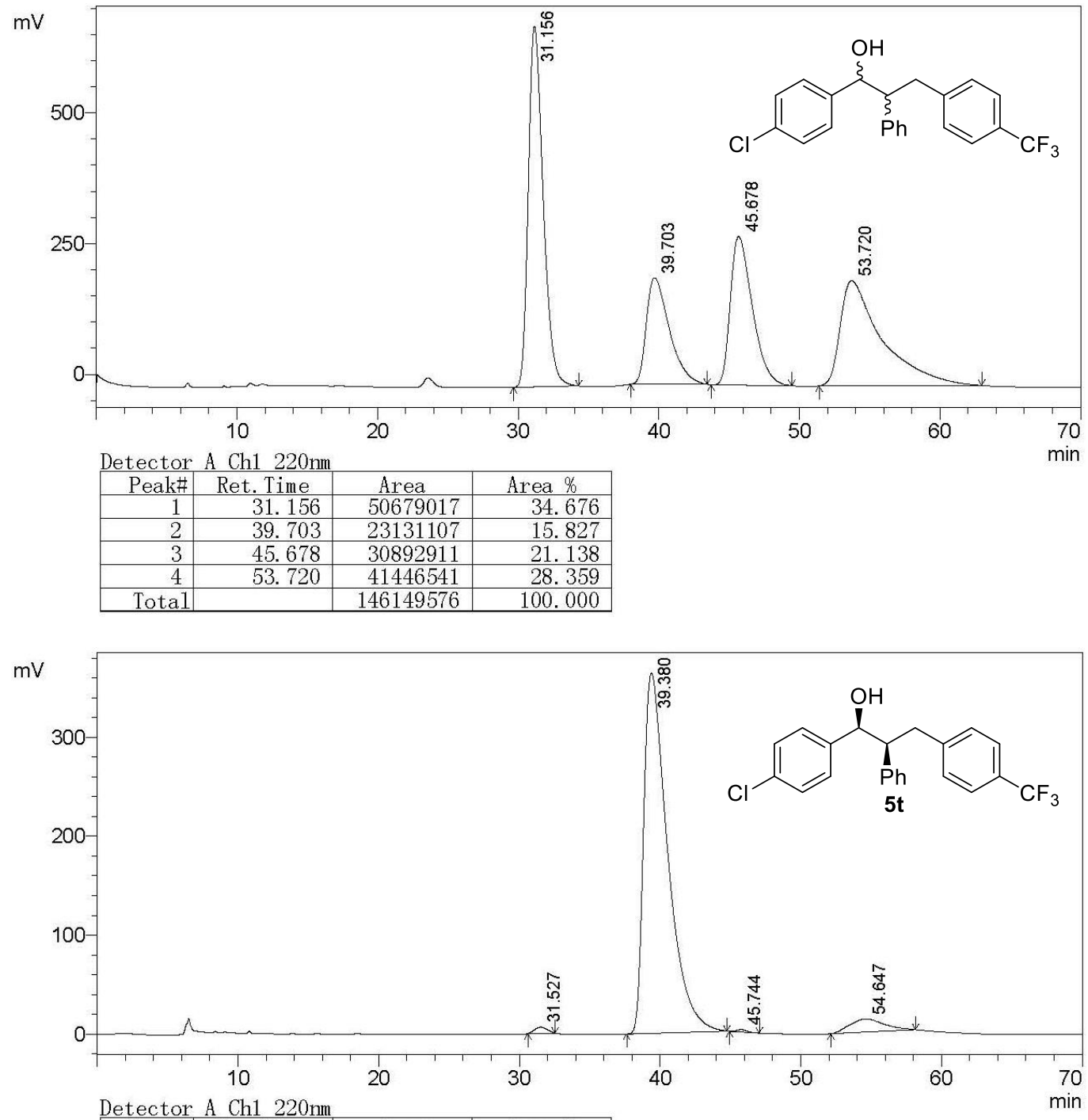

Detector A Ch1 $220 \mathrm{~nm}$
\begin{tabular}{|r|r|r|r|}
\hline Peak\# & Ret. Time & \multicolumn{1}{c|}{ Area } & Area \% \\
\hline 1 & 31.527 & 389430 & 0.845 \\
\hline 2 & 39.380 & 43287476 & 93.979 \\
\hline 3 & 45.744 & 120517 & 0.262 \\
\hline 4 & 54.647 & 2263291 & 4.914 \\
\hline Total & & 46060714 & 100.000 \\
\hline
\end{tabular}



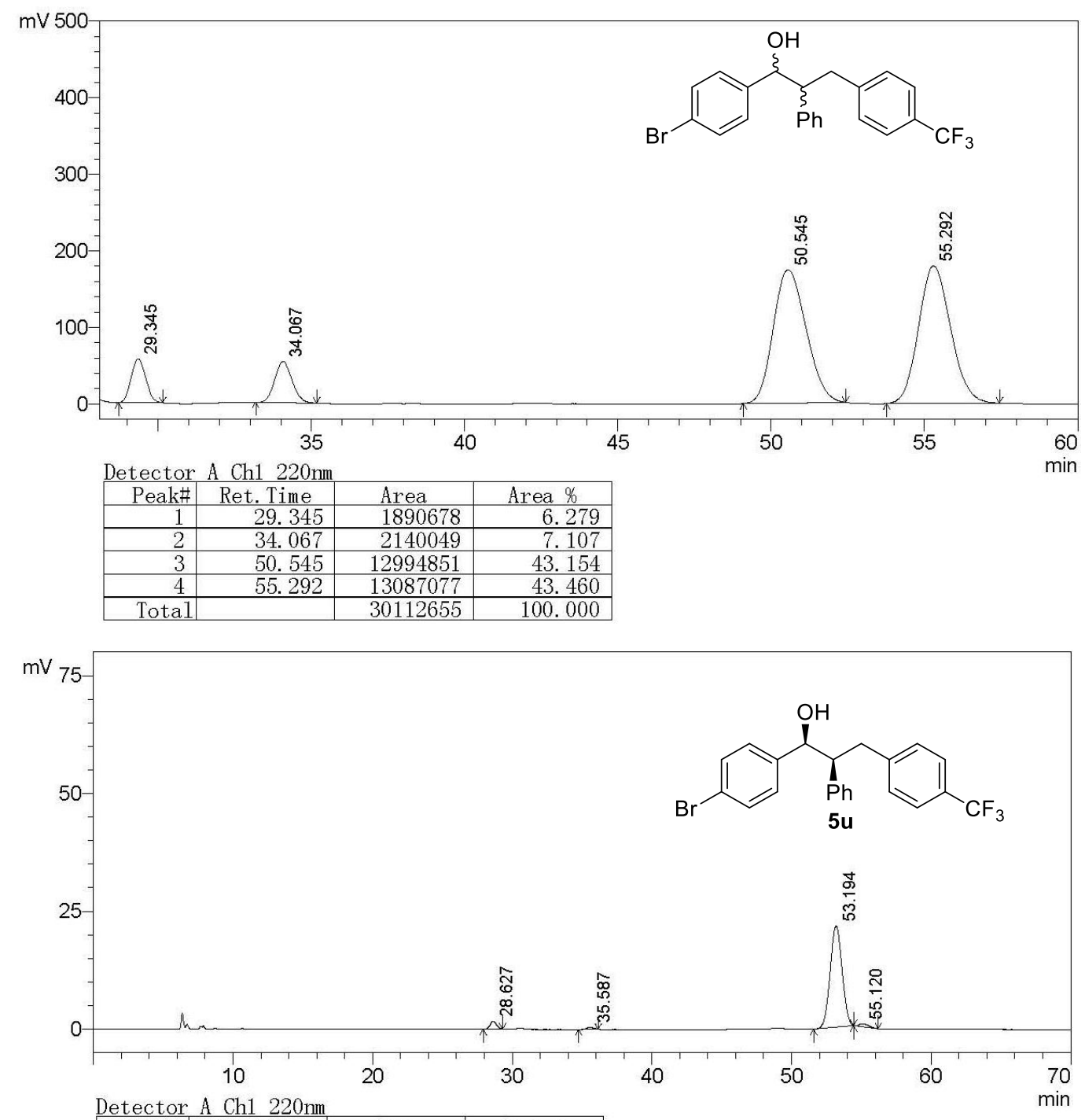

Detector A Ch1 $220 \mathrm{~nm}$
\begin{tabular}{|r|r|r|r|}
\hline Peak\# & Ret. Time & \multicolumn{1}{c|}{ Area } & \multicolumn{1}{c|}{ Area \% } \\
\hline 1 & 28.627 & 53582 & 3.885 \\
\hline 2 & 35.587 & 16359 & 1.186 \\
\hline 3 & 53.194 & 1281681 & 92.941 \\
\hline 4 & 55.120 & 27408 & 1.987 \\
\hline Total & & 1379030 & 100.000 \\
\hline
\end{tabular}



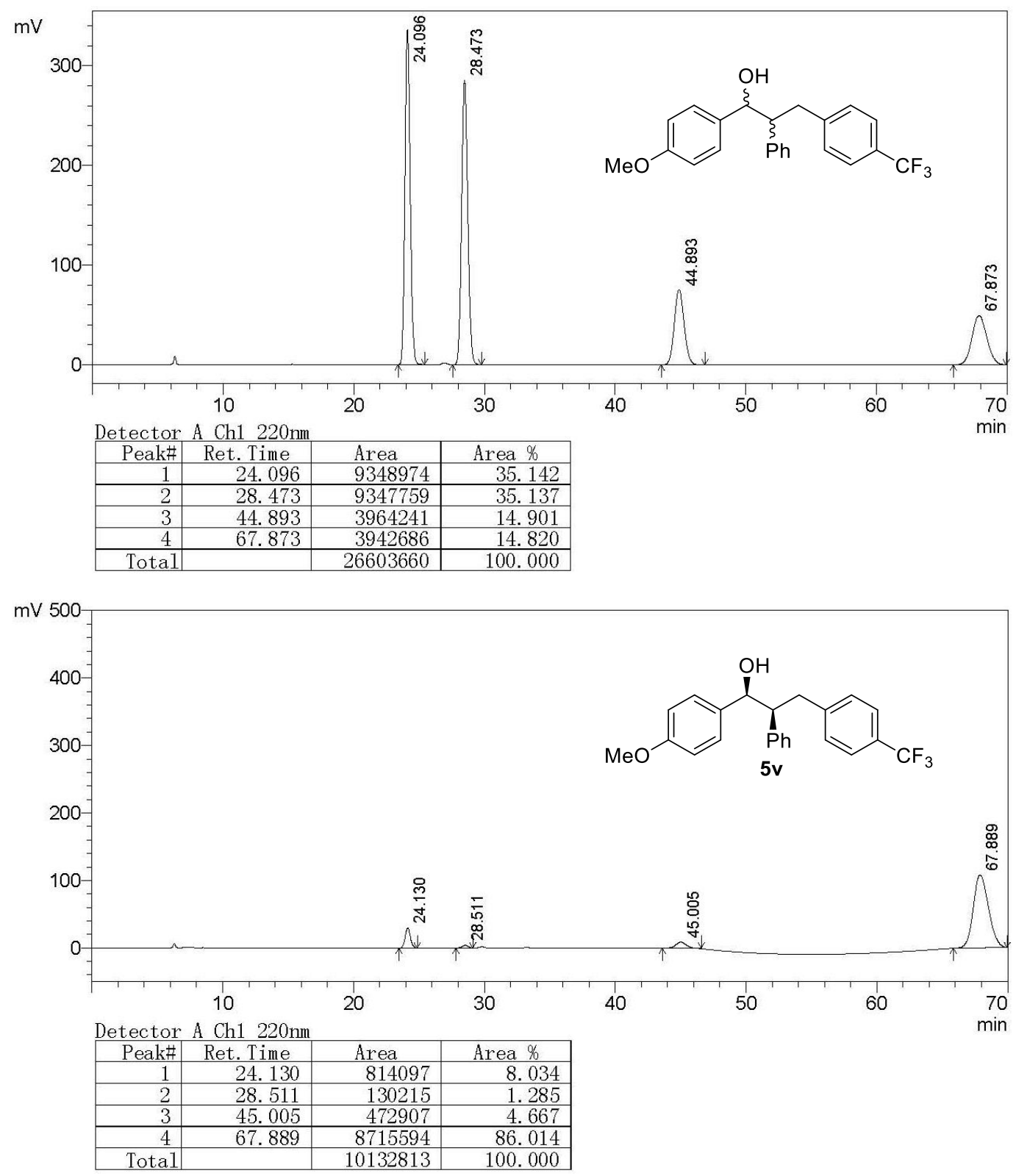

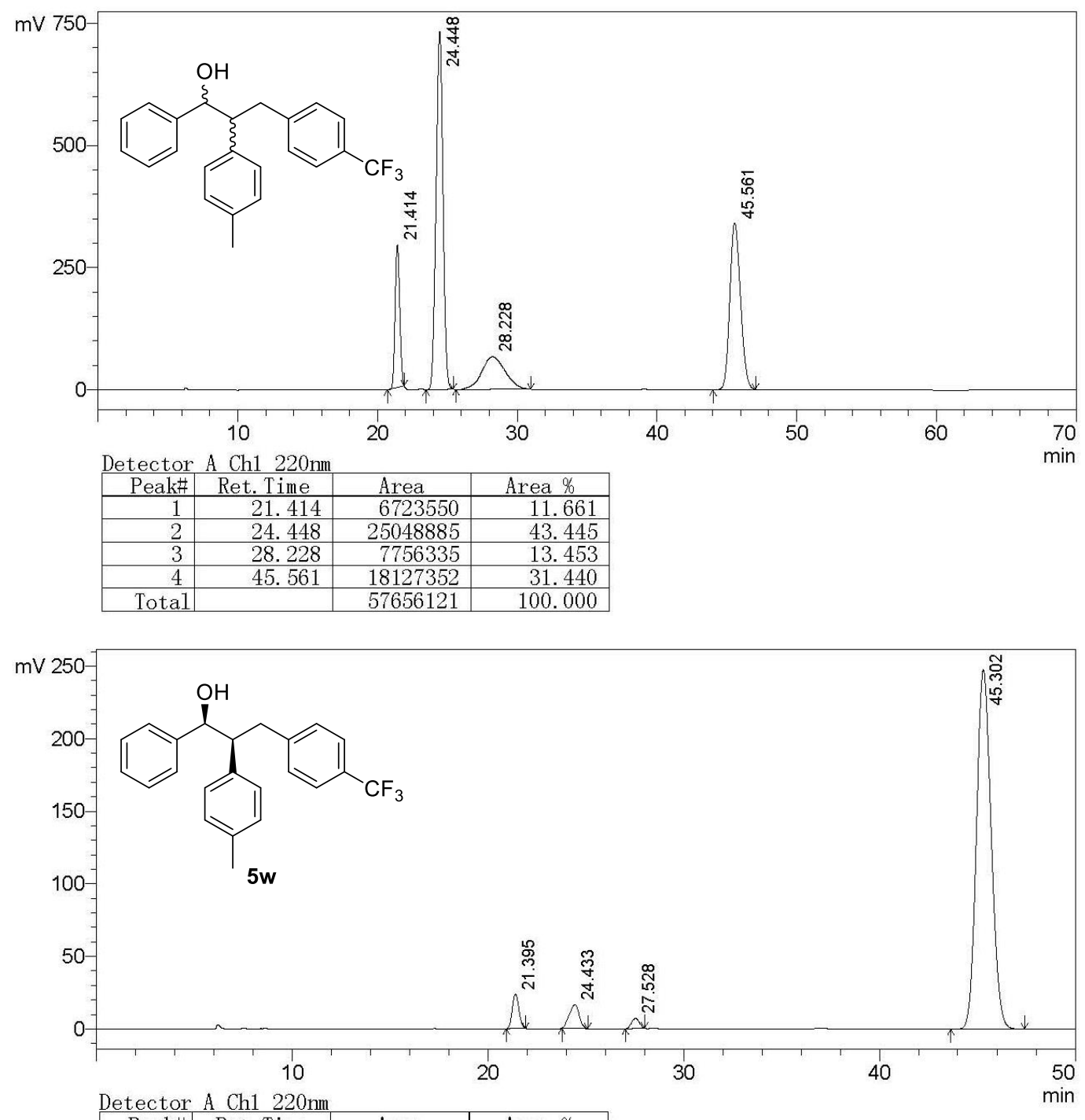

\begin{tabular}{|c|c|c|c|}
\hline Peak\# & Ret. Time & Area & Area \% \\
\hline 1 & 21.395 & 560721 & 3.927 \\
\hline 2 & 24.433 & 583871 & 4.089 \\
\hline 3 & 27.528 & 189384 & 1.326 \\
\hline 4 & 45.302 & 12945737 & 90.658 \\
\hline Total & & 14279713 & 100.000 \\
\hline
\end{tabular}



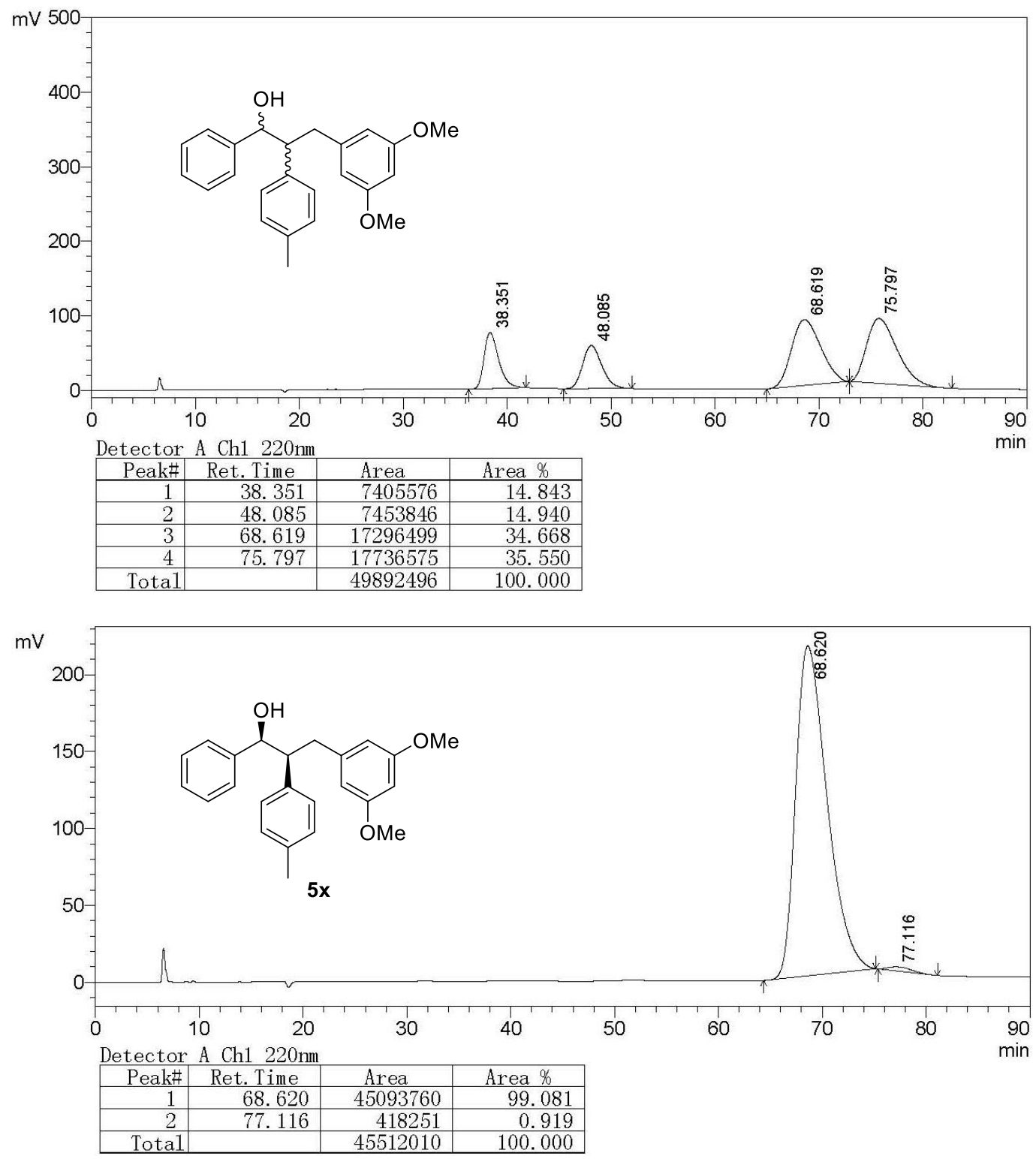

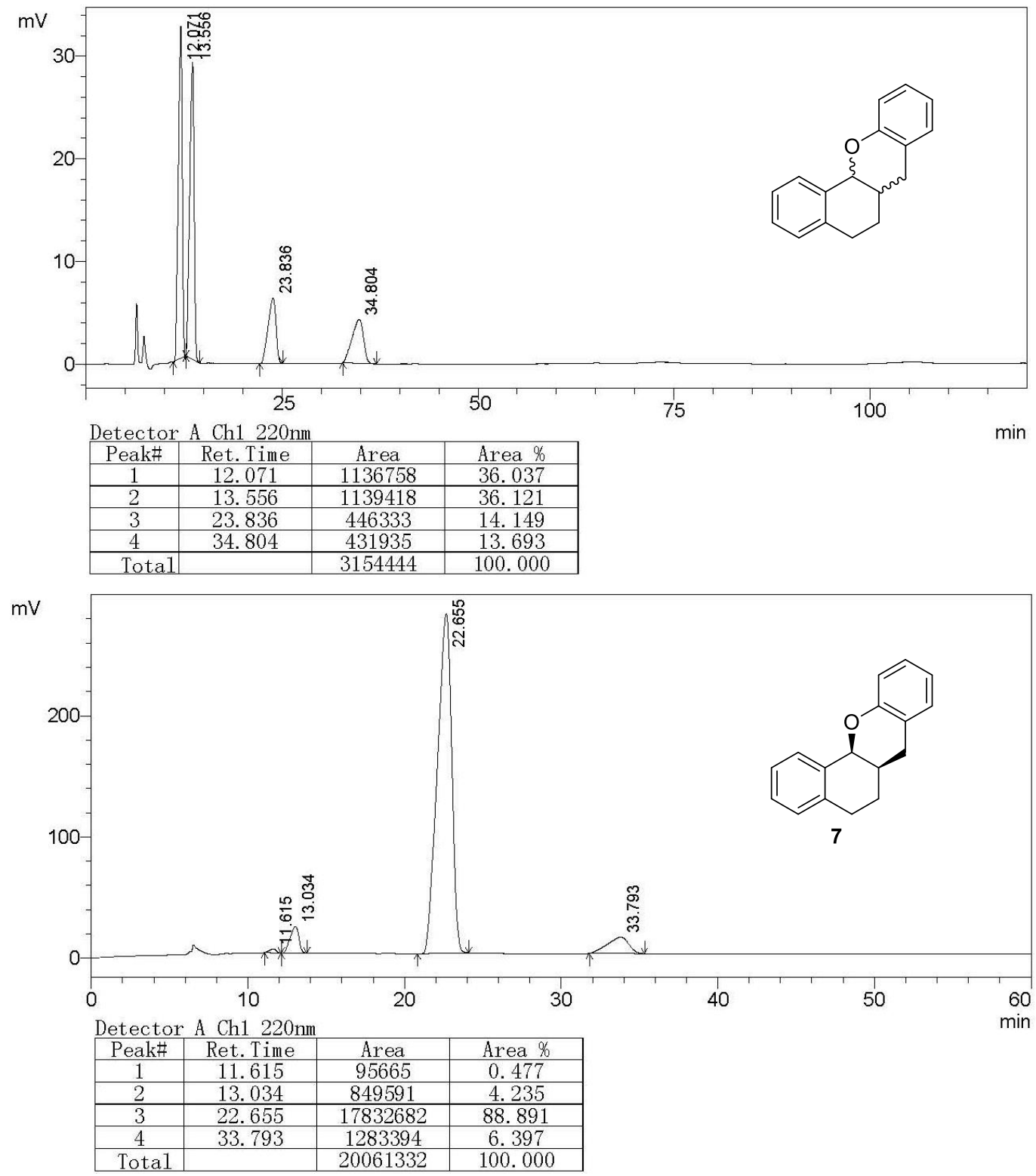

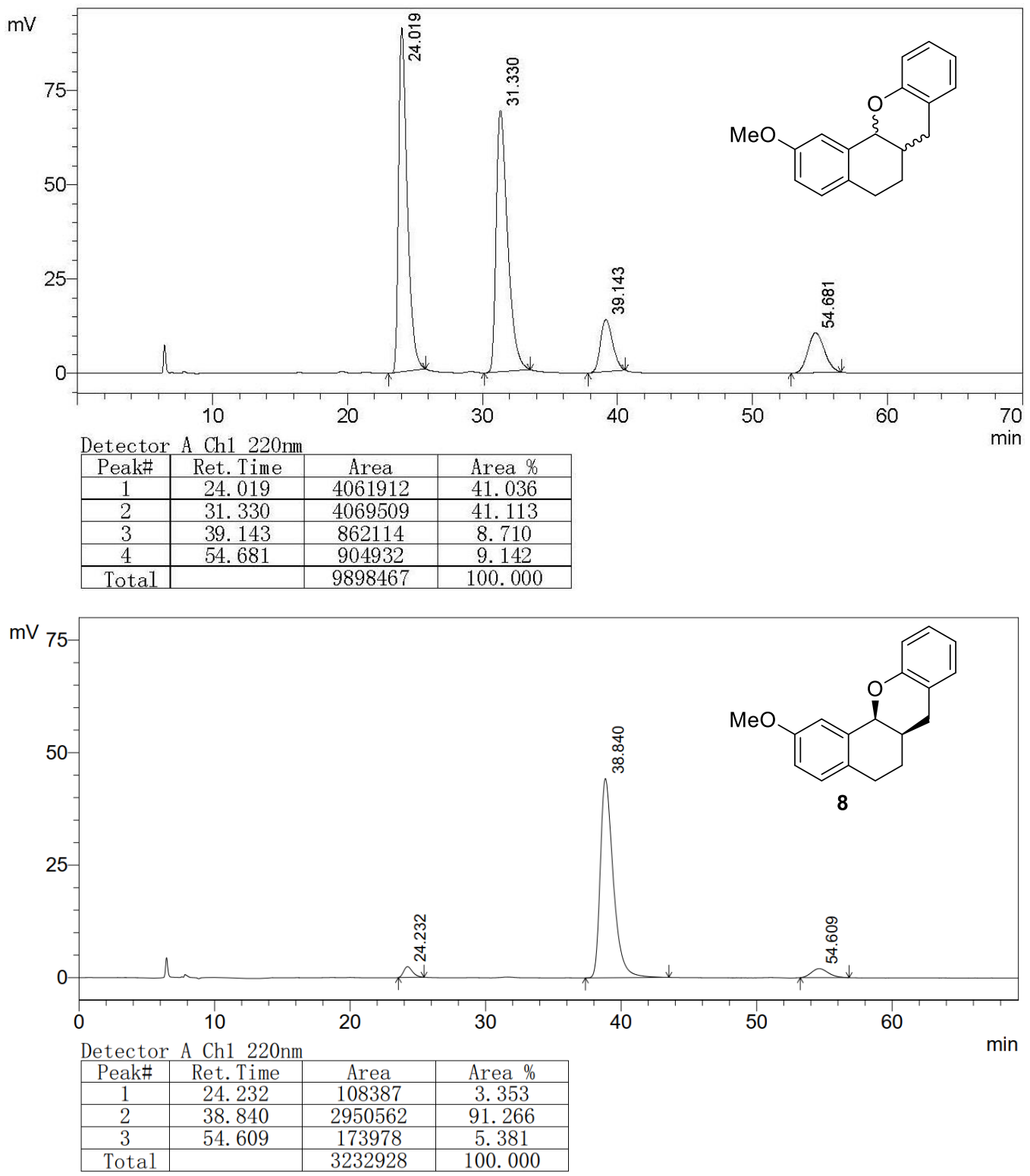

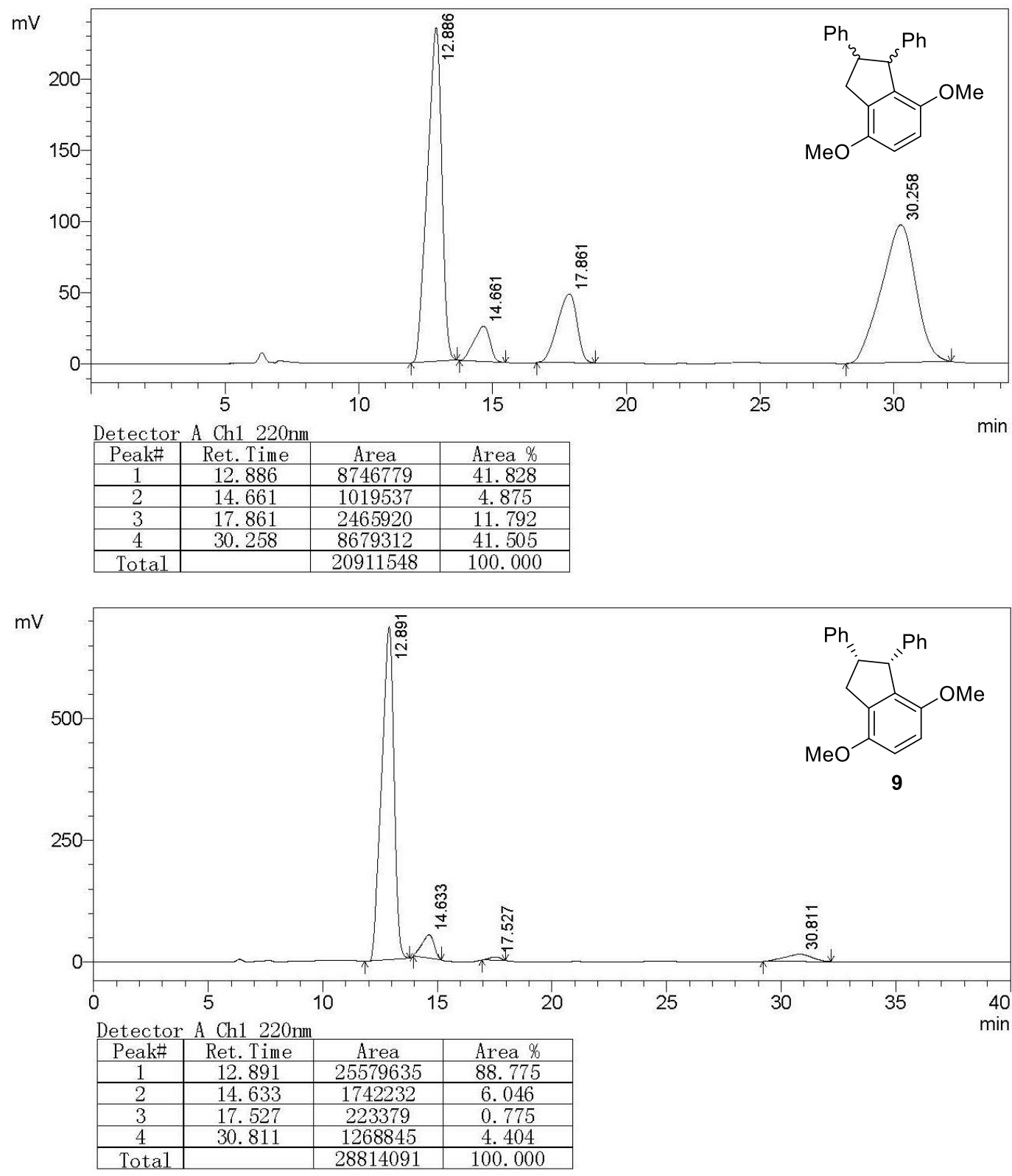

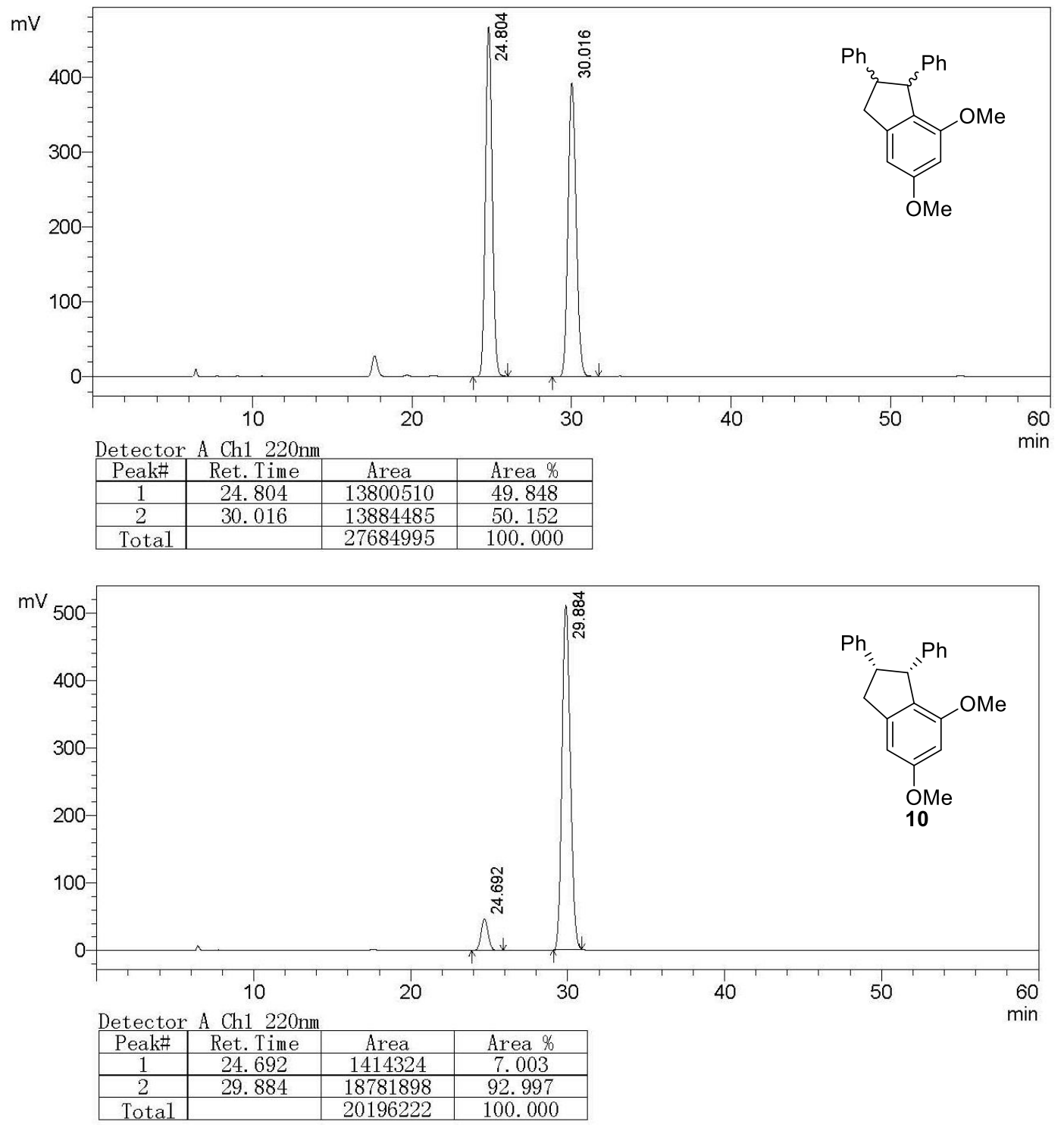

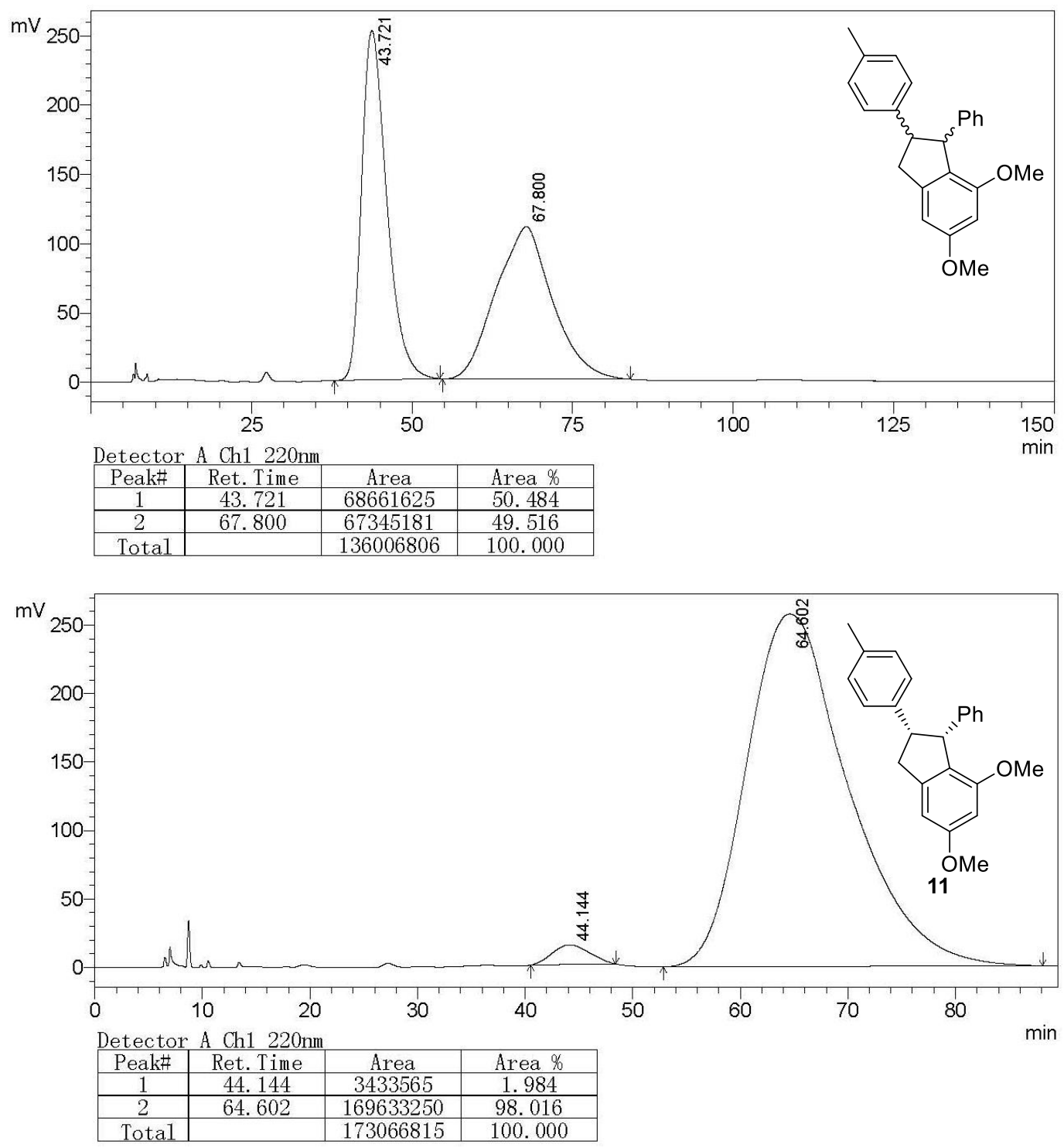